Supporting Information

\title{
Iron-Catalyzed Regiodivergent Alkyne Hydrosilylation
}

Meng-Yang $\mathrm{Hu}^{1}$, Peng $\mathrm{He}^{1}$, Tian-Zhang Qiao ${ }^{1}$, Wei Sun ${ }^{1}$, Wen-Tao $\mathrm{Li}^{1}$, Jie Lian ${ }^{1}$, JinHong $\mathrm{Li}^{1}$, Shou-Fei Zhu, ${ }^{1, *}$

${ }^{1}$ State Key Laboratory and Institute of Elemento-Organic Chemistry, College of Chemistry, Nankai University, Tianjin 300071, China

Email:sfzhu@nankai.edu.cn

\section{Table of Contents}

1. Materials and Methods

2. Synthesis of New Ligands and Catalysts.

3. Optimization of Reaction Conditions.

4. Typical Procedures for Alkyne Hydrosilylation.

5. Analytical Data of Hydrosilylation Products .$S 18$

6. Transformations of Hydrosilylation Products.

.560

7. Mechanistic Studies S70

8. Computational Details .573

9. NMR Spectra of New Compounds. $\mathbf{S 1 0 3}$

10. References 


\section{Materials and Methods}

All manipulations were carried out using standard Schlenk, high-vacuum and glovebox techniques. THF, $\mathrm{Et}_{2} \mathrm{O}, 1,4$-dioxane, and toluene were distilled from sodium benzophenone ketyl prior to use. Iron(II) chloride (99.99\%) and EtMgBr (1.0 M in THF) was purchased from Sigma-Aldrich and used as received. The alkynes and silanes used for hydrosilylation which were commercially available were purchased from SigmaAldrich, Alfa Aesar, Acros, or TCI, and the other alkynes, including but-3-yn-1-yl( $p$ tolyl)sulfane (refer to 3aj), ${ }^{1}$ (pent-4-yn-1-yloxy)benzene (refer to 3ak), ${ }^{2}$ 5-(pent-4-yn1-yloxy)pent-1-ene (refer to 3am), ${ }^{3}$ 2-(but-3-yn-1-yl)-1,3-dioxolane (refer to 3ao), ${ }^{4}$ fluoro-4-(prop-1-yn-1-yl)benzene (refer to 3eb), 5 1-methoxy-4-(prop-1-yn-1yl)benzene (refer to 3ec), ${ }^{6}$ 4-(prop-1-yn-1-yl)-1,1'-biphenyl (refer to 3ed), ${ }^{7} 1$ methoxy-2-(prop-1-yn-1-yl)benzene (refer to 3ee), ${ }^{6}(E)$-pent-1-en-3-yn-1-ylbenzene (refer to 3eg), ${ }^{8}$ 1-(prop-1-yn-1-yl)cyclohex-1-ene (refer to 3ei) ${ }^{9}$ were synthesized according to reported procedures. Alkynes and silanes were dried over $\mathrm{LiAlH}_{4} \mathrm{Or}_{\mathrm{CaH}}$ and distilled prior to use. All the iron-catalyzed hydrosilylation reaction was performed in an argon-filled glovebox and all chemicals and apparatus used in this reaction were thoroughly dried before use. 2,9-Dichloro-1,10-phenanthroline was prepared according to reported procedure. ${ }^{10}$ The known 2,9-disubstituted-1,10-phenanthrolines and these compleses with $\mathrm{FeCl}_{2}$ were prepared according to our previous reports. ${ }^{11}$

Melting points were measured on a RY-I apparatus and uncorrected. Infrared spectra were recorded on a Bruker Fourier transform spectrometric (FT-IR) and reported in wave number. High resolution mass spectrometric (HRMS) analyses spectra were determined on an IonSpec FT-ICR mass spectrometer and Waters GCT Premier mass spectrometer. Trace metal contamination analyses of iron precatalysts by ICPOES (spectro-blue) were performed using a X7 (Thermo Electron Corporation) instruments. Magnetic moment was measured on SQUID VSM (Quantum Design). ${ }^{1} \mathrm{H}$ $\mathrm{NMR},{ }^{13} \mathrm{C} \mathrm{NMR},{ }^{29} \mathrm{Si}$ NMR spectra were recorded with a Bruker AV 400 spectrometer at $400 \mathrm{MHz}\left({ }^{1} \mathrm{H} \mathrm{NMR}\right), 101 \mathrm{MHz}\left({ }^{13} \mathrm{C} \mathrm{NMR}\right), 79 \mathrm{MHz}\left({ }^{29} \mathrm{Si} \mathrm{NMR}\right)$, and $376 \mathrm{MHz}\left({ }^{19} \mathrm{~F}\right.$ NMR). Chemical shifts were reported in ppm down field from internal $\mathrm{Me}_{4} \mathrm{Si}$ ( $\left.{ }^{1} \mathrm{H} \mathrm{NMR}\right)$ 
and $\mathrm{CDCl}_{3}\left({ }^{13} \mathrm{C} \mathrm{NMR}\right)$. Gas Chromatography (GC) analyses were performed using a Hewlett Packard Model HP 7890 Series instruments equipped with an FID detector and a capillary column, HP-5 (Agilent Technologies, $30 \mathrm{~m} \times 0.032 \mathrm{~mm} \times 0.25 \mu \mathrm{m}$ film thickness). Electron paramagnetic resonance (EPR) spectra were recorded on a Bruker ELEXSYS E580 spectrometer (X-band). The molar masses and their distribution for the polymer samples were determined by GPC on a Waters system equipped with a set of three Ultrastyragel columns HT2 $30 \mathrm{~cm}$ x $7.8 \mathrm{~mm}$; $10 \mu \mathrm{m}$ particles; exclusion limits: $100-10000 \mathrm{~g} / \mathrm{mol}$, respectively), THF was used as the mobile phase $(1 \mathrm{~mL} / \mathrm{min})$, and polystyrene samples as the standards in the calibration of the molar masses. 


\section{Synthesis of New Ligands and Catalysts}

\section{Synthesis of 2,9-bis-3,5-bis(2,4,6-trimethylphenyl)phenyl-1,10-phenanthroline}

(L1i)<smiles>Clc1ccc2ccc(Cl)nc2c1</smiles>

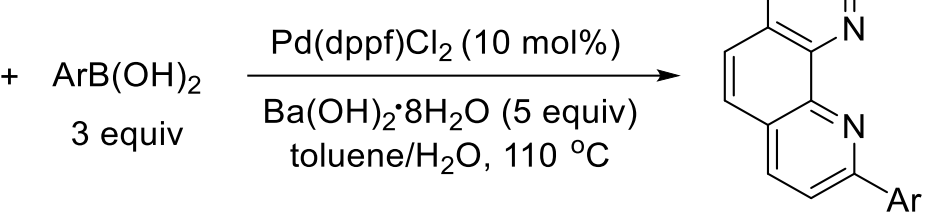<smiles>Cc1cc(C)c(-c2cc(C)cc(C)c2-c2cc(C)cc(C)c2C)c(C)c1</smiles>

A suspension of 2,9-dichloro-1,10-phenanthroline (498 mg, $2 \mathrm{mmol}), \mathrm{Pd}(\mathrm{dppf}) \mathrm{Cl}_{2}$ [147 mg, $0.2 \mathrm{mmol}, 10 \mathrm{~mol} \%$, dppf = 1,1'-bis(diphenylphosphino)ferrocene], $\mathrm{Ba}(\mathrm{OH})_{2} \bullet 8 \mathrm{H}_{2} \mathrm{O}(3.2 \mathrm{~g}, 10 \mathrm{mmol}, 5$ equiv) and aryl boric acid $(2.1 \mathrm{~g}, 6 \mathrm{mmol}, 3$ equiv) in a mixture of toluene $(50 \mathrm{~mL})$ and water $(4 \mathrm{~mL})$ was purged with argon to degas. The mixture was heated up to $110^{\circ} \mathrm{C}$ until the reaction was finished. After cooling to room temperature, the mixture was neutralized with saturated $\mathrm{NH}_{4} \mathrm{Cl}$ (aq., $50 \mathrm{~mL}$ ) and extracted with $\mathrm{CH}_{2} \mathrm{Cl}_{2}(100 \mathrm{~mL} \times 3)$. The combined organic layer was dried over anhydrous $\mathrm{MgSO}_{4}$ and concentrated under reduced pressure. The residue was purified by silica-gel column chromatography with petroleum ether/ethylacetate $(\mathrm{PE} / \mathrm{EA})=20: 1$ $(v / v)$ as eluent and precipitated in $n$-hexane $/ \mathrm{CH}_{2} \mathrm{Cl}_{2}(1: 1, v / v)$ through slow evaporation to give $\mathbf{L} \mathbf{1 i}$.

$1.02 \mathrm{~g}, 63 \%$ yield, white solid, melting point: $190-192{ }^{\circ} \mathrm{C}$.

${ }^{1} \mathrm{H}$ NMR $\left(400 \mathrm{MHz}, \mathrm{CDCl}_{3}\right) \delta 8.30(\mathrm{~d}, J=8.4 \mathrm{~Hz}, 2 \mathrm{H}), 8.06(\mathrm{~d}, J=8.4 \mathrm{~Hz}, 2 \mathrm{H}), 7.96$ $(\mathrm{d}, J=1.5 \mathrm{~Hz}, 4 \mathrm{H}), 7.80(\mathrm{~s}, 2 \mathrm{H}), 7.02(\mathrm{t}, J=1.5 \mathrm{~Hz}, 2 \mathrm{H}), 6.95(\mathrm{~s}, 8 \mathrm{H}), 2.37$ $(\mathrm{s}, 12 \mathrm{H}), 2.05(\mathrm{~s}, 24 \mathrm{H})$.

${ }^{13} \mathrm{C} \mathrm{NMR}\left(101 \mathrm{MHz}, \mathrm{CDCl}_{3}\right) \delta 158.2,146.3,141.6,140.7,138.8,136.9,136.3,136.0$,

$131.4,128.1,127.9,127.3,126.2,121.4,21.1,20.8$.

HRMS (ESI) calcd for $\left[\mathrm{M}+\mathrm{H}, \mathrm{C}_{60} \mathrm{H}_{57} \mathrm{~N}_{2}\right]^{+}:$805.4516, found 805.4518. 


\section{Synthesis of 2,9-bis-3,5-bis(2,4,6-triisopropylph enyl)phenyl-1,10-phenanthroline}

(L1j)

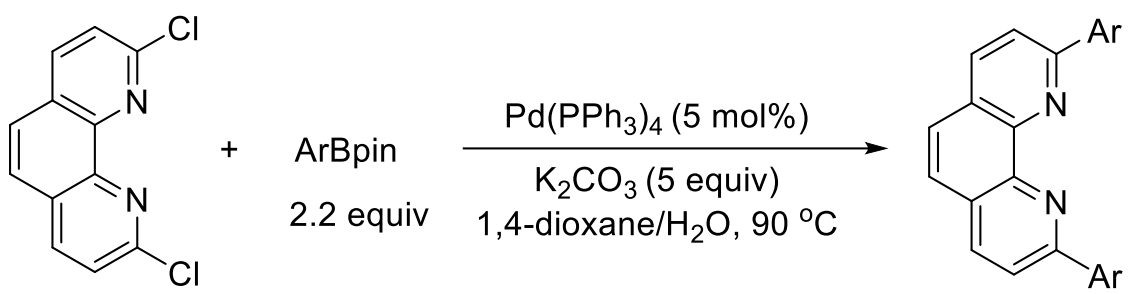<smiles>CCCc1cc(C(C)C)c(-c2cc(-c3c(CC)cc(C(C)C)cc3C(C)C)cc(C(C)C)c2)c(C(C)C)c1</smiles>

A suspension of 2,9-dichloro-1,10-phenanthroline (249 mg, $1 \mathrm{mmol}), \mathrm{Pd}\left(\mathrm{PPh}_{3}\right)_{4}$ (57.8 mg, $0.05 \mathrm{mmol}), \mathrm{K}_{2} \mathrm{CO}_{3}$ (0.69 g, $5 \mathrm{mmol}, 5$ equiv) and ArBpin (1.34 g, $2.2 \mathrm{mmol}$, 2.2 equiv) in a mixture of 1,4-dioxane $(20 \mathrm{~mL})$ and water $(5 \mathrm{~mL})$ was purged with argon to degas. The mixture was heated up to $90{ }^{\circ} \mathrm{C}$ until the reaction was finished. After cooling to room temperature, the mixture was neutralized with saturated $\mathrm{NH}_{4} \mathrm{Cl}$ (aq., $50 \mathrm{~mL})$ and extracted with $\mathrm{CH}_{2} \mathrm{Cl}_{2}(100 \mathrm{~mL} \times 3)$. The combined organic layer was dried over anhydrous $\mathrm{MgSO}_{4}$ and concentrated under reduced pressure. The residue was purified by silica-gel column chromatography with PE/EA $=20: 1(v / v)$ as eluent and precipitated in $n$-hexane $/ \mathrm{CH}_{2} \mathrm{Cl}_{2}(20: 1, v / v)$ through slow evaporation to give $\mathbf{L 1 j}$. $936 \mathrm{mg}, 82 \%$ yield, white solid, melting point: $310-312{ }^{\circ} \mathrm{C}$.

${ }^{1} \mathrm{H}$ NMR $\left(400 \mathrm{MHz}, \mathrm{CDCl}_{3}\right) \delta 8.29(\mathrm{~d}, J=8.4 \mathrm{~Hz}, 2 \mathrm{H}), 8.07(\mathrm{dd}, J=8.4,2.0 \mathrm{~Hz}, 2 \mathrm{H}$,), $8.00(\mathrm{~d}, J=1.7 \mathrm{~Hz}, 4 \mathrm{H}), 7.79(\mathrm{~s}, 2 \mathrm{H}), 7.04-7.00(\mathrm{~m}, 10 \mathrm{H}), 2.98(\mathrm{p}, J=6.9$ $\mathrm{Hz}, 4 \mathrm{H}), 2.82-2.73(\mathrm{~m}, 8 \mathrm{H}), 1.35(\mathrm{dd}, J=6.9,2.4 \mathrm{~Hz}, 24 \mathrm{H}), 1.04-0.95(\mathrm{~m}$, $48 \mathrm{H})$.

${ }^{13} \mathrm{C} \mathrm{NMR}\left(101 \mathrm{MHz}, \mathrm{CDCl}_{3}\right) \delta 158.7,147.6,146.5,146.4,141.1,139.9,137.0,136.7$, $132.9,128.1,127.5,126.3,122.2,120.4,34.3,30.4,24.2,24.1,24.0$.

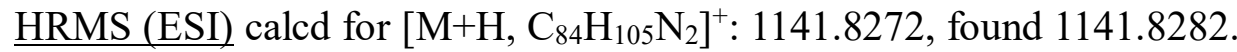




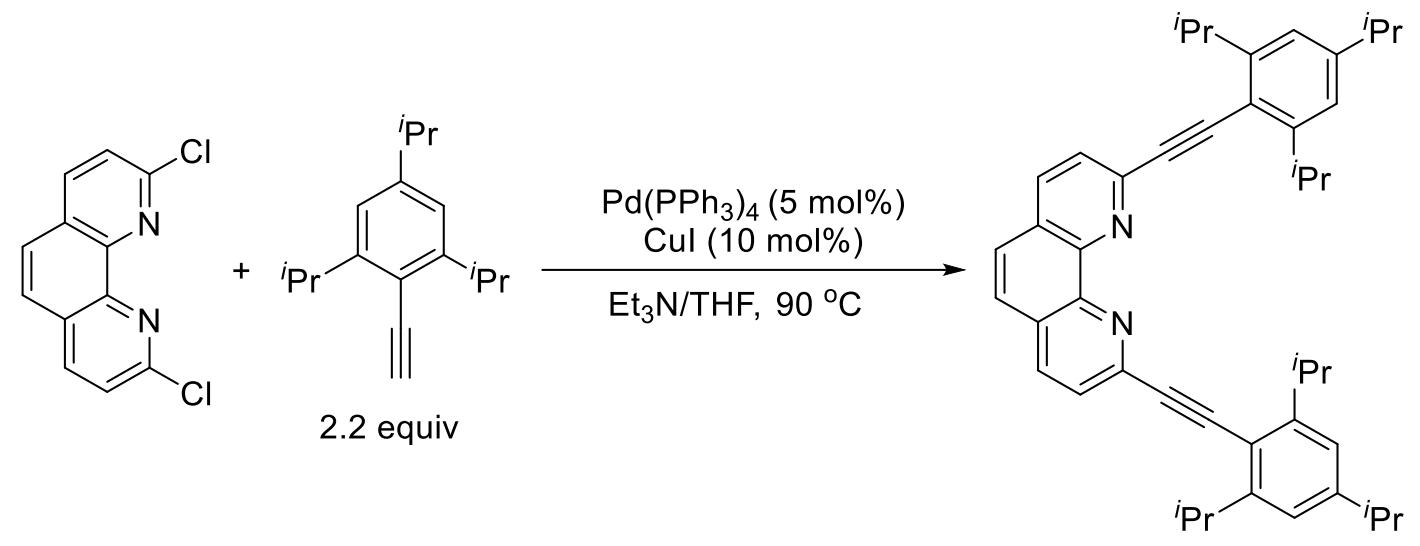

A suspension of 2,9-dichloro-1,10-phenanthroline (1.0 g, $4 \mathrm{mmol}), \mathrm{Pd}\left(\mathrm{PPh}_{3}\right)_{4}(231$ mg, $0.2 \mathrm{mmol}$ ), CuI (76 mg, $0.4 \mathrm{mmol})$ and aryl acetylene $(2.0 \mathrm{~g}, 8.8 \mathrm{mmol})$ were added into a $125 \mathrm{~mL}$ sealing pipe, then charged with argon. After that, $\mathrm{Et}_{3} \mathrm{~N}(8 \mathrm{~mL})$ and anhydrous THF $(24 \mathrm{~mL})$ were injected through a syringe. The mixture was heated up to $90{ }^{\circ} \mathrm{C}$ until the reaction was finished. After cooling to room temperature, the mixture was neutralized with saturated $\mathrm{NH}_{4} \mathrm{Cl}$ (aq., $\left.50 \mathrm{~mL}\right)$ and extracted with $\mathrm{CH}_{2} \mathrm{Cl}_{2}(100 \mathrm{~mL}$ $\times 3$ ). The combined organic layer was dried over anhydrous $\mathrm{MgSO}_{4}$ and concentrated under reduced pressure. The residue was purified by silica-gel column chromatography with $\mathrm{PE} / \mathrm{EA}=20: 1(v / v)$ as eluent and precipitated in $n$-hexane $/ \mathrm{CH}_{2} \mathrm{Cl}_{2}(20: 1, v / v)$ through slow evaporation to give L1k.

$1.82 \mathrm{~g}, 72 \%$ yield, white solid, melting point: $203-207^{\circ} \mathrm{C}$.

래 NMR (400 MHz, $\left.\mathrm{CDCl}_{3}\right) \delta 8.21(\mathrm{~d}, J=8.2 \mathrm{~Hz}, 2 \mathrm{H}), 7.82(\mathrm{~d}, J=8.2 \mathrm{~Hz}, 2 \mathrm{H}), 7.78$ (s, 2H), 7.05 (s, 4H), 3.81 (hept, $J=6.9 \mathrm{~Hz}, 4 \mathrm{H}$ ), 2.93 (hept, $J=6.9 \mathrm{~Hz}, 2 \mathrm{H}$ ), $1.36(\mathrm{~d}, J=6.9 \mathrm{~Hz}, 24 \mathrm{H}), 1.29(\mathrm{~d}, J=6.9 \mathrm{~Hz}, 12 \mathrm{H})$.

${ }^{13} \mathrm{C} \mathrm{NMR}\left(101 \mathrm{MHz}, \mathrm{CDCl}_{3}\right) \delta 151.8,150.1,145.7,144.7,135.9,127.6,127.0,126.5$, $120.5,117.5,97.2,89.2,34.6,31.9,23.9,23.6$.

HRMS (ESI) calcd for [M+H, $\left.\mathrm{C}_{46} \mathrm{H}_{53} \mathrm{~N}_{2}\right]^{+}:$633.4203, found 633.4207. 


\section{Synthesis of complexes of 1,10-phenanthroline ligands with $\mathrm{FeCl}_{2}(\mathrm{C} 1)$}<smiles>[R]c1ccc2ccc3ccc([R])nc3c2n1</smiles>

L1

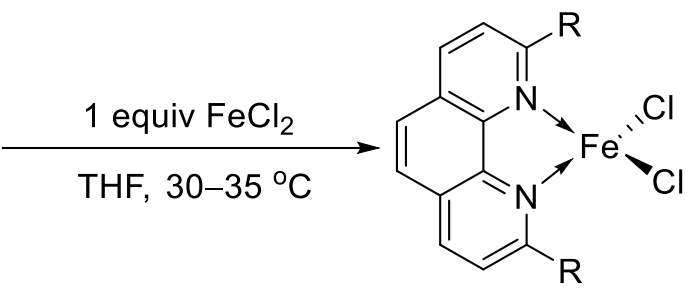

C1

In an argon-filled glovebox, a Schlenk flask $(50 \mathrm{~mL})$ was charged with 2,9disubstituted-1,10-phenanthroline ligands $\mathbf{L 1}$ (2 mmol), $\mathrm{FeCl}_{2}$ (253.5 mg, $2 \mathrm{mmol}$ ) and dry THF $(20 \mathrm{~mL})$. The reaction mixture was stirred at room temperature for $24 \mathrm{~h}$. The solvent was partially removed under vacuum (about $5 \mathrm{~mL}$ left), then dry $n$-hexane (15 $\mathrm{mL}$ ) was added, and solid precipitated. The product was collected by filtration, washed with $20 \mathrm{~mL} n$-hexane, and dried under vacuum.

\section{C1b}

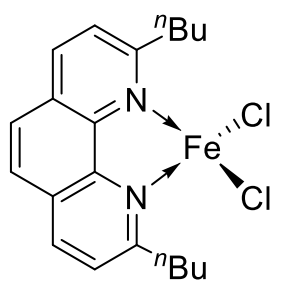

$738 \mathrm{mg}, 88 \%$ yield, orange solid, decomposition temperature: $242-247{ }^{\circ} \mathrm{C}$.

${ }^{1} \mathrm{H} \mathrm{NMR}\left(400 \mathrm{MHz}, \mathrm{CDCl}_{3}\right) \delta 58.11,51.88,27.41,26.39,3.51,2.04,1.73,1.26,1.19$, $1.03,0.86,-1.69,-5.18,-11.83,-15.19$.

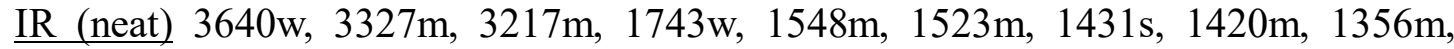
$1322 \mathrm{~m}, 741 \mathrm{~m} \mathrm{~cm}^{-1}$.

\section{C1c}<smiles>CCCc1ccc2ccc3ccc(CCC)n([Te](Cl)(Cl)Cl)c3c12</smiles>

$696 \mathrm{mg}, 89 \%$ yield, orange solid, decomposition temperature: $261-264{ }^{\circ} \mathrm{C}$.

${ }^{1} \mathrm{H}$ NMR $\left(400 \mathrm{MHz}, \mathrm{CDCl}_{3}\right) \delta 58.21,27.67,1.71,-14.68,-17.91$.

IR (neat) $3651 \mathrm{w}, 3316 \mathrm{~m}, 3201 \mathrm{~m}, 1712 \mathrm{w}, 1521 \mathrm{~m}, 1510 \mathrm{~m}, 1437 \mathrm{~s}, 1410 \mathrm{~m}, 1356 \mathrm{~m}$, $1311 \mathrm{~m}, 744 \mathrm{~m} \mathrm{~cm}^{-1}$. 


\section{C1i}<smiles></smiles><smiles>Cc1cc(C)c(-c2cc(C)cc(C)c2-c2cc(C)cc(C)c2C)c(C)c1</smiles>

$1.66 \mathrm{~g}, 89 \%$ yield, orange-red solid, decomposition temperature: $>300{ }^{\circ} \mathrm{C}$.

${ }^{1} \mathrm{H}$ NMR $\left(400 \mathrm{MHz}, \mathrm{CDCl}_{3}\right) \delta 57.53,24.32,7.98,6.93,2.72,2.41,1.41,1.21,0.65,-$ 16.31 .

IR (neat) 2949m, 2916s, 2858m, 2341w, 1612s, 1586s, 1555m, 1498s, 1485s, 1443s, 1378w, 1354w, 1225m, 860s, 797w, 723w cm $\mathrm{cm}^{-1}$.

\section{$\mathbf{C 1 j}$}<smiles></smiles><smiles>CCCc1cc(C(C)C)c(-c2cc(-c3c(CC)cc(C(C)C)cc3C(C)C)cc(C(C)C)c2)c(C(C)C)c1</smiles>

$1.17 \mathrm{~g}, 46 \%$ yield, light pink solid, decomposition temperature: $>300{ }^{\circ} \mathrm{C}$.

${ }^{1} \mathrm{H}$ NMR $\left(400 \mathrm{MHz}, \mathrm{CDCl}_{3}\right) \delta 58.42,24.68,6.67,5.35,3.11,2.76,1.32,1.27,1.23$, $1.21,0.88,-16.13$.

IR (neat) 3552m, 3477m, 3413s, 3234w, 2957w, 2924m, 2853w, 2026w, 1638m, 1616w, 1461w, 1275w, 1261w, 1081w, 763m, 624m cm ${ }^{-1}$.

\section{C1k}

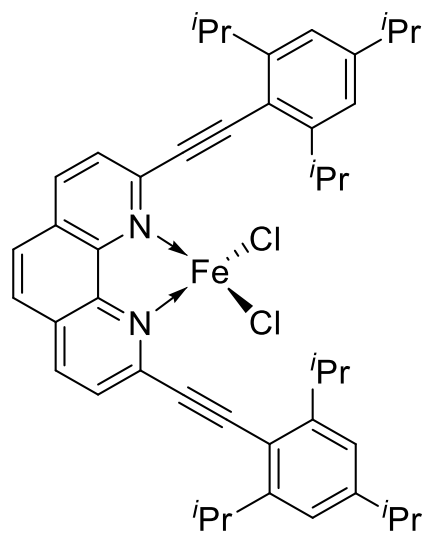

$1.02 \mathrm{~g}, 67 \%$ yield, light pink solid, decomposition temperature: $>300{ }^{\circ} \mathrm{C}$

$\underline{{ }^{1} \mathrm{H} \mathrm{NMR}}\left(400 \mathrm{MHz}, \mathrm{CDCl}_{3}\right) \delta 56.07,26.01,6.21,3.03,2.82,1.26,0.72,-1.50,-17.66$. 
$\underline{\text { IR (neat) } 3666 w, 3523 w, ~ 2919 m, ~ 1746 s, ~ 1612 m, ~ 1588 s, ~ 1556 m, ~ 1511 s, ~ 1481 s, ~ 1445 m, ~}$ $1426 \mathrm{~m}, 1031 \mathrm{~m}, 897 \mathrm{~s}, 865 \mathrm{~m}, 760 \mathrm{w}, 729 \mathrm{w} \mathrm{cm} \mathrm{cm}^{-1}$.

Table S1 | Crystal data and structure refinement for C1g

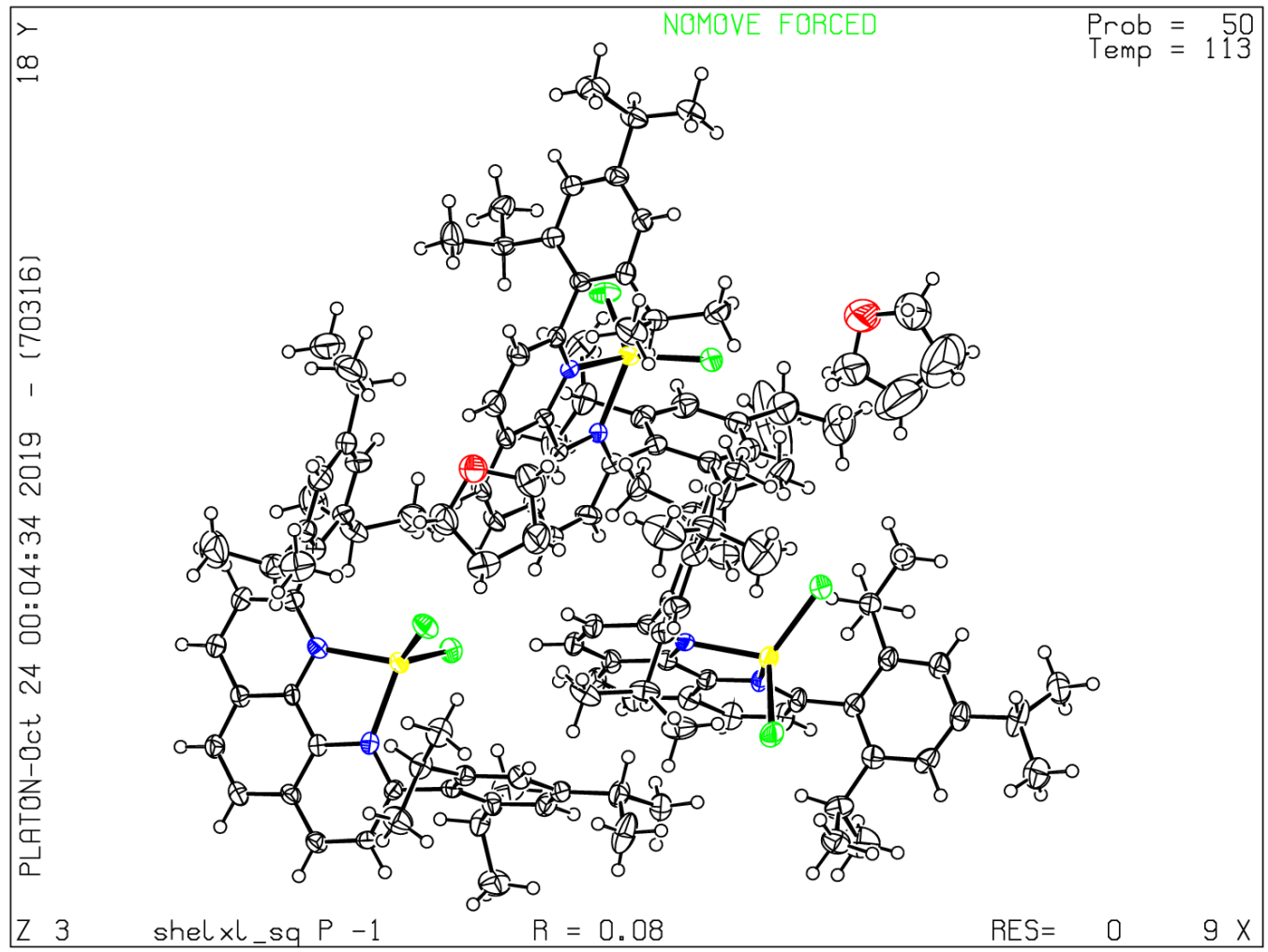

\begin{tabular}{ll}
\hline CCDC number & 1974110 \\
\hline Empirical formula & $\mathrm{C} 44.67 \mathrm{H} 57.33 \mathrm{Cl} 2 \mathrm{Fe}$ N2 O0.67 \\
Moiety formula & $\mathrm{C} 42 \mathrm{H} 52 \mathrm{Cl} 2 \mathrm{Fe} \mathrm{N} 2,0.67(\mathrm{C} 4 \mathrm{H} 8 \mathrm{O})$ \\
Formula weight & 759.67 \\
Temperature & $113(2) \mathrm{K}$ \\
Wavelength & $0.71073 \AA$ \\
Crystal system & triclinic \\
Space group & $\mathrm{P}-1$ \\
Unit cell dimensions & $\mathrm{a}=18.194(4) \mathrm{A}$ alpha $=106.56(3) \mathrm{deg}$. \\
& $\mathrm{b}=18.496(4) \mathrm{A}$ beta $=118.67(3) \mathrm{deg}$. \\
& $\mathrm{c}=25.849(5) \mathrm{A}$ gamma $=118.67(3) \mathrm{deg}$. \\
Volume & $7099(3) \AA^{3}$
\end{tabular}


Calculated density

Absorption coefficient

$\mathrm{F}(000)$

Crystal size

Theta range for data collection

Limiting indices

Reflections collected / unique

Completeness to theta $=25.242$

Absorption correction

Max. and min. transmission

Refinement method

Data / restraints / parameters

Goodness-of-fit on $\mathrm{F}^{2}$

Final $\mathrm{R}$ indices $[\mathrm{I}>2 \operatorname{sigma}(\mathrm{I})]$

$\mathrm{R}$ indices (all data)

Largest diff. peak and hole
$1.066 \mathrm{mg} / \mathrm{m}^{\wedge} 3$

$0.461 \mathrm{~mm}^{-1}$

2428.0

$0.2 \times 0.18 \times 0.12 \mathrm{~mm}$

2.736 to $40.04 \mathrm{deg}$.

$-21<=\mathrm{h}<=21,-21<=\mathrm{k}<=21,-30<=\mathrm{l}<=30$

$58802 / 24600[\mathrm{R}(\mathrm{int})=0.0651]$

$99.9 \%$

Semi-empirical from equivalents

1 and 0.7070

Full-matrix least-squares on $\mathrm{F}^{2}$

$24600 / 42 / 1396$

1.068

$R_{1}=0.0842, w R_{2}=0.2146$

$R_{1}=0.1091, w R_{2}=0.2337$

0.639 and -0.468 e. $\AA^{-3}$ 
Table S2 | Crystal data and structure refinement for C1h

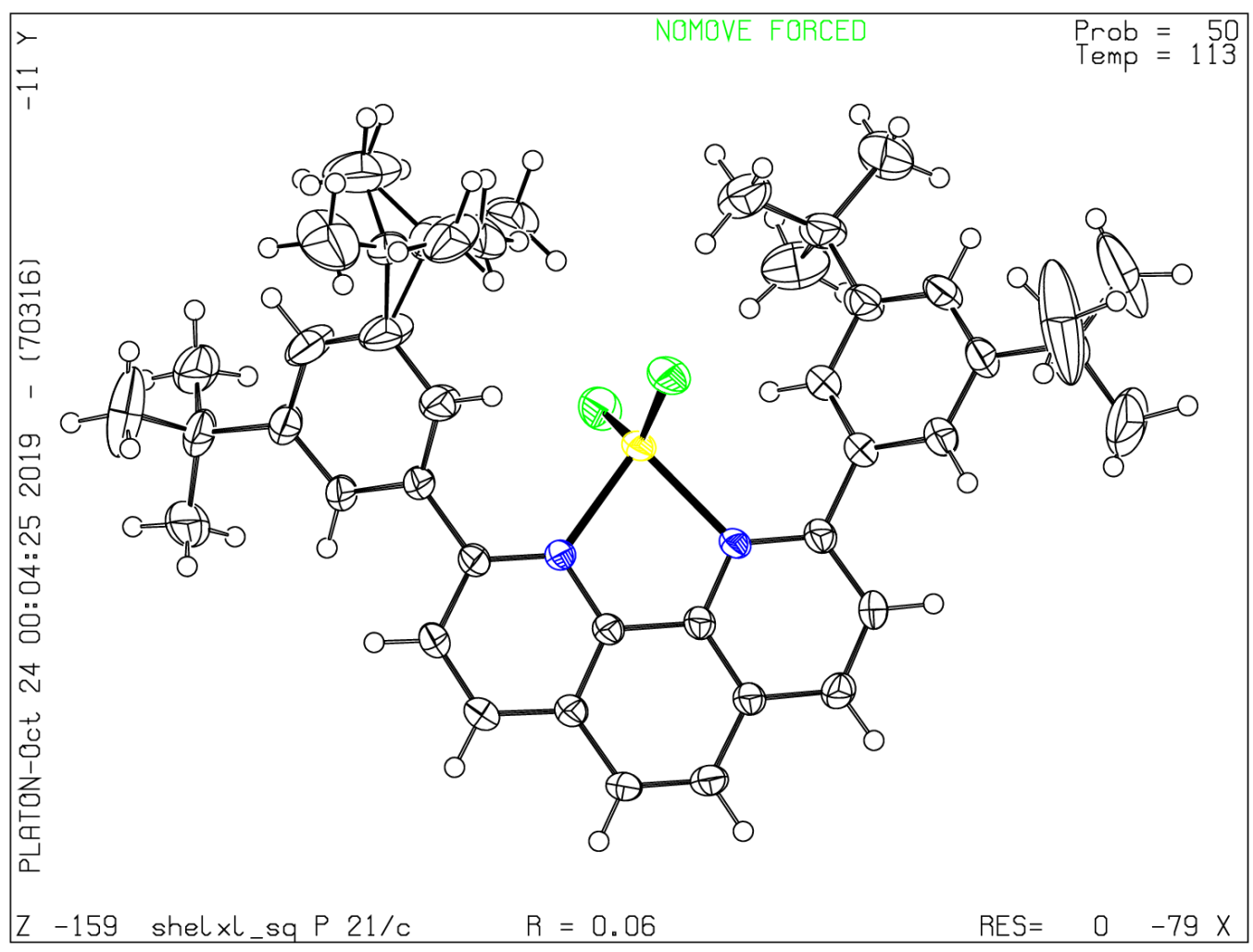

\begin{tabular}{ll}
\hline CCDC number & 1960979 \\
\hline Empirical formula & $\mathrm{C} 40 \mathrm{H} 48 \mathrm{Cl} 2 \mathrm{Fe} \mathrm{N} 2 \mathrm{O} 0$ \\
Moiety formula & $\mathrm{C}_{20} \mathrm{H}_{23} \mathrm{NO}_{3}$ \\
Formula weight & 683.55 \\
Temperature & $113(2) \mathrm{K}$ \\
Wavelength & $0.71073 \AA$ \\
Crystal system & Monoclinic, P2(1)/c \\
Space group & $\mathrm{P} 2(1) 2(1) 2(1)$ \\
Unit cell dimensions & $\mathrm{a}=15.213(3) \mathrm{A} \quad$ alpha $=90 \mathrm{deg}$. \\
& $\mathrm{b}=13.510(3) \mathrm{A} \quad$ beta $=92.82(3) \mathrm{deg}$. \\
& $\mathrm{c}=19.189(4) \mathrm{A} \quad$ gamma $=90 \mathrm{deg}$. \\
Volume & $3939.1(14) \AA^{3}$ \\
Z & 4 \\
Calculated density & $1.153 \mathrm{Mg} / \mathrm{m}^{\wedge} 3$ \\
Absorption coefficient & $0.546 \mathrm{~mm}^{-1}$
\end{tabular}


$\mathrm{F}(000)$

Crystal size

Theta range for data collection

Limiting indices

Reflections collected / unique

Completeness to theta $=25.242$

Absorption correction

Max. and min. transmission

Refinement method

Data / restraints / parameters

Goodness-of-fit on $\mathrm{F}^{2}$

Final $\mathrm{R}$ indices [I $>2 \operatorname{sigma}(\mathrm{I})]$

$\mathrm{R}$ indices (all data)

Largest diff. peak and hole
1448

$0.180 \times 0.120 \times 0.020 \mathrm{~mm}$

2.017 to $27.889 \mathrm{deg}$.

$-19<=\mathrm{h}<=19,-17<=\mathrm{k}<=17,-25<=\mathrm{k}<=22$

$38688 / 9375[\mathrm{R}($ int $)=0.0885]$

$99.9 \%$

Semi-empirical from equivalents

1 and 0.7070

Full-matrix least-squares on $\mathrm{F}^{2}$

9375 / 56 / 443

0.967

$R_{1}=0.0571, w R_{2}=0.1484$

$R_{1}=0.0827, w R_{2}=0.1667$

0.549 and -0.445 e. $\AA^{-3}$ 
Table S3 | ICP-AES analysis of iron precatalysts (ppm)

\begin{tabular}{|c|c|c|c|c|}
\hline \multirow[b]{2}{*}{ Element } & \multirow[b]{2}{*}{ C1e } & \multicolumn{2}{|c|}{$\begin{array}{l}\text { C1e } \mathrm{Ar}=2,4,6-\mathrm{Me}_{3} \mathrm{C}_{6} \mathrm{H}_{2} \\
\text { C1g } \mathrm{Ar}=2,4,6-{ }^{i} \mathrm{Pr}_{3} \mathrm{C}_{6} \mathrm{H}_{2} \\
\text { C1h } \mathrm{Ar}=3,5-{ }^{-} \mathrm{Bu}_{2} \mathrm{C}_{6} \mathrm{H}_{3} \\
\text { C1j } \mathrm{Ar}=3,5-\left(2,4,6-{ }^{-} \mathrm{Pr}_{3} \mathrm{C}_{6} \mathrm{H}_{2}\right)_{2} \mathrm{C}_{6} \mathrm{H}_{3}\end{array}$} & \multirow[b]{2}{*}{$\mathbf{C} \mathbf{1 j}$} \\
\hline & & C1g & C1h & \\
\hline $\mathrm{Fe}$ & 74800 & 89700 & 78300 & 49800 \\
\hline $\mathbf{C u}$ & 28.5 & - & 25.3 & 5.04 \\
\hline $\mathbf{N i}$ & 30.6 & 11.0 & 48.4 & 7.11 \\
\hline $\mathbf{Z n}$ & 77.5 & 11.9 & 55.4 & 26.4 \\
\hline $\mathrm{Cr}$ & 3.21 & 3.43 & 5.52 & - \\
\hline Cd & 6.21 & 8.24 & 10.2 & - \\
\hline Mn & 16.9 & 12.2 & 91.3 & 10.1 \\
\hline $\mathbf{B a}$ & 6.02 & 2.50 & 1.43 & 3.26 \\
\hline Al & - & - & - & - \\
\hline $\mathbf{P b}$ & - & - & - & - \\
\hline $\mathbf{T i}$ & - & - & - & - \\
\hline Co & 10.3 & 4.70 & 8.97 & 4.45 \\
\hline $\mathbf{V}$ & - & - & - & - \\
\hline Pd & - & - & - & - \\
\hline Au & - & - & - & - \\
\hline $\mathbf{P t}$ & - & - & - & - \\
\hline $\mathbf{R u}$ & 7.22 & 2.10 & 1.52 & - \\
\hline
\end{tabular}

'-' means $<1$ ppm 


\section{Optimization of Reaction Conditions}

Table S4 | Effect of additives and solvents

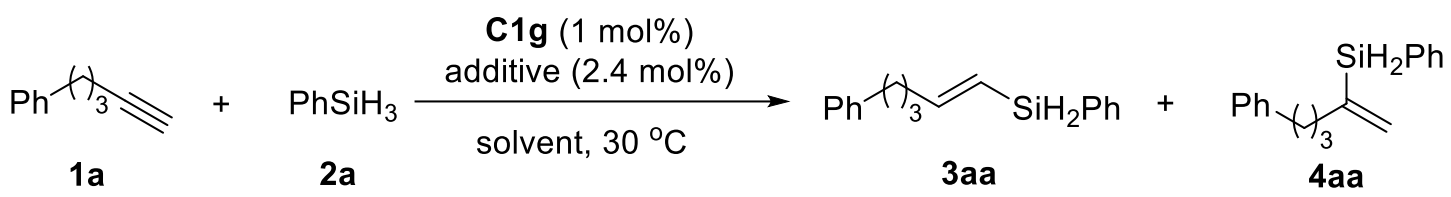

General procedure: In an argon-filled glovebox, a vial $(10 \mathrm{~mL})$ was charged $\mathbf{C 1 g}$ (3.6 $\mathrm{mg}, 0.005 \mathrm{mmol})$ and solvent $(1 \mathrm{~mL})$. The reaction mixture was stirred at room temperature for $1 \mathrm{~min}$, then $\mathbf{1 a}(72.1 \mathrm{mg}, 0.5 \mathrm{mmol}), \mathbf{2 a}(59.5 \mathrm{mg}, 0.55 \mathrm{mmol})$ and additive $(0.012 \mathrm{mmol})$ was added. After stirring for $1 \mathrm{~h}$ at $30^{\circ} \mathrm{C}$, the vial was removed from the glovebox and the reaction mixture was concentrated by rotary evaporation. Iron species were removed by flash column chromatography with hexane as eluent. The raw product was detected by ${ }^{1} \mathrm{H}$ NMR with 1,3,5-trimethoxybenzene as internal standard. The results were listed as following.

\begin{tabular}{|c|c|c|c|c|c|}
\hline entry & additive & solvent & conv. $(\%)$ & yield (\%) & $3 a a / 4 a a$ \\
\hline 1 & $\mathrm{MeMgCl}$ & THF & $>95$ & 92 & $>98: 2$ \\
\hline 2 & $\mathrm{PhMgBr}$ & THF & 59 & 52 & $>98: 2$ \\
\hline 3 & ${ }^{n} \mathrm{BuLi}$ & THF & $>95$ & 92 & $>98: 2$ \\
\hline 4 & $\mathrm{Et}_{2} \mathrm{Zn}$ & THF & trace & $<5$ & $>98: 2$ \\
\hline 5 & $\mathrm{NaHBEt}_{3}$ & THF & trace & $<5$ & $>98: 2$ \\
\hline 6 & $\mathrm{LiAlH}_{4}$ & THF & 90 & 85 & $>98: 2$ \\
\hline 7 & $\mathrm{NaO}^{t} \mathrm{Bu}$ & THF & $>95$ & 88 & $>98: 2$ \\
\hline 8 & EtMgBr & $\mathrm{Et}_{2} \mathrm{O}$ & $>95$ & 90 & $>98: 2$ \\
\hline 9 & $\mathrm{EtMgBr}$ & toluene & $>95$ & 91 & $>98: 2$ \\
\hline
\end{tabular}


Table S5 | Effect of metal salts in the reaction of pent-4-yn-1-yl-benzene

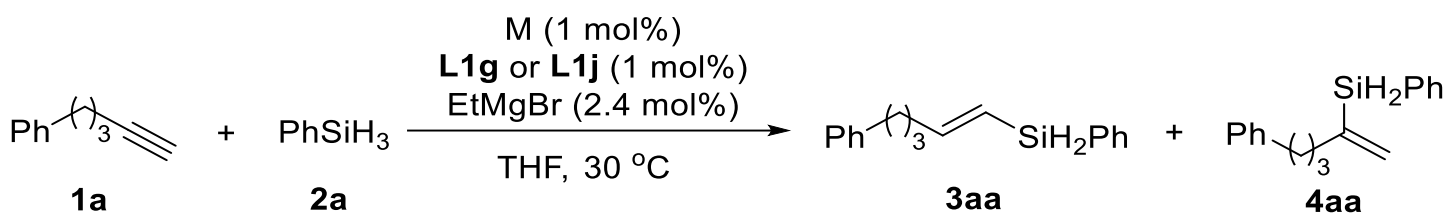

Genearl procedure: In an argon-filled glovebox, a vial $(10 \mathrm{~mL})$ was charged with metal salt (0.005 mmol), L1g (2.9 mg, $0.005 \mathrm{mmol})$ or $\mathbf{L 1 j}$ (5.7 mg, $0.005 \mathrm{mmol})$ and THF (1 mL). The reaction mixture was stirred at room temperature for $1 \mathrm{~h}$, then $\mathbf{1 a}$ (72.1 mg, $0.5 \mathrm{mmol}), 2 \mathrm{a}(59.5 \mathrm{mg}, 0.55 \mathrm{mmol})$, and EtMgBr (1 M in THF, $12 \mu \mathrm{L}, 0.012$ mmol, $2.4 \mathrm{~mol} \%$ ) was added. After stirring for $6 \mathrm{~h}$ at $30{ }^{\circ} \mathrm{C}$, the vial was removed from the glovebox and the reaction mixture was concentrated by rotary evaporation. Iron species were removed by flash column chromatography with hexane as eluent. The raw product was detected by ${ }^{1} \mathrm{H}$ NMR with 1,3,5-trimethoxybenzene as internal standard. The results were listed as following.

\begin{tabular}{llllll}
\hline \multirow{2}{*}{ entry } & metal salt & \multicolumn{3}{l}{ L1g } & \multicolumn{2}{l}{ L1j } \\
\cline { 3 - 6 } & & yield (conv.) & 3aa/4aa & yield (conv.) & 3aa/4aa \\
\hline 1 & $\mathrm{FeCl}_{2}$ & $92 \%(>95 \%)$ & $>98: 2$ & $90 \%(>95 \%)$ & $<2: 98$ \\
2 & none & $0(<5 \%)$ & - & $0(<5 \%)$ & - \\
3 & $\mathrm{CuCl}_{2}$ & $31 \%(76 \%)$ & $89: 11$ & $22 \%(78 \%)$ & $25: 75$ \\
4 & $\mathrm{NiCl}_{2}$ & $53 \%(95 \%)$ & $95: 5$ & $15 \%(72 \%)$ & $28: 72$ \\
5 & $\mathrm{ZnCl}_{2}$ & $0(<5 \%)$ & - & $0(<5 \%)$ & - \\
6 & $\mathrm{CrCl}_{2}$ & $<5 \%(<5 \%)$ & - & $<5 \%(<5 \%)$ & - \\
7 & $\mathrm{MnCl}_{2}$ & $26 \%(66 \%)$ & $87: 13$ & $18 \%(>95 \%)$ & $18: 82$ \\
8 & $\mathrm{BaCl}_{2}$ & $0(<5 \%)$ & - & $0(<5 \%)$ & - \\
9 & $\mathrm{CoCl}_{2}$ & $73 \%(>95 \%)$ & $>98: 2$ & $67 \%(>95 \%)$ & $3: 97$ \\
10 & $\mathrm{RuCl}_{3}$ & $0(14 \%)$ & - & $0(21 \%)$ & - \\
\hline
\end{tabular}


Table S6 | Metal salt effect in the reaction of prop-1-yn-1-ylbenzene

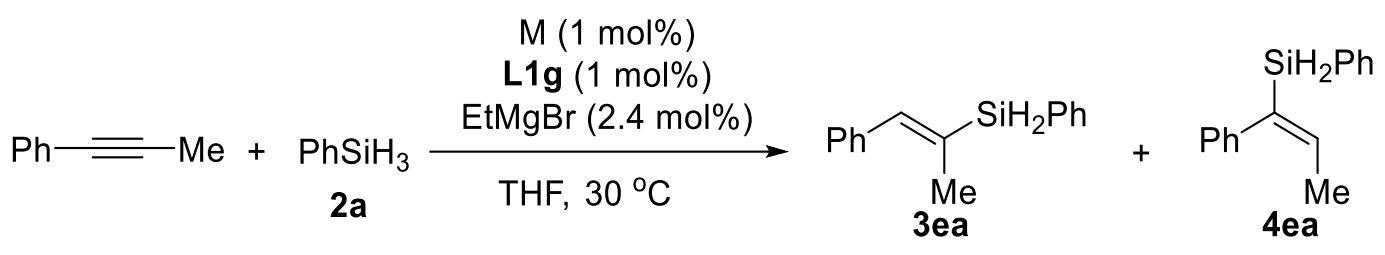

Genearl procedure: In an argon-filled glovebox, a vial $(10 \mathrm{~mL})$ was charged with metal salt (0.005 mmol), L1g $(2.9 \mathrm{mg}, 0.005 \mathrm{mmol})$ and THF $(1 \mathrm{~mL})$. The reaction mixture was stired at room temperature for $1 \mathrm{~h}$, then prop-1-yn-1-ylbenzene $(58.1 \mathrm{mg}$, $0.5 \mathrm{mmol}), 2 \mathrm{a}(59.5 \mathrm{mg}, 0.55 \mathrm{mmol})$ and $\operatorname{EtMgBr}(1 \mathrm{M}$ in THF, $12 \mu \mathrm{L}, 0.012 \mathrm{mmol}$, $2.4 \mathrm{~mol} \%$ ) was added. After stirring for $24 \mathrm{~h}$ at $30{ }^{\circ} \mathrm{C}$, the vial was removed from the glovebox and the reaction mixture was concentrated by rotary evaporation. Iron species were removed by flash column chromatography with hexane as eluent. The raw product was detected by ${ }^{1} \mathrm{H}$ NMR with 1,3,5-trimethoxybenzene as internal standard. The results were listed as following.

\begin{tabular}{llll}
\hline entry & metal salt & yield (conv.) & 3ea/4ea \\
\hline 1 & $\mathrm{FeCl}_{2}$ & $85 \%(>95 \%)$ & $95: 5$ \\
2 & none & $0(<5 \%)$ & - \\
3 & $\mathrm{CuCl}_{2}$ & $0(<5 \%)$ & - \\
4 & $\mathrm{NiCl}_{2}$ & $24 \%(87 \%)$ & $63: 37$ \\
5 & $\mathrm{ZnCl}_{2}$ & $0(<5 \%)$ & - \\
6 & $\mathrm{CrCl}_{2}$ & $0(<5 \%)$ & - \\
7 & $\mathrm{MnCl}_{2}$ & $0(<5 \%)$ & - \\
8 & $\mathrm{BaCl}_{2}$ & $0(<5 \%)$ & - \\
9 & $\mathrm{CoCl}_{2}$ & $76 \%(85 \%)$ & $76: 24$ \\
10 & $\mathrm{RuCl}_{3}$ & $0(<5 \%)$ & - \\
\hline
\end{tabular}




\section{Typical Procedures for Alkyne Hydrosilylation}

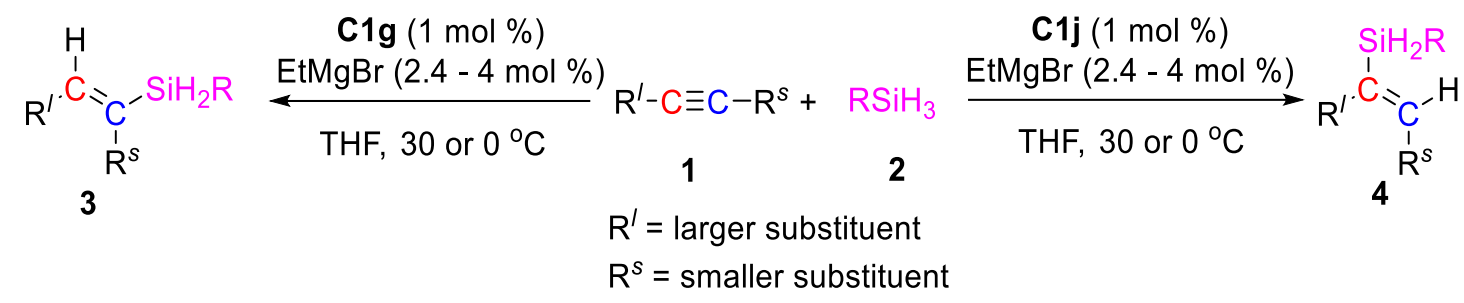

\section{Procedure A (Hydrosilylation of alkyl-substituted acetylenes with primary}

silanes): In an argon-filled glovebox, a vial (10 mL) was charged C1g (3.6 mg, 0.005 mmol, $1 \mathrm{~mol} \%)$ or $\mathbf{C 1 j}$ (6.3 $\mathrm{mg}, 0.005 \mathrm{mmol}, 1 \mathrm{~mol} \%)$ and THF (1 mL). The reaction mixture was stirred at room temperature for $1 \mathrm{~min}$, then EtMgBr $(1 \mathrm{M}$ in THF, $12 \mu \mathrm{L}$, $0.012 \mathrm{mmol}, 2.4 \mathrm{~mol} \%$ ) was added and the reaction mixture was stirred for another 2 min. Then primary silanes $(0.55 \mathrm{mmol}, 1.1$ equiv) and alkynes $(0.5 \mathrm{mmol})$ were added in sequence. After stirring for $10 \mathrm{~min}$ or $1 \mathrm{~h}$ at $30^{\circ} \mathrm{C}$, the vial was removed from the glovebox and the reaction mixture was concentrated by rotary evaporation. The residue was purified by flash column chromatography to afford the desired product.

Procedure B (Hydrosilylation of aryl-substituted acetylenes with phenylsilane): In an argon-filled glovebox, a vial $(10 \mathrm{~mL})$ was charged with THF (1 $\mathrm{mL}$ ) and complex C1g (3.6 mg, $0.005 \mathrm{mmol}, 1 \mathrm{~mol} \%$ ) or C1j (6.3 mg, $0.005 \mathrm{mmol}, 1$ mol\%). The reaction mixture was stirred at $-20^{\circ} \mathrm{C}$ for $1 \mathrm{~min}$, then $\mathrm{EtMgBr}(1 \mathrm{M}$ in THF, $12 \mu \mathrm{L}, 0.012 \mathrm{mmol}, 2.4 \mathrm{~mol} \%$ ) was added and the reaction mixture was stirred for another $2 \mathrm{~min}$. Then $\mathbf{2 a}(59.5 \mathrm{mg}, 0.55 \mathrm{mmol}, 1.1$ equiv) and alkynes ( $0.5 \mathrm{mmol})$ were added in sequence. The vial was sealed and then removed from the glovebox. The reaction mixture was stirred for $3 \mathrm{~h}$ at $0{ }^{\circ} \mathrm{C}$, and concentrated by rotary evaporation. The residue was purified by flash column chromatography to afford the desired product.

Procedure C (Hydrosilylation of internal alkynes with primaryl silanes): In an argon-filled glovebox, a vial $(10 \mathrm{~mL})$ was charged with complexes $\mathbf{C 1 g}$ (3.6 $\mathrm{mg}$, $0.005 \mathrm{mmol}, 1 \mathrm{~mol} \%)$ or C1j (6.3 mg, $0.005 \mathrm{mmol}, 1 \mathrm{~mol} \%)$, dry anhydrous THF (1 $\mathrm{mL})$, internal alkyne $(0.5 \mathrm{mmol})$ and $\mathbf{2 a}(59.5 \mathrm{mg}, 0.55 \mathrm{mmol}, 1.1$ equiv) successivelly. The reaction mixture was stirred at $30^{\circ} \mathrm{C}$ for 3 min before EtMgBr $(1 \mathrm{M}$ in THF, $20 \mu \mathrm{L}$, $0.02 \mathrm{mmol}, 4.0 \mathrm{~mol} \%$ ) was added. After $3 \mathrm{~h}$, the vial was removed from the glovebox and the reaction mixture was concentrated by rotating evaporation. The residue was purified by flash chromatography to afford the desired product. 


\section{Analytical Data of Hydrosilylation Products}

(E)-phenyl(5-phenylpent-1-en-1-yl)silane (3aa) ${ }^{12}$<smiles>S/C=C/CCCc1ccccc1</smiles>

Procedure A, $106.4 \mathrm{mg}, 84 \%$ yield, r.r. > 98:2, colorless oil.

를 NMR (400 MHz, $\left.\mathrm{CDCl}_{3}\right) \delta 7.59-7.53(\mathrm{~m}, 2 \mathrm{H}), 7.43-7.32(\mathrm{~m}, 3 \mathrm{H}), 7.30-7.25$ (m, 2H), $7.21-7.13(\mathrm{~m}, 3 \mathrm{H}), 6.37$ (dt, $J=18.5,6.2 \mathrm{~Hz}, 1 \mathrm{H}), 5.78-5.69$ (m, $1 \mathrm{H}), 4.53(\mathrm{~d}, J=3.1 \mathrm{~Hz}, 2 \mathrm{H}), 2.63(\mathrm{t}, J=7.7 \mathrm{~Hz}, 2 \mathrm{H}), 2.22(\mathrm{q}, J=7.1 \mathrm{~Hz}$, $2 \mathrm{H}), 1.81-1.72(\mathrm{~m}, 2 \mathrm{H})$.

${ }^{13} \mathrm{C}$ NMR $\left(101 \mathrm{MHz}, \mathrm{CDCl}_{3}\right) \delta 153.4$ (1C), 142.2 (1C), 135.3 (2C), 132.2 (1C), 129.6 (1C), 128.4 (2C), 128.3 (2C) 128.0 (2C), 125.7 (1C), 120.5 (1C), 36.3 (1C), 35.3 (1C), 30.1 (1C).

HRMS (EI) calcd for [M, $\mathrm{C}_{17} \mathrm{H}_{20} \mathrm{Si}^{+}:$:252.1334, found 252.1338.

phenyl(5-phenylpent-1-en-2-yl)silane (4aa) ${ }^{13}$<smiles>C=C(CCCc1ccccc1)Sc1ccccc1</smiles>

Procedure A, $117.5 \mathrm{mg}, 93 \%$ yield, r.r. $>98: 2$, colorless oil.

${ }^{1} \mathrm{H}$ NMR $\left(400 \mathrm{MHz}, \mathrm{CDCl}_{3}\right) \delta 7.60-7.53(\mathrm{~m}, 2 \mathrm{H}), 7.44-7.32(\mathrm{~m}, 3 \mathrm{H}), 7.28-7.23$ $(\mathrm{m}, 2 \mathrm{H}), 7.20-7.08(\mathrm{~m}, 3 \mathrm{H}), 5.82-5.76(\mathrm{~m}, 1 \mathrm{H}), 5.58(\mathrm{~d}, J=2.6 \mathrm{~Hz}, 1 \mathrm{H})$, $4.55(\mathrm{~s}, 2 \mathrm{H}), 2.56(\mathrm{t}, J=8.0 \mathrm{~Hz}, 2 \mathrm{H}), 2.30-2.21(\mathrm{~m}, 2 \mathrm{H}), 1.81-1.69(\mathrm{~m}$, $2 \mathrm{H})$.

${ }^{13} \mathrm{C} \mathrm{NMR}\left(101 \mathrm{MHz}, \mathrm{CDCl}_{3}\right) \delta 144.8$ (1C), 142.3 (1C), 135.5 (2C), 131.5 (1C), 129.9 (1C), 129.7 (1C), 128.4 (2C), 128.2 (2C), 128.0 (2C), 125.7 (1C), 37.0 (1C), 35.4 (1C), 30.5 (1C).

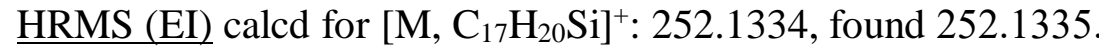

(E)-oct-1-en-1-yl(phenyl)silane (3ab) $)^{14}$<smiles>CCCCCCC/C=C/[SH+]c1ccccc1</smiles> 
Procedure A, $100.5 \mathrm{mg}, 92 \%$ yield, r.r. $>98: 2$, colorless oil.

를 NMR (400 MHz, $\left.\mathrm{CDCl}_{3}\right) \delta 7.57(\mathrm{~d}, J=7.4 \mathrm{~Hz}, 2 \mathrm{H}), 7.45-7.31(\mathrm{~m}, 3 \mathrm{H}), 6.36(\mathrm{dt}$, $J=18.4,6.2 \mathrm{~Hz}, 1 \mathrm{H}), 5.71(\mathrm{~d}, J=18.4 \mathrm{~Hz}, 1 \mathrm{H}), 4.53(\mathrm{~d}, J=3.1 \mathrm{~Hz}, 2 \mathrm{H})$, $2.18(\mathrm{q}, J=6.9 \mathrm{~Hz}, 2 \mathrm{H}), 1.46-1.37(\mathrm{~m}, 2 \mathrm{H}), 1.35-1.23(\mathrm{~m}, 6 \mathrm{H}), 0.88(\mathrm{t}, J$ $=6.6 \mathrm{~Hz}, 3 \mathrm{H})$.

${ }^{13} \mathrm{C} \mathrm{NMR}\left(101 \mathrm{MHz}, \mathrm{CDCl}_{3}\right) \delta 154.2$ (1C), 135.3 (2C), 132.4 (1C), 129.5 (1C), 128.0 (2C), 119.8 (1C), 36.9 (1C), 31.7 (1C), 28.8 (1C), 28.4 (1C), 22.6 (1C), 14.1 (1C).

HRMS (EI) calcd for [M, $\mathrm{C}_{14} \mathrm{H}_{22} \mathrm{Si}^{+}:$218.1491, found 218.1493.

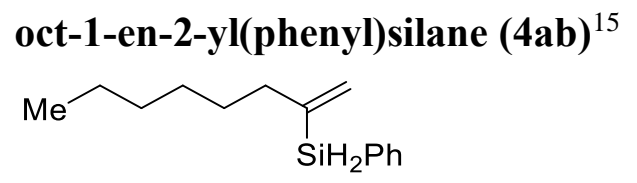

Procedure A, $103.7 \mathrm{mg}, 95 \%$ yield, r.r. > 98:2, colorless oil.

${ }^{1} \mathrm{H}$ NMR $\left(400 \mathrm{MHz}, \mathrm{CDCl}_{3}\right) \delta 7.62-7.52(\mathrm{~m}, 2 \mathrm{H}), 7.44-7.31(\mathrm{~m}, 3 \mathrm{H}), 5.77(\mathrm{~s}, 1 \mathrm{H})$, $5.53(\mathrm{~d}, J=2.9 \mathrm{~Hz}, 1 \mathrm{H}), 4.54(\mathrm{~s}, 2 \mathrm{H}), 2.20(\mathrm{t}, J=7.7 \mathrm{~Hz}, 2 \mathrm{H}), 1.46-1.36$ $(\mathrm{m}, 2 \mathrm{H}), 1.32-1.17(\mathrm{~m}, 6 \mathrm{H}), 0.86(\mathrm{t}, J=6.6 \mathrm{~Hz}, 3 \mathrm{H})$.

${ }^{13} \mathrm{C} \mathrm{NMR}\left(101 \mathrm{MHz}, \mathrm{CDCl}_{3}\right) \delta 145.4$ (1C), 135.5 (2C), 131.7 (1C), 129.7 (1C), 129.4 (1C), 128.0 (2C), 37.4 (1C), 31.6 (1C), 28.9 (1C), 28.8 (1C), 22.6 (1C), 14.1 (1C).

HRMS (EI) calcd for [M, $\left.\mathrm{C}_{14} \mathrm{H}_{22} \mathrm{Si}\right]^{+}:$218.1491, found 218.1495.

\section{(E)-phenyl(prop-1-en-1-yl)silane (3ac)}<smiles>CC=C[SH+]c1ccccc1</smiles>

Procedure A, $64.5 \mathrm{mg}, 87 \%$ yield, r.r. $>98: 2$, colorless oil.

${ }^{1} \mathrm{H}$ NMR $\left(400 \mathrm{MHz}, \mathrm{CDCl}_{3}\right) \delta 7.60-7.52(\mathrm{~m}, 2 \mathrm{H}), 7.42-7.32(\mathrm{~m}, 3 \mathrm{H}), 6.36(\mathrm{dq}, J=$ 18.5, $6.2 \mathrm{~Hz}, 1 \mathrm{H}), 5.75$ (ddq, $J=18.3,3.3,1.6 \mathrm{~Hz}, 1 \mathrm{H}), 4.52$ (d, $J=3.1 \mathrm{~Hz}$, $2 \mathrm{H}), 1.90-1.86(\mathrm{~m}, 3 \mathrm{H})$.

${ }^{13} \mathrm{C} \mathrm{NMR}\left(101 \mathrm{MHz}, \mathrm{CDCl}_{3}\right) \delta 148.8(1 \mathrm{C}), 135.3$ (2C), 132.3 (1C), $129.6(1 \mathrm{C}), 128.0$ (2C), $121.8(1 \mathrm{C}), 22.8(1 \mathrm{C})$.

HRMS (EI) calcd for [M, $\left.\mathrm{C}_{9} \mathrm{H}_{12} \mathrm{Si}\right]^{+}:$148.0708, found 148.0706. 
phenyl(prop-1-en-2-yl)silane (4ac)

$\mathrm{Me}$

$\mathrm{SiH}_{2} \mathrm{Ph}$

Procedure A, $66.7 \mathrm{mg}, 90 \%$ yield, r.r. > 98:2, colorless oil.

${ }^{1} \mathrm{H}$ NMR $\left(400 \mathrm{MHz}, \mathrm{CDCl}_{3}\right) \delta 7.62-7.52(\mathrm{~m}, 2 \mathrm{H}), 7.44-7.29(\mathrm{~m}, 3 \mathrm{H}), 5.77(\mathrm{~s}, 1 \mathrm{H})$, $5.52(\mathrm{~s}, 1 \mathrm{H}), 4.55-4.48(\mathrm{~m}, 2 \mathrm{H}), 1.89(\mathrm{~s}, 3 \mathrm{H})$.

${ }^{13} \mathrm{C} \mathrm{NMR}\left(101 \mathrm{MHz}, \mathrm{CDCl}_{3}\right) \delta 140.5$ (1C), 135.5 (2C), 131.4 (1C), 130.3 (1C), 129.7 (1C), 128.0 (2C), 23.1 (1C).

HRMS (EI) calcd for [M, $\left.\mathrm{C}_{9} \mathrm{H}_{12} \mathrm{Si}\right]^{+}:$148.0708, found 148.0709.

\section{(E)-(2-cyclopropylvinyl)(phenyl)silane (3ad) ${ }^{14}$}

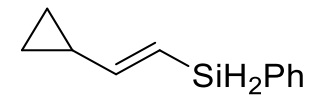

Procedure A, $77.6 \mathrm{mg}, 89 \%$ yield, r.r. > 98:2, colorless oil.

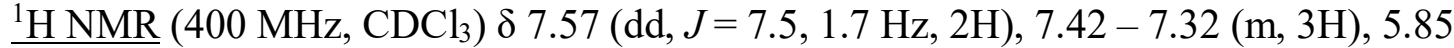
$-5.71(\mathrm{~m}, 2 \mathrm{H}), 4.52(\mathrm{~d}, J=2.4 \mathrm{~Hz}, 2 \mathrm{H}), 1.59-1.51(\mathrm{~m}, 1 \mathrm{H}), 0.83-0.76(\mathrm{~m}$, $2 \mathrm{H}), 0.53-0.46(\mathrm{~m}, 2 \mathrm{H})$.

${ }^{13} \mathrm{C} \mathrm{NMR}\left(101 \mathrm{MHz}, \mathrm{CDCl}_{3}\right) \delta 157.4$ (1C), 135.3 (2C), 132.4 (1C), 129.5 (1C), 128.0 (2C), $116.3(1 \mathrm{C}), 17.8(1 \mathrm{C}), 7.6(2 \mathrm{C})$.

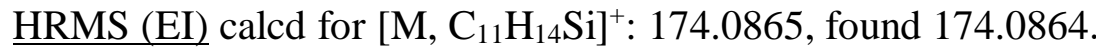

\section{(1-cyclopropylvinyl)(phenyl)silane (4ad) $)^{15}$}<smiles>C=C([SbH]c1ccccc1)C1CC1</smiles>

Procedure A, $78.3 \mathrm{mg}, 90 \%$ yield, r.r. $>98: 2$, colorless oil.

를 NMR $\left(400 \mathrm{MHz}, \mathrm{CDCl}_{3}\right) \delta 7.62-7.56(\mathrm{~m}, 2 \mathrm{H}), 7.42-7.31(\mathrm{~m}, 3 \mathrm{H}), 5.72(\mathrm{~d}, J=$ $2.4 \mathrm{~Hz}, 1 \mathrm{H}), 5.45(\mathrm{~d}, J=2.5 \mathrm{~Hz}, 1 \mathrm{H}), 4.52(\mathrm{~s}, 2 \mathrm{H}), 1.57-1.46(\mathrm{~m}, 1 \mathrm{H}), 0.74$ $-0.59(\mathrm{~m}, 2 \mathrm{H}), 0.56-0.44(\mathrm{~m}, 2 \mathrm{H})$.

${ }^{13} \mathrm{C} \mathrm{NMR}\left(101 \mathrm{MHz}, \mathrm{CDCl}_{3}\right) \delta 146.6$ (1C) 135.5 (2C), 131.6 (1C), 129.7 (1C), 128.0

(2), $126.6(1 \mathrm{C}), 17.3(1 \mathrm{C}), 6.8(2 \mathrm{C})$.

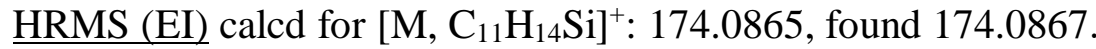


(E)-(2-cyclohexylvinyl)(phenyl)silane (3ae)

$\curvearrowright \mathrm{SiH}_{2} \mathrm{Ph}$

Procedure A, $90.2 \mathrm{mg}, 84 \%$ yield, r.r. > 98:2, colorless oil.

래 NMR (400 MHz, $\left.\mathrm{CDCl}_{3}\right) \delta 7.56(\mathrm{~d}, J=6.4 \mathrm{~Hz}, 2 \mathrm{H}), 7.43-7.32(\mathrm{~m}, 3 \mathrm{H}), 6.32(\mathrm{dd}$, $J=18.6,6.0 \mathrm{~Hz}, 1 \mathrm{H}), 5.66(\mathrm{~d}, J=18.7 \mathrm{~Hz}, 1 \mathrm{H}), 4.53(\mathrm{~d}, J=3.0 \mathrm{~Hz}, 2 \mathrm{H})$, $2.10-2.00(\mathrm{~m}, 1 \mathrm{H}), 1.75(\mathrm{t}, J=11.9 \mathrm{~Hz}, 4 \mathrm{H}), 1.65(\mathrm{~d}, J=12.7 \mathrm{~Hz}, 1 \mathrm{H}), 1.35$ $-1.04(\mathrm{~m}, 5 \mathrm{H})$.

${ }^{13} \mathrm{C} \mathrm{NMR}\left(101 \mathrm{MHz}, \mathrm{CDCl}_{3}\right) \delta 159.5$ (1C), $135.3(2 \mathrm{C}), 132.5$ (1C), $129.6(1 \mathrm{C}), 127.9$ (2C), 116.7 (1C), 44.2 (1C), 32.1 (2C), 26.2 (1C), 25.9 (2C).

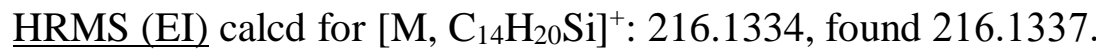

\section{(1-cyclohexylvinyl)(phenyl)silane (4ae)}<smiles>C=C([SnH]c1ccccc1)C1CCCCC1</smiles>

Procedure A, $99.4 \mathrm{mg}, 92 \%$ yield, r.r. > 98:2, colorless oil.

${ }^{1} \mathrm{H}$ NMR $\left(400 \mathrm{MHz}, \mathrm{CDCl}_{3}\right) \delta 7.63-7.50(\mathrm{~m}, 2 \mathrm{H}), 7.47-7.31(\mathrm{~m}, 3 \mathrm{H}), 5.86-5.71$ $(\mathrm{m}, 1 \mathrm{H}), 5.49(\mathrm{dd}, J=2.6,0.8 \mathrm{~Hz}, 1 \mathrm{H}), 4.56(\mathrm{~s}, 2 \mathrm{H}), 2.20-2.05(\mathrm{~m}, 1 \mathrm{H})$, $1.77-1.61(\mathrm{~m}, 4 \mathrm{H}), 1.37-1.04(\mathrm{~m}, 6 \mathrm{H})$.

${ }^{13} \mathrm{C} \mathrm{NMR}\left(101 \mathrm{MHz}, \mathrm{CDCl}_{3}\right) \delta 150.7$ (1C), 135.6 (2C) 132.2 (1C), 129.6 (1C), 127.9 (2C), 127.8 (1C), 45.7 (1C), 33.0 (2C), 26.6 (2C), 26.1 (1C).

HRMS (EI) calcd for [M, $\mathrm{C}_{14} \mathrm{H}_{20} \mathrm{Si}^{+}:$216.1334, found 216.1333.

(E)-phenyl(3-phenylprop-1-en-1-yl)silane (3af) ${ }^{16}$<smiles>[SiH2]C=CCc1ccccc1</smiles>

Procedure A, $101.0 \mathrm{mg}, 90 \%$ yield, r.r. $>98: 2$, colorless oil.

${ }^{1} \mathrm{H} \mathrm{NMR}\left(400 \mathrm{MHz}, \mathrm{CDCl}_{3}\right) \delta 7.56(\mathrm{dd}, J=7.6,1.6 \mathrm{~Hz}, 2 \mathrm{H}), 7.42-7.34(\mathrm{~m}, 3 \mathrm{H}), 7.31$ $(\mathrm{t}, J=7.4 \mathrm{~Hz}, 2 \mathrm{H}), 7.24-7.16(\mathrm{~m}, 3 \mathrm{H}), 6.49(\mathrm{dt}, J=18.3,6.2 \mathrm{~Hz}, 1 \mathrm{H}), 5.77$ (dddd, $J=18.4,4.7,3.1,1.5 \mathrm{~Hz}, 1 \mathrm{H}), 4.54(\mathrm{~d}, J=3.1 \mathrm{~Hz}, 2 \mathrm{H}), 3.52$ (d, $J=$ 
$6.1 \mathrm{~Hz}, 2 \mathrm{H})$.

${ }^{13} \mathrm{C} \mathrm{NMR}\left(101 \mathrm{MHz}, \mathrm{CDCl}_{3}\right) \delta 151.7$ (1C), 139.1 (1C), 135.4 (2C), 131.9 (1C), 129.6

(1C), 128.8 (2C), 128.5 (2C), 128.0 (2C), 126.2 (1C), 121.7 (1C), 43.3 (1C).

HRMS (EI) calcd for [M, $\mathrm{C}_{15} \mathrm{H}_{16} \mathrm{Si}^{+}:$224.1021, found 224.1024.

phenyl(3-phenylprop-1-en-2-yl)silane (4af)

SiH

Procedure A, $102.1 \mathrm{mg}, 91 \%$ yield, r.r. $>98: 2$, colorless oil.

${ }^{1} \mathrm{H}$ NMR $\left(400 \mathrm{MHz}, \mathrm{CDCl}_{3}\right) \delta 7.52-7.43(\mathrm{~m}, 2 \mathrm{H}), 7.42-7.28(\mathrm{~m}, 3 \mathrm{H}), 7.28-7.16$ $(\mathrm{m}, 3 \mathrm{H}), 7.15-7.07(\mathrm{~m}, 2 \mathrm{H}), 5.76-5.71(\mathrm{~m}, 1 \mathrm{H}), 5.66-5.61(\mathrm{~m}, 1 \mathrm{H}), 4.43$ $(\mathrm{s}, 2 \mathrm{H}), 3.51(\mathrm{~s}, 2 \mathrm{H})$.

${ }^{13} \mathrm{C} \mathrm{NMR}\left(101 \mathrm{MHz}, \mathrm{CDCl}_{3}\right) \delta 144.4(1 \mathrm{C}), 139.2(1 \mathrm{C}), 135.6(2 \mathrm{C}), 131.2(1 \mathrm{C}), 130.7$ (1C), 129.7 (1C), 129.2 (2C), 128.3 (2C), 128.0 (2C), 126.2 (1C), 43.6 (1C).

HRMS (EI) calcd for [M, $\mathrm{C}_{15} \mathrm{H}_{16} \mathrm{Si}^{+}:$224.1021, found 224.1024 .

(E)-(3-(4-fluorophenyl)prop-1-en-1-yl)(phenyl)silane (3ag)<smiles>Fc1ccc(C/C=C/[SH2+]c2ccccc2)cc1</smiles>

Procedure A, $105.4 \mathrm{mg}, 87 \%$ yield, r.r. $>98: 2$, colorless oil.

${ }^{1} \mathrm{H}$ NMR $\left(400 \mathrm{MHz}, \mathrm{CDCl}_{3}\right) \delta 7.56(\mathrm{dt}, J=7.5,1.5 \mathrm{~Hz}, 2 \mathrm{H}), 7.45-7.31(\mathrm{~m}, 3 \mathrm{H}), 7.12$ $(\mathrm{dd}, J=8.3,5.5 \mathrm{~Hz}, 2 \mathrm{H}), 6.98(\mathrm{t}, J=8.5 \mathrm{~Hz}, 2 \mathrm{H}), 6.46(\mathrm{dt}, J=18.3,6.1 \mathrm{~Hz}$, 1H), 5.75 (dtd, $J=18.4,3.3,1.6 \mathrm{~Hz}, 1 \mathrm{H}), 4.54(\mathrm{~d}, J=3.2 \mathrm{~Hz}, 2 \mathrm{H}), 3.48$ (d, $J$ $=6.1 \mathrm{~Hz}, 2 \mathrm{H})$.

${ }^{13} \mathrm{C} \mathrm{NMR}\left(101 \mathrm{MHz}, \mathrm{CDCl}_{3}\right) \delta 161.5(\mathrm{~d}, J=243.9 \mathrm{~Hz}, 1 \mathrm{C}), 151.4(1 \mathrm{C}), 135.3(2 \mathrm{C})$, 134.7 (d, $J=3.2 \mathrm{~Hz}, 1 \mathrm{C}), 131.8$ (1C), 130.2 (d, $J=7.7 \mathrm{~Hz}, 2 \mathrm{C}), 129.7$ (1C), 128.0 (2C), 122.0 (1C), 115.2 (d, $J=21.3 \mathrm{~Hz}, 2 \mathrm{C}), 42.3$ (1C).

HRMS (EI) calcd for [M, $\left.\mathrm{C}_{15} \mathrm{H}_{15} \mathrm{FSi}\right]^{+}:$242.0927, found 242.0925. 
(3-(4-fluorophenyl)prop-1-en-2-yl)(phenyl)silane (4ag)<smiles>C=C(Cc1ccc(F)cc1)[SH]Sc1ccccc1</smiles>

Procedure A, $106.6 \mathrm{mg}, 88 \%$ yield, r.r. $>98: 2$, colorless oil.

를 NMR (400 MHz, $\left.\mathrm{CDCl}_{3}\right) \delta 7.47(\mathrm{dt}, J=6.5,1.6 \mathrm{~Hz}, 2 \mathrm{H}), 7.43-7.28(\mathrm{~m}, 3 \mathrm{H}), 7.11$ $-7.00(\mathrm{~m}, 2 \mathrm{H}), 6.99-6.85(\mathrm{~m}, 2 \mathrm{H}), 5.78-5.71(\mathrm{~m}, 1 \mathrm{H}), 5.68-5.57(\mathrm{~m}, 1 \mathrm{H})$, $4.43(\mathrm{~s}, 2 \mathrm{H}), 3.47(\mathrm{~s}, 2 \mathrm{H})$.

${ }^{13} \mathrm{C} \mathrm{NMR}\left(101 \mathrm{MHz}, \mathrm{CDCl}_{3}\right) \delta 161.5(\mathrm{~d}, J=243.9 \mathrm{~Hz}, 1 \mathrm{C}), 144.3(1 \mathrm{C}), 135.5(2 \mathrm{C})$, $134.8(\mathrm{~d}, J=3.2 \mathrm{~Hz}, 1 \mathrm{C}), 131.0(1 \mathrm{C}), 130.8(1 \mathrm{C}), 130.6$ (d, $J=7.8 \mathrm{~Hz}, 2 \mathrm{C})$, 129.7 (1C), 128.0 (2C), 115.0 (d, $J=21.3 \mathrm{~Hz}, 2 \mathrm{C}), 42.8$ (1C).

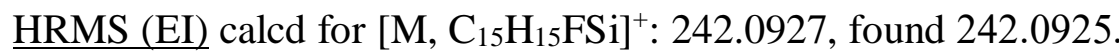

\section{(E)-(3-(4-chlorophenyl)prop-1-en-1-yl)(phenyl)silane (3ah)}<smiles>Clc1ccc(C/C=C/[SnH2]c2ccccc2)cc1</smiles>

Procedure A, $116.5 \mathrm{mg}, 90 \%$ yield, r.r. $>98: 2$, colorless oil.

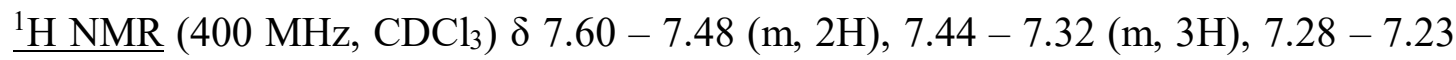
$(\mathrm{m}, 2 \mathrm{H}), 7.15-7.01(\mathrm{~m}, 2 \mathrm{H}), 6.44(\mathrm{dt}, J=18.3,6.1 \mathrm{~Hz}, 1 \mathrm{H}), 5.76(\mathrm{dtt}, J=$ $18.3,3.2,1.6 \mathrm{~Hz}, 1 \mathrm{H}), 4.53(\mathrm{~d}, J=3.2 \mathrm{~Hz}, 2 \mathrm{H}), 3.52-3.43(\mathrm{~m}, 2 \mathrm{H})$.

${ }^{13} \mathrm{C} \mathrm{NMR}\left(101 \mathrm{MHz}, \mathrm{CDCl}_{3}\right) \delta 150.9$ (1C), 137.5 (1C), 135.3 (2C), 132.0 (1C), 131.8 (1C), 130.1 (2C), 129.7 (1C), 128.6 (2C), 128.0 (2C), 122.4 (1C), 42.5 (1C),. HRMS (EI) calcd for [M, $\left.\mathrm{C}_{15} \mathrm{H}_{15} \mathrm{ClSi}\right]^{+}:$258.0632, found 258.0631 .

\section{(3-(4-chlorophenyl)prop-1-en-2-yl)(phenyl)silane (4ah)}<smiles>C=C(Cc1ccc(Cl)cc1)[SH+]c1ccccc1</smiles>

Procedure A, $117.7 \mathrm{mg}, 91 \%$ yield, r.r. > 98:2, colorless oil.

${ }^{1} \mathrm{H}$ NMR (400 MHz, $\left.\mathrm{CDCl}_{3}\right) \delta 7.50-7.43(\mathrm{~m}, 2 \mathrm{H}), 7.42-7.27(\mathrm{~m}, 3 \mathrm{H}), 7.23-7.12$ $(\mathrm{m}, 2 \mathrm{H}), 7.07-6.92(\mathrm{~m}, 2 \mathrm{H}), 5.74(\mathrm{dd}, J=2.7,1.6 \mathrm{~Hz}, 1 \mathrm{H}), 5.64(\mathrm{dd}, J=2.6$, $1.3 \mathrm{~Hz}, 1 \mathrm{H}), 4.43(\mathrm{~s}, 2 \mathrm{H}), 3.46(\mathrm{~s}, 2 \mathrm{H})$. 
${ }^{13} \mathrm{C} \mathrm{NMR}\left(101 \mathrm{MHz}, \mathrm{CDCl}_{3}\right) \delta 144.0$ (1C), 137.6 (1C), 135.5 (2C), 132.0 (1C), 131.0

(1C), 130.9 (1C), 130.5 (2C), 129.8 (1C), 128.4 (2C), 128.0 (2C), 42.9 (1C).

HRMS (EI) calcd for [M, $\mathrm{C}_{15} \mathrm{H}_{15} \mathrm{ClSi}^{+}$: 258.0632, found 258.0634.

\section{(E)-(5-chloropent-1-en-1-yl)(phenyl)silane (3ai)}<smiles>ClCCCC=C[SiH2]c1ccccc1</smiles>

Procedure A, $94.7 \mathrm{mg}, 90 \%$ yield, r.r. $>98: 2$, colorless oil.

${ }^{1} \mathrm{H}$ NMR $\left(400 \mathrm{MHz}, \mathrm{CDCl}_{3}\right) \delta 7.58-7.55(\mathrm{~m}, 2 \mathrm{H}), 7.42-7.34(\mathrm{~m}, 3 \mathrm{H}), 6.31(\mathrm{dt}, J=$ 18.4, $6.3 \mathrm{~Hz}, 1 \mathrm{H}), 5.80$ (ddt, $J=18.5,3.2,1.7 \mathrm{~Hz}, 1 \mathrm{H}), 4.53$ (d, $J=3.1 \mathrm{~Hz}$, 2H), $3.54(\mathrm{t}, J=6.6 \mathrm{~Hz}, 2 \mathrm{H}), 2.34$ (q, $J=6.8 \mathrm{~Hz}, 2 \mathrm{H}), 1.94-1.85(\mathrm{~m}, 2 \mathrm{H})$.

${ }^{13} \mathrm{C} \mathrm{NMR}\left(101 \mathrm{MHz}, \mathrm{CDCl}_{3}\right) \delta 151.4$ (1C), 135.3 (2C), 131.9 (1C), 129.7 (1C), 128.0 (2C), 121.8 (1C), 44.3 (1C), 33.8 (1C), $31.1(1 \mathrm{C})$.

HRMS (EI) calcd for [M, $\left.\mathrm{C}_{11} \mathrm{H}_{15} \mathrm{ClSi}\right]^{+}: 210.0632$, found 210.0637.

\section{(5-chloropent-1-en-2-yl)(phenyl)silane (4ai)}<smiles>C=C(CCCCl)[SH]c1ccccc1</smiles>

Procedure A, $95.9 \mathrm{mg}, 91 \%$ yield, r.r. $>98: 2$, colorless oil.

${ }^{1} \mathrm{H} \mathrm{NMR}\left(400 \mathrm{MHz}, \mathrm{CDCl}_{3}\right) \delta 7.62-7.55(\mathrm{~m}, 2 \mathrm{H}), 7.46-7.33(\mathrm{~m}, 3 \mathrm{H}), 5.83(\mathrm{dt}, J=$ 2.8, $1.5 \mathrm{~Hz}, 1 \mathrm{H}), 5.62(\mathrm{dd}, J=2.5,1.2 \mathrm{~Hz}, 1 \mathrm{H}), 4.55(\mathrm{~s}, 2 \mathrm{H}), 3.49$ (t, $J=6.6$ $\mathrm{Hz}, 2 \mathrm{H}), 2.36(\mathrm{t}, J=7.5 \mathrm{~Hz}, 2 \mathrm{H}), 1.95-1.84(\mathrm{~m}, 2 \mathrm{H})$.

${ }^{13} \mathrm{C} \mathrm{NMR}\left(101 \mathrm{MHz}, \mathrm{CDCl}_{3}\right) \delta 143.4$ (1C), 135.5 (2C), 131.0 (1C), 130.8 (1C), 129.9 (1C), 128.1 (2C), 44.3 (1C), 34.2 (1C), 31.4 (1C).

HRMS (EI) calcd for [M, $\mathrm{C}_{11} \mathrm{H}_{15} \mathrm{ClSi}^{+}:$:210.0632, found 210.0633.

\section{(E)-phenyl(4-(p-tolylthio)but-1-en-1-yl)silane (3aj)}<smiles>Cc1ccc(SCC/C=C/[SH2+]c2ccccc2)cc1</smiles>

Procedure A, $129.4 \mathrm{mg}, 91 \%$ yield, r.r. > 98:2, colorless oil.

$\underline{{ }^{1} \mathrm{H} \mathrm{NMR}}\left(400 \mathrm{MHz}, \mathrm{CDCl}_{3}\right) \delta 7.56(\mathrm{dd}, J=7.7,1.7 \mathrm{~Hz}, 2 \mathrm{H}), 7.37(\mathrm{dd}, J=8.0,2.0 \mathrm{~Hz}$, 3H), $7.29-7.24(\mathrm{~m}, 2 \mathrm{H}), 7.09$ (d, $J=7.9 \mathrm{~Hz}, 2 \mathrm{H}), 6.34(\mathrm{dt}, J=18.5,6.2 \mathrm{~Hz}$, 
1H), 5.79 (ddt, $J=18.5,3.3,1.7 \mathrm{~Hz}, 1 \mathrm{H}), 4.53$ (d, $J=3.2 \mathrm{~Hz}, 2 \mathrm{H}), 2.98-$

$2.93(\mathrm{~m}, 2 \mathrm{H}), 2.47(\mathrm{q}, J=6.8 \mathrm{~Hz}, 1 \mathrm{H}), 2.31(\mathrm{~s}, 3 \mathrm{H})$.

${ }^{13} \mathrm{C} \mathrm{NMR}\left(101 \mathrm{MHz}, \mathrm{CDCl}_{3}\right) \delta 150.8$ (1C), 136.3 (1C), 135.3 (2C), 132.3 (1C), 131.8

(1C), 130.3 (2C), 129.7 (2C+1C), 128.0 (2C), 122.2 (1C), 36.4 (1C), 33.2 (1C), $21.0(1 \mathrm{C})$.

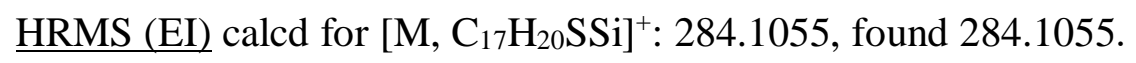

phenyl(4-(p-tolylthio)but-1-en-2-yl)silane (4aj)<smiles>C=C(CCSc1ccc(C)cc1)[SH+]c1ccccc1</smiles>

Procedure A, $128.0 \mathrm{mg}, 90 \%$ yield, r.r. > 98:2, colorless oil.

$\underline{{ }^{1} \mathrm{H} \mathrm{NMR}}\left(400 \mathrm{MHz}, \mathrm{CDCl}_{3}\right) \delta 7.55(\mathrm{~d}, J=6.9 \mathrm{~Hz}, 2 \mathrm{H}), 7.38(\mathrm{dd}, J=15.2,7.4 \mathrm{~Hz}, 3 \mathrm{H})$, $7.21-7.14(\mathrm{~m}, 2 \mathrm{H}), 7.06$ (d, J=7.7 Hz, 2H), 5.83 (s, 1H), 5.63 (s, 1H), 4.56 (s, 2H), $2.93(\mathrm{t}, J=7.9 \mathrm{~Hz}, 2 \mathrm{H}), 2.49(\mathrm{t}, J=7.9 \mathrm{~Hz}, 2 \mathrm{H}), 2.31(\mathrm{~s}, 3 \mathrm{H})$.

${ }^{13} \mathrm{C} \mathrm{NMR}\left(101 \mathrm{MHz}, \mathrm{CDCl}_{3}\right) \delta 143.2$ (1C), 136.1 (1C), 135.6 (2C), 132.4 (1C), 131.2 (1C), 131.0 (1C), 130.0 (2C), 129.9 (1C), 129.6 (2C), 128.1 (2C), 36.9 (1C), 33.5 (1C), $21.0(1 \mathrm{C})$.

HRMS (EI) calcd for [M, $\left.\mathrm{C}_{17} \mathrm{H}_{20} \mathrm{SSi}\right]^{+}: 284.1055$, found 284.1059.

\section{(E)-(5-phenoxypent-1-en-1-yl)(phenyl)silane (3ak)}<smiles>C(=C/Sc1ccccc1)\CCCCOc1ccccc1</smiles>

Procedure A, $124.8 \mathrm{mg}, 93 \%$ yield, r.r. > 98:2, colorless oil.

${ }^{1} \mathrm{H}$ NMR $\left(400 \mathrm{MHz}, \mathrm{CDCl}_{3}\right) \delta 7.61-7.49(\mathrm{~m}, 2 \mathrm{H}), 7.43-7.32(\mathrm{~m}, 3 \mathrm{H}), 7.30-7.24$ $(\mathrm{m}, 3 \mathrm{H}), 6.93(\mathrm{t}, J=7.3 \mathrm{~Hz}, 1 \mathrm{H}), 6.88(\mathrm{~d}, J=8.0 \mathrm{~Hz}, 2 \mathrm{H}), 6.40(\mathrm{dt}, J=18.5$, $6.2 \mathrm{~Hz}, 1 \mathrm{H}), 5.80(\mathrm{~d}, J=18.3 \mathrm{~Hz}, 1 \mathrm{H}), 4.53$ (d, $J=3.1 \mathrm{~Hz}, 2 \mathrm{H}), 3.97$ (t, $J=$ $6.4 \mathrm{~Hz}, 2 \mathrm{H}), 2.38$ (q, $J=7.1 \mathrm{~Hz}, 2 \mathrm{H}), 1.93(\mathrm{p}, J=6.8 \mathrm{~Hz}, 2 \mathrm{H})$.

${ }^{13} \mathrm{C} \mathrm{NMR}\left(101 \mathrm{MHz}, \mathrm{CDCl}_{3}\right) \delta 158.9$ (1C), 152.6 (1C), 135.3 (2C), 132.1 (1C), 129.6 (1C), 129.4 (2C), 128.0 (2C), 121.1 (1C), 120.6 (1C), 114.5 (2C), 67.0 (1C), 33.2 (1C), $28.0(1 \mathrm{C})$.

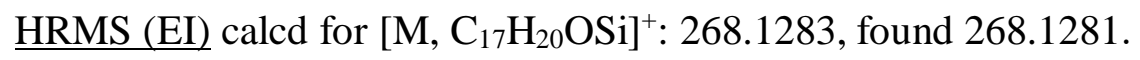




\section{(5-phenoxypent-1-en-2-yl)(phenyl)silane (4ak)}

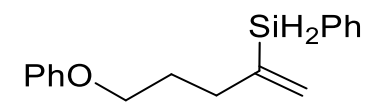

Procedure A, $126.1 \mathrm{mg}, 94 \%$ yield, r.r. > 98:2, colorless oil.

${ }^{1} \mathrm{H}$ NMR $\left(400 \mathrm{MHz}, \mathrm{CDCl}_{3}\right) \delta 7.61-7.56(\mathrm{~m}, 2 \mathrm{H}), 7.44-7.33(\mathrm{~m}, 3 \mathrm{H}), 7.29-7.23$ $(\mathrm{m}, 2 \mathrm{H}), 6.92(\mathrm{t}, J=7.3 \mathrm{~Hz}, 1 \mathrm{H}), 6.86(\mathrm{~d}, J=6.9 \mathrm{~Hz}, 2 \mathrm{H}), 5.83(\mathrm{~s}, 1 \mathrm{H}), 5.60$ $(\mathrm{d}, J=2.7 \mathrm{~Hz}, 1 \mathrm{H}), 4.57(\mathrm{~s}, 2 \mathrm{H}), 3.91(\mathrm{t}, J=6.4 \mathrm{~Hz}, 2 \mathrm{H}), 2.39(\mathrm{t}, J=7.6 \mathrm{~Hz}$, $2 \mathrm{H}), 1.97-1.86(\mathrm{~m}, 2 \mathrm{H})$.

${ }^{13} \mathrm{C} \mathrm{NMR}\left(101 \mathrm{MHz}, \mathrm{CDCl}_{3}\right) \delta 159.0$ (1C), 144.2 (1C), $135.6(2 \mathrm{C}), 131.3$ (1C), 130.2 (1C), 129.8 (1C), 129.4 (2C), 128.1 (2C), 120.5 (1C), 114.5 (2C), 67.0 (1C), $33.4(1 \mathrm{C}), 28.3(1 \mathrm{C})$.

HRMS (EI) calcd for [M, $\left.\mathrm{C}_{17} \mathrm{H}_{20} \mathrm{OSi}\right]^{+}:$268.1283, found 268.1284.

\section{(E)-2-methyl-8-((5-(phenylsilyl)pent-4-en-1-yl)oxy)quinolone (3al)}<smiles>Cc1ccc2cccc(OCCC/C=C/[SH2+]c3ccccc3)c2n1</smiles>

Procedure A, 150.0 mg, 90\% yield, r.r. $>98: 2$, colorless oil.

를 NMR $\left(400 \mathrm{MHz}, \mathrm{CDCl}_{3}\right) \delta 7.98(\mathrm{~d}, J=8.4 \mathrm{~Hz}, 1 \mathrm{H}), 7.60-7.50(\mathrm{~m}, 2 \mathrm{H}), 7.41-$ $7.24(\mathrm{~m}, 6 \mathrm{H}), 7.02(\mathrm{dd}, J=7.2,1.9 \mathrm{~Hz}, 1 \mathrm{H}), 6.45(\mathrm{dt}, J=18.5,6.2 \mathrm{~Hz}, 1 \mathrm{H})$, $5.82(\mathrm{ddt}, J=18.4,3.3,1.7 \mathrm{~Hz}, 1 \mathrm{H}), 4.54(\mathrm{~d}, J=3.1 \mathrm{~Hz}, 2 \mathrm{H}), 4.25(\mathrm{t}, J=7.0$ $\mathrm{Hz}, 2 \mathrm{H}), 2.77(\mathrm{~s}, 3 \mathrm{H}), 2.45(\mathrm{q}, J=7.1 \mathrm{~Hz}, 2 \mathrm{H}), 2.17(\mathrm{p}, J=7.2 \mathrm{~Hz}, 2 \mathrm{H})$.

${ }^{13} \mathrm{C} \mathrm{NMR}\left(101 \mathrm{MHz}, \mathrm{CDCl}_{3}\right) \delta 158.0$ (1C), 154.2 (1C), 152.6 (1C), 139.9 (1C), 135.9

(1C), 135.3 (2C), 132.0 (1C), $129.6(1 \mathrm{C}), 127.9$ (2C), 127.6 (1C), 125.6

(1C), 122.4 (1C), 121.0 (1C), 119.4 (1C), 109.1 (1C), 68.3 (1C), 33.2 (1C), $27.5(1 \mathrm{C}), 25.8(1 \mathrm{C})$.

HRMS (EI) calcd for [M, $\left.\mathrm{C}_{21} \mathrm{H}_{23} \mathrm{NOSi}\right]^{+}: 333.1549$, found 333.1548 . 
2-methyl-8-((4-(phenylsilyl)pent-4-en-1-yl)oxy)quinolone (4al)<smiles>C=C(CCCOc1cccc2ccc(C)nc12)Sc1ccccc1</smiles>

Procedure A, $155.1 \mathrm{mg}, 93 \%$ yield, r.r. > 98:2, colorless oil.

$\underline{{ }^{1} \mathrm{H} \text { NMR }}\left(400 \mathrm{MHz}, \mathrm{CDCl}_{3}\right) \delta 7.98(\mathrm{~d}, J=8.4 \mathrm{~Hz}, 1 \mathrm{H}), 7.59(\mathrm{~d}, J=7.2 \mathrm{~Hz}, 2 \mathrm{H}), 7.42$ $-7.22(\mathrm{~m}, 6 \mathrm{H}), 6.97(\mathrm{~d}, J=7.0 \mathrm{~Hz}, 1 \mathrm{H}), 5.87(\mathrm{~s}, 1 \mathrm{H}), 5.61(\mathrm{~s}, 1 \mathrm{H}), 4.59$ (s, 2H), 4.19 (t, $J=7.0 \mathrm{~Hz}, 2 \mathrm{H}), 2.77(\mathrm{~s}, 3 \mathrm{H}), 2.45$ (t, $J=7.7 \mathrm{~Hz}, 2 \mathrm{H}), 2.18$ (p, $J$ $=7.3 \mathrm{~Hz}, 2 \mathrm{H})$.

${ }^{13} \mathrm{C} \mathrm{NMR}\left(101 \mathrm{MHz}, \mathrm{CDCl}_{3}\right) \delta 158.0$ (1C), 154.2 (1C), 144.1 (1C), 139.9 (1C), 135.9 (1C), 135.5 (2C), $131.2(1 \mathrm{C}), 130.2$ (1C), 129.7 (1C), $128.0(2 \mathrm{C}), 127.6(1 \mathrm{C})$, 125.6 (1C), 122.4 (1C), 119.3 (1C), 109.1 (1C), 68.4 (1C), 33.3 (1C), 27.8 (1C), 25.8 (1C).

HRMS (EI) calcd for [M, $\left.\mathrm{C}_{21} \mathrm{H}_{23} \mathrm{NOSi}\right]^{+}: 333.1549$, found 333.1550.

\section{(E)-(5-(pent-4-en-1-yloxy)pent-1-en-1-yl)(phenyl)silane (3am)}<smiles>C=CCCCOCCC/C=C/[SH-]c1ccccc1</smiles>

Procedure A, $118.4 \mathrm{mg}, 91 \%$ yield, r.r. > 98:2, colorless oil.

$\underline{{ }^{1} \mathrm{H} \mathrm{NMR}}\left(400 \mathrm{MHz}, \mathrm{CDCl}_{3}\right) \delta 7.60-7.51(\mathrm{~m}, 2 \mathrm{H}), 7.43-7.30(\mathrm{~m}, 3 \mathrm{H}), 6.36(\mathrm{dt}, J=$ $18.4,6.3 \mathrm{~Hz}, 1 \mathrm{H}), 5.88-5.69(\mathrm{~m}, 2 \mathrm{H}), 5.07-4.91(\mathrm{~m}, 2 \mathrm{H}), 4.53(\mathrm{~d}, J=3.2$ $\mathrm{Hz}, 2 \mathrm{H}), 3.41(\mathrm{td}, J=6.5,3.4 \mathrm{~Hz}, 4 \mathrm{H}), 2.26(\mathrm{q}, J=7.1 \mathrm{~Hz}, 2 \mathrm{H}), 2.11(\mathrm{q}, J=$ $7.2 \mathrm{~Hz}, 2 \mathrm{H}), 1.77-1.61(\mathrm{~m}, 4 \mathrm{H})$.

${ }^{13} \mathrm{C} \mathrm{NMR}\left(101 \mathrm{MHz}, \mathrm{CDCl}_{3}\right) \delta 153.2$ (1C), 138.3 (1C), 135.3 (2C), $132.2(1 \mathrm{C}), 129.6$ (1C), 128.0 (2C), 120.4 (1C), 114.7 (1C), 70.1 (1C), 70.0 (1C), 33.4 (1C), 30.3 (1C), 28.9 (1C), 28.4 (1C).

HRMS (EI) calcd for [M-H, $\left.\mathrm{C}_{16} \mathrm{H}_{23} \mathrm{OSi}\right]^{+}:$259.1513, found 259.1512. 
(5-(pent-4-en-1-yloxy)pent-1-en-2-yl)(phenyl)silane (4am)<smiles>C=CCCCOCCCC(=C)[SiH2]c1ccccc1</smiles>

Procedure A, $117.0 \mathrm{mg}, 90 \%$ yield, r.r. $>98: 2$, colorless oil

$\underline{{ }^{1} \mathrm{H} \text { NMR }}\left(400 \mathrm{MHz}, \mathrm{CDCl}_{3}\right) \delta 7.61-7.54(\mathrm{~m}, 2 \mathrm{H}), 7.43-7.33(\mathrm{~m}, 3 \mathrm{H}), 5.87-5.75$

$(\mathrm{m}, 2 \mathrm{H}), 5.56(\mathrm{dd}, J=2.7,1.2 \mathrm{~Hz}, 1 \mathrm{H}), 5.06-4.92(\mathrm{~m}, 2 \mathrm{H}), 4.55(\mathrm{~s}, 2 \mathrm{H})$,

$3.36(\mathrm{td}, J=6.6,1.1 \mathrm{~Hz}, 4 \mathrm{H}), 2.27(\mathrm{t}, J=7.8 \mathrm{~Hz}, 2 \mathrm{H}), 2.15-2.05(\mathrm{~m}, 2 \mathrm{H})$,

$1.76-1.59(\mathrm{~m}, 4 \mathrm{H})$.

${ }^{13} \mathrm{C} \mathrm{NMR}\left(101 \mathrm{MHz}, \mathrm{CDCl}_{3}\right) \delta 144.6$ (1C), 138.3 (1C), 135.5 (2C), 131.4 (1C), 129.8

(1C), 129.7 (1C), 128.0 (2C), 114.6 (1C), 70.1 (2C), 33.6 (1C), 30.3 (1C), 28.9 (1C), $28.7(1 \mathrm{C})$.

HRMS (EI) calcd for [M-H, $\left.\mathrm{C}_{16} \mathrm{H}_{23} \mathrm{OSi}\right]^{+}:$259.1513, found 259.1515.

(E)-phenyl(4-((4-(prop-1-en-2-yl)cyclohex-1-en-1-yl)methoxy)but-1-en-1-yl)silane (3an)

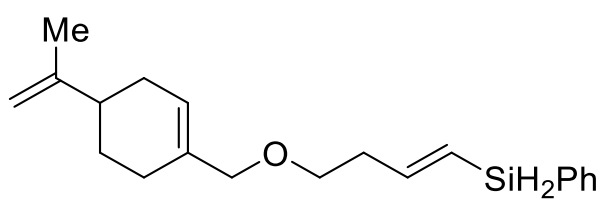

Procedure A, $135.9 \mathrm{mg}, 87 \%$ yield, r.r. > 98:2, colorless oil.

${ }^{1} \mathrm{H}$ NMR $\left(400 \mathrm{MHz}, \mathrm{CDCl}_{3}\right) \delta 7.61-7.51(\mathrm{~m}, 2 \mathrm{H}), 7.43-7.31(\mathrm{~m}, 3 \mathrm{H}), 6.36(\mathrm{dt}, J=$ 18.5, $6.3 \mathrm{~Hz}, 1 \mathrm{H}), 5.82(\mathrm{~d}, J=18.5 \mathrm{~Hz} 1 \mathrm{H}), 5.69(\mathrm{~d}, J=4.4 \mathrm{~Hz}, 1 \mathrm{H}), 4.72(\mathrm{~d}$, $J=5.4 \mathrm{~Hz}, 2 \mathrm{H}), 4.53(\mathrm{~d}, J=3.1 \mathrm{~Hz}, 2 \mathrm{H}), 3.84(\mathrm{~s}, 2 \mathrm{H}), 3.47(\mathrm{t}, J=6.7 \mathrm{~Hz}$, 2H), $2.47(\mathrm{q}, J=6.6 \mathrm{~Hz}, 2 \mathrm{H}), 2.17-1.80(\mathrm{~m}, 6 \mathrm{H}), 1.74(\mathrm{~s}, 3 \mathrm{H}), 1.47(\mathrm{qd}, J$ $=11.4,6.2 \mathrm{~Hz}, 1 \mathrm{H})$.

${ }^{13} \mathrm{C} \mathrm{NMR}\left(101 \mathrm{MHz}, \mathrm{CDCl}_{3}\right) \delta 150.1$ (1C), 149.9 (1C), 135.4 (2C), 134.7 (1C), 132.1 (1C), 129.6 (1C), 128.0 (2C), 124.3 (1C), 122.4 (1C), 108.6 (1C), 75.2 (1C), 68.5 (1C), 41.1 (1C), 37.2 (1C), 30.5 (1C), 27.5 (1C), 26.4 (1C), 20.8 (1C). HRMS (EI) calcd for [M, $\left.\mathrm{C}_{20} \mathrm{H}_{28} \mathrm{OSi}\right]^{+}: 312.1909$, found 312.1910. 
Phenyl(4-((4-(prop-1-en-2-yl)cyclohex-1-en-1-yl)methoxy)but-1-en-2-yl)silane (4an)<smiles>C=C(CCOCC1=CCC(C(=C)C)CC1)[SnH2]c1ccccc1</smiles>

Procedure A, $140.4 \mathrm{mg}, 90 \%$ yield, r.r. $>98: 2$, colorless oil.

래 NMR (400 MHz, $\left.\mathrm{CDCl}_{3}\right) \delta 7.58(\mathrm{~d}, J=6.9 \mathrm{~Hz}, 2 \mathrm{H}), 7.37(\mathrm{~d}, J=7.6 \mathrm{~Hz}, 3 \mathrm{H}), 5.86$ (s, 1H), $5.65(\mathrm{~s}, 1 \mathrm{H}), 5.61(\mathrm{~s}, 1 \mathrm{H}), 4.71(\mathrm{~d}, J=5.5 \mathrm{~Hz}, 2 \mathrm{H}), 4.55(\mathrm{~d}, J=2.4$ $\mathrm{Hz}, 2 \mathrm{H}), 3.77$ (s, 2H), $3.42(\mathrm{t}, J=6.9 \mathrm{~Hz}, 2 \mathrm{H}), 2.50(\mathrm{t}, J=7.2 \mathrm{~Hz}, 2 \mathrm{H}), 2.18$ $-1.79(\mathrm{~m}, 6 \mathrm{H}), 1.73(\mathrm{~s}, 3 \mathrm{H}), 1.52-1.38(\mathrm{~m}, 1 \mathrm{H})$.

${ }^{13} \mathrm{C}$ NMR $\left(101 \mathrm{MHz}, \mathrm{CDCl}_{3}\right) \delta 149.9$ (1C), 142.1 (1C), 135.6 (2C), 134.7 (1C), 131.6 (1C), 129.7 (1C), 128.0 (2C), 127.8 (1C), 124.2 (1C), 108.6 (1C), 75.1 (1C), 69.0 (1C), 41.1 (1C), 37.7 (1C), 30.5 (1C), 27.5 (1C), 26.4 (1C), 20.8 (1C).

HRMS (EI) calcd for [M, $\left.\mathrm{C}_{20} \mathrm{H}_{28} \mathrm{OSi}\right]^{+}:$312.1909, found 312.1912.

(E)-(4-(1,3-dioxolan-2-yl)but-1-en-1-yl)(phenyl)silane (3ao)<smiles>C(=C/Sc1ccccc1)\CCC1OCCO1</smiles>

Procedure A, $109.1 \mathrm{mg}, 93 \%$ yield, r.r. > 98:2, colorless oil.

$\underline{{ }^{1} \mathrm{H} \text { NMR }}\left(400 \mathrm{MHz}, \mathrm{CDCl}_{3}\right) \delta 7.60-7.52(\mathrm{~m}, 2 \mathrm{H}), 7.43-7.32(\mathrm{~m}, 3 \mathrm{H}), 6.38(\mathrm{dt}, J=$ 18.4, 6.2 Hz, 1H), 5.78 (dtt, $J=18.5,3.3,1.5 \mathrm{~Hz}, 1 \mathrm{H}), 4.89$ (t, $J=4.7 \mathrm{~Hz}$, $1 \mathrm{H}), 4.53(\mathrm{~d}, J=3.3 \mathrm{~Hz}, 2 \mathrm{H}), 4.01-3.91(\mathrm{~m}, 2 \mathrm{H}), 3.90-3.81(\mathrm{~m}, 2 \mathrm{H}), 2.36$ $-2.28(\mathrm{~m}, 2 \mathrm{H}), 1.86-1.75(\mathrm{~m}, 2 \mathrm{H})$.

${ }^{13} \mathrm{C} \mathrm{NMR}\left(101 \mathrm{MHz}, \mathrm{CDCl}_{3}\right) \delta 152.5$ (1C), 135.3 (2C), 132.1 (1C), 129.6 (1C), 128.0 (2C), 120.5 (1C), 103.8 (1C), 64.9 (2C), 32.5 (1C), 31.1 (1C).

HRMS (EI) calcd for [M, $\left.\mathrm{C}_{13} \mathrm{H}_{18} \mathrm{O}_{2} \mathrm{Si}\right]^{+}:$234.1076, found 234.1074 .

(4-(1,3-dioxolan-2-yl)but-1-en-2-yl)(phenyl)silane (4ao)<smiles>C=C(CCC1OCCO1)[SnH2]c1ccccc1</smiles>

Procedure A, $106.8 \mathrm{mg}, 91 \%$ yield, r.r. > 98:2, colorless oil. 
${ }^{1} \mathrm{H} \mathrm{NMR}\left(400 \mathrm{MHz}, \mathrm{CDCl}_{3}\right) \delta 7.57(\mathrm{dt}, J=6.4,1.7 \mathrm{~Hz}, 2 \mathrm{H}), 7.42-7.32(\mathrm{~m}, 3 \mathrm{H}), 5.82$ $(\mathrm{q}, J=1.9 \mathrm{~Hz}, 1 \mathrm{H}), 5.57(\mathrm{dd}, J=2.6,1.3 \mathrm{~Hz}, 1 \mathrm{H}), 4.83(\mathrm{t}, J=4.7 \mathrm{~Hz}, 1 \mathrm{H})$, $4.55(\mathrm{~s}, 2 \mathrm{H}), 3.97-3.87(\mathrm{~m}, 2 \mathrm{H}), 3.86-3.77(\mathrm{~m}, 2 \mathrm{H}), 2.40-2.28(\mathrm{~m}, 2 \mathrm{H})$, $1.86-1.73(\mathrm{~m}, 2 \mathrm{H})$.

${ }^{13} \mathrm{C} \mathrm{NMR}\left(101 \mathrm{MHz}, \mathrm{CDCl}_{3}\right) \delta 144.2$ (1C), 135.5 (2C), 131.2 (1C), 129.7 (2C), 128.0 (2C), 104.0 (1C), 64.8 (2C), 33.0 (1C), 31.3 (1C).

HRMS (EI) calcd for [M, $\left.\mathrm{C}_{13} \mathrm{H}_{18} \mathrm{O}_{2} \mathrm{Si}\right]^{+}:$234.1076, found 234.1078

\section{(E)-(3-(2,2-dimethyl-1,3-dioxan-5-yl)prop-1-en-1-yl)(phenyl)silane (3ap)}

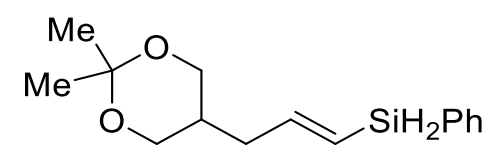

Procedure A, $115.6 \mathrm{mg}, 88 \%$ yield, r.r. $>98: 2$, colorless oil.

를 NMR (400 MHz, $\left.\mathrm{CDCl}_{3}\right) \delta 7.55(\mathrm{dt}, J=6.3,1.7 \mathrm{~Hz}, 2 \mathrm{H}), 7.44-7.31(\mathrm{~m}, 3 \mathrm{H}), 6.25$ $(\mathrm{dt}, J=18.4,6.7 \mathrm{~Hz}, 1 \mathrm{H}), 5.79(\mathrm{dtt}, J=18.3,3.1,1.5 \mathrm{~Hz}, 1 \mathrm{H}), 4.52(\mathrm{~d}, J=$ $3.2 \mathrm{~Hz}, 2 \mathrm{H}), 3.92-3.83(\mathrm{~m}, 2 \mathrm{H}), 3.63-3.55(\mathrm{~m}, 2 \mathrm{H}), 2.19-2.11(\mathrm{~m}, 2 \mathrm{H})$, $1.97-1.88(\mathrm{~m}, 1 \mathrm{H}), 1.42(\mathrm{~s}, 3 \mathrm{H}), 1.41$ (s, $3 \mathrm{H})$.

${ }^{13} \mathrm{C} \mathrm{NMR}\left(101 \mathrm{MHz}, \mathrm{CDCl}_{3}\right) \delta 149.8$ (1C), 135.3 (2C), 131.7 (1C), 129.7 (1C), 128.0 (2C), 123.2 (1C), 97.8 (1C), 64.4 (2C), 36.3 (1C), 33.5 (1C), 26.6 (1C), 21.3 (1C).

$\underline{\mathrm{HRMS}(\mathrm{EI})}$ calcd for [M, $\mathrm{C}_{15} \mathrm{H}_{22} \mathrm{O}_{2} \mathrm{Si}^{+}:$: 262.1389, found 262.1388 .

\section{(3-(2,2-dimethyl-1,3-dioxan-5-yl)prop-1-en-2-yl)(phenyl)silane (4ap)}

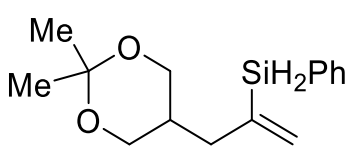

Procedure A, $112.8 \mathrm{mg}, 86 \%$ yield, r.r. $>98: 2$, colorless oil.

${ }^{1} \mathrm{H}$ NMR $\left(400 \mathrm{MHz}, \mathrm{CDCl}_{3}\right) \delta 7.56(\mathrm{~d}, J=7.0 \mathrm{~Hz}, 2 \mathrm{H}), 7.45-7.35(\mathrm{~m}, 3 \mathrm{H}), 5.80(\mathrm{~s}$, 1H), $5.61(\mathrm{~s}, 1 \mathrm{H}), 4.55(\mathrm{~s}, 2 \mathrm{H}), 3.81(\mathrm{dd}, J=11.7,4.5 \mathrm{~Hz}, 2 \mathrm{H}), 3.52$ (dd, $J=$ 11.6, $8.8 \mathrm{~Hz}, 2 \mathrm{H}), 2.13(\mathrm{~d}, J=7.4 \mathrm{~Hz}, 2 \mathrm{H}), 2.06-1.94(\mathrm{~m}, 1 \mathrm{H}), 1.40$ (d, $J=$ $4.5 \mathrm{~Hz}, 6 \mathrm{H})$.

${ }^{13} \mathrm{C} \mathrm{NMR}\left(101 \mathrm{MHz}, \mathrm{CDCl}_{3}\right) \delta 141.9$ (1C), 135.6 (2C), 131.7 (1C), $130.8(1 \mathrm{C}), 130.0$ 
(1C), 128.2 (2C), 97.8 (1C), 64.5 (2C), 36.7 (1C), 32.9 (1C), 26.8 (1C), 21.1 (1C).

HRMS (EI) calcd for [M, $\mathrm{C}_{15} \mathrm{H}_{22} \mathrm{O}_{2} \mathrm{Si}^{+}$: 262.1389, found 262.1387.

$((E)-4-(((3 S, 8 S, 9 S, 10 R, 13 R, 14 S, 17 R)-10,13-d i m e t h y l-17-((R)-6-m e t h y l h e p t a n-2-$ yl)-2,3,4,7,8,9,10,11,12,13,14,15,16,17-tetradecahydro-1Hcyclopenta[a]phenanthren-3-yl)oxy)but-1-en-1-yl)(phenyl)silane (3aq)<smiles>CC(C)CCCCC1CC[C@H]2[C@H]3CC=C4C[C@@H](OCC/C=C/Sc5ccccc5)CCC4(C)[C@H]3CC[C@]12C</smiles>

Procedure A, $248.7 \mathrm{mg}, 91 \%$ yield, r.r. $>98: 2$, white solid, melting point: $78-80{ }^{\circ} \mathrm{C}$. ${ }^{1} \mathrm{H}$ NMR $\left(400 \mathrm{MHz}, \mathrm{CDCl}_{3}\right) \delta 7.58-7.53(\mathrm{~m}, 2 \mathrm{H}), 7.42-7.32(\mathrm{~m}, 3 \mathrm{H}), 6.36(\mathrm{dt}, J=$ 18.5, 6.2 Hz, 1H), 5.82 (d, $J=18.5 \mathrm{~Hz}, 1 \mathrm{H}), 5.36-5.31(\mathrm{~m}, 1 \mathrm{H}), 4.53$ (d, $J$ $=3.2 \mathrm{~Hz}, 2 \mathrm{H}), 3.55(\mathrm{td}, J=6.9,1.4 \mathrm{~Hz}, 2 \mathrm{H}), 3.14(\mathrm{tt}, J=11.2,4.4 \mathrm{~Hz}, 1 \mathrm{H})$, $2.45(\mathrm{q}, J=6.7 \mathrm{~Hz}, 2 \mathrm{H}), 2.38-2.31(\mathrm{~m}, 1 \mathrm{H}), 2.24-2.14(\mathrm{~m}, 1 \mathrm{H}), 2.04-$ $1.92(\mathrm{~m}, 2 \mathrm{H}), 1.91-1.78(\mathrm{~m}, 3 \mathrm{H}), 1.63-1.02(\mathrm{~m}, 24 \mathrm{H}), 0.92(\mathrm{~d}, J=6.5 \mathrm{~Hz}$, $3 \mathrm{H}), 0.87(\mathrm{~d}, J=6.6 \mathrm{~Hz}, 3 \mathrm{H}), 0.86(\mathrm{~d}, J=6.6 \mathrm{~Hz}, 3 \mathrm{H}), 0.68(\mathrm{~s}, 3 \mathrm{H})$.

${ }^{13} \mathrm{C} \mathrm{NMR}\left(100 \mathrm{MHz}, \mathrm{CDCl}_{3}\right) \delta 150.1,141.0,135.4,132.0,129.6,128.0,122.3,121.5$, 79.1, 66.8, 56.8, 56.2, 50.2, 42.3, 39.8, 39.5, 39.1, 37.6, 37.3, 36.9, 36.2, 35.8, $32.0,31.9,28.4,28.2,28.0,24.3,23.8,22.8,22.6,21.1,19.4,18.7,11.9$.

HRMS (ESI) calcd for [M+H, $\left.\mathrm{C}_{37} \mathrm{H}_{59} \mathrm{OSi}\right]^{+}:$547.4330, found 547.4332.

(4-(((3S,8S,9S,10R,13R,14S,17R)-10,13-dimethyl-17-((R)-6-methylheptan-2-yl)-

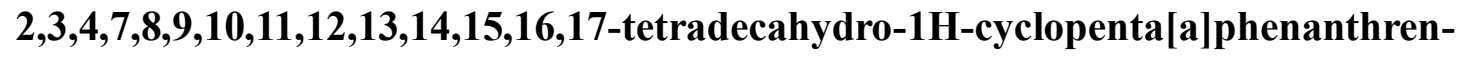
3-yl)oxy)but-1-en-2-yl)(phenyl)silane (4aq)

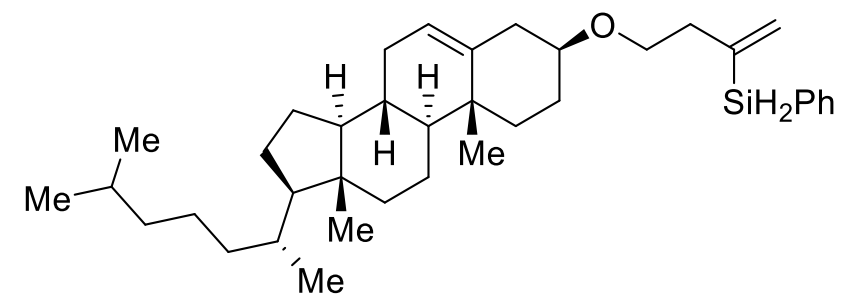


Procedure A, $248.9 \mathrm{mg}, 91 \%$ yield, r.r. $>98: 2$, white solid, melting point: $74-77^{\circ} \mathrm{C}$. ${ }^{1} \mathrm{H}$ NMR $\left(400 \mathrm{MHz}, \mathrm{CDCl}_{3}\right) \delta 7.61-7.54(\mathrm{~m}, 2 \mathrm{H}), 7.43-7.33(\mathrm{~m}, 3 \mathrm{H}), 5.85(\mathrm{~s}, 1 \mathrm{H})$, $5.60(\mathrm{~d}, J=2.8 \mathrm{~Hz}, 1 \mathrm{H}), 5.32(\mathrm{dd}, J=4.9,2.3 \mathrm{~Hz}, 1 \mathrm{H}), 4.56(\mathrm{~s}, 2 \mathrm{H}), 3.51(\mathrm{t}$, $J=7.1 \mathrm{~Hz}, 2 \mathrm{H}), 3.09(\mathrm{tt}, J=11.3,4.3 \mathrm{~Hz}, 1 \mathrm{H}), 2.48(\mathrm{t}, J=6.9 \mathrm{~Hz}, 2 \mathrm{H}), 2.31$ $(\mathrm{dd}, J=13.1,3.0 \mathrm{~Hz}, 1 \mathrm{H}), 2.19-2.09(\mathrm{~m}, 1 \mathrm{H}), 1.98(\mathrm{tt}, J=18.0,2.9 \mathrm{~Hz}, 2 \mathrm{H})$, $1.90-1.75(\mathrm{~m}, 3 \mathrm{H}), 1.65-0.95(\mathrm{~m}, 24 \mathrm{H}), 0.91(\mathrm{~d}, J=6.5 \mathrm{~Hz}, 3 \mathrm{H}), 0.86(\mathrm{dd}$, $J=6.6,1.8 \mathrm{~Hz}, 6 \mathrm{H}), 0.67(\mathrm{~s}, 3 \mathrm{H})$.

${ }^{13} \mathrm{C} \mathrm{NMR}\left(101 \mathrm{MHz}, \mathrm{CDCl}_{3}\right) \delta 142.1,141.0,135.6,131.6,131.5,129.7,128.0,121.4$, 79.1, 67.2, 56.7, 56.1, 50.2, 42.3, 39.7, 39.5, 39.0, 38.1, 37.2, 36.9, 36.2, 35.8, $31.9,31.8,28.3,28.2,28.0,24.3,23.8,22.8,22.6,21.0,19.4,18.7,11.8$. HRMS (ESI) calcd for [M+H, $\left.\mathrm{C}_{37} \mathrm{H}_{59} \mathrm{OSi}\right]^{+}:$547.4330, found 547.4337.

\section{(1E,7E)-1,8-bis(phenylsilyl)octa-1,7-diene (3ar)}

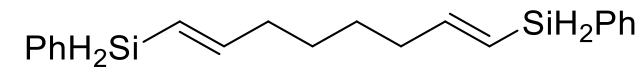

Procedure A; 2.2 equiv 2a (1.1 mmol) was used; $150.0 \mathrm{mg}, 93 \%$ yield, r.r. $>$ 98:2, colorless oil.

${ }^{1} \mathrm{H}$ NMR $\left(400 \mathrm{MHz}, \mathrm{CDCl}_{3}\right) \delta 7.64-7.50(\mathrm{~m}, 4 \mathrm{H}), 7.45-7.27(\mathrm{~m}, 6 \mathrm{H}), 6.34(\mathrm{dt}, J=$ 18.4, $6.3 \mathrm{~Hz}, 2 \mathrm{H}), 5.72$ (dt, $J=18.6,3.4 \mathrm{~Hz}, 2 \mathrm{H}), 4.53(\mathrm{~d}, J=3.1 \mathrm{~Hz}, 4 \mathrm{H})$, $2.18(\mathrm{q}, J=6.3 \mathrm{~Hz}, 4 \mathrm{H}), 1.50-1.40(\mathrm{~m}, 4 \mathrm{H})$.

${ }^{13} \mathrm{C} \mathrm{NMR}\left(101 \mathrm{MHz}, \mathrm{CDCl}_{3}\right) \delta 153.7$ (2C), 135.3 (4C), 132.3 (2C), 129.6 (2C), 128.0 (4C), 120.2 (2C), 36.7 (2C), 27.9 (2C).

HRMS (EI) calcd for [M, $\left.\mathrm{C}_{20} \mathrm{H}_{26} \mathrm{Si}_{2}\right]^{+}:$322.1573, found 322.1576.

octa-1,7-diene-2,7-diylbis(phenylsilane) (4ar)<smiles>C=C([Hg])CCCCC(=C)[SnH2]c1ccccc1</smiles>

Procedure A; 2.2 equiv 2a (1.1 mmol) was used; $148.2 \mathrm{mg}, 92 \%$ yield, r.r. $>$ 98:2, colorless oil. 
${ }^{1} \mathrm{H} \mathrm{NMR}\left(400 \mathrm{MHz}, \mathrm{CDCl}_{3}\right) \delta 7.66-7.52(\mathrm{~m}, 4 \mathrm{H}), 7.42-7.30(\mathrm{~m}, 6 \mathrm{H}), 5.73(\mathrm{~d}, J=$ $2.5 \mathrm{~Hz}, 2 \mathrm{H}), 5.52(\mathrm{~d}, J=2.9 \mathrm{~Hz}, 2 \mathrm{H}), 4.52(\mathrm{~s}, 4 \mathrm{H}), 2.17(\mathrm{~d}, J=6.6 \mathrm{~Hz}, 4 \mathrm{H})$, $1.45-1.33(\mathrm{~m}, 4 \mathrm{H})$.

${ }^{13} \mathrm{C} \mathrm{NMR}\left(101 \mathrm{MHz}, \mathrm{CDCl}_{3}\right) \delta 145.1$ (2C), 135.5 (4C), 131.6 (2C), 129.7 (2C), 129.6 (2C), $128.0(4 \mathrm{C}), 37.1(2 \mathrm{C}), 28.3$ (2C).

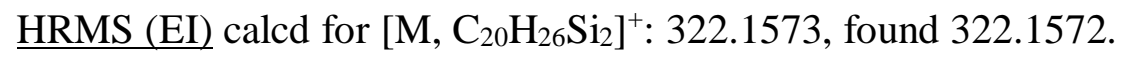

(E)-phenyl(styryl)silane (3ba) ${ }^{16}$<smiles>C(=C/c1ccccc1)\[SiH2]c1ccccc1</smiles>

Procedure B, $96.5 \mathrm{mg}, 92 \%$ yield, r.r. $>98: 2$, colorless oil.

${ }^{1} \mathrm{H}$ NMR $\left(400 \mathrm{MHz}, \mathrm{CDCl}_{3}\right) \delta 7.64-7.61(\mathrm{~m}, 2 \mathrm{H}), 7.48-7.26(\mathrm{~m}, 8 \mathrm{H}), 7.16(\mathrm{~d}, J=$ $19.0 \mathrm{~Hz}, 1 \mathrm{H}), 6.51$ (dt, $J=19.0,3.3 \mathrm{~Hz}, 1 \mathrm{H}), 4.70(\mathrm{~d}, J=3.2 \mathrm{~Hz}, 2 \mathrm{H})$.

${ }^{13} \mathrm{C} \mathrm{NMR}\left(101 \mathrm{MHz}, \mathrm{CDCl}_{3}\right) \delta 149.3$ (1C), 137.7 (1C), 135.5 (2C), 131.6 (1C), 129.8 (1C), $128.6(3 \mathrm{C}), 128.1(2 \mathrm{C}), 126.7$ (2C), $119.4(1 \mathrm{C})$.

HRMS (EI) calcd for [M, $\left.\mathrm{C}_{14} \mathrm{H}_{14} \mathrm{Si}\right]^{+}: 210.0865$, found 210.0867.

\section{phenyl(1-phenylvinyl)silane (4ba) ${ }^{17}$}<smiles>C=C([SH2+]c1ccccc1)c1ccccc1</smiles>

Procedure B, $96.8 \mathrm{mg}, 92 \%$ yield, r.r. $=96: 4$ (determined by ${ }^{1} \mathrm{H}$ NMR of crude product through the peak area ratio of $=\mathrm{C}_{2} /=\mathrm{C} \underline{H S i}$ ), colorless oil.

$\underline{{ }^{1} \mathrm{H} \mathrm{NMR}}\left(400 \mathrm{MHz}, \mathrm{CDCl}_{3}\right) \delta 7.60-7.56(\mathrm{~m}, 2 \mathrm{H}), 7.43-7.22(\mathrm{~m}, 8 \mathrm{H}), 6.23(\mathrm{~s}, 1 \mathrm{H})$, $5.83(\mathrm{~s}, 1 \mathrm{H}), 4.83(\mathrm{~s}, 2 \mathrm{H})$.

${ }^{13} \mathrm{C} \mathrm{NMR}\left(101 \mathrm{MHz}, \mathrm{CDCl}_{3}\right) \delta 144.2$ (1C), 142.3 (1C), 135.6 (2C), 131.3 (1C), 131.2 (1C), 129.9 (1C), 128.5 (2C), 128.1 (2C), 127.3 (1C), 126.5 (2C).

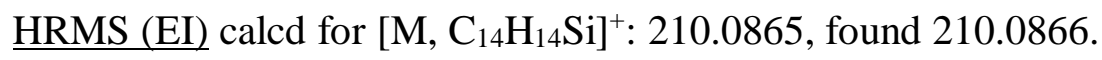




\section{(E)-(2-methylstyryl)(phenyl)silane (3bb) $)^{16}$}<smiles>Cc1ccccc1/C=C/[SnH2]c1ccccc1</smiles>

Procedure B, 101.0 mg, 90\% yield, r.r. > 98:2, colorless oil.

${ }^{1} \mathrm{H}$ NMR $\left(400 \mathrm{MHz}, \mathrm{CDCl}_{3}\right) \delta$ 7.66-7.60 (m, 2H), $7.56-7.51(\mathrm{~m}, 1 \mathrm{H}), 7.46-7.34(\mathrm{~m}$, 4H), $7.21-7.11(\mathrm{~m}, 3 \mathrm{H}), 6.61-6.23(\mathrm{~m}, 1 \mathrm{H}), 4.70(\mathrm{~d}, J=3.3 \mathrm{~Hz}, 2 \mathrm{H}), 2.37$ $(\mathrm{s}, 3 \mathrm{H})$.

${ }^{13} \mathrm{C}$ NMR $\left(101 \mathrm{MHz}, \mathrm{CDCl}_{3}\right) \delta 147.2$ (1C), 137.1 (1C), 135.6 (1C), 135.4 (2C), 131.8 (1C), 130.4 (1C), 129.8 (1C), 128.4 (1C), 128.1 (2C), 126.1 (1C), 125.5 (1C), $121.0(1 \mathrm{C}), 19.6(1 \mathrm{C})$.

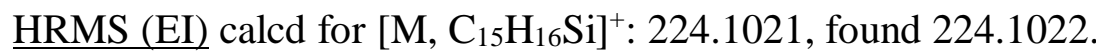

\section{phenyl(1-(o-tolyl)vinyl)silane (4bb) ${ }^{17}$}<smiles>C=C([SnH+]c1ccccc1)c1ccccc1C</smiles>

Procedure B, $104.3 \mathrm{mg}, 93 \%$ yield, r.r. $=93: 7$ (determined by ${ }^{1} \mathrm{H}$ NMR of crude product through the peak area ratio of $=\underline{\mathrm{CH}}_{2} /=\mathrm{C} \underline{\mathrm{HS}}$ ), colorless oil.

${ }^{1} \mathrm{H}$ NMR $\left(400 \mathrm{MHz}, \mathrm{CDCl}_{3}\right) \delta 7.56-7.51(\mathrm{~m}, 2 \mathrm{H}), 7.43-7.31(\mathrm{~m}, 3 \mathrm{H}), 7.17-7.07$ $(\mathrm{m}, 3 \mathrm{H}), 6.99-6.95(\mathrm{~m}, 1 \mathrm{H}), 5.91(\mathrm{~d}, J=3.1 \mathrm{~Hz}, 1 \mathrm{H}), 5.86(\mathrm{~d}, J=3.1 \mathrm{~Hz}$, 1H), 4.69 (s, 2H), 2.23 (s, 3H).

${ }^{13} \mathrm{C} \mathrm{NMR}\left(101 \mathrm{MHz}, \mathrm{CDCl}_{3}\right) \delta 146.5$ (1C), 143.1 (1C), 135.6 (2C), 134.4 (1C), 133.0 (1C), $131.1(1 \mathrm{C}), 130.1$ (1C), 129.8 (1C), 128.0 (2C), 127.9 (1C), 126.5 (1C), $125.6(1 \mathrm{C}), 20.2(1 \mathrm{C})$.

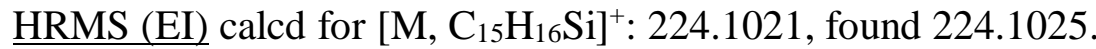

\section{(E)-(2-fluorostyryl)(phenyl)silane (3bc) $)^{18}$}<smiles>Fc1ccccc1/C=C/Sc1ccccc1</smiles>

Procedure B, $102.8 \mathrm{mg}, 90 \%$ yield, r.r. > 98:2, colorless oil.

${ }^{1} \mathrm{H}$ NMR $\left(400 \mathrm{MHz}, \mathrm{CDCl}_{3}\right) \delta 7.66-7.60(\mathrm{~m}, 2 \mathrm{H}), 7.54(\mathrm{t}, J=7.7 \mathrm{~Hz}, 1 \mathrm{H}), 7.44-7.33$ 
(m, 4H), $7.28-7.21(\mathrm{~m}, 1 \mathrm{H}), 7.11(\mathrm{t}, J=7.7 \mathrm{~Hz}, 1 \mathrm{H}), 7.03(\mathrm{t}, J=9.5 \mathrm{~Hz}$, $1 \mathrm{H}), 6.63-6.54(\mathrm{~m}, 1 \mathrm{H}), 4.73-4.68(\mathrm{~m}, 2 \mathrm{H})$.

${ }^{13} \mathrm{C} \mathrm{NMR}\left(101 \mathrm{MHz}, \mathrm{CDCl}_{3}\right) \delta 160.4(\mathrm{~d}, J=251.2 \mathrm{~Hz}, 1 \mathrm{C}), 141.0(\mathrm{~d}, J=4.4 \mathrm{~Hz}, 1 \mathrm{C})$, 135.5 (2C), 131.4 (1C), 130.0 (d, $J=8.8 \mathrm{~Hz}, 1 \mathrm{C}), 129.9$ (1C), 128.1 (2C), $127.1(\mathrm{~d}, J=3.3 \mathrm{~Hz}, 1 \mathrm{C}), 125.6(\mathrm{~d}, J=11.7 \mathrm{~Hz}, 1 \mathrm{C}), 124.1$ (d, $J=3.5 \mathrm{~Hz}$, 1C), $122.6(\mathrm{~d}, J=3.8 \mathrm{~Hz}, 1 \mathrm{C}), 115.6(\mathrm{~d}, J=22.1 \mathrm{~Hz}, 1 \mathrm{C})$.

HRMS (EI) calcd for [M, $\left.\mathrm{C}_{14} \mathrm{H}_{13} \mathrm{FSi}\right]^{+}:$228.0771, found 228.0770.

\section{(1-(2-fluorophenyl)vinyl)(phenyl)silane (4bc) ${ }^{15}$}<smiles>C=C([SnH2])c1ccccc1F</smiles>

Procedure B, $106.2 \mathrm{mg}, 93 \%$ yield, r.r. $=95: 5$ (determined by ${ }^{1} \mathrm{H}$ NMR of crude product through the peak area ratio of $\left.=\mathrm{C}_{2} /=\mathrm{C} \underline{H S i}\right)$, colorless oil.

${ }^{1} \mathrm{H} \mathrm{NMR}\left(400 \mathrm{MHz}, \mathrm{CDCl}_{3}\right) \delta 7.61-7.56(\mathrm{~m}, 2 \mathrm{H}), 7.41-7.31(\mathrm{~m}, 3 \mathrm{H}), 7.29-7.16(\mathrm{~m}$, 2H), $7.23-7.18(\mathrm{~m}, 1 \mathrm{H}), 7.07(\mathrm{t}, J=7.5 \mathrm{~Hz}, 1 \mathrm{H}), 6.22(\mathrm{~s}, 1 \mathrm{H}), 5.93(\mathrm{~d}, J=$ $2.3 \mathrm{~Hz}, 1 \mathrm{H}), 4.79(\mathrm{~d}, J=7.5 \mathrm{~Hz}, 2 \mathrm{H})$.

${ }^{13} \mathrm{C} \mathrm{NMR}\left(101 \mathrm{MHz}, \mathrm{CDCl}_{3}\right) \delta 159.7$ (d, $\left.J=246.3 \mathrm{~Hz}, 1 \mathrm{C}\right), 139.5$ (1C), 135.6 (2C), 134.3 (1C), 131.4 (d, $J=1.7 \mathrm{~Hz}, 1 \mathrm{C}), 130.3$ (d, $J=15.0 \mathrm{~Hz}, 1 \mathrm{C}), 129.8$ (1C), 129.1 (d, $J=4.1 \mathrm{~Hz}, 1 \mathrm{C}), 128.8$ (d, $J=8.1 \mathrm{~Hz}, 1 \mathrm{C}), 128.0(2 \mathrm{C}), 124.2$ (d, $J$ $=3.5 \mathrm{~Hz}, 1 \mathrm{C}), 115.6(\mathrm{~d}, J=22.0 \mathrm{~Hz}, 1 \mathrm{C})$.

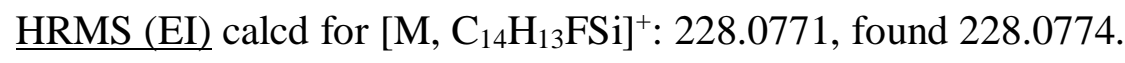

\section{(E)-(2-methoxystyryl)(phenyl)silane (3bd)}<smiles>COc1ccccc1/C=C/[SnH]c1ccccc1</smiles>

Procedure B, $107.0 \mathrm{mg}, 89 \%$ yield, r.r. $>98: 2$, colorless oil.

$\underline{{ }^{1} \mathrm{H} \mathrm{NMR}}\left(400 \mathrm{MHz}, \mathrm{CDCl}_{3}\right) \delta 7.65-7.61(\mathrm{~m}, 2 \mathrm{H}), 7.54(\mathrm{dd}, J=7.7,1.7 \mathrm{~Hz}, 1 \mathrm{H}), 7.42$ - $7.35(\mathrm{~m}, 3 \mathrm{H}), 7.29-7.24(\mathrm{~m}, 1 \mathrm{H}), 6.94(\mathrm{t}, J=7.5 \mathrm{~Hz}, 1 \mathrm{H}), 6.88(\mathrm{~d}, J=8.3$ $\mathrm{Hz}, 1 \mathrm{H}), 6.49$ (dt, $J=19.2,3.5 \mathrm{~Hz}, 1 \mathrm{H}), 4.70$ (d, $J=3.5 \mathrm{~Hz}, 2 \mathrm{H}), 3.85$ (s, 3H). ${ }^{13} \mathrm{C} \mathrm{NMR}\left(101 \mathrm{MHz}, \mathrm{CDCl}_{3}\right) \delta 156.9$ (1C), 143.8 (1C), 135.5 (2C), 132.1 (1C), 129.7 
(1C), 128.0 (2C), 126.9 (1C), 126.7 (1C), 120.6 (1C), 119.7 (1C), 111.0 (1C), $55.5(1 \mathrm{C})$.

HRMS (EI) calcd for [M, $\left.\mathrm{C}_{15} \mathrm{H}_{16} \mathrm{OSi}\right]^{+}:$240.0970, found 240.0971.

\section{(1-(2-methoxyphenyl)vinyl)(phenyl)silane (4bd) ${ }^{17}$}<smiles>C=C([SnH+]c1ccccc1)c1ccccc1OC</smiles>

Procedure B, $107.2 \mathrm{mg}, 89 \%$ yield, r.r. $=92: 8$ (determined by ${ }^{1} \mathrm{H}$ NMR of crude product through the peak area ratio of $=\mathrm{C}_{2} /=\mathrm{C} \underline{H S i}$ ), colorless oil.

${ }^{1} \mathrm{H}$ NMR $\left(400 \mathrm{MHz}, \mathrm{CDCl}_{3}\right) \delta 7.58-7.54(\mathrm{~m}, 2 \mathrm{H}), 7.38-7.29(\mathrm{~m}, 4 \mathrm{H}), 7.25-7.20$ $(\mathrm{m}, 1 \mathrm{H}), 6.95(\mathrm{td}, J=7.5,0.9 \mathrm{~Hz}, 1 \mathrm{H}), 6.79(\mathrm{~d}, J=8.2 \mathrm{~Hz}, 1 \mathrm{H}), 6.21(\mathrm{~d}, J=$ $2.5 \mathrm{~Hz}, 1 \mathrm{H}), 5.77(\mathrm{~d}, J=2.5 \mathrm{~Hz}, 1 \mathrm{H}), 4.72(\mathrm{~s}, 2 \mathrm{H}), 3.64(\mathrm{~s}, 3 \mathrm{H})$.

${ }^{13} \mathrm{C} \mathrm{NMR}\left(101 \mathrm{MHz}, \mathrm{CDCl}_{3}\right) \delta 156.0$ (1C), $141.6(1 \mathrm{C}), 135.1$ (2C), 133.5 (1C), 132.2 (1C), 131.7 (1C), 129.2 (1C), 128.6 (1C), 127.7 (2C), 127.5 (1C), 121.1 (1C), $110.4(1 \mathrm{C}), 54.7(1 \mathrm{C})$.

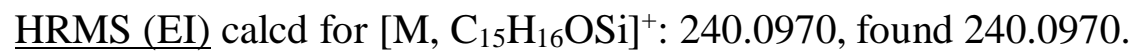

\section{(E)-(3-methylstyryl)(phenyl)silane (3be) ${ }^{16}$}<smiles>Cc1cccc(/C=C/[SH2]c2ccccc2)c1</smiles>

Procedure B, $105.5 \mathrm{mg}, 94 \%$ yield, r.r. > 98:2, colorless oil.

${ }^{1} \mathrm{H}$ NMR $\left(400 \mathrm{MHz}, \mathrm{CDCl}_{3}\right) \delta 7.64-7.59(\mathrm{~m}, 2 \mathrm{H}), 7.45-7.34(\mathrm{~m}, 3 \mathrm{H}), 7.28-7.20$ (m, 3H), 7.16- $7.08(\mathrm{~m}, 2 \mathrm{H}), 6.49(\mathrm{dt}, J=19.2,3.4 \mathrm{~Hz}, 1 \mathrm{H}), 4.69(\mathrm{~d}, J=2.9$ $\mathrm{Hz}, 2 \mathrm{H}), 2.35$ (s, 3H).

${ }^{13} \mathrm{C} \mathrm{NMR}\left(101 \mathrm{MHz}, \mathrm{CDCl}_{3}\right) \delta 149.5$ (1C), 138.2 (1C), 137.7 (1C), 135.5 (2C), 131.7 (1C), 129.8 (1C), 129.4 (1C), 128.5 (1C), 128.1 (2C), 127.4 (1C), 123.9 (1C), 119.1 (1C), $21.3(1 \mathrm{C})$.

HRMS (EI) calcd for [M, $\left.\mathrm{C}_{15} \mathrm{H}_{16} \mathrm{Si}\right]^{+}:$224.1021, found 224.1025. 
phenyl(1-(m-tolyl)vinyl)silane (4be) $)^{17}$<smiles>C=C([SH+]c1ccccc1)c1cccc(C)c1</smiles>

Procedure B, 99.8 mg, 89\% yield, r.r. $=96: 4$ (determined by ${ }^{1} \mathrm{H}$ NMR of crude product through the peak area ratio of $\left.=\mathrm{C}_{2} /=\mathrm{C} \underline{H S i}\right)$, colorless oil.

${ }^{1} \mathrm{H}$ NMR $\left(400 \mathrm{MHz}, \mathrm{CDCl}_{3}\right) \delta 7.61-7.56(\mathrm{~m}, 2 \mathrm{H}), 7.41-7.32(\mathrm{~m}, 3 \mathrm{H}), 7.22-7.14$ (m, 3H), $7.08-7.03(\mathrm{~m}, 1 \mathrm{H}), 6.21(\mathrm{~d}, J=2.1 \mathrm{~Hz}, 1 \mathrm{H}), 5.79(\mathrm{~d}, J=2.1 \mathrm{~Hz}$, $1 \mathrm{H}), 4.82(\mathrm{~s}, 2 \mathrm{H}), 2.32(\mathrm{~s}, 3 \mathrm{H})$.

${ }^{13} \mathrm{C} \mathrm{NMR}\left(101 \mathrm{MHz}, \mathrm{CDCl}_{3}\right) \delta 149.4$ (1C), 144.2 (1C), 142.2 (1C), 138.0 (1C), 135.6 (2C), 131.1 (1C), 129.8 (1C), 128.4 (1C), 128.1 (2C), 128.0 (1C), 127.1 (1C), 123.6 (1C), 21.4 (1C).

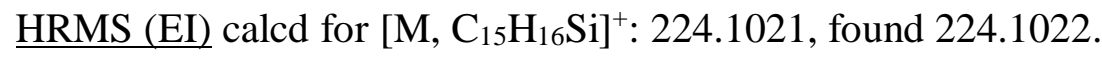

(E)-(2-(naphthalen-2-yl)vinyl)(phenyl)silane (3bf)<smiles>[SH-]c1ccccc1</smiles>

Procedure B, $115.9 \mathrm{mg}, 89 \%$ yield, r.r. $>98: 2$, colorless oil.

${ }^{1} \mathrm{H}$ NMR $\left(400 \mathrm{MHz}, \mathrm{CDCl}_{3}\right) \delta 7.83-7.77(\mathrm{~m}, 4 \mathrm{H}), 7.69-7.63(\mathrm{~m}, 3 \mathrm{H}), 7.49-7.37$ (m, 5H), $7.31(\mathrm{~d}, J=18.9 \mathrm{~Hz}, 2 \mathrm{H}), 6.62(\mathrm{dt}, J=18.9,3.2 \mathrm{~Hz}, 1 \mathrm{H}), 4.74$ (d, $J$ $=3.2 \mathrm{~Hz}, 2 \mathrm{H})$.

${ }^{13} \mathrm{C} \mathrm{NMR}\left(101 \mathrm{MHz}, \mathrm{CDCl}_{3}\right) \delta 149.3$ (1C), 135.5 (2C), 135.2 (1C), 133.5 (2C), 131.6 (1C), 129.8 (1C), 128.3 (2C), 128.1 (2C), 127.7 (1C), 127.3 (1C), 126.3 (2C), 123.2 (1C), 119.8 (1C).

HRMS (EI) calcd for [M, $\mathrm{C}_{18} \mathrm{H}_{16} \mathrm{Si}^{+}: 260.1021$, found 260.1026.

(1-(naphthalen-2-yl)vinyl)(phenyl)silane (4bf) ${ }^{17}$<smiles>C=C([SH+]c1ccccc1)c1ccc2ccccc2c1</smiles> 
Procedure B, 118.5 mg, 91\% yield, r.r. = 96:4 (determined by ${ }^{1} \mathrm{H}$ NMR of crude product through the peak area ratio of $=\mathrm{CH}_{2} /=\mathrm{CHSi}$ ), colorless oil.

${ }^{1} \mathrm{H}$ NMR $\left(400 \mathrm{MHz}, \mathrm{CDCl}_{3}\right) \delta 7.82-7.77(\mathrm{~m}, 4 \mathrm{H}), 7.64-7.58(\mathrm{~m}, 2 \mathrm{H}), 7.56-7.52$ (m, 1H), $7.46-7.30(\mathrm{~m}, 5 \mathrm{H}), 6.35(\mathrm{~d}, J=1.8 \mathrm{~Hz}, 1 \mathrm{H}), 5.91(\mathrm{~d}, J=2.3 \mathrm{~Hz}$, $1 \mathrm{H}), 4.91(\mathrm{~s}, 2 \mathrm{H})$.

${ }^{13} \mathrm{C} \mathrm{NMR}\left(101 \mathrm{MHz}, \mathrm{CDCl}_{3}\right) \delta 144.0(1 \mathrm{C}), 139.6(1 \mathrm{C}), 135.6(2 \mathrm{C}), 133.4(1 \mathrm{C}), 132.6$ (1C), $131.6(1 \mathrm{C}), 131.1$ (1C), 129.9 (1C), 128.1 (4C), 127.5 (1C), 126.1 (1C), 125.8 (1C), 125.6 (1C), 124.5 (1C).

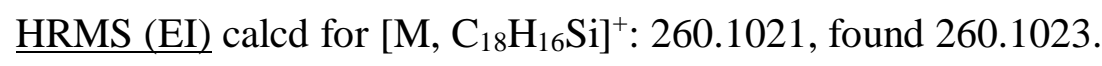

\section{(E)-(2-(ferrocenylvinyl))(phenyl)silane (3bg)}

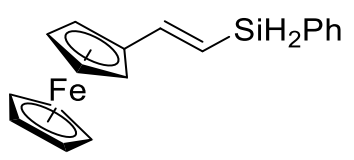

Procedure B, $144.8 \mathrm{mg}, 91 \%$ yield, r.r. $>98: 2$, colorless oil.

${ }^{1} \mathrm{H}$ NMR $\left(400 \mathrm{MHz}, \mathrm{CDCl}_{3}\right) \delta 7.68-7.56(\mathrm{~m}, 2 \mathrm{H}), 7.44-7.35(\mathrm{~m}, 3 \mathrm{H}), 6.96(\mathrm{~d}, J=$ $18.8 \mathrm{~Hz}, 1 \mathrm{H}), 6.03$ (dt, $J=18.8,3.4 \mathrm{~Hz}, 1 \mathrm{H}), 4.61$ (d, $J=3.3 \mathrm{~Hz}, 2 \mathrm{H}), 4.42$ (t, $J=1.9 \mathrm{~Hz}, 2 \mathrm{H}), 4.26(\mathrm{t}, J=1.9 \mathrm{~Hz}, 2 \mathrm{H}), 4.11(\mathrm{~s}, 5 \mathrm{H})$.

${ }^{13} \mathrm{C} \mathrm{NMR}\left(101 \mathrm{MHz}, \mathrm{CDCl}_{3}\right) \delta 148.6$ (1C), $135.4(2 \mathrm{C}), 132.3$ (1C), 129.6 (1C), 128.0 (2C), 115.7 (1C), $83.6(1 \mathrm{C}), 69.4$ (7C), 67.2 (2C).

HRMS (ESI) calcd for [M+H, $\left.\mathrm{C}_{18} \mathrm{H}_{19} \mathrm{FeSi}\right]^{+}: 319.0600$, found 319.0611 .

\section{(1-(ferrocenylvinyl))(phenyl)silane (4bg)}

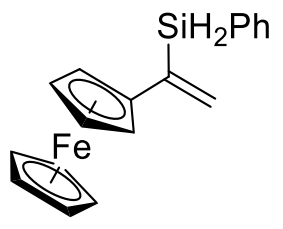

Procedure B, $136.9 \mathrm{mg}, 86 \%$ yield, r.r. $>98: 2$, colorless oil.

$\underline{{ }^{1} \mathrm{H} \mathrm{NMR}}\left(400 \mathrm{MHz}, \mathrm{CDCl}_{3}\right) \delta 7.71-7.65(\mathrm{~m}, 2 \mathrm{H}), 7.46-7.36(\mathrm{~m}, 3 \mathrm{H}), 6.13(\mathrm{~s}, 1 \mathrm{H})$, $5.88(\mathrm{~s}, 1 \mathrm{H}), 4.80(\mathrm{~s}, 2 \mathrm{H}), 4.37(\mathrm{~s}, 2 \mathrm{H}), 4,21(\mathrm{~s}, 2 \mathrm{H}), 4.03(\mathrm{~s}, 5 \mathrm{H})$.

${ }^{13} \mathrm{C} \mathrm{NMR}\left(101 \mathrm{MHz}, \mathrm{CDCl}_{3}\right) \delta 140.7$ (1C), 135.7 (2C), 131.7 (1C), 129.9 (1C), 128.1 (2C), 126.4 (1C), 86.7 (1C), 69.4 (5C), 68.7 (2C), 66.5 (2C). 


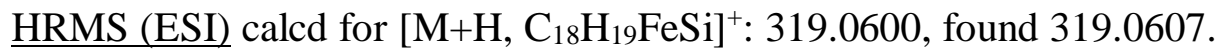

(E)-(3-methylbuta-1,3-dien-1-yl)(phenyl)silane (3ca) ${ }^{19}$<smiles>C=C(C)C=C[SnH2]c1ccccc1</smiles>

Procedure B, $69.7 \mathrm{mg}, 80 \%$ yield, r.r. $>98: 2$, colorless oil.

$\underline{{ }^{1} \mathrm{H} \mathrm{NMR}}\left(400 \mathrm{MHz}, \mathrm{CDCl}_{3}\right) \delta 7.68-7.56(\mathrm{~m}, 2 \mathrm{H}), 7.44-7.34(\mathrm{~m}, 3 \mathrm{H}), 6.88(\mathrm{~d}, J=$ $18.8 \mathrm{~Hz}, 1 \mathrm{H}), 5.88$ (dt, $J=18.8,3.3 \mathrm{~Hz}, 1 \mathrm{H}), 5.13(\mathrm{~s}, 1 \mathrm{H}), 5.07(\mathrm{~s}, 1 \mathrm{H}), 4.61$ $(\mathrm{d}, J=3.2 \mathrm{~Hz}, 2 \mathrm{H}), 1.87(\mathrm{~s}, 3 \mathrm{H})$.

${ }^{13} \mathrm{C} \mathrm{NMR}\left(101 \mathrm{MHz}, \mathrm{CDCl}_{3}\right) \delta 152.1$ (1C), 143.1 (1C), 135.4 (2C), 131.8 (1C), 129.7 (1C), 128.0 (2C), 119.2 (1C), 118.8 (1C), 17.8 (1C).

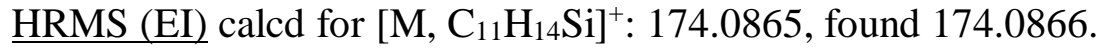

\section{(3-methylbuta-1,3-dien-2-yl)(phenyl)silane (4ca)}<smiles>C=C(C)C(=C)[SbH2]c1ccccc1</smiles>

Procedure B, $73.2 \mathrm{mg}, 84 \%$ yield, r.r. $>98: 2$, colorless oil.

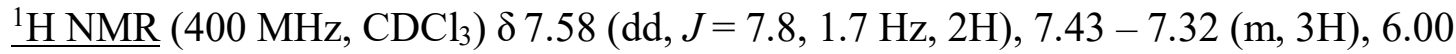
(s, 1H), $5.71(\mathrm{~s}, 1 \mathrm{H}), 5.10(\mathrm{~s}, 1 \mathrm{H}), 5.07(\mathrm{t}, J=1.5 \mathrm{~Hz}, 1 \mathrm{H}), 4.71(\mathrm{~s}, 2 \mathrm{H}), 1.94$ (s, 3H).

${ }^{13} \mathrm{C}$ NMR $\left(101 \mathrm{MHz}, \mathrm{CDCl}_{3}\right) \delta 144.8$ (1C), 144.1 (1C), 135.5 (2C), 131.7 (1C), 130.1 (1C), 129.7 (1C), 128.0 (2C), 117.0 (1C), 20.4 (1C).

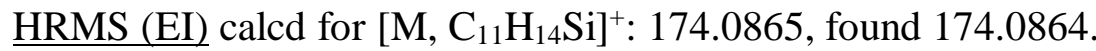

(E)-(2-(cyclohex-1-en-1-yl)vinyl)(phenyl)silane (3cb) ${ }^{16}$<smiles>C1=C(/C=C/Sc2ccccc2)CCCC1</smiles>

Procedure B, 97.5 mg, 91\% yield, r.r. > 98:2, colorless oil.

$\underline{{ }^{1} \mathrm{H} \mathrm{NMR}}\left(400 \mathrm{MHz}, \mathrm{CDCl}_{3}\right) \delta 7.62-7.54(\mathrm{~m}, 2 \mathrm{H}), 7.43-7.33(\mathrm{~m}, 3 \mathrm{H}), 6.78(\mathrm{~d}, J=$ $18.8 \mathrm{~Hz}, 1 \mathrm{H}), 5.88(\mathrm{t}, J=6.5 \mathrm{~Hz}, 1 \mathrm{H}), 5.72(\mathrm{dt}, J=18.8,3.2 \mathrm{~Hz}, 1 \mathrm{H}), 4.60$ 
$(\mathrm{d}, J=3.3 \mathrm{~Hz}, 2 \mathrm{H}), 2.17(\mathrm{dd}, J=6.0,4.6 \mathrm{~Hz}, 4 \mathrm{H}), 1.72-1.64(\mathrm{~m}, 2 \mathrm{H}), 1.64$ $-1.56(\mathrm{~m}, 2 \mathrm{H})$.

${ }^{13} \mathrm{C} \mathrm{NMR}\left(101 \mathrm{MHz}, \mathrm{CDCl}_{3}\right) \delta 153.1$ (1C), 137.2 (1C), 135.4 (2C), 132.9 (1C), 132.4 (1C), 129.6 (1C), 128.0 (2C), 114.0 (1C), 26.0 (1C), 23.9 (1C), 22.4 (1C), $22.3(1 \mathrm{C})$.

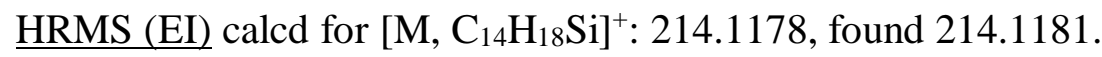

\section{(1-(cyclohex-1-en-1-yl)vinyl)(phenyl)silane $(4 \mathrm{cb})^{15}$}<smiles>C=C([SiH2]c1ccccc1)C1=CCCCC1</smiles>

Procedure B, $95.4 \mathrm{mg}, 89 \%$ yield, r.r. > 98:2, colorless oil.

$\underline{{ }^{1} \mathrm{H} \mathrm{NMR}}\left(400 \mathrm{MHz}, \mathrm{CDCl}_{3}\right) \delta 7.58(\mathrm{~d}, J=6.8 \mathrm{~Hz}, 2 \mathrm{H}), 7.43-7.31(\mathrm{~m}, 3 \mathrm{H}), 5.91(\mathrm{~s}$, 2H), $5.53(\mathrm{~s}, 1 \mathrm{H}), 4.69$ (s, 2H), $2.21(\mathrm{~d}, J=6.6 \mathrm{~Hz}, 2 \mathrm{H}), 2.11(\mathrm{~d}, J=5.8 \mathrm{~Hz}$, 2H), $1.68(\mathrm{q}, J=5.9 \mathrm{~Hz}, 2 \mathrm{H}), 1.57(\mathrm{q}, J=5.9 \mathrm{~Hz}, 2 \mathrm{H})$.

${ }^{13} \mathrm{C} \mathrm{NMR}\left(101 \mathrm{MHz}, \mathrm{CDCl}_{3}\right) \delta 144.4$ (1C), 138.4 (1C), 135.5 (2C), 132.1 (1C), 129.7 (1C), 129.5 (1C), 128.0 (2C), 126.3 (1C), 26.1 (1C), 25.5 (1C), 22.7 (1C), $22.2(1 \mathrm{C})$.

HRMS (EI) calcd for [M, $\mathrm{C}_{14} \mathrm{H}_{18} \mathrm{Si}^{+}: 214.1178$, found 214.1179.

\section{(E)-oct-1-en-1-yl(o-tolyl)silane (3da)}<smiles>CCCCC=C[SiH2]c1ccccc1C</smiles>

Procedure A, $104.6 \mathrm{mg}, 90 \%$ yield, r.r. > 98:2, colorless oil.

를 NMR (400 MHz, $\left.\mathrm{CDCl}_{3}\right) \delta 7.53(\mathrm{dd}, J=7.6,1.5 \mathrm{~Hz}, 1 \mathrm{H}), 7.31(\mathrm{td}, J=7.5,1.5 \mathrm{~Hz}$, 1H), $7.21-7.13(\mathrm{~m}, 2 \mathrm{H}), 6.32(\mathrm{dt}, J=18.4,6.3 \mathrm{~Hz}, 1 \mathrm{H}), 5.72(\mathrm{ddt}, J=18.4$, 3.3, $1.7 \mathrm{~Hz}, 1 \mathrm{H}), 4.56(\mathrm{~d}, J=3.2 \mathrm{~Hz}, 2 \mathrm{H}), 2.43(\mathrm{~s}, 3 \mathrm{H}), 2.16$ (q, $J=6.8 \mathrm{~Hz}$, $2 \mathrm{H}), 1.45-1.37(\mathrm{~m}, 2 \mathrm{H}), 1.34-1.23(\mathrm{~m}, 6 \mathrm{H}), 0.91-0.85(\mathrm{~m}, 3 \mathrm{H})$.

${ }^{13} \mathrm{C}$ NMR $\left(101 \mathrm{MHz}, \mathrm{CDCl}_{3}\right) \delta 153.8$ (1C), 144.2 (1C), 136.5 (1C), 131.8 (1C), 130.1 (1C), 129.3 (1C), 125.1 (1C), 119.8 (1C), 36.9 (1C), 31.7 (1C), 28.8 (1C), 
28.4 (1C), $22.6(1 \mathrm{C}), 22.4(1 \mathrm{C}), 14.1(1 \mathrm{C})$.

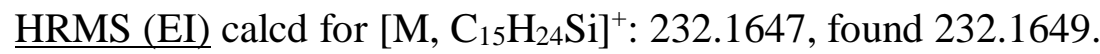

oct-1-en-2-yl(o-tolyl)silane (4da)<smiles>C=C([SiH2]CCCCCCCC)c1ccccc1C</smiles>

Procedure A, $103.4 \mathrm{mg}, 89 \%$ yield, r.r. $>98: 2$, colorless oil.

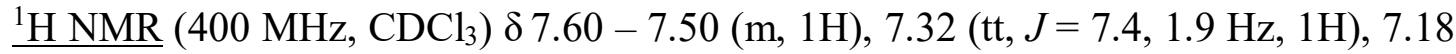
$(\mathrm{d}, J=7.1 \mathrm{~Hz}, 2 \mathrm{H}), 5.80-5.71(\mathrm{~m}, 1 \mathrm{H}), 5.48(\mathrm{~d}, J=3.1 \mathrm{~Hz}, 1 \mathrm{H}), 4.57(\mathrm{~s}$, 2H), 2.43 (s, 3H), 2.20 (t, J=7.0 Hz, 2H), $1.42(\mathrm{~s}, 2 \mathrm{H}), 1.32-1.20(\mathrm{~m}, 6 \mathrm{H})$, $0.91-0.83(\mathrm{~m}, 3 \mathrm{H})$.

${ }^{13} \mathrm{C}$ NMR $\left(101 \mathrm{MHz}, \mathrm{CDCl}_{3}\right) \delta 145.2$ (1C), 144.5 (1C), 137.0 (1C), 131.0 (1C), 130.2 (1C), 129.4 (1C), 129.1 (1C), 125.1 (1C), 37.5 (1C), 31.6 (1C), 28.9 (1C), 28.8 (1C), $22.6(1 \mathrm{C}), 22.5$ (1C), 14.1 (1C).

HRMS (EI) calcd for [M, $\mathrm{C}_{15} \mathrm{H}_{24} \mathrm{Si}^{+}:$232.1647, found 232.1648 .

(E)-oct-1-en-1-yl(p-tolyl)silane (3db)<smiles>Cc1ccc([SiH2]C=Cc2ccccc2)cc1</smiles>

Procedure A, $110.4 \mathrm{mg}, 95 \%$ yield, r.r. $>98: 2$, colorless oil.

${ }^{1} \mathrm{H}$ NMR $\left(400 \mathrm{MHz}, \mathrm{CDCl}_{3}\right) \delta 7.46(\mathrm{~d}, J=7.6 \mathrm{~Hz}, 2 \mathrm{H}), 7.18(\mathrm{~d}, J=7.5 \mathrm{~Hz}, 2 \mathrm{H}), 6.34$ $(\mathrm{dt}, J=18.4,6.3 \mathrm{~Hz}, 1 \mathrm{H}), 5.77-5.64(\mathrm{~m}, 1 \mathrm{H}), 4.51(\mathrm{~d}, J=3.2 \mathrm{~Hz}, 2 \mathrm{H}), 2.35$ $(\mathrm{s}, 3 \mathrm{H}), 2.24-2.10(\mathrm{~m}, 2 \mathrm{H}), 1.48-1.18(\mathrm{~m}, 8 \mathrm{H}), 0.88(\mathrm{t}, J=6.6 \mathrm{~Hz}, 3 \mathrm{H})$.

${ }^{13} \mathrm{C} \mathrm{NMR}\left(101 \mathrm{MHz}, \mathrm{CDCl}_{3}\right) \delta 153.9$ (1C), 139.5 (1C), 135.4 (2C), $128.8(2 \mathrm{C}), 128.6$ (1C), 120.1 (1C), 36.9 (1C), 31.7 (1C), 28.8 (1C), 28.4 (1C), 22.6 (1C), 21.5 (1C), $14.1(1 \mathrm{C})$.

HRMS (EI) calcd for [M, $\mathrm{C}_{15} \mathrm{H}_{24} \mathrm{Si}^{+}:$232.1647, found 232.1650. 
oct-1-en-2-yl(p-tolyl)silane (4db)<smiles>C=C(CCCCCC)[SiH2]c1ccc(C)cc1</smiles>

Procedure A, $104.6 \mathrm{mg}, 90 \%$ yield, r.r. $>98: 2$, colorless oil.

${ }^{1} \mathrm{H}$ NMR $\left(400 \mathrm{MHz}, \mathrm{CDCl}_{3}\right) \delta 7.47(\mathrm{~d}, J=7.6 \mathrm{~Hz}, 2 \mathrm{H}), 7.18(\mathrm{~d}, J=7.4 \mathrm{~Hz}, 2 \mathrm{H}), 5.80$ - $5.71(\mathrm{~m}, 1 \mathrm{H}), 5.52(\mathrm{~d}, J=2.7 \mathrm{~Hz}, 1 \mathrm{H}), 4.52(\mathrm{~s}, 2 \mathrm{H}), 2.35(\mathrm{~s}, 3 \mathrm{H}), 2.23-$ $2.15(\mathrm{~m}, 2 \mathrm{H}), 1.45-1.36(\mathrm{~m}, 2 \mathrm{H}), 1.25(\mathrm{dt}, J=11.0,5.7 \mathrm{~Hz}, 6 \mathrm{H}), 0.86(\mathrm{t}, J$ $=6.7 \mathrm{~Hz}, 3 \mathrm{H})$.

${ }^{13} \mathrm{C} \mathrm{NMR}\left(101 \mathrm{MHz}, \mathrm{CDCl}_{3}\right) \delta 145.7$ (1C), $139.6(1 \mathrm{C}), 135.6(2 \mathrm{C}), 129.1$ (1C), 128.9 (2C), 127.9 (1C), 37.4 (1C), 31.7 (1C), 28.9 (1C), 28.8 (1C), 22.6 (1C), 21.5 (1C), $14.1(1 \mathrm{C})$.

$\underline{\mathrm{HRMS}(\mathrm{EI})}$ calcd for [M, $\left.\mathrm{C}_{15} \mathrm{H}_{24} \mathrm{Si}\right]^{+}:$232.1647, found 232.1651 .

\section{(E)-(4-methoxyphenyl)(oct-1-en-1-yl)silane (3dc)}<smiles>COc1ccc([SiH2]C=Cc2ccccc2)cc1</smiles>

Procedure A, $114.3 \mathrm{mg}, 92 \%$ yield, r.r. > 98:2, colorless oil.

래 NMR (400 MHz, $\left.\mathrm{CDCl}_{3}\right) \delta 7.49(\mathrm{~d}, J=8.5 \mathrm{~Hz}, 2 \mathrm{H}), 6.91(\mathrm{~d}, J=8.5 \mathrm{~Hz}, 2 \mathrm{H}), 6.33$ $(\mathrm{dt}, J=18.4,6.3 \mathrm{~Hz}, 1 \mathrm{H}), 5.78-5.62(\mathrm{~m}, 1 \mathrm{H}), 4.51(\mathrm{~d}, J=3.2 \mathrm{~Hz}, 2 \mathrm{H}), 3.81$ $(\mathrm{s}, 3 \mathrm{H}), 2.25-2.10(\mathrm{~m}, 2 \mathrm{H}), 1.49-1.15(\mathrm{~m}, 8 \mathrm{H}), 0.88(\mathrm{t}, J=6.7 \mathrm{~Hz}, 3 \mathrm{H})$.

${ }^{13} \mathrm{C} \mathrm{NMR}\left(101 \mathrm{MHz}, \mathrm{CDCl}_{3}\right) \delta 160.9$ (1C), 153.8 (1C), 136.8 (2C), 122.9 (1C), 120.3 (1C), 113.8 (2C), 55.0 (1C), 36.9 (1C), 31.7 (1C), 28.8 (1C), 28.4 (1C), 22.6 (1C), $14.1(1 \mathrm{C})$.

HRMS (EI) calcd for [M, $\mathrm{C}_{15} \mathrm{H}_{24} \mathrm{SiO}^{+}:$: 248.1596, found 248.1597. 
<smiles>C=C(CCCCCCCC)[SiH2]c1ccc(OC)cc1</smiles>

Procedure A, $113.0 \mathrm{mg}, 91 \%$ yield, r.r. $>98: 2$, colorless oil.

${ }^{1} \mathrm{H}$ NMR $\left(400 \mathrm{MHz}, \mathrm{CDCl}_{3}\right) \delta 7.50(\mathrm{~d}, J=8.5 \mathrm{~Hz}, 2 \mathrm{H}), 6.92(\mathrm{~d}, J=7.9 \mathrm{~Hz}, 2 \mathrm{H}), 5.78$ $-5.72(\mathrm{~m}, 1 \mathrm{H}), 5.51(\mathrm{~d}, J=2.9 \mathrm{~Hz}, 1 \mathrm{H}), 4.51(\mathrm{~s}, 2 \mathrm{H}), 3.82(\mathrm{~s}, 3 \mathrm{H}), 2.18(\mathrm{t}, J$ $=7.7 \mathrm{~Hz}, 2 \mathrm{H}), 1.48-1.12(\mathrm{~m}, 8 \mathrm{H}), 0.86(\mathrm{t}, J=6.6 \mathrm{~Hz}, 3 \mathrm{H})$.

${ }^{13} \mathrm{C} \mathrm{NMR}\left(101 \mathrm{MHz}, \mathrm{CDCl}_{3}\right) \delta 161.0$ (1C), 145.9 (1C), 137.1 (2C), 129.0 (1C), 122.2 (1C), 113.8 (2C), 55.0 (1C), 37.4 (1C), 31.7 (1C), 28.9 (1C), 28.8 (1C), 22.6 (1C), $14.1(1 \mathrm{C})$.

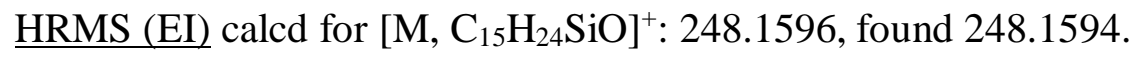

\section{(E)-(4-fluorophenyl)(oct-1-en-1-yl)silane (3dd)}<smiles>Fc1ccc([SiH2]C=Cc2ccccc2)cc1</smiles>

Procedure A, $107.6 \mathrm{mg}, 91 \%$ yield, r.r. $>98: 2$, colorless oil.

${ }^{1} \mathrm{H}$ NMR $\left(400 \mathrm{MHz}, \mathrm{CDCl}_{3}\right) \delta 7.59-7.48(\mathrm{~m}, 2 \mathrm{H}), 7.11-7.01(\mathrm{~m}, 2 \mathrm{H}), 6.35(\mathrm{dt}, J=$ 18.4, $6.3 \mathrm{~Hz}, 1 \mathrm{H}), 5.69$ (dtt, $J=18.4,3.2,1.6 \mathrm{~Hz}, 1 \mathrm{H}), 4.52$ (d, $J=3.2 \mathrm{~Hz}$, $2 \mathrm{H}), 2.18(\mathrm{q}, J=6.5 \mathrm{~Hz}, 2 \mathrm{H}), 1.47-1.38(\mathrm{~m}, 2 \mathrm{H}), 1.36-1.21(\mathrm{~m}, 6 \mathrm{H}), 0.88$ $(\mathrm{t}, J=6.8 \mathrm{~Hz}, 3 \mathrm{H})$.

${ }^{13} \mathrm{C} \mathrm{NMR}\left(101 \mathrm{MHz}, \mathrm{CDCl}_{3}\right) \delta 164.1(\mathrm{~d}, J=248.8 \mathrm{~Hz}, 1 \mathrm{C}), 154.5(1 \mathrm{C}), 137.3(\mathrm{~d}, J=$ $7.5 \mathrm{~Hz}, 2 \mathrm{C}), 127.8$ (d, $J=3.7 \mathrm{~Hz}, 1 \mathrm{C}), 119.6(1 \mathrm{C}), 115.2$ (d, $J=19.9 \mathrm{~Hz}, 2 \mathrm{C})$, 36.9 (1C), 31.7 (1C), 28.8 (1C), 28.4 (1C), 22.6 (1C), 14.1 (1C).

HRMS (EI) calcd for [M, $\left.\mathrm{C}_{14} \mathrm{H}_{21} \mathrm{SiF}\right]^{+}:$236.1397, found 236.1396. 


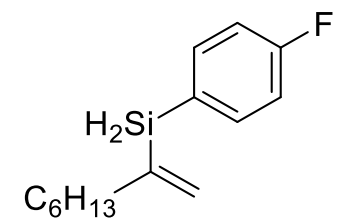

Procedure A, $111.1 \mathrm{mg}, 94 \%$ yield, r.r. > 98:2, colorless oil.

${ }^{1} \mathrm{H}$ NMR $\left(400 \mathrm{MHz}, \mathrm{CDCl}_{3}\right) \delta 7.59-7.51(\mathrm{~m}, 2 \mathrm{H}), 7.11-7.02(\mathrm{~m}, 2 \mathrm{H}), 5.77(\mathrm{~s}, 1 \mathrm{H})$, $5.52(\mathrm{~d}, J=2.8 \mathrm{~Hz}, 1 \mathrm{H}), 4.53(\mathrm{~s}, 2 \mathrm{H}), 2.22-2.12(\mathrm{~m}, 2 \mathrm{H}), 1.45-1.35(\mathrm{~m}$, 2H), $1.32-1.16(\mathrm{~m}, 6 \mathrm{H}), 0.86(\mathrm{t}, J=6.8 \mathrm{~Hz}, 3 \mathrm{H})$.

${ }^{13} \mathrm{C} \mathrm{NMR}\left(101 \mathrm{MHz}, \mathrm{CDCl}_{3}\right) \delta 164.2(\mathrm{~d}, J=249.1 \mathrm{~Hz}, 1 \mathrm{C}), 145.2(1 \mathrm{C}), 137.5(\mathrm{~d}, J=$ $7.9 \mathrm{~Hz}, 2 \mathrm{C}), 129.6(1 \mathrm{C}), 127.1$ (d, $J=4.0 \mathrm{~Hz}, 1 \mathrm{C}), 115.3$ (d, $J=19.9 \mathrm{~Hz}, 2 \mathrm{C}$ ), 37.3 (1C), 31.6 (1C), 28.9 (1C), 28.8 (1C), 22.6 (1C), 14.1 (1C).

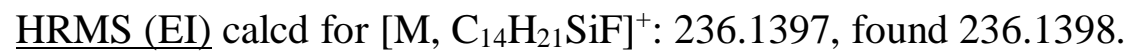

\section{(E)-(4-chlorophenyl)(oct-1-en-1-yl)silane (3de)}<smiles>Clc1ccc([SiH2]C=Cc2ccccc2)cc1</smiles>

Procedure A, $113.8 \mathrm{mg}, 90 \%$ yield, r.r. $>98: 2$, colorless oil.

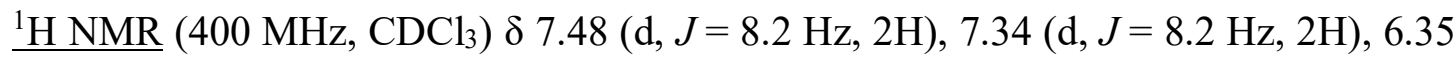
$(\mathrm{dt}, J=18.5,6.3 \mathrm{~Hz}, 1 \mathrm{H}), 5.68(\mathrm{dtt}, J=18.4,3.1,1.6 \mathrm{~Hz}, 1 \mathrm{H}), 4.51(\mathrm{~d}, J=$ $3.2 \mathrm{~Hz}, 2 \mathrm{H}), 2.18(\mathrm{q}, J=6.7 \mathrm{~Hz}, 2 \mathrm{H}), 1.46-1.37(\mathrm{~m}, 2 \mathrm{H}), 1.35-1.22(\mathrm{~m}$, $6 \mathrm{H}), 0.92-0.84(\mathrm{~m}, 3 \mathrm{H})$.

${ }^{13} \mathrm{C} \mathrm{NMR}\left(101 \mathrm{MHz}, \mathrm{CDCl}_{3}\right) \delta 154.7$ (1C), 136.7 (2C), 136.0 (1C), 130.7 (1C), 128.2 (2C), 119.3 (1C), 36.9 (1C), 31.7 (1C), 28.8 (1C), 28.3 (1C), 22.6 (1C), 14.1 $(1 \mathrm{C})$.

HRMS (EI) calcd for [M, $\left.\mathrm{C}_{14} \mathrm{H}_{21} \mathrm{SiCl}\right]^{+}: 252.1101$, found252.1105. 


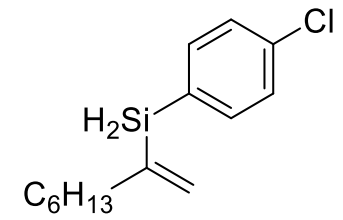

Procedure A, $115.1 \mathrm{mg}, 91 \%$ yield, r.r. > 98:2, colorless oil.

${ }^{1} \mathrm{H}$ NMR $\left(400 \mathrm{MHz}, \mathrm{CDCl}_{3}\right) \delta 7.55-7.45(\mathrm{~m}, 2 \mathrm{H}), 7.39-7.29(\mathrm{~m}, 2 \mathrm{H}), 5.78(\mathrm{dt}, J=$ 3.0, $1.5 \mathrm{~Hz}, 1 \mathrm{H}), 5.53(\mathrm{~d}, J=2.9 \mathrm{~Hz}, 1 \mathrm{H}), 4.52(\mathrm{~s}, 2 \mathrm{H}), 2.23-2.12(\mathrm{~m}, 2 \mathrm{H})$, $1.41-1.35(\mathrm{~m}, 2 \mathrm{H}), 1.33-1.18(\mathrm{~m}, 6 \mathrm{H}), 0.90-0.82(\mathrm{~m}, 3 \mathrm{H})$.

${ }^{13} \mathrm{C} \mathrm{NMR}\left(101 \mathrm{MHz}, \mathrm{CDCl}_{3}\right) \delta 144.9$ (1C), 136.9 (2C), 136.2 (1C), 130.0 (1C), 129.8 (1C), 128.3 (2C), 37.3 (1C), 31.6 (1C), 28.9 (1C), 28.8 (1C), 22.6 (1C), 14.1 (1C).

$\underline{\operatorname{HRMS}(\mathrm{EI})}$ calcd for [M, $\left.\mathrm{C}_{14} \mathrm{H}_{21} \mathrm{SiCl}\right]^{+}:$252.1101, found 252.1103 .

\section{(E)-dodecyl(oct-1-en-1-yl)silane (3df)}

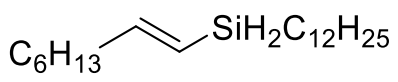

Procedure A, $147.5 \mathrm{mg}, 95 \%$ yield, r.r. $>98: 2$, colorless oil.

를 NMR (400 MHz, $\left.\mathrm{CDCl}_{3}\right) \delta 6.31-6.19(\mathrm{~m}, 1 \mathrm{H}), 5.62-5.50(\mathrm{~m}, 1 \mathrm{H}), 3.90-3.86$ $(\mathrm{m}, 2 \mathrm{H}), 2.13(\mathrm{q}, J=7.0 \mathrm{~Hz}, 2 \mathrm{H}), 1.44-1.20(\mathrm{~m}, 28 \mathrm{H}), 0.88(\mathrm{t}, J=6.6 \mathrm{~Hz}$, $6 \mathrm{H}), 0.72(\mathrm{tt}, J=7.9,3.7 \mathrm{~Hz}, 2 \mathrm{H})$.

${ }^{13} \mathrm{C} \mathrm{NMR}\left(101 \mathrm{MHz}, \mathrm{CDCl}_{3}\right) \delta 152.3$ (1C), 120.9 (1C), 36.9 (1C), 32.8 (1C), $31.9(1 \mathrm{C})$, 31.7 (1C), 29.7 (3C), 29.6 (1C), 29.4 (1C), 29.3 (1C), 28.8 (1C), 28.5 (1C), 25.2 (1C), 22.7 (1C), 22.6 (1C), 14.1 (2C), 9.9 (1C).

HRMS (EI) calcd for $\left[\mathrm{M}, \mathrm{C}_{20} \mathrm{H}_{42} \mathrm{Si}^{+}: 310.3056\right.$, found 310.3057 .

\section{dodecyl(oct-1-en-2-yl)silane (4df)}

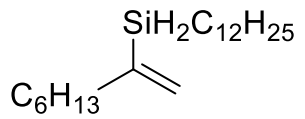

Procedure A, $146.0 \mathrm{mg}, 94 \%$ yield, r.r. $>98: 2$, colorless oil.

${ }^{1} \mathrm{H}$ NMR $\left(400 \mathrm{MHz}, \mathrm{CDCl}_{3}\right) \delta 5.68-5.62(\mathrm{~m}, 1 \mathrm{H}), 5.44(\mathrm{~d}, J=3.0 \mathrm{~Hz}, 1 \mathrm{H}), 3.89(\mathrm{t}, J$ $=3.7 \mathrm{~Hz}, 2 \mathrm{H}), 2.21-2.13(\mathrm{~m}, 2 \mathrm{H}), 1.45-1.23(\mathrm{~m}, 28 \mathrm{H}), 0.91-0.85(\mathrm{~m}, 6 \mathrm{H})$, 
$0.75(\mathrm{tt}, J=11.8,3.7 \mathrm{~Hz}, 2 \mathrm{H})$.

${ }^{13} \mathrm{C} \mathrm{NMR}\left(101 \mathrm{MHz}, \mathrm{CDCl}_{3}\right) \delta 146.2(1 \mathrm{C}), 127.5$ (1C), $37.9(1 \mathrm{C}), 32.8(1 \mathrm{C}), 31.9(1 \mathrm{C})$,

31.7 (1C), 29.7 (4C), 29.5 (1C), 29.4 (1C), 29.3 (1C), 29.0 (1C), 28.9 (1C),

25.2 (1C), 22.7 (1C), 22.6 (1C), 14.1 (1C), 9.3 (1C).

HRMS (EI) calcd for $\left[\mathrm{M}, \mathrm{C}_{20} \mathrm{H}_{42} \mathrm{Si}^{+}: 310.3056\right.$, found 310.3054 .

\section{(E)-phenyl(1-phenylprop-1-en-2-yl)silane (3ea) ${ }^{20}$}<smiles>C/C(=C\c1ccccc1)[SiH2]c1ccccc1</smiles>

Procedure C, $95.6 \mathrm{mg}, 86 \%$ yield, r.r. $=95: 5$ (determined by ${ }^{1} \mathrm{H}$ NMR of crude product through the peak area ratio of $=\mathrm{C} \underline{\mathrm{HPh}} /=\mathrm{C} \underline{\mathrm{HMe}}$ ), colorless oil.

를 (m, 1H), $7.00(\mathrm{q}, J=1.8 \mathrm{~Hz}, 1 \mathrm{H}), 4.65(\mathrm{~s}, 2 \mathrm{H}), 2.03(\mathrm{~d}, J=1.8 \mathrm{~Hz}, 3 \mathrm{H})$.

${ }^{13} \mathrm{C}$ NMR $\left(101 \mathrm{MHz}, \mathrm{CDCl}_{3}\right) \delta 142.4$ (1C), 137.7 (1C), 135.6 (2C), 132.7 (1C), 131.6 (1C), 129.8 (1C), 129.0 (2C), 128.1 (4C), 127.0 (1C), 17.5 (1C).

HRMS (EI) calcd for [M, $\left.\mathrm{C}_{15} \mathrm{H}_{16} \mathrm{Si}\right]^{+}: 224.1021$, found 224.1017.

\section{(E)-phenyl(1-phenylprop-1-en-1-yl)silane (4ea) ${ }^{21}$}<smiles>CC=C(c1ccccc1)c1ccccc1</smiles>

Procedure C, 99.9 mg, 89\% yield, r.r. $>98: 2$, colorless oil.

${ }^{1} \mathrm{H} \mathrm{NMR}\left(400 \mathrm{MHz}, \mathrm{CDCl}_{3}\right) \delta 7.52(\mathrm{~d}, J=6.6 \mathrm{~Hz}, 2 \mathrm{H}), 7.44-7.22(\mathrm{~m}, 5 \mathrm{H}), 7.18(\mathrm{t}, J$ $=7.3 \mathrm{~Hz}, 1 \mathrm{H}), 7.06(\mathrm{~d}, J=7.5 \mathrm{~Hz}, 2 \mathrm{H}), 6.36(\mathrm{q}, J=6.6 \mathrm{~Hz}, 1 \mathrm{H}), 4.67(\mathrm{~s}, 2 \mathrm{H})$, $1.72(\mathrm{~d}, J=6.7 \mathrm{~Hz}, 3 \mathrm{H})$.

${ }^{13} \mathrm{C} \mathrm{NMR}\left(101 \mathrm{MHz}, \mathrm{CDCl}_{3}\right) \delta 141.5$ (1C), 140.8 (1C), 137.3 (1C), $135.6(2 \mathrm{C}), 131.9$ (1C), 129.6 (1C), 128.2 (4C), 127.9 (2C), 126.0 (1C), 16.4 (1C).

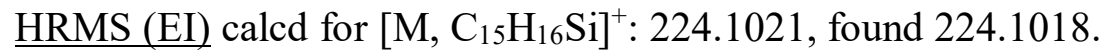




\section{(E)-(1-(4-fluorophenyl)prop-1-en-2-yl)(phenyl)silane (3eb)}<smiles>C/C(=C\c1ccc(F)cc1)[SH2+]c1ccccc1</smiles>

Procedure C, $118.2 \mathrm{mg}, 98 \%$ yield, r.r. $=95: 5$ (determined by ${ }^{1} \mathrm{H}$ NMR of crude product through the peak area ratio of $=\mathrm{C} \underline{\mathrm{HAr}} /=\mathrm{C} \underline{\mathrm{HMe}})$, colorless oil.

${ }^{1} \mathrm{H} \mathrm{NMR}\left(400 \mathrm{MHz}, \mathrm{CDCl}_{3}\right) \delta 7.63(\mathrm{dd}, J=7.6,1.6 \mathrm{~Hz}, 2 \mathrm{H}), 7.41(\mathrm{~m}, 3 \mathrm{H}), 7.29(\mathrm{dd}, J$ $=8.6,5.6 \mathrm{~Hz}, 2 \mathrm{H}), 7.09-7.00(\mathrm{~m}, 2 \mathrm{H}), 6.95(\mathrm{~s}, 1 \mathrm{H}), 4.64(\mathrm{~s}, 2 \mathrm{H}), 2.01(\mathrm{~d}, J$ $=1.7 \mathrm{~Hz}, 3 \mathrm{H})$.

${ }^{13} \mathrm{C} \mathrm{NMR}\left(101 \mathrm{MHz}, \mathrm{CDCl}_{3}\right) \delta 161.7$ (d, $\left.J=252.5 \mathrm{~Hz}, 1 \mathrm{C}\right), 141.1$ (1C), $135.6(2 \mathrm{C})$, $133.8(\mathrm{~d}, J=4.0 \mathrm{~Hz}, 1 \mathrm{C}), 132.6(1 \mathrm{C}), 131.4(1 \mathrm{C}), 130.6$ (d, $J=8.1 \mathrm{~Hz}, 2 \mathrm{C})$, 129.8 (1C), 128.1 (2C), 115.0 (d, $J=21.2 \mathrm{~Hz}, 2 \mathrm{C}), 17.4$ (1C).

HRMS (EI) calcd for [M, $\left.\mathrm{C}_{15} \mathrm{H}_{15} \mathrm{FSi}\right]^{+}:$242.0927, found 242.0922.

\section{(E)-(1-(4-fluorophenyl)prop-1-en-1-yl)(phenyl)silane (4eb)}<smiles>C/C=C(\c1ccccc1)c1ccc(F)cc1</smiles>

Procedure C, $107.2 \mathrm{mg}, 88 \%$ yield, r.r. $>98: 2$, colorless oil.

${ }^{1} \mathrm{H}$ NMR $\left(400 \mathrm{MHz}, \mathrm{CDCl}_{3}\right) \delta 7.50(\mathrm{dd}, J=7.8,1.5 \mathrm{~Hz}, 2 \mathrm{H}), 7.42-7.30(\mathrm{~m}, 3 \mathrm{H}), 7.03$ - $6.94(\mathrm{~m}, 4 \mathrm{H}), 6.37$ (q, $J=6.6 \mathrm{~Hz}, 1 \mathrm{H}), 4.66(\mathrm{~s}, 2 \mathrm{H}), 1.70(\mathrm{~d}, J=6.4 \mathrm{~Hz}$, $3 \mathrm{H})$.

${ }^{13} \mathrm{C} \mathrm{NMR}\left(101 \mathrm{MHz}, \mathrm{CDCl}_{3}\right) \delta 161.3(\mathrm{~d}, J=244.5 \mathrm{~Hz}, 1 \mathrm{C}), 141.9(1 \mathrm{C}), 136.5(\mathrm{~d}, J=$ $4.0 \mathrm{~Hz}, 1 \mathrm{C}), 136.4$ (1C), 135.5 (2C), $131.6(1 \mathrm{C}), 129.7$ (1C), 129.6 (d, $J=8.1$ Hz, 2C), 128.0 (2C), 115.1 (d, $J=21.2 \mathrm{~Hz}, 2 \mathrm{C}), 16.3$ (1C).

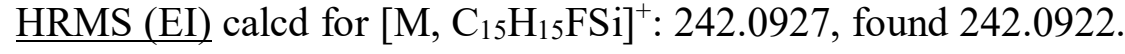

\section{(E)-(1-(4-methoxyphenyl)prop-1-en-2-yl)(phenyl)silane (3ec)}<smiles>COc1ccc(/C=C(\C)[SH+]c2ccccc2)cc1</smiles>

Procedure C, $116.4 \mathrm{mg}$, 92\% yield, r.r. = 97:3 (determined by ${ }^{1} \mathrm{H}$ NMR of crude product through the peak area ratio of $=\mathrm{CHAr} /=\mathrm{CHMe})$, colorless oil. 
${ }^{1} \mathrm{H}$ NMR $\left(400 \mathrm{MHz}, \mathrm{CDCl}_{3}\right) \delta 7.66-7.59(\mathrm{~m}, 2 \mathrm{H}), 7.45-7.35(\mathrm{~m}, 3 \mathrm{H}), 7.32-7.27$ $(\mathrm{m}, 2 \mathrm{H}), 6.94(\mathrm{~d}, J=2.0 \mathrm{~Hz}, 1 \mathrm{H}), 6.89(\mathrm{~d}, J=8.7 \mathrm{~Hz}, 2 \mathrm{H}), 4.64(\mathrm{~s}, 2 \mathrm{H}), 3.82$ (s, 3H), 2.03 (d, $J=1.8 \mathrm{~Hz}, 3 \mathrm{H})$.

${ }^{13} \mathrm{C} \mathrm{NMR}\left(101 \mathrm{MHz}, \mathrm{CDCl}_{3}\right) \delta 158.6(1 \mathrm{C}), 141.9(1 \mathrm{C}), 135.6(2 \mathrm{C}), 131.8(1 \mathrm{C}), 130.6$ (1C), 130.4 (1C), 130.3 (2C), 129.7 (1C), 128.1 (2C), 113.5 (2C), 55.2 (1C), $17.5(1 \mathrm{C})$.

HRMS (EI) calcd for $\left[\mathrm{M}, \mathrm{C}_{16} \mathrm{H}_{18} \mathrm{OSi}\right]^{+}: 254.1127$, found 254.1122.

\section{(E)-(1-(4-methoxyphenyl)prop-1-en-1-yl)(phenyl)silane (4ec)}<smiles>C/C=C(\c1ccccc1)c1ccc(OC)cc1</smiles>

Procedure C, $120.2 \mathrm{mg}, 95 \%$ yield, r.r. $>98: 2$, colorless oil.

${ }^{1} \mathrm{H} \mathrm{NMR}\left(400 \mathrm{MHz}, \mathrm{CDCl}_{3}\right) \delta 7.52(\mathrm{~d}, J=6.3 \mathrm{~Hz}, 2 \mathrm{H}), 7.40-7.27(\mathrm{~m}, 3 \mathrm{H}), 7.00(\mathrm{~d}, J$ $=8.6 \mathrm{~Hz}, 2 \mathrm{H}), 6.84(\mathrm{~d}, J=8.6 \mathrm{~Hz}, 2 \mathrm{H}), 6.33(\mathrm{q}, J=6.5 \mathrm{~Hz}, 1 \mathrm{H}), 4.66(\mathrm{~s}, 2 \mathrm{H})$, $3.79(\mathrm{~s}, 3 \mathrm{H}), 1.73(\mathrm{~d}, J=6.6 \mathrm{~Hz}, 3 \mathrm{H})$.

${ }^{13} \mathrm{C} \mathrm{NMR}\left(101 \mathrm{MHz}, \mathrm{CDCl}_{3}\right) \delta 157.8$ (1C), 141.3 (1C), 136.6 (1C), $135.6(2 \mathrm{C}), 132.9$ (1C), 132.1 (1C), 129.6 (1C), 129.3 (2C), 127.9 (2C), 113.7 (2C), 55.1 (1C), $16.3(1 \mathrm{C})$.

HRMS (EI) calcd for [M, $\left.\mathrm{C}_{16} \mathrm{H}_{18} \mathrm{OSi}\right]^{+}: 254.1127$, found 254.1118.

\section{(E)-(1-([1,1'-biphenyl]-4-yl)prop-1-en-2-yl)(phenyl)silane (3ed)}<smiles>C/C(=C\c1ccc(-c2ccccc2)cc1)[SH+]c1ccccc1</smiles>

Procedure C, $138.6 \mathrm{mg}, 92 \%$ yield, r.r. $=94: 6$ (determined by ${ }^{1} \mathrm{H}$ NMR of crude product through the peak area ratio of $=\mathrm{C} \underline{\mathrm{HAr}} /=\mathrm{CHMe})$, colorless oil.

$\underline{{ }^{1} \mathrm{H} \mathrm{NMR}}\left(400 \mathrm{MHz}, \mathrm{CDCl}_{3}\right) \delta 7.68-7.57(\mathrm{~m}, 6 \mathrm{H}), 7.48-7.32(\mathrm{~m}, 8 \mathrm{H}), 7.04(\mathrm{~s}, 1 \mathrm{H})$, $4.67(\mathrm{~s}, 2 \mathrm{H}), 2.08(\mathrm{~s}, 3 \mathrm{H})$.

${ }^{13} \mathrm{C} \mathrm{NMR}\left(101 \mathrm{MHz}, \mathrm{CDCl}_{3}\right) \delta 141.9$ (1C), 140.7 (1C), 139.8 (1C), 136.8 (1C), 135.6 (2C), $133.0(1 \mathrm{C}), 131.6(1 \mathrm{C}), 129.8$ (1C), $129.5(2 \mathrm{C}), 128.8(2 \mathrm{C}), 128.1(2 \mathrm{C})$, 
127.3 (1C), $127.0(2 \mathrm{C}), 126.8(2 \mathrm{C}), 17.7$ (1C).

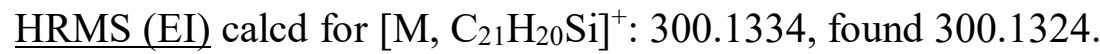

\section{(E)-(1-([1,1'-biphenyl]-4-yl)prop-1-en-1-yl)(phenyl)silane (4ed)}<smiles>C/C=C(\c1ccccc1)c1ccc(-c2ccccc2)cc1</smiles>

Procedure C, $130.2 \mathrm{mg}, 87 \%$ yield, r.r. $>98: 2$, colorless oil.

${ }^{1} \mathrm{H}$ NMR $\left(400 \mathrm{MHz}, \mathrm{CDCl}_{3}\right) \delta 7.63-7.51(\mathrm{~m}, 6 \mathrm{H}), 7.46-7.30(\mathrm{~m}, 6 \mathrm{H}), 7.14(\mathrm{~d}, J=$ $8.2 \mathrm{~Hz}, 2 \mathrm{H}), 6.38$ (q, $J=6.7 \mathrm{~Hz}, 1 \mathrm{H}), 4.71(\mathrm{~s}, 2 \mathrm{H}), 1.79(\mathrm{~s}, 3 \mathrm{H})$.

${ }^{13} \mathrm{C} \mathrm{NMR}\left(101 \mathrm{MHz}, \mathrm{CDCl}_{3}\right) \delta 141.8$ (1C), 140.9 (1C), 139.8 (1C), 138.7 (1C), 136.9 (2C), $135.6(2 \mathrm{C}), 131.9$ (1C), 129.7 (1C), 128.7 (2C), $128.6(2 \mathrm{C}), 128.0(2 \mathrm{C})$, 127.1 (1C), 126.9 (3C), 16.5 (1C).

HRMS (EI) calcd for [M, $\mathrm{C}_{21} \mathrm{H}_{20} \mathrm{Si}^{+}: 300.1334$, found 300.1328 .

\section{(E)-(1-(2-methoxyphenyl)prop-1-en-2-yl)(phenyl)silane (3ee)}<smiles>COc1ccccc1/C=C(\C)[SnH]c1ccccc1</smiles>

Procedure C, $5 \mathrm{~mol} \% \mathrm{C1g}$ was used as catalyst; $105.2 \mathrm{mg}, 83 \%$ yield, r.r. $=97: 3$ (determined by ${ }^{1} \mathrm{H}$ NMR of crude product through the peak area ratio of $=\mathrm{C} \underline{\mathrm{H} A r} /=\mathrm{C} \underline{\mathrm{HMe}})$, colorless oil.

$\underline{{ }^{1} \mathrm{H} \text { NMR }}\left(400 \mathrm{MHz}, \mathrm{CDCl}_{3}\right) \delta 7.68-7.62(\mathrm{~m}, 2 \mathrm{H}), 7.44-7.35(\mathrm{~m}, 3 \mathrm{H}), 7.27-7.20$ (m, 2H), $7.14(\mathrm{~s}, 1 \mathrm{H}), 7.05-6.86(\mathrm{~m}, 2 \mathrm{H}), 4.67(\mathrm{~s}, 2 \mathrm{H}), 3.82(\mathrm{~s}, 3 \mathrm{H}), 1.95(\mathrm{~s}$, $3 \mathrm{H})$.

${ }^{13} \mathrm{C} \mathrm{NMR}\left(101 \mathrm{MHz}, \mathrm{CDCl}_{3}\right) \delta 157.1$ (1C), $138.2(1 \mathrm{C}), 135.6$ (2C), 132.3 (1C), 131.9 (1C), 130.1 (1C), 129.7 (1C), 128.5 (1C), 128.0 (2C), 126.5 (1C), 119.8 (1C), 110.5 (1C), $55.4(1 \mathrm{C}), 17.6(1 \mathrm{C})$.

HRMS (EI) calcd for [M, $\left.\mathrm{C}_{16} \mathrm{H}_{18} \mathrm{OSi}\right]^{+}:$254.1127, found 254.1122. 


\section{(E)-(1-(2-methoxyphenyl)prop-1-en-1-yl)(phenyl)silane (4ee)}<smiles>C/C=C(\c1ccccc1)c1ccccc1OC</smiles>

Procedure C, $114.5 \mathrm{mg}, 90 \%$ yield, r.r. $>98: 2$, colorless oil.

${ }^{1} \mathrm{H}$ NMR $\left(400 \mathrm{MHz}, \mathrm{CDCl}_{3}\right) \delta 7.65-7.50(\mathrm{~m}, 2 \mathrm{H}), 7.43-7.26(\mathrm{~m}, 3 \mathrm{H}), 7.18(\mathrm{t}, J=$ $7.8 \mathrm{~Hz}, 1 \mathrm{H}), 7.00$ (dd, $J=7.5,1.8 \mathrm{~Hz}, 1 \mathrm{H}), 6.91$ (d, $J=7.4 \mathrm{~Hz}, 1 \mathrm{H}), 6.83$ (d, $J=8.2 \mathrm{~Hz}, 1 \mathrm{H}), 6.36(\mathrm{qd}, J=6.7,1.9 \mathrm{~Hz}, 1 \mathrm{H}), 4.62(\mathrm{~d}, J=2.0 \mathrm{~Hz}, 2 \mathrm{H}), 3.66$ (d, $J=1.7 \mathrm{~Hz}, 3 \mathrm{H}), 1.72(\mathrm{~d}, J=6.7 \mathrm{~Hz}, 3 \mathrm{H})$.

${ }^{13} \mathrm{C} \mathrm{NMR}\left(101 \mathrm{MHz}, \mathrm{CDCl}_{3}\right) \delta 156.2$ (1C), 142.0 (1C), 135.4 (2C), 133.5 (1C), 133.0 (1C), 129.7 (1C), 129.5 (1C), 129.3 (1C), 127.7 (2C), 127.6 (1C), 120.3 (1C), 110.6 (1C), $55.1(1 \mathrm{C}), 16.7(1 \mathrm{C})$.

HRMS (EI) calcd for [M-H, $\left.\mathrm{C}_{16} \mathrm{H}_{17} \mathrm{OSi}\right]^{+}:$253.1043, found 253.1042.

\section{(E)-phenyl(1-phenylbut-1-en-2-yl)silane (3ef)}<smiles>CC/C(=C\c1ccccc1)Sc1ccccc1</smiles>

Procedure C, $5 \mathrm{~mol} \%$ C1g was used as catalyst; $107.4 \mathrm{mg}, 90 \%$ yield. r.r. $=78: 22$ (determined by ${ }^{1} \mathrm{H}$ NMR of crude product through the peak area ratio of $=\mathrm{CHEt} /=\mathrm{C} \underline{H P h})$, colorless oil.

를 NMR (400 MHz, $\left.\mathrm{CDCl}_{3}\right) \delta 7.65(\mathrm{dt}, J=6.2,1.8 \mathrm{~Hz}, 2 \mathrm{H}), 7.45-7.23(\mathrm{~m}, 8 \mathrm{H}), 6.98$ (s, 1H), $4.68(\mathrm{~s}, 2 \mathrm{H}), 2.47(\mathrm{qd}, J=7.6,1.3 \mathrm{~Hz}, 2 \mathrm{H}), 1.09(\mathrm{t}, J=7.6 \mathrm{~Hz}, 3 \mathrm{H})$

${ }^{13} \mathrm{C} \mathrm{NMR}\left(101 \mathrm{MHz}, \mathrm{CDCl}_{3}\right) \delta 142.1$ (1C), 139.7 (1C), 137.7 (1C), 135.7 (2C), 132.0 (1C), 129.7 (1C), 128.7 (2C), 128.2 (2C), 128.1 (2), 127.0 (1C), 24.5 (1C) $14.2(1 \mathrm{C})$

HRMS (EI) calcd for [M, $\left.\mathrm{C}_{16} \mathrm{H}_{18} \mathrm{Si}\right]^{+}: 238.1178$, Found 238.1173. 


\section{(E)-phenyl(1-phenylbut-1-en-1-yl)silane (4ef) ${ }^{15}$}<smiles>CC=C(c1ccccc1)c1ccccc1</smiles>

Procedure C, $114.5 \mathrm{mg}, 96 \%$ yield, r.r. $=97: 3$ (determined by ${ }^{1} \mathrm{H}$ NMR of crude product through the peak area ratio of $=\mathrm{CHEt} /=\mathrm{C} \underline{\mathrm{HPh}})$, colorless oil.

를 NMR (400 MHz, $\left.\mathrm{CDCl}_{3}\right) \delta 7.55-7.48(\mathrm{~m}, 2 \mathrm{H}), 7.41-7.23(\mathrm{~m}, 5 \mathrm{H}), 7.20-7.13$ (m, 1H), $7.07-7.01(\mathrm{~m}, 2 \mathrm{H}), 6.25(\mathrm{t}, J=7.1 \mathrm{~Hz}, 1 \mathrm{H}), 4.68(\mathrm{~s}, 2 \mathrm{H}), 2.10(\mathrm{p}$, $J=7.4 \mathrm{~Hz}, 2 \mathrm{H}), 0.96(\mathrm{t}, J=7.5 \mathrm{~Hz}, 3 \mathrm{H})$.

${ }^{13} \mathrm{C} \mathrm{NMR}\left(101 \mathrm{MHz}, \mathrm{CDCl}_{3}\right) \delta 148.9$ (1C), 141.0 (1C), 135.5 (3C), 131.9 (1C), 129.6 (1C), 128.2 (2C), 128.1 (2C), 127.9 (2C), 125.9 (1C), 23.7 (1C), 14.0 (1C).

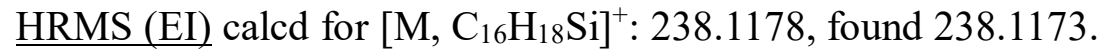

\section{phenyl((2E,4E)-5-phenylpenta-2,4-dien-2-yl)silane (3eg)}<smiles>C/C(=C\C=C\c1ccccc1)Sc1ccccc1</smiles>

Procedure C; C1k was used as catalyst; $83.9 \mathrm{mg}, 66 \%$ yield, r.r. $=91: 9$ (determined by ${ }^{1} \mathrm{H}$ NMR of crude product through the peak area ratio of $=\mathrm{C} \underline{\mathrm{HC}}=\mathrm{C} /=\mathrm{C} \underline{\mathrm{HMe}}$ ), colorless oil.

${ }^{1} \mathrm{H}$ NMR $\left(400 \mathrm{MHz}, \mathrm{CDCl}_{3}\right) \delta 7.64-7.57(\mathrm{~m}, 2 \mathrm{H}), 7.47-7.28(\mathrm{~m}, 7 \mathrm{H}), 7.26-7.10$ (m, 2H), $6.71(\mathrm{dd}, J=10.8,1.9 \mathrm{~Hz}, 1 \mathrm{H}), 6.61(\mathrm{~d}, J=15.5 \mathrm{~Hz}, 1 \mathrm{H}), 4.60$ (s, 2H), 1.99 (s, 3H).

${ }^{13} \mathrm{C} \mathrm{NMR}\left(101 \mathrm{MHz}, \mathrm{CDCl}_{3}\right) \delta 142.2$ (1C), 137.3 (1C), 135.6 (2C), 134.4 (1C), 132.4 (1C), 131.5 (1C), 129.8 (1C), $128.6(2 \mathrm{C}), 128.1$ (2C), 127.8 (1C), $126.6(2 \mathrm{C})$, 124.0 (1C), 16.4 (1C).

$\underline{\operatorname{HRMS}(\mathrm{EI})}$ calcd for $\left[\mathrm{M}, \mathrm{C}_{17} \mathrm{H}_{18} \mathrm{Si}^{+}:\right.$: 250.1178, found 250.1174.

phenyl((1E,3E)-1-phenylpenta-1,3-dien-3-yl)silane (4eg)<smiles>C/C=C(\C=C\c1ccccc1)[SH+]c1ccccc1</smiles> 
Procedure C, $84.1 \mathrm{mg}, 67 \%$ yield, r.r. $=98: 2$ (determined by ${ }^{1} \mathrm{H}$ NMR of crude product through the peak area ratio of $=\mathrm{C} \underline{H M e} /=\mathrm{C} \underline{H C}=\mathrm{C}$ ), colorless oil.

${ }^{1} \mathrm{H} \mathrm{NMR}\left(400 \mathrm{MHz}, \mathrm{CDCl}_{3}\right) \delta 7.62(\mathrm{~d}, J=7.3 \mathrm{~Hz}, 2 \mathrm{H}), 7.43-7.27$ (m, 8H), $7.19(\mathrm{t}, J$ $=7.3 \mathrm{~Hz}, 1 \mathrm{H}), 6.65(\mathrm{~d}, J=16.3 \mathrm{~Hz}, 1 \mathrm{H}), 6.25(\mathrm{q}, J=6.8 \mathrm{~Hz}, 1 \mathrm{H}), 4.76(\mathrm{~s}$, 2H), $1.97(\mathrm{~d}, J=6.9 \mathrm{~Hz}, 3 \mathrm{H})$.

${ }^{13} \mathrm{C} \mathrm{NMR}\left(101 \mathrm{MHz}, \mathrm{CDCl}_{3}\right) \delta 143.3$ (1C), 137.7 (1C), 135.5 (2C), $132.2(1 \mathrm{C}), 132.1$

(1C), 131.8 (1C), 129.7 (1C), 128.5 (2C), $128.0(2 \mathrm{C}), 127.4(1 \mathrm{C}), 127.1$ (1C), $126.3(2 \mathrm{C}), 15.3(1 \mathrm{C})$.

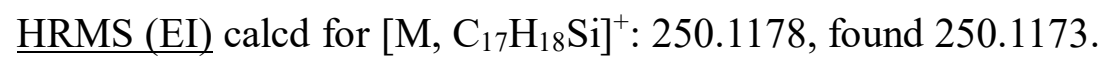

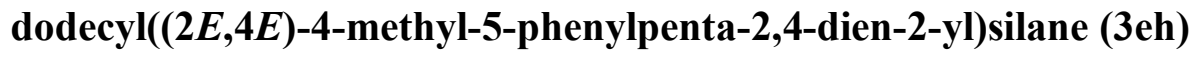<smiles>CCCCCCCCCCCCCCCC(C)=Cc1ccccc1</smiles>

Procedure C, $169.3 \mathrm{mg}, 95 \%$ yield, r.r. $=90: 10$ (determined by ${ }^{1} \mathrm{H}$ NMR of crude product through the peak area ratio of $=\mathrm{C} \underline{\mathrm{HC}}=\mathrm{C} /=\mathrm{C} \underline{H M e})$, colorless oil.

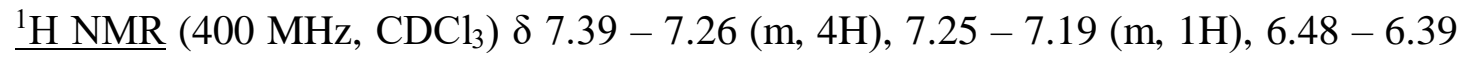
$(\mathrm{m}, 2 \mathrm{H}), 3.96(\mathrm{t}, J=3.7 \mathrm{~Hz}, 2 \mathrm{H}), 2.02(\mathrm{dd}, J=10.9,1.6 \mathrm{~Hz}, 5 \mathrm{H}), 1.50-1.19$ $(\mathrm{m}, 21 \mathrm{H}), 0.88(\mathrm{t}, J=6.6 \mathrm{~Hz}, 3 \mathrm{H}), 0.83(\mathrm{~s}, 2 \mathrm{H})$.

${ }^{13} \mathrm{C} \mathrm{NMR}\left(101 \mathrm{MHz}, \mathrm{CDCl}_{3}\right) \delta 145.2$ (1C), 137.8 (1C), 135.6 (1C), 131.8 (1C), 130.6 (1C), 129.1 (2C), 128.1 (2C), 126.4 (1C), 32.9 (1C), 31.9 (1C), 29.7 (3C), 29.6 (1C), 29.4 (1C), 29.3 (1C), 25.2 (1C), 22.7 (1C), 18.6 (1C), 17.9 (1C), $14.1(1 \mathrm{C}), 9.2(1 \mathrm{C})$.

HRMS (EI) calcd for [M, $\mathrm{C}_{24} \mathrm{H}_{40} \mathrm{Si}^{+}: 356.2899$, found 356.2893.

\section{((1E,3E)-2-methyl-1-phenylpenta-1,3-dien-3-yl)(phenyl)silane (4eh)}<smiles>CC=C(/C(C)=C/c1ccccc1)/C(C)=C/c1ccccc1</smiles>

Procedure C, $117.3 \mathrm{mg}, 89 \%$ yield, r.r. $=93: 7$ (determined by ${ }^{1} \mathrm{H}$ NMR of crude product through the peak area ratio of $=\mathrm{C} \underline{\mathrm{HMe}} /=\mathrm{C} \underline{\mathrm{HC}}=\mathrm{C})$, colorless oil.

${ }^{1} \mathrm{H}$ NMR $\left(400 \mathrm{MHz}, \mathrm{CDCl}_{3}\right) \delta 7.66-7.57(\mathrm{~m}, 2 \mathrm{H}), 7.46-7.26(\mathrm{~m}, 5 \mathrm{H}), 7.25-7.06$ 
$(\mathrm{m}, 3 \mathrm{H}), 6.18-6.05(\mathrm{~m}, 2 \mathrm{H}), 4.62(\mathrm{~s}, 2 \mathrm{H}), 1.90(\mathrm{~d}, J=1.4 \mathrm{~Hz}, 3 \mathrm{H}), 1.80(\mathrm{~d}$, $J=6.6 \mathrm{~Hz}, 3 \mathrm{H})$.

${ }^{13} \mathrm{C} \mathrm{NMR}\left(101 \mathrm{MHz}, \mathrm{CDCl}_{3}\right) \delta 141.5$ (1C), 139.5 (1C), 138.1 (1C), 138.0 (1C), 135.6 (2C), $132.2(1 \mathrm{C}), 129.6(1 \mathrm{C}), 128.8(2 \mathrm{C}), 128.0(2 \mathrm{C}), 127.9(2 \mathrm{C}), 126.8(1 \mathrm{C})$, 126.1 (1C), $18.8(1 \mathrm{C}), 16.2(1 \mathrm{C})$.

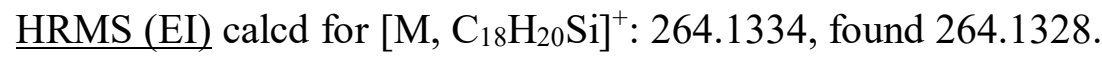

(E)-(1-(cyclohex-1-en-1-yl)prop-1-en-2-yl)(phenyl)silane (3ei)<smiles>C/C(=C\C1=CCCCC1)[SiH2]c1ccccc1</smiles>

Procedure C, C1k was used as catalyst; $99.1 \mathrm{mg}, 86 \%$ yield, r.r. $=93: 7$ (determined by ${ }^{1} \mathrm{H}$ NMR of crude product through the peak area ratio of $\left.=\mathrm{C} \underline{\mathrm{HC}}=\mathrm{C} /=\mathrm{C} \underline{\mathrm{HMe}}\right)$, colorless oil.

${ }^{1} \mathrm{H}$ NMR $\left(400 \mathrm{MHz}, \mathrm{CDCl}_{3}\right) \delta 7.64-7.51(\mathrm{~m}, 2 \mathrm{H}), 7.45-7.29(\mathrm{~m}, 3 \mathrm{H}), 6.33(\mathrm{~s}, 1 \mathrm{H})$, $5.71(\mathrm{~s}, 1 \mathrm{H}), 4.54(\mathrm{~s}, 2 \mathrm{H}), 2.21-2.10(\mathrm{~m}, 4 \mathrm{H}), 1.93(\mathrm{~s}, 3 \mathrm{H}), 1.67-1.54(\mathrm{~m}$, $4 \mathrm{H})$.

${ }^{13} \mathrm{C} \mathrm{NMR}\left(101 \mathrm{MHz}, \mathrm{CDCl}_{3}\right) \delta 145.6$ (1C), 136.1 (1C), 135.5 (2C), 134.1 (1C), 132.2 (1C), 129.6 (1C), 129.3 (1C), 128.0 (2C), 28.9 (1C), 25.6 (1C), 22.9 (1C), $22.0(1 \mathrm{C}), 17.6(1 \mathrm{C})$.

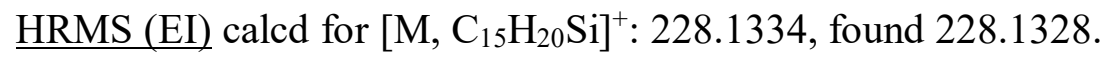

\section{(E)-(1-(cyclohex-1-en-1-yl)prop-1-en-1-yl)(phenyl)silane (4ei)}<smiles>C/C=C(/[SH2+]c1ccccc1)C1=CCCCC1</smiles>

Procedure C, $105.8 \mathrm{mg}, 92 \%$ yield, r.r. $=93: 7$ (determined by ${ }^{1} \mathrm{H}$ NMR of crude product through the peak area ratio of $=\mathrm{C} \underline{\mathrm{HMe}} /=\mathrm{C} \underline{\mathrm{HC}}=\mathrm{C})$, colorless oil.

${ }^{1} \mathrm{H}$ NMR $\left(400 \mathrm{MHz}, \mathrm{CDCl}_{3}\right) \delta 7.72-7.49(\mathrm{~m}, 2 \mathrm{H}), 7.46-7.28(\mathrm{~m}, 3 \mathrm{H}), 6.02(\mathrm{q}, J=$ $6.6 \mathrm{~Hz}, 1 \mathrm{H}), 5.30(\mathrm{~s}, 1 \mathrm{H}), 4.52(\mathrm{~s}, 2 \mathrm{H}), 2.05-2.01(\mathrm{~m}, 2 \mathrm{H}), 1.93-1.89(\mathrm{~m}$, $2 \mathrm{H}), 1.72(\mathrm{~d}, J=6.6 \mathrm{~Hz}, 3 \mathrm{H}), 1.63-1.50(\mathrm{~m}, 4 \mathrm{H})$.

${ }^{13} \mathrm{C} \mathrm{NMR}\left(101 \mathrm{MHz}, \mathrm{CDCl}_{3}\right) \delta 139.6$ (1C), 139.3 (1C), 137.2 (1C), 135.5 (2C), 132.6 
(1C), 129.4 (1C), 127.8 (2C), 123.4 (1C), 29.2 (1C), 25.3 (1C), 22.9 (1C), $22.2(1 \mathrm{C}), 16.2(1 \mathrm{C})$.

HRMS (EI) calcd for [M, $\left.\mathrm{C}_{15} \mathrm{H}_{20} \mathrm{Si}^{+}\right]^{+}$:228.1334, found 228.1328.

\section{(E)-(4-methylbenzyl)(oct-2-en-2-yl)silane (3ej)}<smiles>CC(=C[CH+]c1ccccc1)[SiH2]Cc1ccc(C)cc1</smiles>

Procedure C, C1e was used as catalyst and toluene $(1.5 \mathrm{~mL})$ was used as solvent; 111.0 mg, 90\% yield, r.r. $=91: 9$ (determined by ${ }^{1} \mathrm{H}$ NMR of crude product through the peak area ratio of $\left.=\mathrm{CHCH}_{2} /=\mathrm{C} \underline{H M e}\right)$, colorless oil.

${ }^{1} \mathrm{H}$ NMR $\left(400 \mathrm{MHz}, \mathrm{CDCl}_{3}\right) \delta 7.03(\mathrm{~d}, J=7.9 \mathrm{~Hz}, 2 \mathrm{H}), 6.98(\mathrm{~d}, J=8.0 \mathrm{~Hz}, 2 \mathrm{H}), 5.93$ $-5.85(\mathrm{~m}, 2 \mathrm{H}) 3.98(\mathrm{t}, J=3.8 \mathrm{~Hz}, 2 \mathrm{H}), 2.29(\mathrm{~s}, 3 \mathrm{H}), 2.24(\mathrm{t}, J=3.8 \mathrm{~Hz}, 2 \mathrm{H})$, $2.07(\mathrm{q}, J=7.1 \mathrm{~Hz}, 2 \mathrm{H}), 1.69-1.65(\mathrm{~m}, 3 \mathrm{H}), 1.39-1.20(\mathrm{~m}, 6 \mathrm{H}), 0.89(\mathrm{t}, J$ $=7.0 \mathrm{~Hz}, 3 \mathrm{H})$.

${ }^{13} \mathrm{C} \mathrm{NMR}\left(101 \mathrm{MHz}, \mathrm{CDCl}_{3}\right) \delta 145.2$ (1C), 136.5 (1C), 133.8 (1C), 129.1 (2C), 128.6 (1C), 128.1 (2C), 31.5 (1C), 28.6 (1C), 22.6 (1C), 20.9 (1C), 18.7 (1C), 15.6 (1C), $14.0(1 \mathrm{C})$.

HRMS (EI) calcd for $\left[\mathrm{M}, \mathrm{C}_{16} \mathrm{H}_{26} \mathrm{Si}\right]^{+}:$246.1804, found 246.1798 .

\section{(E)-oct-2-en-3-yl(phenyl)silane (4ej)}<smiles>C/C=C(/[SH2+])c1ccccc1</smiles>

Procedure C, $102.6 \mathrm{mg}, 93 \%$ yield, r.r. $=97: 3$ (determined by ${ }^{1} \mathrm{H}$ NMR of crude product through the peak area ratio of $=\mathrm{CHCH}_{2} /=\mathrm{C} \underline{\mathrm{HMe}}$ ), colorless oil.

${ }^{1} \mathrm{H} \mathrm{NMR}\left(400 \mathrm{MHz}, \mathrm{CDCl}_{3}\right) \delta 7.65-7.50(\mathrm{~m}, 2 \mathrm{H}), 7.42-7.32(\mathrm{~m}, 3 \mathrm{H}), 6.10$ (q, $J=$ $6.6 \mathrm{~Hz}, 1 \mathrm{H}), 4.52(\mathrm{~s}, 2 \mathrm{H}), 2.19(\mathrm{t}, J=7.7 \mathrm{~Hz}, 2 \mathrm{H}), 1.73(\mathrm{~d}, J=6.5 \mathrm{~Hz}, 3 \mathrm{H})$, $1.43-1.15(\mathrm{~m}, 6 \mathrm{H}), 0.85(\mathrm{t}, J=6.7 \mathrm{~Hz}, 3 \mathrm{H})$.

${ }^{13} \mathrm{C}$ NMR $\left(400 \mathrm{MHz}, \mathrm{CDCl}_{3}\right) \delta 139.9$ (1C), 135.5 (2C), 135.3 (1C), 132.6 (1C), 129.5 (1C), 127.9 (2C), 31.8 (1C), 29.9 (1C), 28.9 (1C), 22.5 (1C), 14.6 (1C), 14.0 
(1C).

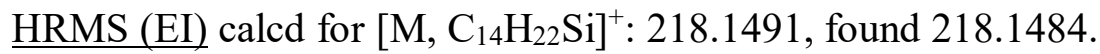

\section{(E)-(4-methylbenzyl)(6-phenylhex-2-en-2-yl)silane (3ek)}<smiles>CC(=CCCCc1ccccc1)[SiH2]Cc1ccc(C)cc1</smiles>

Procedure C; C1e was used as catalyst and toluene $(1.5 \mathrm{~mL})$ was used as solvent; 139.9 mg, 95\% yield, r.r. $=91: 9$ (determined by ${ }^{1} \mathrm{H}$ NMR of crude product through the peak area ratio of $\left.=\mathrm{CHCH}_{2} /=\mathrm{CHMe}\right)$, colorless oil.

${ }^{1} \mathrm{H}$ NMR $\left(400 \mathrm{MHz}, \mathrm{CDCl}_{3}\right) \delta 7.28(\mathrm{~d}, J=7.4 \mathrm{~Hz}, 1 \mathrm{H}), 7.25(\mathrm{~d}, J=3.1 \mathrm{~Hz}, 1 \mathrm{H}), 7.21$ $-7.13(\mathrm{~m}, 3 \mathrm{H}), 7.03(\mathrm{~d}, J=8.1 \mathrm{~Hz}, 2 \mathrm{H}), 6.98(\mathrm{~d}, J=8.1 \mathrm{~Hz}, 2 \mathrm{H}), 5.91$ (tq, $J$ $=6.9,1.8 \mathrm{~Hz}, 1 \mathrm{H}), 3.99(\mathrm{t}, J=3.8 \mathrm{~Hz}, 2 \mathrm{H}), 2.60-2.54(\mathrm{~m}, 2 \mathrm{H}), 2.27(\mathrm{~s}, 3 \mathrm{H})$, $2.25(\mathrm{t}, J=3.9 \mathrm{~Hz}, 2 \mathrm{H}), 2.12(\mathrm{q}, J=7.3 \mathrm{~Hz}, 2 \mathrm{H}), 1.73-1.62(\mathrm{~m}, 5 \mathrm{H})$.

${ }^{13} \mathrm{C} \mathrm{NMR}\left(101 \mathrm{MHz}, \mathrm{CDCl}_{3}\right) \delta 144.4$ (1C), 142.3 (1C), 136.4 (1C), 133.8 (1C), 129.3 (1C), 129.1 (2C), 128.4 (2C), 128.2 (2C), 128.1 (2C), 125.7 (1C), 35.4 (1C), 30.6 (1C), 28.2 (1C), 20.9 (1C), 18.7 (1C), 15.6 (1C).

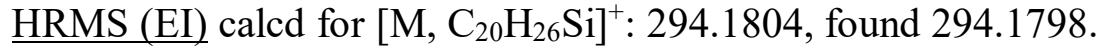

\section{(E)-phenyl(6-phenylhex-2-en-3-yl)silane (4ek)}<smiles>C/C=C(\CCCc1ccccc1)[Hg]c1ccccc1</smiles>

Procedure C, the reaction time was $2 \mathrm{~h}, 82.3 \mathrm{mg}$ (0.524 mmol) alkyne was used, 138.2 mg, 99\% yield, r.r. $>98: 2$, colorless oil.

$\underline{{ }^{1} \mathrm{H} \mathrm{NMR}}\left(400 \mathrm{MHz}, \mathrm{CDCl}_{3}\right) \delta 7.55(\mathrm{~d}, J=6.4 \mathrm{~Hz}, 2 \mathrm{H}), 7.41-7.33(\mathrm{~m}, 3 \mathrm{H}), 7.30-$ $7.21(\mathrm{~m}, 2 \mathrm{H}), 7.16(\mathrm{t}, J=7.3 \mathrm{~Hz}, 1 \mathrm{H}), 7.10(\mathrm{~d}, J=7.5 \mathrm{~Hz}, 2 \mathrm{H}), 6.13(\mathrm{q}, J=$ $6.6 \mathrm{~Hz}, 1 \mathrm{H}), 4.54(\mathrm{~s}, 2 \mathrm{H}), 2.56(\mathrm{t}, J=7.8 \mathrm{~Hz}, 2 \mathrm{H}), 2.24(\mathrm{t}, J=7.9 \mathrm{~Hz}, 2 \mathrm{H})$, $1.86-1.63(\mathrm{~m}, 5 \mathrm{H})$.

${ }^{13} \mathrm{C} \mathrm{NMR}\left(101 \mathrm{MHz}, \mathrm{CDCl}_{3}\right) \delta 142.3$ (1C), $140.4(1 \mathrm{C}), 135.6$ (2C), 134.8 (1C), 132.5 (1C), 129.5 (1C), 128.3 (2C), 128.2 (2C), 128.0 (2C), 125.6 (1C), 35.8 (1C), 
30.8 (1C), $29.6(1 \mathrm{C}), 14.7(1 \mathrm{C})$

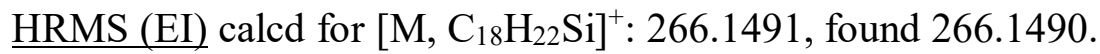

\section{(E)-(1-cyclohexylprop-1-en-2-yl)(phenyl)silane (3el) ${ }^{22}$}<smiles>CC(=CC1CCCCC1)[SnH2]c1ccccc1</smiles>

Procedure C; C1e was used as catalyst and toluene $(1.5 \mathrm{~mL})$ was used as solvent; the reaction time was $2 \mathrm{~h} ; 109.0 \mathrm{mg}, 95 \%$ yield, r.r. $=96: 4$ (determined by ${ }^{1} \mathrm{H}$ NMR of crude product through the peak area ratio of $=\mathrm{CHCH} /=\mathrm{CHMe})$, colorless oil.

${ }^{1} \mathrm{H}$ NMR $\left(400 \mathrm{MHz}, \mathrm{CDCl}_{3}\right) \delta 7.62-7.52(\mathrm{~m}, 2 \mathrm{H}), 7.45-7.32(\mathrm{~m}, 3 \mathrm{H}), 5.87(\mathrm{~d}, J=$ $5.2 \mathrm{~Hz}, 1 \mathrm{H}), 4.49$ (s, 2H), $2.47-2.35(\mathrm{~m}, 1 \mathrm{H}), 1.71-1.59$ (m, 8H), $1.36-$ $1.02(\mathrm{~m}, 5 \mathrm{H})$.

${ }^{13} \mathrm{C} \mathrm{NMR}\left(101 \mathrm{MHz}, \mathrm{CDCl}_{3}\right) \delta 151.8(1 \mathrm{C}), 135.5(2 \mathrm{C}), 132.3$ (1C), 129.5 (1C), 127.9 (2C), 126.3 (1C), 37.7 (1C), 32.5 (2C), 26.1 (1C), 25.9 (2C), 15.5 (1C).

HRMS (EI) calcd for [M, $\left.\mathrm{C}_{15} \mathrm{H}_{22} \mathrm{Si}\right]^{+}:$230.1491, found 230.1486.

\section{(E)-(1-cyclohexylprop-1-en-1-yl)(phenyl)silane (4el)}<smiles>C/C=C(/S)C1CCCCC1</smiles>

Procedure C; C1i was used as catalyst, reaction time was 2 h; 104.9 mg, 90\% yield, r.r. $=91: 9$ (determined by ${ }^{1} \mathrm{H}$ NMR of crude product through the peak area ratio of $=\mathrm{C} \underline{\mathrm{HMe}} /=\mathrm{C} \underline{\mathrm{HCH}})$, colorless oil.

를 NMR $\left(400 \mathrm{MHz}, \mathrm{CDCl}_{3}\right) \delta 7.61-7.52(\mathrm{~m}, 2 \mathrm{H}), 7.44-7.29(\mathrm{~m}, 3 \mathrm{H}), 6.01(\mathrm{q}, J=$ $6.6 \mathrm{~Hz}, 1 \mathrm{H}), 4.55(\mathrm{~s}, 2 \mathrm{H}), 2.59-2.52(\mathrm{~m}, 1 \mathrm{H}), 1.79-1.47(\mathrm{~m}, 8 \mathrm{H}), 1.32-$ $1.17(\mathrm{~m}, 4 \mathrm{H}), 1.16-1.04(\mathrm{~m}, 1 \mathrm{H})$.

${ }^{13} \mathrm{C} \mathrm{NMR}\left(101 \mathrm{MHz}, \mathrm{CDCl}_{3}\right) \delta 141.1$ (1C), 139.4 (1C), 135.6 (2C), 133.6 (1C), 129.3 (1C), 127.8 (2C), 40.2 (1C), 32.8 (2C), 26.6 (2C), 26.0 (1C), 14.6 (1C).

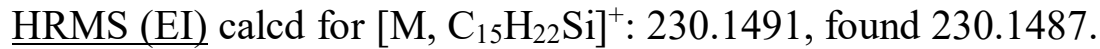




\section{(E)-(4,4-dimethylpent-2-en-2-yl)(dodecyl)silane (3em)}

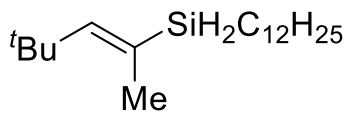

Procedure $\mathrm{C}, \mathrm{C1e}$ was used as catalyst and toluene $(1.5 \mathrm{~mL})$ was used as solvent, the reaction time was $2 \mathrm{~h}, 49.1 \mathrm{mg}(0.510 \mathrm{mmol})$ alkyne was used, $152.9 \mathrm{mg}$, 99\% yield, r.r. $>98: 2$, colorless oil.

${ }^{1} \mathrm{H}$ NMR $\left(400 \mathrm{MHz}, \mathrm{CDCl}_{3}\right) \delta 5.87(\mathrm{~d}, J=1.8 \mathrm{~Hz}, 1 \mathrm{H}), 3.84(\mathrm{t}, J=3.6 \mathrm{~Hz}, 2 \mathrm{H}), 1.85$ $(\mathrm{d}, J=1.8 \mathrm{~Hz}, 3 \mathrm{H}), 1.26(\mathrm{~s}, 20 \mathrm{H}), 1.13(\mathrm{~s}, 9 \mathrm{H}), 0.88(\mathrm{t}, J=6.7 \mathrm{~Hz}, 3 \mathrm{H}), 0.77$ $-0.70(\mathrm{~m}, 2 \mathrm{H})$.

${ }^{13} \mathrm{C} \mathrm{NMR}\left(101 \mathrm{MHz}, \mathrm{CDCl}_{3}\right) \delta 152.8(1 \mathrm{C}), 128.4(1 \mathrm{C}), 34.9(1 \mathrm{C}), 32.8(1 \mathrm{C}), 31.9(1 \mathrm{C})$, 30.6 (3C), 29.7 (2C), 29.7 (1C), 29.6 (1C), 29.4 (1C), 29.3 (1C), 25.1 (1C), 22.7 (1C), 16.5 (1C), 14.1 (1C), 9.3 (1C).

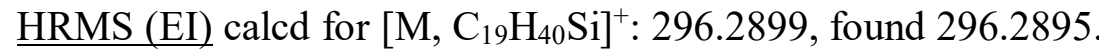

\section{(E)-(4,4-dimethylpent-2-en-3-yl)(phenyl)silane (4em)}<smiles>C/C=C(\[SH2+])[SnH2]c1ccccc1</smiles>

Procedure C; C1h was used as catalyst; the reaction time was $2 \mathrm{~h} ; 90.1 \mathrm{mg}, 86 \%$ yield, r.r. $=86: 14$ (determined by ${ }^{1} \mathrm{H}$ NMR of crude product through the peak area ratio of $\left.=\underline{\mathrm{H}}^{t} \mathrm{Bu} /=\mathrm{C} \underline{\mathrm{HMe}}\right)$, colorless oil.

$\underline{{ }^{1} \mathrm{H} \mathrm{NMR}}\left(400 \mathrm{MHz}, \mathrm{CDCl}_{3}\right) \delta 7.58(\mathrm{~d}, J=9.4 \mathrm{~Hz}, 2 \mathrm{H}), 7.40-7.31(\mathrm{~m}, 3 \mathrm{H}), 6.17(\mathrm{q}, J$ $=7.1 \mathrm{~Hz}, 1 \mathrm{H}), 4.60(\mathrm{~s}, 2 \mathrm{H}), 1.93-1.86(\mathrm{~m}, 3 \mathrm{H}), 1.18(\mathrm{~s}, 9 \mathrm{H})$.

${ }^{13} \mathrm{C} \mathrm{NMR}\left(101 \mathrm{MHz}, \mathrm{CDCl}_{3}\right) \delta 144.7$ (1C), 141.5 (1C), 135.3 (2C), 134.5 (1C), 129.2 (1C), 127.9 (2C), 36.0 (1C), 31.1 (3C), 18.1 (1C).

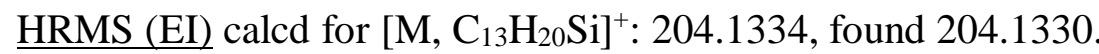

\section{(E)-(1-cyclohexylbut-1-en-2-yl)(dodecyl)silane (3en)}

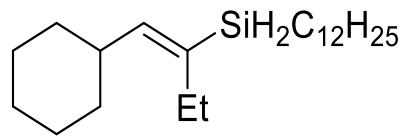

Procedure C, C1e was used as catalyst and toluene (1.5 mL) was used as solvent; 154.7 
mg, 92\% yield, r.r. $=93: 7$ (determined by ${ }^{1} \mathrm{H}$ NMR of crude product through the peak area ratio of $=\mathrm{CHCH} /=\mathrm{CHCH}_{2}$ ), colorless oil.

$\underline{{ }^{1} \mathrm{H} \mathrm{NMR}}\left(400 \mathrm{MHz}, \mathrm{CDCl}_{3}\right) \delta 5.68(\mathrm{~d}, J=9.1 \mathrm{~Hz}, 1 \mathrm{H}), 3.87(\mathrm{t}, J=3.7 \mathrm{~Hz}, 2 \mathrm{H}), 2.42-$ $2.28(\mathrm{~m}, 1 \mathrm{H}), 2.17(\mathrm{q}, J=7.5 \mathrm{~Hz}, 2 \mathrm{H}), 1.76-1.54(\mathrm{~m}, 6 \mathrm{H}), 1.43-1.03(\mathrm{~m}$, 24H), $0.98(\mathrm{t}, J=7.6 \mathrm{~Hz}, 3 \mathrm{H}), 0.88$ (t, $J=6.7 \mathrm{~Hz}, 3 \mathrm{H}), 0.77-0.69(\mathrm{~m}, 2 \mathrm{H})$.

${ }^{13} \mathrm{C} \mathrm{NMR}\left(101 \mathrm{MHz}, \mathrm{CDCl}_{3}\right) \delta 149.4$ (1C), 133.9 (1C), 37.4 (1C), 33.0 (2C), 32.8 (1C), 31.9 (1C), 29.7 (3C), 29.6 (1C), 29.4 (1C), 29.3 (1C), 26.1 (1C), 25.9 (2C), 25.3 (1C), 23.8 (1C), 22.7 (1C), 14.9 (1C), 14.1 (1C), 9.7 (1C).

HRMS (EI) calcd for [M, $\mathrm{C}_{22} \mathrm{H}_{44} \mathrm{Si}^{+}$: 336.3212 , found 336.3203 .

\section{(E)-(1-cyclohexylbut-1-en-1-yl)(phenyl)silane (4en)}<smiles>C/C=C(/[SH2+]c1ccccc1)C1CCCCC1</smiles>

Procedure C, C1i was used as catalyst; the reaction time was $2 \mathrm{~h} ; 111.5 \mathrm{mg}, 90 \%$ yield r.r. $=84: 16$ (determined by ${ }^{1} \mathrm{H}$ NMR of crude product through the peak area ratio of $\left.=\mathrm{CHCH}_{2} /=\mathrm{CHCH}\right)$, colorless oil.

${ }^{1} \mathrm{H}$ NMR $\left(400 \mathrm{MHz}, \mathrm{CDCl}_{3}\right) \delta 7.59-7.55(\mathrm{~m}, 2 \mathrm{H}), 7.42-7.29(\mathrm{~m}, 3 \mathrm{H}), 5.92(\mathrm{t}, J=$ $6.9 \mathrm{~Hz}, 1 \mathrm{H}), 4.56(\mathrm{~s}, 2 \mathrm{H}), 2.62-2.46(\mathrm{~m}, 1 \mathrm{H}), 2.18$ (p, J=7.4 Hz, 2H), 1.79 - 1.45 (m, 5H), $1.33-1.05(\mathrm{~m}, 5 \mathrm{H}), 0.99$ (t, $J=7.5 \mathrm{~Hz}, 3 \mathrm{H})$.

${ }^{13} \mathrm{C} \mathrm{NMR}\left(101 \mathrm{MHz}, \mathrm{CDCl}_{3}\right) \delta 147.3$ (1C), 139.4 (1C), 135.6 (1C), 133.7 (2C), 129.3 (1C), 127.8 (2C), 40.6 (1C), 33.2 (2C), 26.6 (2C), 26.0 (1C), 22.0 (1C), 14.1 (1C).

HRMS (EI) calcd for [M, $\mathrm{C}_{16} \mathrm{H}_{24} \mathrm{Si}^{+}$: 244.1647, found 244.1641.

\section{(E)-(2-methyloct-3-en-4-yl)(phenyl)silane （3eo）}<smiles>C=C(C)C=C(CCC)SCc1ccccc1</smiles>

Procedure C; $99.7 \mathrm{mg}, 86 \%$ yield, r.r. $=83: 17$ (determined by ${ }^{1} \mathrm{H}$ NMR of crude product through the peak area ratio of $=\mathrm{CHCH} /=\mathrm{CHCH}_{2}$ ), colorless oil. 
$\underline{{ }^{1} \mathrm{H} \text { NMR }}\left(400 \mathrm{MHz}, \mathrm{CDCl}_{3}\right) \delta 7.61-7.55(\mathrm{~m}, 2 \mathrm{H}), 7.42-7.32(\mathrm{~m}, 3 \mathrm{H}), 5.85-5.76$ $(\mathrm{m}, 1 \mathrm{H}), 4.51(\mathrm{~s}, 2 \mathrm{H}), 280-2.68(\mathrm{~m}, 1 \mathrm{H}), 2.21-2.13(\mathrm{~m}, 2 \mathrm{H}), 1.38-1.22$ $(\mathrm{m}, 4 \mathrm{H}), 0.97(\mathrm{~d}, J=6.6 \mathrm{~Hz}, 6 \mathrm{H}), 0.87-0.81(\mathrm{~m}, 3 \mathrm{H})$.

${ }^{13} \mathrm{C} \mathrm{NMR}\left(101 \mathrm{MHz}, \mathrm{CDCl}_{3}\right) \delta 153.3$ (1C), 135.5 (1C), 132.7 (2C), 131.1 (1C), 129.4 (1C), 127.9 (2C), 32.1 (1C), 30.1 (1C), 27.7 (1C), 22.8 (2C), 22.7 (1C), 13.9 (1C).

HRMS (EI) calcd for [M, $\mathrm{C}_{15} \mathrm{H}_{24} \mathrm{Si}^{+}:$: 232.1647, found 232.1643.

\section{(E)-(2-methyloct-3-en-3-yl)(phenyl)silane (4eo)}

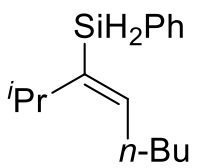

Procedure C; $102.3 \mathrm{mg}, 88 \%$ yield, r.r. $=65: 35$ (determined by ${ }^{1} \mathrm{H}$ NMR of crude product through the peak area ratio of $=\mathrm{CHCH} /=\mathrm{CHCH}_{2}$ ), colorless oil. ${ }^{1} \mathrm{H}$ NMR $\left(400 \mathrm{MHz}, \mathrm{CDCl}_{3}\right) \delta 7.60-7.54(\mathrm{~m}, 2 \mathrm{H}), 7.41-7.31(\mathrm{~m}, 3 \mathrm{H}), 5.91(\mathrm{t}, J=$ $6.9 \mathrm{~Hz}, 1 \mathrm{H}), 4.56(\mathrm{~s}, 2 \mathrm{H}), 2.98-2.85(\mathrm{~m}, 1 \mathrm{H}), 2.21-2.12(\mathrm{~m}, 2 \mathrm{H}), 1.40-$ $1.22(\mathrm{~m}, 4 \mathrm{H}), 0.99(\mathrm{~d}, J=6.9 \mathrm{~Hz}, 6 \mathrm{H}), 0.91(\mathrm{t}, J=7.0 \mathrm{~Hz}, 3 \mathrm{H})$.

${ }^{13} \mathrm{C} \mathrm{NMR}\left(101 \mathrm{MHz}, \mathrm{CDCl}_{3}\right) \delta 145.3$ (1C), 140.9 (1C), 135.6 (2C),133.7 $\quad(1 \mathrm{C})$ 129.3 (1C), 127.8 (2C), 31.6. (1C), 29.9 (1C), 28.4 (1C), 26.0 (1C), 23.0 (2C), 22.5 (1C), 14.0 (1C).

HRMS (EI) calcd for [M, $\mathrm{C}_{15} \mathrm{H}_{24} \mathrm{Si}^{+}: 232.1647$, found 232.1640 . 


\section{Transformations of Hydrosilylation Products}

6.1 Evaluation of the reaction efficiency

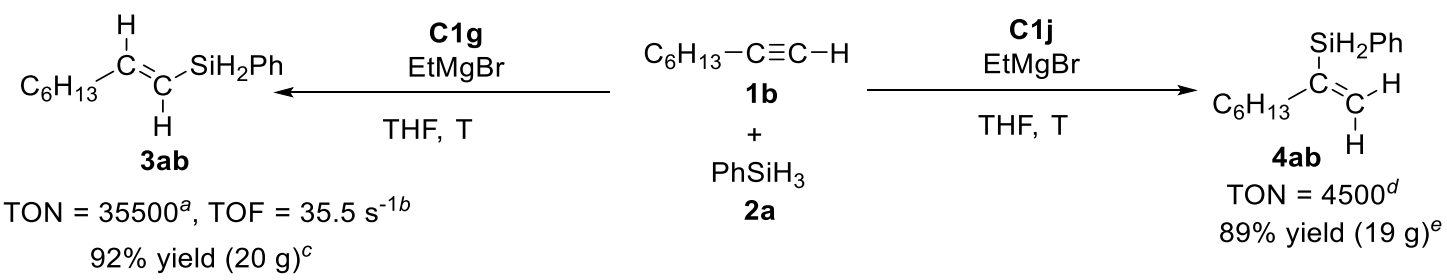

TON measurement of left equation: In an argon-filled glovebox, a bottle (100 $\mathrm{mL})$ was charged with $\mathbf{1 b}(5.51 \mathrm{~g}, 50 \mathrm{mmol}), \mathbf{2 a}(5.95 \mathrm{~g}, 55 \mathrm{mmol}), 20 \mathrm{~mL}$ THF, and a solution of $\mathbf{C 1 g}(1 \mathrm{~mL}, 0.001 \mathrm{M}$ in THF). $\operatorname{EtMgBr}(5 \mu \mathrm{L}, 1 \mathrm{M}$ in THF) was added to the reaction mixture at $10{ }^{\circ} \mathrm{C}$ with stirring. After $2 \mathrm{~h}$, the bottle was warm to room temperature and stirred for another $24 \mathrm{~h}$. Then the reaction mixture was concentrated under vacuum. The residue was purified by vacuum distillation $\left(78-80{ }^{\circ} \mathrm{C}\right.$ in 0.02 torr $)$ to give 3ab (7.74 g, 71\% yield).

TOF measurement of left equation: In an argon-filled glovebox, a bottle (100 $\mathrm{mL})$ was charged with $\mathbf{1 b}(0.551 \mathrm{~g}, 5 \mathrm{mmol}), \mathbf{2 a}(0.595 \mathrm{~g}, 5.5 \mathrm{mmol})$, complex C1g (7.2 $\mathrm{mg}, 0.01 \mathrm{mmol})$, and $20 \mathrm{~mL}$ THF. EtMgBr solution $(24 \mu \mathrm{L}, 1 \mathrm{M}$ in THF) was added to the reaction mixture at room temperature with stirring. After 10 seconds, water was added to quench the reaction. The mixture was concentrated under vacuum. The residue was purified by flash chromatography to afford $\mathbf{3 a b}(0.758 \mathrm{~g}, 71 \%$ yield $)$.

Scale-up experiment of left equation: In an argon-filled glovebox, a bottle (100 $\mathrm{mL})$ was charged with $\mathbf{1 b}(11.0 \mathrm{~g}, 100 \mathrm{mmol}), \mathbf{2 a}(11.9 \mathrm{~g}, 110 \mathrm{mmol})$, complex C1g (36 mg, $0.05 \mathrm{mmol}$ ), and $20 \mathrm{~mL}$ THF. EtMgBr solution (120 $\mu \mathrm{L}, 1 \mathrm{M}$ in THF) was added to the reaction mixture at romm temperature with stirring. After $4 \mathrm{~h}$, the bottle was removed from the glovebox and the reaction mixture was concentrated under vacuum. The residue was purified by vacuum distillation $\left(78-80{ }^{\circ} \mathrm{C}\right.$ in 0.02 torr) to obtain 4 ab (20.04 g, 92\% yield).

TON measurement of right equation: In an argon-filled glovebox, a bottle (100 $\mathrm{mL}$ ) was charged with $\mathbf{1 b}(5.51 \mathrm{~g}, 50 \mathrm{mmol}), \mathbf{2 a}(5.95 \mathrm{~g}, 55 \mathrm{mmol})$, complex C1j (6.3 $\mathrm{mg}, 0.005 \mathrm{mmol})$, and $20 \mathrm{~mL}$ THF. EtMgBr solution (12 $\mu \mathrm{L}, 1 \mathrm{M}$ in THF) was added 
to the reaction mixture at $10{ }^{\circ} \mathrm{C}$ with stirring. After $2 \mathrm{~h}$, the bottle was warm to room temperature and stirred for another $24 \mathrm{~h}$. Then the reaction mixture was concentrated under vacuum. The residue was purified by vacuum distillation $\left(76-79^{\circ} \mathrm{C}\right.$ in 0.02 torr) to afford $4 \mathbf{a b}(4.90 \mathrm{~g}, 45 \%$ yield $)$.

Scale-up experiment of right equation: In an argon-filled glovebox, a bottle (100 $\mathrm{mL}$ ) was charged with $\mathbf{1 b}$ (11.0 g, $100 \mathrm{mmol}), \mathbf{2 a}$ (11.9 g, $110 \mathrm{mmol})$, complex C1j (63 $\mathrm{mg}, 0.05 \mathrm{mmol}$ ) and $10 \mathrm{~mL}$ THF. The reaction mixture was stirred at room temperature for $3 \mathrm{~min}$, then the EtMgBr solution $(120 \mu \mathrm{L}, 1 \mathrm{M}$ in THF) was added to the reaction mixture. After $12 \mathrm{~h}$, the bottle was removed from the glovebox and the reaction mixture was concentrated under vacuum. The residue was purified by vacuum distillation ( $76-$ $79{ }^{\circ} \mathrm{C}$ in 0.02 torr) to afford $\mathbf{4 a b}(19.4 \mathrm{~g}, 89 \%$ yield).

\subsection{Preparation of value-added 2-methyl styrene derivatives from propyne}

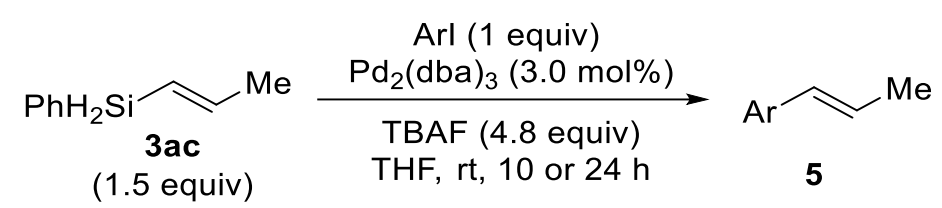

In an argon-filled glovebox, a Schlenk flask $(10 \mathrm{~mL})$ was charged with 3ac (66.6 mg, $0.45 \mathrm{mmol}$ ) and TBAF (tetra- $n$-butylammonium fluoride, $1.44 \mathrm{~mL}, 1.44 \mathrm{mmol}, 1.0$ $M$ in THF). The resulted mixture was stirred for $3 \mathrm{~min} . \mathrm{Pd}_{2}(\mathrm{dba})_{3}(8.2 \mathrm{mg}, 9 \mu \mathrm{mol})$ and ArI $(0.3 \mathrm{mmol})$ was then introduced and the reaction mixture was stirred at room temperature till the reaction was accomplished. The mixture was concentrated under vacuum. And the residue was purified by column chromatography using PE/EA (50:1 $-10: 1, v / v)$ as eluent to give the desired product 5 .

\section{Anethole $^{23}$}<smiles>C/C=C/c1ccc(OC)cc1</smiles>

$43.1 \mathrm{mg}$, 97\% yield (reaction time: $10 \mathrm{~h}$; flash chromatography eluent: $\mathrm{PE} / \mathrm{EA}=50: 1$, $v / v)$, colorless oil.

${ }^{11} \mathrm{H}$ NMR $\left(400 \mathrm{MHz}, \mathrm{CDCl}_{3}\right) \delta 7.29-7.22(\mathrm{~m}, 2 \mathrm{H}), 6.88-6.78(\mathrm{~m}, 2 \mathrm{H}), 6.34(\mathrm{dd}, \mathrm{J}=$ 15.9, $2.1 \mathrm{~Hz}, 1 \mathrm{H}), 6.09$ (dq, $J=15.5,6.6 \mathrm{~Hz}, 1 \mathrm{H}), 3.80$ (d, $J=1.2 \mathrm{~Hz}, 3 \mathrm{H})$, 
$1.85(\mathrm{dd}, J=6.6,1.6 \mathrm{~Hz}, 3 \mathrm{H})$.

${ }^{13} \mathrm{C} \mathrm{NMR}\left(101 \mathrm{MHz}, \mathrm{CDCl}_{3}\right) \delta 158.6$ (1C), 130.8 (1C), 130.4 (1C), 126.9 (2C), 123.5

(1C), 113.9 (2C), 55.3 (1C), 18.4 (1C).

isosafrole $^{23}$<smiles>CC=Cc1ccc2c(c1)OCO2</smiles>

$46.2 \mathrm{mg}$, 95\% yield (reaction time: $10 \mathrm{~h}$, flash chromatography eluent: $\mathrm{PE} / \mathrm{EA}=20: 1$, $v / v)$, colorless oil.

${ }^{1} \mathrm{H}$ NMR $\left(400 \mathrm{MHz}, \mathrm{CDCl}_{3}\right) \delta 6.88(\mathrm{~s}, 1 \mathrm{H}), 6.74(\mathrm{~d}, J=1.5 \mathrm{~Hz}, 2 \mathrm{H}), 6.31(\mathrm{dd}, J=15.7$, $1.8 \mathrm{~Hz}, 1 \mathrm{H}), 6.11-6.01(\mathrm{~m}, 1 \mathrm{H}), 5.93(\mathrm{~s}, 2 \mathrm{H}), 1.85(\mathrm{dd}, J=6.6,1.7 \mathrm{~Hz}, 3 \mathrm{H})$.

${ }^{13} \mathrm{C} \mathrm{NMR}\left(101 \mathrm{MHz}, \mathrm{CDCl}_{3}\right): \delta 147.9(1 \mathrm{C}), 146.5$ (1C), 132.5 (1C), $130.6(1 \mathrm{C}), 123.9$ (1C), 120.0 (1C), 108.2 (1C), 105.3 (1C), 100.9 (1C), 18.3 (1C).

(E)-1,2,3-trimethoxy-5-(prop-1-en-1-yl)benzene ${ }^{23}$<smiles>CC=Cc1cc(OC)c(OC)c(OC)c1</smiles>

$61.2 \mathrm{mg}$, 98\% yield (reaction time: $10 \mathrm{~h}$; flash chromatography eluent: $\mathrm{PE} / \mathrm{EA}=20: 1$, $v / v)$, colorless oil.

$\underline{{ }^{1} \mathrm{H} \mathrm{NMR}}\left(400 \mathrm{MHz}, \mathrm{CDCl}_{3}\right) \delta 6.55(\mathrm{~d}, J=1.7 \mathrm{~Hz}, 2 \mathrm{H}), 6.38-6.28(\mathrm{~m}, 1 \mathrm{H}), 6.22-$ 6.09 (m, 1H), 3.86 (d, $J=3.8 \mathrm{~Hz}, 6 \mathrm{H}), 3.83$ (d, $J=1.2 \mathrm{~Hz}, 3 \mathrm{H}), 1.88$ (ddd, $J$ $=6.6,2.8,1.5 \mathrm{~Hz}, 3 \mathrm{H})$.

${ }^{13} \mathrm{C}$ NMR (101 MHz, $\left.\mathrm{CDCl}_{3}\right): 153.3$ (1C), 137.3(2C), 133.8 (1C), 130.9 (1C), 102.9 (2C), $60.9(1 \mathrm{C}), 56.1(2 \mathrm{C}), 18.3(1 \mathrm{C})$.

\subsection{Preparation of a key intermediate of antifungal drugs}
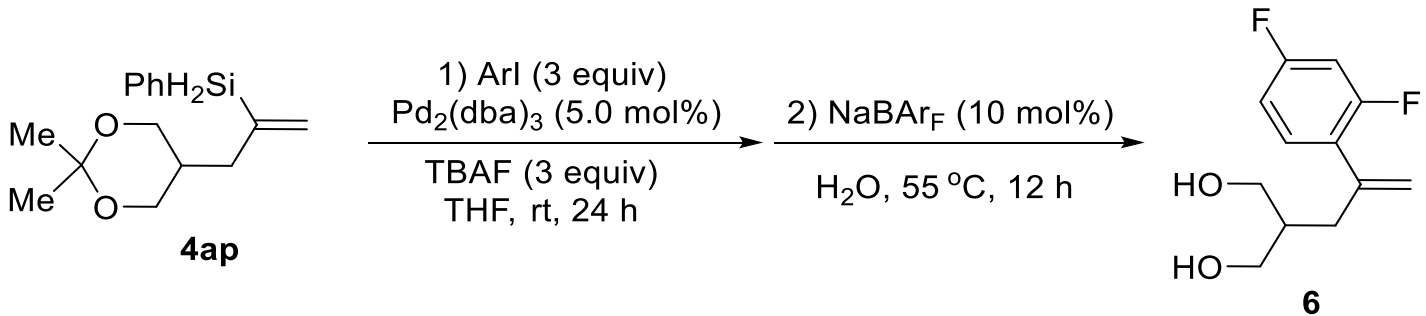

In an argon-filled glovebox, a Schlenk tube was charged with $\mathrm{Pd}_{2}(\mathrm{dba})_{3}(45.8 \mathrm{mg}$, S62 
$0.05 \mathrm{mmol})$, ArI (3 mmol), 4ap (262 mg, $1 \mathrm{mmol})$ and THF (5 mL). Then TBAF (3 mL, 1.0 $\mathrm{M}$ in THF) was added. And the mixture was stirred at room temperature for $24 \mathrm{~h}$. The mixture was extracted with $\mathrm{Et}_{2} \mathrm{O}$ and then the organic layer was separated and washed with brine, dried over $\mathrm{Na}_{2} \mathrm{SO}_{4}$ and concentrated under vacuum. The residue was purified by flash column chromatography using PE/EA $=10: 1(v / v)$ as the eluent. To the 25-mL flask charged the ketal was added a mixture of $\operatorname{NaBAr}_{\mathrm{F}}$ (sodium tetrakis[3,5-bis(trifluoromethyl)phenyl]borate, $0.05 \mathrm{mmol})$ in $\mathrm{H}_{2} \mathrm{O}(5 \mathrm{~mL})$. The resulting suspension was stirred vigorously at $55{ }^{\circ} \mathrm{C}$ for $12 \mathrm{~h}$ till the reaction was accomplished. The mixture was extracted with EA and then the organic layer was separated and washed with brine, dried over $\mathrm{Na}_{2} \mathrm{SO}_{4}$ and concentrated under vacuum. The residue was purified by column chromatography using PE/EA $=10: 1-3: 1(v / v)$ as the eluent to afford compound $\mathbf{6}^{24}(121 \mathrm{mg}, 53 \%$ yield) as a white solid, melting point: $45-47^{\circ} \mathrm{C}$.

$\underline{{ }^{1} \mathrm{H} \text { NMR }}\left(400 \mathrm{MHz}, \mathrm{CDCl}_{3}\right) \delta 7.26-7.21(\mathrm{~m}, 1 \mathrm{H}), 6.86-6.81(\mathrm{~m}, 1 \mathrm{H}), 6.80-6.78$ $(\mathrm{m}, 1 \mathrm{H}), 5.27(\mathrm{~s}, 1 \mathrm{H}), 5.20(\mathrm{~s}, 1 \mathrm{H}), 3.79-3.76(\mathrm{~m}, 2 \mathrm{H}), 3.67-3.63(\mathrm{~m}, 2 \mathrm{H})$, $2.49(\mathrm{~d}, J=7.4 \mathrm{~Hz}, 2 \mathrm{H}), 2.19-2.12$ (brs, $2 \mathrm{H}), 1.78-1.74(\mathrm{~m}, 1 \mathrm{H})$.

${ }^{13} \mathrm{C} \mathrm{NMR}\left(101 \mathrm{MHz}, \mathrm{CDCl}_{3}\right) \delta 162.2(\mathrm{dd}, J=247.1,11.9 \mathrm{~Hz}, 1 \mathrm{C}), 159.9(\mathrm{dd}, J=248.5$, $11.8 \mathrm{~Hz}, 1 \mathrm{C}), 141.7$ (1C), 130.7 (dd, $J=9.4,6.1 \mathrm{~Hz}, 1 \mathrm{C}), 125.2(\mathrm{dd}, J=14.2$, $4.1 \mathrm{~Hz}, 1 \mathrm{C}), 118.0$ (d, $J=1.5 \mathrm{~Hz}, 1 \mathrm{C}), 111.3$ (dd, $J=20.7,3.7 \mathrm{~Hz}, 1 \mathrm{C}), 104.2$ (t, $J=26.7 \mathrm{~Hz}, 1 \mathrm{C}), 65.2(2 \mathrm{C}), 40.0$ (1C), 34.9 (1C).

\subsection{Preparation of deuterium labelled alkenes}

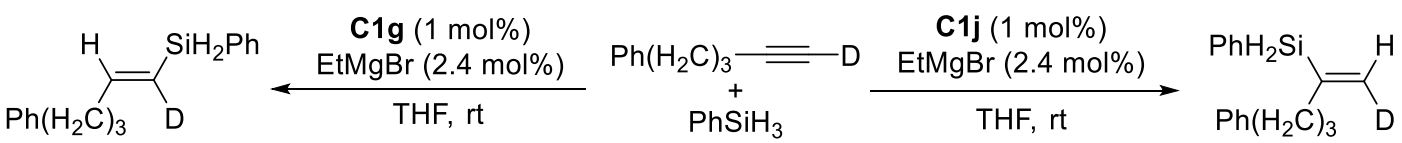

3aa-d-A, $91 \%$ yield

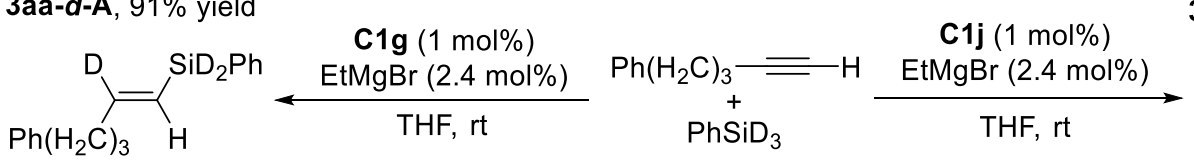
3aa-d-B, 93\% yield

3aa-d-C, $92 \%$ yield

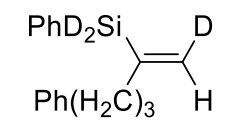
3aa-d-D, $90 \%$ yield

In an argon-filled glovebox, a vial $(10 \mathrm{~mL})$ was charged with alkyne (2a or $\mathbf{2 a -} \boldsymbol{d}$, $0.5 \mathrm{mmol}), \mathrm{PhSiH}_{3}$ or $\mathrm{PhSiD}_{3}(0.55 \mathrm{mmol})$ and complex $\mathbf{C 1}(0.005 \mathrm{mmol})$. The reaction mixture was stirred at room temperature for $3 \mathrm{~min}$, then the EtMgBr solution (12 $\mu \mathrm{L}, 1$ 
$\mathrm{M}$ in THF) was added to the reaction mixture. After $10 \mathrm{~min}$, the vial was removed from the glovebox and the reaction mixture was concentrated under vacuum. The residue was purified by flash chromatography to afford the desired deuterium product as a colorless oil.

\section{3aa-d-A}

$115 \mathrm{mg}, 91 \%$ yield, colorless oil, with C1g as catalyst.

$\underline{{ }^{1} \mathrm{H} \text { NMR }}\left(400 \mathrm{MHz}, \mathrm{CDCl}_{3}\right) \delta 7.59-7.54(\mathrm{~m}, 2 \mathrm{H}), 7.42-7.32(\mathrm{~m}, 3 \mathrm{H}), 7.30-7.24$ $(\mathrm{m}, 2 \mathrm{H}), 7.20-7.14(\mathrm{~m}, 3 \mathrm{H}), 6.39-6.31(\mathrm{~m}, 1 \mathrm{H}), 4.53(\mathrm{~s}, 2 \mathrm{H}), 2.66-2.60$ $(\mathrm{m}, 2 \mathrm{H}), 2.22(\mathrm{q}, J=7.0 \mathrm{~Hz}, 2 \mathrm{H}), 1.76(\mathrm{p}, J=7.5 \mathrm{~Hz}, 2 \mathrm{H})$.

${ }^{13} \mathrm{C} \mathrm{NMR}\left(101 \mathrm{MHz}, \mathrm{CDCl}_{3}\right) \delta 153.4$ (1C), $142.2(1 \mathrm{C}), 135.3$ (2C), 132.2 (1C), 129.6 (1C), 128.4 (2C), 128.3 (2C) 128.0 (2C), 125.7 (1C), 120.1 (1C), 36.2 (1C), 35.3 (1C), $30.1(1 \mathrm{C})$.

HRMS (EI) calcd for [M, $\left.\mathrm{C}_{17} \mathrm{H}_{19} \mathrm{DSi}\right]^{+}:$253.1397, found 253.1390.

\section{3aa-d-B}

$118 \mathrm{mg}, 93 \%$ yield, colorless oil, with $\mathbf{C 1} \mathbf{j}$ as catalyst.

$\underline{{ }^{1} \mathrm{H} \text { NMR }}\left(400 \mathrm{MHz}, \mathrm{CDCl}_{3}\right) \delta 7.59-7.55(\mathrm{~m}, 2 \mathrm{H}), 7.43-7.32(\mathrm{~m}, 3 \mathrm{H}), 7.28-7.22$ (m, 2H), $7.19-7.14(\mathrm{~m}, 1 \mathrm{H}), 7.11(\mathrm{~d}, J=7.2 \mathrm{~Hz}, 2 \mathrm{H}), 5.56(\mathrm{~s}, 1 \mathrm{H}), 4.55(\mathrm{~s}$, 2H), $2.60-2.53(\mathrm{~m}, 2 \mathrm{H}), 2.26(\mathrm{t}, J=7.6 \mathrm{~Hz}, 2 \mathrm{H}), 1.75(\mathrm{p}, J=7.7 \mathrm{~Hz}, 2 \mathrm{H})$.

${ }^{13} \mathrm{C} \mathrm{NMR}\left(101 \mathrm{MHz}, \mathrm{CDCl}_{3}\right) \delta 144.7$ (1C), 142.3 (1C), 135.5 (2C), 131.5 (1C), 129.7 (1C), 129.6 (1C), 128.4 (2C), 128.2 (2C), 128.0 (2C), 125.7 (1C), 36.9 (1C), 35.4 (1C), $30.5(1 \mathrm{C})$.

HRMS (EI) calcd for [M, $\left.\mathrm{C}_{17} \mathrm{H}_{19} \mathrm{DSi}\right]^{+}:$253.1397, found 253.1393.

\section{3aa-d-C}

$118 \mathrm{mg}$, 92\% yield, colorless oil, with C1g as catalyst.

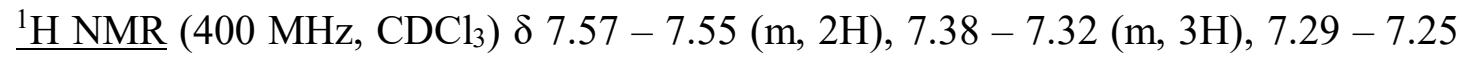
$(\mathrm{m}, 2 \mathrm{H}), 7.19-7.15(\mathrm{~m}, 3 \mathrm{H}), 5.73(\mathrm{~s}, 1 \mathrm{H}), 2.66-2.60(\mathrm{~m}, 2 \mathrm{H}), 2.21(\mathrm{t}, J=$ $7.3 \mathrm{~Hz}, 2 \mathrm{H}), 1.81-1.72(\mathrm{~m}, 2 \mathrm{H})$.

${ }^{13} \mathrm{C} \mathrm{NMR}\left(101 \mathrm{MHz}, \mathrm{CDCl}_{3}\right) \delta 153.0$ (1C), $142.2(1 \mathrm{C}), 135.3$ (2C), 129.6 (1C), 128.4 S64 
(2C), 128.3 (2C), 128.0 (2C), 125.7 (1C), 120.3 (1C), 36.2 (1C), 35.3 (1C), $30.0(1 \mathrm{C})$.

HRMS (EI) calcd for [M, $\mathrm{C}_{17} \mathrm{H}_{17} \mathrm{D}_{3} \mathrm{Si}^{+}:$255.1523, found 255.1520.

\section{3aa-d-D}

$115 \mathrm{mg}$, 90\% yield, colorless oil, with $\mathbf{C 1} \mathbf{j}$ as catalyst.

$\underline{{ }^{1} \mathrm{H} \mathrm{NMR}}\left(400 \mathrm{MHz}, \mathrm{CDCl}_{3}\right) \delta 7.57(\mathrm{~d}, J=7.4 \mathrm{~Hz}, 1 \mathrm{H}), 7.43-7.32(\mathrm{~m}, 3 \mathrm{H}), 7.28-$

$7.21(\mathrm{~m}, 2 \mathrm{H}), 7.20-7.14(\mathrm{~m}, 1 \mathrm{H}), 7.11(\mathrm{~d}, J=7.4 \mathrm{~Hz}, 2 \mathrm{H}), 5.78(\mathrm{~s}, 1 \mathrm{H})$,

$2.56(\mathrm{t}, J=7.8 \mathrm{~Hz}, 2 \mathrm{H}), 2.25(\mathrm{t}, J=7.7 \mathrm{~Hz}, 2 \mathrm{H}), 1.75(\mathrm{p}, J=7.6 \mathrm{~Hz}, 2 \mathrm{H})$.

${ }^{13} \mathrm{C} \mathrm{NMR}\left(101 \mathrm{MHz}, \mathrm{CDCl}_{3}\right) \delta 144.7$ (1C), 142.3 (1C), 135.6 (2C), 131.4 (1C), 129.7

(1C), 129.6 (1C), 128.4 (2C), 128.2 (2C) 128.0 (2C), 125.7 (1C), 36.9 (1C),

35.4 (1C), 30.5 (1C).

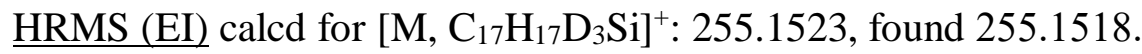

\subsection{Preparation of polyorganosiloxanes}
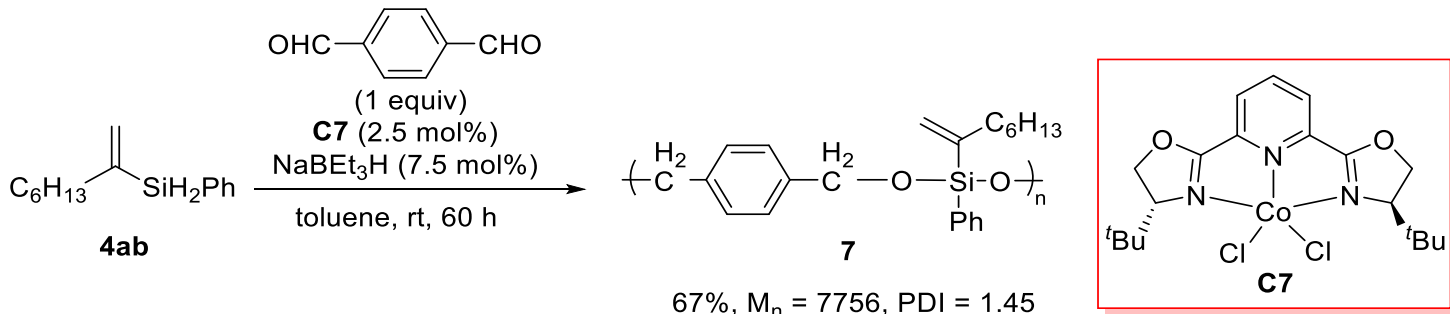

In an argon-filled glovebox, to a solution of $\mathbf{C} 7$ catalyst $(5.7 \mathrm{mg}, 0.0125 \mathrm{mmol})$ in $2.0 \mathrm{~mL}$ of toluene, a solution of $\mathrm{NaBEt}_{3} \mathrm{H}(1.0 \mathrm{M}$ in THF, $37.5 \mu \mathrm{L}, 0.0375 \mathrm{mmol})$ was slowly added. After stirring for $5 \mathrm{~min}, 4 \mathbf{a b}(109 \mathrm{mg}, 0.5 \mathrm{mmol}$,) and terephthalaldehyde (67 mg, $0.5 \mathrm{mmol}$ ) were added. The mixture was allowed to stir at room temperature for $60 \mathrm{~h}$. Then the solvent was removed by vacuum pump. The residue was washed and filtered with hexane, collecting filtrate and removing the solvent under vacuum pump to afford the product as brown viscous liquid. The $M_{w}$ and $M_{w} / M_{n}$ values were determined by GPC with THF solvent and polystyrene standards, $M_{n}=7756, M_{w} / M_{n}=$ 1.45 . 


\subsection{Preparation of $\alpha$-hydroxy ketones}

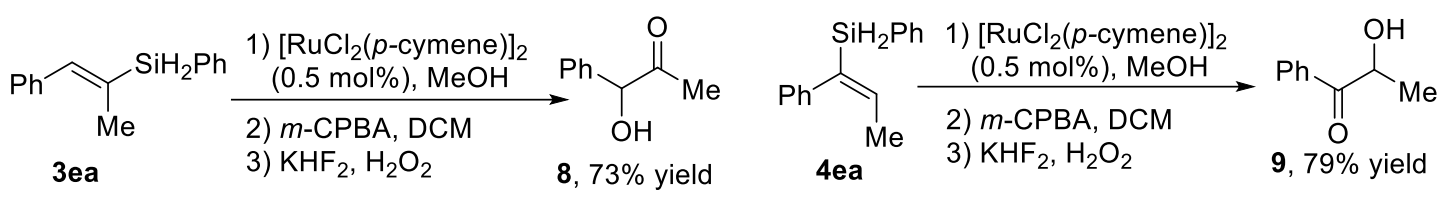

The 3ea or 4ea $(1 \mathrm{mmol})$ in $\mathrm{MeOH}$ solution was cooled to $0{ }^{\circ} \mathrm{C}$ (ice bath). Then $\left[\mathrm{RuCl}_{2}(p \text {-cymene })\right]_{2}(0.005 \mathrm{mmol}, 0.5 \mathrm{~mol} \%)$ was added. The reaction mixture was stirred at $0{ }^{\circ} \mathrm{C}$ for $30 \mathrm{~min}$ and the progress was monitored by TLC till the reaction was accomplished. The mixture was added hexane and filtered. The desired product was obtained by concentrating the filtrate under vacuum, which was used without further purification. To a solution of the residue in dichloromethane $(10 \mathrm{~mL})$ was added $m$ CPBA (3-chloroperoxybenzoic acid, $80 \%$ purity, $323 \mathrm{mg}, 1.5 \mathrm{mmol}$ ) at $0{ }^{\circ} \mathrm{C}$ with stirring. After $5 \mathrm{~h}$ stirring at $0{ }^{\circ} \mathrm{C}$, the mixture was diluted with hexane $(10 \mathrm{~mL})$ and filtered with suction, the filter cake being washed with cold hexane. The solvents in the combined filtrate were evaporated under reduced pressure at $45^{\circ} \mathrm{C}$. To the remaining oil, essentially pure epoxide, were added successively $\mathrm{KHF}_{2}$ (156 mg, $2.0 \mathrm{mmol}$ ), $\mathrm{KHCO}_{3}(300 \mathrm{mg}, 3.0 \mathrm{mmol}), \mathrm{MeOH}(4 \mathrm{~mL}), \mathrm{THF}(4 \mathrm{~mL})$ and $30 \% \mathrm{H}_{2} \mathrm{O}_{2}(0.25 \mathrm{~mL}, 2.5$ mmol). The mixture was stirred at room temperature for $3 \mathrm{~h}$. The resulting white mixture was poured into an aqueous solution of $\mathrm{NaHCO}_{3}(250 \mathrm{mg}$ in $20 \mathrm{~mL})$ at $0{ }^{\circ} \mathrm{C}$ and extracted with ether for five times. The ether layer was washed with saturated $\mathrm{NaHCO}_{3}$ solution and water and then dried over $\mathrm{Na}_{2} \mathrm{SO}_{4}$. After evaporation of the solvent, the remaining oil was subjected to preparative TLC to give $\mathbf{8}$ or $\mathbf{9}$.

\section{1-hydroxy-1-phenylpropan-2-one $(8)^{25}$}<smiles>CC(=O)C(O)c1ccccc1</smiles>

$110 \mathrm{mg}, 73 \%$ yield, light yellow transparent liquid.

를 NMR $\left(400 \mathrm{MHz}, \mathrm{CDCl}_{3}\right) \delta 7.42-7.30(\mathrm{~m}, 5 \mathrm{H}), 5.09(\mathrm{~d}, J=4.2 \mathrm{~Hz}, 1 \mathrm{H}), 4.29(\mathrm{~d}, J$ $=4.2 \mathrm{~Hz}, 1 \mathrm{H}), 2.09(\mathrm{~s}, 3 \mathrm{H})$.

${ }^{13} \mathrm{C} \mathrm{NMR}\left(101 \mathrm{MHz}, \mathrm{CDCl}_{3}\right) \delta 207.2$ (1C), 138.1 (1C), 129.1 (2C), 128.8 (2C), 127.5 (1C), 80.2 (1C), $25.3(1 \mathrm{C})$. 


\section{2-hydroxy-1-phenylpropan-1-one $(9)^{26}$}<smiles>CC(O)C(=O)c1ccccc1</smiles>

$119 \mathrm{mg}, 79 \%$ yield, light yellow transparent liquid.

$\underline{{ }^{1} \mathrm{H} \mathrm{NMR}}\left(400 \mathrm{MHz}, \mathrm{CDCl}_{3}\right) \delta 7.93(\mathrm{dd}, J=8.3,1.5 \mathrm{~Hz}, 2 \mathrm{H}), 7.63(\mathrm{tt}, J=7.4,1.6 \mathrm{~Hz}$, 1H), 7.51 (t, $J=7.3 \mathrm{~Hz}, 2 \mathrm{H}), 5.23-5.13(\mathrm{~m}, 1 \mathrm{H}), 3.77$ (d, $J=6.6 \mathrm{~Hz}, 1 \mathrm{H})$, $1.46(\mathrm{~d}, J=6.9 \mathrm{~Hz}, 3 \mathrm{H})$.

${ }^{13} \mathrm{C}$ NMR (101 MHz, $\left.\mathrm{CDCl}_{3}\right) \delta 202.5$ (1C), 134.1 (1C), 133.5 (1C), 129.0 (2C), 128.8 (2C), 69.5 (1C), 22.4 (1C).

\subsection{Synthesis of natural product asnipyrone}

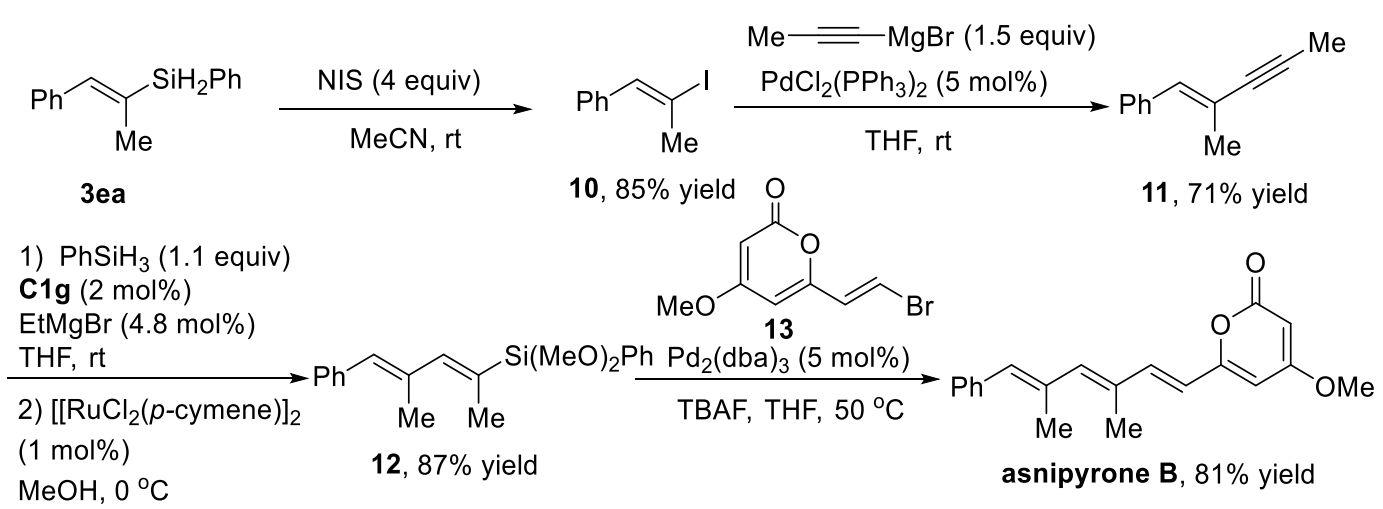

To a solution of 3ea $(2.5 \mathrm{mmol})$ in $\mathrm{MeCN}(5 \mathrm{~mL})$ was added NIS ( $N$ iodosuccinimide, $10 \mathrm{mmol}, 4$ equiv) at $0{ }^{\circ} \mathrm{C}$ (ice bath) under an air atmosphere. The reaction mixture was stirred at $0{ }^{\circ} \mathrm{C}$ for $10 \mathrm{~min}$ then warmed to room temperature and the progress was monitored by GC-MS till the reaction was accomplished. The mixture was extracted with $\mathrm{Et}_{2} \mathrm{O}$ and then the organic layer was separated and washed with brine, dried over $\mathrm{Na}_{2} \mathrm{SO}_{4}$ and concentrated under vacuum. The residue was purified by column chromatography using PE as the eluent to give $0.519 \mathrm{~g}(2.13 \mathrm{mmol}, 85 \%$ yield $)$ of $\mathbf{1 0}^{27}$ as colorless oil.

를 NMR (400 MHz, $\left.\mathrm{CDCl}_{3}\right) \delta 7.36-7.32(\mathrm{~m}, 2 \mathrm{H}), 7.29-7.25(\mathrm{~m}, 2 \mathrm{H}), 7.21(\mathrm{~d}, J=$ $7.5 \mathrm{~Hz}, 2 \mathrm{H}), 2.63(\mathrm{~d}, J=1.5 \mathrm{~Hz}, 3 \mathrm{H})$.

${ }^{13} \mathrm{C} \mathrm{NMR}\left(101 \mathrm{MHz}, \mathrm{CDCl}_{3}\right) \delta 140.8(1 \mathrm{C}), 137.5(1 \mathrm{C}), 128.4(2 \mathrm{C}), 128.2(2 \mathrm{C}), 127.1$ (1C), 98.5 (1C), 29.4 (1C). 
To a solution of $10(244 \mathrm{mg})$ and $\mathrm{Pd}\left(\mathrm{PPh}_{3}\right)_{2} \mathrm{Cl}_{2}(35.1 \mathrm{mg})$ in $\mathrm{THF}(10 \mathrm{~mL})$ was added 1-propynylmagnesium bromide ( $3 \mathrm{~mL}, 0.5 \mathrm{M}$ in THF, $1.5 \mathrm{mmol}, 1.5$ equiv) at room temperture under argon atmosphere. The reaction mixture was stirred and the progress was monitored by GC-MS. After $18 \mathrm{~h}$, weter $(10 \mathrm{~mL})$ was added to the mixture then extracted with $\mathrm{Et}_{2} \mathrm{O}$. The organic layer was separated and washed with brine, dried over $\mathrm{Na}_{2} \mathrm{SO}_{4}$ and concentrated under vacuum. The residue was purified by column chromatography using PE as the eluent to give $100.5 \mathrm{mg}(0.71 \mathrm{mmol}, 71 \%$ yield $)$ of $\mathbf{1 1}$ as colorless oil.

를 NMR (400 MHz, $\left.\mathrm{CDCl}_{3}\right) \delta 7.36-7.30(\mathrm{~m}, 2 \mathrm{H}), 7.27-7.19(\mathrm{~m}, 3 \mathrm{H}), 6.76(\mathrm{~s}, 1 \mathrm{H})$, $2.03(\mathrm{~d}, J=1.4 \mathrm{~Hz}, 3 \mathrm{H}), 2.00(\mathrm{~s}, 3 \mathrm{H})$.

${ }^{13} \mathrm{C} \mathrm{NMR}\left(101 \mathrm{MHz}, \mathrm{CDCl}_{3}\right) \delta 137.0$ (1C), $134.6(1 \mathrm{C}), 128.9$ (1C), $128.2(1 \mathrm{C}), 126.8$ (1C), 120.5 (1C), 84.7 (1C), 83.4 (1C), 19.4 (1C), 4.3 (1C).

In an argon-filled glovebox, a vial $(10 \mathrm{ml})$ was charged with $11(78.0 \mathrm{mg}, 0.5$ mmol), 2a (59.4 mg, $0.55 \mathrm{mmol})$ and complex C1g $(0.01 \mathrm{mmol})$. The reaction mixture was stirred at room temperature for $3 \mathrm{~min}$, then the EtMgBr solution $(24 \mu \mathrm{L} 1 \mathrm{M}$ in THF, ) was added to the reaction mixture. After $10 \mathrm{~min}$, the vial was removed from the glovebox and the reaction mixture was concentrated under vacuum. To the residue $\mathrm{MeOH}(5 \mathrm{mmol})$ was added and cooled to $0{ }^{\circ} \mathrm{C}$ (ice bath). Then $\left[\mathrm{RuCl}_{2}(p \text {-cymene })\right]_{2}$ $(0.005 \mathrm{mmol}, 1 \mathrm{~mol} \%)$ was added. The reaction mixture was stirred at $0{ }^{\circ} \mathrm{C}$ for $10 \mathrm{~min}$ and the progress was monitored by GC-MS till the reaction was accomplished. The mixture was added hexane and filtered through gel. The desired product 12 was obtained in $87 \%$ isolated yield by concentrating the filtrate under vacuum, which was used for next transition without further purified.

(E)-6-(2-bromovinyl)-4-methoxy-2H-pyran-2-one (13) was synthesized according to reference. ${ }^{28}$

래 NMR $\left(400 \mathrm{MHz}, \mathrm{CDCl}_{3}\right) \delta .7 .28(\mathrm{~d}, J=13.9 \mathrm{~Hz}, 1 \mathrm{H}), 6.62(\mathrm{~d}, J=13.3 \mathrm{~Hz}, 1 \mathrm{H})$, $5.83(\mathrm{~d}, J=2.5 \mathrm{~Hz}, 1 \mathrm{H}), 5.49(\mathrm{~d}, J=2.6 \mathrm{~Hz}, 1 \mathrm{H}), 3.80(\mathrm{~s}, 3 \mathrm{H})$.

${ }^{13} \mathrm{C} \mathrm{NMR}\left(101 \mathrm{MHz}, \mathrm{CDCl}_{3}\right) \delta 164.6$ (1C), 163.9 (1C), 154.0 (1C), 128.9 (1C), 104.9 (1C), 98.1 (1C), 96.3 (1C), 56.4 (1C).

In an argon-filled glovebox, a vial $(10 \mathrm{ml})$ was charged with $\mathrm{Pd}_{2}(\mathrm{dba})_{3}(23 \mathrm{mg}$, S68 
$0.025 \mathrm{mmol}), \mathrm{TBAF}(1.5 \mathrm{~mL}, 1.0 \mathrm{M}$ in THF), vinyl bromide 13 (1 mmol), 12 (162 mg, $0.5 \mathrm{mmol})$, and THF $(5 \mathrm{~mL})$. The resulted mixture was heated up to $50{ }^{\circ} \mathrm{C}$ and the reaction was monitored by TLC till the reaction was accomplished. the mixture was extracted with $\mathrm{Et}_{2} \mathrm{O}$ and then the organic layer was separated and washed with brine, dried over $\mathrm{Na}_{2} \mathrm{SO}_{4}$ and concentrated under vacuum. The residue was purified by column chromatography using (hexane/EA $=9: 1$ ) as the eluent.

asnipyrone $\mathbf{B}^{29}$<smiles>COc1cc(/C=C/C(C)=C/C(C)=C/c2ccccc2)oc(=O)c1</smiles>

$125 \mathrm{mg}, 81 \%$ yield, white solid, melting point: $87-89^{\circ} \mathrm{C}$.

${ }^{1} \mathrm{H} \mathrm{NMR}\left(400 \mathrm{MHz}, \mathrm{CDCl}_{3}\right) \delta 7.29-7.23(\mathrm{~m}, 4 \mathrm{H}), 7.18-7.15(\mathrm{~m}, 2 \mathrm{H}), 6.46(\mathrm{~s}, 1 \mathrm{H})$, $6.31(\mathrm{~s}, 1 \mathrm{H}), 5.98(\mathrm{~d}, J=15.5 \mathrm{~Hz}, 1 \mathrm{H}), 5.79(\mathrm{~d}, J=2.0 \mathrm{~Hz}, 1 \mathrm{H}), 5.38(\mathrm{~d}, J=$ $2.0 \mathrm{~Hz}, 1 \mathrm{H}), 3.73(\mathrm{~s}, 3 \mathrm{H}), 2.02(\mathrm{~s}, 3 \mathrm{H}), 1.98(\mathrm{~s}, 3 \mathrm{H})$.

${ }^{13} \mathrm{C} \mathrm{NMR}\left(101 \mathrm{MHz}, \mathrm{CDCl}_{3}\right) \delta 171.3$ (1C), 164.3 (1C), 159.4 (1C), 142.5 (1C), 141.8 (1C), 137.5 (1C), 135.2 (1C), 133.2 (1C), 133.1 (1C), 129.3 (2C), 128.3 (2C), 126.8 (1C), 117.5 (1C), $100.6(1 \mathrm{C}), 88.4$ (1C), 55.9 (1C), 18.9 (1C), 14.1 (1C). 


\section{Mechanistic Studies}

\subsection{Deuterium labelling experiments}

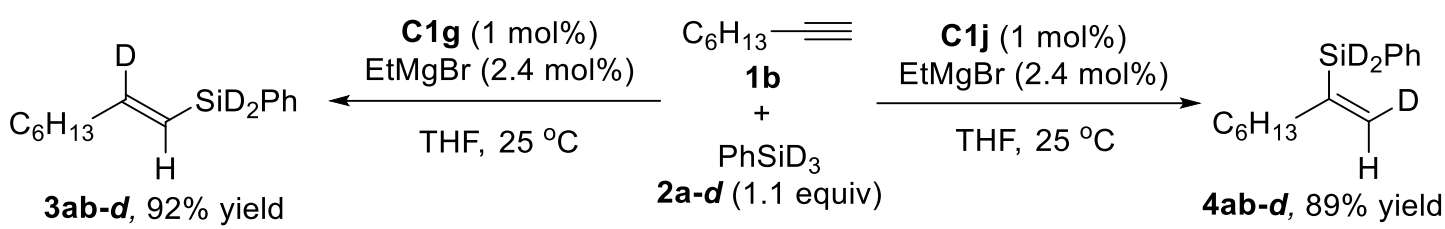

In an argon-filled glovebox, a vial $(10 \mathrm{~mL})$ was charged with $\mathbf{1 b}(55.1 \mathrm{mg}, 0.5$ mmol), $\mathrm{PhSiD}_{3}(61.2 \mathrm{mg}, 0.55 \mathrm{mmol})$, complex C1g or C1j (0.005 mmol) and THF (1 $\mathrm{mL}$ ). The reaction mixture was stirred at room temperature for $3 \mathrm{~min}$, then the $\mathrm{EtMgBr}$ solution (12 $\mu \mathrm{L}, 1 \mathrm{M}$ in THF) was added to the reaction mixture. After $10 \mathrm{~min}$, the vial was removed from the glovebox and the reaction mixture was concentrated under vacuum. The residue was purified by column chromatography to afford 3ab- $\boldsymbol{d}, 101.8$ mg (92\% yield) or $\mathbf{4 a b}-\boldsymbol{d}, 98.6 \mathrm{mg}(89 \%$ yield $)$.

\section{(E)-(oct-1-en-1-yl-2- $d$ ) (phenyl)silane- $d_{2}(3 \mathrm{ab}-d)$}<smiles>[2H]/C(=C\c1ccccc1)[SeH]c1ccccc1</smiles>

$102.0 \mathrm{mg}, 92 \%$ yield, colorless oil.

${ }^{1} \mathrm{H}$ NMR $\left(400 \mathrm{MHz}, \mathrm{CDCl}_{3}\right) \delta 7.57(\mathrm{~d}, J=7.4 \mathrm{~Hz}, 2 \mathrm{H}), 7.45-7.31(\mathrm{~m}, 3 \mathrm{H}), 5.71(\mathrm{~s}$, $1 \mathrm{H}), 2.18(\mathrm{t}, J=6.8 \mathrm{~Hz}, 2 \mathrm{H}), 1.43-1.36(\mathrm{~m}, 2 \mathrm{H}), 1.35-1.23(\mathrm{~m}, 6 \mathrm{H}), 0.86$ $(\mathrm{t}, J=6.7 \mathrm{~Hz}, 3 \mathrm{H})$.

${ }^{13} \mathrm{C} \mathrm{NMR}\left(101 \mathrm{MHz}, \mathrm{CDCl}_{3}\right) \delta 145.2(1 \mathrm{C}), 135.5(2 \mathrm{C}), 129.7(1 \mathrm{C}), 129.1(\mathrm{t}, J=24.3$ $\mathrm{Hz}, 1 \mathrm{C}), 128.8$ (2C), 128.0 (1C), 37.3 (1C), 31.6 (1C), 28.9 (1C), 28.8 (1C), $22.6(1 \mathrm{C}), 14.1(1 \mathrm{C})$.

HRMS (EI) calcd for[M, $\mathrm{C}_{14} \mathrm{H}_{19} \mathrm{D}_{3} \mathrm{Si}^{+}$: 221.1679, found 221.1678.

\section{(Z)-(oct-1-en-2-yl-1- $d$ )(phenyl)silane- $d_{2}(4 \mathrm{ab}-d)$}<smiles>[2H]/[PH+](=C/c1ccccc1)C(=O)c1ccccc1</smiles> 
$98.5 \mathrm{mg}, 89 \%$ yield, colorless oil.

${ }^{1} \mathrm{H}$ NMR $\left(400 \mathrm{MHz}, \mathrm{CDCl}_{3}\right) \delta 7.60-7.54(\mathrm{~m}, 2 \mathrm{H}), 7.40-7.32(\mathrm{~m}, 3 \mathrm{H}), 5.70(\mathrm{~s}, 1 \mathrm{H})$, $2.17(\mathrm{t}, J=7.3 \mathrm{~Hz}, 2 \mathrm{H}), 1.47-1.36(\mathrm{~m}, 2 \mathrm{H}), 1.35-1.23(\mathrm{~m}, 6 \mathrm{H}), 0.91-0.86$ $(\mathrm{m}, 3 \mathrm{H})$.

${ }^{13} \mathrm{C} \mathrm{NMR}\left(101 \mathrm{MHz}, \mathrm{CDCl}_{3}\right) \delta 153.8(\mathrm{t}, J=25.3,1 \mathrm{C}), 135.3(2 \mathrm{C}), 132.4(1 \mathrm{C}), 129.5$

(1C), 127.9 (2C), 119.6 (1C), 36.8 (1C), 31.7 (1C), 28.8 (1C), 28.3 (1C), 22.6

(1C), $14.1(1 \mathrm{C})$.

HRMS (EI) calcd for [M, $\left.\mathrm{C}_{14} \mathrm{H}_{19} \mathrm{D}_{3} \mathrm{Si}^{+}\right]^{+}$221.1679, found 221.1677.

\subsection{Deuterium labelling experiments using mixed silanes}

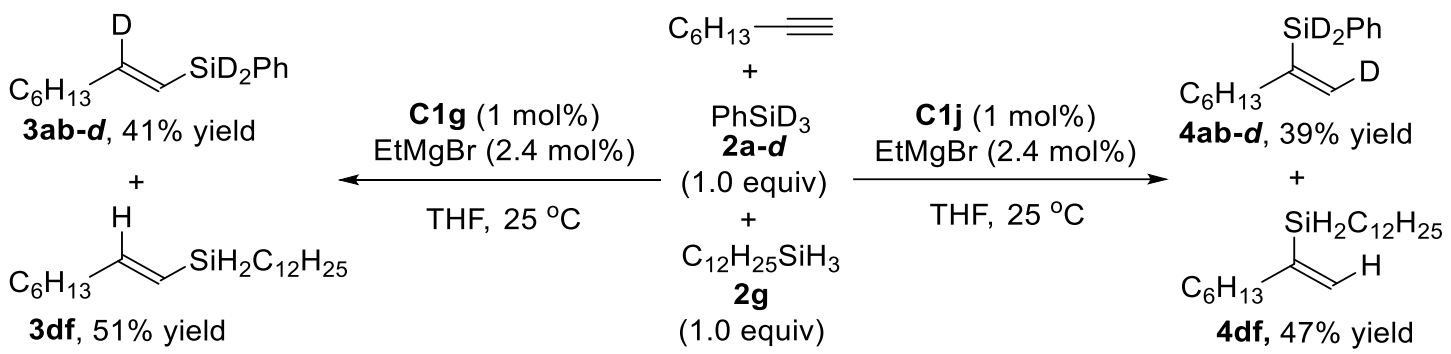

Left equation: In an argon-filled glovebox, a mixture of $\mathbf{1 b}(55.1 \mathrm{mg}, 0.5 \mathrm{mmol})$, 2a-d (55.6 mg, $0.5 \mathrm{mmol}), 2 \mathrm{~g}$ (100.2 mg, $0.5 \mathrm{mmol})$, complex C1g (3.6 mg, $0.005 \mathrm{mmol})$ and THF $(1 \mathrm{~mL})$ in a vial $(10 \mathrm{~mL})$ was stirred at room temperature for $3 \mathrm{~min}$. After the addition of EtMgBr solution ( $12 \mu \mathrm{L}, 1 \mathrm{M}$ in THF), the reaction mixture was stirred for another $10 \mathrm{~min}$, removed from the glovebox, and concentrated under vacuum. The residue was purified by column chromatography to afford 3ab-d, $45.5 \mathrm{mg}$ ( $41 \%$ yield) and 3df, $79.2 \mathrm{mg}$ (51\% yield).

Right equation: In an argon-filled glovebox, a vial $(10 \mathrm{~mL})$ was charged with $\mathbf{1 b}$ (55.1 mg, $0.5 \mathrm{mmol}), \mathbf{2 a}-\boldsymbol{d}$ (55.6 mg, $0.5 \mathrm{mmol}), 2 \mathrm{~g}$ (100.2 mg, $0.5 \mathrm{mmol})$, complex C1j (6.3 mg, $0.005 \mathrm{mmol})$ and THF (1 mL). The reaction mixture was stirred at room temperature for $3 \mathrm{~min}$, then the EtMgBr solution (12 $\mu \mathrm{L}, 1 \mathrm{M}$ in THF) was added to the reaction mixture. After $10 \mathrm{~min}$, the vial was removed from the glovebox and the reaction mixture was concentrated under vacuum. The residue was purified by column chromatography to afford 4ab-d, $43.3 \mathrm{mg}$ (39\% yield) and $\mathbf{4 d f}, 73.0 \mathrm{mg}$ (47\% yield). 


\subsection{Kinetic isotopic effect experiments}

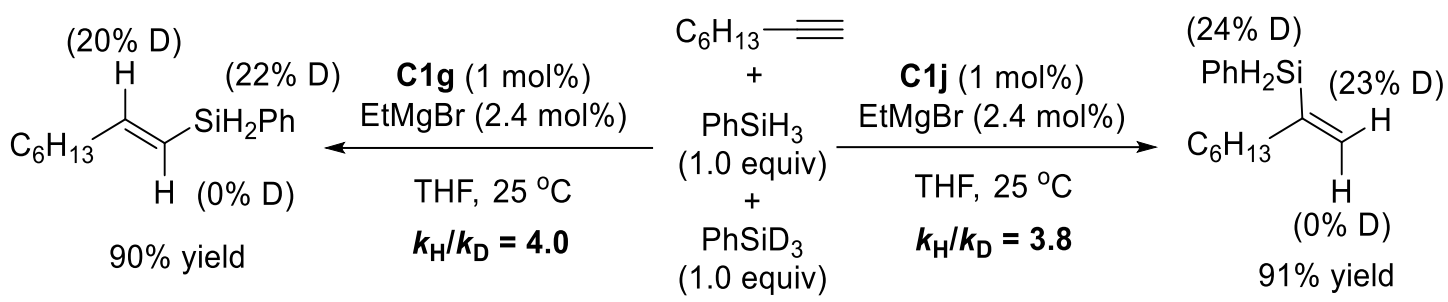

Left equation: In an argon-filled glovebox, a vial (10 ml) was charged with alkyne $1 \mathbf{b}(55.1 \mathrm{mg}, 0.5 \mathrm{mmol}), \mathbf{2 a}(54.1 \mathrm{mg}, 0.5 \mathrm{mmol}), \mathbf{2 a}-\mathbf{d}(55.6 \mathrm{mg}, 0.5 \mathrm{mmol})$ and complex C1g (3.6 mg, $0.005 \mathrm{mmol}$ ). The reaction mixture was stirred at room temperature for $3 \mathrm{~min}$, then the EtMgBr solution ( $12 \mu \mathrm{L}, 1 \mathrm{M}$ in THF) was added to the reaction mixture. After $10 \mathrm{~min}$, the vial was removed from the glovebox and the reaction mixture was concentrated under vacuum. The residue was purified by flash chromatography to afford the mixture of 3ab and 3ab- $\boldsymbol{d}$.

90\% yield, colorless oil. The ratio of deuterium substitution was detected by ${ }^{1} \mathrm{H}$ NMR.

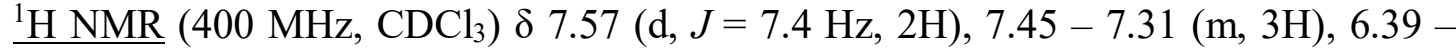
$6.34(\mathrm{~m}, 0.80 \mathrm{H}), 5.73-5.69(\mathrm{~m}, 0.78 \mathrm{H}), 4.57-4.52(\mathrm{~m}, 1 \mathrm{H}), 2.18(\mathrm{t}, J=6.8$ $\mathrm{Hz}, 2 \mathrm{H}), 1.42(\mathrm{dt}, J=14.4,7.1 \mathrm{~Hz}, 2 \mathrm{H}), 1.35-1.23(\mathrm{~m}, 6 \mathrm{H}), 0.88(\mathrm{t}, J=6.6$ $\mathrm{Hz}, 3 \mathrm{H})$.

Right equation: In an argon-filled glovebox, a vial $(10 \mathrm{~mL})$ was charged with $\mathbf{1 b}$ (55.1 mg, $0.5 \mathrm{mmol}), \mathbf{2 a}(54.1 \mathrm{mg}, 0.5 \mathrm{mmol}), \mathbf{2 a}-\mathbf{d}(55.6 \mathrm{mg}, 0.5 \mathrm{mmol})$ and complex C1j (6.3 mg, $0.005 \mathrm{mmol}$ ). The reaction mixture was stirred at room temperature for 3 min, then the EtMgBr solution ( $12 \mu \mathrm{L}, 1 \mathrm{M}$ in THF) was added to the reaction mixture. After $10 \mathrm{~min}$, the vial was removed from the glovebox and the reaction mixture was concentrated under vacuum. The residue was purified by flash chromatography to afford the mixture of $\mathbf{4 a b}$ and $\mathbf{4 a b}-\boldsymbol{d}$.

91\% yield, colorless oil,. The ratio of deuterium substitution was detected by ${ }^{1} \mathrm{H}$ NMR. ${ }^{1} \mathrm{H}$ NMR $\left(400 \mathrm{MHz}, \mathrm{CDCl}_{3}\right) \delta 7.57(\mathrm{~d}, J=7.4 \mathrm{~Hz}, 2 \mathrm{H}), 7.46-7.33(\mathrm{~m}, 3 \mathrm{H}), 5.79-$ $5.72(\mathrm{~m}, 1 \mathrm{H}), 5.53(\mathrm{~s}, 0.77 \mathrm{H}), 4.53(\mathrm{~s}, 0.76 \mathrm{H}), 3.72(\mathrm{t}, J=4.8 \mathrm{~Hz}, 4 \mathrm{H}), 2.21$ $(\mathrm{t}, J=6.7 \mathrm{~Hz}, 2 \mathrm{H}), 1.35-1.20(\mathrm{~m}, 6 \mathrm{H}), 0.88(\mathrm{t}, J=6.6 \mathrm{~Hz}, 3 \mathrm{H})$. 


\section{Computational Details}

All stationary points were fully optimized at the density functional theory level in Gaussian $09,{ }^{30}$ using the unrestricted $\omega \mathrm{B} 97 \mathrm{XD}^{31}$ functional without symmetry constraints. The triple- $\zeta$ valence basis set TZVP ${ }^{32,33}$ were used for Fe, and the 6-31G(d) basis set was used for $\mathrm{H}, \mathrm{C}, \mathrm{N}$ and $\mathrm{Si}$. The energies were further evaluated using a larger basis set def2-TZVPP 34,35 for all atoms involving the solvation effect with an implicit description of THF using the CPCM treatment, ${ }^{36,37}$ where the United Atom Topological Model (UAHF) was used to define the solute cavity. For selected key steps of energy profile were checked by M06/def2-TZVPP ${ }^{38}$ and SMD solvent correction ${ }^{39}$ at single point energy calculations. All optimized species were verified as either minima or transition structures by the presence of zero or a single imaginary vibrational frequency. Saddle points were connected to minima in the usual way with intrinsic reaction coordinate (IRC) calculations. ${ }^{40,41}$ Computed structures are displayed with CYLview. ${ }^{42}$ All of the Cartesian coordinates can be found in XYZ files based on the label in Table S7. 


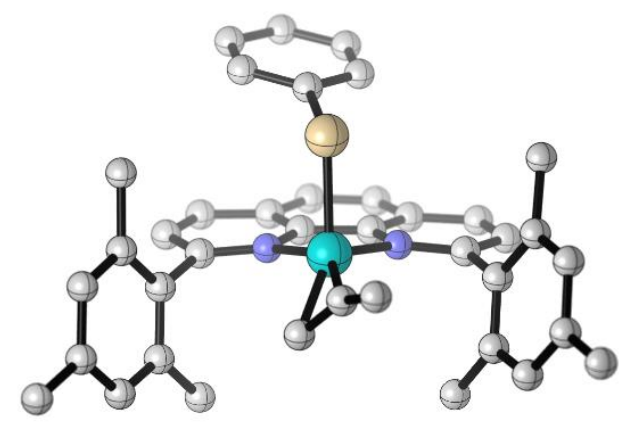

$E=-3172.433673$

$\mathrm{E}_{\mathrm{CPCM}}=-3173.062534$

$E_{S M D}=-3172.781024$

${ }^{3}$ TS-1a

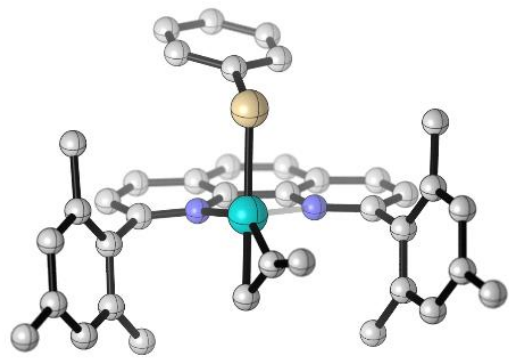

$\mathrm{E}_{\mathrm{CPCM}}=-3173.075015$

$E_{S M D}=-3172.987412$

${ }^{5}$ TS-1a

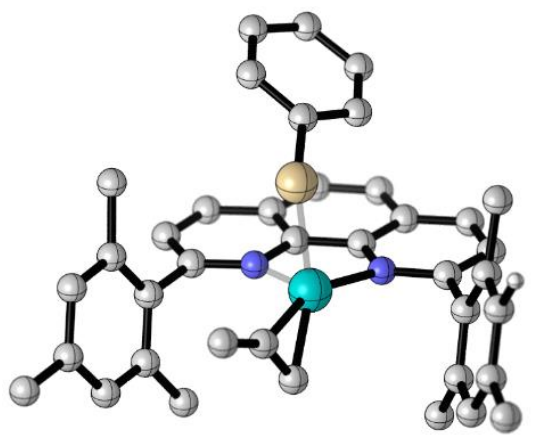

$E=-3172.450769$

$\mathrm{E}_{\mathrm{CPCM}}=-3173.053971$

$E_{S M D}=-3172.823944$

Ts-1a'

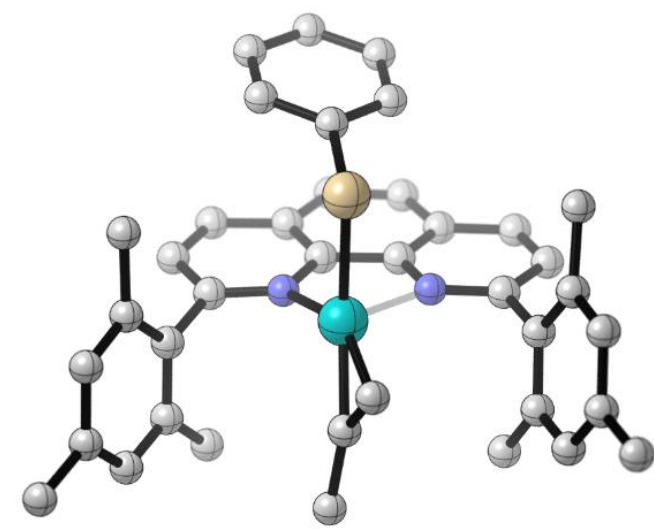

$E=-3172.462643$

$\mathrm{E}_{\mathrm{CPCM}}=-3173.071577$

$E_{S M D}=-3172.983243$ 
Ts-1c

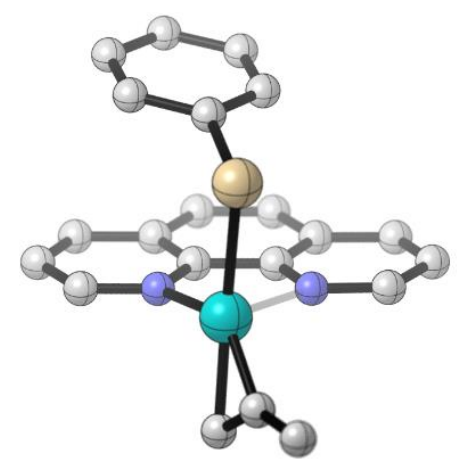

Ts-1c'

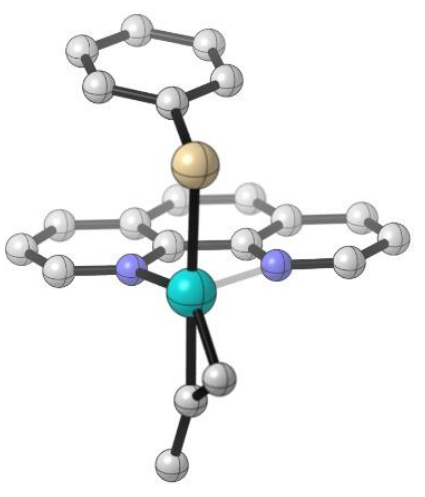

Ts-1b

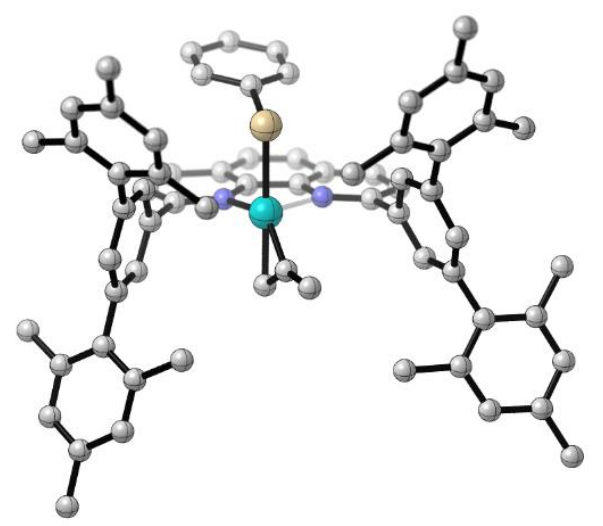

Ts-1b'

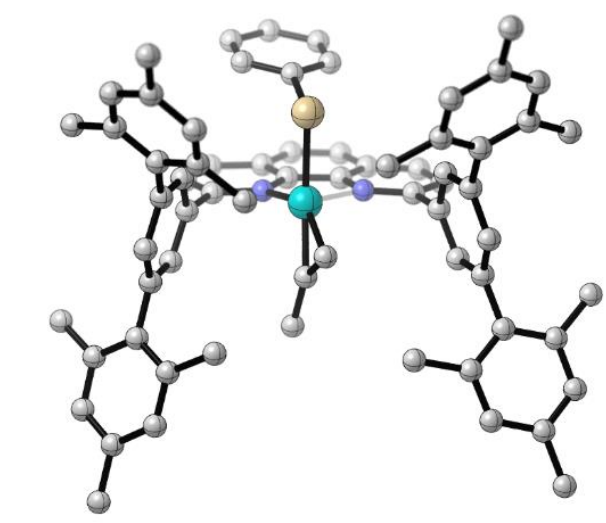

$E=-2474.729570 \quad E_{\mathrm{CPCM}}=-2475.025979$

$E_{\text {SMD }}=-2475.0131232$

$E=-2474.729788$

$\mathrm{E}_{\mathrm{CPCM}}=-2475.026040$

$E_{S M D}=-2475.016534$

$$
\begin{array}{ll}
E=-4332.170065 & E_{\mathrm{CPCM}}=-4333.235279 \\
& E_{\mathrm{SMD}}=-4333.203421
\end{array}
$$


Ts-1d

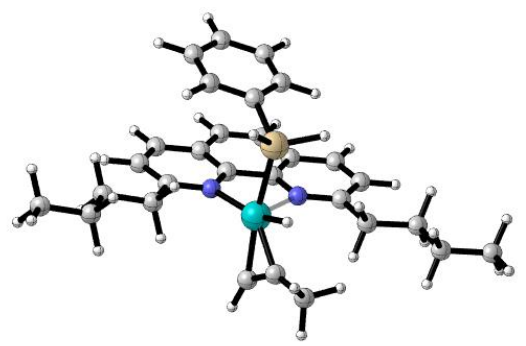

$\mathrm{E}=-2789.071932 \quad \mathrm{E}_{\mathrm{CPCM}}=-2789.583721$

Ts-1d'

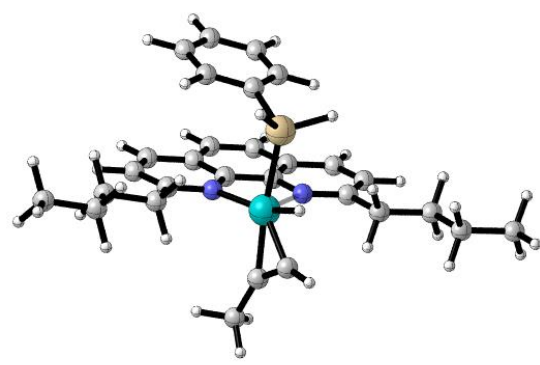

$\mathrm{E}=-2789.07182 \quad \mathrm{E}_{\mathrm{CPCM}}=-2789.582994$

Ts-1e

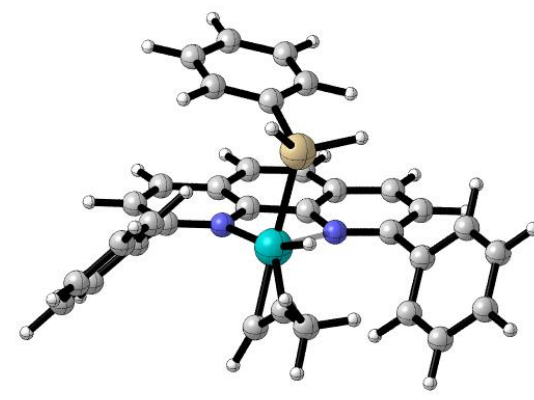

$\mathrm{E}=-2936.702012 \quad \mathrm{E}_{\mathrm{CPCM}}=-2937.150353$

Ts-1e'

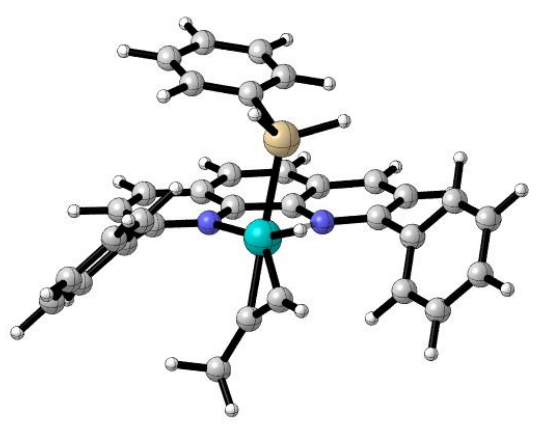

$E=-2936.701954 \quad E_{C P C M}=-2937.150397$ 


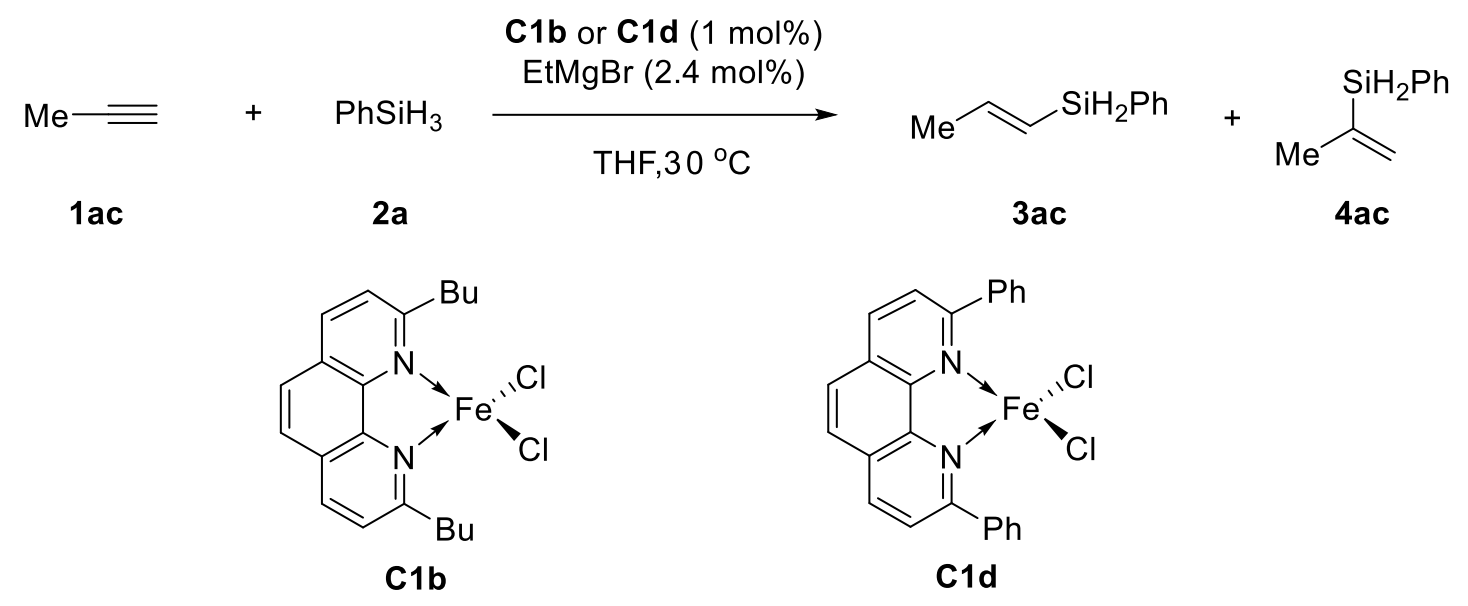

$82 \%$ yield, $3 \mathrm{ac} / 4 \mathrm{ac}=52: 48$.

$89 \%$ yield, 3 ac $/ 4 a c=54: 46$.
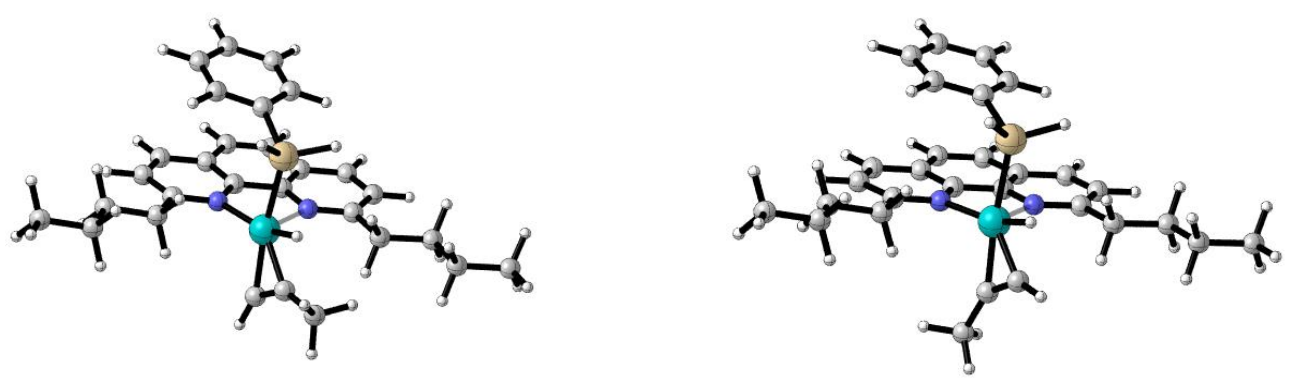

Ts-1d (0 kcal $/ \mathrm{mol})$

Ts-1d' (0.07 kcal $/ \mathrm{mol})$

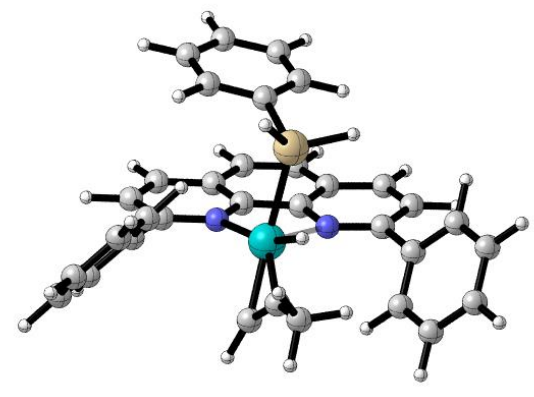

Ts-1e $(0 \mathrm{kcal} / \mathrm{mol})$

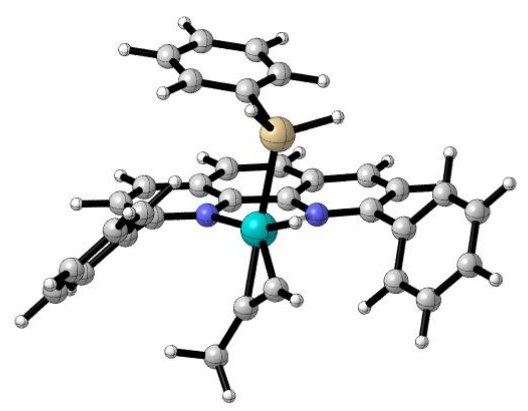

Ts-1e' (0.04 kcal/mol)

Figure S1 Regioselectivity of C1b and C1d 


\section{Cartesian coordinates of structures}

${ }^{1}$ TS-1

$\mathrm{Fe}$

$\mathrm{N}$

0.25503800

0.63894200

$-0.27861100$

$\mathrm{N}$

1.19923500

$-0.85208400$

0.58886700

C

$-1.33399200$

$-0.26890100$

0.55392600

C

3.46178300

0.05414500

0.34537400

C

4.25442100

$-0.04359900$

$-0.80554600$

$\mathrm{C}$

5.16269600

0.97941200

$-1.08262700$

C

5.77056800

0.91285800

$-1.98257200$

5.31043000

2.07744800

$-0.23817600$

$\mathrm{C}$

4.52381400

2.13809400

0.91242900

$\mathrm{H}$

4.63343300

2.98376200

1.58831900

C

3.59810200

1.14499500

1.22137000

C

2.52259500

$-1.03950200$

0.71999600

$\mathrm{C}$

3.03800600

$-2.21931900$

1.28269500

$\mathrm{H}$

4.11549100

$-2.32311400$

1.35351400

C

2.19885200

$-3.20814100$

1.75041300

2.59728600

$-4.12264300$

2.18028500

0.81319900

$-3.00077300$

1.68795700

$-0.16488900$

$-3.93837500$

2.16423400

0.18246100

$-4.87431700$

2.59222200

$-1.49057400$

$-3.67041400$

2.07099500

$-2.22561300$

$-4.39049000$

2.41841500

$-1.95990700$

$-2.42773300$

1.52480600

$-3.31252400$

$-2.06751600$

1.43916500

$-4.08514800$

$-2.75989000$

1.76006200

$-3.62973000$

$-0.80929200$

0.97805700

$-4.65931100$

$-0.46811700$

0.94797800

$-2.62507700$

0.09098800

0.57645500

$-1.02032900$

$-1.49627100$

1.05437800

0.37033000

$-1.79928100$

1.11016100

$-3.00409600$

1.51144400

0.32081600

$-2.87447300$

2.41178200

1.39415300

$-3.26300300$

3.73527200

1.21244500

$-3.15494300$

4.43611000

2.03837600

$-3.78289300$

4.18698200

$-0.00186400$

$-3.92206700$

3.27057900

$-1.03890300$

$-4.33356200$

3.60159800

$-1.99012900$

$-3.55033500$

1.93227100

$-0.89586900$

$-2.28763500$

1.96229500

2.70861800

$-2.30778300$

2.77116000

3.44459300

$-2.83475800$

1.10963500

3.12700700

$-1.24718600$

1.64693400

2.56738100

$-3.72430500$

0.97742700

$-2.04745900$ 


\begin{tabular}{|c|c|c|c|}
\hline $\mathrm{H}$ & -4.22809400 & 0.05609400 & -1.73552600 \\
\hline $\mathrm{H}$ & -4.31570100 & 1.43146600 & -2.84775600 \\
\hline $\mathrm{H}$ & -2.75327200 & 0.69566100 & -2.46754300 \\
\hline $\mathrm{C}$ & 4.14484400 & -1.23270100 & -1.72697100 \\
\hline $\mathrm{H}$ & 4.68334900 & -1.05367600 & -2.66177300 \\
\hline $\mathrm{H}$ & 4.56668700 & -2.13448400 & -1.26665000 \\
\hline $\mathrm{H}$ & 3.10187500 & -1.44659400 & -1.97793500 \\
\hline $\mathrm{C}$ & 2.76842900 & 1.24227200 & 2.47655800 \\
\hline $\mathrm{H}$ & 3.10424100 & 2.07385100 & 3.10256500 \\
\hline $\mathrm{H}$ & 1.71480900 & 1.40577100 & 2.22481800 \\
\hline $\mathrm{H}$ & 2.83015400 & 0.32316400 & 3.07042000 \\
\hline $\mathrm{C}$ & 6.26867700 & 3.19216400 & -0.57396200 \\
\hline $\mathrm{H}$ & 7.05355900 & 2.85330900 & -1.25704100 \\
\hline $\mathrm{H}$ & 5.74293800 & 4.02266900 & -1.06109300 \\
\hline $\mathrm{H}$ & 6.74747100 & 3.59182500 & 0.32580300 \\
\hline $\mathrm{C}$ & -4.20576000 & 5.62494200 & -0.16818800 \\
\hline $\mathrm{H}$ & -5.13724300 & 5.82671600 & 0.37433000 \\
\hline $\mathrm{H}$ & -3.44568400 & 6.31046800 & 0.22169500 \\
\hline $\mathrm{H}$ & -4.37453300 & 5.87064200 & -1.22087800 \\
\hline $\mathrm{C}$ & 0.08946200 & 2.44906200 & 0.28759100 \\
\hline $\mathrm{C}$ & 0.98974800 & 2.35433900 & -0.64004500 \\
\hline $\mathrm{H}$ & -0.41048500 & 3.23641900 & 0.83613000 \\
\hline $\mathrm{C}$ & 1.92033400 & 3.11468800 & -1.51554300 \\
\hline $\mathrm{H}$ & 1.83992100 & 4.19250000 & -1.34052800 \\
\hline $\mathrm{H}$ & 2.95266900 & 2.80042600 & -1.33130100 \\
\hline $\mathrm{H}$ & 1.70248700 & 2.91092000 & -2.56980800 \\
\hline $\mathrm{H}$ & 1.37147300 & 0.81509900 & -1.24830100 \\
\hline $\mathrm{Si}$ & 0.02437500 & -0.60277900 & -2.22407900 \\
\hline $\mathrm{H}$ & -0.79212700 & 0.11586800 & -3.26313000 \\
\hline $\mathrm{H}$ & 1.25656600 & -0.99103100 & -2.99392800 \\
\hline $\mathrm{C}$ & -0.83609800 & -2.28443300 & -2.00295200 \\
\hline $\mathrm{C}$ & -0.10158400 & -3.40026800 & -1.57249300 \\
\hline $\mathrm{C}$ & -2.22138100 & -2.44887200 & -2.14188200 \\
\hline $\mathrm{C}$ & -0.71704800 & -4.61385900 & -1.27861300 \\
\hline $\mathrm{H}$ & 0.97828200 & -3.31660600 & -1.45470600 \\
\hline $\mathrm{C}$ & -2.84777000 & -3.65830700 & -1.85151200 \\
\hline $\mathrm{H}$ & -2.82575900 & -1.61669900 & -2.49072200 \\
\hline $\mathrm{C}$ & -2.09719800 & -4.74564900 & -1.41191700 \\
\hline $\mathrm{H}$ & -0.11974600 & -5.45669500 & -0.94016100 \\
\hline $\mathrm{H}$ & -3.92390700 & -3.75416000 & -1.97270200 \\
\hline $\mathrm{H}$ & -2.58209300 & -5.69089500 & -1.18318400 \\
\hline
\end{tabular}

${ }^{3}$ TS-1

$\mathrm{Fe}$

$\begin{array}{lll}0.08835000 & 0.77729400 & -0.29431000\end{array}$ 


\begin{tabular}{|c|c|c|c|}
\hline $\mathrm{N}$ & 1.20322300 & -0.91426200 & 0.67803500 \\
\hline $\mathrm{N}$ & -1.39422300 & -0.27390600 & 0.60817200 \\
\hline $\mathrm{C}$ & 3.45731200 & -0.09076900 & 0.35730900 \\
\hline $\mathrm{C}$ & 4.16811600 & -0.16253300 & -0.84766700 \\
\hline $\mathrm{C}$ & 5.04550500 & 0.87410700 & -1.16931800 \\
\hline $\mathrm{H}$ & 5.58917600 & 0.82965400 & -2.11079800 \\
\hline $\mathrm{C}$ & 5.23967900 & 1.96035500 & -0.31866100 \\
\hline $\mathrm{C}$ & 4.54078700 & 1.99069600 & 0.88762300 \\
\hline $\mathrm{H}$ & 4.69049000 & 2.82552900 & 1.56922400 \\
\hline $\mathrm{C}$ & 3.64918900 & 0.98212900 & 1.24299200 \\
\hline $\mathrm{C}$ & 2.50863800 & -1.17251400 & 0.74683200 \\
\hline $\mathrm{C}$ & 2.99263500 & -2.39992700 & 1.23522400 \\
\hline $\mathrm{H}$ & 4.06452500 & -2.56418200 & 1.26962200 \\
\hline $\mathrm{C}$ & 2.10855100 & -3.35804500 & 1.68125200 \\
\hline $\mathrm{H}$ & 2.46625000 & -4.30872600 & 2.06767900 \\
\hline $\mathrm{C}$ & 0.73089800 & -3.09087100 & 1.64783700 \\
\hline $\mathrm{C}$ & -0.26596900 & -4.02188300 & 2.09359900 \\
\hline $\mathrm{H}$ & 0.05884900 & -4.98332000 & 2.48135400 \\
\hline $\mathrm{C}$ & -1.58291800 & -3.71512700 & 2.01895000 \\
\hline $\mathrm{H}$ & -2.33616000 & -4.42662400 & 2.34449200 \\
\hline $\mathrm{C}$ & -2.02153700 & -2.44355700 & 1.51537700 \\
\hline $\mathrm{C}$ & -3.37335600 & -2.06889600 & 1.45187000 \\
\hline $\mathrm{H}$ & -4.14515200 & -2.76852000 & 1.75897900 \\
\hline $\mathrm{C}$ & -3.69285500 & -0.79697100 & 1.03260500 \\
\hline $\mathrm{H}$ & -4.72094500 & -0.45051000 & 1.01679000 \\
\hline $\mathrm{C}$ & -2.68145200 & 0.09562000 & 0.64082000 \\
\hline $\mathrm{C}$ & -1.06703800 & -1.50995100 & 1.07393900 \\
\hline $\mathrm{C}$ & 0.33034400 & -1.84309800 & 1.12954100 \\
\hline $\mathrm{C}$ & -2.99678800 & 1.52622100 & 0.36093800 \\
\hline $\mathrm{C}$ & -2.78276700 & 2.44599500 & 1.40535400 \\
\hline $\mathrm{C}$ & -3.04246100 & 3.79199600 & 1.17805500 \\
\hline $\mathrm{H}$ & -2.87267700 & 4.50662900 & 1.98118600 \\
\hline $\mathrm{C}$ & -3.51562600 & 4.24961200 & -0.05351700 \\
\hline $\mathrm{C}$ & -3.75734000 & 3.31555800 & -1.05465000 \\
\hline $\mathrm{H}$ & -4.14980600 & 3.65073500 & -2.01256800 \\
\hline $\mathrm{C}$ & -3.51188700 & 1.95283900 & -0.86781300 \\
\hline $\mathrm{C}$ & -2.28669700 & 1.97746900 & 2.75066200 \\
\hline $\mathrm{H}$ & -2.18641400 & 2.81696100 & 3.44486900 \\
\hline $\mathrm{H}$ & -2.97492500 & 1.25003300 & 3.19742600 \\
\hline $\mathrm{H}$ & -1.31086400 & 1.49074500 & 2.65187200 \\
\hline $\mathrm{C}$ & -3.79012700 & 0.98567500 & -1.98998700 \\
\hline $\mathrm{H}$ & -4.21341200 & 0.04594100 & -1.62188700 \\
\hline $\mathrm{H}$ & -4.49320000 & 1.41545100 & -2.70966800 \\
\hline $\mathrm{H}$ & -2.86908400 & 0.74163400 & -2.53006400 \\
\hline
\end{tabular}




\begin{tabular}{|c|c|c|c|}
\hline $\mathrm{C}$ & 4.00873700 & -1.33909300 & -1.77845500 \\
\hline $\mathrm{H}$ & 4.49561900 & -1.14704200 & -2.73856900 \\
\hline $\mathrm{H}$ & 4.45521600 & -2.24648700 & -1.35378700 \\
\hline $\mathrm{H}$ & 2.95444200 & -1.55091900 & -1.97758500 \\
\hline $\mathrm{C}$ & 2.91547000 & 1.03938900 & 2.55848300 \\
\hline $\mathrm{H}$ & 3.24833200 & 1.89410600 & 3.15410300 \\
\hline $\mathrm{H}$ & 1.83784900 & 1.13616200 & 2.38999600 \\
\hline $\mathrm{H}$ & 3.08036200 & 0.12892900 & 3.14655200 \\
\hline $\mathrm{C}$ & 6.14962800 & 3.09731700 & -0.70894200 \\
\hline $\mathrm{H}$ & 5.57779300 & 3.90309600 & -1.18592500 \\
\hline $\mathrm{H}$ & 6.65205800 & 3.52536700 & 0.16437700 \\
\hline $\mathrm{H}$ & 6.91581800 & 2.77215400 & -1.41948500 \\
\hline $\mathrm{C}$ & -3.74505100 & 5.72148400 & -0.28610800 \\
\hline $\mathrm{H}$ & -4.37619900 & 5.89577000 & -1.16273500 \\
\hline $\mathrm{H}$ & -4.22482900 & 6.19216100 & 0.57863000 \\
\hline $\mathrm{H}$ & -2.79302800 & 6.23907400 & -0.45406200 \\
\hline $\mathrm{C}$ & 0.38139900 & 2.41281500 & 0.72159500 \\
\hline $\mathrm{C}$ & 1.05556800 & 2.48735100 & -0.37188500 \\
\hline $\mathrm{H}$ & 0.17181400 & 3.03456700 & 1.58281300 \\
\hline $\mathrm{C}$ & 1.95088900 & 3.32193500 & -1.21764800 \\
\hline $\mathrm{H}$ & 2.22323100 & 4.24857600 & -0.70129000 \\
\hline $\mathrm{H}$ & 2.86696400 & 2.77489700 & -1.46355800 \\
\hline $\mathrm{H}$ & 1.45628500 & 3.57874300 & -2.16155900 \\
\hline $\mathrm{H}$ & 0.96899300 & 1.19457900 & -1.45994000 \\
\hline $\mathrm{Si}$ & -0.04848300 & -0.47145400 & -2.32430500 \\
\hline $\mathrm{H}$ & -0.90399000 & 0.17462200 & -3.37868900 \\
\hline $\mathrm{H}$ & 1.21892400 & -0.77705400 & -3.06632500 \\
\hline $\mathrm{C}$ & -0.83112600 & -2.18958300 & -2.08633700 \\
\hline $\mathrm{C}$ & -0.05918000 & -3.27758400 & -1.65146300 \\
\hline $\mathrm{C}$ & -2.21194200 & -2.39959000 & -2.21335000 \\
\hline $\mathrm{C}$ & -0.63348300 & -4.50988100 & -1.35071000 \\
\hline $\mathrm{H}$ & 1.01728000 & -3.15775200 & -1.53398400 \\
\hline $\mathrm{C}$ & -2.79747200 & -3.62638600 & -1.91144600 \\
\hline $\mathrm{H}$ & -2.84583100 & -1.59055200 & -2.56519000 \\
\hline $\mathrm{C}$ & -2.00896900 & -4.68775700 & -1.47458700 \\
\hline $\mathrm{H}$ & -0.00634800 & -5.33046600 & -1.01135100 \\
\hline $\mathrm{H}$ & -3.87097400 & -3.75640700 & -2.02366200 \\
\hline $\mathrm{H}$ & -2.46160600 & -5.64697400 & -1.23829000 \\
\hline
\end{tabular}

${ }^{5}$ TS-1

$\begin{array}{lrrr}\mathrm{Fe} & 0.24807500 & -0.70080500 & -0.05126600 \\ \mathrm{~N} & 1.45894500 & 0.70657800 & -0.78306200 \\ \mathrm{~N} & -1.22448100 & 0.65719400 & -0.90133600 \\ \mathrm{C} & 3.43134600 & -0.59572300 & -0.32420200\end{array}$




\begin{tabular}{|c|c|c|c|}
\hline $\mathrm{C}$ & 3.93468500 & -0.77702300 & 0.97362300 \\
\hline $\mathrm{C}$ & 4.38759700 & -2.04228800 & 1.35015300 \\
\hline $\mathrm{H}$ & 4.76531900 & -2.18724000 & 2.36048200 \\
\hline $\mathrm{C}$ & 4.36906500 & -3.12139100 & 0.46954600 \\
\hline $\mathrm{C}$ & 3.89403700 & -2.91021800 & -0.82426600 \\
\hline $\mathrm{H}$ & 3.88770800 & -3.73787300 & -1.53136000 \\
\hline $\mathrm{C}$ & 3.42660800 & -1.66504700 & -1.23810700 \\
\hline $\mathrm{C}$ & 2.83026900 & 0.70390800 & -0.73684900 \\
\hline $\mathrm{C}$ & 3.56648900 & 1.81042300 & -1.07523000 \\
\hline $\mathrm{H}$ & 4.64921000 & 1.75841000 & -1.02815200 \\
\hline $\mathrm{C}$ & 2.91423900 & 2.99988000 & -1.49708700 \\
\hline $\mathrm{H}$ & 3.48792600 & 3.88292200 & -1.76092900 \\
\hline $\mathrm{C}$ & 1.51949500 & 3.01447500 & -1.59051300 \\
\hline $\mathrm{C}$ & 0.75408600 & 4.16292300 & -1.99999500 \\
\hline $\mathrm{H}$ & 1.29637100 & 5.06496700 & -2.27195700 \\
\hline $\mathrm{C}$ & -0.60625400 & 4.14667000 & -2.03700900 \\
\hline $\mathrm{H}$ & -1.15963400 & 5.03126700 & -2.34139500 \\
\hline $\mathrm{C}$ & -1.33392200 & 2.97038000 & -1.68111200 \\
\hline $\mathrm{C}$ & -2.72637400 & 2.86722900 & -1.70504500 \\
\hline $\mathrm{H}$ & -3.32183600 & 3.72573500 & -2.00661500 \\
\hline $\mathrm{C}$ & -3.34159800 & 1.67506000 & -1.35696300 \\
\hline $\mathrm{H}$ & -4.41959500 & 1.56266200 & -1.38399100 \\
\hline $\mathrm{C}$ & -2.54828800 & 0.58506700 & -0.95476400 \\
\hline $\mathrm{C}$ & -0.59610900 & 1.81892200 & -1.28090900 \\
\hline $\mathrm{C}$ & 0.80987900 & 1.84131900 & -1.23307100 \\
\hline $\mathrm{C}$ & -3.16848700 & -0.72663000 & -0.60209700 \\
\hline $\mathrm{C}$ & -3.09745700 & -1.78067600 & -1.52633200 \\
\hline $\mathrm{C}$ & -3.64737800 & -3.01478400 & -1.18426300 \\
\hline $\mathrm{H}$ & -3.59002900 & -3.83285100 & -1.89931600 \\
\hline $\mathrm{C}$ & -4.26614900 & -3.22586100 & 0.04657000 \\
\hline $\mathrm{C}$ & -4.33812100 & -2.15917200 & 0.94077300 \\
\hline $\mathrm{H}$ & -4.82345500 & -2.30270000 & 1.90485600 \\
\hline $\mathrm{C}$ & -3.80412300 & -0.90696400 & 0.63447000 \\
\hline $\mathrm{C}$ & -2.43320900 & -1.58918300 & -2.86637700 \\
\hline $\mathrm{H}$ & -2.58420100 & -2.46287400 & -3.50680300 \\
\hline $\mathrm{H}$ & -2.83148200 & -0.70985700 & -3.38436200 \\
\hline $\mathrm{H}$ & -1.35582500 & -1.42956500 & -2.74819200 \\
\hline $\mathrm{C}$ & -3.94813400 & 0.22500800 & 1.62049300 \\
\hline $\mathrm{H}$ & -3.17852600 & 0.98725700 & 1.48376500 \\
\hline $\mathrm{H}$ & -4.91999400 & 0.71995300 & 1.50207500 \\
\hline $\mathrm{H}$ & -3.88611900 & -0.14318100 & 2.64956600 \\
\hline $\mathrm{C}$ & 4.01113600 & 0.36912800 & 1.95216500 \\
\hline $\mathrm{H}$ & 4.75667400 & 1.10764900 & 1.63811000 \\
\hline $\mathrm{H}$ & 3.05687600 & 0.89561600 & 2.03842500 \\
\hline
\end{tabular}




$\begin{array}{lccc}\mathrm{H} & 4.28588500 & 0.01219700 & 2.94905200 \\ \mathrm{C} & 2.94014800 & -1.46460800 & -2.65191600 \\ \mathrm{H} & 1.87738800 & -1.20246800 & -2.67048500 \\ \mathrm{H} & 3.47740800 & -0.64140400 & -3.13569400 \\ \mathrm{H} & 3.08694400 & -2.37019600 & -3.24830000 \\ \mathrm{C} & 4.88285500 & -4.47429300 & 0.89474800 \\ \mathrm{H} & 4.73956600 & -4.63515100 & 1.96796700 \\ \mathrm{H} & 4.37264600 & -5.28166700 & 0.35949400 \\ \mathrm{H} & 5.95588200 & -4.57073400 & 0.68858100 \\ \mathrm{C} & -4.83944100 & -4.57152300 & 0.41462600 \\ \mathrm{H} & -4.34941400 & -4.97845100 & 1.30695800 \\ \mathrm{H} & -5.90987900 & -4.49774200 & 0.63717400 \\ \mathrm{H} & -4.71423800 & -5.29607200 & -0.39548000 \\ \mathrm{C} & 0.18237900 & -2.51015500 & -0.79638200 \\ \mathrm{C} & -0.33330400 & -2.66256200 & 0.36695700 \\ \mathrm{H} & 0.42769600 & -3.09391000 & -1.66892100 \\ \mathrm{C} & -0.90530400 & -3.62816600 & 1.34866900 \\ \mathrm{H} & -0.26601500 & -3.69981700 & 2.23545400 \\ \mathrm{H} & -1.89899400 & -3.30043900 & 1.66920700 \\ \mathrm{H} & -0.98932300 & -4.61660100 & 0.88933500 \\ \mathrm{H} & -0.44976200 & -1.48433600 & 1.19154000 \\ \mathrm{Si} & -0.02671300 & 0.04133800 & 2.29489300 \\ \mathrm{H} & -1.15282000 & -0.45343800 & 3.14774800 \\ \mathrm{H} & 1.21693800 & -0.33226700 & 3.03243100 \\ \mathrm{C} & -0.23763500 & 1.91408300 & 2.27306300 \\ \mathrm{C} & -1.39324800 & 2.49306900 & 2.81783800 \\ \mathrm{C} & 0.71273700 & 2.77336400 & 1.70294100 \\ \mathrm{C} & -1.61127300 & 3.86734000 & 2.76068900 \\ \mathrm{H} & -2.13467000 & 1.86179200 & 3.30069500 \\ \mathrm{C} & 0.50215300 & 4.14682200 & 1.64486100 \\ \mathrm{H} & 1.62787200 & 2.37246300 & 1.27785700 \\ \mathrm{C} & -0.66690900 & 4.69687400 & 2.16343700 \\ \mathrm{H} & -2.51856600 & 4.28901700 & 3.18432400 \\ \mathrm{H} & 1.24802700 & 4.78255100 & 1.17711800 \\ & & 5.76811600 & 2.10614300\end{array}$

${ }^{3}$ Ts-1a

$\mathrm{Fe}$

$\mathrm{N}$

$\mathrm{N}$

C

C

C

$\mathrm{H}$

0.08835000
1.20322300
-1.39422300
3.45731200
4.16811600
5.04550500
5.58917600

$$
\begin{array}{cc}
0.77729400 & -0.29431000 \\
-0.91426200 & 0.67803500 \\
-0.27390600 & 0.60817200 \\
-0.09076900 & 0.35730900 \\
-0.16253300 & -0.84766700 \\
0.87410700 & -1.16931800 \\
0.82965400 & -2.11079800
\end{array}
$$




\begin{tabular}{|c|c|c|c|}
\hline $\mathrm{C}$ & 5.23967900 & 1.96035500 & -0.31866100 \\
\hline $\mathrm{C}$ & 4.54078700 & 1.99069600 & 0.88762300 \\
\hline $\mathrm{H}$ & 4.69049000 & 2.82552900 & 1.56922400 \\
\hline $\mathrm{C}$ & 3.64918900 & 0.98212900 & 1.24299200 \\
\hline $\mathrm{C}$ & 2.50863800 & -1.17251400 & 0.74683200 \\
\hline $\mathrm{C}$ & 2.99263500 & -2.39992700 & 1.23522400 \\
\hline $\mathrm{H}$ & 4.06452500 & -2.56418200 & 1.26962200 \\
\hline $\mathrm{C}$ & 2.10855100 & -3.35804500 & 1.68125200 \\
\hline $\mathrm{H}$ & 2.46625000 & -4.30872600 & 2.06767900 \\
\hline $\mathrm{C}$ & 0.73089800 & -3.09087100 & 1.64783700 \\
\hline $\mathrm{C}$ & -0.26596900 & -4.02188300 & 2.09359900 \\
\hline $\mathrm{H}$ & 0.05884900 & -4.98332000 & 2.48135400 \\
\hline $\mathrm{C}$ & -1.58291800 & -3.71512700 & 2.01895000 \\
\hline $\mathrm{H}$ & -2.33616000 & -4.42662400 & 2.34449200 \\
\hline $\mathrm{C}$ & -2.02153700 & -2.44355700 & 1.51537700 \\
\hline $\mathrm{C}$ & -3.37335600 & -2.06889600 & 1.45187000 \\
\hline $\mathrm{H}$ & -4.14515200 & -2.76852000 & 1.75897900 \\
\hline $\mathrm{C}$ & -3.69285500 & -0.79697100 & 1.03260500 \\
\hline $\mathrm{H}$ & -4.72094500 & -0.45051000 & 1.01679000 \\
\hline $\mathrm{C}$ & -2.68145200 & 0.09562000 & 0.64082000 \\
\hline $\mathrm{C}$ & -1.06703800 & -1.50995100 & 1.07393900 \\
\hline $\mathrm{C}$ & 0.33034400 & -1.84309800 & 1.12954100 \\
\hline $\mathrm{C}$ & -2.99678800 & 1.52622100 & 0.36093800 \\
\hline $\mathrm{C}$ & -2.78276700 & 2.44599500 & 1.40535400 \\
\hline $\mathrm{C}$ & -3.04246100 & 3.79199600 & 1.17805500 \\
\hline $\mathrm{H}$ & -2.87267700 & 4.50662900 & 1.98118600 \\
\hline $\mathrm{C}$ & -3.51562600 & 4.24961200 & -0.05351700 \\
\hline $\mathrm{C}$ & -3.75734000 & 3.31555800 & -1.05465000 \\
\hline $\mathrm{H}$ & -4.14980600 & 3.65073500 & -2.01256800 \\
\hline $\mathrm{C}$ & -3.51188700 & 1.95283900 & -0.86781300 \\
\hline $\mathrm{C}$ & -2.28669700 & 1.97746900 & 2.75066200 \\
\hline $\mathrm{H}$ & -2.18641400 & 2.81696100 & 3.44486900 \\
\hline $\mathrm{H}$ & -2.97492500 & 1.25003300 & 3.19742600 \\
\hline $\mathrm{H}$ & -1.31086400 & 1.49074500 & 2.65187200 \\
\hline $\mathrm{C}$ & -3.79012700 & 0.98567500 & -1.98998700 \\
\hline $\mathrm{H}$ & -4.21341200 & 0.04594100 & -1.62188700 \\
\hline $\mathrm{H}$ & -4.49320000 & 1.41545100 & -2.70966800 \\
\hline $\mathrm{H}$ & -2.86908400 & 0.74163400 & -2.53006400 \\
\hline $\mathrm{C}$ & 4.00873700 & -1.33909300 & -1.77845500 \\
\hline $\mathrm{H}$ & 4.49561900 & -1.14704200 & -2.73856900 \\
\hline $\mathrm{H}$ & 4.45521600 & -2.24648700 & -1.35378700 \\
\hline $\mathrm{H}$ & 2.95444200 & -1.55091900 & -1.97758500 \\
\hline $\mathrm{C}$ & 2.91547000 & 1.03938900 & 2.55848300 \\
\hline $\mathrm{H}$ & 3.24833200 & 1.89410600 & 3.15410300 \\
\hline
\end{tabular}




\begin{tabular}{|c|c|c|c|}
\hline $\mathrm{H}$ & 1.83784900 & 1.13616200 & 2.38999600 \\
\hline $\mathrm{H}$ & 3.08036200 & 0.12892900 & 3.14655200 \\
\hline $\mathrm{C}$ & 6.14962800 & 3.09731700 & -0.70894200 \\
\hline $\mathrm{H}$ & 5.57779300 & 3.90309600 & -1.18592500 \\
\hline $\mathrm{H}$ & 6.65205800 & 3.52536700 & 0.16437700 \\
\hline $\mathrm{H}$ & 6.91581800 & 2.77215400 & -1.41948500 \\
\hline $\mathrm{C}$ & -3.74505100 & 5.72148400 & -0.28610800 \\
\hline $\mathrm{H}$ & -4.37619900 & 5.89577000 & -1.16273500 \\
\hline $\mathrm{H}$ & -4.22482900 & 6.19216100 & 0.57863000 \\
\hline $\mathrm{H}$ & -2.79302800 & 6.23907400 & -0.45406200 \\
\hline $\mathrm{C}$ & 0.38139900 & 2.41281500 & 0.72159500 \\
\hline $\mathrm{C}$ & 1.05556800 & 2.48735100 & -0.37188500 \\
\hline $\mathrm{H}$ & 0.17181400 & 3.03456700 & 1.58281300 \\
\hline $\mathrm{C}$ & 1.95088900 & 3.32193500 & -1.21764800 \\
\hline $\mathrm{H}$ & 2.22323100 & 4.24857600 & -0.70129000 \\
\hline $\mathrm{H}$ & 2.86696400 & 2.77489700 & -1.46355800 \\
\hline $\mathrm{H}$ & 1.45628500 & 3.57874300 & -2.16155900 \\
\hline $\mathrm{H}$ & 0.96899300 & 1.19457900 & -1.45994000 \\
\hline $\mathrm{Si}$ & -0.04848300 & -0.47145400 & -2.32430500 \\
\hline $\mathrm{H}$ & -0.90399000 & 0.17462200 & -3.37868900 \\
\hline $\mathrm{H}$ & 1.21892400 & -0.77705400 & -3.06632500 \\
\hline $\mathrm{C}$ & -0.83112600 & -2.18958300 & -2.08633700 \\
\hline $\mathrm{C}$ & -0.05918000 & -3.27758400 & -1.65146300 \\
\hline $\mathrm{C}$ & -2.21194200 & -2.39959000 & -2.21335000 \\
\hline $\mathrm{C}$ & -0.63348300 & -4.50988100 & -1.35071000 \\
\hline $\mathrm{H}$ & 1.01728000 & -3.15775200 & -1.53398400 \\
\hline $\mathrm{C}$ & -2.79747200 & -3.62638600 & -1.91144600 \\
\hline $\mathrm{H}$ & -2.84583100 & -1.59055200 & -2.56519000 \\
\hline $\mathrm{C}$ & -2.00896900 & -4.68775700 & -1.47458700 \\
\hline $\mathrm{H}$ & -0.00634800 & -5.33046600 & -1.01135100 \\
\hline $\mathrm{H}$ & -3.87097400 & -3.75640700 & -2.02366200 \\
\hline $\mathrm{H}$ & -2.46160600 & -5.64697400 & -1.23829000 \\
\hline \multicolumn{4}{|c|}{${ }^{3} \mathrm{Ts}-1 \mathrm{a}^{\prime}$} \\
\hline $\mathrm{Fe}$ & -0.03343300 & -0.80662100 & -0.38090600 \\
\hline $\mathrm{N}$ & -1.39295300 & 0.64288500 & 0.67694800 \\
\hline $\mathrm{N}$ & 1.28089800 & 0.45081000 & 0.55022200 \\
\hline $\mathrm{C}$ & -3.48627400 & -0.50004800 & 0.26767300 \\
\hline $\mathrm{C}$ & -4.17702000 & -0.43653900 & -0.95086900 \\
\hline $\mathrm{C}$ & -4.83103100 & -1.57949000 & -1.40708700 \\
\hline $\mathrm{H}$ & -5.35015400 & -1.54252600 & -2.36281700 \\
\hline $\mathrm{C}$ & -4.83438600 & -2.76550900 & -0.67349700 \\
\hline $\mathrm{C}$ & -4.17361500 & -2.78745300 & 0.55272700 \\
\hline $\mathrm{H}$ & -4.17370100 & -3.70184200 & 1.14242300 \\
\hline
\end{tabular}




\begin{tabular}{|c|c|c|c|}
\hline $\mathrm{C}$ & -3.49948000 & -1.67002400 & 1.04033800 \\
\hline $\mathrm{C}$ & -2.72364500 & 0.67899500 & 0.76712400 \\
\hline $\mathrm{C}$ & -3.39405000 & 1.76608300 & 1.35511000 \\
\hline $\mathrm{H}$ & -4.47771400 & 1.74712000 & 1.40496100 \\
\hline $\mathrm{C}$ & -2.67307300 & 2.82242700 & 1.86768400 \\
\hline $\mathrm{H}$ & -3.17457100 & 3.67097600 & 2.32521600 \\
\hline $\mathrm{C}$ & -1.27155000 & 2.79295800 & 1.80151600 \\
\hline $\mathrm{C}$ & -0.43829800 & 3.85268300 & 2.29317200 \\
\hline $\mathrm{H}$ & -0.91254000 & 4.71606400 & 2.75118300 \\
\hline $\mathrm{C}$ & 0.90819400 & 3.78755700 & 2.16844000 \\
\hline $\mathrm{H}$ & 1.53815600 & 4.59824900 & 2.52291300 \\
\hline $\mathrm{C}$ & 1.54346500 & 2.64779200 & 1.56914500 \\
\hline $\mathrm{C}$ & 2.93627300 & 2.53481500 & 1.43818100 \\
\hline $\mathrm{H}$ & 3.58070000 & 3.34557900 & 1.76521500 \\
\hline $\mathrm{C}$ & 3.46282600 & 1.37241500 & 0.92281300 \\
\hline $\mathrm{H}$ & 4.53492000 & 1.22388200 & 0.84799000 \\
\hline $\mathrm{C}$ & 2.61537600 & 0.32898300 & 0.51595500 \\
\hline $\mathrm{C}$ & 0.75453700 & 1.58343500 & 1.09478000 \\
\hline $\mathrm{C}$ & -0.67757400 & 1.66812000 & 1.19304700 \\
\hline $\mathrm{C}$ & 3.19768800 & -1.00036200 & 0.17065200 \\
\hline $\mathrm{C}$ & 3.23537200 & -1.95938000 & 1.19782200 \\
\hline $\mathrm{C}$ & 3.79291000 & -3.20706200 & 0.93441400 \\
\hline $\mathrm{H}$ & 3.81960900 & -3.95163100 & 1.72778200 \\
\hline $\mathrm{C}$ & 4.31893600 & -3.52325000 & -0.31781800 \\
\hline $\mathrm{C}$ & 4.29813700 & -2.54483200 & -1.30794900 \\
\hline $\mathrm{H}$ & 4.72113000 & -2.76756900 & -2.28543800 \\
\hline $\mathrm{C}$ & 3.75648200 & -1.27850300 & -1.08336100 \\
\hline $\mathrm{C}$ & 2.69567400 & -1.63362000 & 2.56764800 \\
\hline $\mathrm{H}$ & 2.77980900 & -2.49319200 & 3.23838200 \\
\hline $\mathrm{H}$ & 3.23935500 & -0.79512500 & 3.01911400 \\
\hline $\mathrm{H}$ & 1.64105300 & -1.34744100 & 2.50570900 \\
\hline $\mathrm{C}$ & 3.78087000 & -0.24988300 & -2.18403700 \\
\hline $\mathrm{H}$ & 4.07743500 & 0.73536300 & -1.80948700 \\
\hline $\mathrm{H}$ & 4.48339600 & -0.53888000 & -2.97089500 \\
\hline $\mathrm{H}$ & 2.79262400 & -0.14246200 & -2.64265800 \\
\hline $\mathrm{C}$ & -4.23207200 & 0.83997500 & -1.75330900 \\
\hline $\mathrm{H}$ & -4.66450400 & 0.66168700 & -2.74173300 \\
\hline $\mathrm{H}$ & -4.84742100 & 1.59719800 & -1.25202100 \\
\hline $\mathrm{H}$ & -3.23718800 & 1.26833900 & -1.90082000 \\
\hline $\mathrm{C}$ & -2.80387400 & -1.71766700 & 2.37642300 \\
\hline $\mathrm{H}$ & -2.89179100 & -2.70936700 & 2.82906400 \\
\hline $\mathrm{H}$ & -1.74043600 & -1.48643300 & 2.26515400 \\
\hline $\mathrm{H}$ & -3.23206100 & -0.98654300 & 3.07305500 \\
\hline $\mathrm{C}$ & -5.56517300 & -3.98149500 & -1.18570600 \\
\hline
\end{tabular}




$\begin{array}{lrrr}\mathrm{H} & -5.23848300 & -4.89016700 & -0.67107300 \\ \mathrm{H} & -6.64650900 & -3.88270400 & -1.03156100 \\ \mathrm{H} & -5.39988800 & -4.12157800 & -2.25906000 \\ \mathrm{C} & 4.93778600 & -4.87338700 & -0.57914500 \\ \mathrm{H} & 4.53627800 & -5.63372000 & 0.09777700 \\ \mathrm{H} & 4.75509800 & -5.20219500 & -1.60703200 \\ \mathrm{H} & 6.02431200 & -4.84046600 & -0.43222900 \\ \mathrm{C} & -0.14172400 & -2.56144800 & 0.51279300 \\ \mathrm{C} & -0.75639400 & -2.58529700 & -0.62062100 \\ \mathrm{H} & -0.79743600 & -1.19668800 & -1.64089300 \\ \mathrm{Si} & -0.15775300 & 0.56203600 & -2.34063100 \\ \mathrm{H} & 0.79259400 & 0.13735900 & -3.42302000 \\ \mathrm{H} & -1.46316500 & 0.68395500 & -3.06646300 \\ \mathrm{C} & 0.31940200 & 2.37212800 & -2.00148400 \\ \mathrm{C} & -0.61401800 & 3.28764400 & -1.49262700 \\ \mathrm{C} & 1.63886200 & 2.82356400 & -2.14910500 \\ \mathrm{C} & -0.25041100 & 4.58463200 & -1.14049800 \\ \mathrm{H} & -1.65075100 & 2.98169900 & -1.35857200 \\ \mathrm{C} & 2.01450600 & 4.11708500 & -1.79597300 \\ \mathrm{H} & 2.38973900 & 2.15328700 & -2.55742100 \\ \mathrm{C} & 1.06981400 & 5.00333500 & -1.28557200 \\ \mathrm{H} & -0.99782900 & 5.26795100 & -0.74541500 \\ \mathrm{H} & 3.04612800 & 4.43506400 & -1.92474200 \\ \mathrm{H} & 1.35711700 & 6.01426700 & -1.00916200 \\ \mathrm{C} & 0.14600800 & -3.51626200 & 1.62218200 \\ \mathrm{H} & -0.51139000 & -4.39416200 & 1.58614400 \\ \mathrm{H} & 1.18123600 & -3.86746600 & 1.54536700 \\ \mathrm{H} & 0.03899600 & -3.04995000 & 2.60737100 \\ \mathrm{H} & -1.33669300 & -3.20683500 & -1.28598900\end{array}$

${ }^{3}$ Ts-1c

$\mathrm{Fe}$

$\mathrm{N}$

$\begin{array}{rcc}0.08526000 & 0.71582100 & -0.42801500 \\ 1.20234000 & -0.93585700 & 0.51431500 \\ -1.38728200 & -0.35202100 & 0.42864100 \\ 2.49925200 & -1.20920000 & 0.50430100 \\ 3.03361900 & -2.37359700 & 1.06917300 \\ 4.10331700 & -2.54768700 & 1.03114800 \\ 2.18105700 & -3.28487500 & 1.66197200 \\ 2.56504800 & -4.19979700 & 2.10481300 \\ 0.80097600 & -3.02624700 & 1.67924600 \\ -0.17505500 & -3.92137300 & 2.23780300 \\ 0.16961800 & -4.84583700 & 2.69236900 \\ -1.49725800 & -3.62990600 & 2.18517300 \\ -2.23015500 & -4.31882700 & 2.59514900\end{array}$




\begin{tabular}{|c|c|c|c|}
\hline $\mathrm{C}$ & -1.97162900 & -2.41484200 & 1.57901100 \\
\hline $\mathrm{C}$ & -3.33083600 & -2.07661300 & 1.48695700 \\
\hline $\mathrm{H}$ & -4.08551500 & -2.74976300 & 1.88282100 \\
\hline $\mathrm{C}$ & -3.68216300 & -0.88669100 & 0.88300600 \\
\hline $\mathrm{H}$ & -4.72056000 & -0.58841400 & 0.79062900 \\
\hline $\mathrm{C}$ & -2.68387500 & -0.05406000 & 0.37052000 \\
\hline $\mathrm{C}$ & -1.03656100 & -1.51533000 & 1.03549600 \\
\hline $\mathrm{C}$ & 0.36359900 & -1.82630500 & 1.08500900 \\
\hline $\mathrm{C}$ & 0.26005400 & 2.23105700 & 0.76286600 \\
\hline $\mathrm{C}$ & 1.00773800 & 2.44653100 & -0.26406000 \\
\hline $\mathrm{H}$ & -0.00656000 & 2.71340400 & 1.69399500 \\
\hline $\mathrm{C}$ & 1.93388100 & 3.41157300 & -0.92028700 \\
\hline $\mathrm{H}$ & 2.08379500 & 4.29601400 & -0.29291500 \\
\hline $\mathrm{H}$ & 2.90876900 & 2.94994000 & -1.11287000 \\
\hline $\mathrm{H}$ & 1.53405100 & 3.73379600 & -1.88838400 \\
\hline $\mathrm{H}$ & 1.01135100 & 1.31936000 & -1.48140700 \\
\hline $\mathrm{Si}$ & 0.06180600 & -0.57509600 & -2.44242800 \\
\hline $\mathrm{H}$ & -0.66267400 & 0.03242800 & -3.61140200 \\
\hline $\mathrm{H}$ & 1.40564900 & -0.96743100 & -2.98746600 \\
\hline $\mathrm{C}$ & -0.82086100 & -2.23325300 & -2.14914000 \\
\hline $\mathrm{C}$ & -0.12865300 & -3.35421200 & -1.66588400 \\
\hline $\mathrm{C}$ & -2.21807100 & -2.33584400 & -2.23687000 \\
\hline $\mathrm{C}$ & -0.79373300 & -4.51689100 & -1.28313700 \\
\hline $\mathrm{H}$ & 0.95616200 & -3.31387500 & -1.57638500 \\
\hline $\mathrm{C}$ & -2.89266100 & -3.49268600 & -1.85622800 \\
\hline $\mathrm{H}$ & -2.79277700 & -1.49015600 & -2.61058200 \\
\hline $\mathrm{C}$ & -2.18139300 & -4.58900800 & -1.37313200 \\
\hline $\mathrm{H}$ & -0.22831500 & -5.36624300 & -0.90802300 \\
\hline $\mathrm{H}$ & -3.97580300 & -3.54084600 & -1.93775100 \\
\hline $\mathrm{H}$ & -2.70388300 & -5.49365800 & -1.07379200 \\
\hline $\mathrm{H}$ & 3.13496800 & -0.47363200 & 0.02049100 \\
\hline $\mathrm{H}$ & -2.93310800 & 0.88485900 & -0.11264400 \\
\hline \multicolumn{4}{|c|}{${ }^{3}$ Ts-1 $1 c^{\prime}$} \\
\hline $\mathrm{Fe}$ & 0.08967800 & 0.72747600 & -0.48288300 \\
\hline $\mathrm{N}$ & 1.19335000 & -0.91009500 & 0.48519500 \\
\hline $\mathrm{N}$ & -1.39151100 & -0.32380800 & 0.38962300 \\
\hline $\mathrm{C}$ & 2.49097200 & -1.18168900 & 0.48476700 \\
\hline $\mathrm{C}$ & 3.02439600 & -2.33294800 & 1.07676200 \\
\hline $\mathrm{H}$ & 4.09433000 & -2.50690000 & 1.04511500 \\
\hline $\mathrm{C}$ & 2.17139900 & -3.23250100 & 1.68714700 \\
\hline $\mathrm{H}$ & 2.55530900 & -4.13764200 & 2.14966400 \\
\hline $\mathrm{C}$ & 0.79117600 & -2.97453200 & 1.69605200 \\
\hline $\mathrm{C}$ & -0.18673000 & -3.85706100 & 2.27151500 \\
\hline
\end{tabular}




$\begin{array}{lccc}\mathrm{H} & 0.15631200 & -4.77220200 & 2.74574800 \\ \mathrm{C} & -1.50881600 & -3.56622100 & 2.21051900 \\ \mathrm{H} & -2.24269300 & -4.24625600 & 2.63333400 \\ \mathrm{C} & -1.98146800 & -2.36342000 & 1.57884900 \\ \mathrm{C} & -3.33985200 & -2.02454600 & 1.47848700 \\ \mathrm{H} & -4.09648800 & -2.68841600 & 1.88608300 \\ \mathrm{C} & -3.68830600 & -0.84581300 & 0.85077500 \\ \mathrm{H} & -4.72613800 & -0.54810900 & 0.75050000 \\ \mathrm{C} & -2.68793700 & -0.02512600 & 0.32367700 \\ \mathrm{C} & -1.04435500 & -1.47636000 & 1.01867700 \\ \mathrm{C} & 0.35488500 & -1.78797100 & 1.07512400 \\ \mathrm{C} & 0.30479200 & 2.19572500 & 0.76611600 \\ \mathrm{C} & 1.00288500 & 2.44067400 & -0.28710900 \\ \mathrm{H} & 0.97377900 & 1.36707200 & -1.55727500 \\ \mathrm{Si} & 0.05674500 & -0.57635200 & -2.48546600 \\ \mathrm{H} & -0.68126300 & 0.00680600 & -3.65821600 \\ \mathrm{H} & 1.39872700 & -0.96986400 & -3.03426600 \\ \mathrm{C} & -0.81620000 & -2.23524000 & -2.16270400 \\ \mathrm{C} & -0.11819300 & -3.34471800 & -1.66140900 \\ \mathrm{C} & -2.21317200 & -2.34612000 & -2.24534700 \\ \mathrm{C} & -0.77708300 & -4.50370600 & -1.25712300 \\ \mathrm{H} & 0.96662200 & -3.29798900 & -1.57498600 \\ \mathrm{C} & -2.88178900 & -3.49917200 & -1.84316700 \\ \mathrm{H} & -2.79247400 & -1.50970800 & -2.63268900 \\ \mathrm{C} & -2.16460400 & -4.58381400 & -1.34262800 \\ \mathrm{H} & -0.20688800 & -5.34387900 & -0.86869000 \\ \mathrm{H} & -3.96487100 & -3.55375600 & -1.92174300 \\ \mathrm{H} & -2.68223700 & -5.48547500 & -1.02631100 \\ \mathrm{H} & 3.12626900 & -0.45577700 & -0.01386000 \\ \mathrm{H} & -2.93593000 & 0.90334000 & -0.18041100 \\ \mathrm{C} & -0.07101900 & 2.69134100 & 2.11347500 \\ \mathrm{H} & 0.27877100 & 1.98790900 & 2.87799600 \\ \mathrm{H} & 0.34535400 & 3.68084600 & 2.33832800 \\ & & 2.74302900 & 2.21440900 \\ \mathrm{H} & 3.16763900 & -0.76417700\end{array}$

${ }^{3}$ Ts-1b

$\mathrm{Fe}$

$\mathrm{N}$

$\mathrm{N}$

C

C

C

C

$\begin{array}{rcc}-0.41364600 & -0.16759500 & 0.76772600 \\ 1.08675100 & -0.56626900 & 2.39855400 \\ -1.58879000 & -0.53730900 & 2.35426600 \\ 3.13037400 & -0.11435400 & 1.15661400 \\ 3.21923600 & -1.01387200 & 0.09494400 \\ 3.97966100 & -0.70842100 & -1.03469300 \\ 4.62954300 & 0.52798800 & -1.09694500 \\ & \text { S89 } & \end{array}$


4.55669600

1.44010600

$-0.04178800$

3.80626400

1.10228100

1.08755600

2.41402000

$-0.49321400$

2.40719300

3.16313300

$-0.77494300$

3.56689800

4.24431300

$-0.70033200$

3.52340900

2.51880900

$-1.15681900$

4.72232300

3.08165100

$-1.39520600$

5.62082400

1.11645500

$-1.23601300$

4.73426100

0.35338300

$-1.62399000$

5.88760700

0.88672500

$-1.89326700$

6.79493900

$-1.00046900$

$-1.65422600$

5.84822200

$-1.57263900$

$-1.95075500$

6.72258400

$-1.71579200$

$-1.28132400$

4.65965700

$-3.11767100$

$-1.23616500$

4.58475700

$-3.71510100$

$-1.52006700$

5.44631300

$-3.71487400$

$-0.81560100$

3.41663000

$-4.79345300$

$-0.75055000$

3.32471400

$-2.92187600$

$-0.46634500$

2.31369100

$-0.99168800$

$-0.91880200$

3.50984600

0.44675800

$-0.91113400$

3.53894800

$-3.51519800$

0.02367200

1.03995300

$-3.89838400$

1.35896000

0.92190300

$-4.36289300$

1.85754900

$-0.29518700$

$-4.44805400$

0.99309800

$-1.38862700$

$-4.07031400$

$-0.34524300$

$-1.28837500$

$-3.61088600$

$-0.82437100$

$-0.06133400$

$-0.46110300$

1.76471900

0.72320600

0.13178300

1.36157000

$-0.34577600$

0.32912000

$-0.27591500$

$-0.55843100$

$-0.36299700$

$-2.47808100$

0.11282000

$-1.36225200$

$-2.87788800$

$-0.93730800$

$-3.05447100$

$-0.40007000$

$-0.78582000$

$-3.57485900$

1.60955700

0.19890500

$-3.92209500$

2.54777000

$-2.11084400$

$-3.90978900$

1.92799900

$-0.11909100$

$-4.55885800$

3.74489200

1.24127800

$-3.68084000$

2.34303300

$-2.44160800$

$-4.54110500$

3.12441200

$-2.90738700$

$-3.67922400$

1.22319600

$-1.44507000$

$-4.86393200$

4.04199100

0.66790800

$-4.80943800$

4.45161300

$-3.47968300$

$-4.78169400$

3.33985000

$-1.69776000$

$-5.35437000$

4.97817300

5.23213500

0.76907400 
$\mathrm{H}$

$\mathrm{H}$

$\mathrm{H}$

$\mathrm{H}$

$\mathrm{H}$

C

C

C

C

C

C

$\mathrm{H}$

$\mathrm{H}$

C

C

C

C

C

C

$\mathrm{H}$

$\mathrm{H}$

C

C

C

C

C

C

$\mathrm{H}$

$\mathrm{H}$

C

C

C

C

C

C

$\mathrm{H}$

$\mathrm{H}$

C

$\mathrm{H}$

$\mathrm{H}$

$\mathrm{H}$

C

$\mathrm{H}$

$\mathrm{H}$
2.71708400

$-1.97347400$

0.15560100

3.76143900

1.79628600

$-3.79263400$

2.02895600

1.92325900

$-3.30091800$

$-1.86034700$

1.77106900

$-4.79881700$

1.37659200

$-4.71135000$

3.30047700

3.80734500

4.15047600

5.16105400

5.49687100

6.02154100

5.55136800

6.15579900

$-1.24092800$

$-2.08546800$

$-1.23273600$

$-2.91381100$

$-2.07560700$

$-2.92523900$

$-3.56709700$

$-2.07151600$

2.71447200

2.67211700

3.94899600

3.86416200

5.11748900

5.09735800

3.82591200

6.07157400

$-1.73721300$

$-2.50631100$

$-1.96589300$

$-3.48516400$

$-2.95409700$

$-3.72250300$

$-4.08322200$

$-3.13218300$

$-2.29894200$

$-1.32327500$

$-2.32855400$

$-3.06971300$

$-1.15319200$

0.01963800

$-2.34373600$

$-0.44352200$

0.13070200

$-1.16401800$

$-0.02421200$

$-1.29038300$

$-0.73071700$

0.41749700

$-1.84409500$

$-2.48119400$

$-2.70757000$

$-3.37110400$

$-3.82975200$

$-4.48011100$

$-4.72531400$

$-4.00827100$

$-5.17062400$

$-0.06118600$

0.16242100

$-0.26906400$

0.17384900

$-0.24968300$

$-0.02795800$

0.35001200

$-0.41507700$

$-2.10232200$

$-2.09066000$

$-3.08425000$

$-3.06718700$

$-4.04235600$

$-4.05394200$

$-3.04990600$

$-4.79778900$

$-1.02888500$

$-1.13292900$

$-0.02405300$

$-1.09054600$

$-3.12134800$

$-1.18078600$

$-2.16146000$

$-0.10137100$

$-3.34144600$ 
$\mathrm{H}$

C

$\mathrm{H}$

$\mathrm{H}$

$\mathrm{H}$

C

$\mathrm{H}$

$\mathrm{H}$

$\mathrm{H}$

C

$\mathrm{H}$

$\mathrm{H}$

$\mathrm{H}$

C

$\mathrm{H}$

$\mathrm{H}$

$\mathrm{H}$

C

$\mathrm{H}$

$\mathrm{H}$

$\mathrm{H}$

C

$\mathrm{H}$

$\mathrm{H}$

$\mathrm{H}$

C

$\mathrm{H}$

$\mathrm{H}$

$\mathrm{H}$

C

$\mathrm{H}$

$\mathrm{H}$

$\mathrm{H}$

C

$\mathrm{H}$

$\mathrm{H}$

$\mathrm{H}$

C

$\mathrm{H}$

$\mathrm{H}$

$\mathrm{H}$

C

$\mathrm{H}$

$\mathrm{H}$
1.24952400

4.80481600

5.19680800

5.52445400

3.87029100

3.22126600

3.01462000

2.62416900

2.86229000

7.44264900

6.87467900

7.59406100

8.42750900

7.62758500

7.27653700

7.53070000

8.69250400

$-6.82322700$

$-7.04245100$

$-6.38790400$

$-7.76892900$

$-2.58591600$

$-2.05348200$

$-2.80332000$

$-1.91031600$

$-5.69320600$

$-5.87206400$

$-6.59309400$

$-4.87511900$

$-1.82821900$

$-2.11794000$

$-1.36279200$

$-1.07095400$

$-6.36697000$

$-6.06136400$

$-6.80339800$

$-7.15058800$

$-4.12043300$

$-3.62487200$

$-3.58561700$

$-5.13831600$

0.76426200

1.82653300

0.29395300
$-1.52828300$

$-4.76983400$

$-4.31903300$

$-5.52599700$

$-5.27910500$

4.02373100

3.65202800

3.41562400

5.05568000

1.36227200

0.69250800

0.83152000

1.52790400

6.37983400

7.09319600

6.86441000

6.19855400

2.90904900

1.98975700

2.60500000

3.41783200

3.62818400

2.93420600

3.08543600

4.45126800

7.48110700

7.72134600

7.75091900

8.12295600

$-0.33578200$

0.72136400

$-0.55423600$

$-0.46497000$

$-2.09703400$

$-2.41966900$

$-1.09762500$

$-2.77592600$

$-3.85159900$

$-3.39232200$

$-4.78130100$

$-4.12204200$

1.79116000

1.52175400

1.29799900
$-3.88823900$

$-5.11653900$

$-6.03652200$

$-4.78768100$

$-5.37325800$

$-0.56304100$

$-1.57312400$

0.12359300

$-0.50702200$

0.38269600

1.03568000

$-0.56482500$

0.82939300

0.01922300

$-0.73368900$

0.99844900

$-0.15578400$

0.90311400

0.34958700

1.86205300

1.11162400

$-1.79915700$

$-1.14131700$

$-2.72739500$

$-2.05207800$

$-0.90209900$

$-1.95663700$

$-0.34104000$

$-0.55636800$

$-3.12584200$

$-3.10381900$

$-2.15828100$

$-3.90527000$

$-1.75458500$

$-0.75257500$

$-1.64535200$

$-2.10331900$

$-5.91580100$

$-6.77733200$

$-5.68643700$

$-6.21417900$

$-1.62017300$

$-1.62753700$

$-2.47733600$ 
$\mathrm{H}$

${ }^{3}$ Ts-1 $1 b^{\prime}$

$\mathrm{Fe}$

$\mathrm{N}$

$\mathrm{N}$

C

C

C

C

C

C

C

C

$\mathrm{H}$

C

$\mathrm{H}$

C

C

$\mathrm{H}$

$\mathrm{C}$

$\mathrm{H}$

C

C

$\mathrm{H}$

$\mathrm{C}$

$\mathrm{H}$

C

C

C

C

C

C

C

C

C

C

C

$\mathrm{H}$

$\mathrm{Si}$

$\mathrm{H}$

$\mathrm{H}$

C

\section{(1)}

$\mathrm{N}$

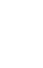

0.67375800

2.87475300

$-1.74735700$

$-0.77589700$

2.68527800

1.19597900

C.

C

$-0.34176000$

1.08910800

$-0.29772200$

0.82088700

$-1.56602000$

$-0.69324000$

2.49461000

3.14269600

3.25711400

$-0.49384600$

2.41113500

$-0.37070000$

1.25546700

3.95012900

$-1.30704500$

0.22948800

4.51383100

$-0.99391200$

$-0.93980400$

4.41134200

0.27710300

$-1.07130900$

$1.22551200-0.05289700$

3.72714700

0.88660100

1.11600900

2.41921100

$-0.71240100$

2.51240100

3.13865200

$-1.03019600$

3.67929100

4.22267400

$-1.03563400$

3.64210400

2.46094500

$-1.33908700$

4.83934300

3.00036900

$-1.59978200$

5.74594200

1.05685800

$-1.30912500$

4.84493600

0.25588000

$-1.61008900$

5.99912700

0.75903400

$-1.89311700$

6.91939300

$-1.09658500$

$-1.54524100$

5.94420800

$-1.69634300$

$-1.77877200$

6.81919800

$-1.77374500$

$-1.15879100$

4.73714100

$-3.16846200$

$-1.02942500$

4.63803000

$-3.79545700$

$-1.25042600$

5.49690800

$-3.72139700$

$-0.61146000$

3.44634800

$-4.79266300$

$-0.48558000$

3.33450400

$-2.89276800$

$-0.34714600$

2.34660100

$-1.01316800$

$-0.87674000$

3.58863500

0.42009900

$-0.96573300$

3.63703400

$-3.42933600$

0.14745200

1.05036100

$-3.75124500$

1.49627400

0.91634000

$-4.13035700$

2.01752600

$-0.32065900$

$-4.21841800$

1.15432400

$-1.41412200$

$-3.91696600$

$-0.20254300$

$-1.29583300$

$-3.52013900$

$-0.69903000$

$-0.05362700$

$-0.18195100$

1.62930900

0.60768200

0.34190400

1.04200100

$-0.41181600$

0.37250900

$-0.62178300$

$-0.48503700$

$-0.40941800$

$-2.62811200$

0.29848600

$-1.37774400$

$-3.01797400$

$-0.78445000$

0.86810500

$-3.30210500$

$-0.11184700$

$-0.97206800$

$-3.62217400$

1.81920400 


\begin{tabular}{|c|c|c|c|}
\hline $\mathrm{C}$ & -0.05071200 & -4.00301900 & 2.80749200 \\
\hline $\mathrm{C}$ & -2.32924000 & -3.84789600 & 2.09531600 \\
\hline $\mathrm{C}$ & -0.45922100 & -4.57081500 & 4.01178500 \\
\hline $\mathrm{H}$ & 1.01310800 & -3.84448600 & 2.63558600 \\
\hline $\mathrm{C}$ & -2.74954100 & -4.41000300 & 3.29813900 \\
\hline $\mathrm{H}$ & -3.07989000 & -3.58558900 & 1.35220000 \\
\hline $\mathrm{C}$ & -1.81396600 & -4.77052400 & 4.26472900 \\
\hline $\mathrm{H}$ & 0.28066200 & -4.85114600 & 4.75704800 \\
\hline $\mathrm{H}$ & -3.80931000 & -4.57009800 & 3.47998600 \\
\hline $\mathrm{H}$ & -2.13756500 & -5.20909800 & 5.20481700 \\
\hline $\mathrm{H}$ & 5.05456900 & 0.52790300 & -1.98097300 \\
\hline $\mathrm{H}$ & 2.80360800 & -2.28703700 & 0.33753000 \\
\hline $\mathrm{H}$ & 3.63929100 & 1.61854000 & 1.91497800 \\
\hline $\mathrm{H}$ & -3.65650200 & 2.15993300 & 1.77205300 \\
\hline $\mathrm{H}$ & -3.25977800 & -1.74788800 & 0.03927800 \\
\hline $\mathrm{H}$ & -4.50769300 & 1.55114300 & -2.38404400 \\
\hline $\mathrm{C}$ & -4.36863900 & 3.48103800 & -0.47705000 \\
\hline $\mathrm{C}$ & -5.49968200 & 4.08043000 & 0.10685300 \\
\hline $\mathrm{C}$ & -3.44922900 & 4.26146400 & -1.19567900 \\
\hline $\mathrm{C}$ & -5.68926200 & 5.45188000 & -0.03560100 \\
\hline $\mathrm{C}$ & -3.67355100 & 5.63587500 & -1.30817200 \\
\hline $\mathrm{C}$ & -4.78435200 & 6.24914400 & -0.73824400 \\
\hline $\mathrm{H}$ & -6.56810700 & 5.91327600 & 0.41140800 \\
\hline $\mathrm{H}$ & -2.95640000 & 6.24116900 & -1.85932900 \\
\hline $\mathrm{C}$ & -3.96717200 & -1.09851500 & -2.48826600 \\
\hline $\mathrm{C}$ & -5.09129800 & -1.90058400 & -2.72348600 \\
\hline $\mathrm{C}$ & -2.87463000 & -1.12974800 & -3.37162200 \\
\hline $\mathrm{C}$ & -5.10972600 & -2.72762200 & -3.84788000 \\
\hline $\mathrm{C}$ & -2.93055500 & -1.96902600 & -4.48101600 \\
\hline $\mathrm{C}$ & -4.03912800 & -2.77683600 & -4.73621400 \\
\hline $\mathrm{H}$ & -5.98445800 & -3.34818100 & -4.03238900 \\
\hline $\mathrm{H}$ & -2.08398100 & -1.99725600 & -5.16474600 \\
\hline $\mathrm{C}$ & 5.04589700 & 2.56912400 & -0.20078900 \\
\hline $\mathrm{C}$ & 6.40795400 & 2.72803100 & 0.09271100 \\
\hline $\mathrm{C}$ & 4.28492400 & 3.66653800 & -0.63726500 \\
\hline $\mathrm{C}$ & 6.99117100 & 3.98774800 & -0.05152400 \\
\hline $\mathrm{C}$ & 4.90260000 & 4.90832600 & -0.76785700 \\
\hline $\mathrm{C}$ & 6.25535500 & 5.08987800 & -0.47904500 \\
\hline $\mathrm{H}$ & 8.04854300 & 4.10967200 & 0.17522800 \\
\hline $\mathrm{H}$ & 4.31312000 & 5.75793100 & -1.10823100 \\
\hline $\mathrm{C}$ & 4.13120200 & -2.01844100 & -2.01161400 \\
\hline $\mathrm{C}$ & 5.30210900 & -2.79267500 & -2.02820300 \\
\hline $\mathrm{C}$ & 3.14265500 & -2.21481400 & -2.98775700 \\
\hline $\mathrm{C}$ & 5.46474300 & -3.75688600 & -3.02221300 \\
\hline
\end{tabular}


C

C

$\mathrm{H}$

$\mathrm{H}$

C

$\mathrm{H}$

$\mathrm{H}$

$\mathrm{H}$

C

$\mathrm{H}$

$\mathrm{H}$

$\mathrm{H}$

C

$\mathrm{H}$

$\mathrm{H}$

$\mathrm{H}$

C

$\mathrm{H}$

$\mathrm{H}$

$\mathrm{H}$

C

$\mathrm{H}$

$\mathrm{H}$

$\mathrm{H}$

C

$\mathrm{H}$

$\mathrm{H}$

$\mathrm{H}$

C

$\mathrm{H}$

$\mathrm{H}$

$\mathrm{H}$

C

$\mathrm{H}$

$\mathrm{H}$

$\mathrm{H}$

C

$\mathrm{H}$

$\mathrm{H}$

$\mathrm{H}$

C

$\mathrm{H}$

$\mathrm{H}$

$\mathrm{H}$
3.34348300

4.49682700

6.37017900

2.57350600

6.37197000

6.83224200

5.95748900

7.16296900

1.86839400

1.21506900

2.06659000

1.31482200

4.69780700

5.08179200

5.41584200

3.75668000

2.82626500

2.70355600

2.26444700

2.36395300

7.23844000

6.79432800

7.30893200

8.25426200

6.89369200

6.69942200

6.49717800

7.97798200

$-6.50962300$

$-6.79538200$

$-6.10998000$

$-7.41443000$

$-2.23470300$

$-1.81537100$

$-2.48591800$

$-1.45090300$

$-5.01887600$

$-5.92894400$

$-5.14248900$

$-4.18366000$

$-1.65193100$

$-1.89560600$

$-1.19010700$

$-0.90608300$
$-3.18615400$

$-3.96755500$

$-4.36093200$

$-3.34020800$

$-2.59473700$

$-1.60308900$

$-2.66768600$

$-3.34358400$

$-1.40940600$

$-1.72405700$

$-0.34091600$

$-1.54054600$

$-4.99825700$

$-4.53514100$

$-5.76476300$

$-5.49687500$

3.49876900

2.83669000

3.04113800

4.46047800

1.55433300

1.07124300

0.78660700

1.86791600

6.44913700

6.87720400

7.15225100

6.39665200

3.25528600

2.35818900

2.91450800

3.83588500

3.65332700

2.82191800

3.26186700

4.40551200

7.73268700

7.93592300

8.20768400

8.22334300

$-0.28728200$

0.77928900

$-0.55543300$

$-0.42232400$
$-3.96746900$

$-4.00204600$

$-3.02881600$

$-4.72121600$

$-0.98130700$

$-1.06369000$

0.03005000

$-1.08261400$

$-2.97575300$

$-2.15411500$

$-2.83877500$

$-3.91000500$

$-5.08508000$

$-6.00229900$

$-4.77749700$

$-5.33865500$

$-0.98250300$

$-1.84830000$

$-0.16231100$

$-1.22419400$

0.55326600

1.43054900

$-0.22579300$

0.81104800

$-0.62228700$

$-1.61193300$

0.11980900

$-0.48502800$

0.86876200

0.30926000

1.83085600

1.07114100

$-1.85858000$

$-1.28498700$

$-2.85251700$

$-1.99267100$

$-0.87693300$

$-1.45337800$

0.10288500

$-1.38564800$

$-3.11120200$

$-3.05159100$

$-2.15517700$

$-3.90000500$ 


$\begin{array}{lrrr}\mathrm{C} & -6.26771200 & -1.87374700 & -1.77794600 \\ \mathrm{H} & -5.99158100 & -2.25391500 & -0.78749700 \\ \mathrm{H} & -6.64122700 & -0.85377800 & -1.63323400 \\ \mathrm{H} & -7.08971400 & -2.48825900 & -2.15647500 \\ \mathrm{C} & -4.06059200 & -3.68561900 & -5.93999200 \\ \mathrm{H} & -3.83985800 & -3.13239200 & -6.85977400 \\ \mathrm{H} & -3.30742000 & -4.47687600 & -5.84744700 \\ \mathrm{H} & -5.03615900 & -4.16598800 & -6.06197300 \\ \mathrm{C} & -0.46166200 & 2.95444000 & 1.21602400 \\ \mathrm{H} & 0.08391600 & 3.77134700 & 0.72703300 \\ \mathrm{H} & -1.53241800 & 3.17796200 & 1.13982700 \\ \mathrm{H} & -0.21166800 & 2.95446400 & 2.28262000 \\ \mathrm{H} & 0.82999000 & 1.19569300 & -1.36259400\end{array}$

\section{Ts-1d}

$\mathrm{Fe}$

0.17603300

$-1$

.39270200

$-0.20921200$

1.44963700

0.37825400

$-0.64479400$

$\mathrm{N}$

$-1.18717100$

$-0.03108400$

$-0.79919400$

C

2.76637700

0.53541400

$-0.51266800$

3.37294100

1.78168200

$-0.75310400$

4.44413600

1.89298700

$-0.63286500$

2.60318600

2.85912300

$-1.13856300$

3.06176500

3.82688400

$-1.32379000$

1.21618200

2.70537500

$-1.28467100$

0.32841800

3.77089100

$-1.65789700$

0.75129900

4.75091400

$-1.86036700$

$-1.00743200$

3.56216200

$-1.74138400$

$-1.67888000$

4.37171400

$-2.01297400$

$-1.57615900$

2.27270500

$-1.46083400$

$-2.95401400$

2.01072000

$-1.50085300$

$-3.64862800$

2.80153300

$-1.76931100$

$-3.41071300$

0.75192400

$-1.17911800$

$-4.47298500$

0.53856200

$-1.18822000$

$-2.50540300$

$-0.25807500$

$-0.81704000$

$-0.72968600$

1.20789600

$-1.10665800$

0.68877800

1.42724000

$-1.01855700$

0.38378400

$-2.54541700$

$-1.75460300$

1.03302700

$-3.07227900$

$-0.77229500$

0.96780800

$-2.33058200$

0.69770400

$\mathrm{Si}$

0.10944200

$-0.67795300$

2.06712100

$-0.70160800$

$-1.51860400$

3.01542000

1.44275800

$-0.52439600$

2.74622300

$-0.67177100$

1.04998400

2.17731900

0.10141300

2.21153000

2.02998000 


\begin{tabular}{|c|c|c|c|}
\hline $\mathrm{C}$ & -2.06552100 & 1.21061300 & 2.21254700 \\
\hline $\mathrm{C}$ & -0.48376000 & 3.47031600 & 1.91424600 \\
\hline $\mathrm{H}$ & 1.18654100 & 2.12853700 & 1.98902100 \\
\hline $\mathrm{C}$ & -2.66046400 & 2.46392500 & 2.09736800 \\
\hline $\mathrm{H}$ & -2.70232600 & 0.33421000 & 2.32668900 \\
\hline $\mathrm{C}$ & -1.86973500 & 3.60061900 & 1.94318100 \\
\hline $\mathrm{H}$ & 0.14295200 & 4.35012500 & 1.79266700 \\
\hline $\mathrm{H}$ & -3.74347700 & 2.55538700 & 2.12481100 \\
\hline $\mathrm{H}$ & -2.33064100 & 4.57994200 & 1.84684800 \\
\hline $\mathrm{C}$ & 1.88799800 & -4.21534700 & -0.34563900 \\
\hline $\mathrm{H}$ & 1.41574200 & -4.76937500 & 0.47349600 \\
\hline $\mathrm{H}$ & 2.06723500 & -4.90489300 & -1.17678000 \\
\hline $\mathrm{H}$ & 2.85565700 & -3.85701800 & 0.02710100 \\
\hline $\mathrm{H}$ & 0.18790300 & -2.75502100 & -2.79834400 \\
\hline $\mathrm{C}$ & 3.53554000 & -0.69375300 & -0.10047400 \\
\hline $\mathrm{H}$ & 3.06012700 & -1.08149200 & 0.80976300 \\
\hline $\mathrm{H}$ & 3.35950900 & -1.46065900 & -0.86650200 \\
\hline $\mathrm{C}$ & -2.93123500 & -1.63974200 & -0.39844700 \\
\hline $\mathrm{H}$ & -2.45647900 & -2.35816300 & -1.08128500 \\
\hline $\mathrm{H}$ & -2.47727300 & -1.83194000 & 0.58669200 \\
\hline $\mathrm{C}$ & -4.43291300 & -1.90031000 & -0.31794100 \\
\hline $\mathrm{H}$ & -4.89762400 & -1.18508100 & 0.37503800 \\
\hline $\mathrm{H}$ & -4.89810900 & -1.73663600 & -1.29999000 \\
\hline $\mathrm{C}$ & 5.03386100 & -0.52226700 & 0.12857800 \\
\hline $\mathrm{H}$ & 5.21042000 & 0.24205100 & 0.89817100 \\
\hline $\mathrm{H}$ & 5.52093500 & -0.16456300 & -0.78937700 \\
\hline $\mathrm{C}$ & 5.69385400 & -1.83161000 & 0.56445500 \\
\hline $\mathrm{H}$ & 5.51259200 & -2.59832500 & -0.20107700 \\
\hline $\mathrm{H}$ & 5.20900000 & -2.19075500 & 1.48166500 \\
\hline $\mathrm{C}$ & 7.19601500 & -1.68645700 & 0.79957400 \\
\hline $\mathrm{H}$ & 7.40099000 & -0.94374000 & 1.57926900 \\
\hline $\mathrm{H}$ & 7.64430000 & -2.63440600 & 1.11401100 \\
\hline $\mathrm{H}$ & 7.70700500 & -1.36005100 & -0.11375200 \\
\hline $\mathrm{C}$ & -4.74232600 & -3.32337400 & 0.14928500 \\
\hline $\mathrm{H}$ & -4.28080300 & -4.03893000 & -0.54379500 \\
\hline $\mathrm{H}$ & -4.26995900 & -3.49208300 & 1.12578400 \\
\hline $\mathrm{C}$ & -6.24155800 & -3.59769300 & 0.24940400 \\
\hline $\mathrm{H}$ & -6.43666800 & -4.62105500 & 0.58560400 \\
\hline $\mathrm{H}$ & -6.71931300 & -2.91411500 & 0.96083700 \\
\hline $\mathrm{H}$ & -6.73231200 & -3.46431600 & -0.72180800 \\
\hline
\end{tabular}

\section{Ts-1d'}

$\mathrm{Fe}$

$\mathrm{N}$
$\begin{array}{lll}0.13798100 & -1.46678500 & -0.00321000\end{array}$
$\begin{array}{lll}1.52878900 & 0.17291800 & -0.56732500\end{array}$


N

C

C

$\mathrm{H}$

C

$\mathrm{H}$

C

C

$\mathrm{H}$

C

$\mathrm{H}$

C

C

$\mathrm{H}$

C

$\mathrm{H}$

C

C

C

C

C

$\mathrm{H}$

$\mathrm{Si}$

$\mathrm{H}$

$\mathrm{H}$

C

C

C

C

$\mathrm{H}$

C

$\mathrm{H}$

C

$\mathrm{H}$

$\mathrm{H}$

$\mathrm{H}$

C

$\mathrm{H}$

$\mathrm{H}$

$\mathrm{H}$

$\mathrm{H}$

C

$\mathrm{H}$

$\mathrm{H}$

$\begin{array}{ccc}-1.12873800 & -0.05942000 & -0.69388900 \\ 2.85396100 & 0.25036900 & -0.44030700 \\ 3.54523700 & 1.42550000 & -0.78829400 \\ 4.62212200 & 1.47044600 & -0.67575200 \\ 2.85227200 & 2.51771700 & -1.26643000 \\ 3.37686500 & 3.43130900 & -1.53365600 \\ 1.45695000 & 2.45121000 & -1.39829100 \\ 0.64410800 & 3.54302500 & -1.85686000 \\ 1.13327600 & 4.47143400 & -2.13806100 \\ -0.70365300 & 3.42325700 & -1.92441700 \\ -1.31746600 & 4.25425000 & -2.26006100 \\ -1.35986000 & 2.20385500 & -1.54127600 \\ -2.75252300 & 2.03509200 & -1.56300100 \\ -3.39211700 & 2.84837200 & -1.89390700 \\ -3.29399000 & 0.83983700 & -1.14427200 \\ -4.36844000 & 0.70011200 & -1.13956500 \\ -2.45945000 & -0.19817100 & -0.70106800 \\ -0.58772000 & 1.11524200 & -1.10005800 \\ 0.84287100 & 1.23954100 & -1.02809700 \\ 0.21750300 & -2.67828900 & -1.51448900 \\ 0.78081400 & -3.24082600 & -0.50112500 \\ 0.84097100 & -2.44359900 & 0.94507300 \\ 0.12829500 & -0.59585500 & 2.21833800 \\ -0.74712800 & -1.29308900 & 3.22396400 \\ 1.46677300 & -0.49207300 & 2.89670100 \\ -0.52063300 & 1.19030700 & 2.19322400 \\ 0.33262000 & 2.27678000 & 1.94755200 \\ -1.89846400 & 1.45573000 & 2.22223900 \\ -0.16120400 & 3.56178000 & 1.73369100 \\ 1.40869800 & 2.11314500 & 1.90882900 \\ -2.40273400 & 2.73578200 & 2.00937600 \\ -2.59645500 & 0.64120900 & 2.41258200 \\ -1.53353400 & 3.79526700 & 1.75891700 \\ 0.52722900 & 4.37999600 & 1.53781100 \\ -3.47600600 & 2.90734300 & 2.03606600 \\ -1.92343900 & 4.79516000 & 1.58829500 \\ -0.13484800 & -2.84380800 & -2.94635200 \\ -2.51346600 & -1.67502400 & 0.80495600\end{array}$




$\begin{array}{lrrr}\mathrm{C} & 3.53528600 & -0.98067700 & 0.09967700 \\ \mathrm{H} & 3.05792600 & -1.21518400 & 1.05996500 \\ \mathrm{H} & 3.27437700 & -1.81825300 & -0.55998500 \\ \mathrm{C} & 5.04915600 & -0.90370100 & 0.27447600 \\ \mathrm{H} & 5.30909800 & -0.07251300 & 0.94467800 \\ \mathrm{H} & 5.53280200 & -0.69478300 & -0.69023600 \\ \mathrm{C} & 5.62149000 & -2.20113600 & 0.84846500 \\ \mathrm{H} & 5.14260700 & -2.40728300 & 1.81435300 \\ \mathrm{H} & 5.35444300 & -3.03618700 & 0.18737600 \\ \mathrm{C} & 7.13769200 & -2.14959500 & 1.02561600 \\ \mathrm{H} & 7.42669100 & -1.33974100 & 1.70551900 \\ \mathrm{H} & 7.52309400 & -3.08682500 & 1.44008200 \\ \mathrm{H} & 7.63998500 & -1.97529300 & 0.06683800 \\ \mathrm{C} & -4.49199900 & -1.64989700 & -0.05153900 \\ \mathrm{H} & -4.96932700 & -1.51499700 & -1.03217100 \\ \mathrm{H} & -4.88112700 & -0.85634200 & 0.60133500 \\ \mathrm{C} & -4.89747200 & -3.01089900 & 0.51604300 \\ \mathrm{H} & -4.41285400 & -3.15389300 & 1.49053800 \\ \mathrm{H} & -4.51213300 & -3.80349400 & -0.13871100 \\ \mathrm{C} & -6.41010500 & -3.15896900 & 0.66798500 \\ \mathrm{H} & -6.91621300 & -3.04774400 & -0.29809000 \\ \mathrm{H} & -6.67391700 & -4.14060900 & 1.07424000 \\ \mathrm{H} & -6.81286200 & -2.39712200 & 1.34551200\end{array}$

$\begin{array}{cccc}\text { Ts-1e } & & & \\ \text { Fe } & -0.33969600 & 1.12303700 & -0.06169600 \\ \mathrm{~N} & -1.28777700 & -0.79901700 & -0.72427400 \\ \mathrm{~N} & 1.25636200 & 0.06893600 & -0.72111800 \\ \mathrm{C} & -3.60343600 & -0.23736700 & -0.29303300 \\ \mathrm{C} & -4.50827000 & -0.48155500 & 0.74297800 \\ \mathrm{C} & -5.51574200 & 0.43526600 & 1.02496300 \\ \mathrm{C} & -5.63665300 & 1.59427200 & 0.26232800 \\ \mathrm{C} & -4.74244600 & 1.83645000 & -0.77826000 \\ \mathrm{C} & -3.72611600 & 0.92784300 & -1.05239100 \\ \mathrm{C} & -2.54725500 & -1.22644200 & -0.61634000 \\ \mathrm{C} & -2.89576600 & -2.57235400 & -0.83587600 \\ \mathrm{H} & -3.93455200 & -2.86898800 & -0.73903700 \\ \mathrm{C} & -1.92944300 & -3.47695600 & -1.21275700 \\ \mathrm{H} & -2.18317300 & -4.51491500 & -1.40971100 \\ \mathrm{C} & -0.60007500 & -3.04671200 & -1.34458900 \\ \mathrm{C} & 0.47166700 & -3.92192300 & -1.72186400 \\ \mathrm{H} & 0.24039800 & -4.95961500 & -1.94502800 \\ \mathrm{C} & 1.74550600 & -3.46741900 & -1.78534200 \\ \mathrm{H} & 2.55827100 & -4.13175900 & -2.06425800 \\ & & & \\ & & \mathrm{~S} 99 & \end{array}$




\begin{tabular}{|c|c|c|c|}
\hline $\mathrm{C}$ & 2.06128600 & -2.10573100 & -1.46153100 \\
\hline $\mathrm{C}$ & 3.37337100 & -1.60729900 & -1.47646800 \\
\hline $\mathrm{H}$ & 4.19824100 & -2.26022400 & -1.74640100 \\
\hline $\mathrm{C}$ & 3.59182400 & -0.29522100 & -1.13103800 \\
\hline $\mathrm{H}$ & 4.59351700 & 0.11983200 & -1.11409100 \\
\hline $\mathrm{C}$ & 2.51527000 & 0.52606000 & -0.75181000 \\
\hline $\mathrm{C}$ & 1.03142300 & -1.22171400 & -1.08804800 \\
\hline $\mathrm{C}$ & -0.32765800 & -1.69218900 & -1.06106300 \\
\hline $\mathrm{C}$ & 2.77387800 & 1.93893600 & -0.38007900 \\
\hline $\mathrm{C}$ & 3.55055500 & 2.74175300 & -1.22076600 \\
\hline $\mathrm{C}$ & 3.79339000 & 4.07261900 & -0.89859800 \\
\hline $\mathrm{C}$ & 3.27068500 & 4.61316100 & 0.27393400 \\
\hline $\mathrm{C}$ & 2.50850200 & 3.81561100 & 1.12273200 \\
\hline $\mathrm{C}$ & 2.26318800 & 2.48593400 & 0.79930400 \\
\hline $\mathrm{C}$ & -0.49039600 & 2.55472300 & -1.35662700 \\
\hline $\mathrm{C}$ & -1.21444400 & 2.86713400 & -0.33795500 \\
\hline $\mathrm{H}$ & -1.35612700 & 1.76592600 & 0.88218500 \\
\hline $\mathrm{Si}$ & -0.42062200 & 0.12141800 & 2.10418700 \\
\hline $\mathrm{H}$ & 0.02341400 & 0.98488700 & 3.25567600 \\
\hline $\mathrm{H}$ & -1.74163300 & -0.44834900 & 2.53388200 \\
\hline $\mathrm{C}$ & 0.76026800 & -1.36780000 & 2.16095700 \\
\hline $\mathrm{C}$ & 0.30486000 & -2.67100600 & 1.91474200 \\
\hline $\mathrm{C}$ & 2.14909400 & -1.18694900 & 2.26537900 \\
\hline $\mathrm{C}$ & 1.18647500 & -3.74082400 & 1.77191900 \\
\hline $\mathrm{H}$ & -0.76478100 & -2.85242000 & 1.82005500 \\
\hline $\mathrm{C}$ & 3.03845800 & -2.24762900 & 2.12323900 \\
\hline $\mathrm{H}$ & 2.54928100 & -0.19224400 & 2.45661700 \\
\hline $\mathrm{C}$ & 2.55881200 & -3.53186000 & 1.87119900 \\
\hline $\mathrm{H}$ & 0.79979200 & -4.73787000 & 1.57651800 \\
\hline $\mathrm{H}$ & 4.10807900 & -2.07325600 & 2.20817000 \\
\hline $\mathrm{H}$ & 3.25071400 & -4.36209500 & 1.75702600 \\
\hline $\mathrm{H}$ & -6.42492400 & 2.30889900 & 0.48007600 \\
\hline $\mathrm{H}$ & -4.40165400 & -1.37825600 & 1.34723000 \\
\hline $\mathrm{H}$ & -3.01868600 & 1.12061300 & -1.85133200 \\
\hline $\mathrm{H}$ & 3.94289600 & 2.32724900 & -2.14568100 \\
\hline $\mathrm{H}$ & 1.67801100 & 1.86854400 & 1.47497600 \\
\hline $\mathrm{H}$ & 3.45728500 & 5.65336900 & 0.52359200 \\
\hline $\mathrm{C}$ & -2.05895400 & 3.91898100 & 0.29169000 \\
\hline $\mathrm{H}$ & -1.59706400 & 4.27373100 & 1.22026200 \\
\hline $\mathrm{H}$ & -2.18445300 & 4.77230300 & -0.38300200 \\
\hline $\mathrm{H}$ & -3.04718400 & 3.52267500 & 0.54921400 \\
\hline $\mathrm{H}$ & -0.16771500 & 2.99452000 & -2.29099100 \\
\hline $\mathrm{H}$ & 2.10137100 & 4.22596400 & 2.04167700 \\
\hline $\mathrm{H}$ & 4.38633300 & 4.69000000 & -1.56676400 \\
\hline
\end{tabular}


$\mathrm{H}$

$\mathrm{H}$

\section{Ts-1e'}

$\mathrm{Fe}$

$\mathrm{N}$

$\mathrm{N}$

C

C

C

C

C

C

C

C

$\mathrm{H}$

C

$\mathrm{H}$

C

C

$\mathrm{H}$

C

$\mathrm{H}$

C

C

$\mathrm{H}$

C

$\mathrm{H}$

C

C

C

C

C

C

C

C

C

C

C

$\mathrm{H}$

$\mathrm{Si}$

$\mathrm{H}$

$\mathrm{H}$

C

,

.

C

(

C

C

C

C

.

C

(1)

(1)

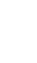

.

C

C

C

C

C

.

H

H
$-6.20412400$

0.24597000

1.84302200

$-4.83196900$

2.73849400

$-1.37624500$

0.13888300

1.47939000

$-1.21871500$

0.09600700

$-1.19982300$

$0.40260300 \quad-0.70504100$

3.62872800

$0.11274100 \quad-0.62137700$

$-0.60057200 \quad-0.20489300$

$\begin{array}{lll}4.57646700 & -0.45743300 & 0.81200900\end{array}$

$\begin{array}{lll}5.36358800 & -1.53846000 & 1.19420100\end{array}$

$\begin{array}{lll}5.21896300 & -2.76689100 & 0.55395100\end{array}$

$4.28219400 \quad-2.91127100 \quad-0.46647700$

$3.48922900 \quad-1.83343600 \quad-0.84399800$

$\begin{array}{lll}2.80254500 & 0.55775100 & -0.62233700\end{array}$

$\begin{array}{lll}3.42779700 & 1.77795800 & -0.94132800\end{array}$

$\begin{array}{lll}4.50674300 & 1.85365100 & -0.86148700\end{array}$

$\begin{array}{lll}2.67236000 & 2.83725100 & -1.38912900\end{array}$

$3.14053800 \quad 3.77859000 \quad-1.66346600$

$\begin{array}{lll}1.27978600 & 2.69464700 & -1.48872600\end{array}$

$\begin{array}{lll}0.41765300 & 3.75253800 & -1.92868200\end{array}$

$\begin{array}{lll}0.86601200 & 4.69437100 & -2.23194000\end{array}$

$\begin{array}{lll}-0.92515700 & 3.58365500 & -1.95260200\end{array}$

$\begin{array}{lll}-1.58117200 & 4.38590300 & -2.27770500\end{array}$

$\begin{array}{lll}-1.52183500 & 2.35171900 & -1.52294000\end{array}$

$\begin{array}{lll}-2.91146700 & 2.15781100 & -1.49147900\end{array}$

$\begin{array}{lll}-3.57818100 & 2.95640300 & -1.80322900\end{array}$

$\begin{array}{lll}-3.40667100 & 0.95636400 & -1.04589600\end{array}$

$\begin{array}{lll}-4.47507500 & 0.78118100 & -0.98631500\end{array}$

$\begin{array}{lll}-2.53046100 & -0.05454200 & -0.61268200\end{array}$

$\begin{array}{lll}-0.70298100 & 1.29191200 & -1.08814500\end{array}$

$\begin{array}{lll}0.72695700 & 1.45503600 & -1.10390500\end{array}$

$\begin{array}{lll}-3.08927800 & -1.33983800 & -0.12776200\end{array}$

$\begin{array}{lll}-4.09935900 & -1.97911500 & -0.85567900\end{array}$

$\begin{array}{lll}-4.62443100 & -3.19091100 & -0.41921700\end{array}$

$\begin{array}{lll}-4.15308500 & -3.77405500 & 0.75504100\end{array}$

$\begin{array}{lll}-3.15860500 & -3.13731800 & 1.49123700\end{array}$

$\begin{array}{lll}-2.63209400 & -1.92759900 & 1.05338200\end{array}$

$\begin{array}{lll}-0.09337500 & -2.67949500 & -1.15754900\end{array}$

$\begin{array}{lll}0.64014400 & -3.08499800 & -0.18153000\end{array}$

$\begin{array}{lll}1.03975700 & -2.02934400 & 1.03450700\end{array}$

$\begin{array}{lll}0.50882200 & -0.12804400 & 2.18946000\end{array}$

$\begin{array}{lll}-0.08504500 & -0.78872700 & 3.40592000\end{array}$

$\begin{array}{lll}1.93360700 & 0.16218000 & 2.56381100\end{array}$

$\begin{array}{lll}-0.31151800 & 1.58796100 & 2.15100900\end{array}$ 


$\begin{array}{lrrr}\mathrm{C} & 0.41418300 & 2.73723800 & 1.80681800 \\ \mathrm{C} & -1.70367700 & 1.72738300 & 2.27581700 \\ \mathrm{C} & -0.21239700 & 3.96298500 & 1.59049100 \\ \mathrm{H} & 1.49526500 & 2.66977600 & 1.69349300 \\ \mathrm{C} & -2.33968300 & 2.94639200 & 2.06278400 \\ \mathrm{H} & -2.30799700 & 0.86087400 & 2.54175500 \\ \mathrm{C} & -1.59445200 & 4.07150500 & 1.71373500 \\ \mathrm{H} & 0.37956500 & 4.83348400 & 1.31852200 \\ \mathrm{H} & -3.41919800 & 3.02077400 & 2.16755500 \\ \mathrm{H} & -2.08718700 & 5.02502600 & 1.54367300 \\ \mathrm{C} & -0.78898000 & -3.09346300 & -2.40092400 \\ \mathrm{H} & -1.85830300 & -3.21431900 & -2.18919900 \\ \mathrm{H} & -0.70452600 & -2.32404600 & -3.17599600 \\ \mathrm{H} & -0.41276800 & -4.04150500 & -2.80521800 \\ \mathrm{H} & 1.16935100 & -3.92962300 & 0.23304700 \\ \mathrm{H} & 5.83447400 & -3.61069200 & 0.85194000 \\ \mathrm{H} & 4.67308600 & 0.49524600 & 1.32544100 \\ \mathrm{H} & 2.74952900 & -1.94625200 & -1.62852500 \\ \mathrm{H} & -4.45399000 & -1.53871600 & -1.78389700 \\ \mathrm{H} & -1.86685100 & -1.43232300 & 1.64512100 \\ \mathrm{H} & -4.56213700 & -4.72083900 & 1.09470000 \\ \mathrm{H} & -5.39731700 & -3.68400400 & -1.00124100 \\ \mathrm{H} & -2.78672000 & -3.57967500 & 2.40998800 \\ \mathrm{H} & 4.16495300 & -3.86659200 & -0.96941300 \\ \mathrm{H} & 6.08562300 & -1.42327300 & 1.99691600\end{array}$




\section{NMR Spectra of New Compounds}

2,9-bis-3,5-bis(2,4,6-trimethylphenyl)phenyl-1,10-phenanthroline (L1i)

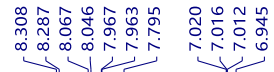<smiles>[Li]c1ccc2ccc3ccc(Br)nc3c2n1</smiles><smiles>Cc1cc(C)c(-c2cc(C)cc(C)c2-c2cc(C)cc(C)c2C)c(C)c1</smiles>

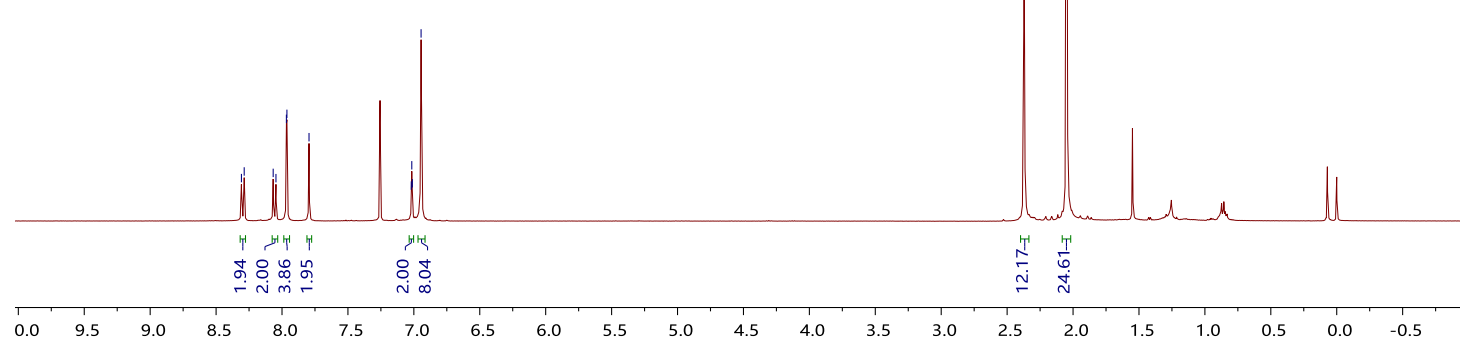
I<smiles>Brc1ccc2ccc3ccc(Br)nc3c2n1</smiles><smiles>Cc1cc(C)c(-c2cc(C)cc(C)c2-c2cc(C)cc(C)c2C)c(C)c1</smiles>

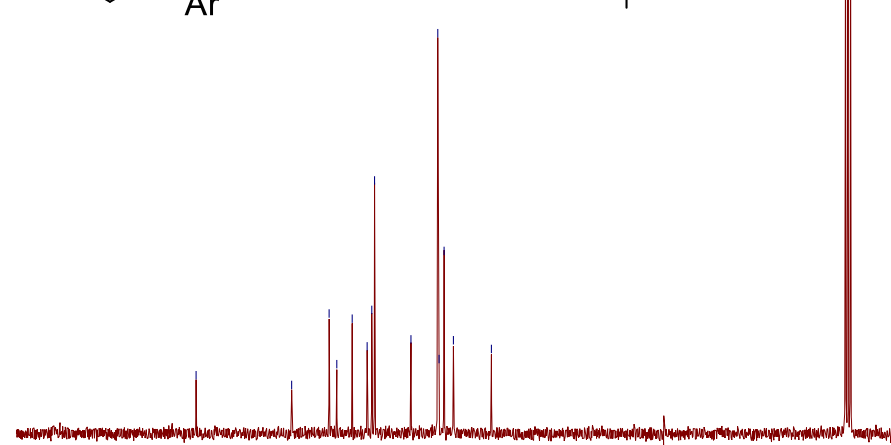

$80 \quad 170 \quad 160$

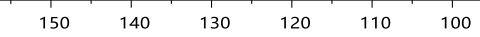

$90 \quad 80$ 
2,9-bis-3,5-bis(2,4,6-triisopropylphenyl)phenyl-1,10-phenanthroline (L1j)

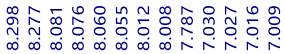

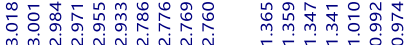

(N)<smiles>CCCc1cc(C(C)C)c(-c2cc(-c3c(CCC)cc(C(C)C)cc3C(C)C)cc(C(C)C)c2)c(C(C)C)c1</smiles>

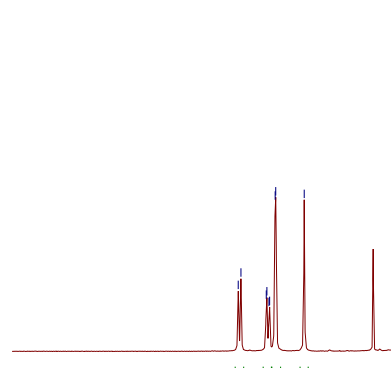

T北
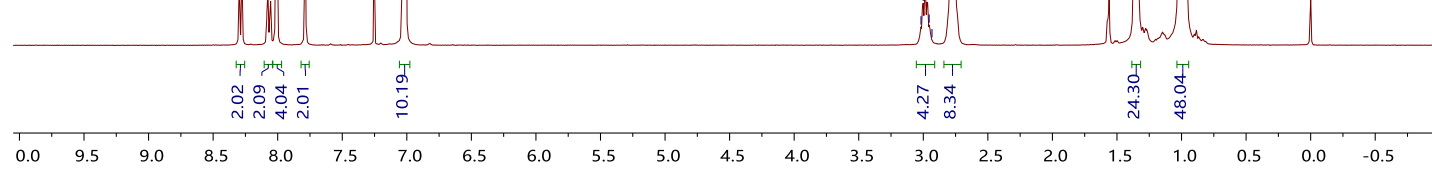<smiles>Brc1ccc2ccc3ccc([Te][Te])nc3c2n1</smiles>
$\underbrace{3}$

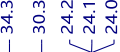<smiles>CCCc1cc(C(C)C)c(-c2cc(-c3c(CC)cc(C(C)C)cc3C(C)C)cc(C(C)C)c2)c(C(C)C)c1</smiles>

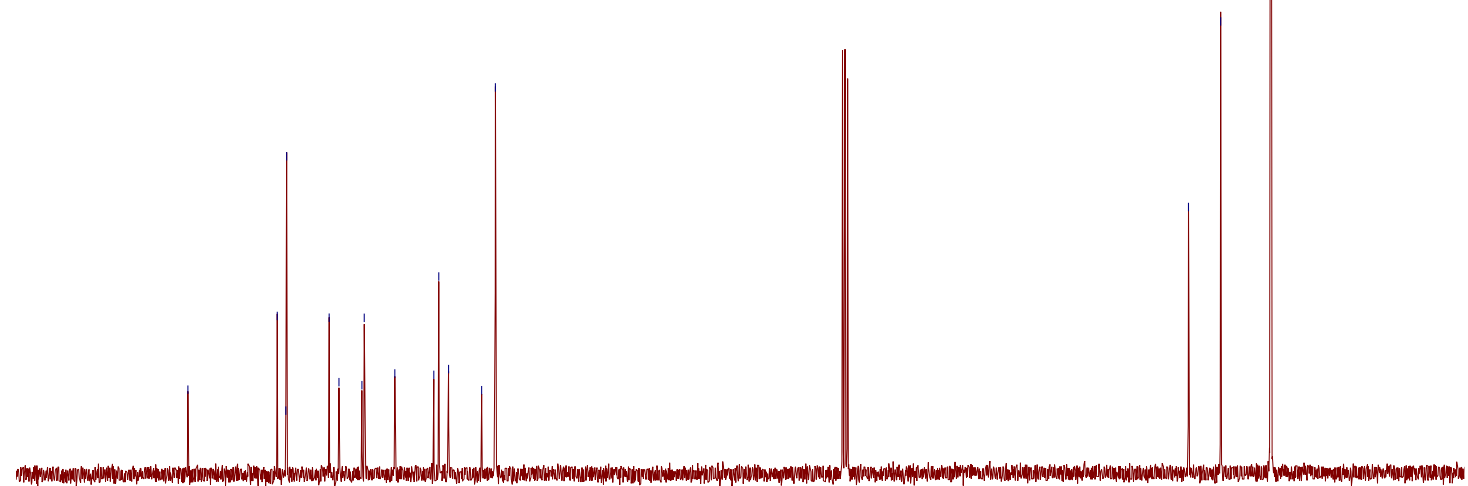

80

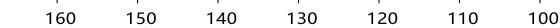

$90 \quad 80$ 
2,9-bis((2,4,6-triisopropylphenyl)ethynyl)-1,10-phenanthroline (L1k)
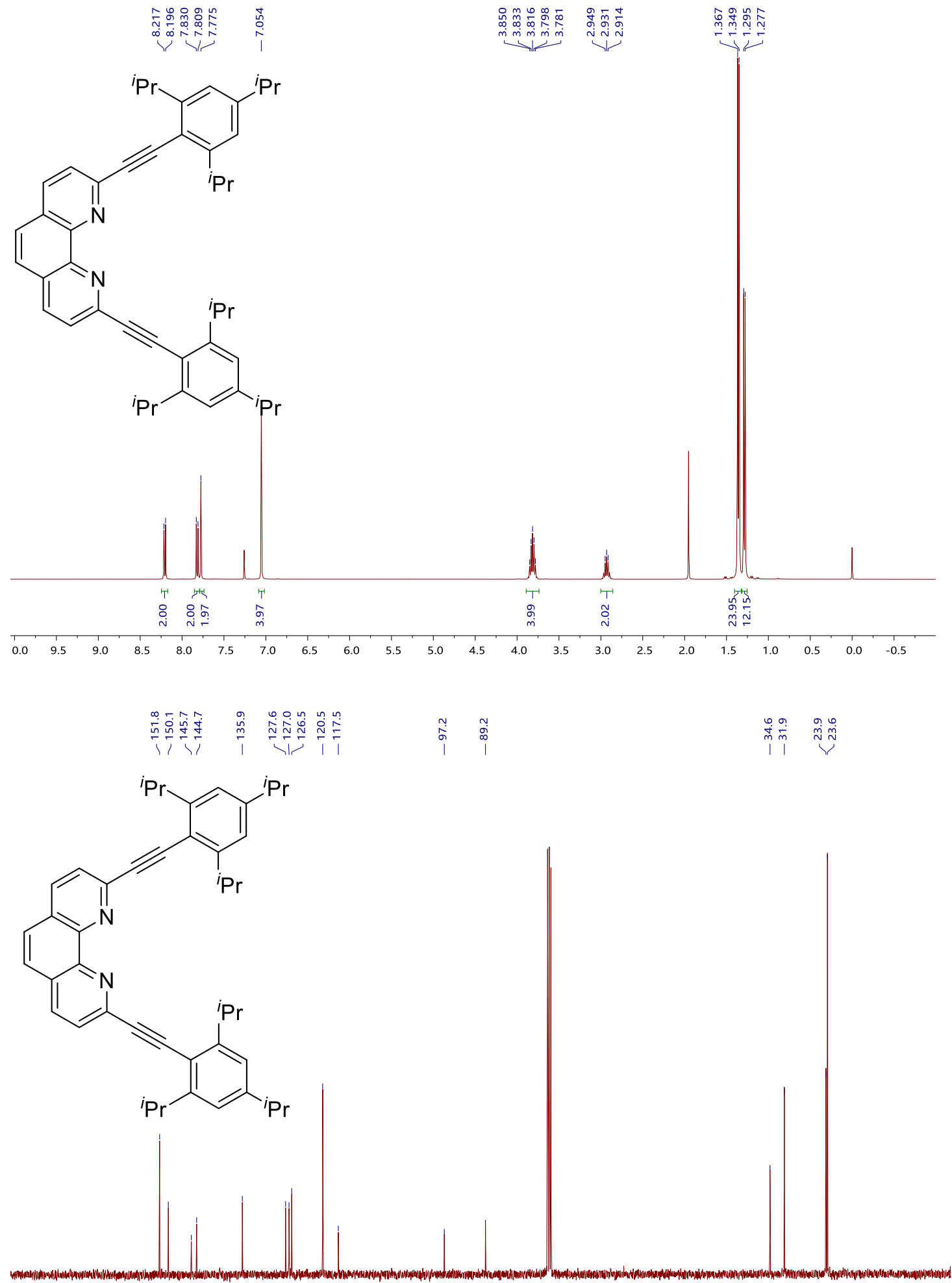

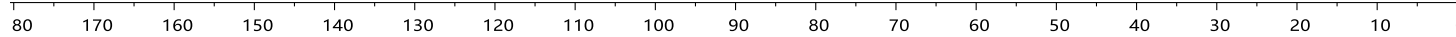




\section{(E)-phenyl(5-phenylpent-1-en-1-yl)silane (3aa)}
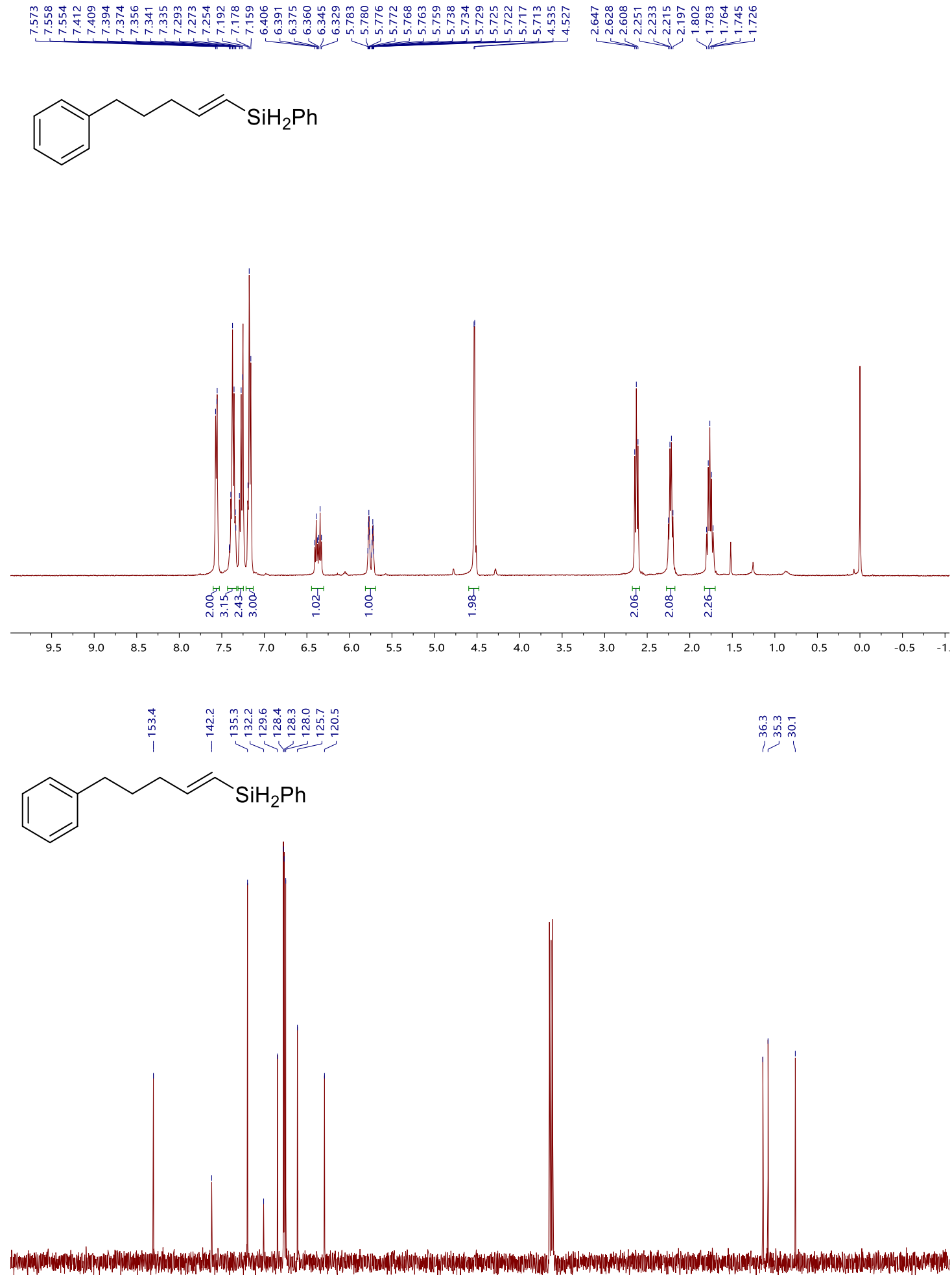

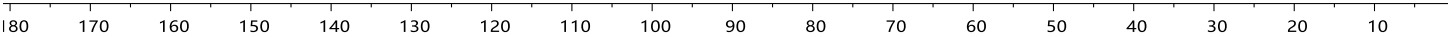


phenyl(5-phenylpent-1-en-2-yl)silane (4aa)

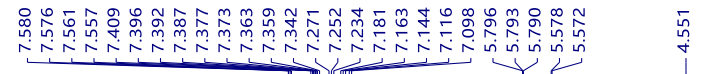

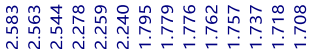

PiH

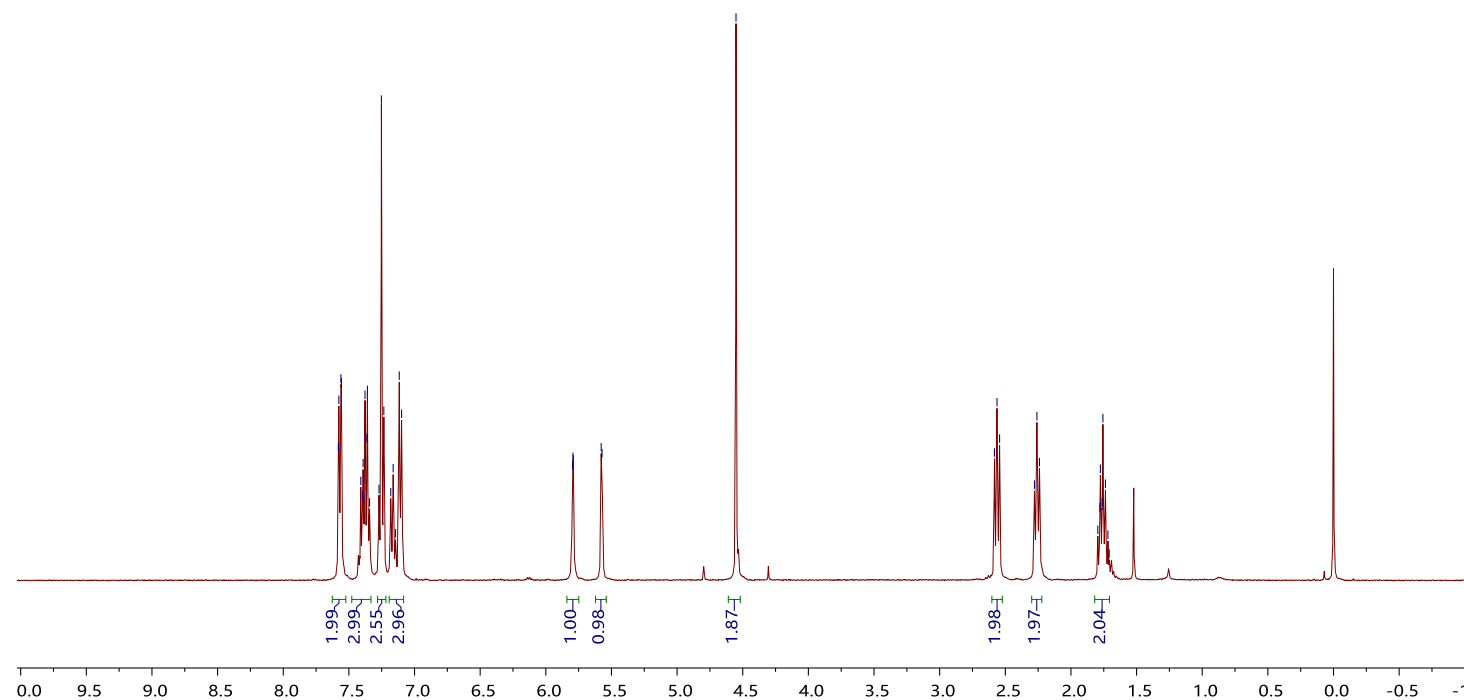

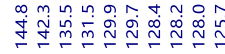

11 工

品势路

PiH

$\mathrm{SiH}_{2} \mathrm{Ph}$

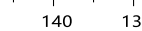

$120 \quad 110 \quad 100$

$90 \quad 80$

$70 \quad 60$

$50 \quad 40$

$\begin{array}{lll}30 & 20 & 10\end{array}$ 
(E)-oct-1-en-1-yl(phenyl)silane (3ab)

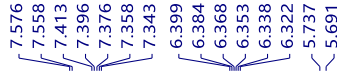

隻

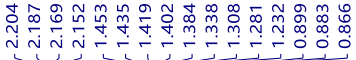

$\mathrm{Me}_{\mathrm{SiH}_{2} \mathrm{Ph}}$

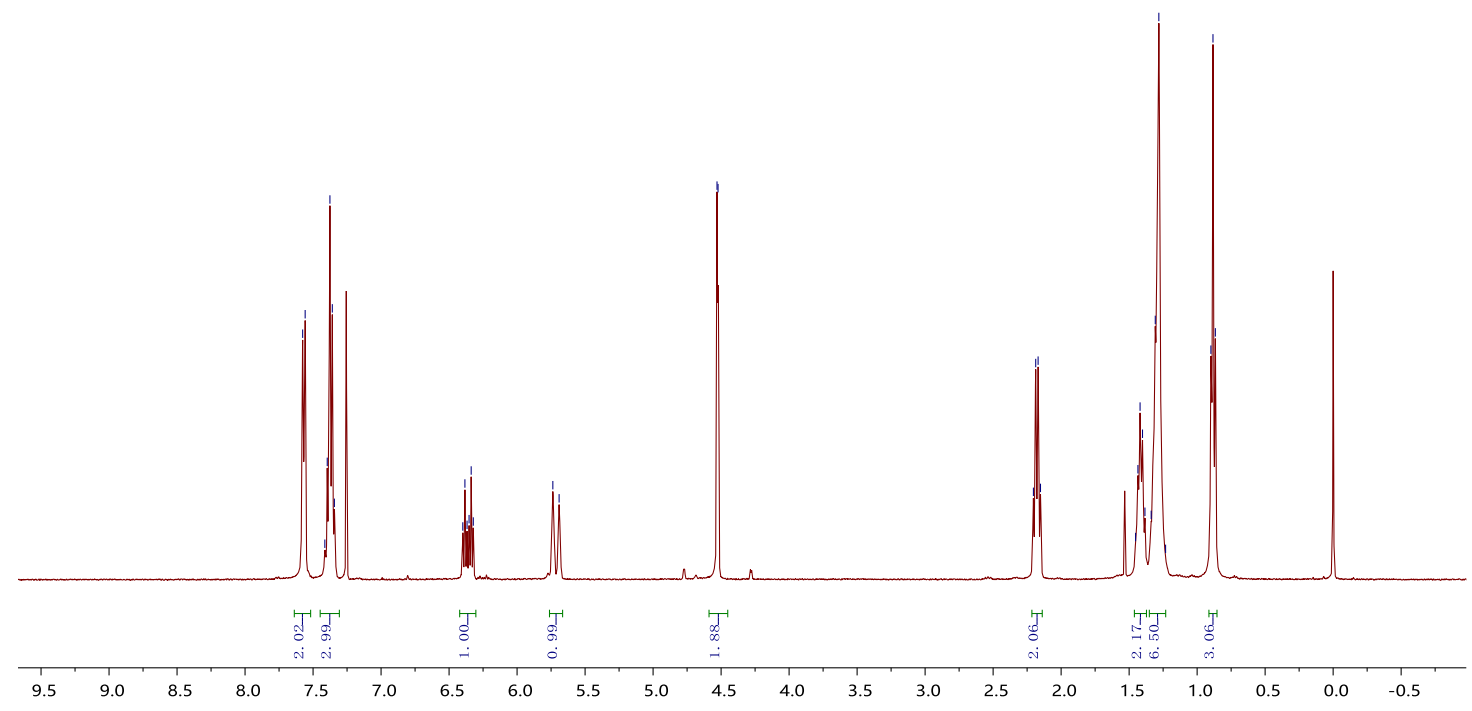

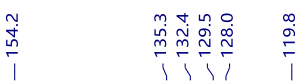

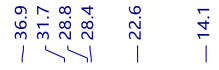

$\overbrace{\mathrm{SiH}_{2} \mathrm{Ph}}$

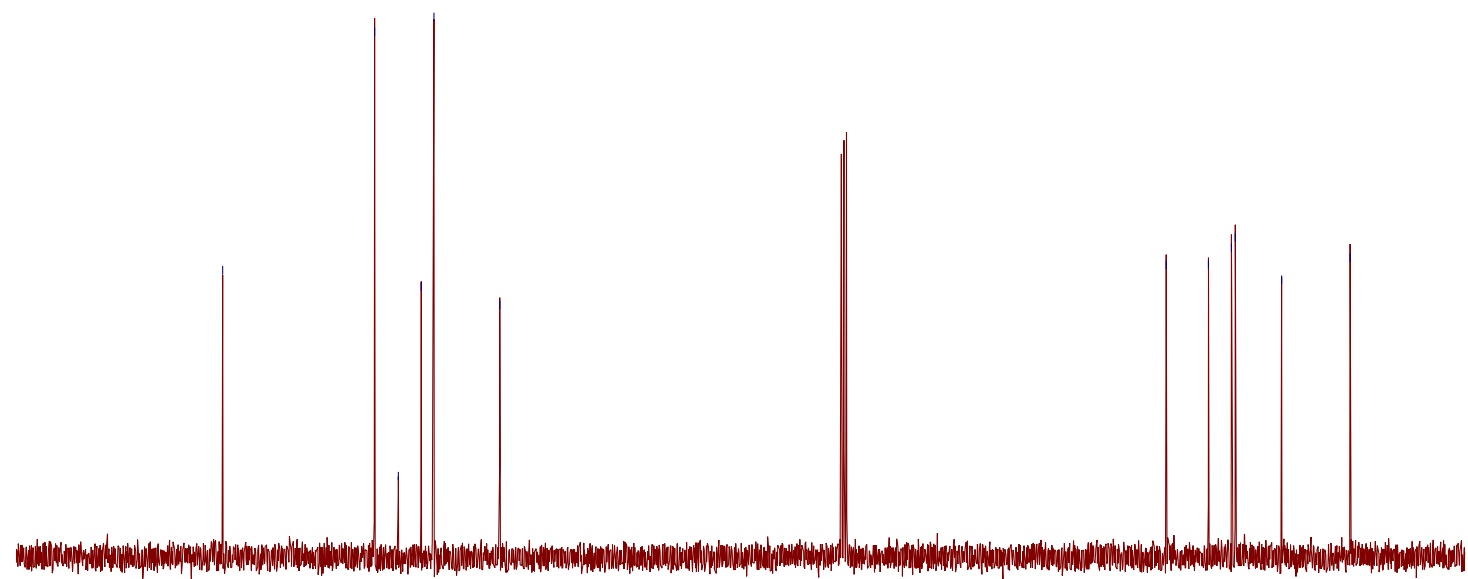

$170 \quad 160$

140

120

100

$90 \quad 80$

$70 \quad 60$

$50 \quad 40$

$30 \quad 20 \quad 10$ 
oct-1-en-2-yl(phenyl)silane (4ab)

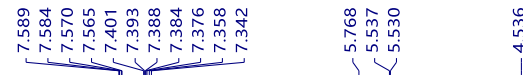

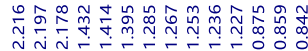

$\mathrm{NiH}_{\mathrm{SiHh}}$
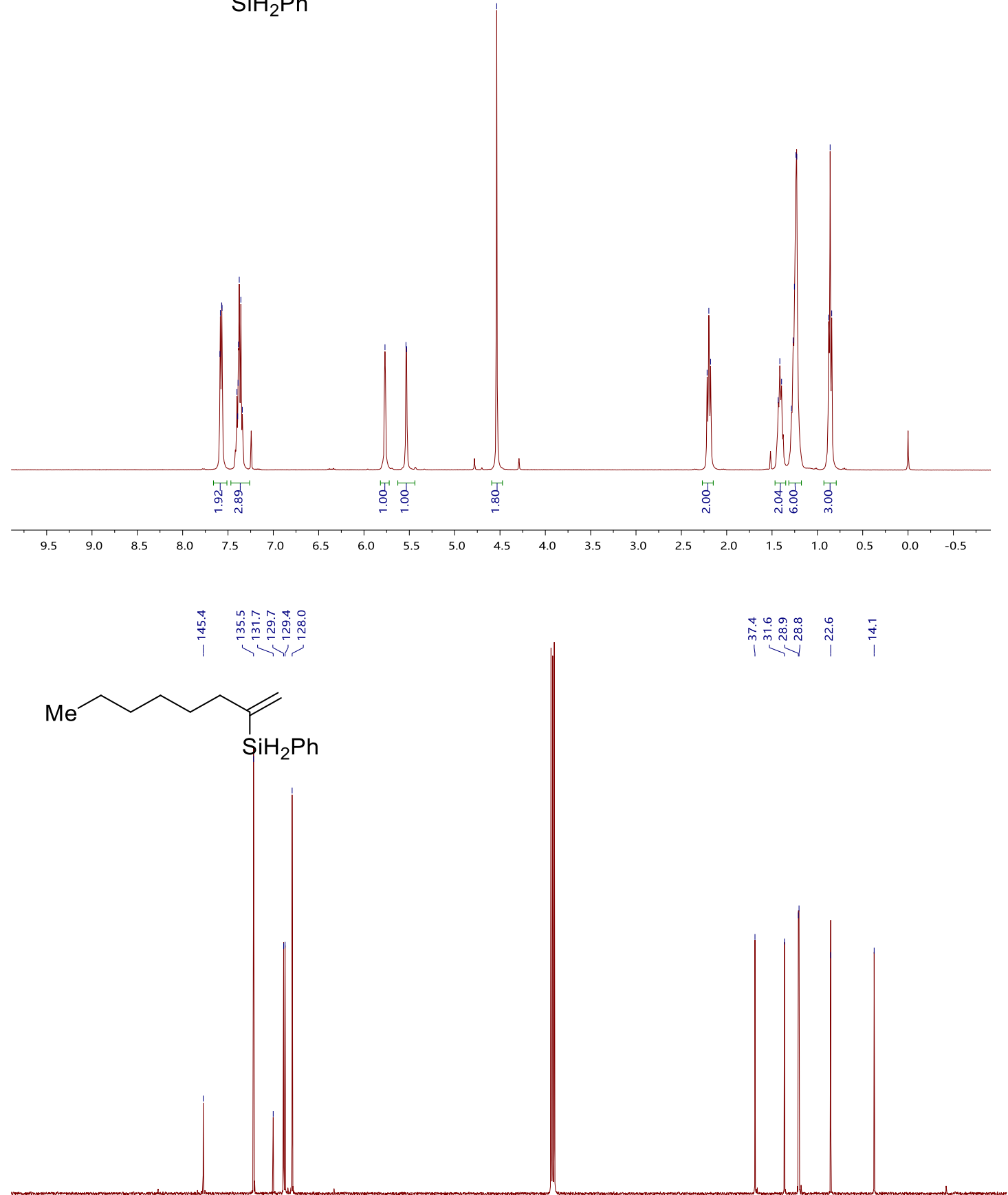

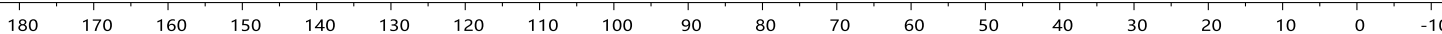




\section{(E)-phenyl(prop-1-en-1-yl)silane (3ac)}

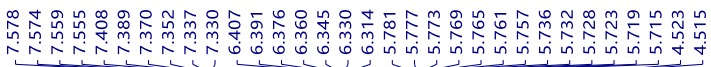

$\mathrm{Me} / \mathrm{SiH}_{2} \mathrm{Ph}$

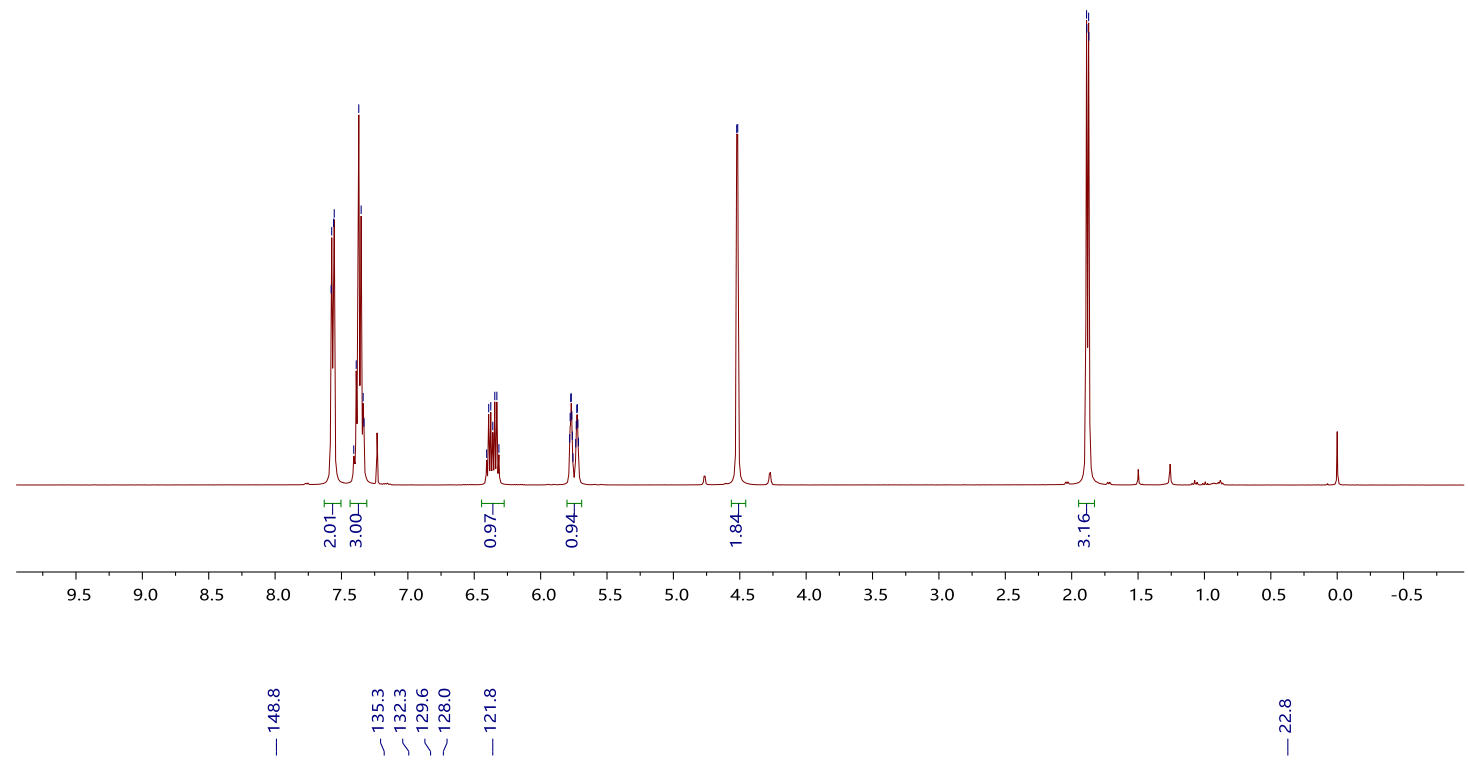

$\mathrm{Me} / \mathrm{SiH}_{2} \mathrm{Ph}$
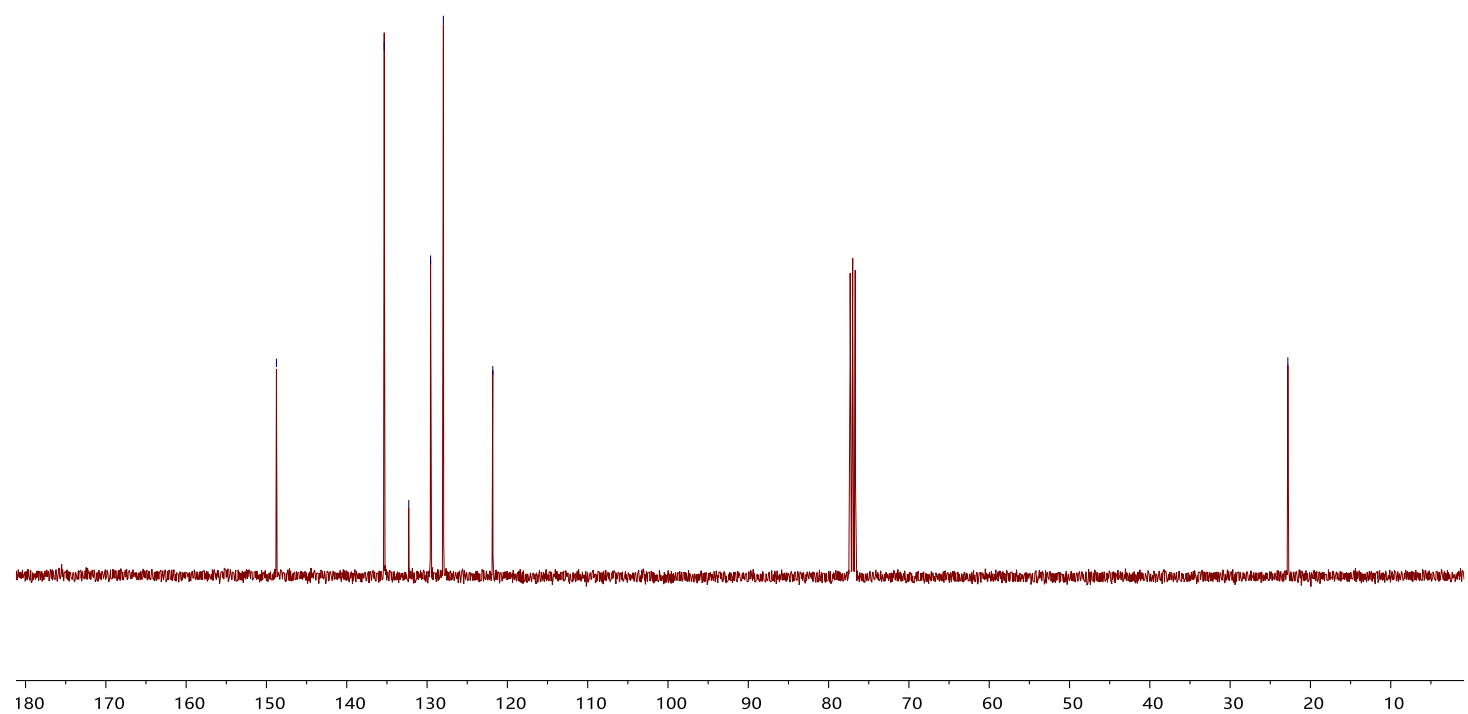

90

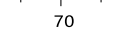

50

$\begin{array}{llll}40 & 30 \quad 20 \quad 10\end{array}$ 
phenyl(prop-1-en-2-yl)silane (4ac)

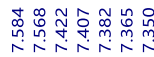

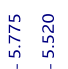

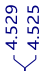

$\mathrm{SiH}_{2} \mathrm{Ph}$
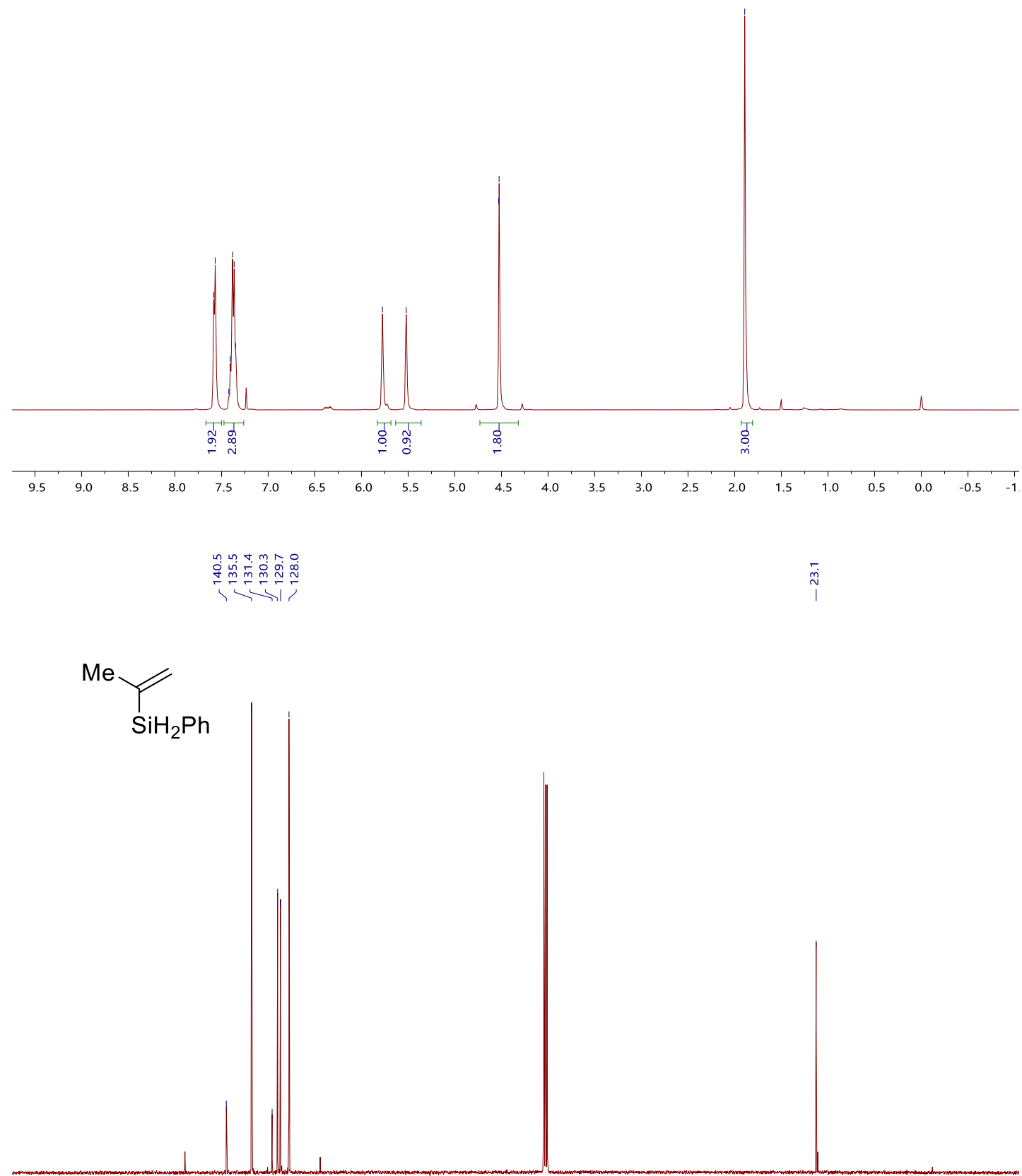

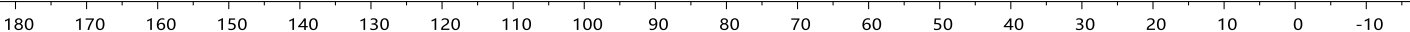


(E)-(2-cyclopropylvinyl)(phenyl)silane (3ad)

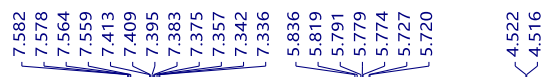

$\triangle \mathrm{SiH}_{2} \mathrm{Ph}$

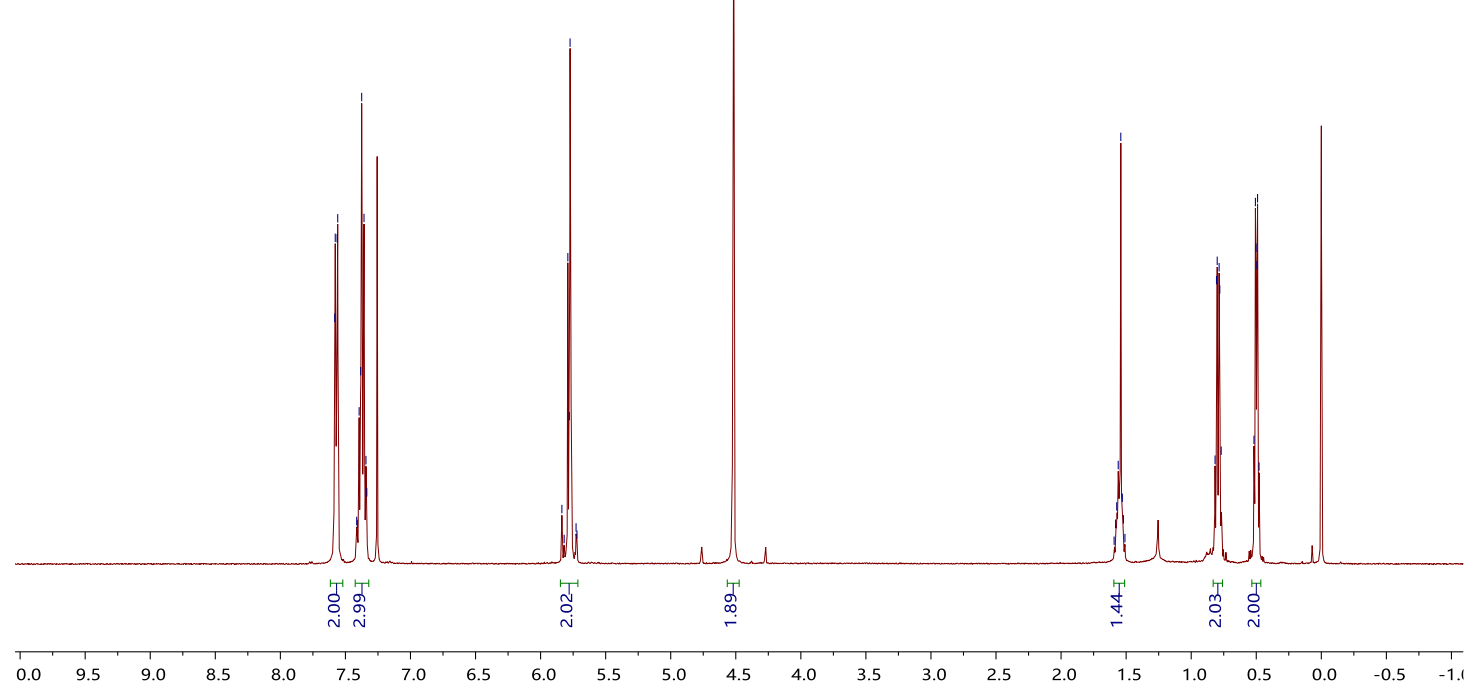

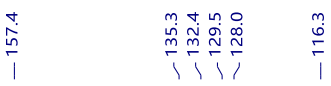

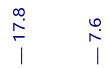

$\triangle \triangle_{\mathrm{SiH}_{2} \mathrm{Ph}}$

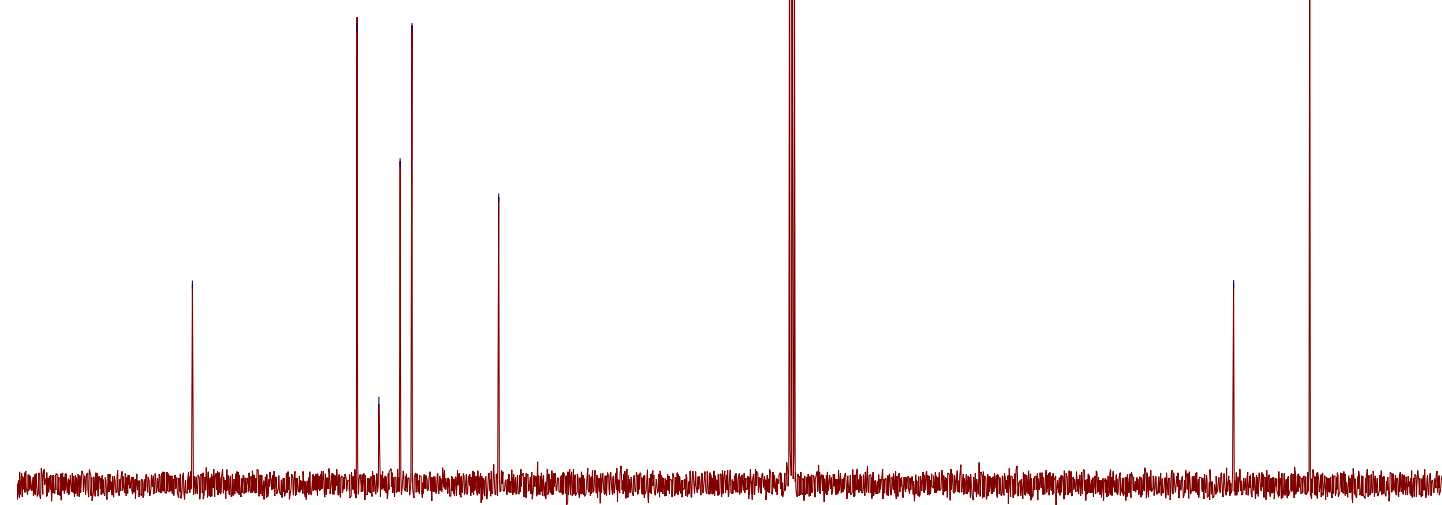

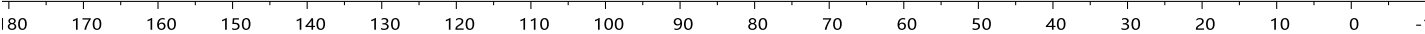




\section{(1-cyclopropylvinyl)(phenyl)silane (4ad)}

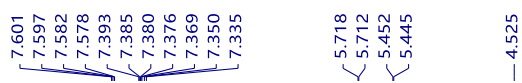
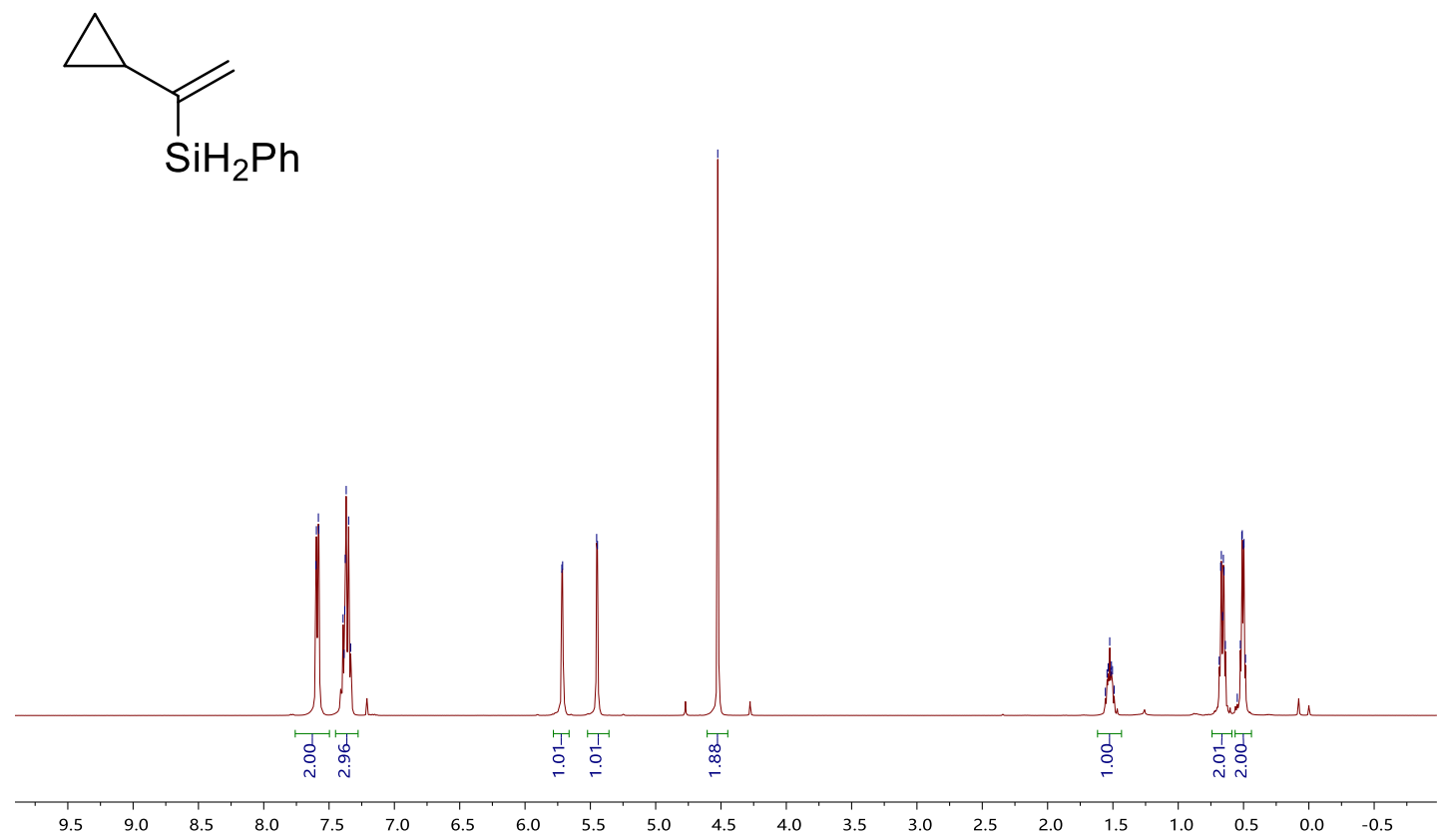

إن
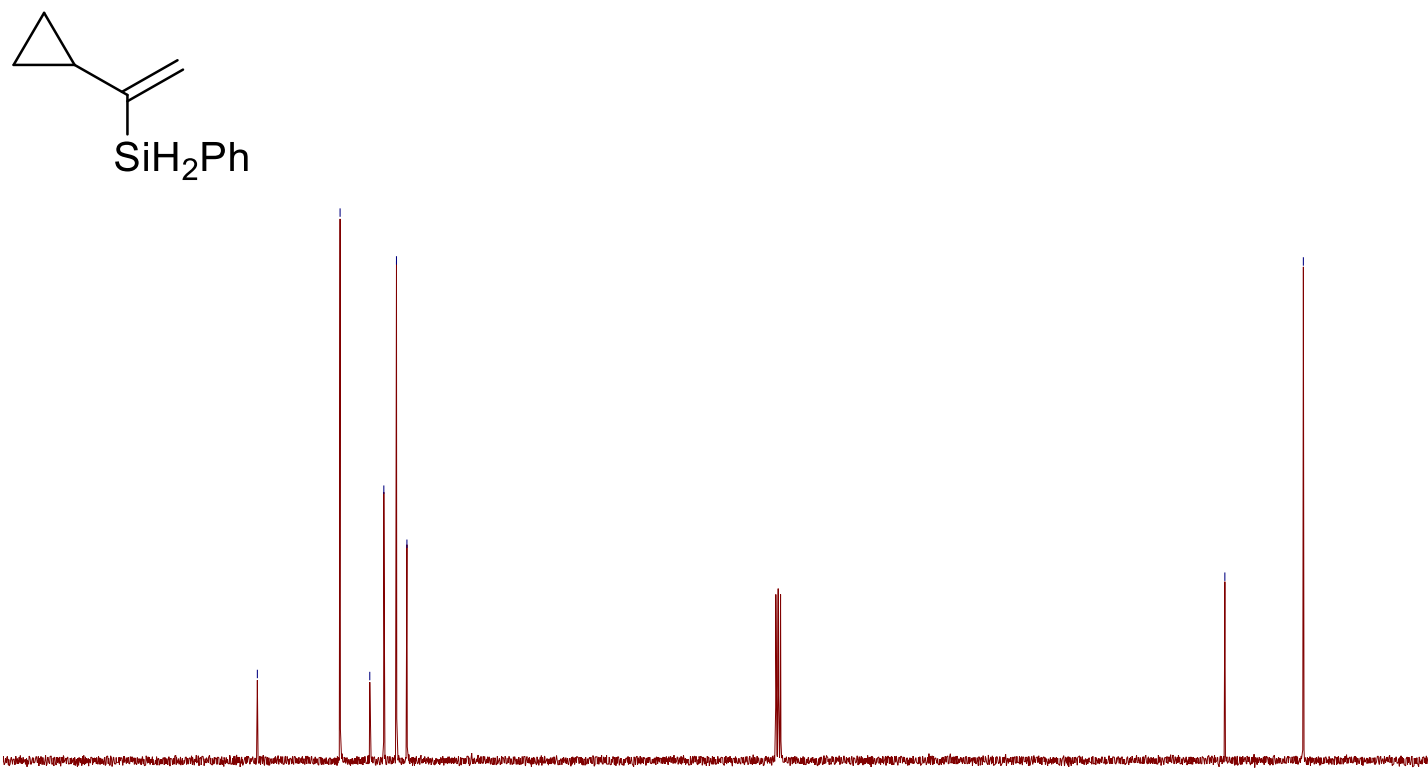

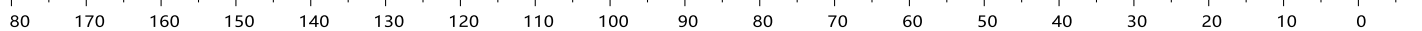


(E)-(2-cyclohexylvinyl)(phenyl)silane (3ae)

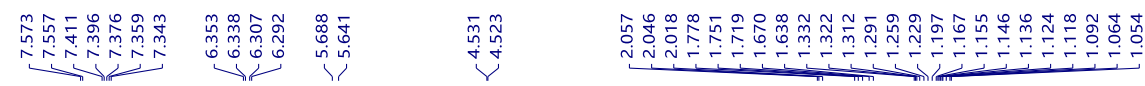<smiles>C(=C/C1CCCCC1)\[SH-]c1ccccc1</smiles>
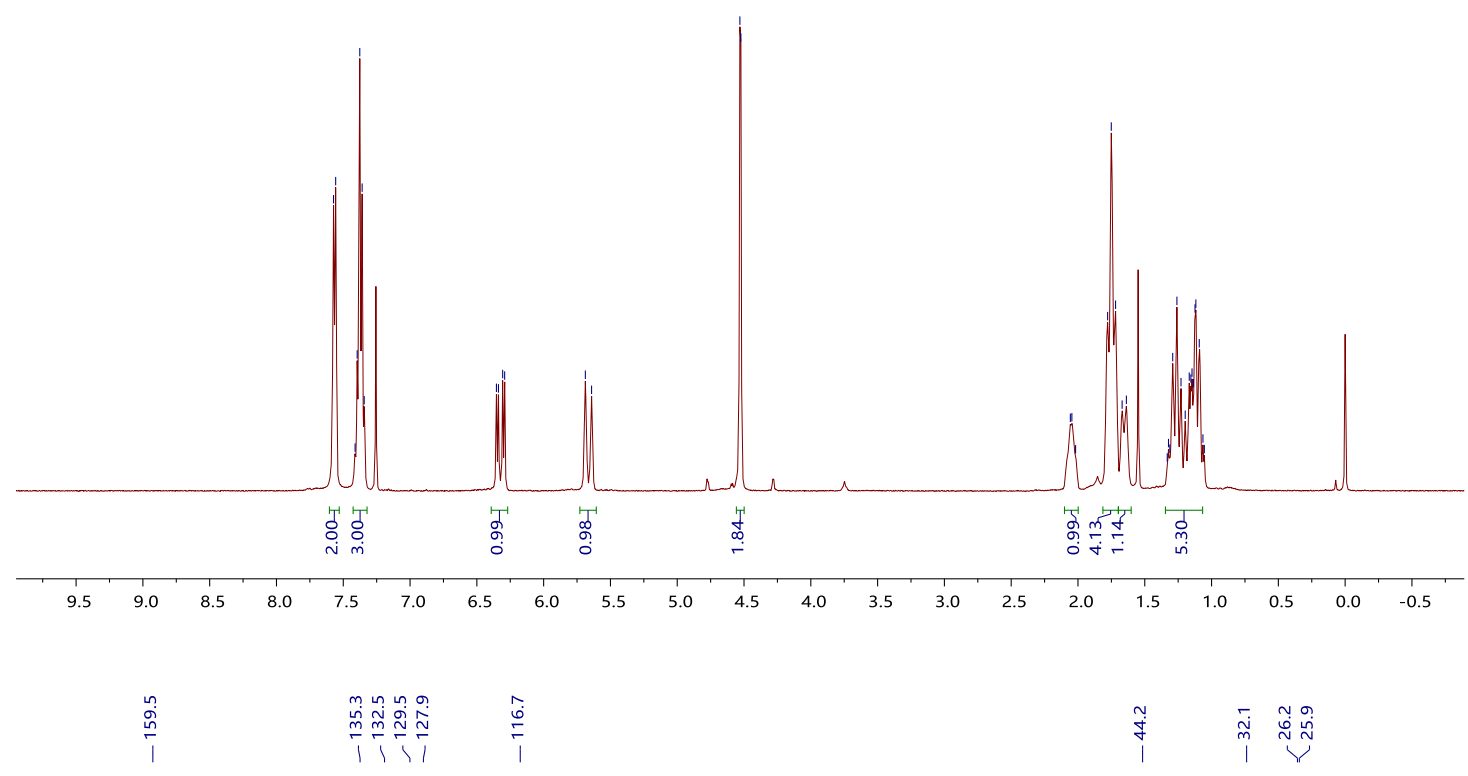

$\sim \mathrm{SiH}_{2} \mathrm{Ph}$

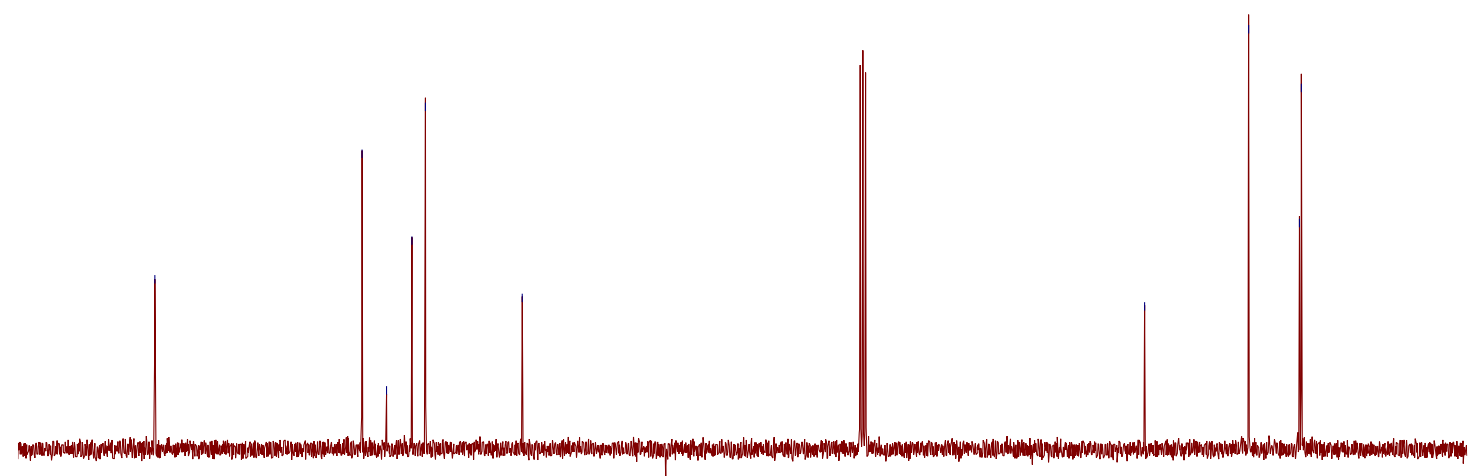

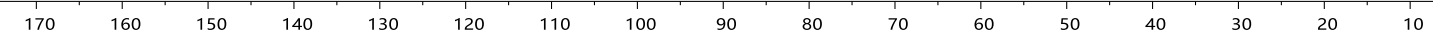


(1-cyclohexylvinyl)(phenyl)silane (4ae)

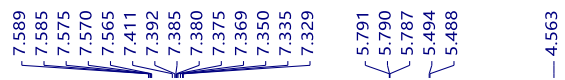

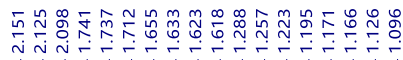<smiles>C=C([SH2+]c1ccccc1)C1CCCCC1</smiles>

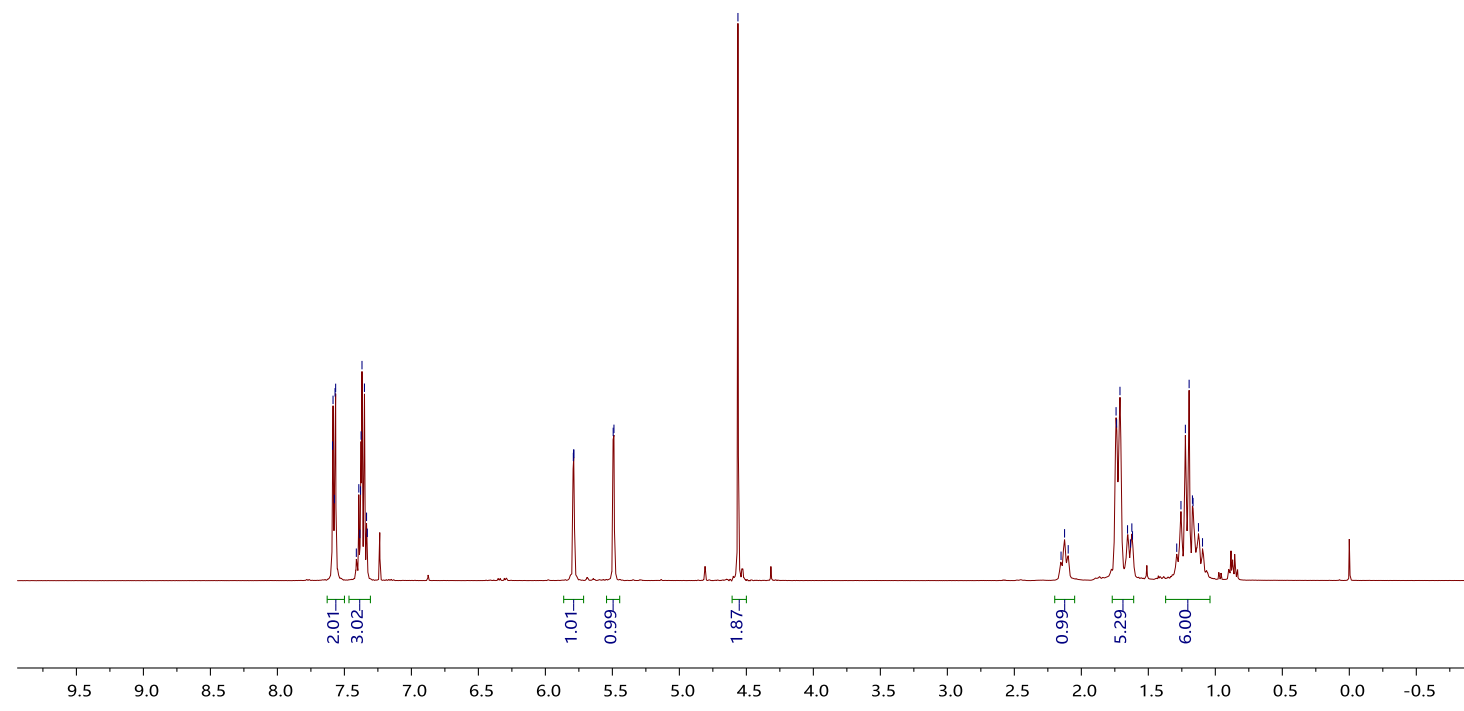

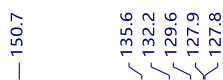

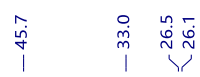<smiles>C=C([SH2+]c1ccccc1)C1CCCCC1</smiles>

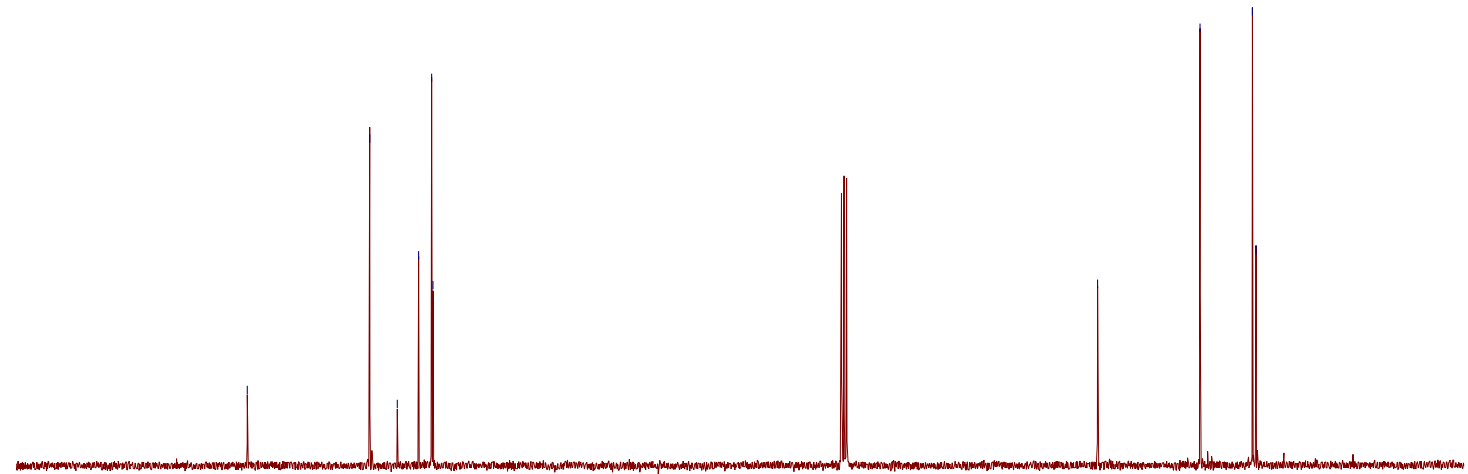

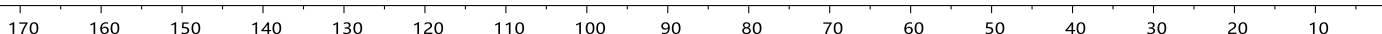


(E)-phenyl(3-phenylprop-1-en-1-yl)silane (3af)

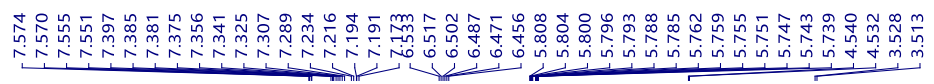
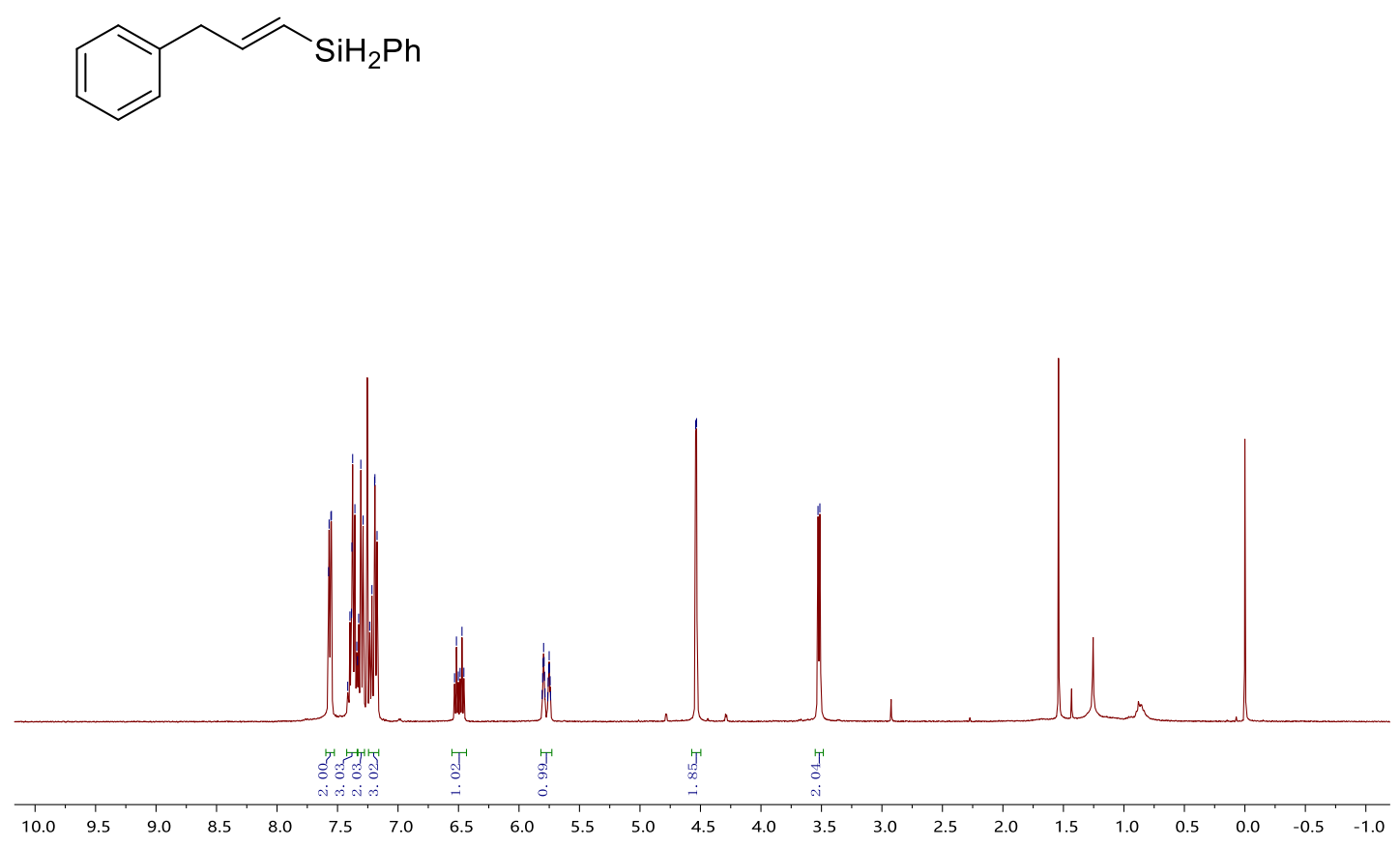

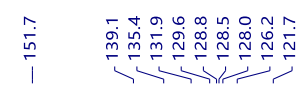

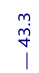
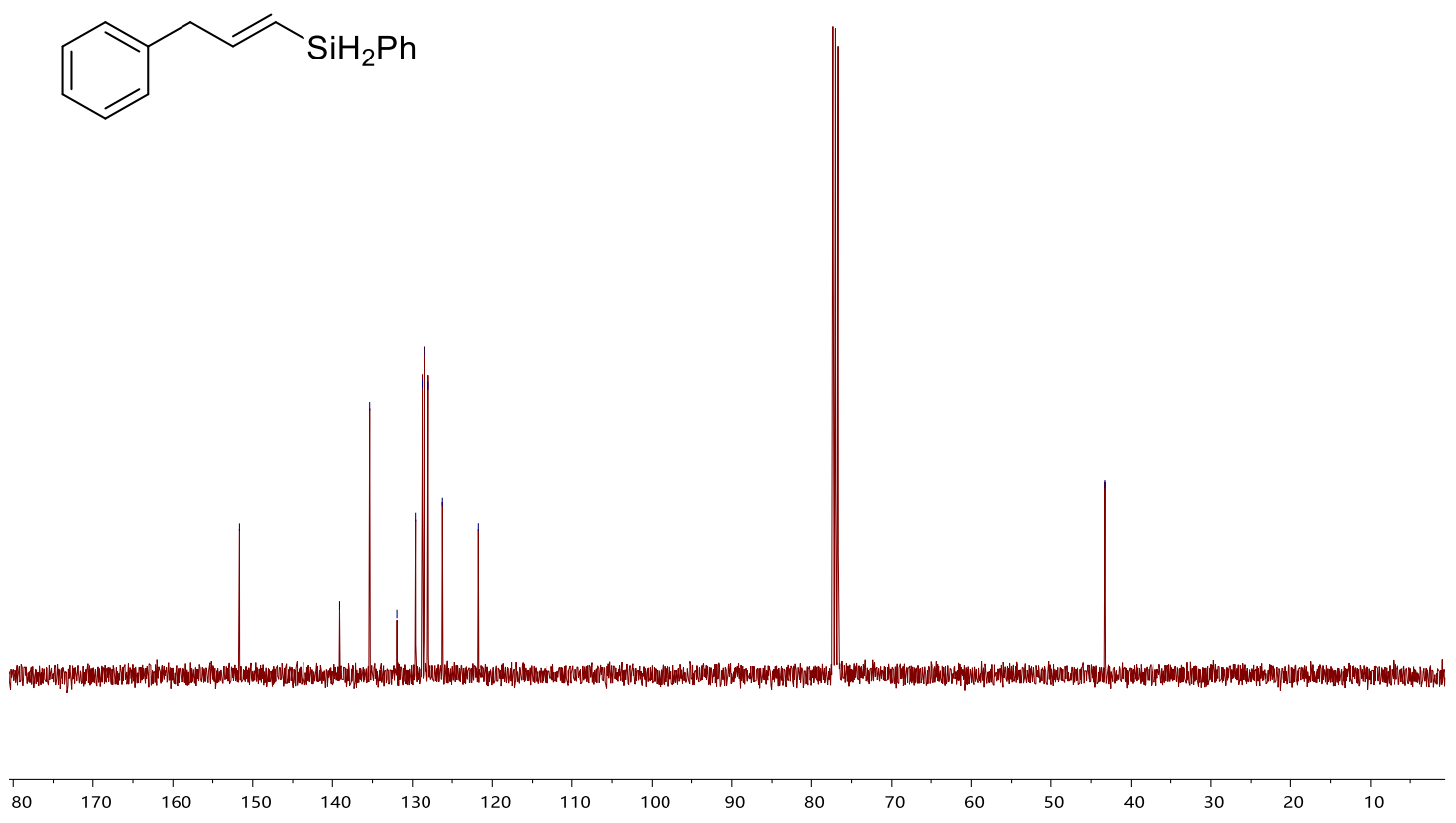

S116 
phenyl(3-phenylprop-1-en-2-yl)silane (4af)

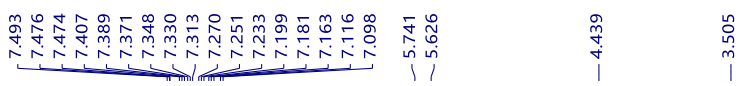

P
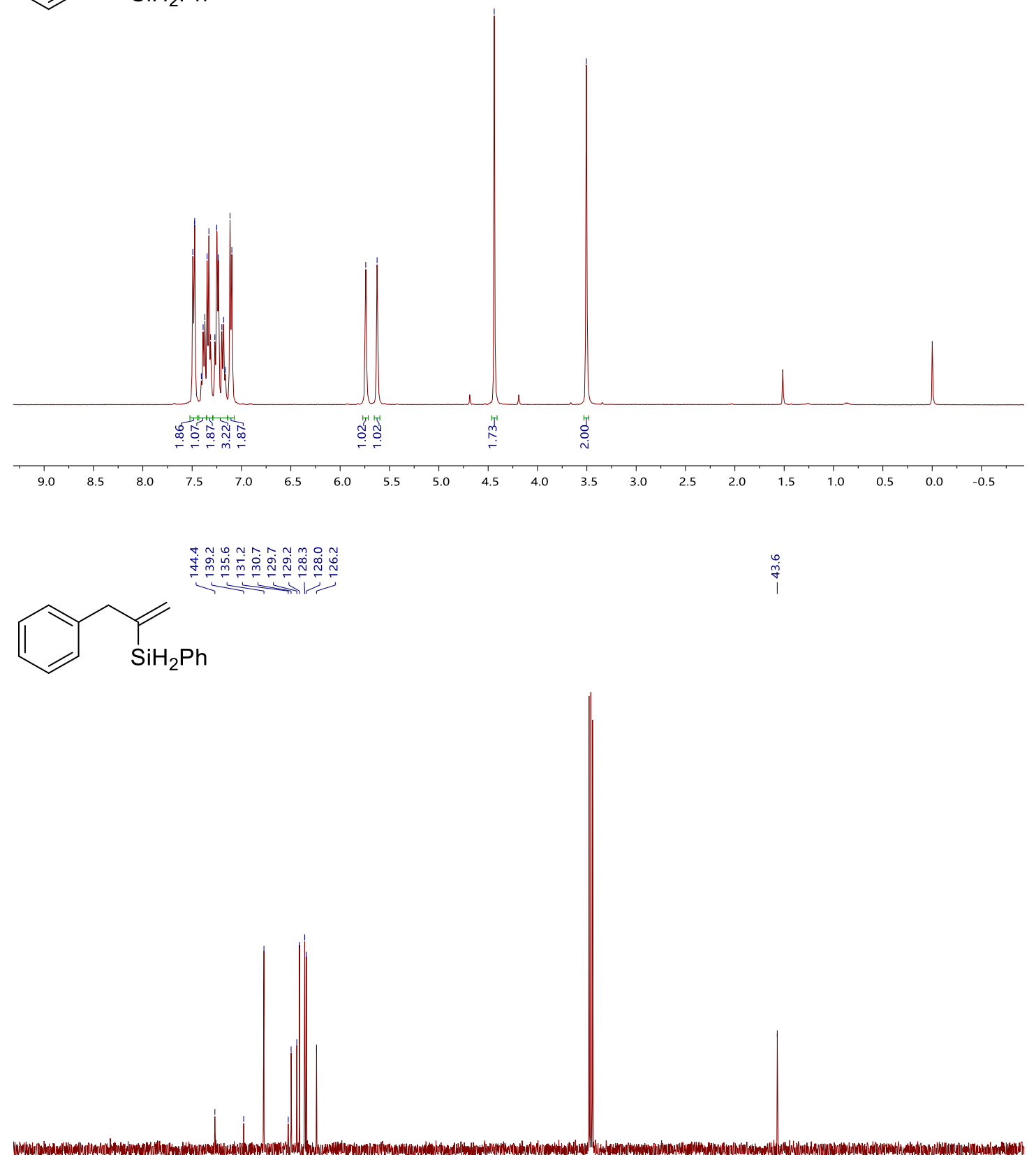

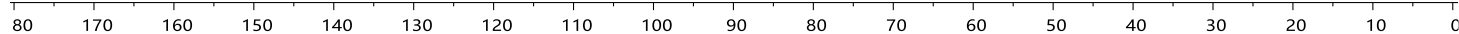


(E)-(3-(4-fluorophenyl)prop-1-en-1-yl)(phenyl)silane (3ag)

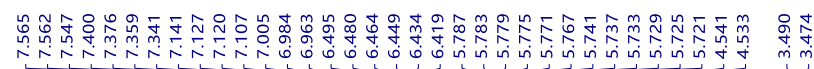<smiles>Fc1ccc(C/C=C/[SH2+]c2ccccc2)cc1</smiles>

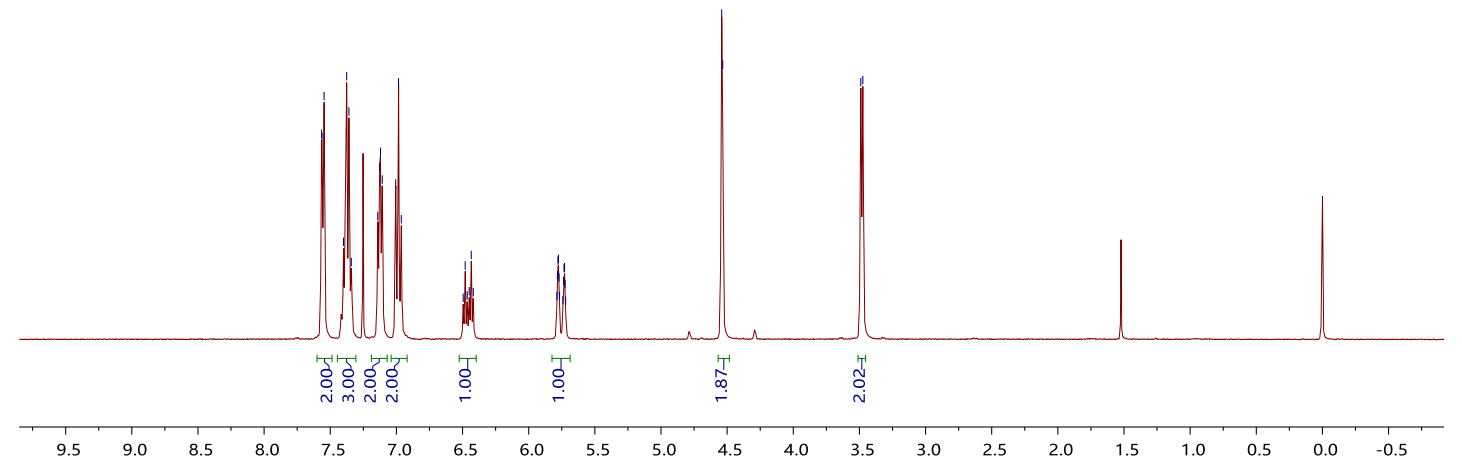

1T<smiles>Fc1ccc(C/C=C/[SH2+]c2ccccc2)cc1</smiles>

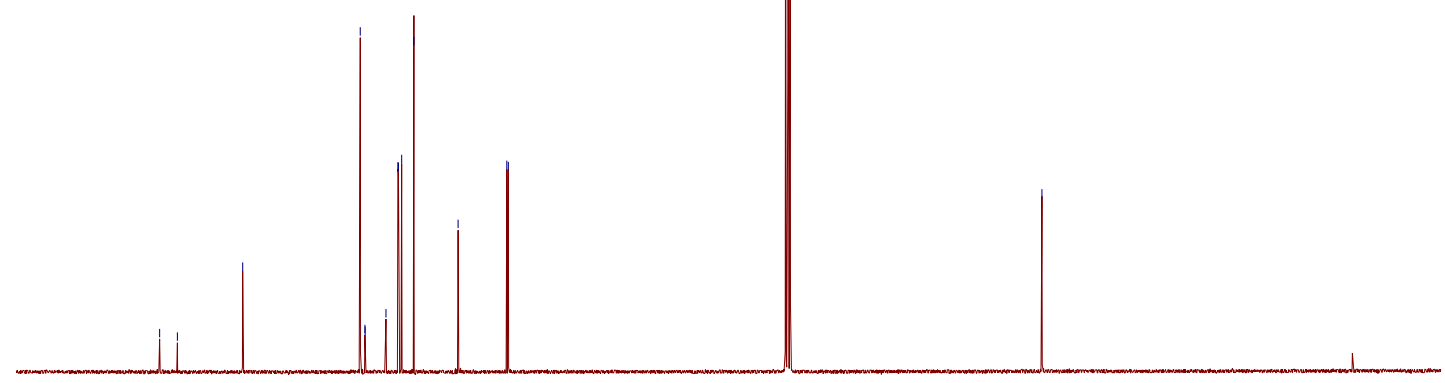

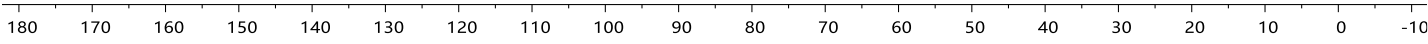


(3-(4-fluorophenyl)prop-1-en-2-yl)(phenyl)silane (4ag)

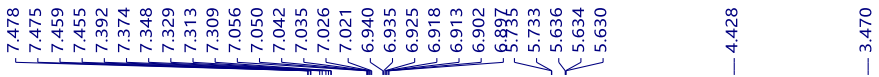

$\overbrace{\mathrm{SiH}_{2} \mathrm{Ph}}^{\mathrm{C}}$

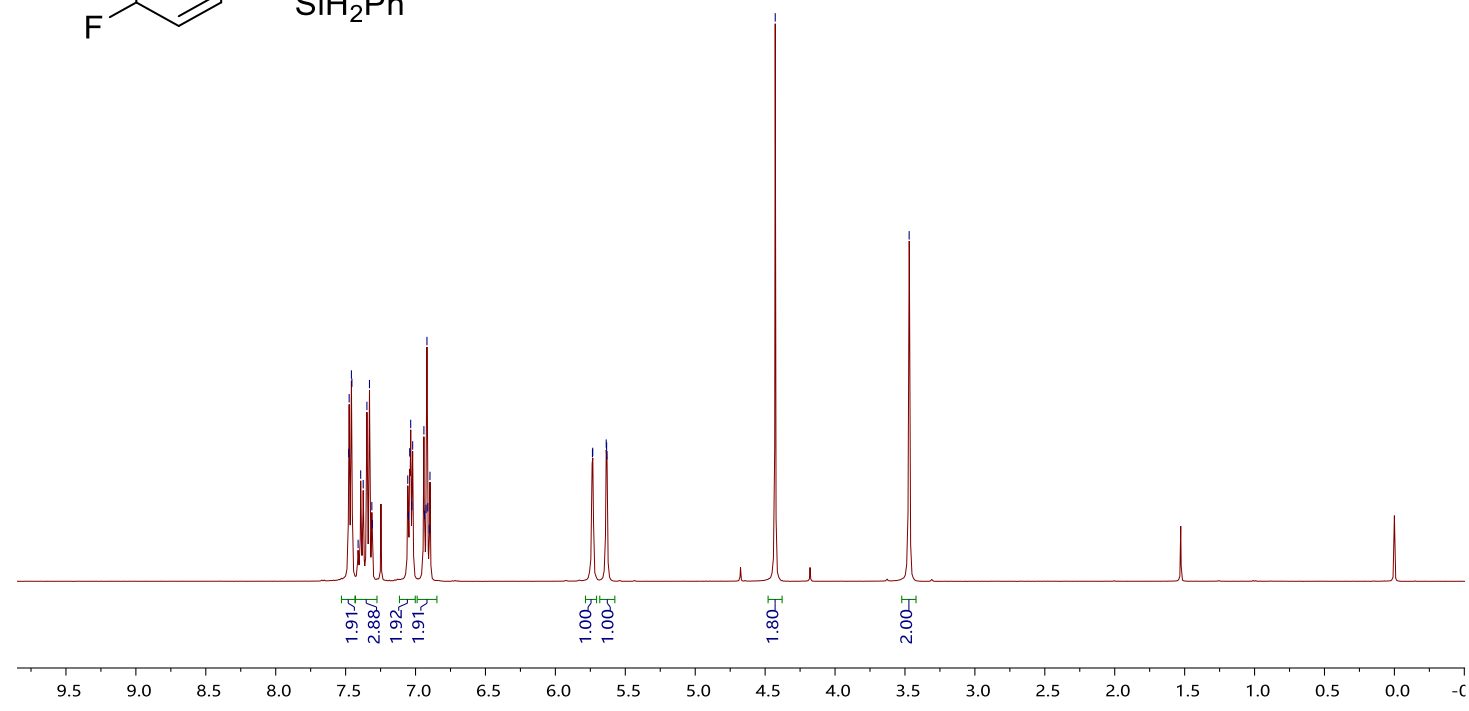

ll

$\overbrace{\mathrm{SiH}_{2} \mathrm{Ph}}^{\mathrm{C}}$
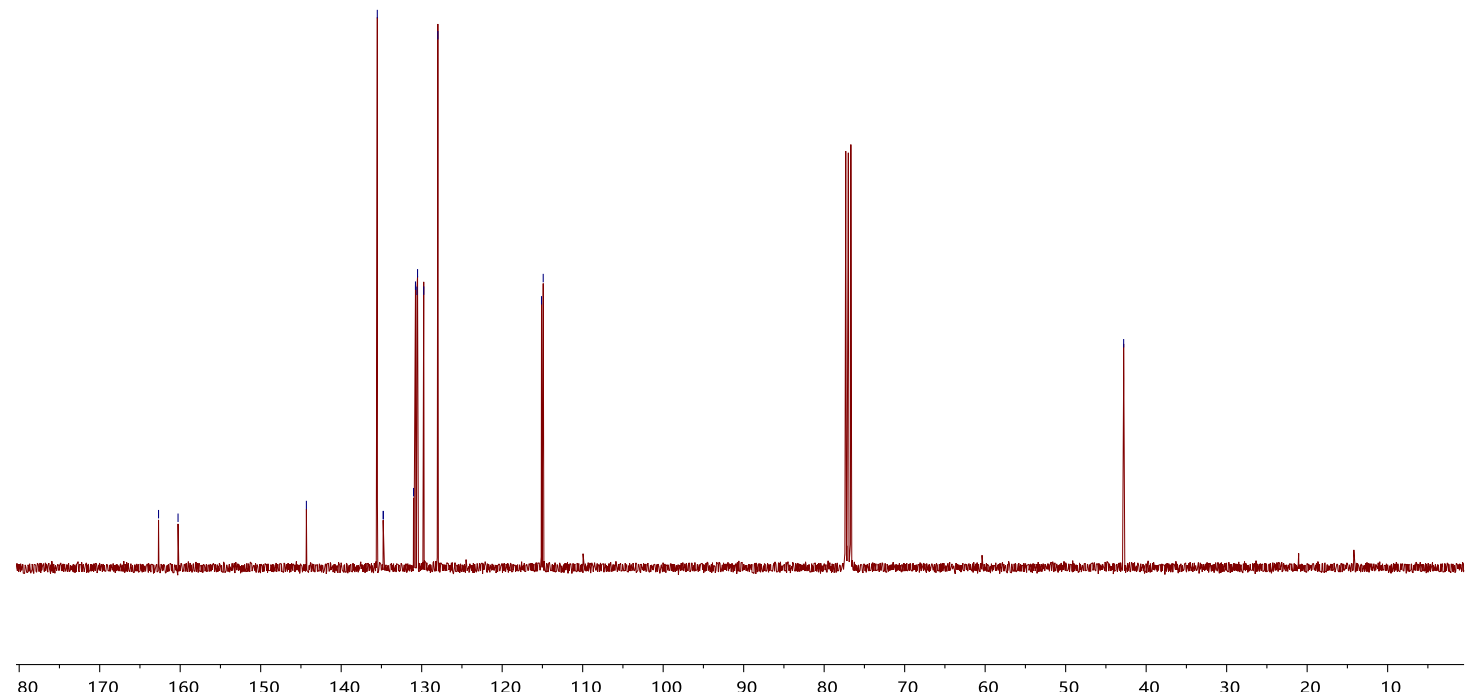

120

$110 \quad 100$

$90 \quad 80$

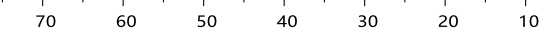




\section{(E)-(3-(4-chlorophenyl)prop-1-en-1-yl)(phenyl)silane (3ah)}

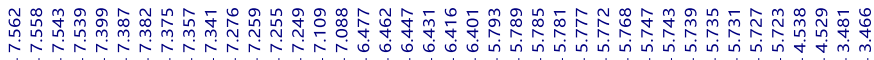

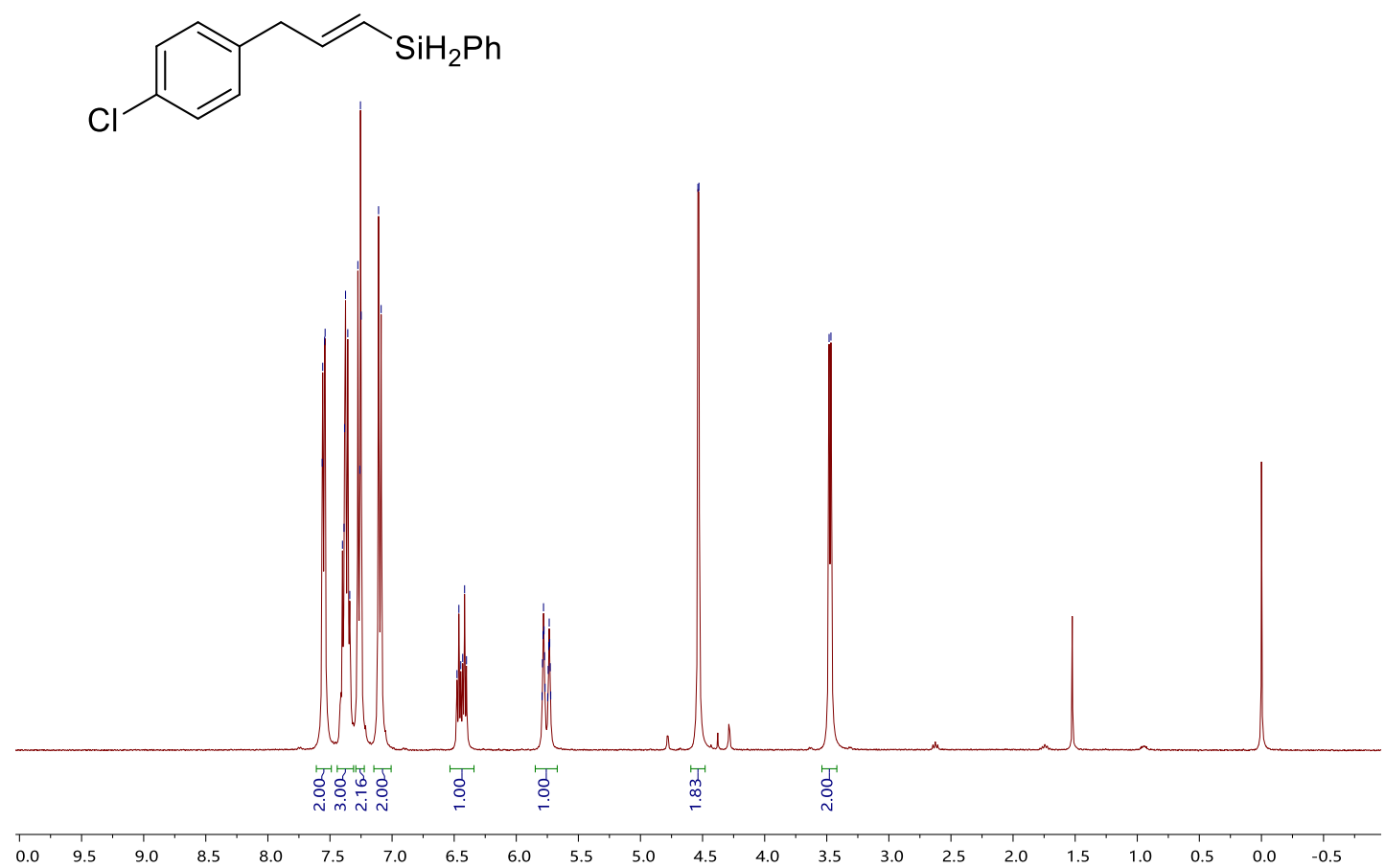

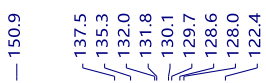<smiles>Clc1ccc(C/C=C/[SnH2]c2ccccc2)cc1</smiles>

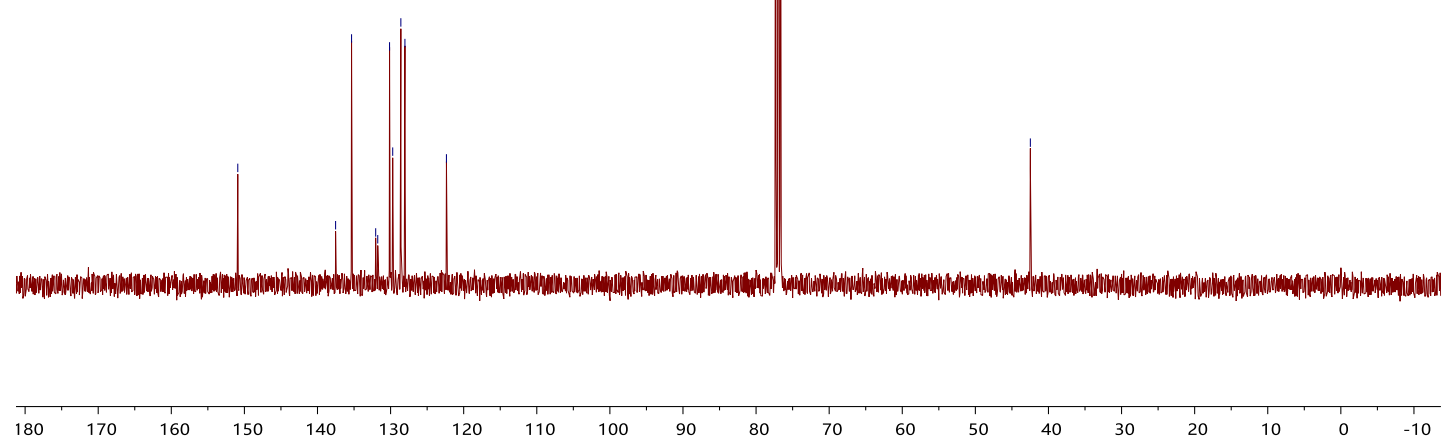


(3-(4-chlorophenyl)prop-1-en-2-yl)(phenyl)silane (4ah)

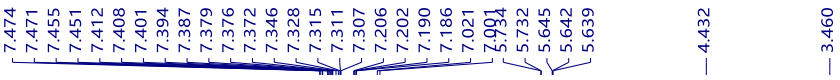<smiles>C=C(Cc1ccc(Cl)cc1)[SH]c1ccccc1</smiles>

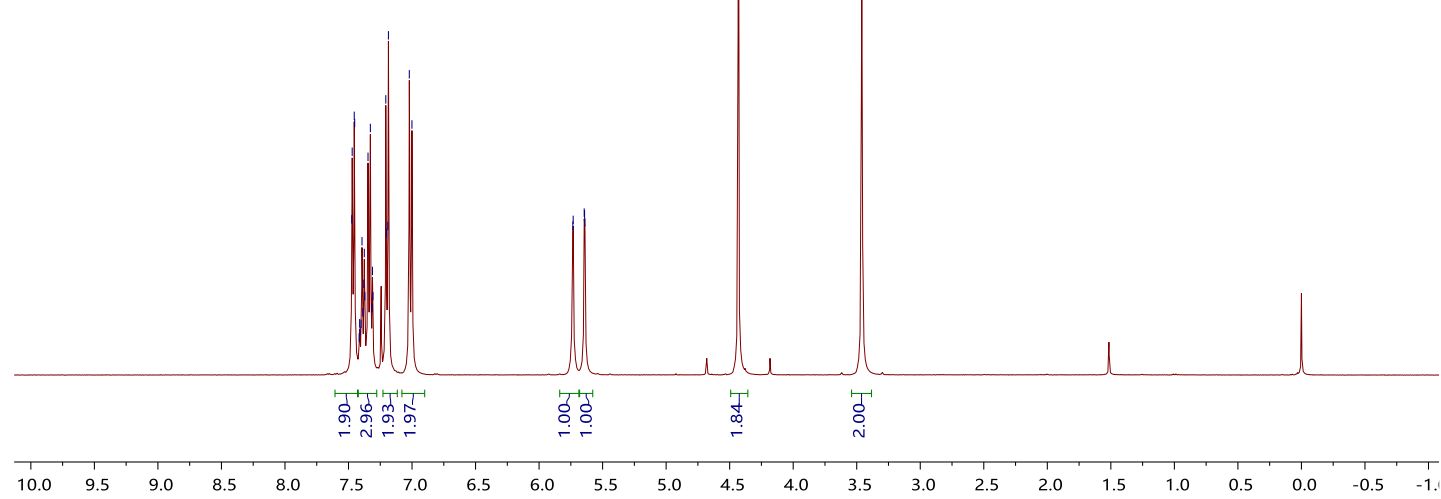

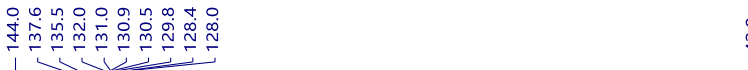<smiles>C=C(Cc1ccc(Cl)cc1)[SiH2]c1ccccc1</smiles>

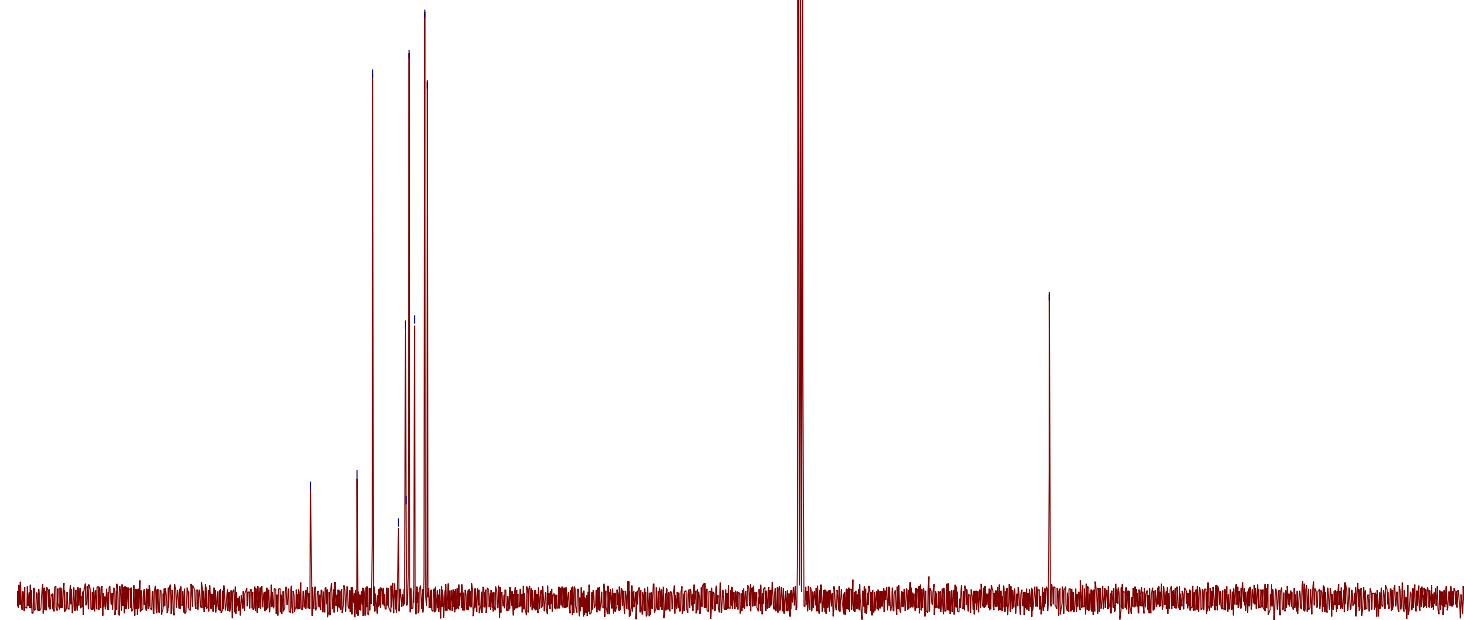

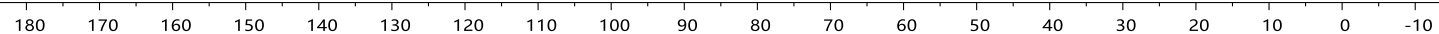




\section{(E)-(5-chloropent-1-en-1-yl)(phenyl)silane (3ai)}

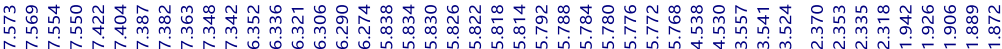

$\frown \mathrm{SiH}_{2} \mathrm{Ph}$

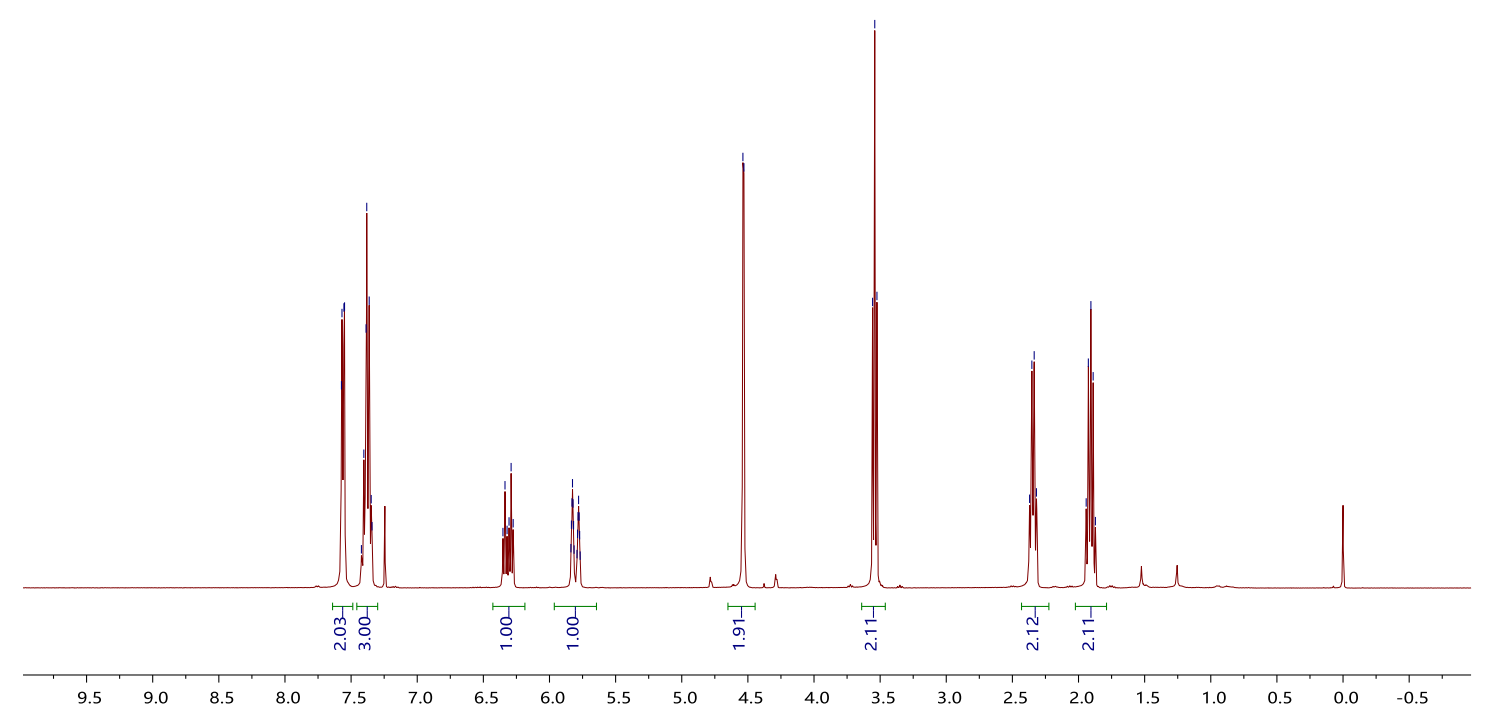

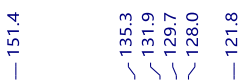

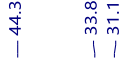

$\mathrm{Cl}_{\mathrm{SiH}_{2} \mathrm{Ph}}$

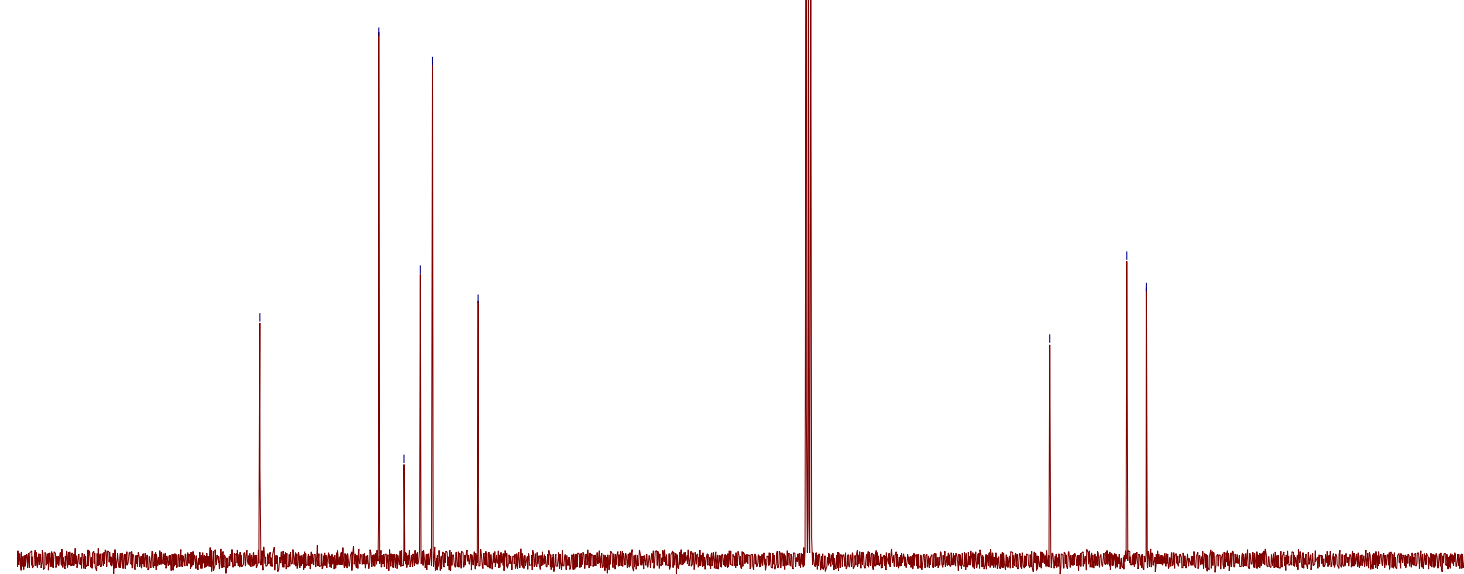


(5-chloropent-1-en-2-yl)(phenyl)silane (4ai)

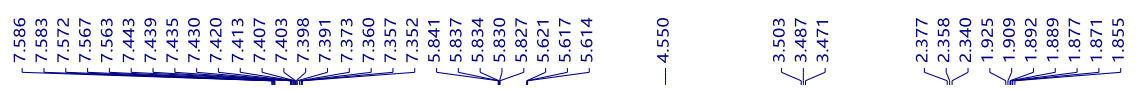

$\overbrace{\mathrm{SiH}_{2} \mathrm{Ph}}$

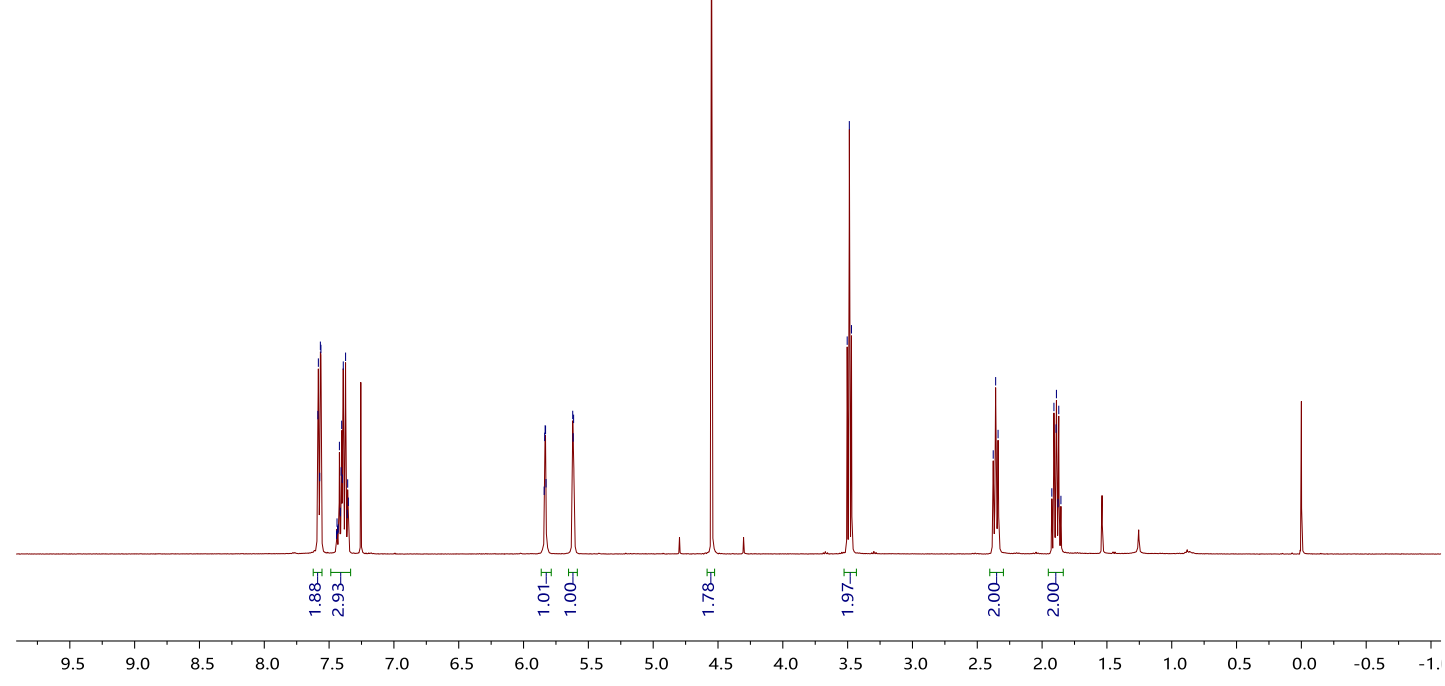

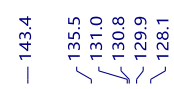

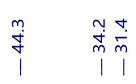

$\overbrace{\mathrm{SiH}_{2} \mathrm{Ph}}$

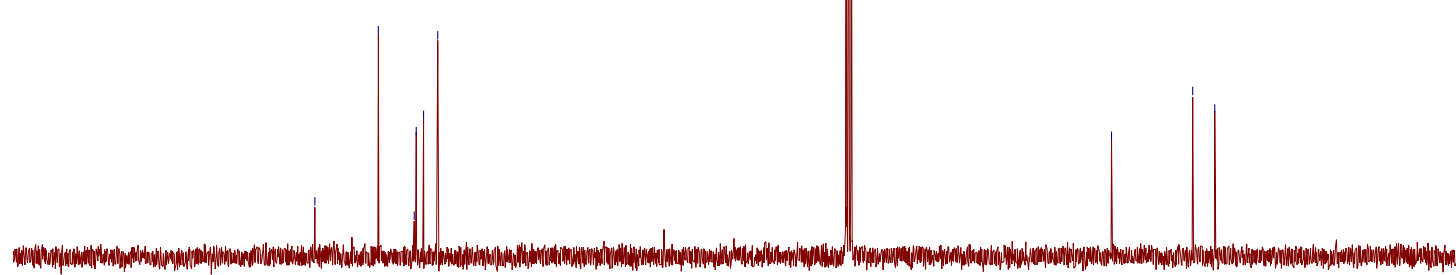

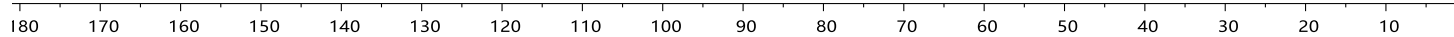


(E)-phenyl(4-(p-tolylthio)but-1-en-1-yl)silane (3aj)

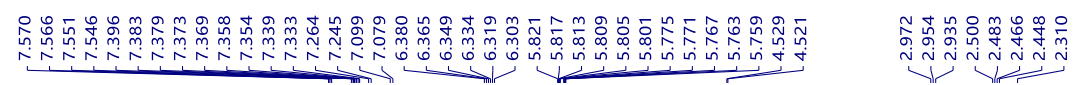



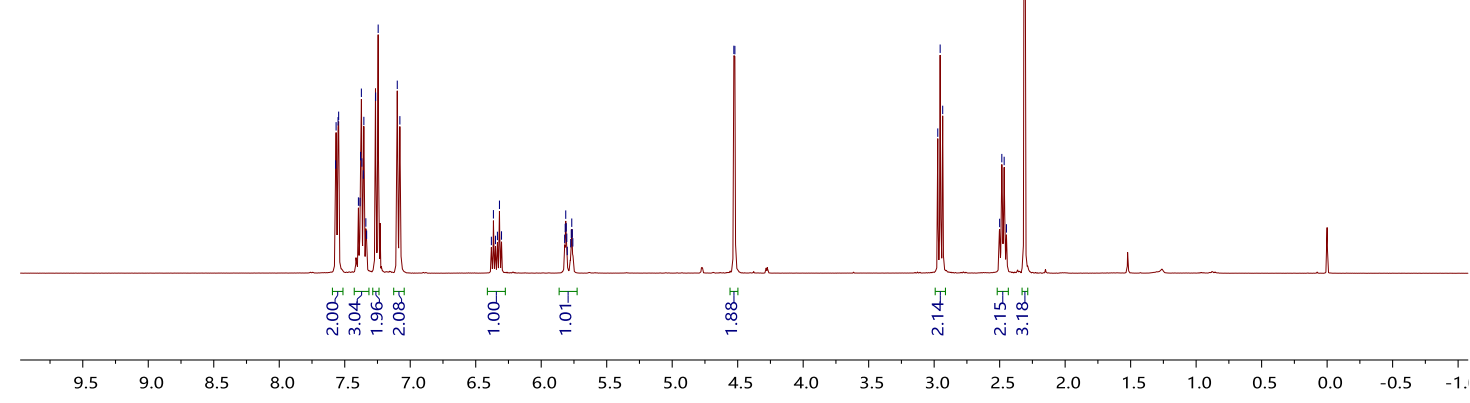

ind

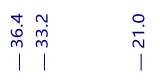

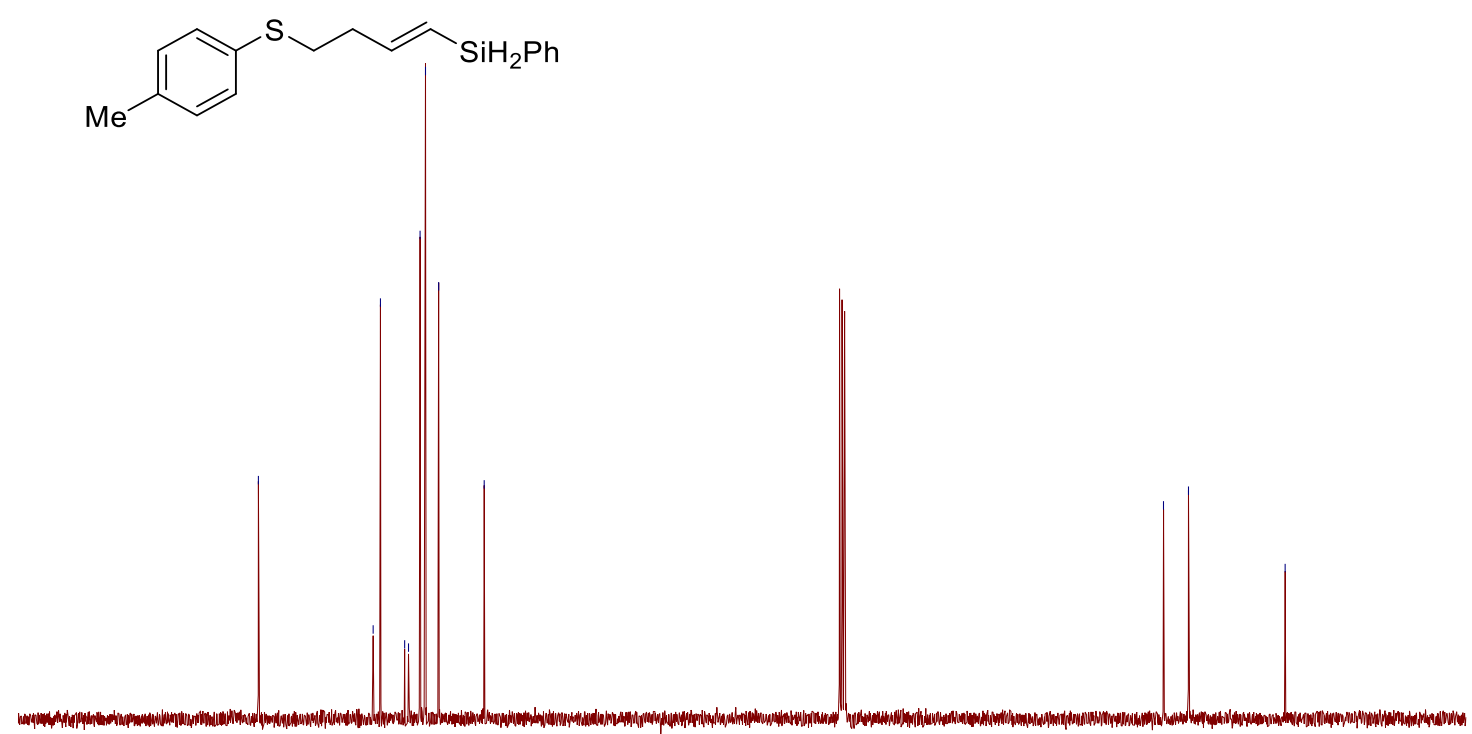

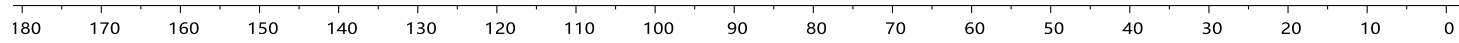


phenyl(4-(p-tolylthio)but-1-en-2-yl)silane (4aj)

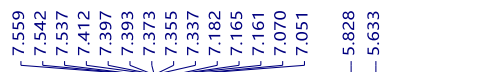

商

$\overbrace{\mathrm{SiH}_{2} \mathrm{Ph}}^{\mathrm{S}}$

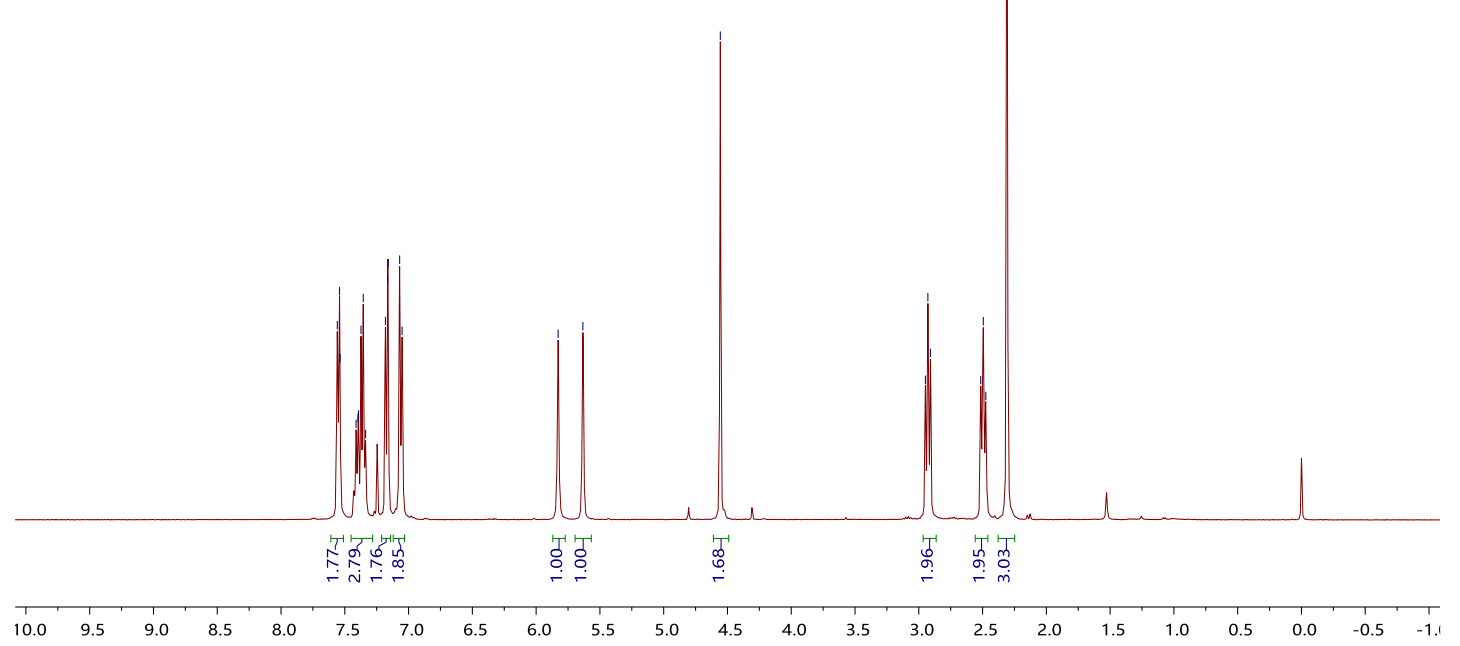

\section{|}<smiles>C=C(CCSc1ccc(C)cc1)Sc1ccccc1</smiles>

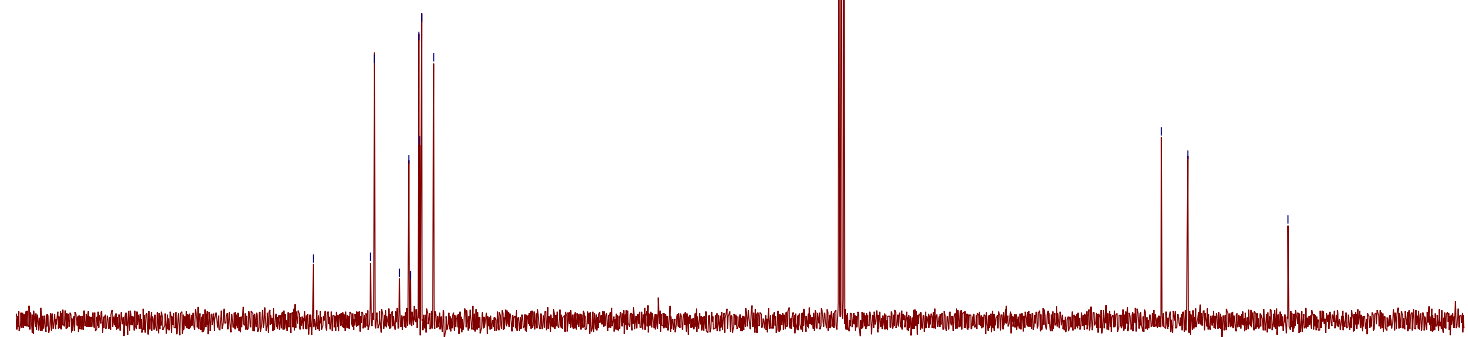

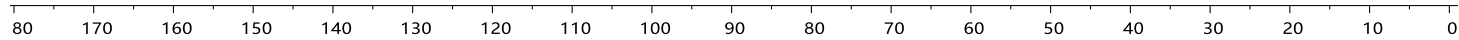


(E)-(5-phenoxypent-1-en-1-yl)(phenyl)silane (3ak)

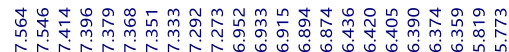

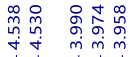

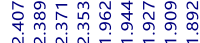

nin-

$\mathrm{PhO}^{\sim} \mathrm{SiH}_{2} \mathrm{Ph}$

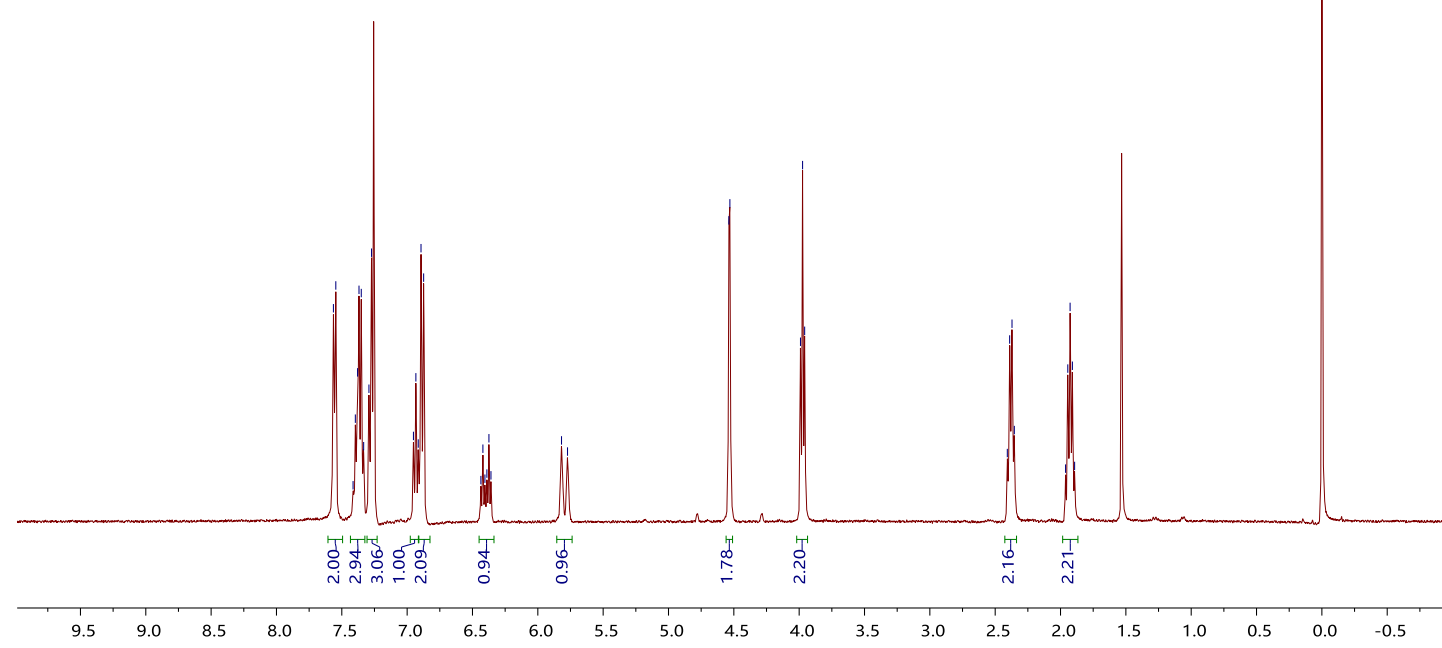

I

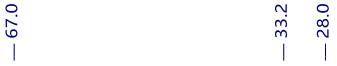

$\mathrm{PhO}$ $\mathrm{SiH}_{2} \mathrm{Ph}$

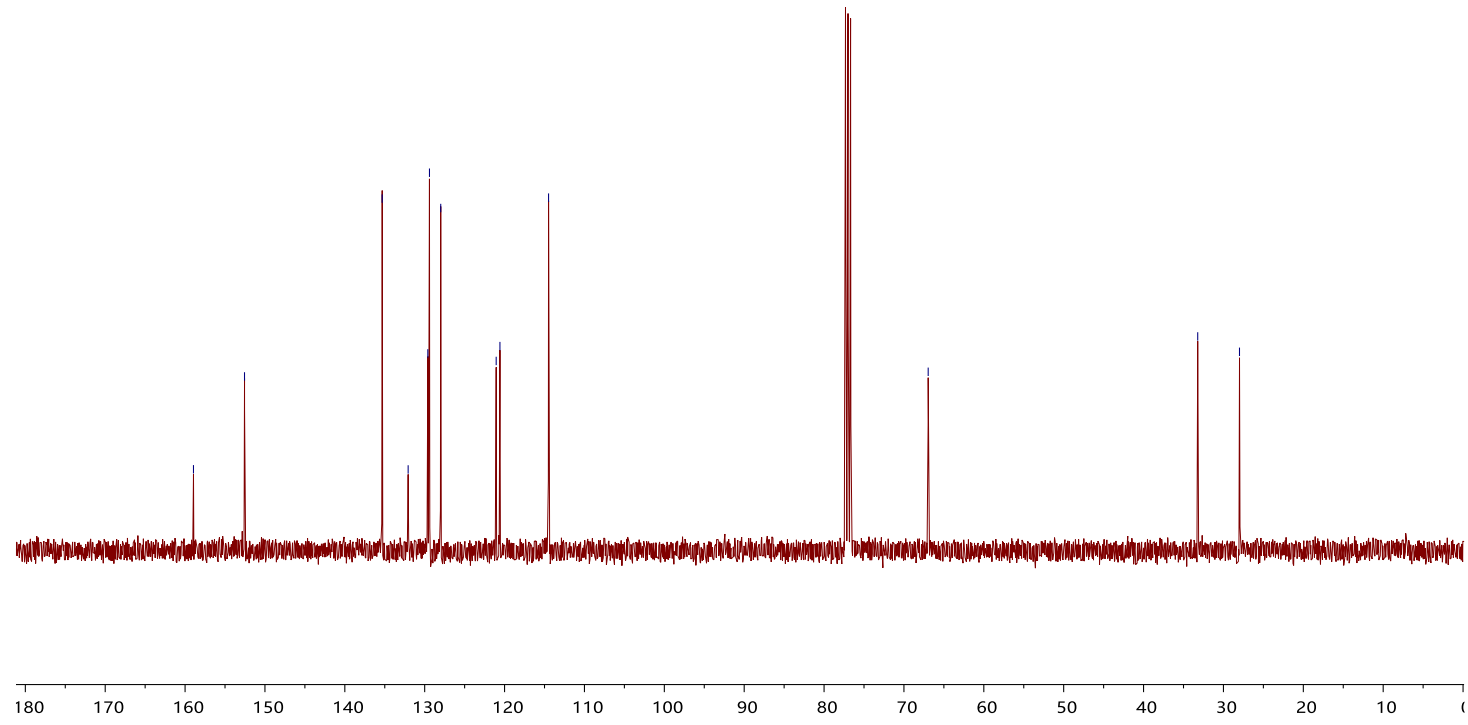




\section{(5-phenoxypent-1-en-2-yl)(phenyl)silane (4ak)}

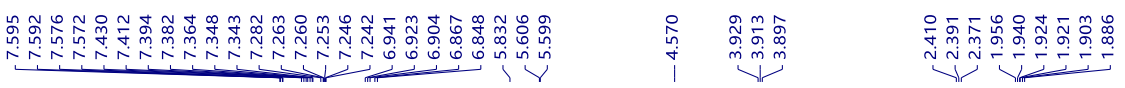<smiles>C=C(CCCOc1ccccc1)[SH2+]c1ccccc1</smiles>
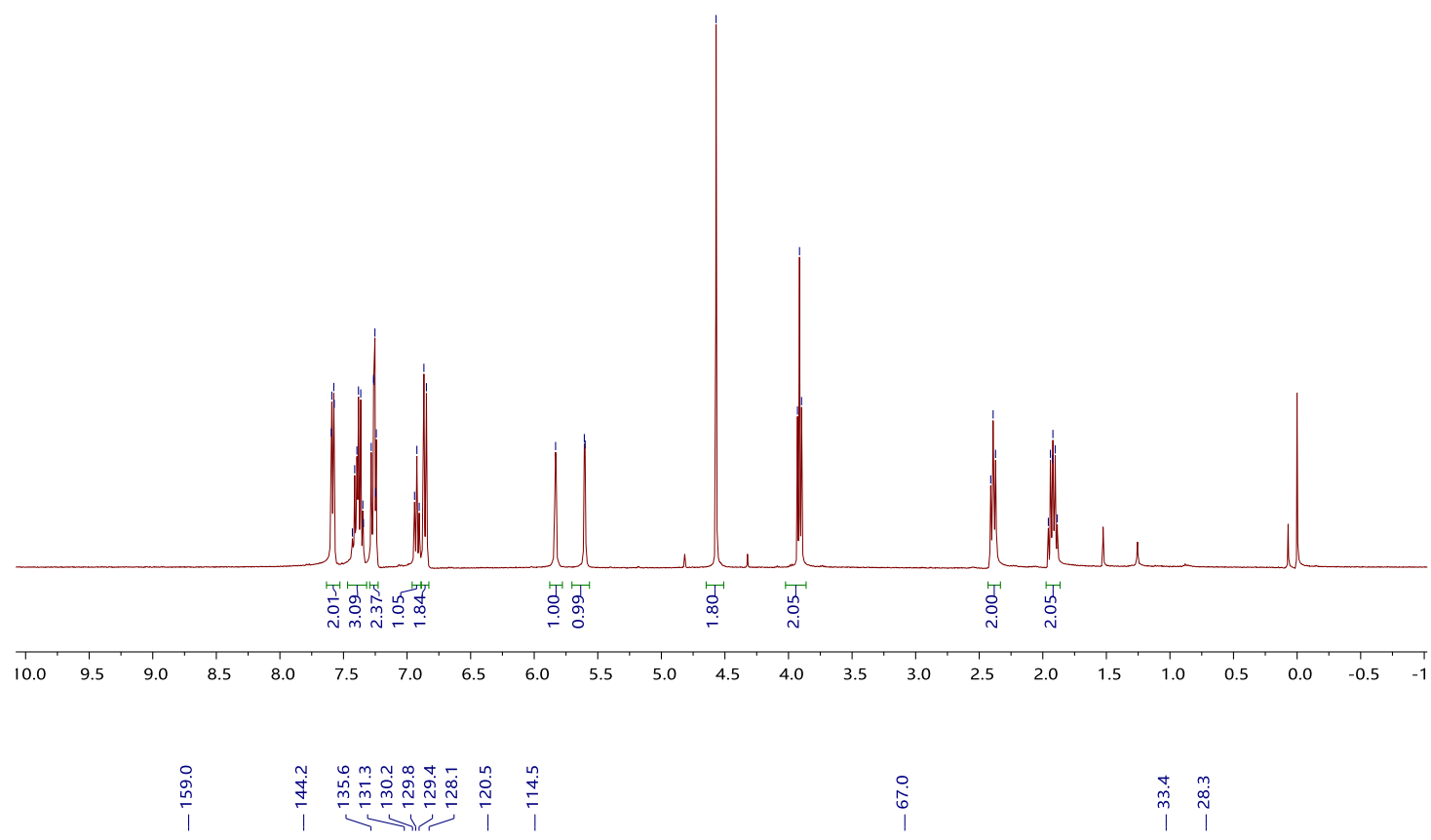<smiles>C=C(CCCOc1ccccc1)[SiH2]c1ccccc1</smiles>

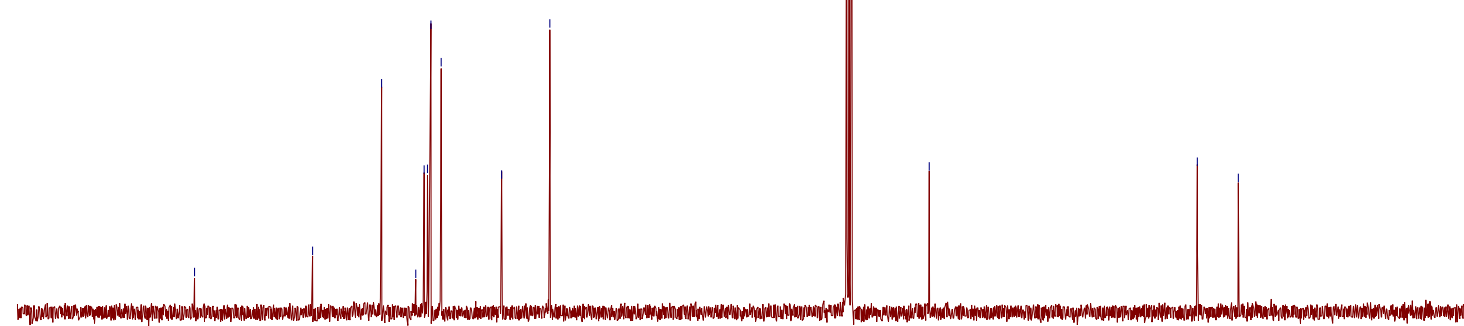

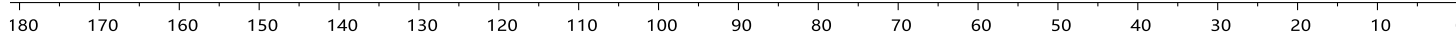


(E)-2-methyl-8-((5-(phenylsilyl)pent-4-en-1-yl)oxy)quinolone (3al)

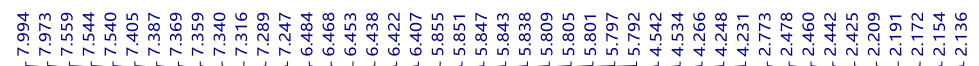<smiles>Cc1ccc2cccc(OCCC/C=C/[SH2+]c3ccccc3)c2n1</smiles>

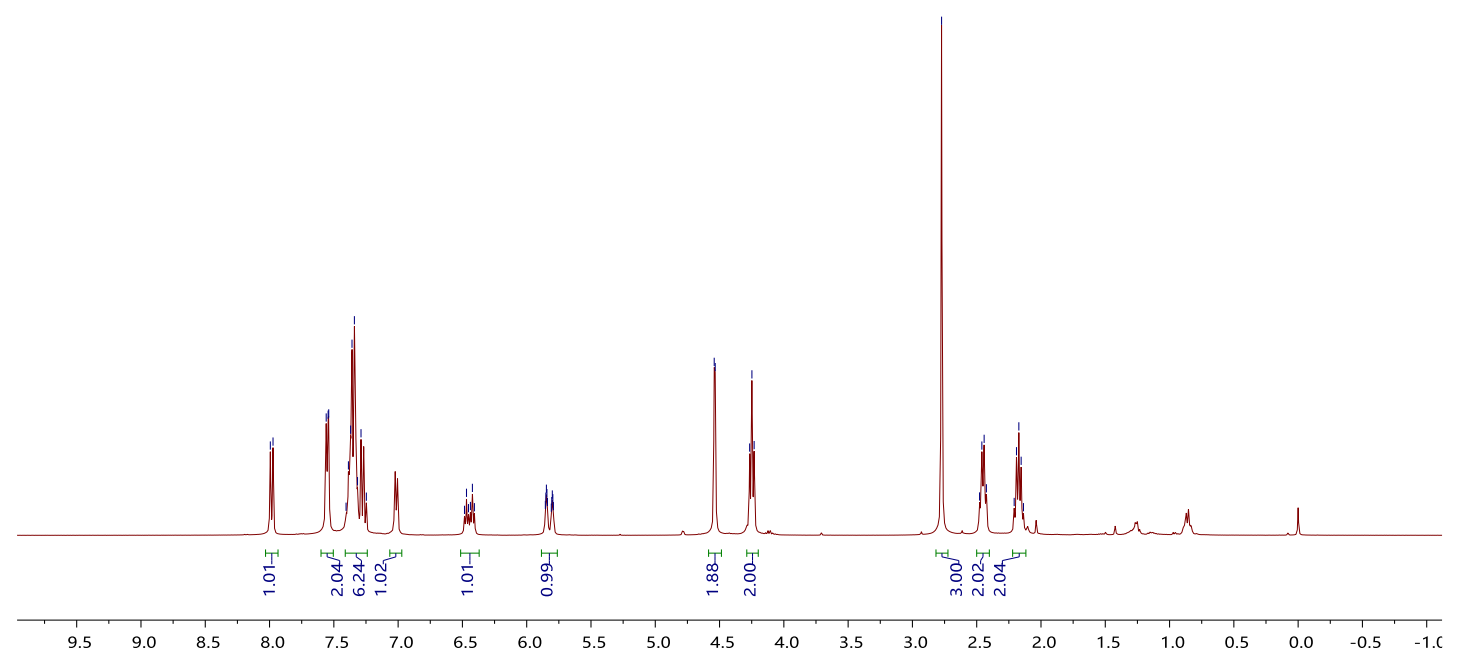

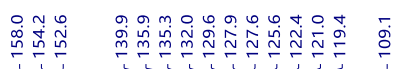

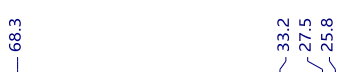

Me) $\mathrm{SiH}_{2} \mathrm{Ph}$

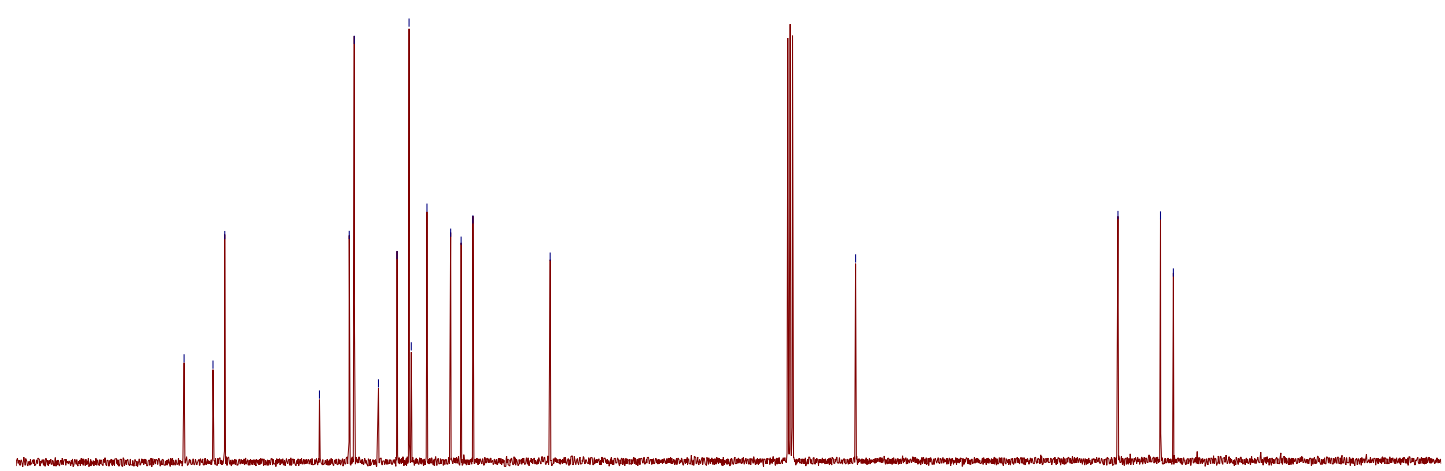

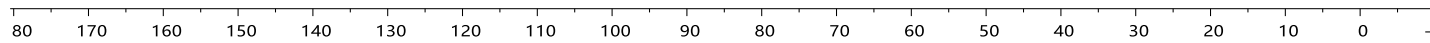


2-methyl-8-((4-(phenylsilyl)pent-4-en-1-yl)oxy)quinolone (4al)

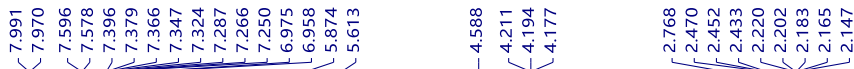

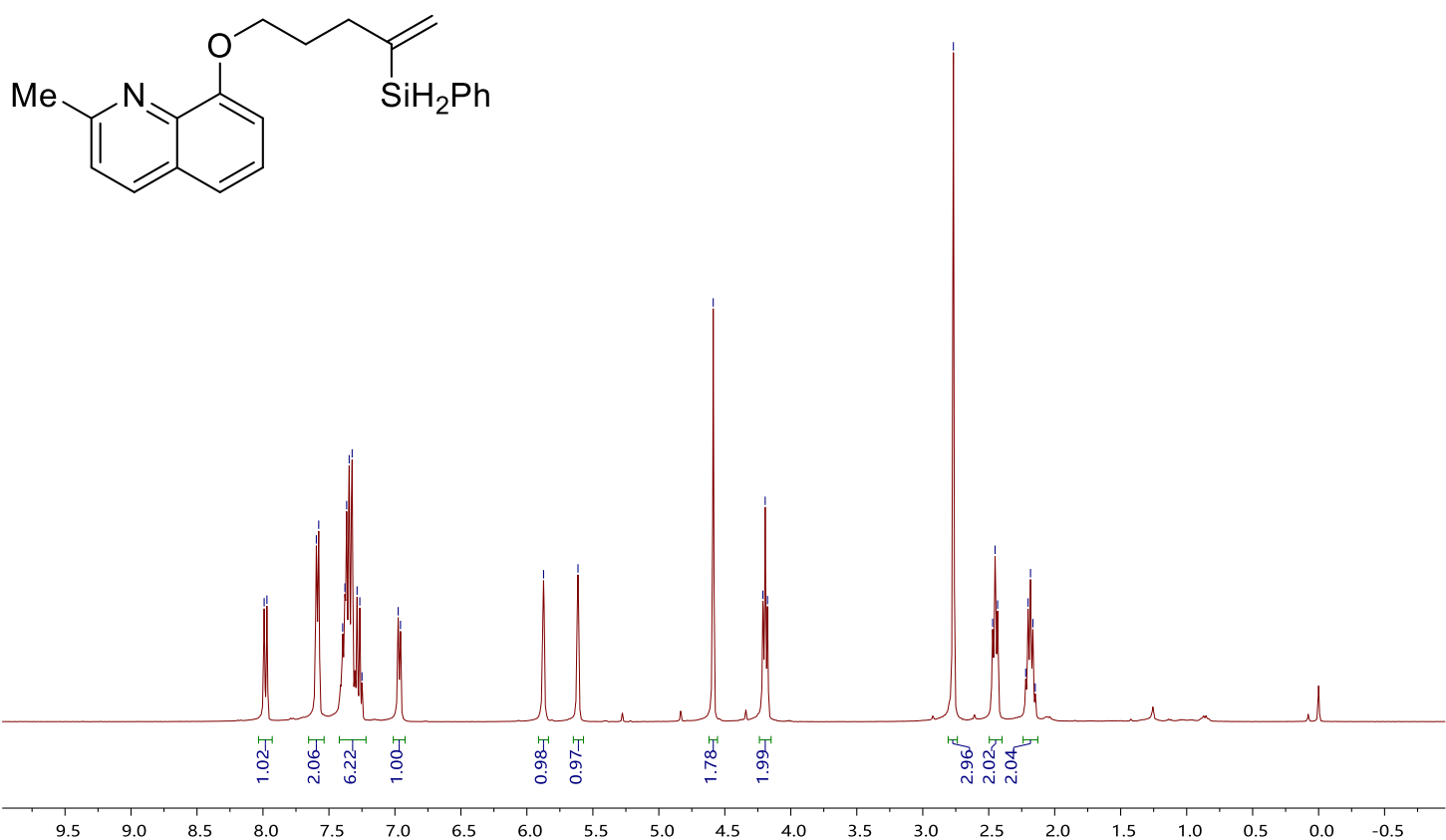

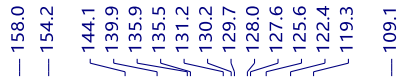

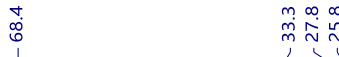<smiles>C=C(CCCOC(=C)C=NCC)CSc1ccccc1</smiles><smiles>c1ccc([SnH]CCCc2ccc3ccccc3c2)cc1</smiles>

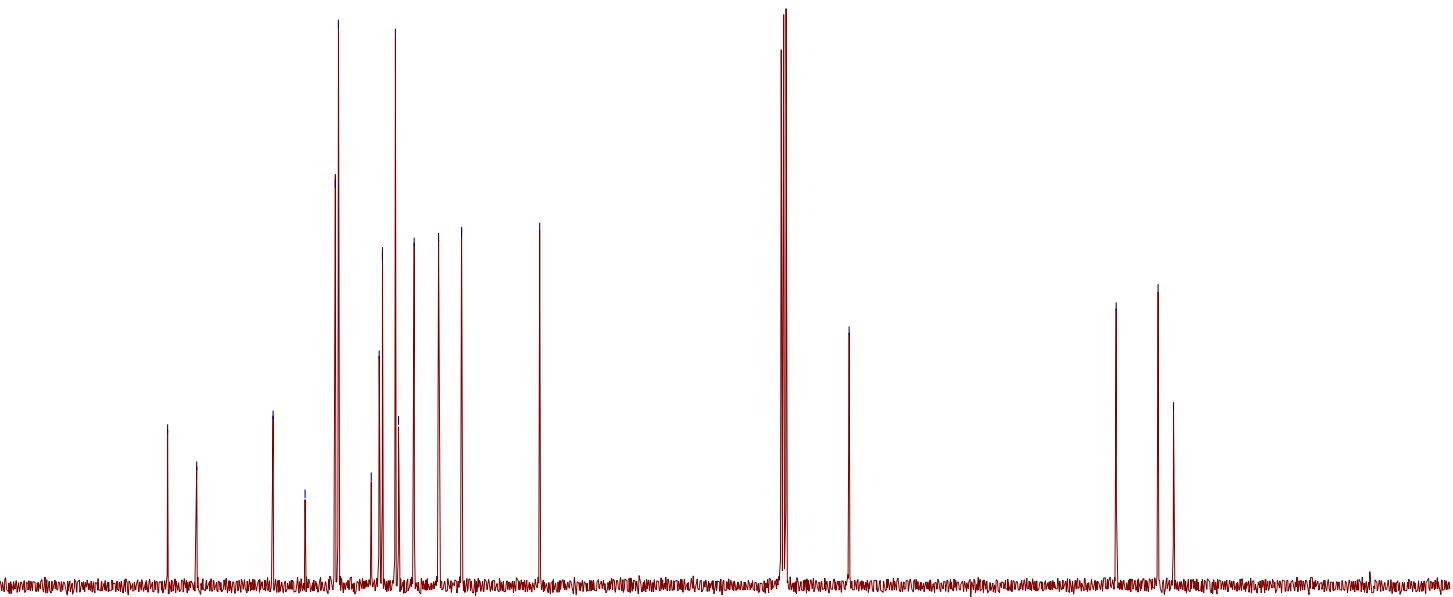

160

150

$\begin{array}{llll}130 & 120 & 110 & 100\end{array}$

80

50 
(E)-(5-(pent-4-en-1-yloxy)pent-1-en-1-yl)(phenyl)silane (3am)

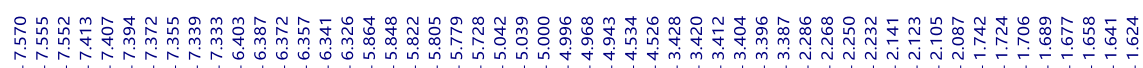

$\curvearrowright \overbrace{\mathrm{O}} \mathrm{SiH}_{2} \mathrm{Ph}$
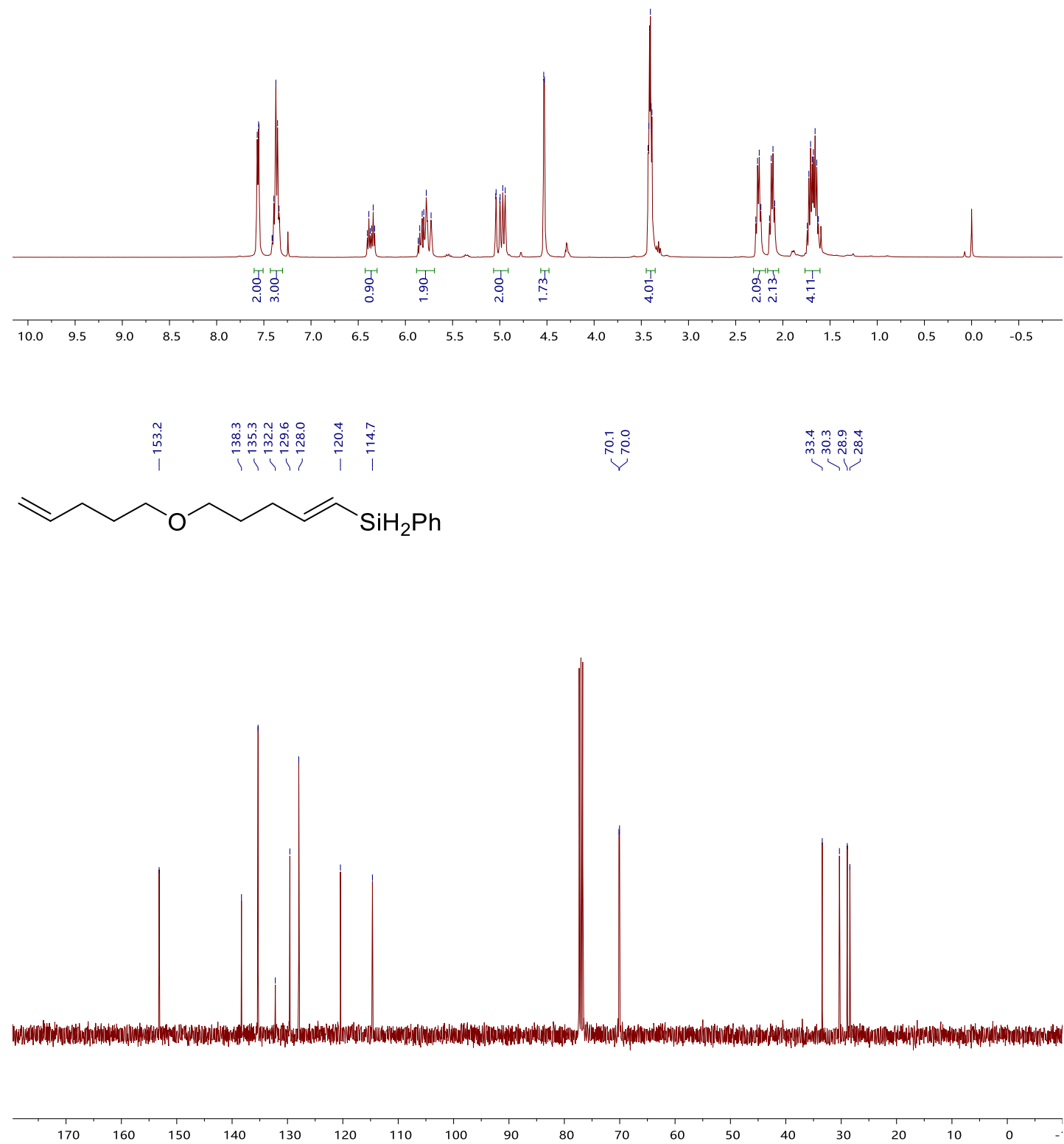
(5-(pent-4-en-1-yloxy)pent-1-en-2-yl)(phenyl)silane (4am)

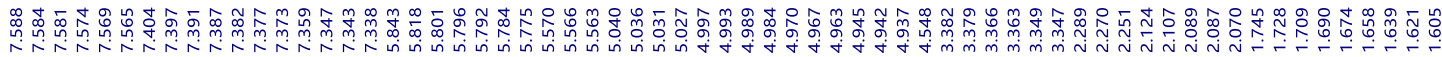

$\overbrace{\mathrm{SiH}_{2} \mathrm{Ph}}$

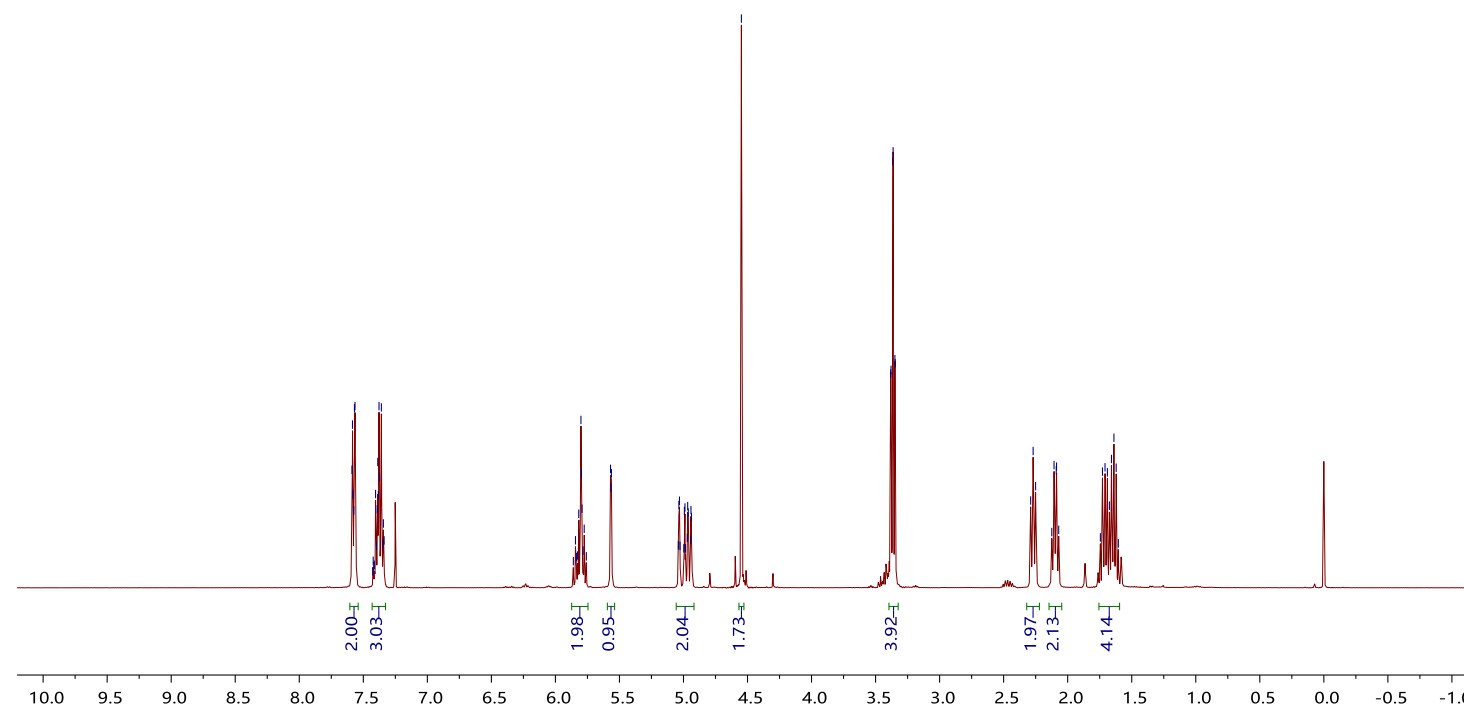

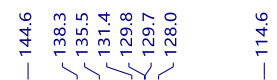

产官

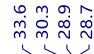

$\overbrace{\mathrm{SiH}_{2} \mathrm{Ph}}$

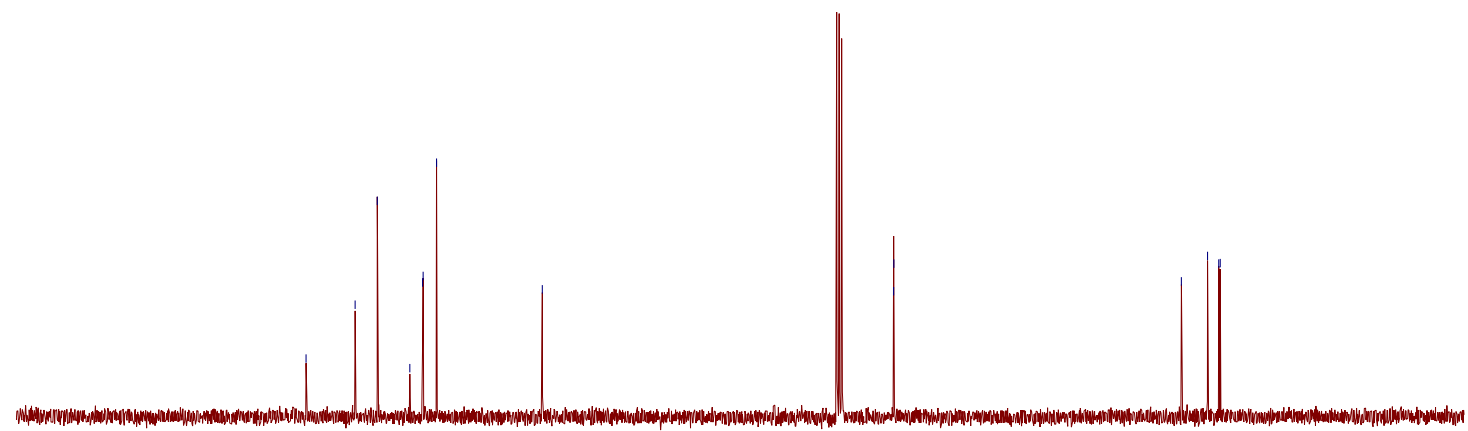

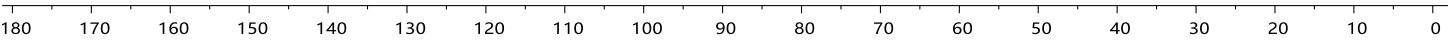


(E)-phenyl(4-((4-(prop-1-en-2-yl)cyclohex-1-en-1-yl)methoxy)but-1-en-1-yl)silane (3an)

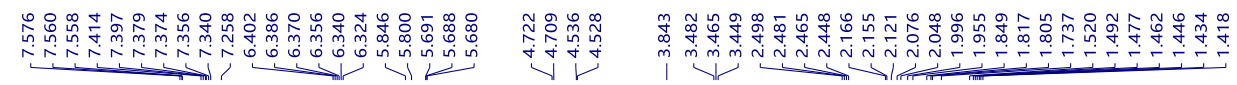

舟

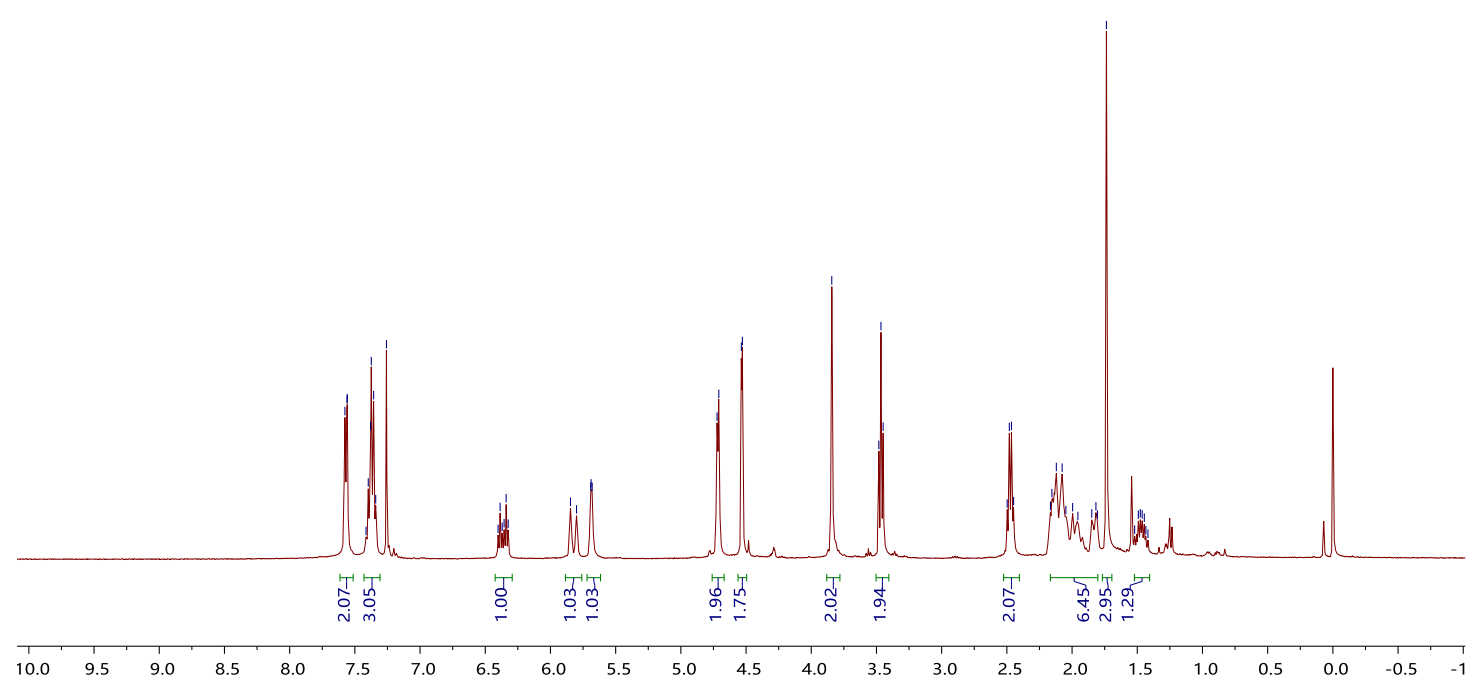

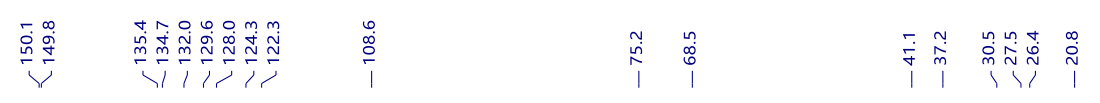

(NhH

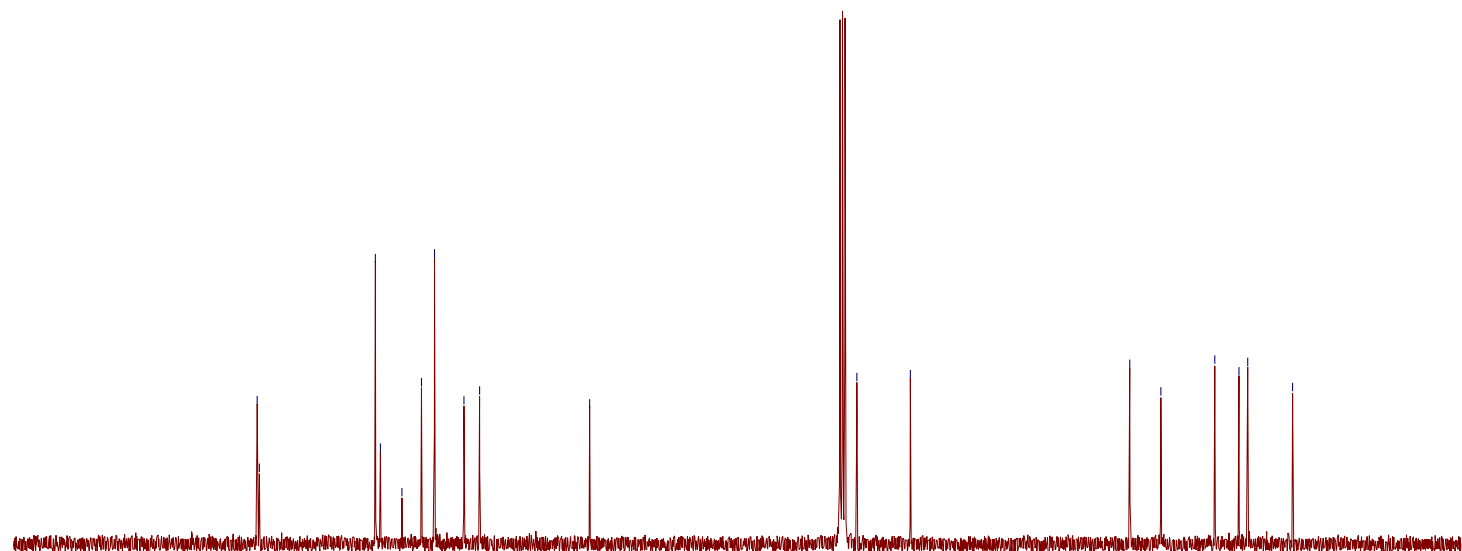

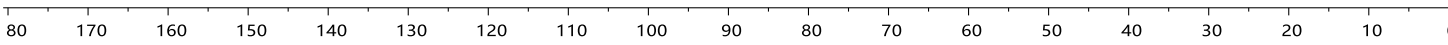


Phenyl(4-((4-(prop-1-en-2-yl)cyclohex-1-en-1-yl)methoxy)but-1-en-2-yl)silane (4an)
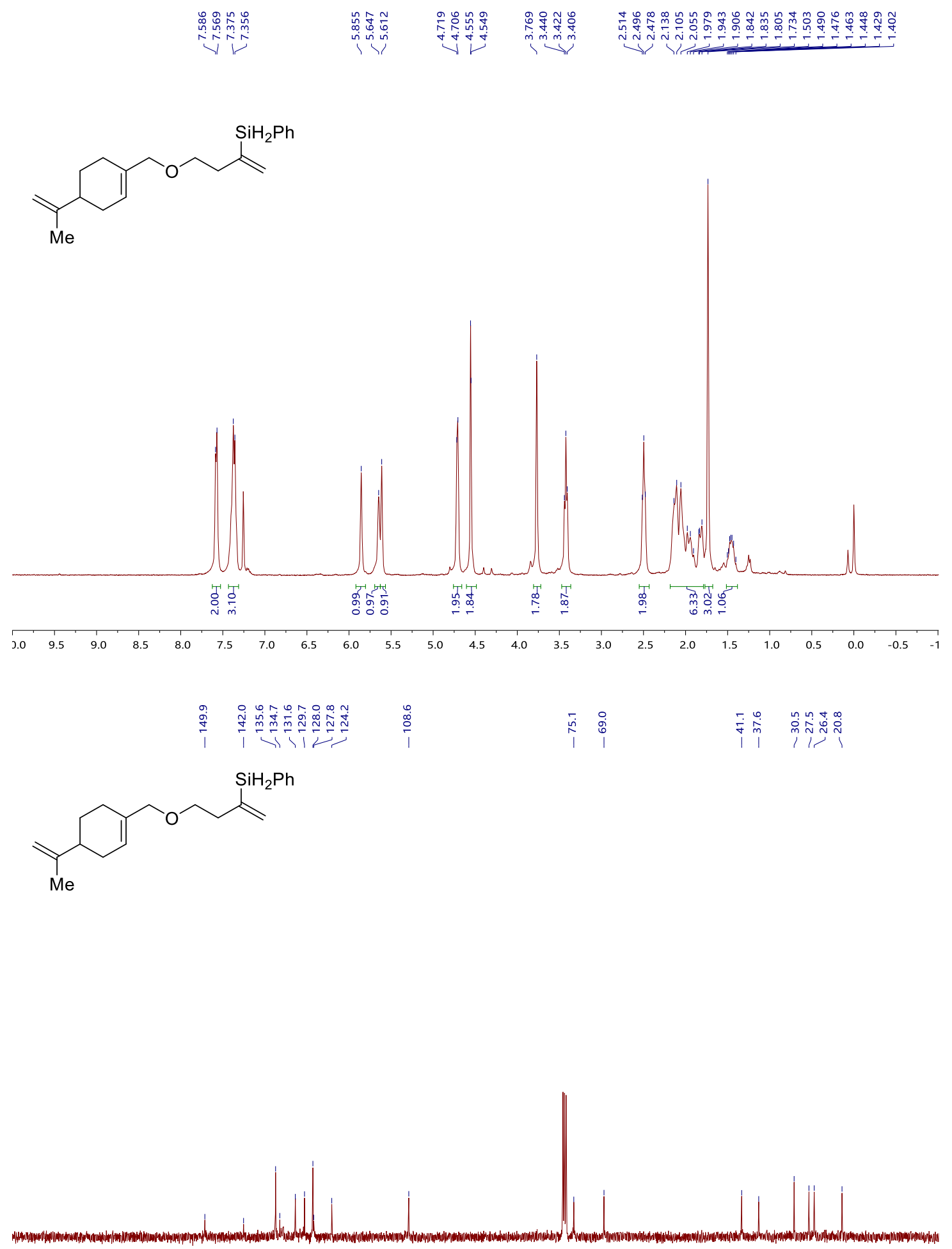

180

$\begin{array}{llllllllll}1 & 1 & 1 & 1 & 1 & 1 & 1 & 1 & 1 & 1 \\ 170 & 160 & 150 & 140 & 130 & 120 & 110 & 100 & 90\end{array}$

$80 \quad 70$

$60 \quad 50$

$40 \quad 30 \quad 20 \quad 10$ 
(E)-(4-(1,3-dioxolan-2-yl)but-1-en-1-yl)(phenyl)silane (3ao)

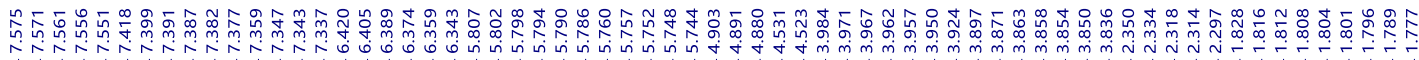

$\overbrace{\mathrm{O}}^{\mathrm{O}} \mathrm{SiH}_{2} \mathrm{Ph}$

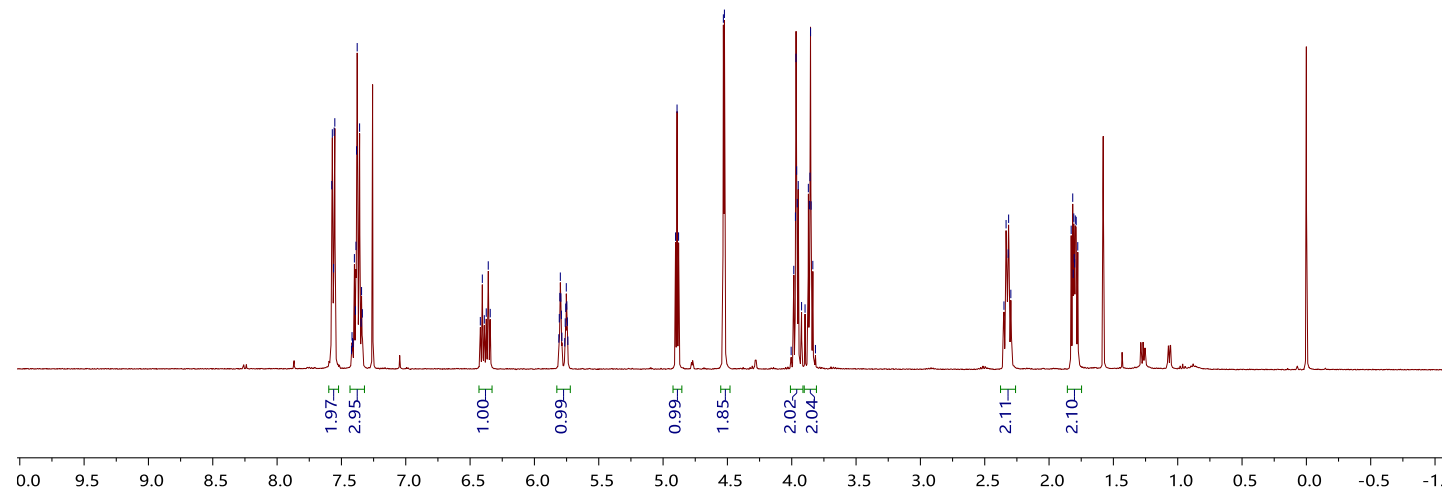

等

$\overbrace{\mathrm{O}}^{\mathrm{O}} \mathrm{SiH}_{2} \mathrm{Ph}$

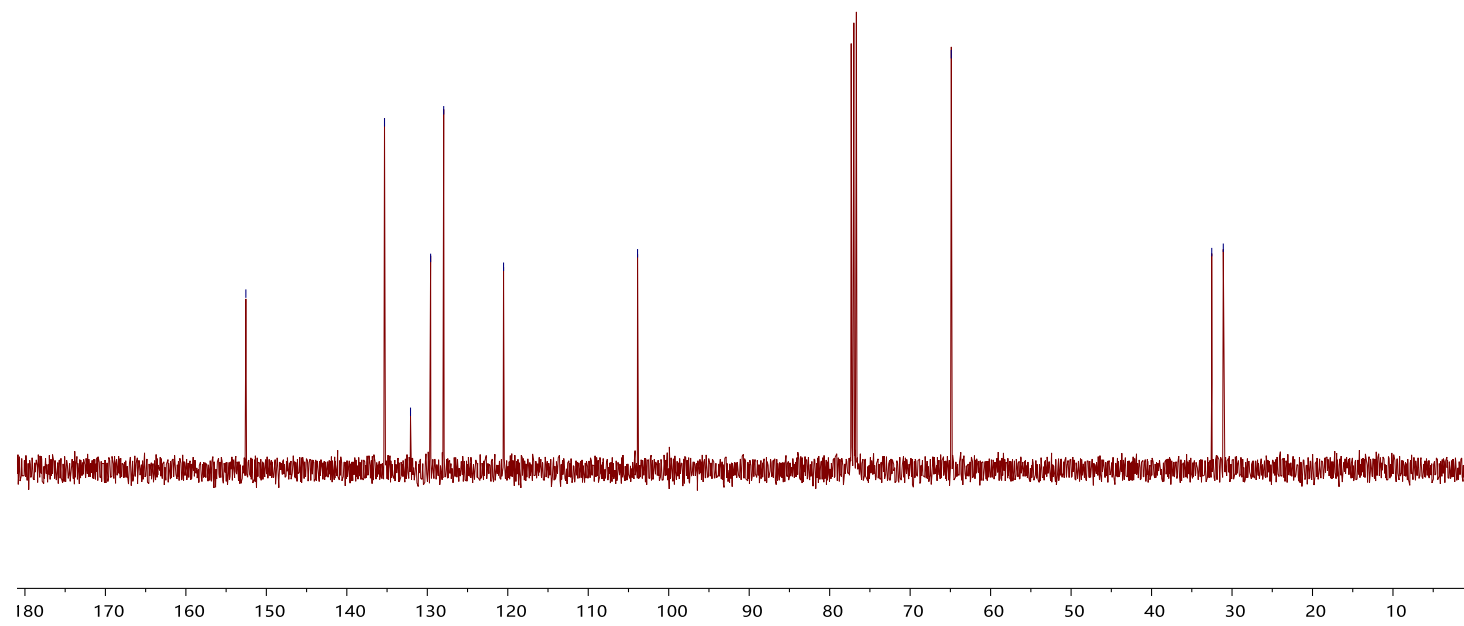


(4-(1,3-dioxolan-2-yl)but-1-en-2-yl)(phenyl)silane (4ao)

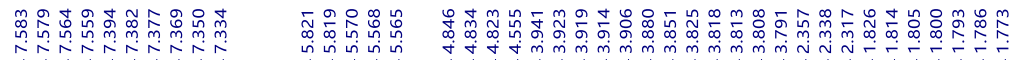

$\overbrace{\mathrm{SiH}_{2} \mathrm{Ph}}^{\mathrm{O}}$

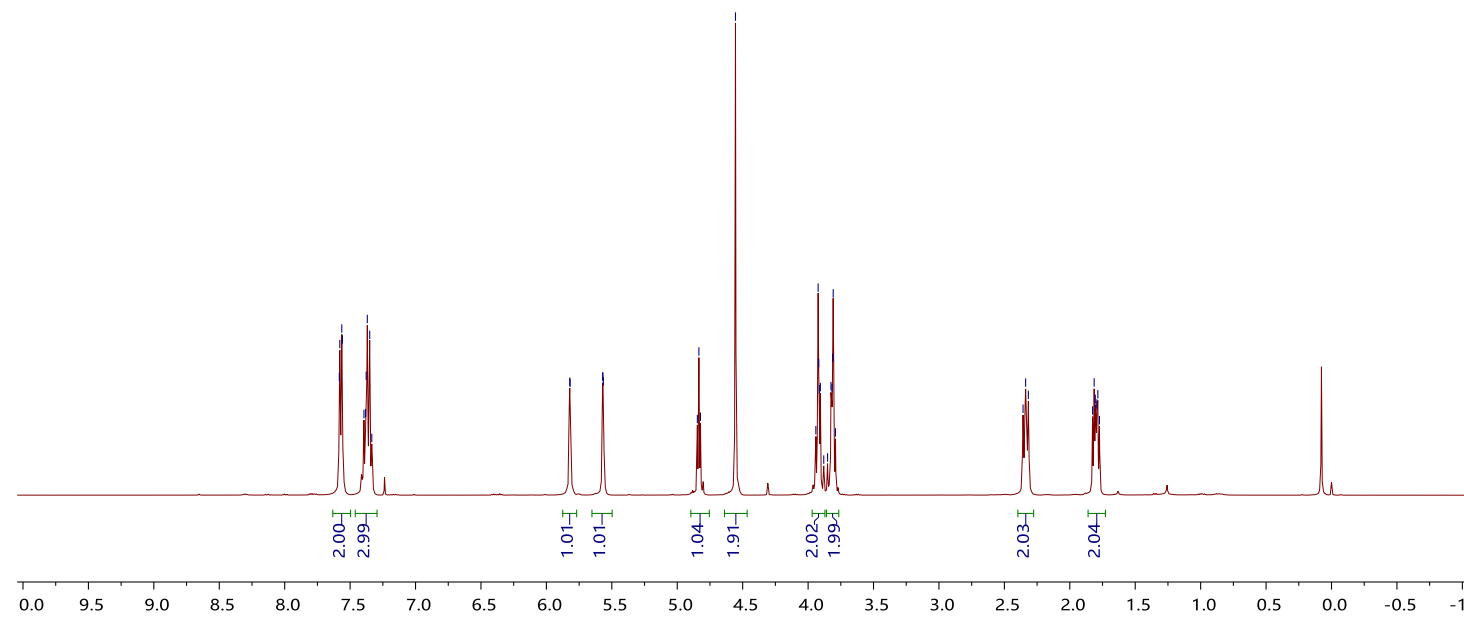

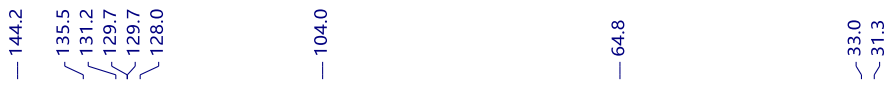

$\overbrace{\mathrm{SiH}_{2} \mathrm{Ph}}^{\mathrm{O}}$

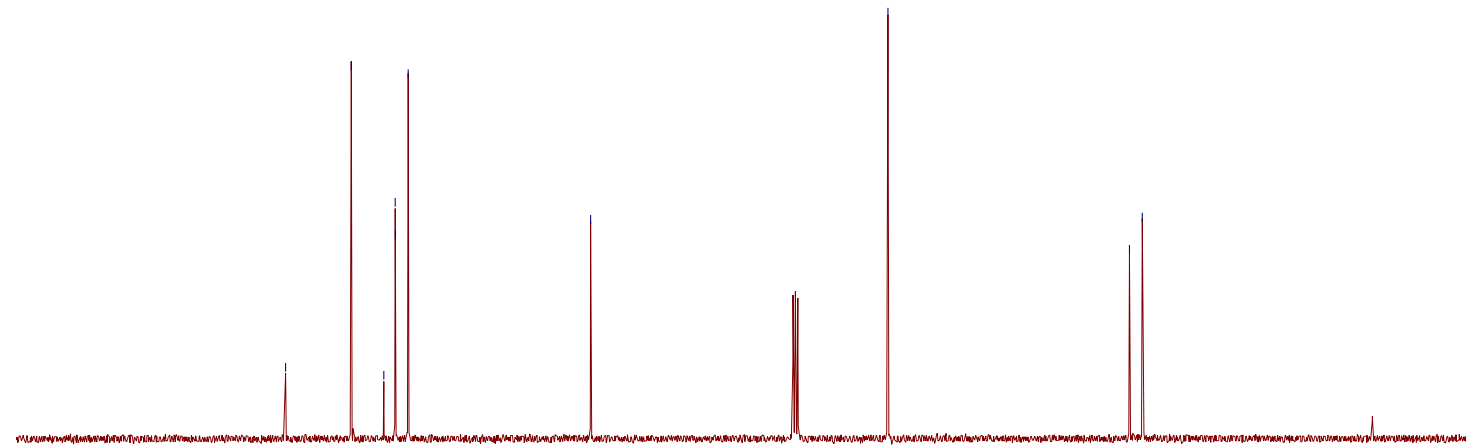

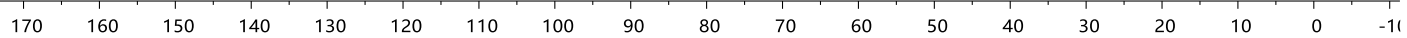


(E)-(3-(2,2-dimethyl-1,3-dioxan-5-yl)prop-1-en-1-yl)(phenyl)silane (3ap)

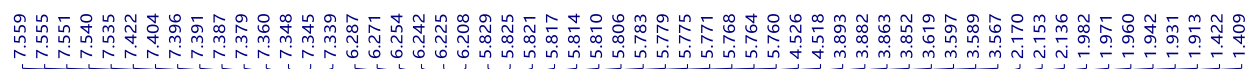<smiles>CC1(C)OCC(C/C=C/[SH+]c2ccccc2)CO1</smiles>
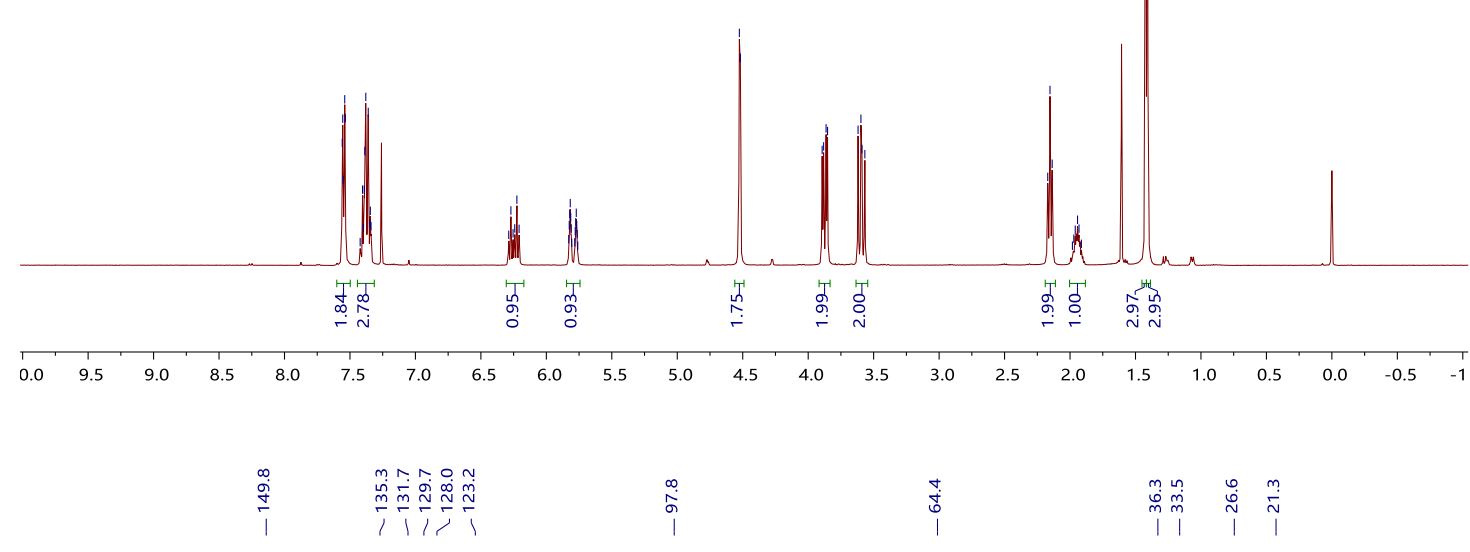<smiles>CC1(C)OCC(C/C=C/[SH2+]c2ccccc2)CO1</smiles>

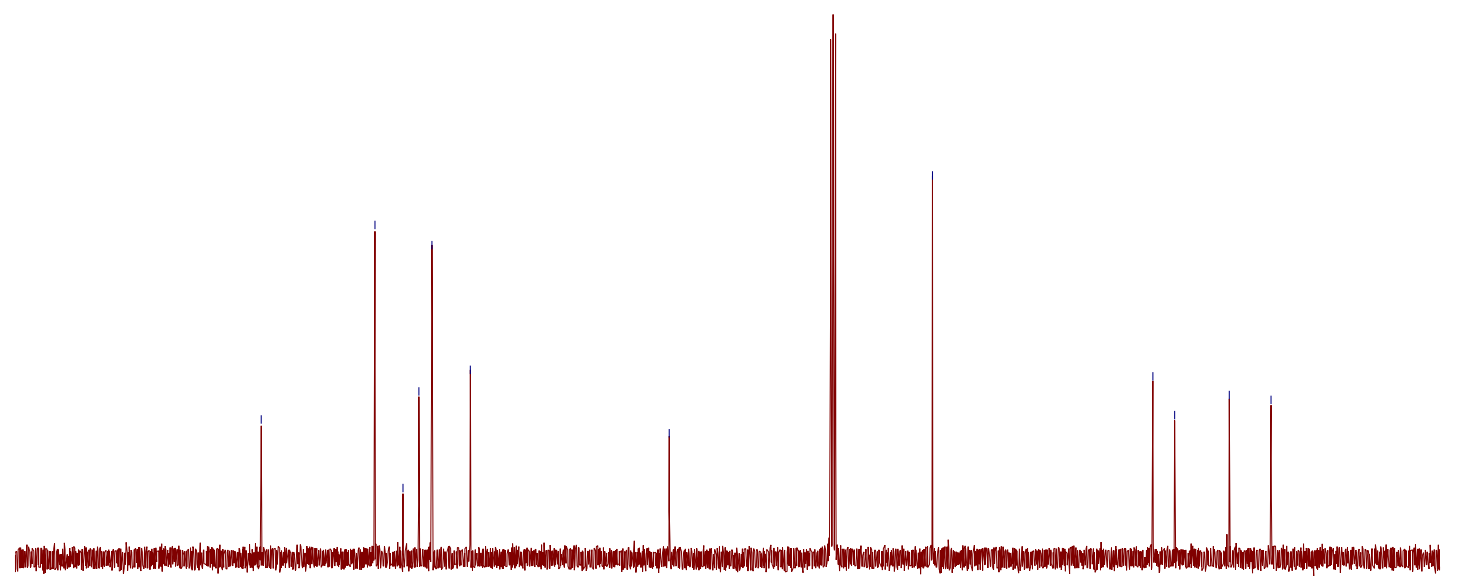

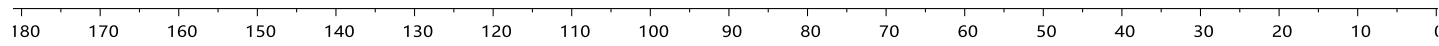


(3-(2,2-dimethyl-1,3-dioxan-5-yl)prop-1-en-2-yl)(phenyl)silane (4ap)

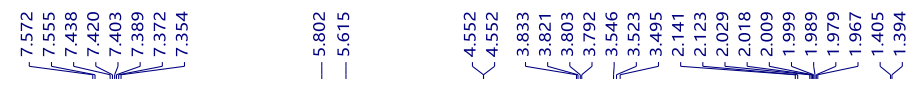<smiles>C=C(CC1COC(C)(C)OC1)[SiH2]c1ccccc1</smiles>
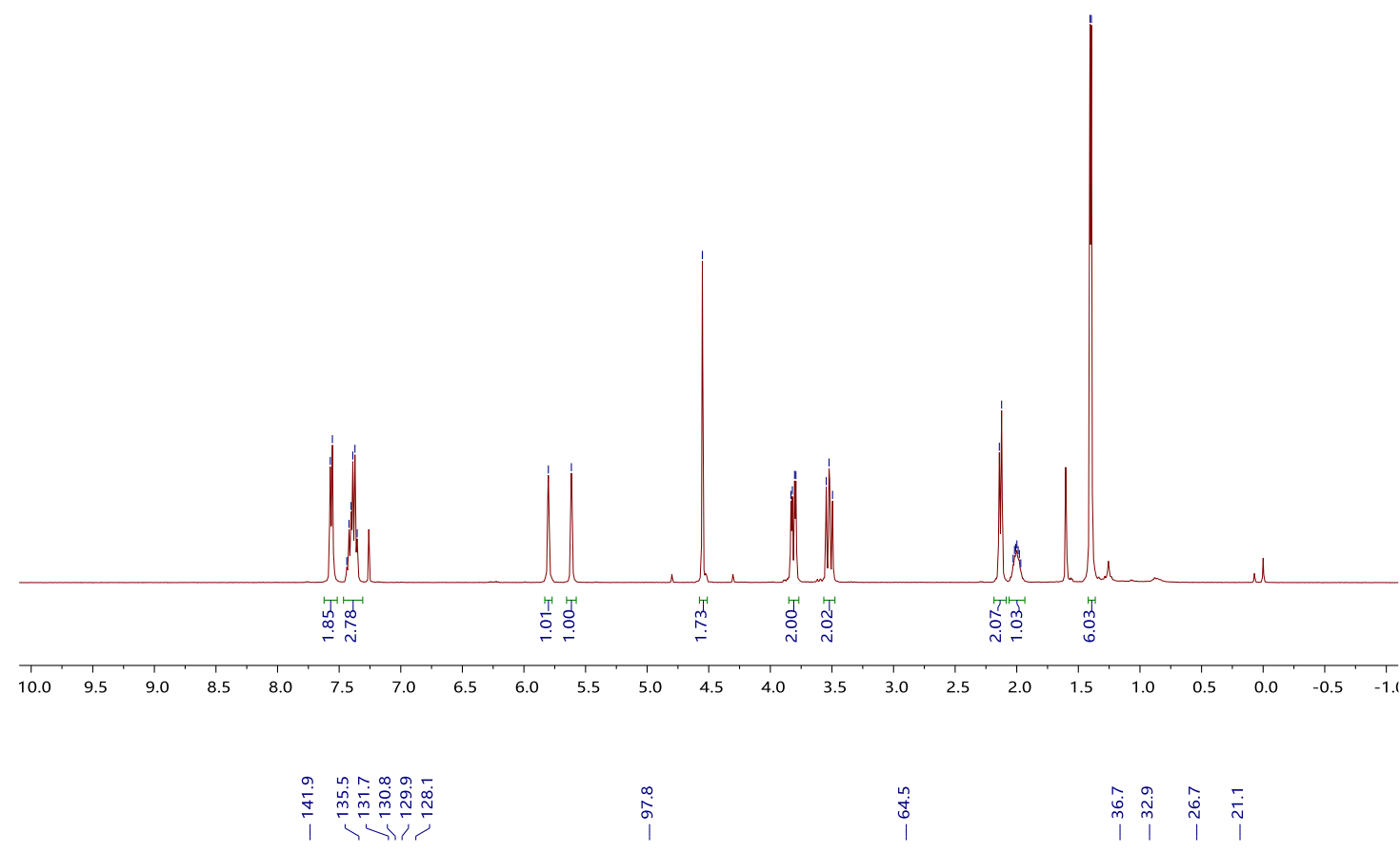<smiles>C=C(CC1COC(C)(C)OC1)[SiH2]c1ccccc1</smiles>

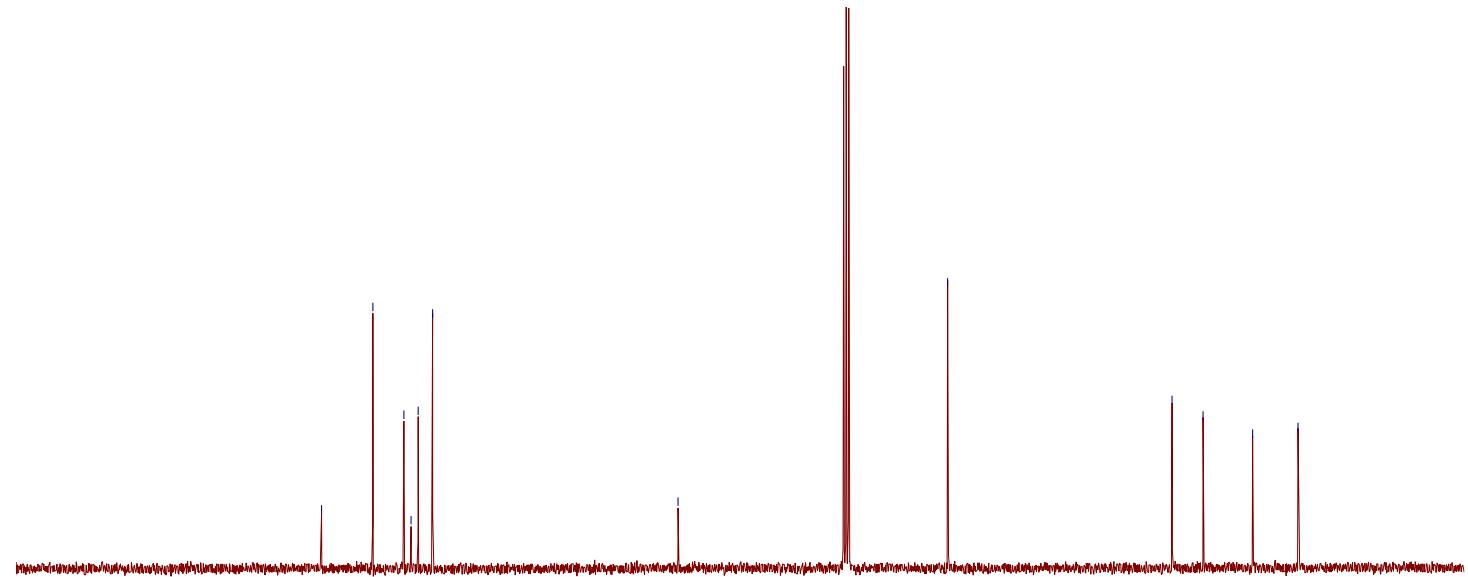

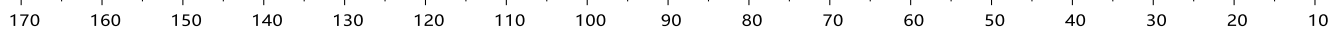


$((E)-4-(((3 S, 8 S, 9 S, 10 R, 13 R, 14 S, 17 R)-10,13-d i m e t h y l-17-((R)-6-m e t h y l h e p t a n-2-$ yl)-2,3,4,7,8,9,10,11,12,13,14,15,16,17-tetradecahydro-1Hcyclopenta[a]phenanthren-3-yl)oxy)but-1-en-1-yl)(phenyl)silane (3aq)

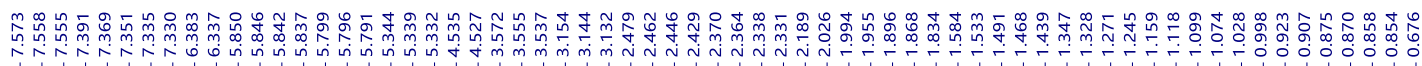
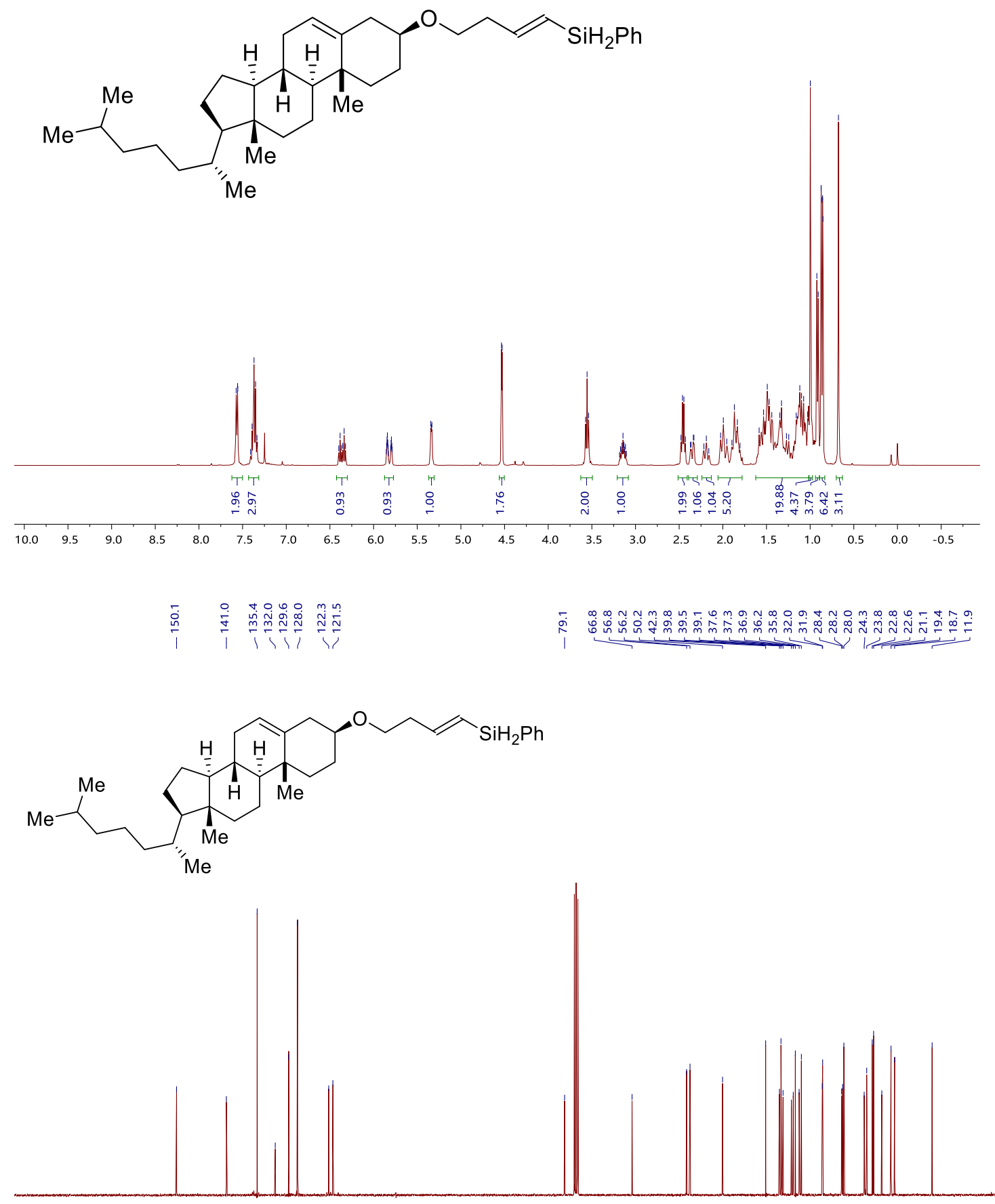

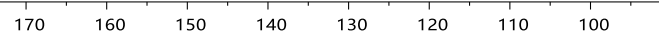

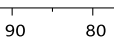


(4-(((3S,8S,9S,10R,13R,14S,17R)-10,13-dimethyl-17-((R)-6-methylheptan-2-yl)$2,3,4,7,8,9,10,11,12,13,14,15,16,17$-tetradecahydro-1H-cyclopenta[a]phenanthren3-yl)oxy)but-1-en-2-yl)(phenyl)silane (4aq)

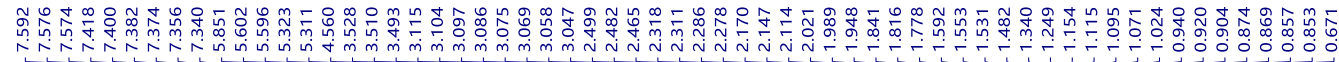
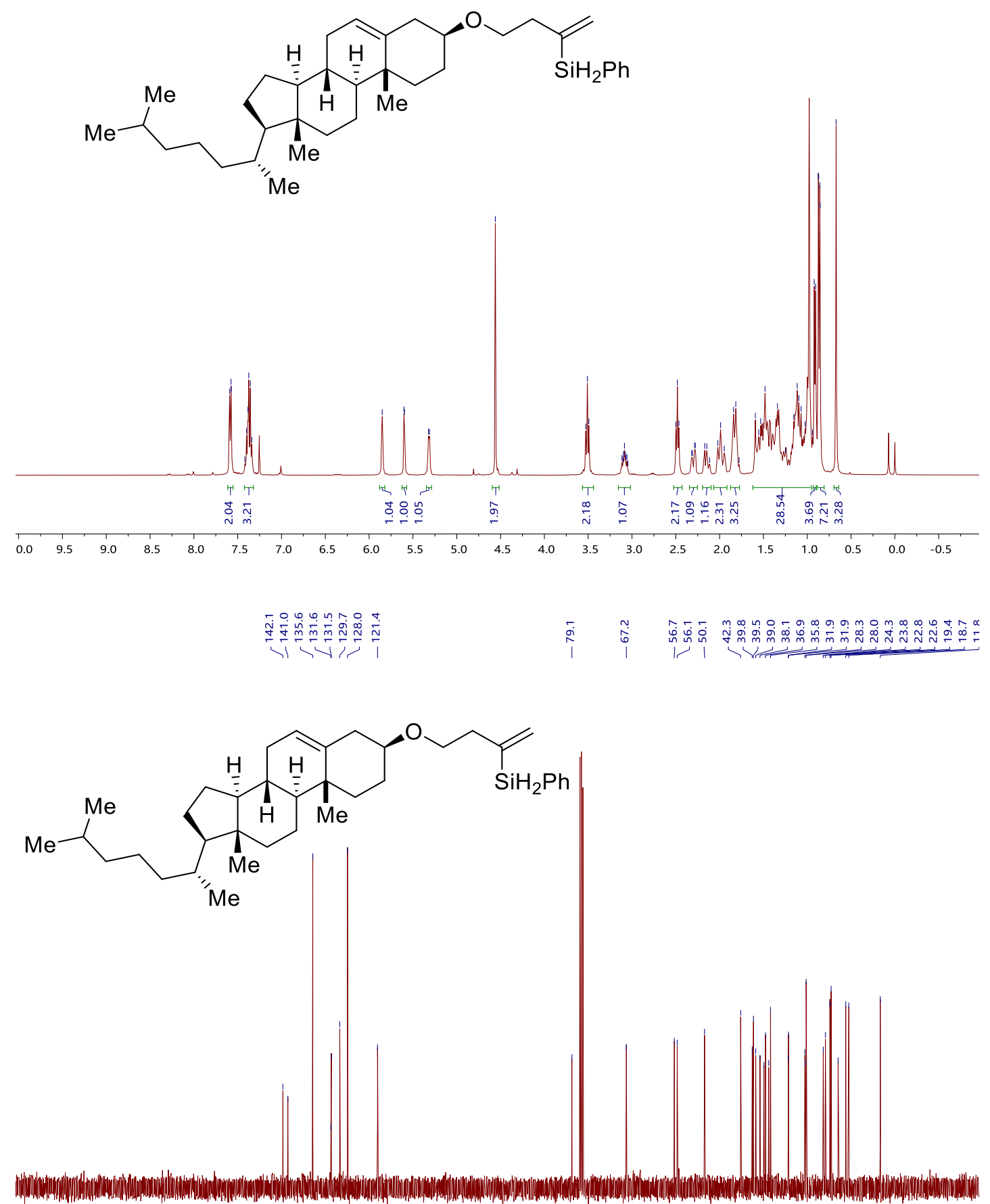

$\begin{array}{llllllllllll}00 & 190 & 180 & 170 & 160 & 150 & 140 & 130 & 120 & 110 & 100 & 90\end{array}$ 
(1E,7E)-1,8-bis(phenylsilyl)octa-1,7-diene (3ar)

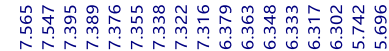

紷

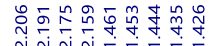

$\sqrt{n+4}$

$\mathrm{PhH}_{2} \mathrm{Si} \curvearrowright \mathrm{SiH}_{2} \mathrm{Ph}$

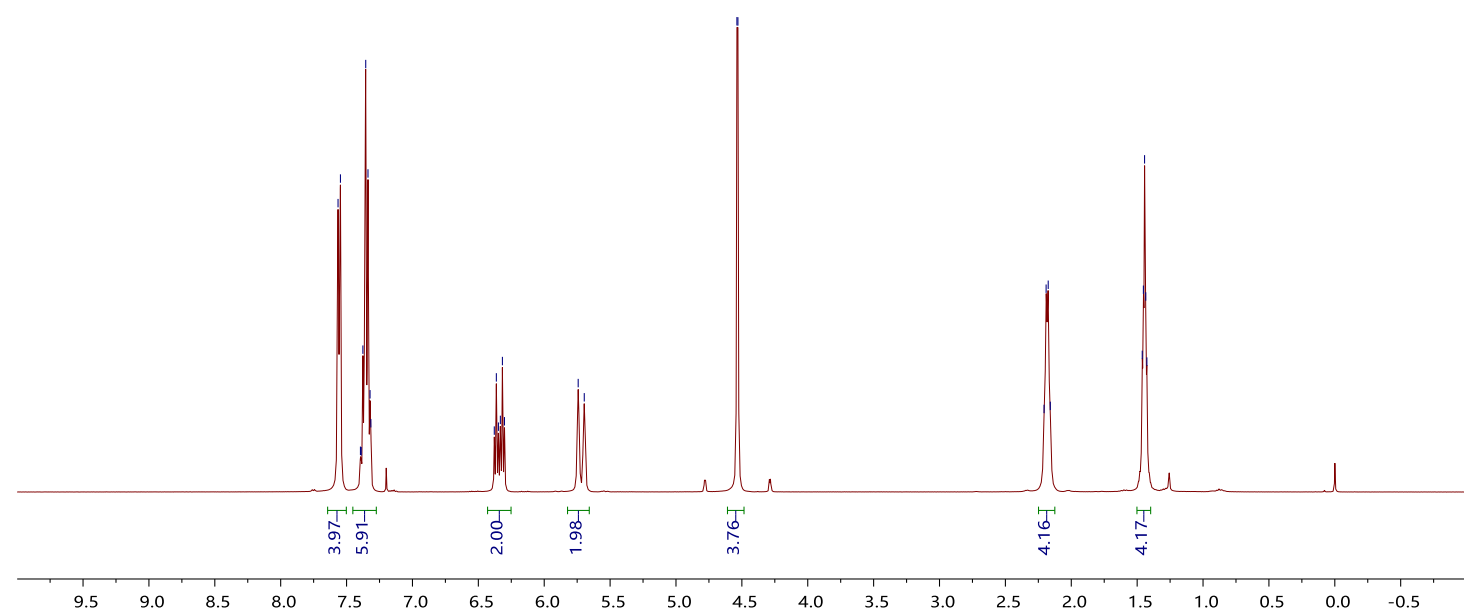

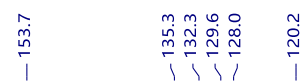

$\stackrel{\substack{i \\ i}}{i}$

$\mathrm{PhH}_{2} \mathrm{Si} \curvearrowright \mathrm{SiH}_{2} \mathrm{Ph}$
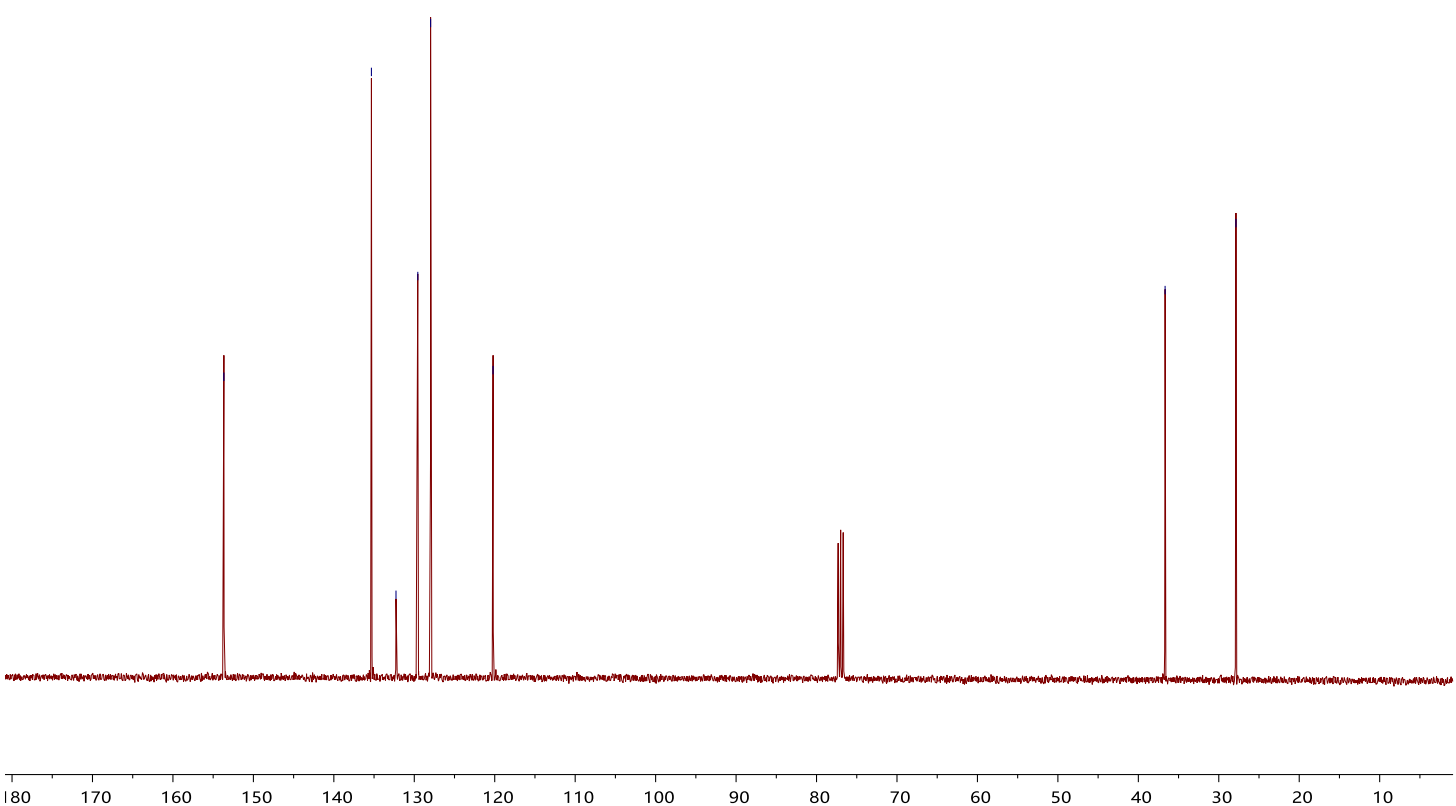

$120 \quad 110 \quad 100$

$\begin{array}{lllllllll}90 & 80 & 70 & 60 & 50 & 40 & 30 & 20 & 10\end{array}$ 
octa-1,7-diene-2,7-diylbis(phenylsilane) (4ar)

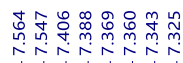

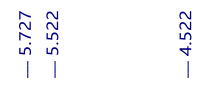

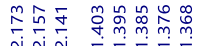

每<smiles>C=C([SiH3])CCCCC(=C)[SnH+]c1ccccc1</smiles>
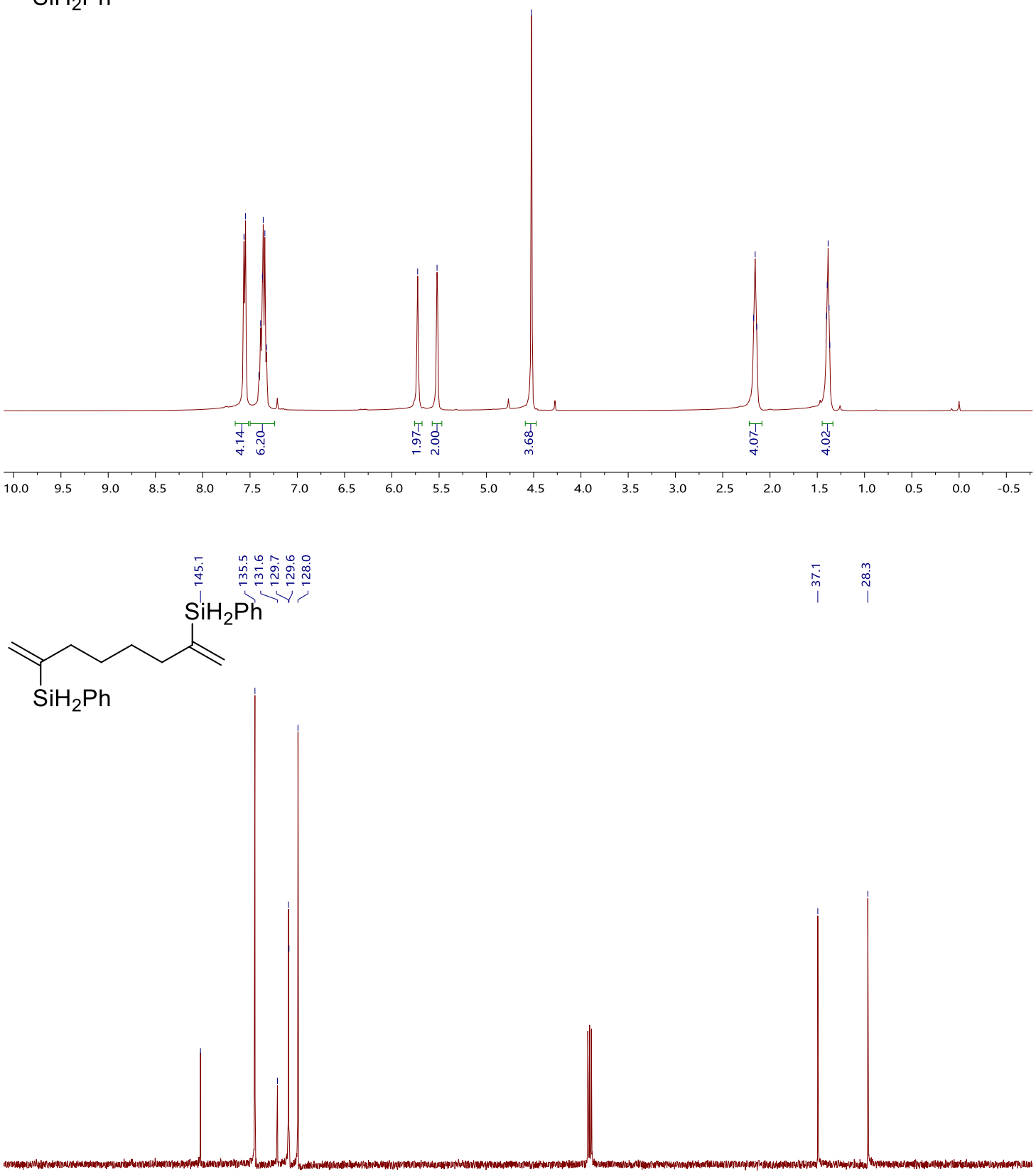

$170 \quad 1$

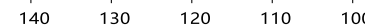

90

80

60

$30 \quad 20,10$ 


\section{(E)-phenyl(styryl)silane (3ba)}

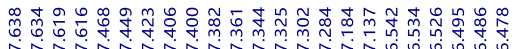

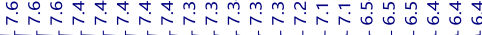

这染

$\sim \mathrm{SiH}_{2} \mathrm{Ph}$

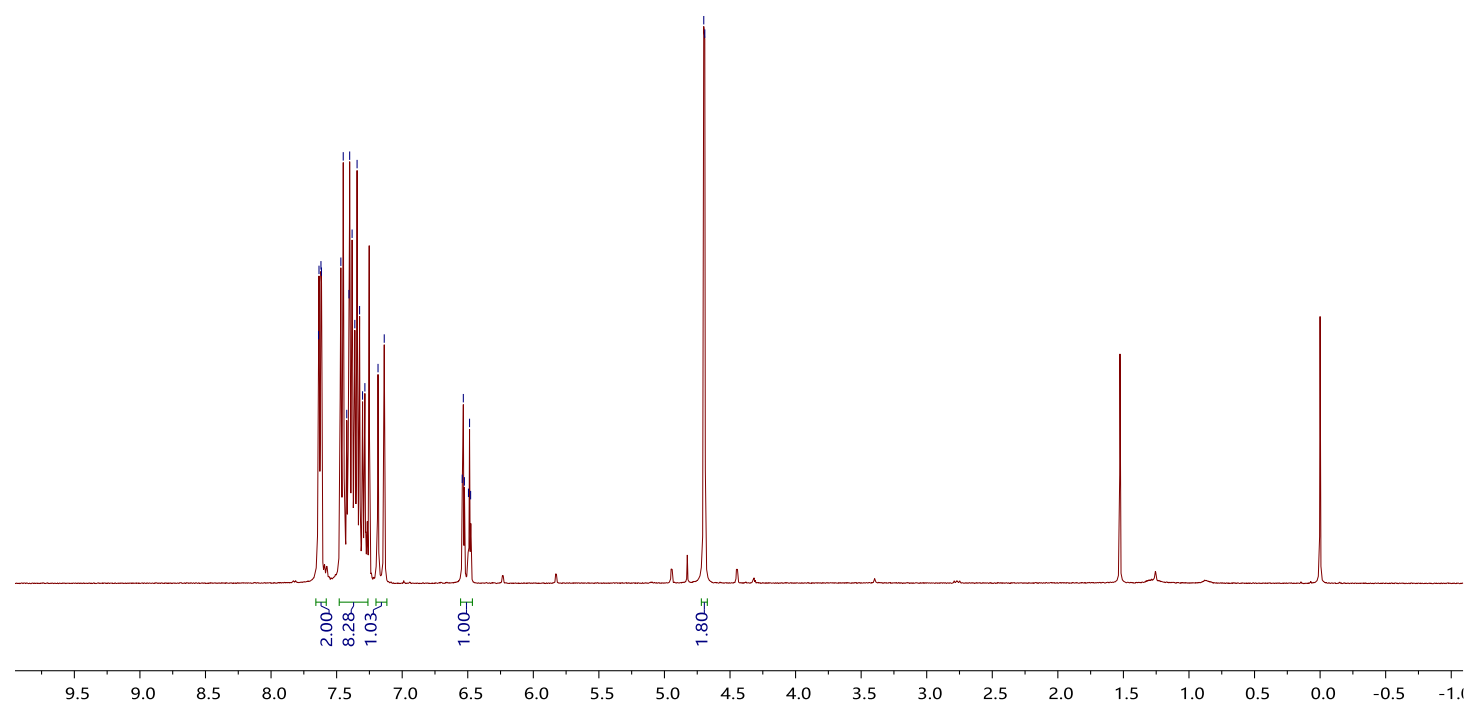

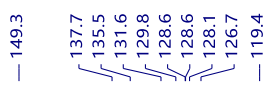

$\sim \mathrm{SiH}_{2} \mathrm{Ph}$

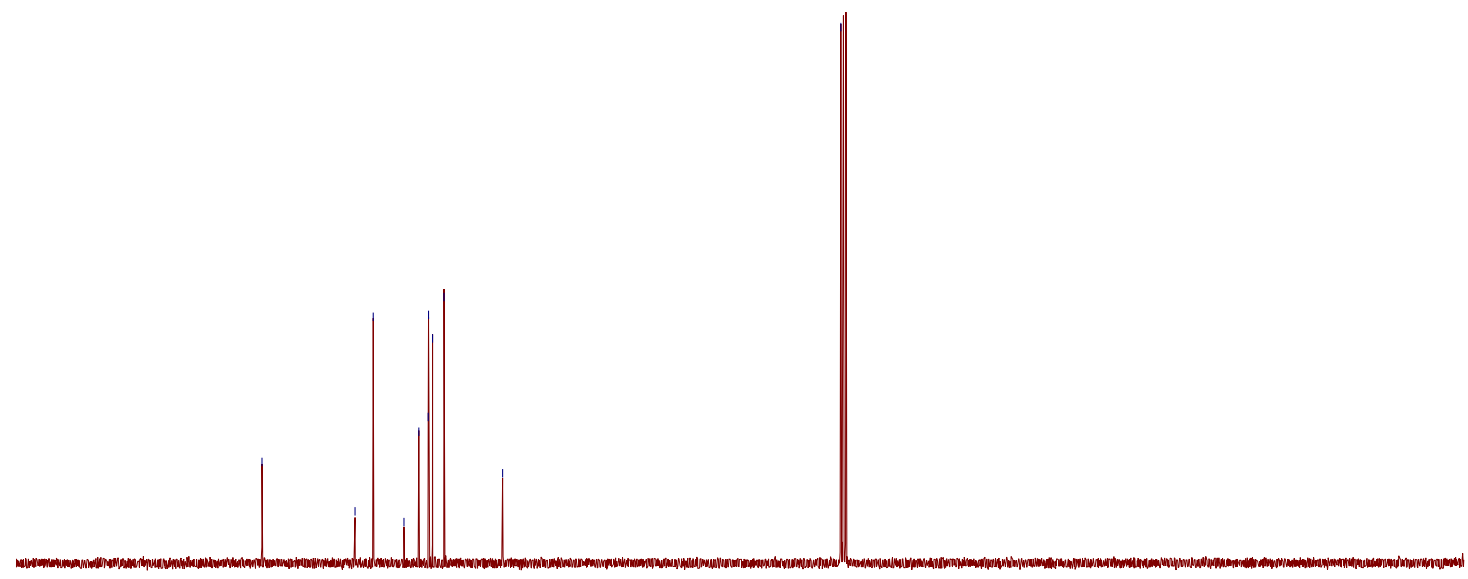

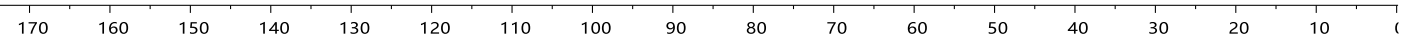


phenyl(1-phenylvinyl)silane (4ba)

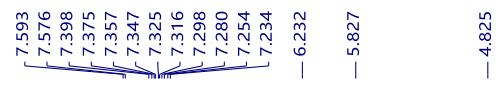

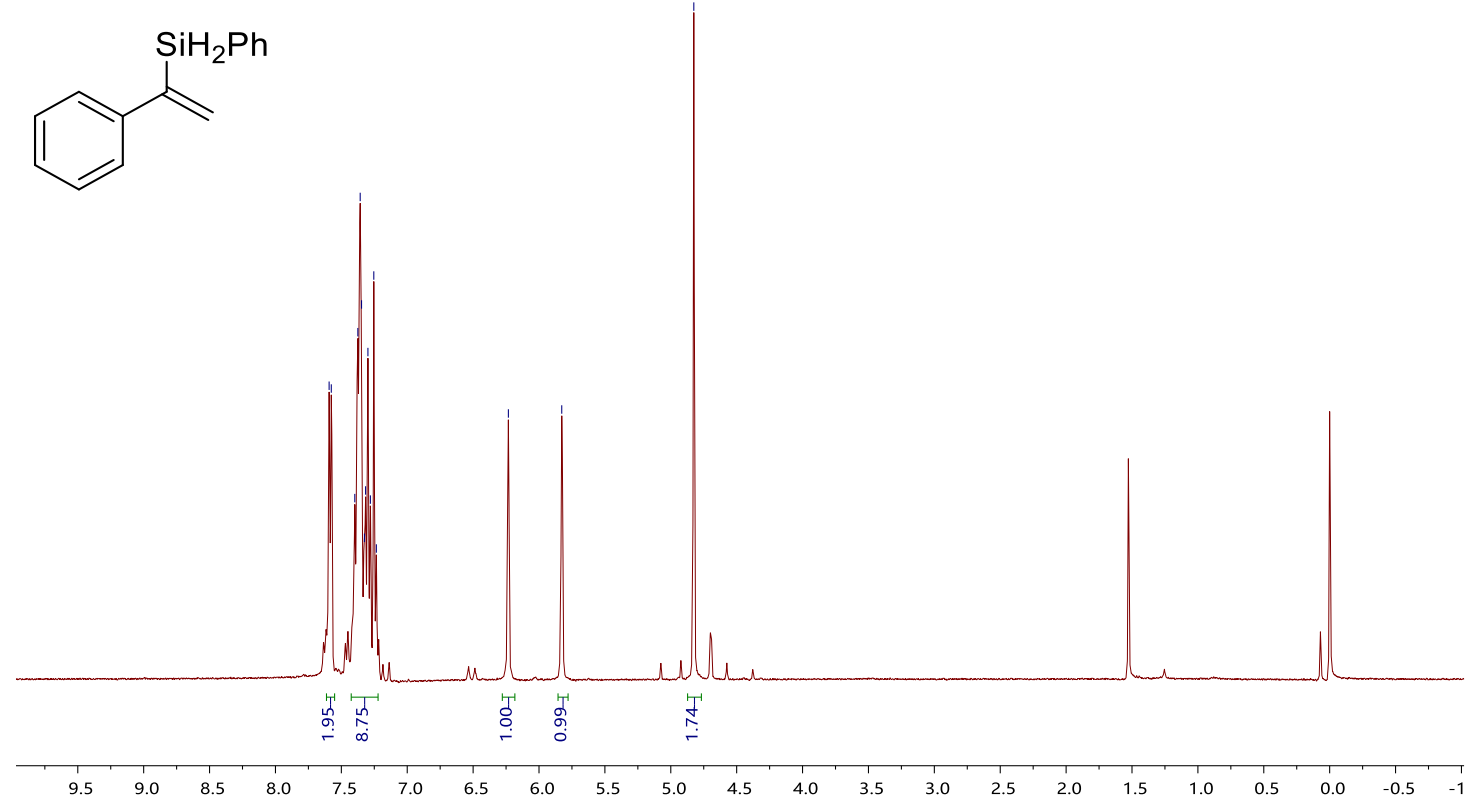

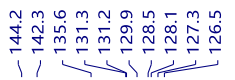<smiles>C=C([SiH2]c1ccccc1)c1ccccc1</smiles>

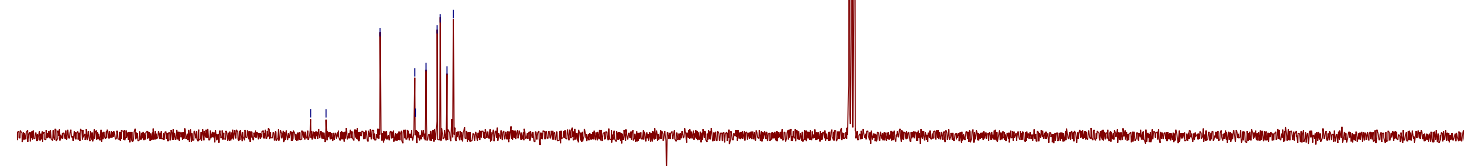

80

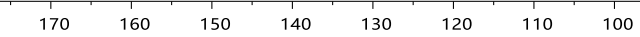

$90 \quad 80$

$70 \quad 60$

50

$30 \quad 20 \quad 10$ 


\section{(E)-(2-methylstyryl)(phenyl)silane (3bb)}

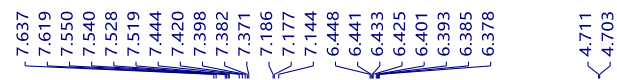<smiles>Cc1ccccc1/C=C/Sc1ccccc1</smiles>

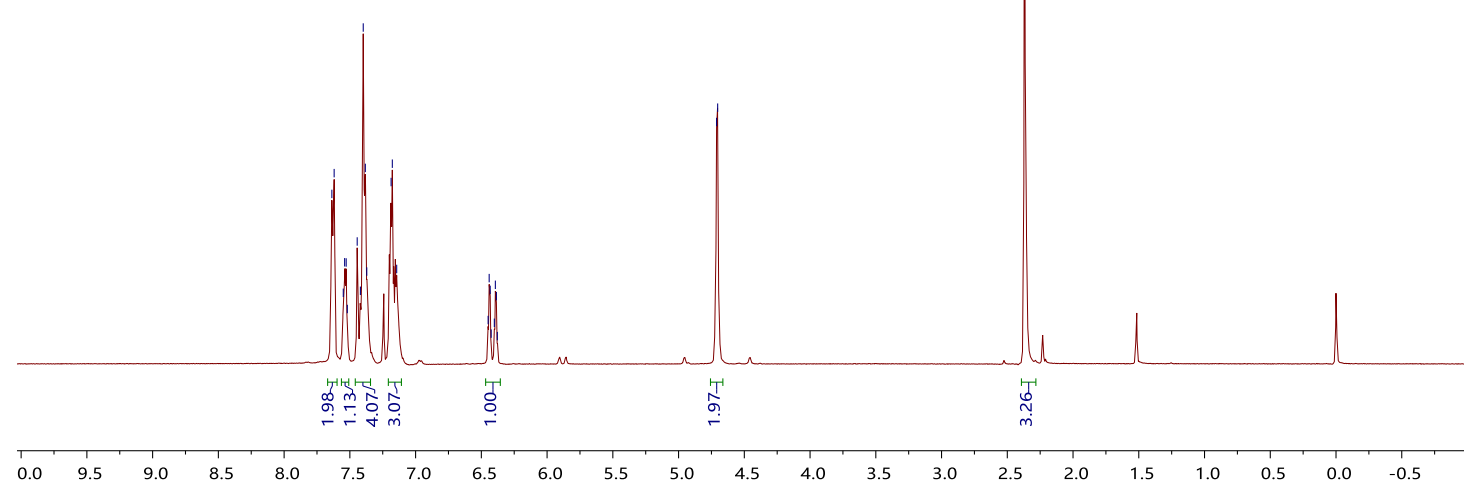

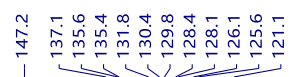<smiles>Cc1ccccc1/C=C/Sc1ccccc1</smiles>

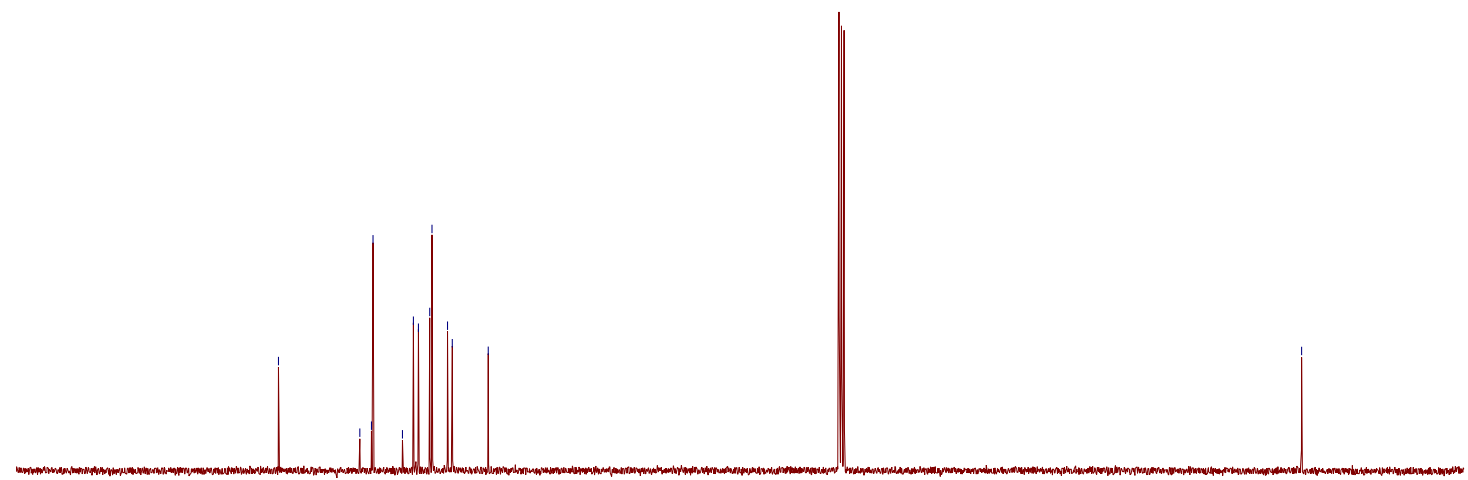

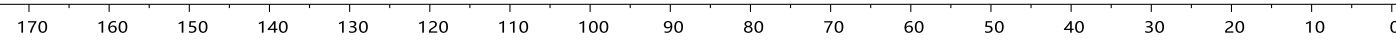


phenyl(1-(o-tolyl)vinyl)silane (4bb)
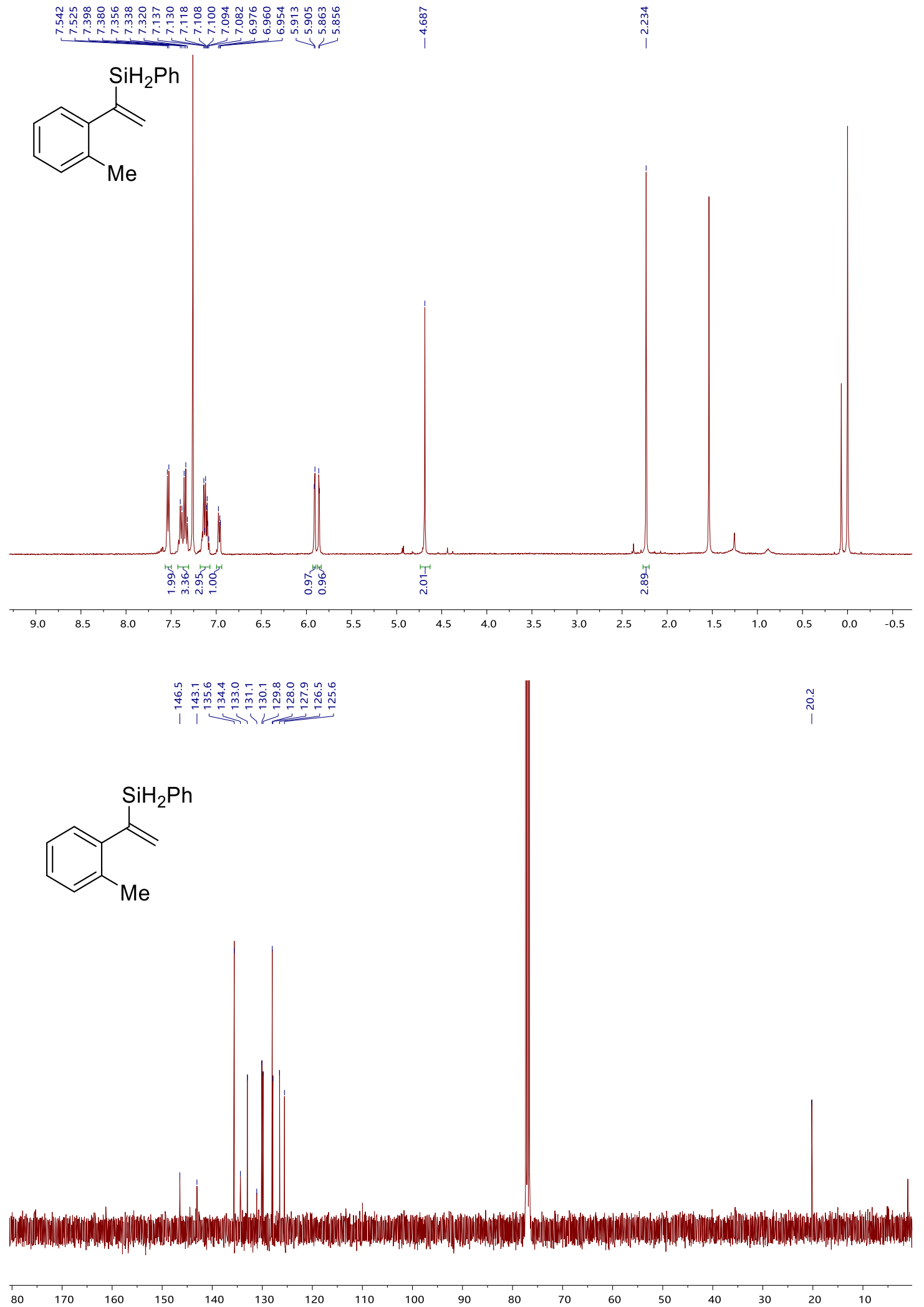


\section{(E)-(2-fluorostyryl)(phenyl)silane (3bc)}
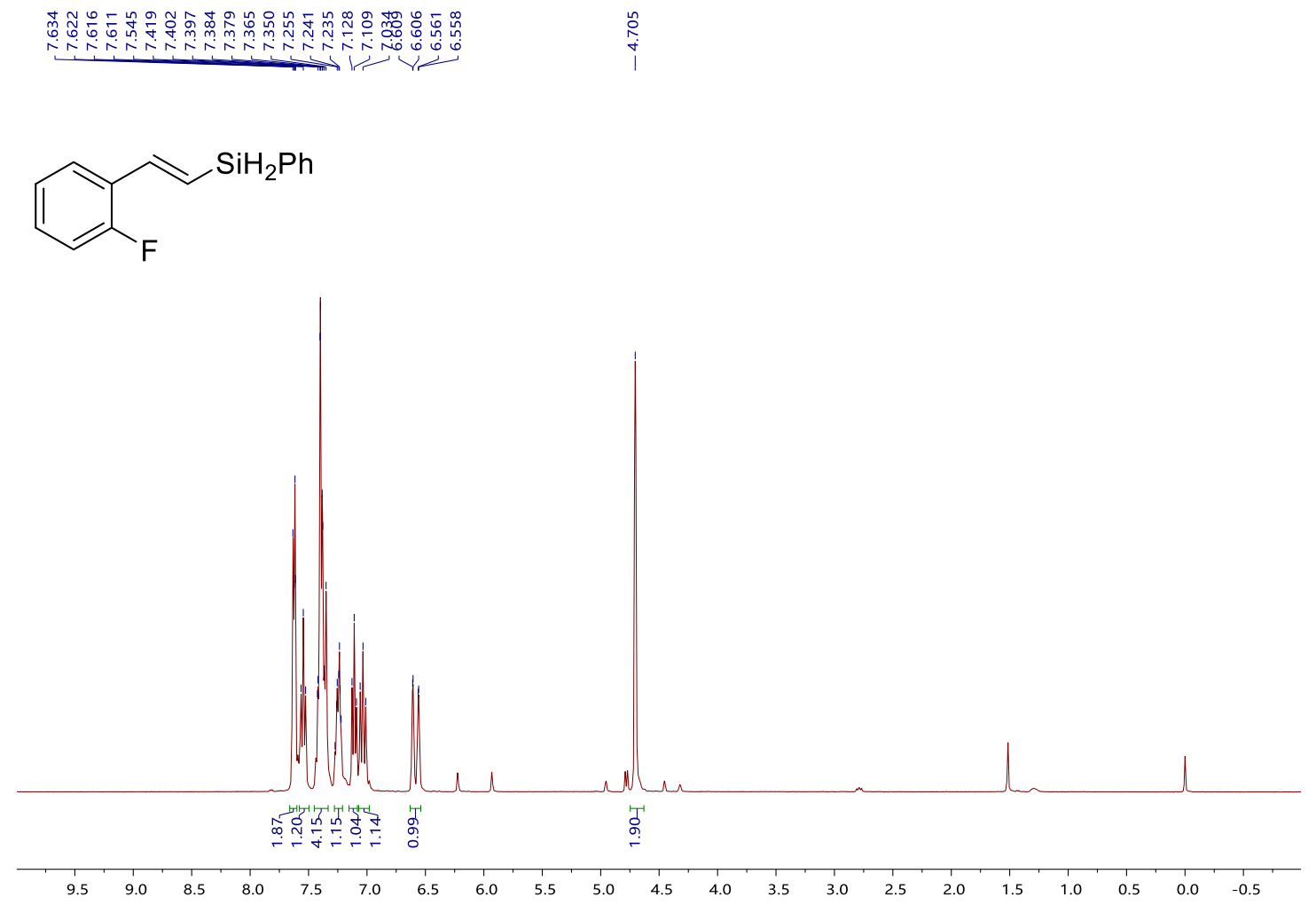

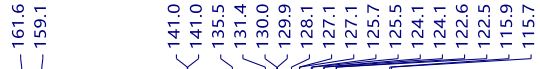
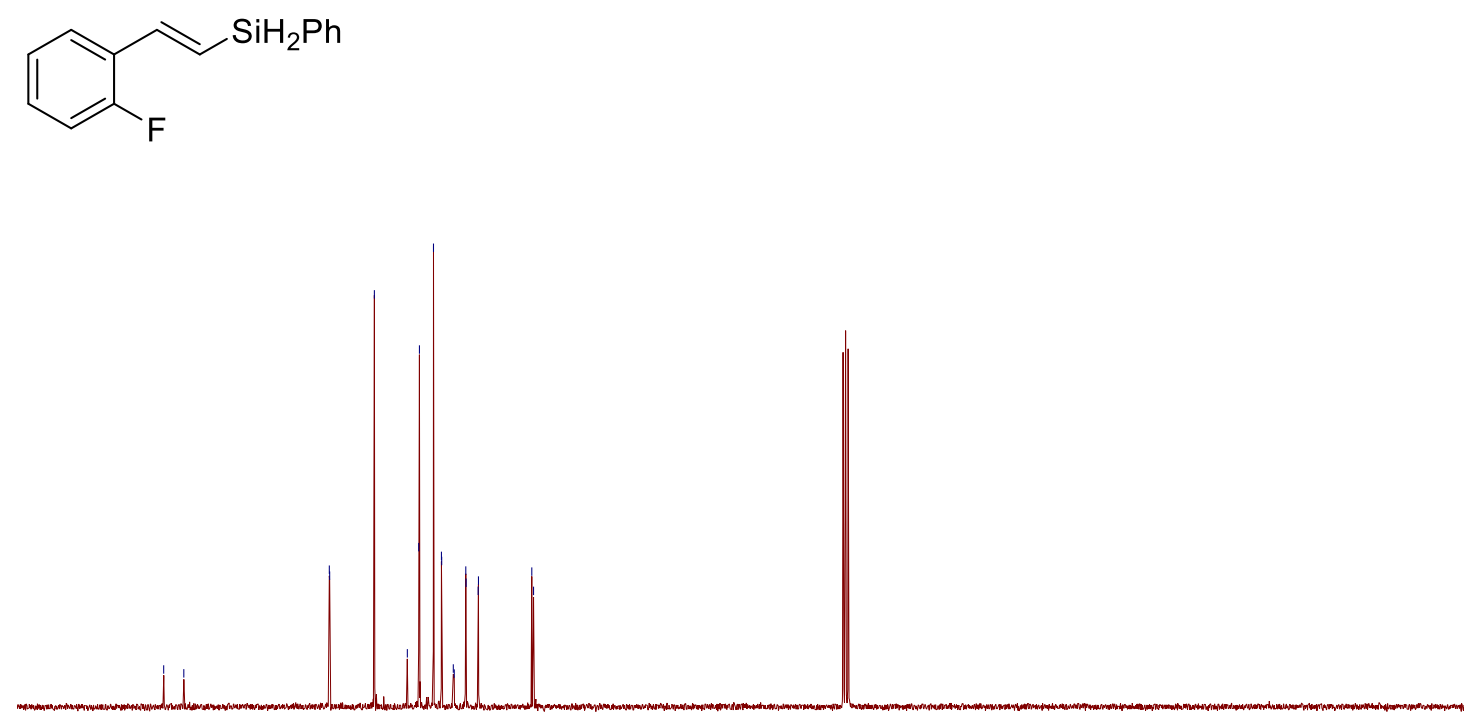


\section{(1-(2-fluorophenyl)vinyl)(phenyl)silane (4bc)}

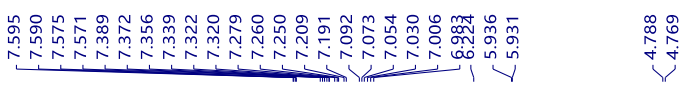<smiles>C=C([SnH2])c1ccccc1F</smiles>

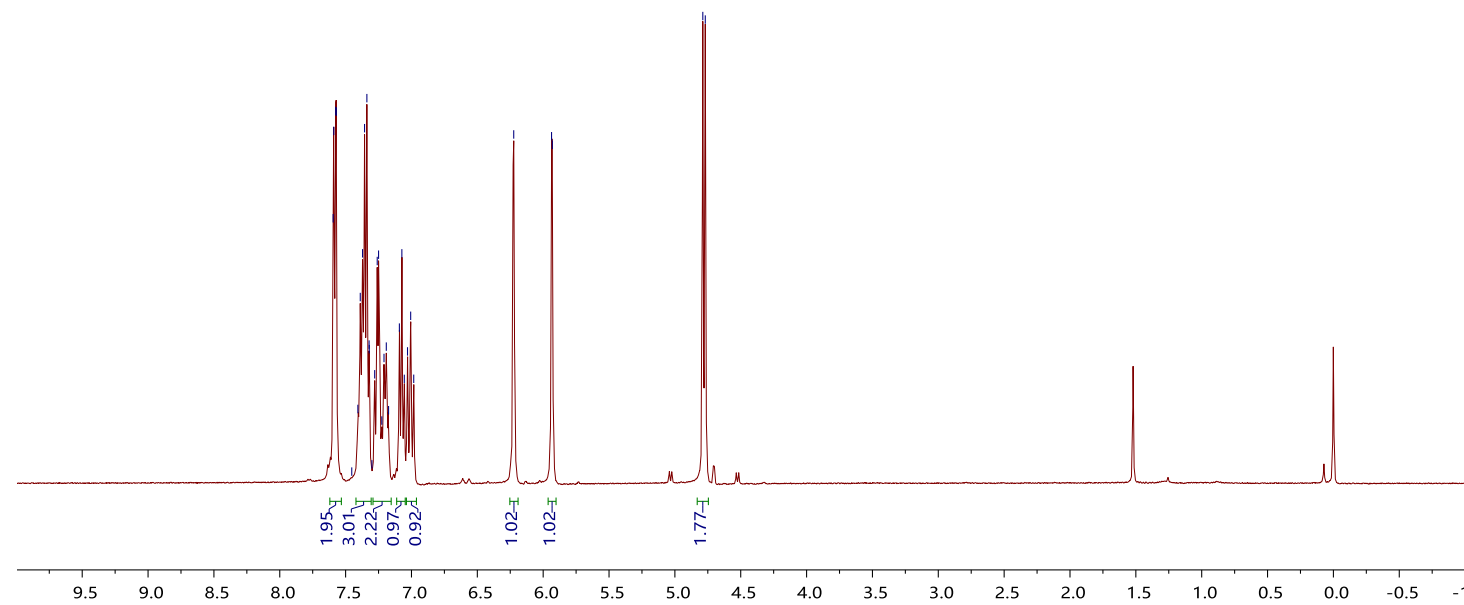<smiles>C=C([SnH2])c1ccccc1F</smiles>

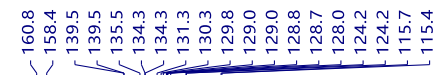

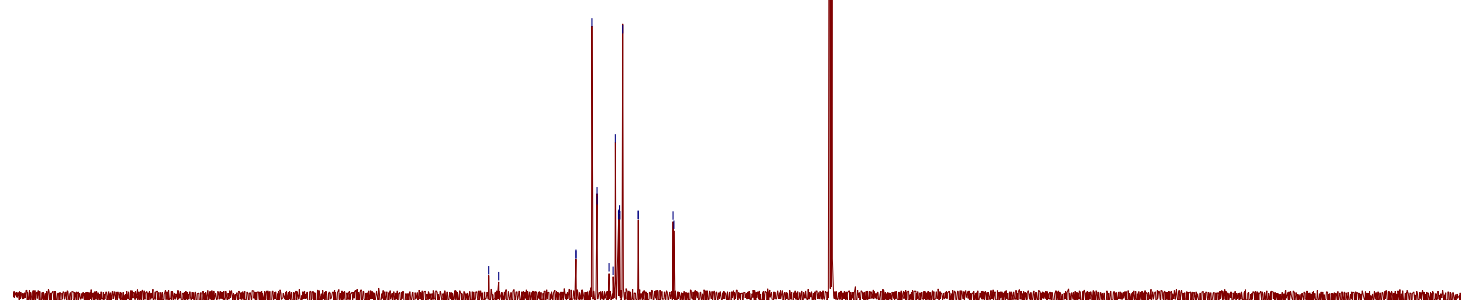


(E)-(2-methoxystyryl)(phenyl)silane (3bd)

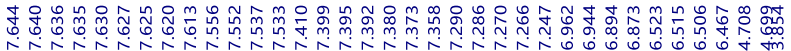

$\overbrace{\mathrm{OMe}}^{\mathrm{SiH}_{2} \mathrm{Ph}}$

$\overbrace{\mathrm{OMe}}^{\mathrm{SiH}_{2} \mathrm{Ph}}$

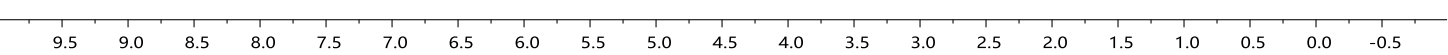

l l

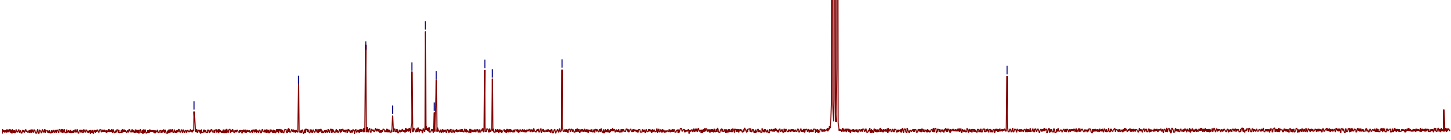

180

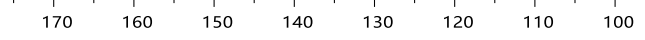

$90 \quad 80$ 
(1-(2-methoxyphenyl)vinyl)(phenyl)silane (4bd)

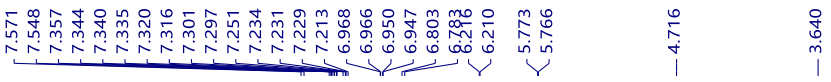<smiles>C=C([SH+]c1ccccc1)c1ccccc1OC</smiles>

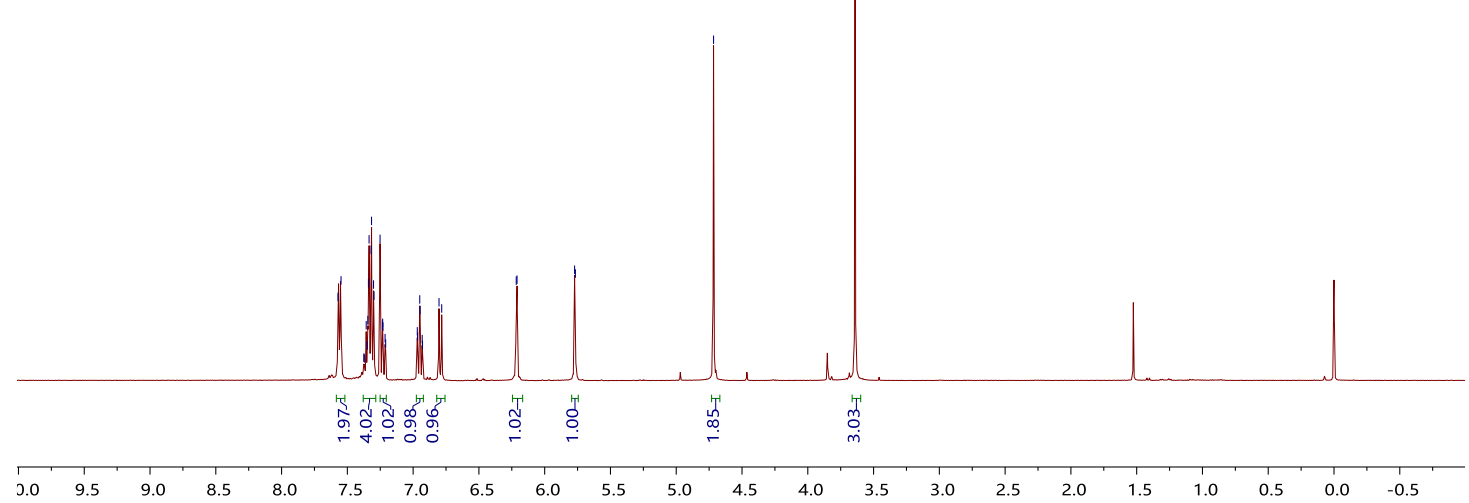

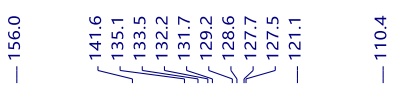<smiles>C=C([SH+]c1ccccc1)c1ccccc1OC</smiles>

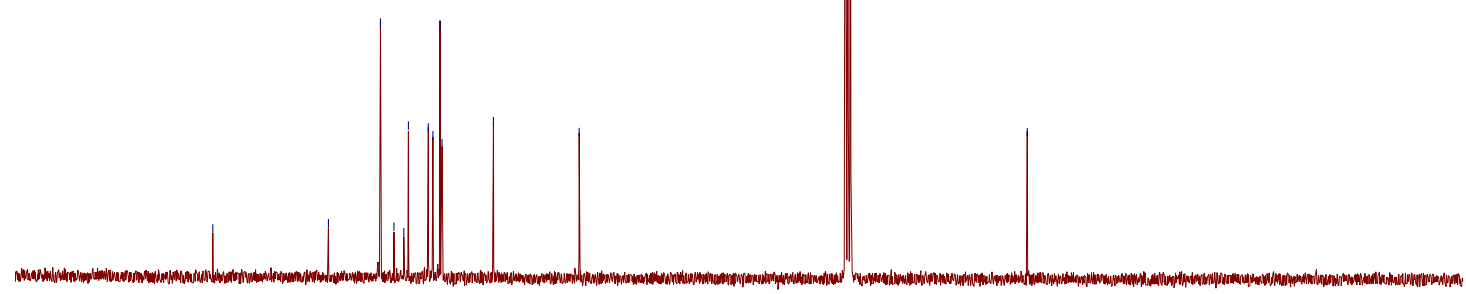

80

$90 \quad 80$ 
(E)-(3-methylstyryl)(phenyl)silane (3be)
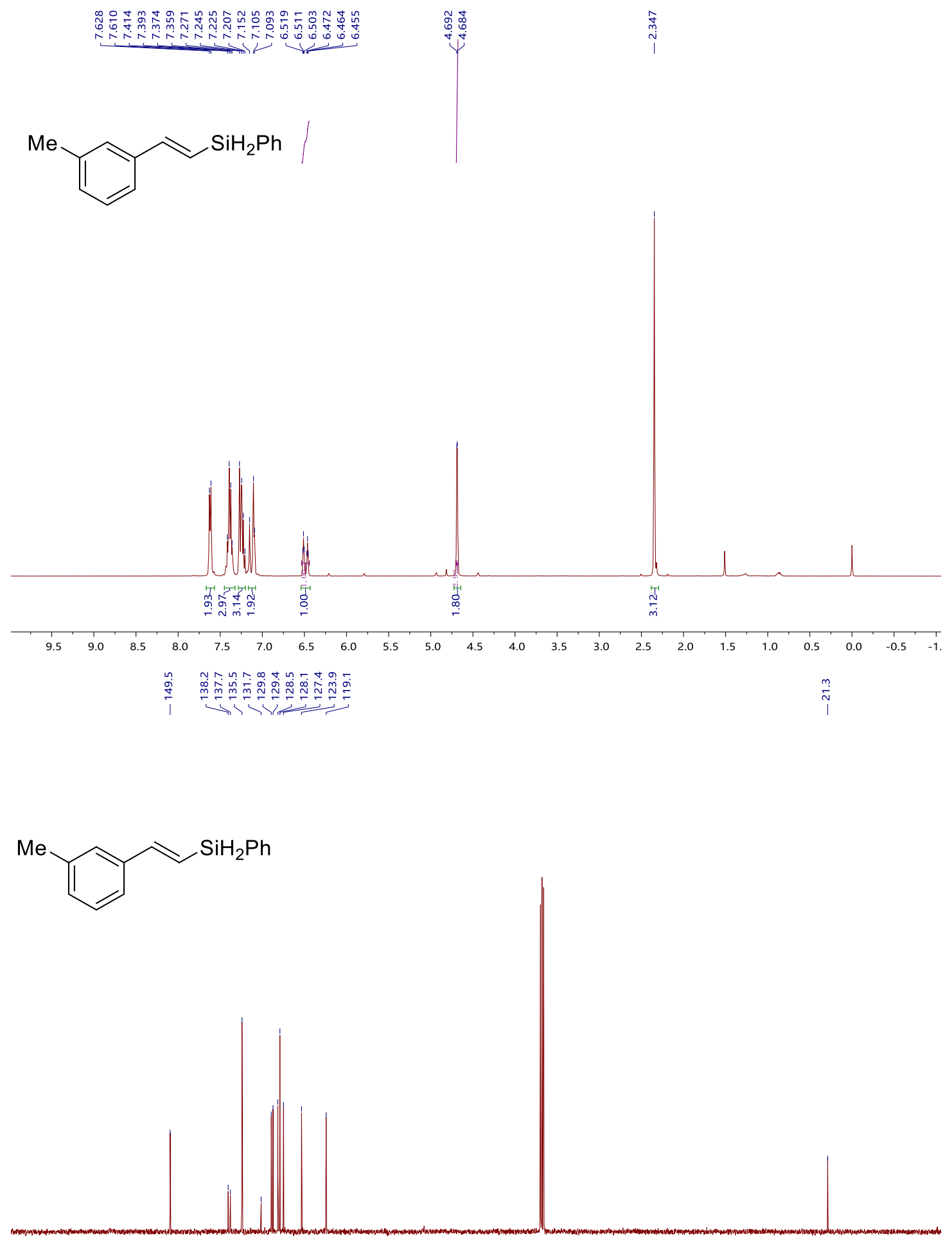

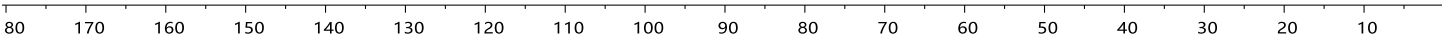


phenyl(1-(m-tolyl)vinyl)silane (4be)

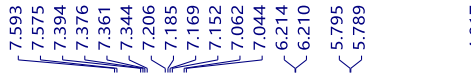<smiles>C=C([SH+]c1ccccc1)c1cccc(C)c1</smiles>
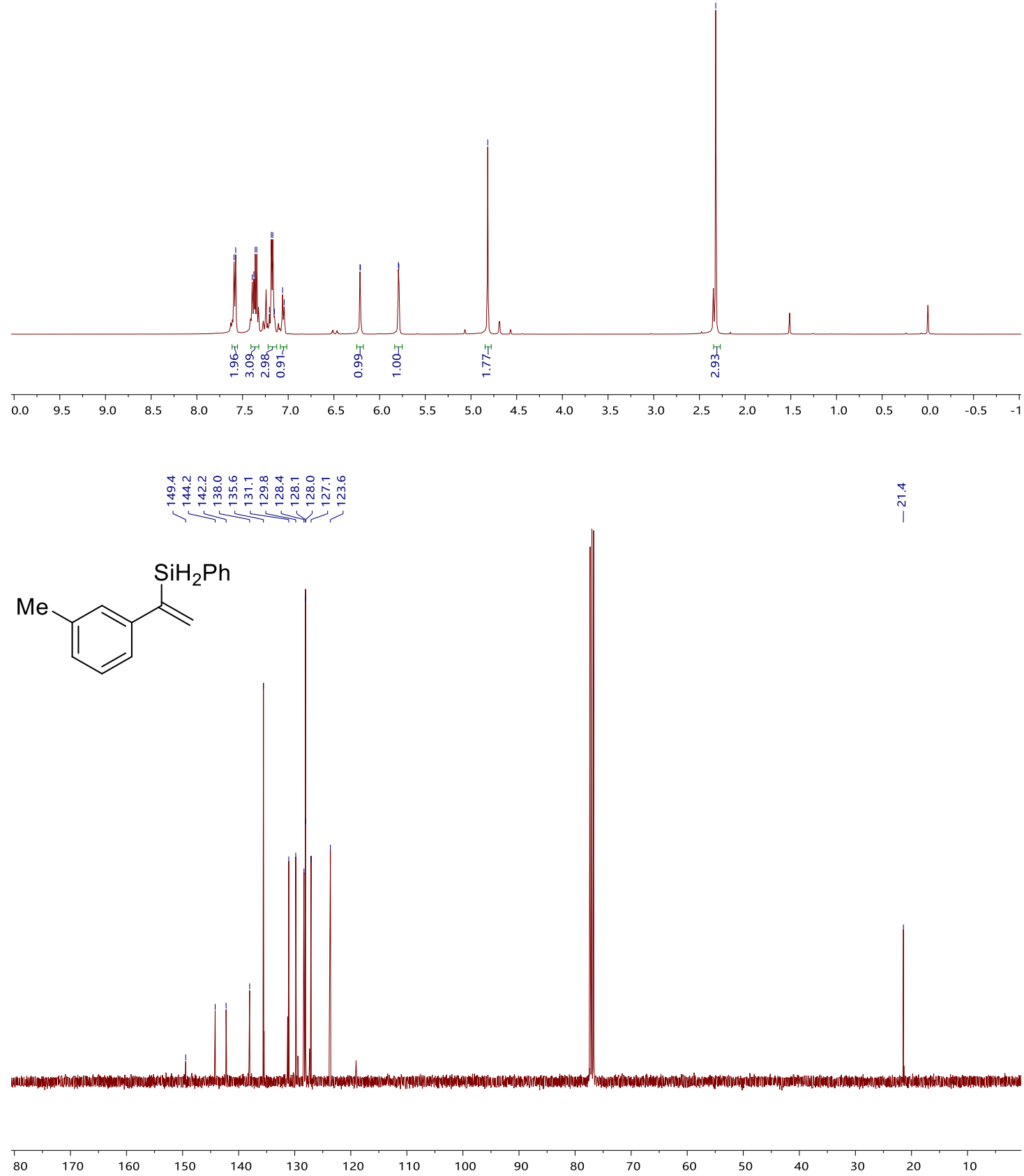
<smiles>C(=C/[SH+](c1ccccc1)c1ccc2ccccc2c1)\c1ccccc1</smiles>

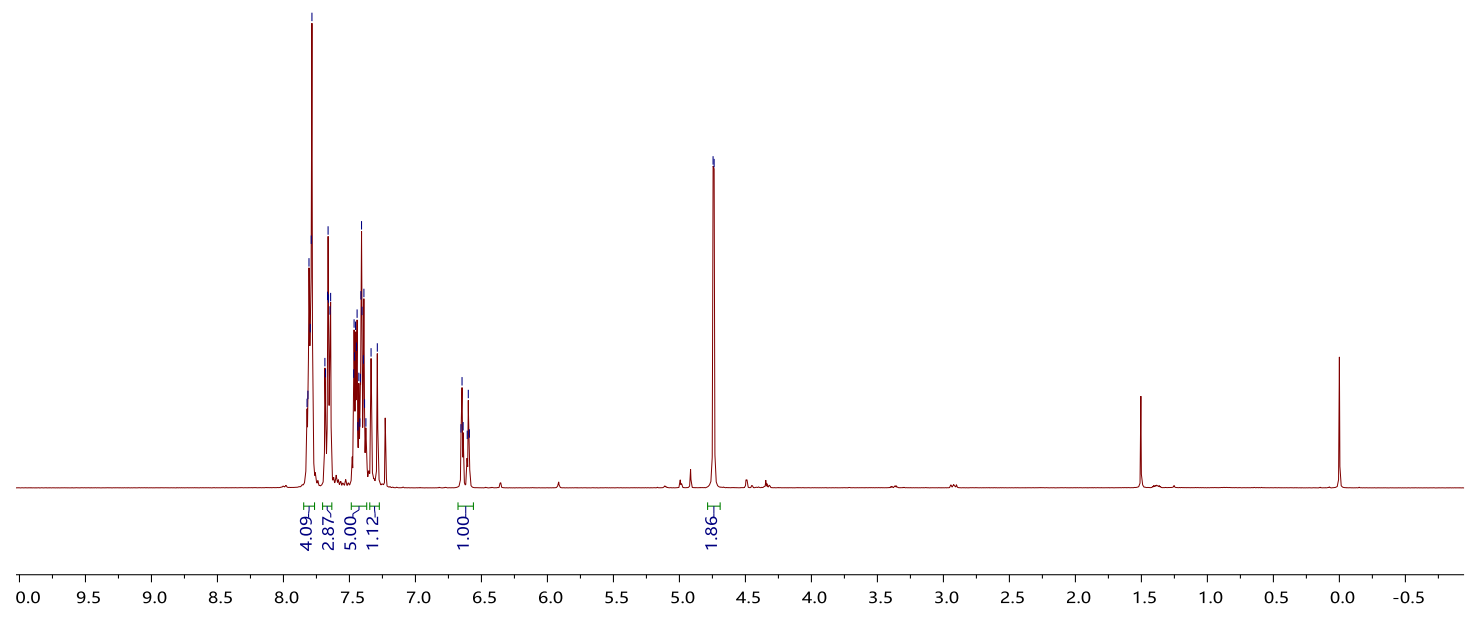

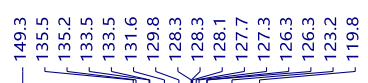<smiles>C(=C/c1ccccc1)\[SH+]c1ccc2ccccc2c1</smiles>
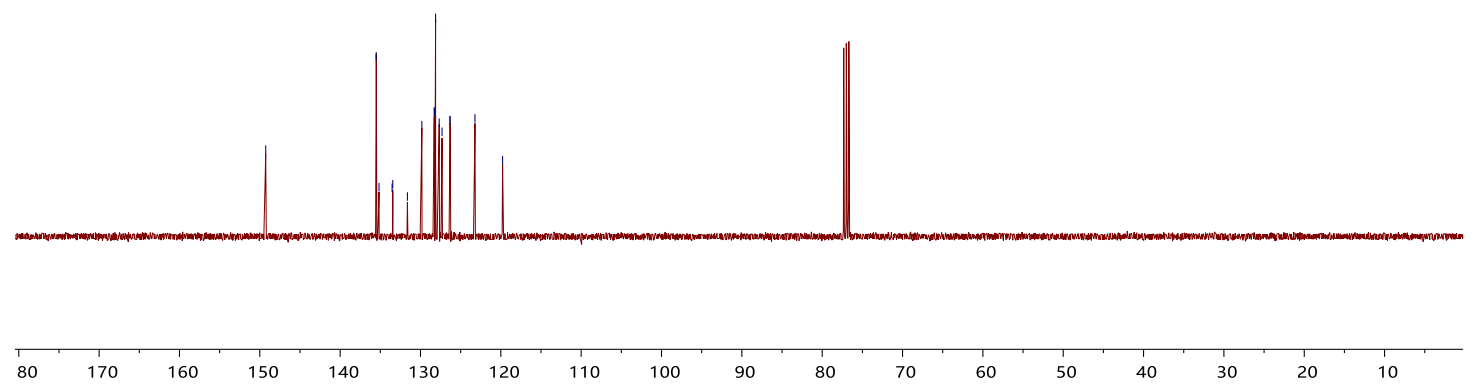
(1-(naphthalen-2-yl)vinyl)(phenyl)silane (4bf)

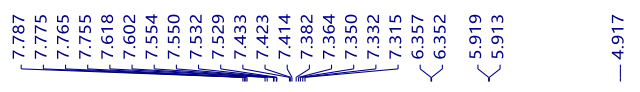<smiles>C=C(c1ccccc1)c1ccc2ccccc2c1</smiles>

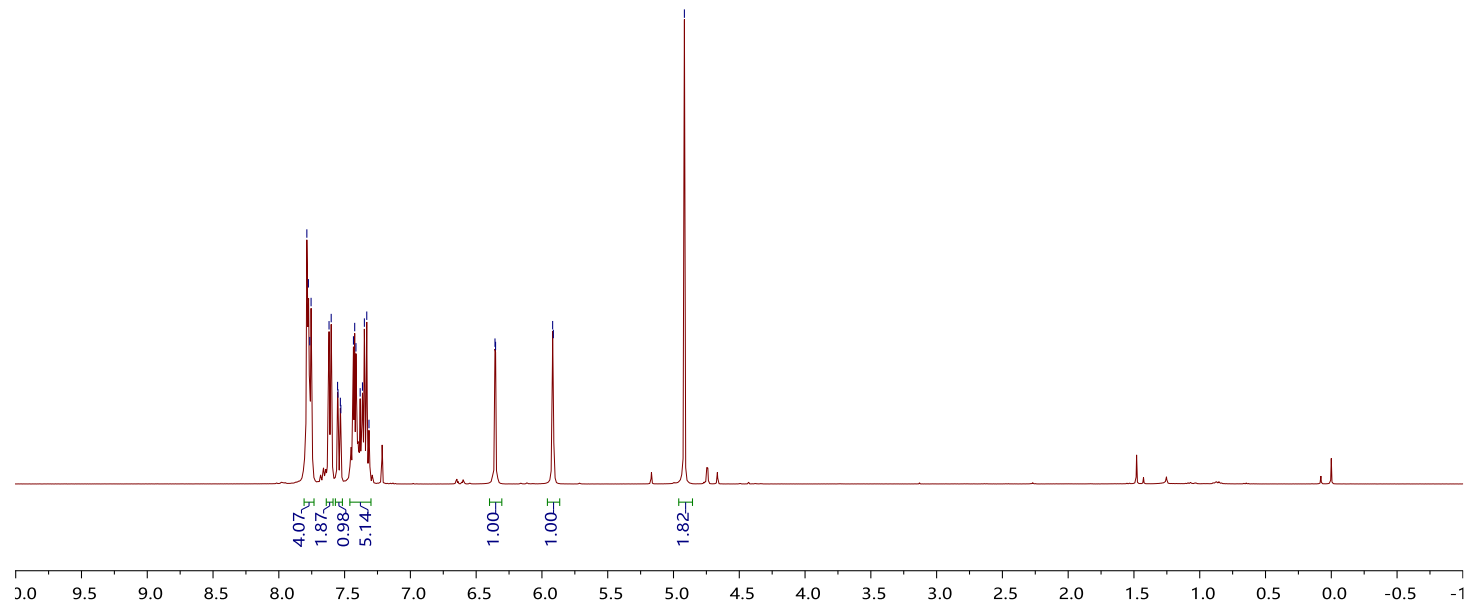

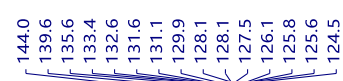<smiles>C=C([SnH]c1ccccc1)c1ccc2ccccc2c1</smiles>
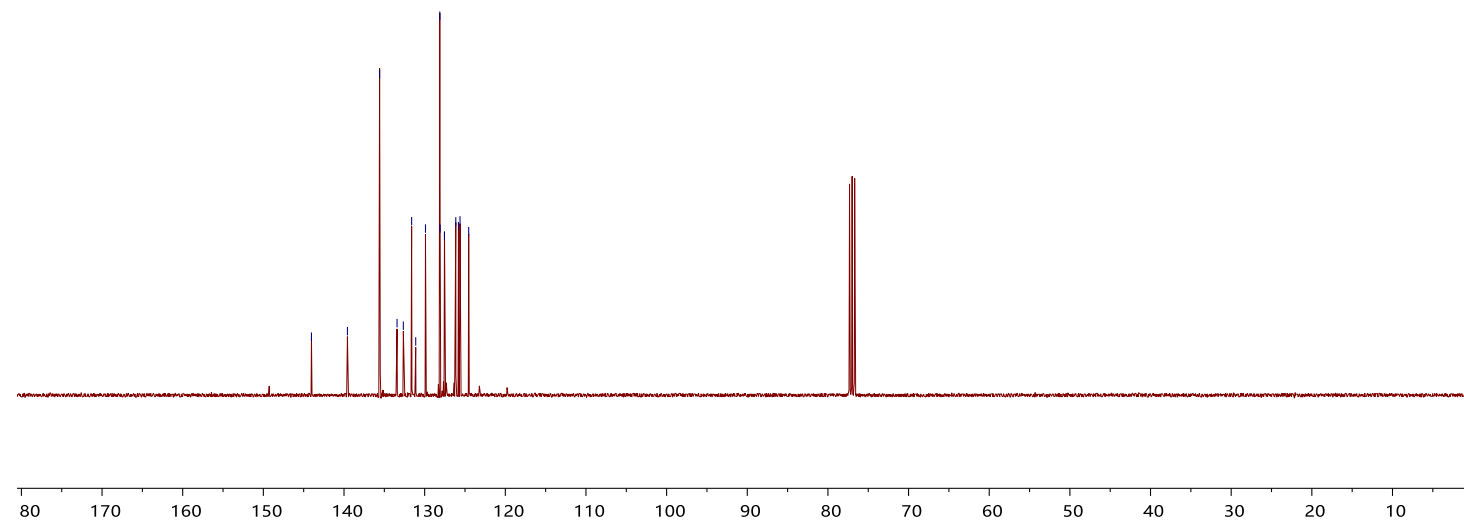
(E)-(2-(ferrocenylvinyl))(phenyl)silane (3bg)

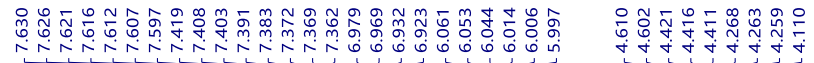

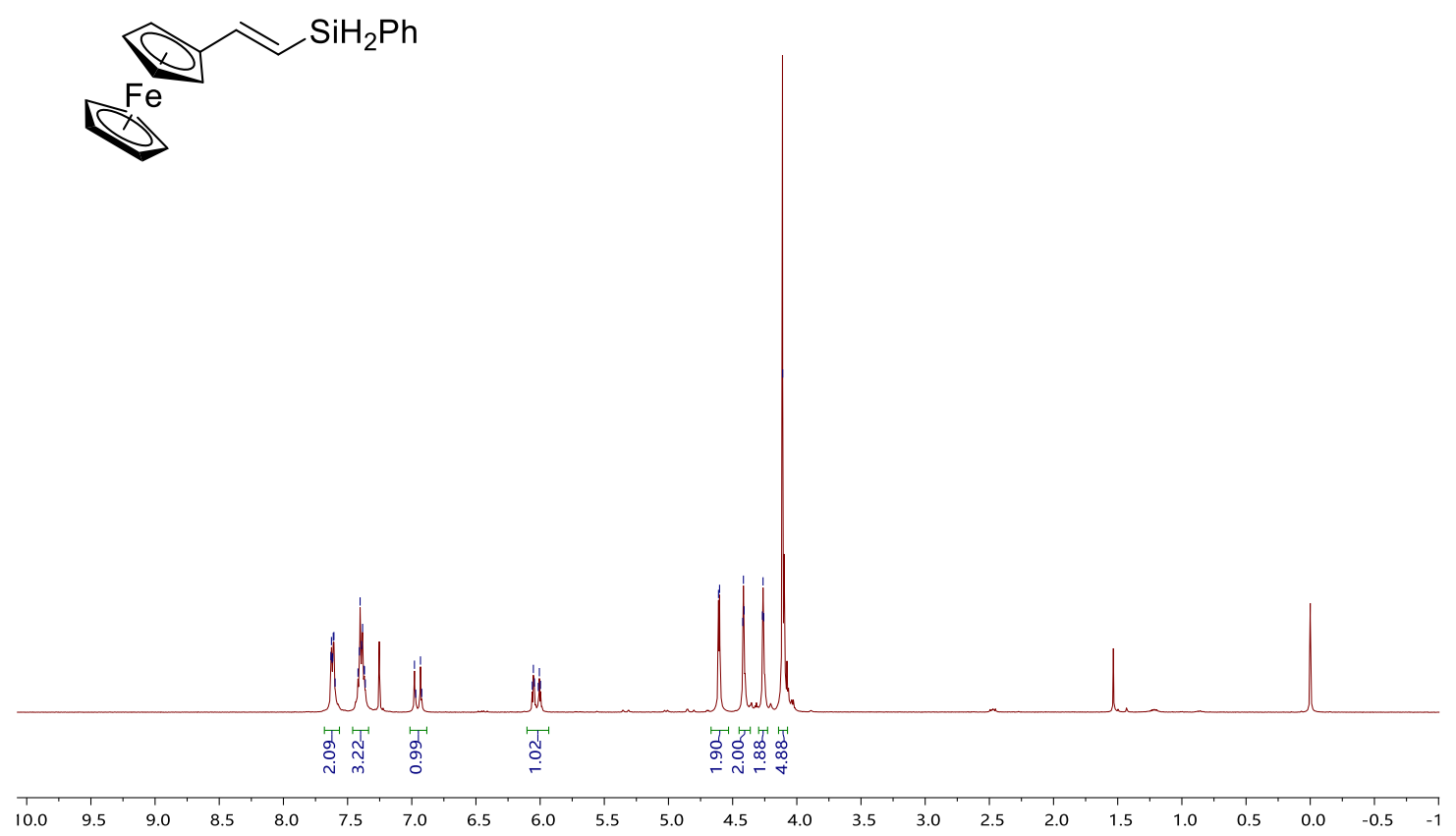

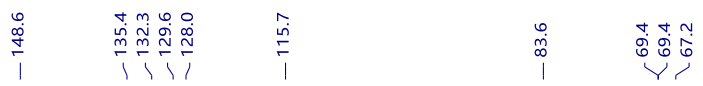

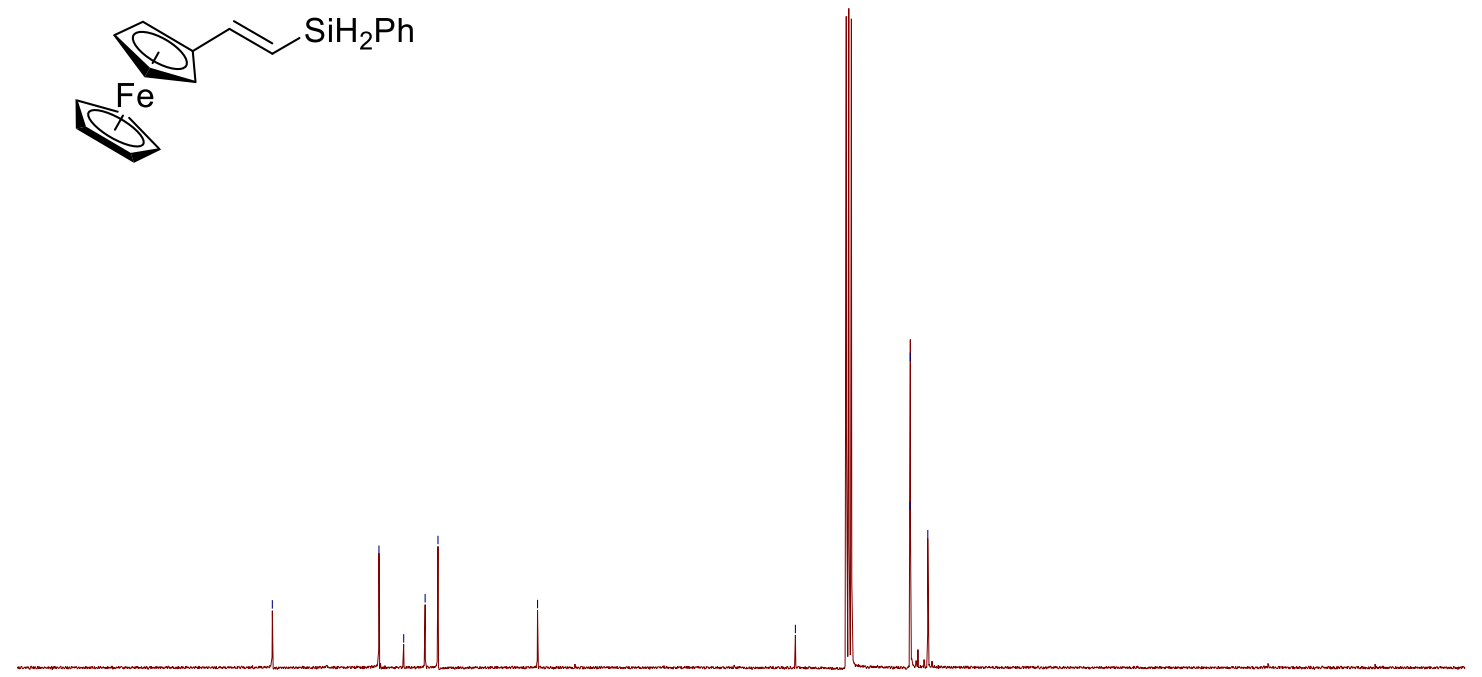

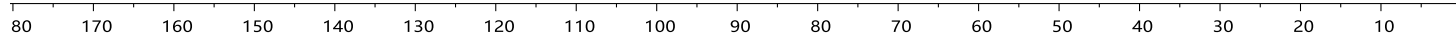


(1-(ferrocenylvinyl))(phenyl)silane (4bg)

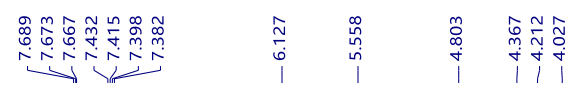
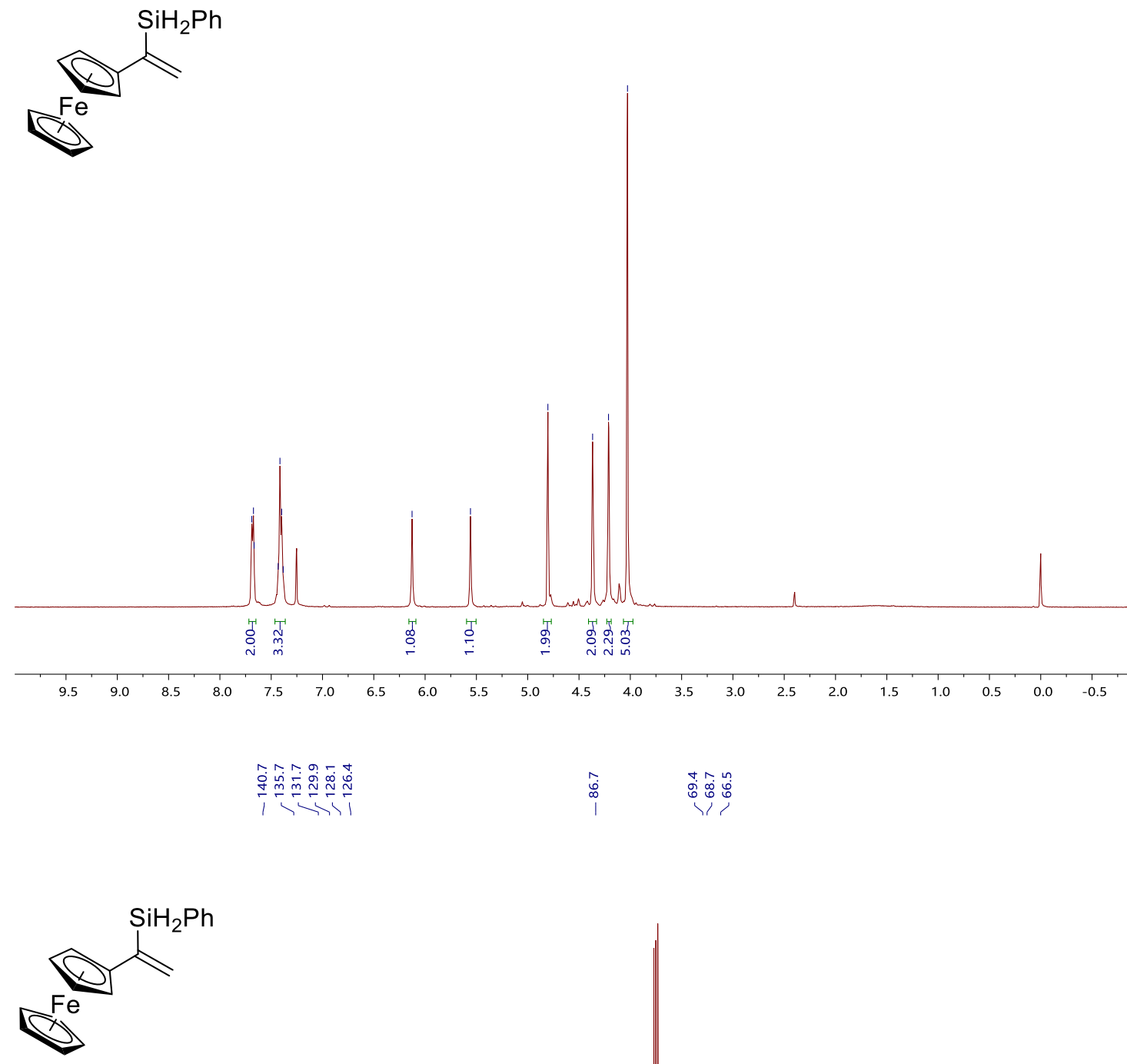
(E)-(3-methylbuta-1,3-dien-1-yl)(phenyl)silane (3ca)

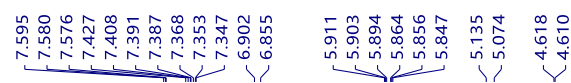

$\overbrace{\mathrm{SiH}_{2} \mathrm{Ph}}^{\mathrm{Me}}$

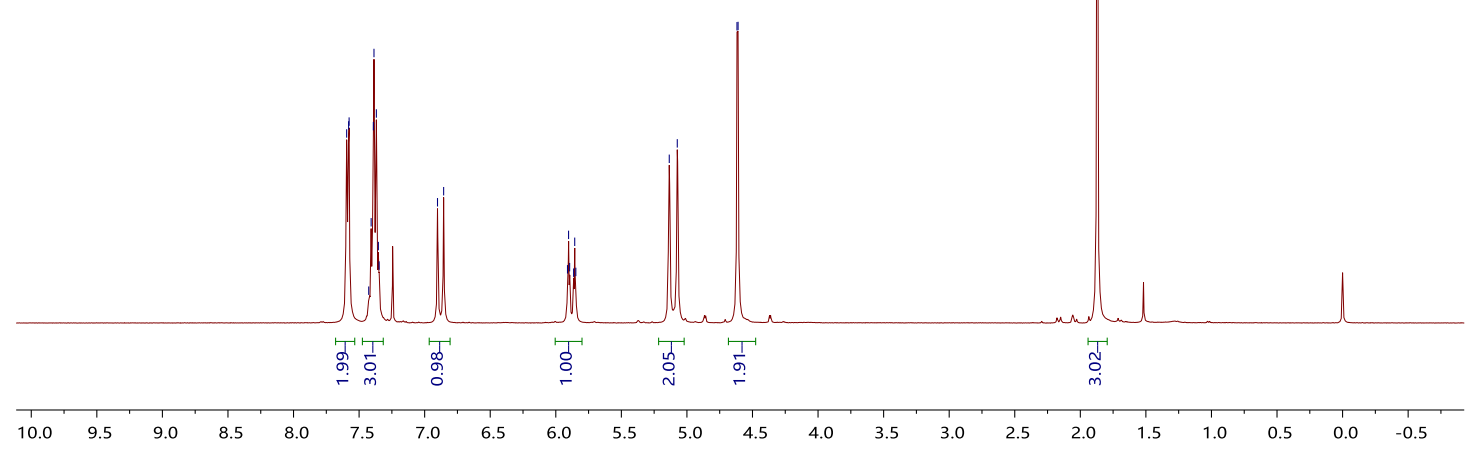

$$
\text { I }
$$

$\overbrace{\mathrm{SiH}_{2} \mathrm{Ph}}^{\mathrm{Me}}$

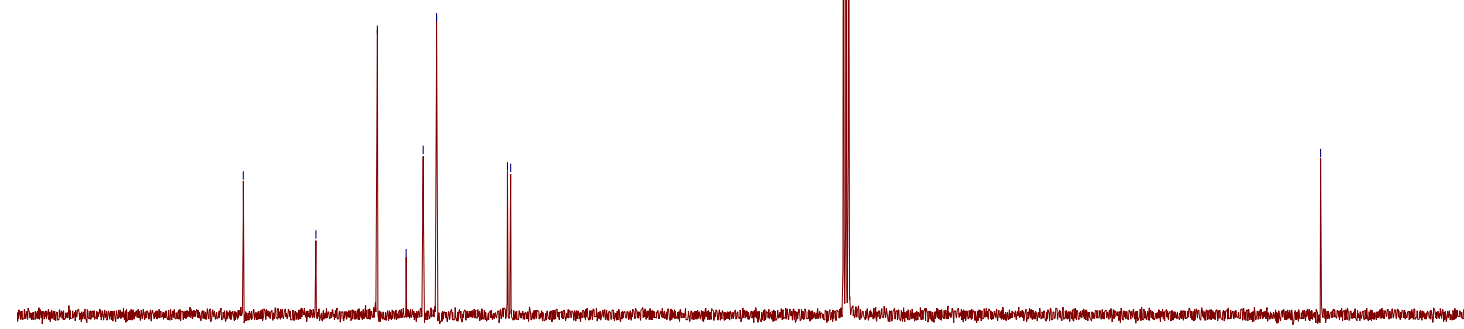

80

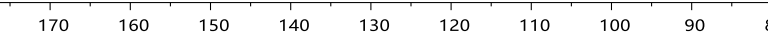

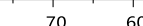

$\begin{array}{lllll}50 & 40 & 30 & 20 & 10\end{array}$ 
(3-methylbuta-1,3-dien-2-yl)(phenyl)silane (4ca)

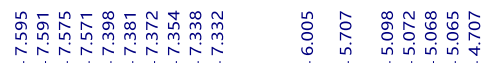<smiles>C=C(C)C(=C)[SnH2]c1ccccc1</smiles>

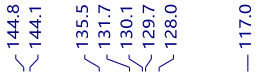<smiles>C=C(C)C(=C)[SnH2]c1ccccc1</smiles>

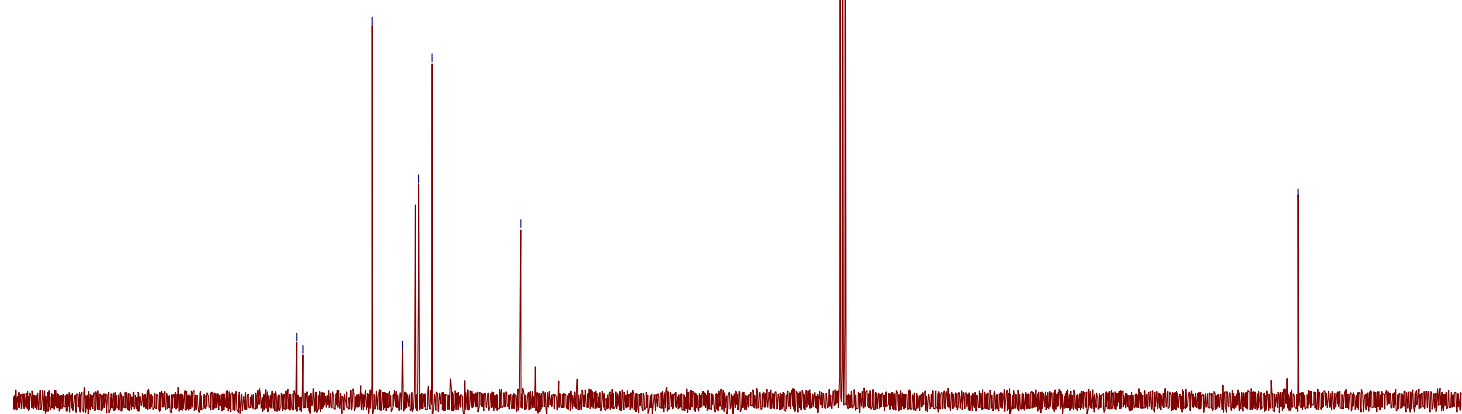

$\begin{array}{llll}170 \quad 160 \quad 150 & 140 & 130\end{array}$

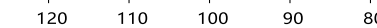

$70 \quad 60$

$\begin{array}{lllll}50 & 40 & 30 & 20 & 10\end{array}$ 
(E)-(2-(cyclohex-1-en-1-yl)vinyl)(phenyl)silane (3cb)

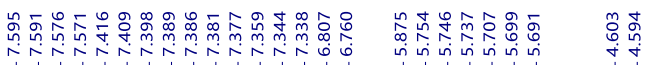

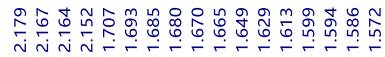

$\curvearrowright \mathrm{SiH}_{2} \mathrm{Ph}$

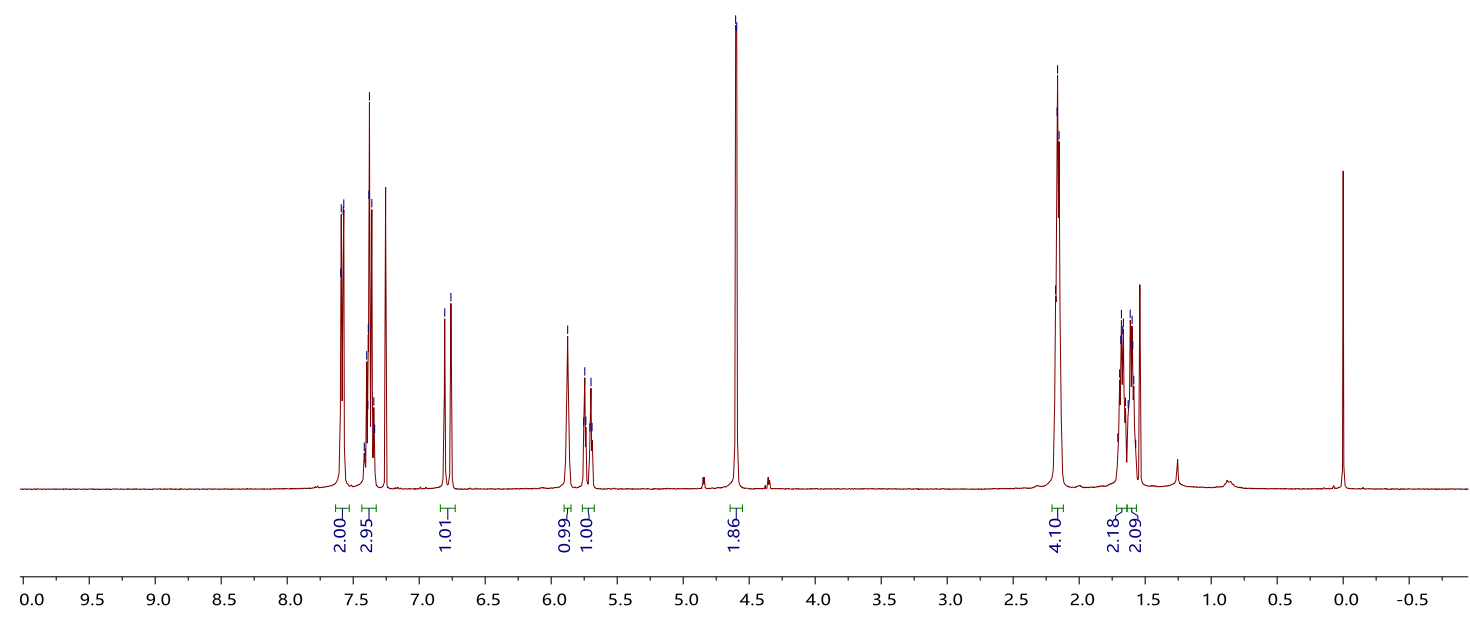

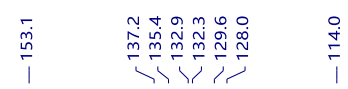

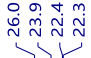

$\curvearrowright \mathrm{SiH}_{2} \mathrm{Ph}$

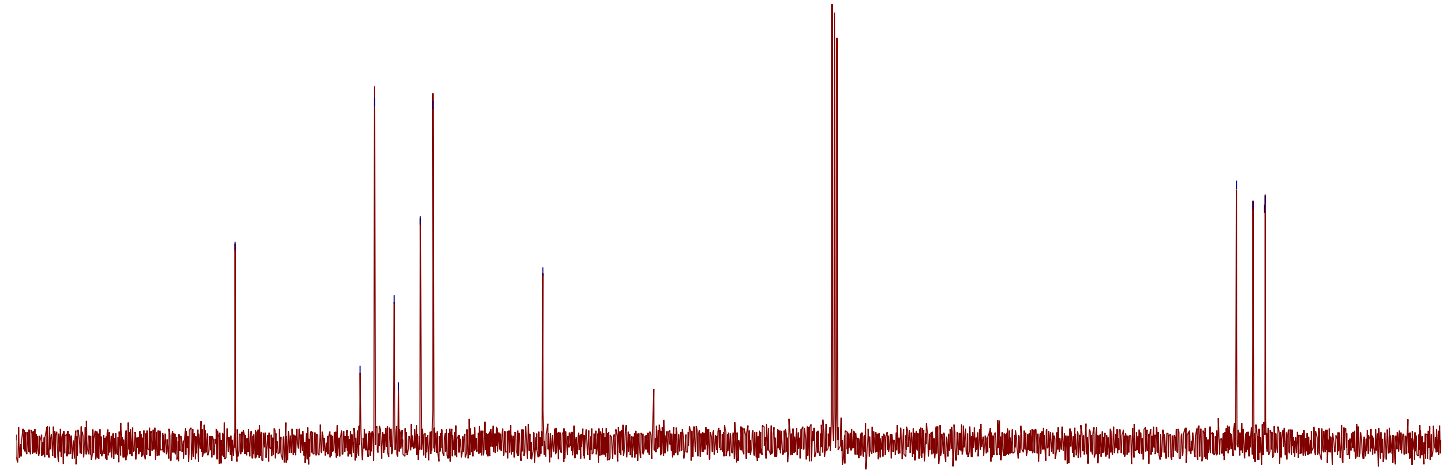

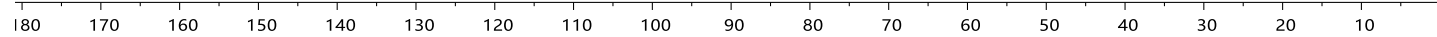


(1-(cyclohex-1-en-1-yl)vinyl)(phenyl)silane (4cb)
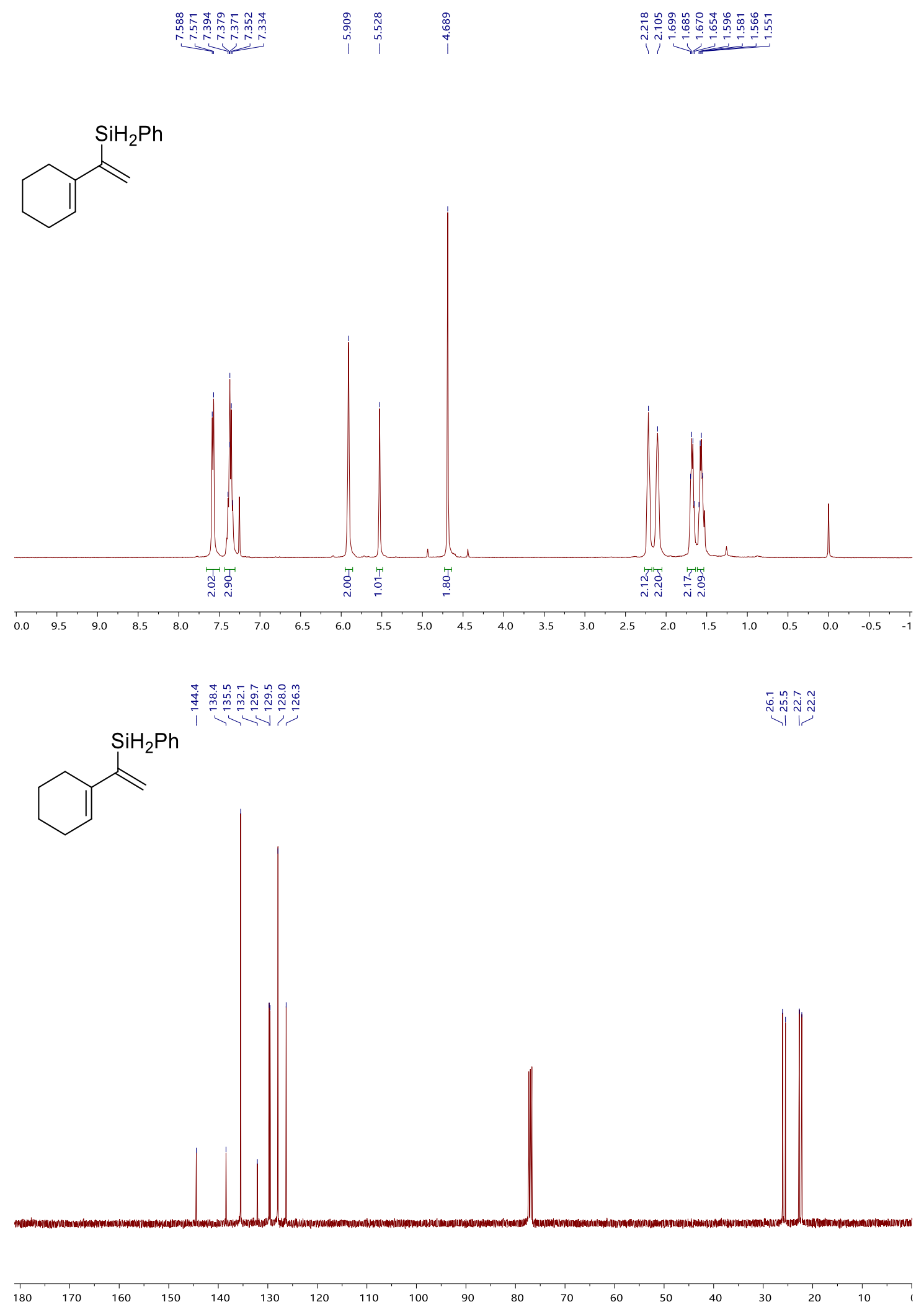
(E)-oct-1-en-1-yl(o-tolyl)silane (3da)

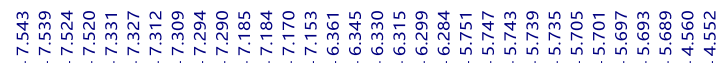

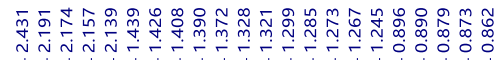

$\mathrm{C}_{6} \mathrm{H}_{13} \leadsto{ }^{\mathrm{H}}$

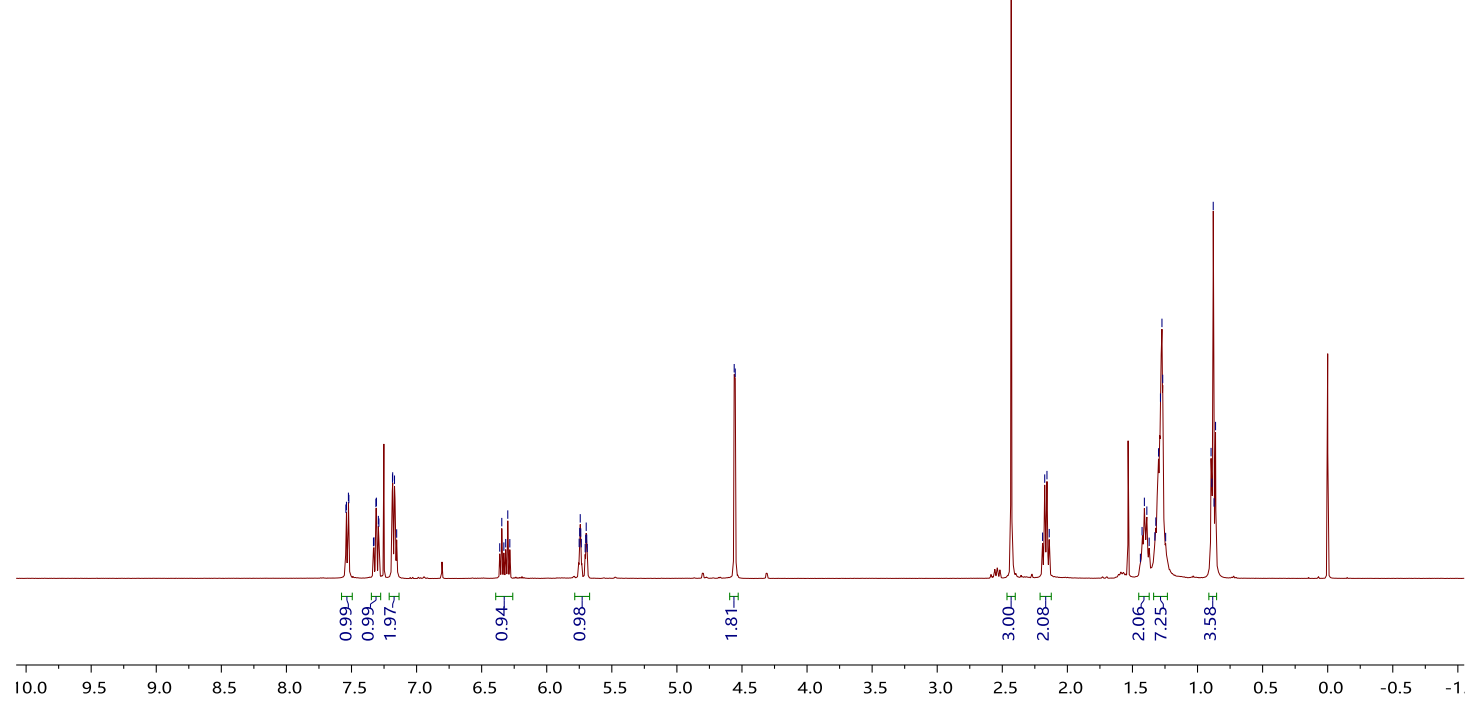

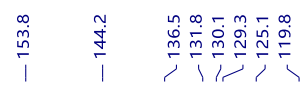

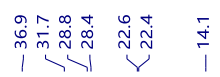

$\mathrm{C}_{6} \mathrm{H}_{13} \curvearrowright{ }^{\mathrm{Si}}$

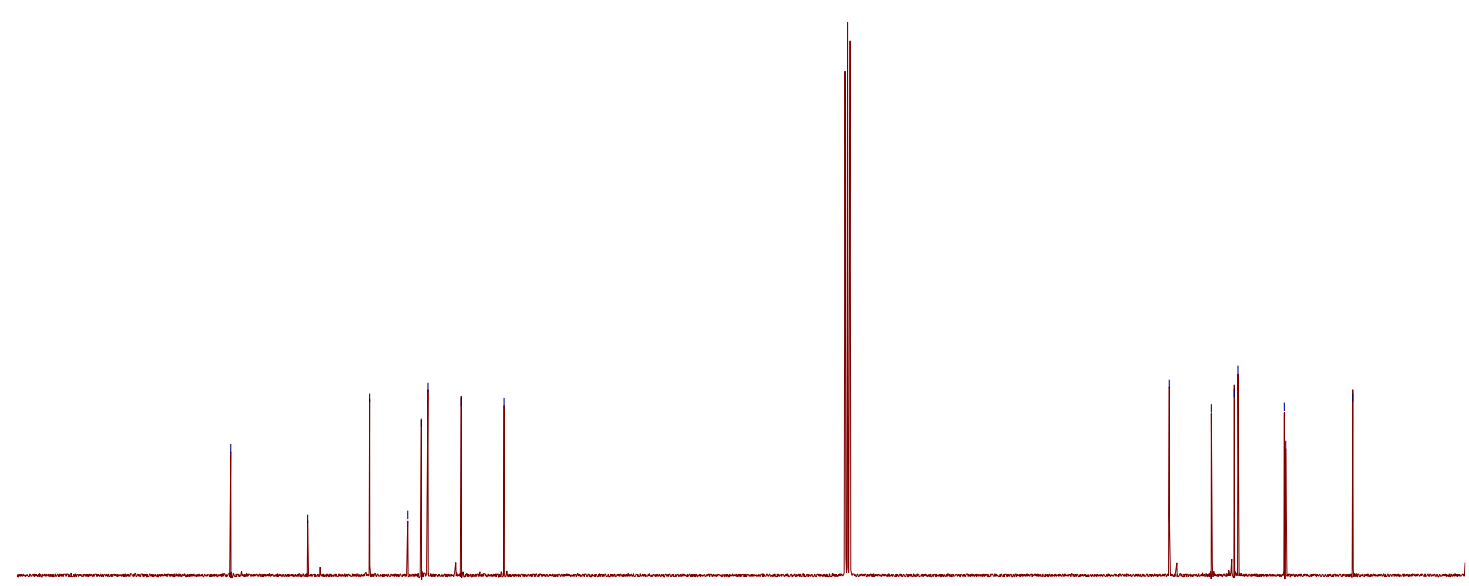

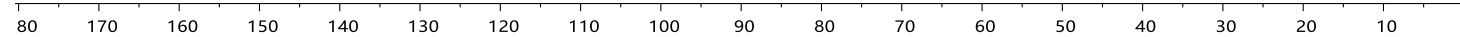




\section{oct-1-en-2-yl(o-tolyl)silane (4da)}

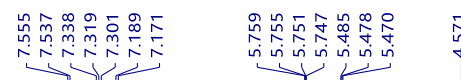

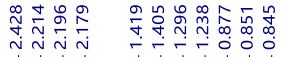<smiles>C=C([Hg])[SiH2]c1ccccc1C</smiles>

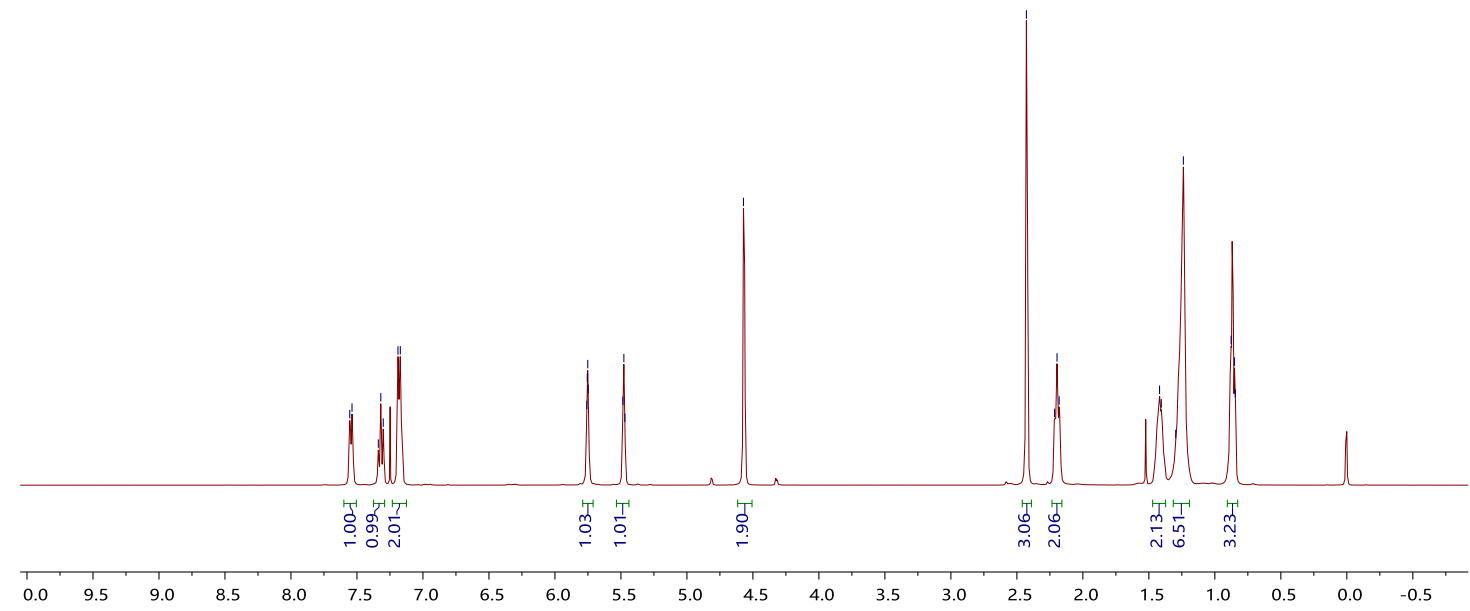

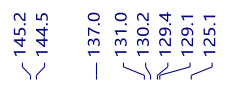

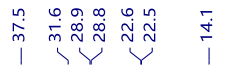<smiles>C=C(CCCCCCCC)[SiH2]c1ccccc1C</smiles>

180

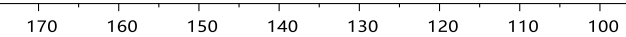

$90 \quad 80$

$70 \quad 60$

$50 \quad 40$

$30 \quad 20$

10 


\section{(E)-oct-1-en-1-yl $(p$-tolyl)silane (3db)}

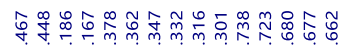

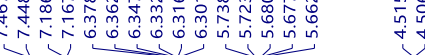

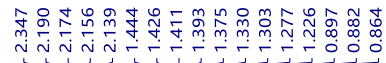

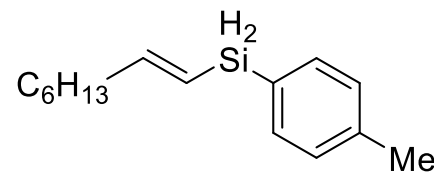

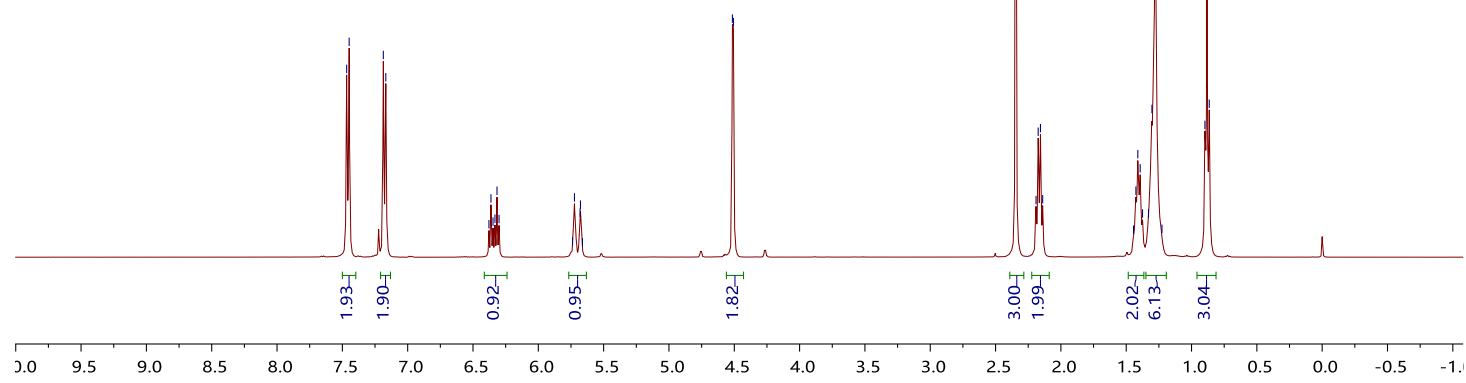

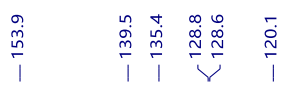

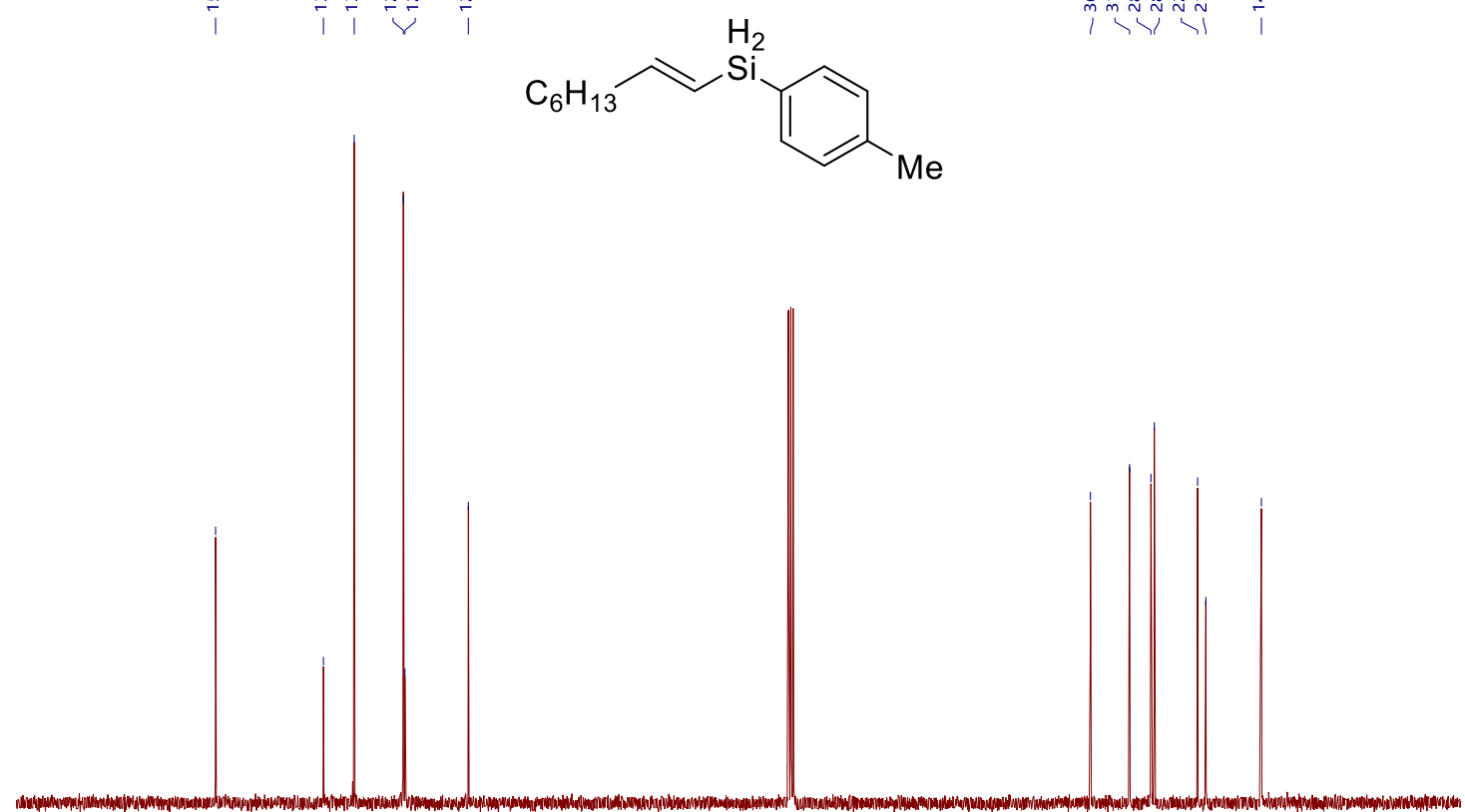

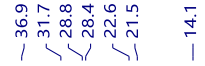

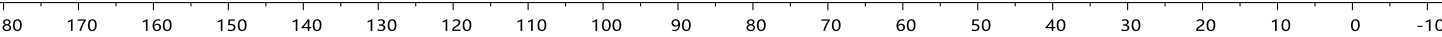


oct-1-en-2-yl(p-tolyl)silane (4db)

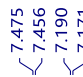

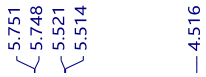

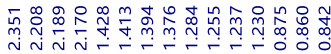<smiles>C=C(CCCCCC)[SiH2]c1ccc(C)cc1</smiles>

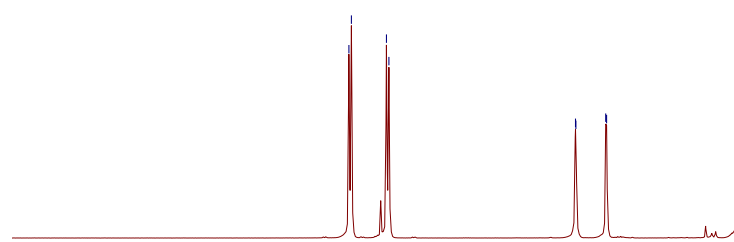

पर

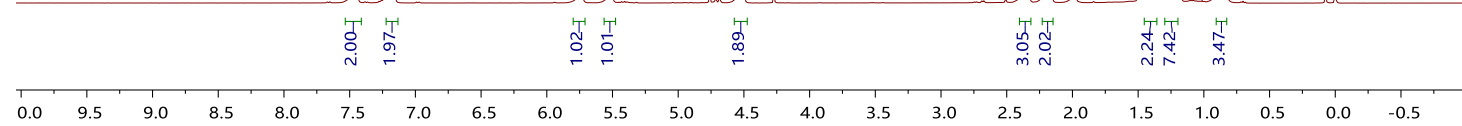

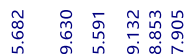

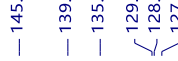

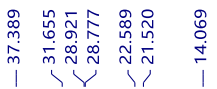<smiles>C=C(CCCCCC)[SiH2]c1ccc(C)cc1</smiles>

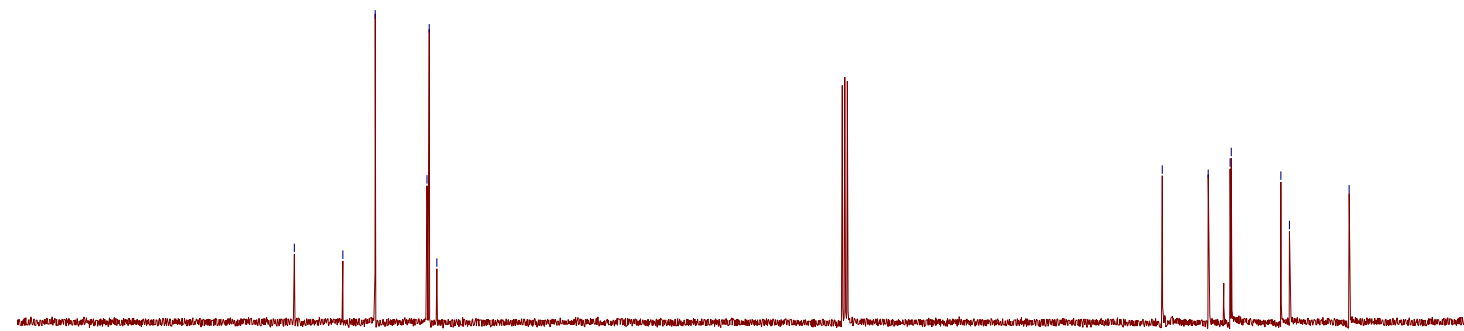

$90 \quad 80$

$\begin{array}{lllllll}70 & 60 & 50 & 40 & 30 & 20 & 10\end{array}$ 
(E)-(4-methoxyphenyl)(oct-1-en-1-yl)silane (3dc)

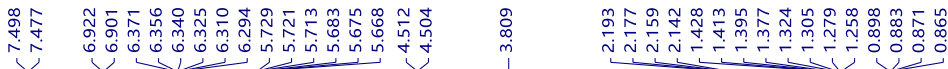

$\mathrm{C}_{6} \mathrm{H}_{13} \curvearrowright \mathrm{Si}_{\mathrm{OMe}}^{\mathrm{Si}_{2}}$

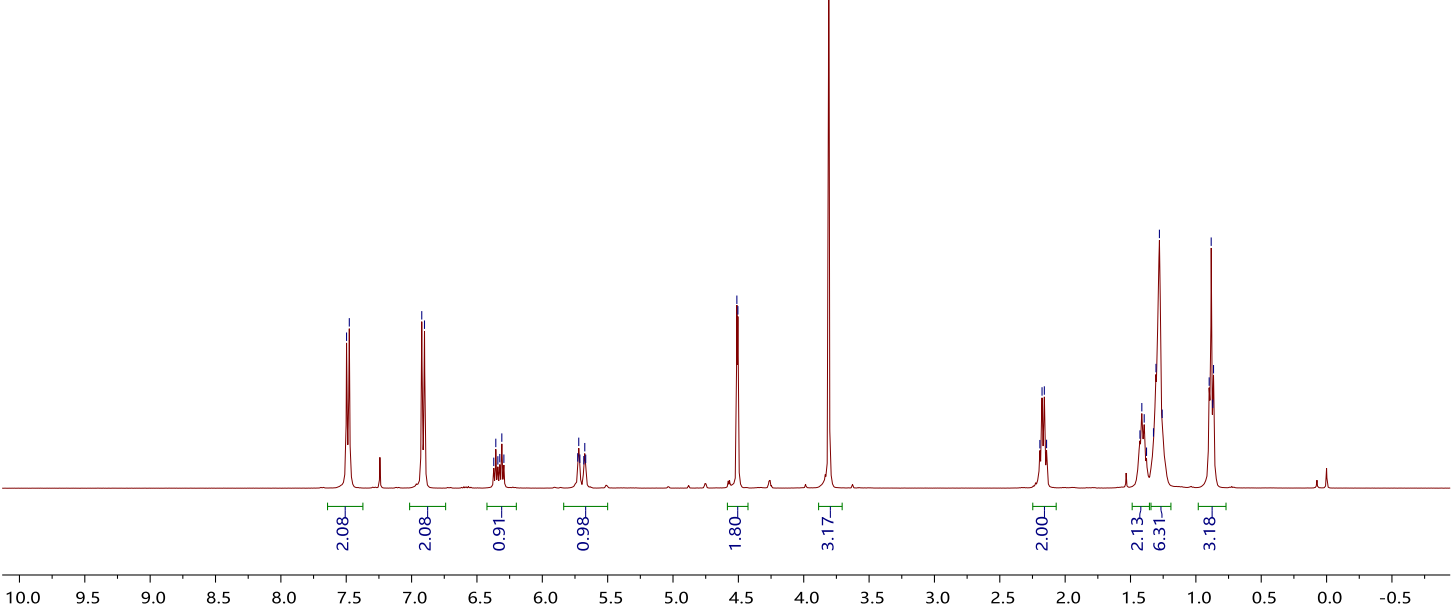

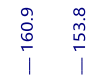

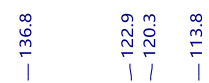
覀

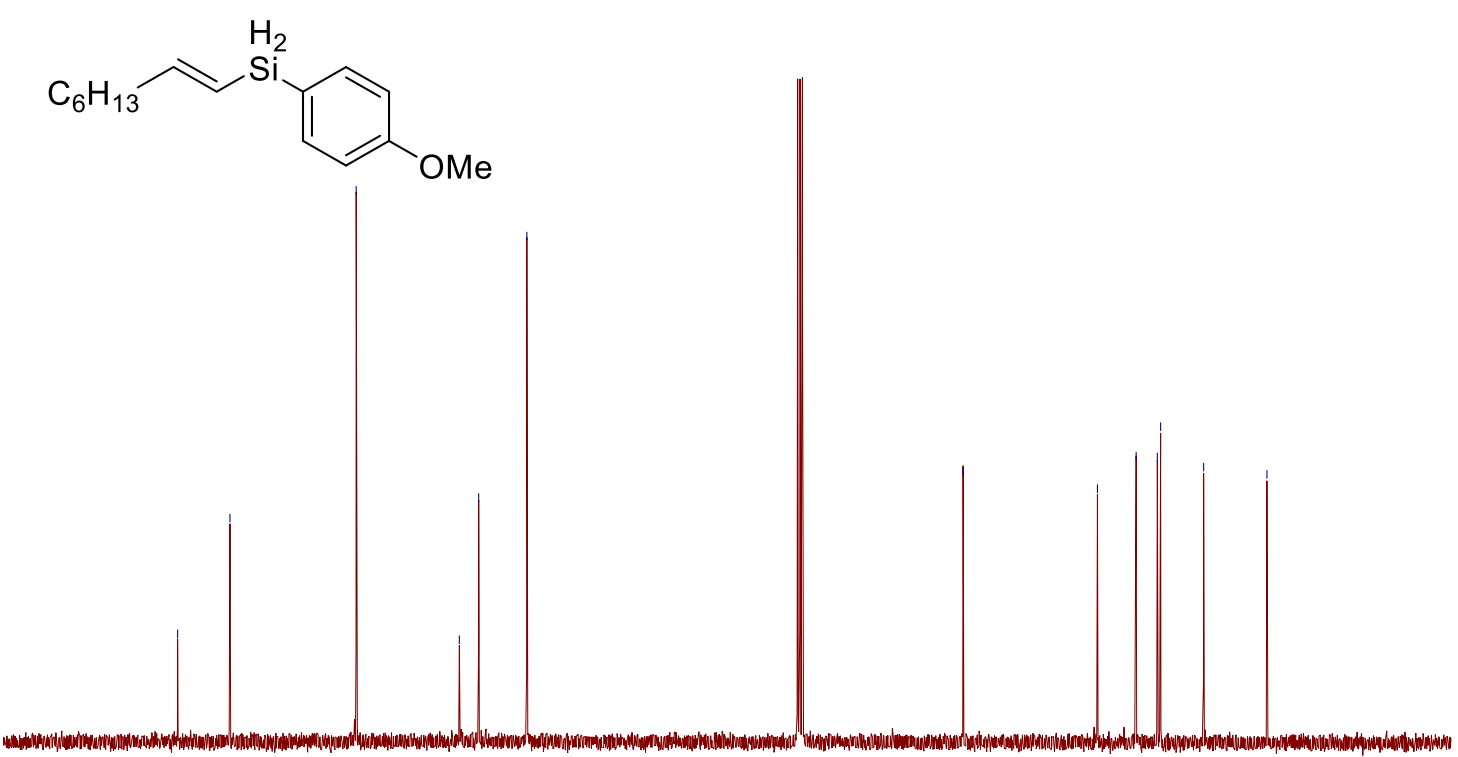

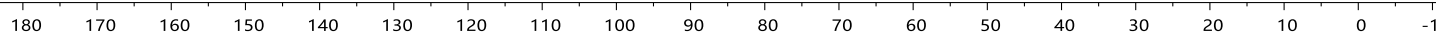


(4-methoxyphenyl)(oct-1-en-2-yl)silane (4dc)

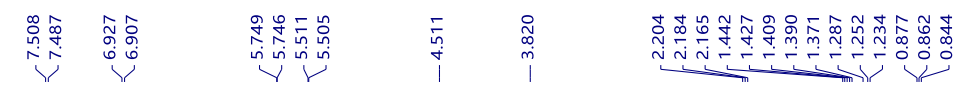

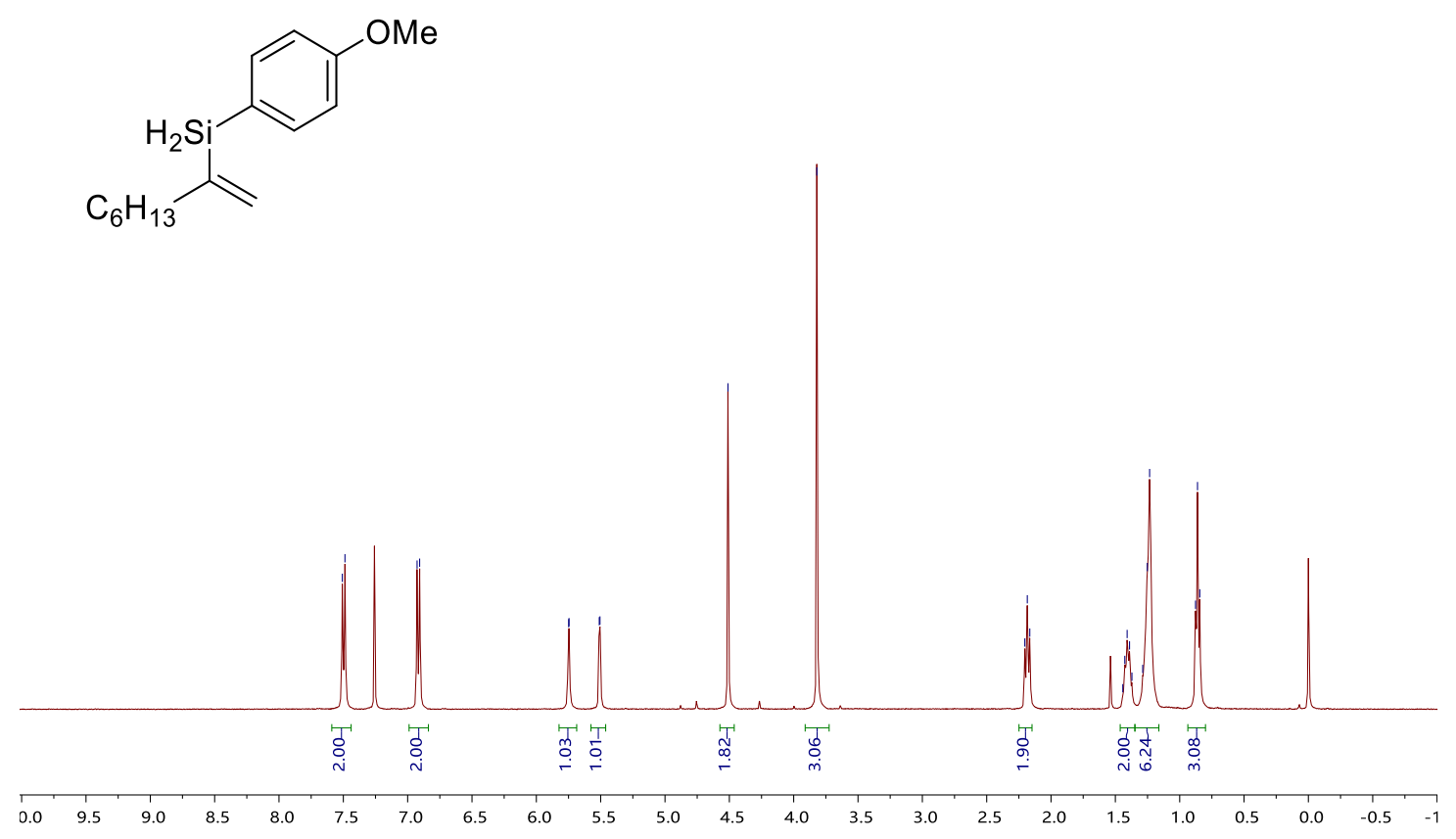

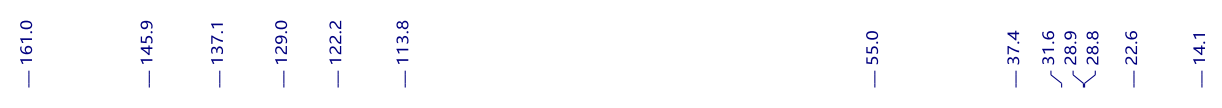<smiles>C=C(NCCCCCCC)[SiH2]c1ccc(OC)cc1</smiles>

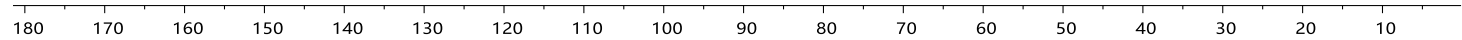




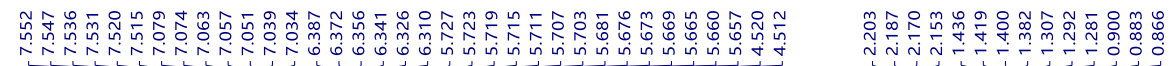<smiles>Fc1ccc([SiH2]C=C[AsH2]c2ccccc2)cc1</smiles>

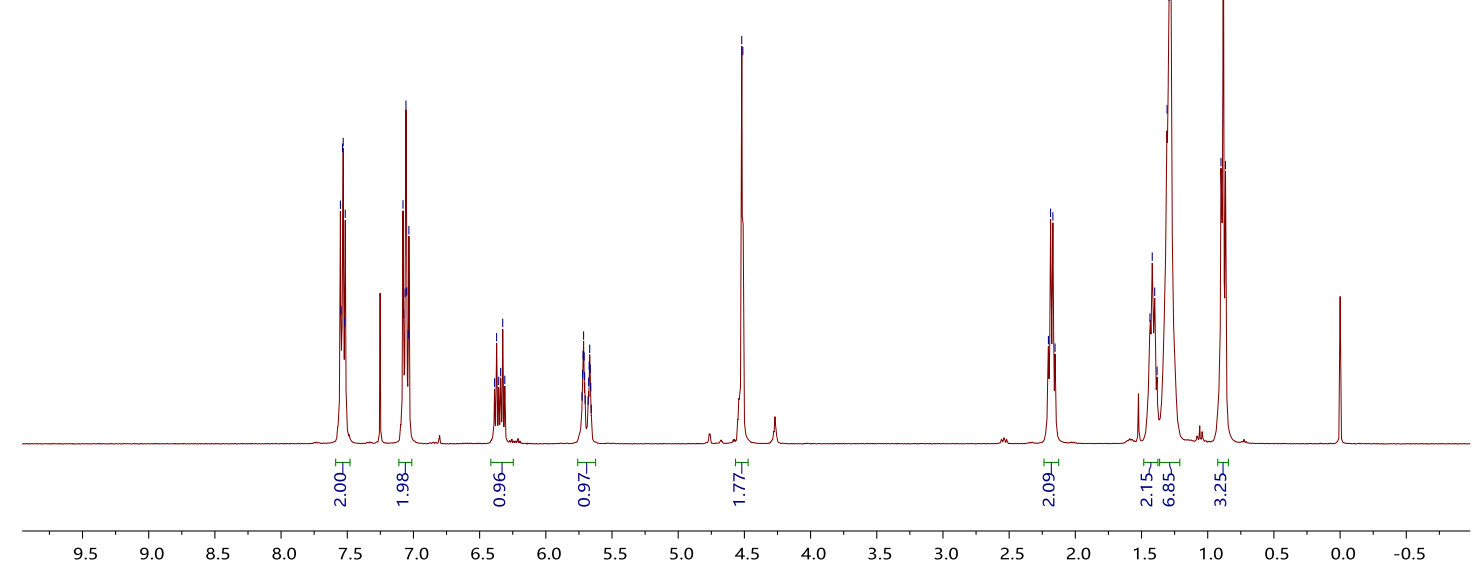

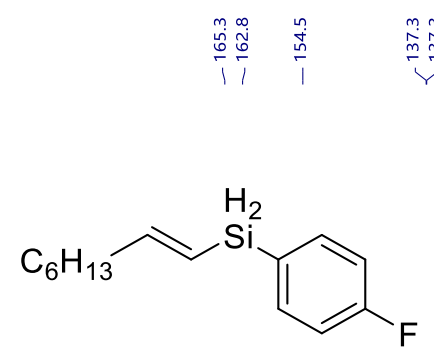

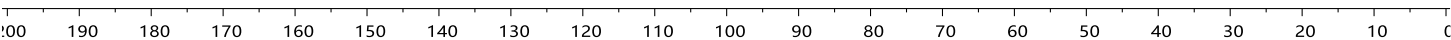


(4-fluorophenyl)(oct-1-en-2-yl)silane (4dd)

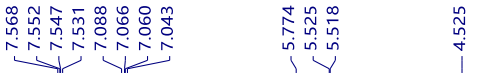

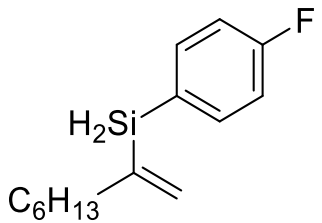
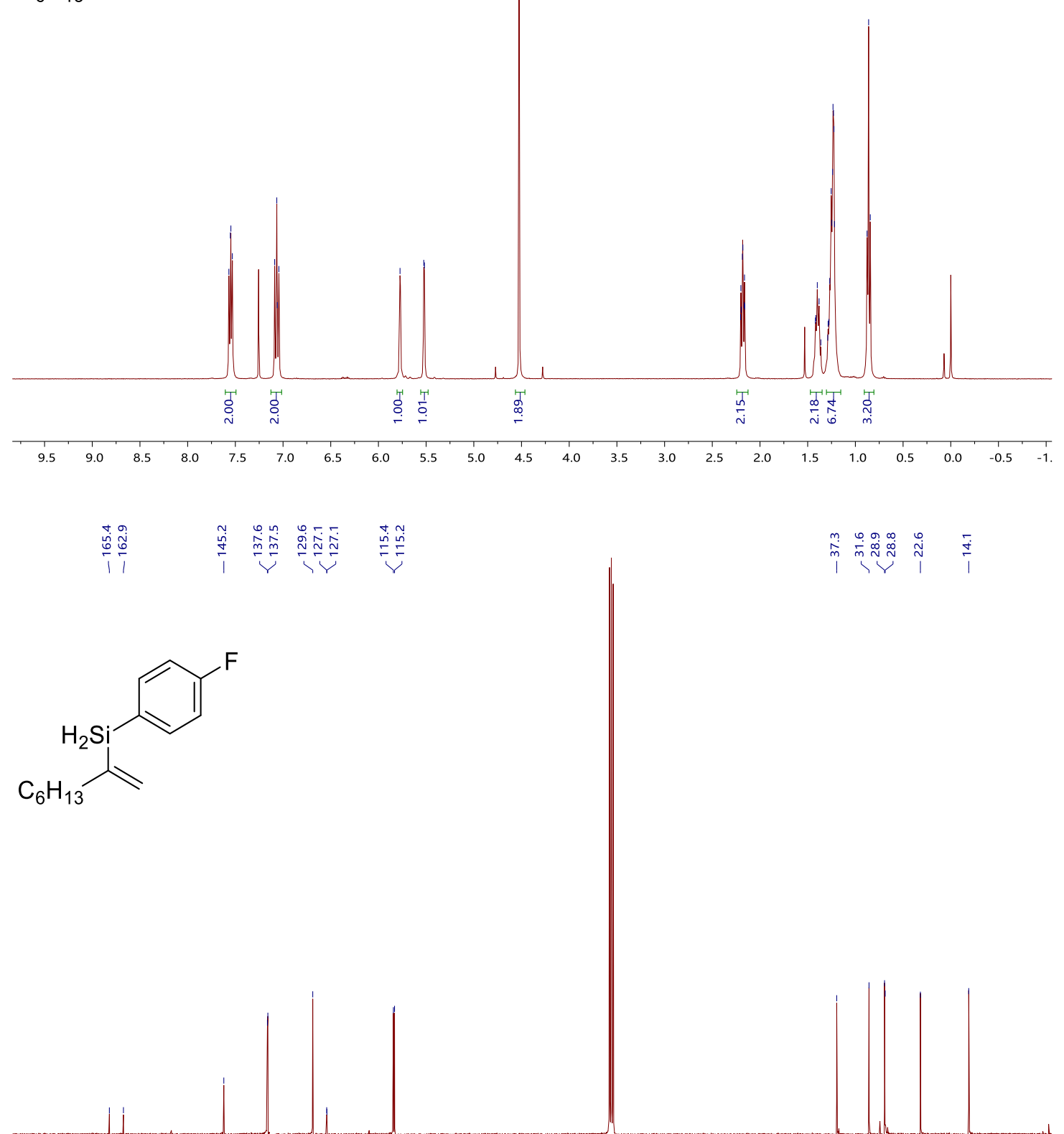

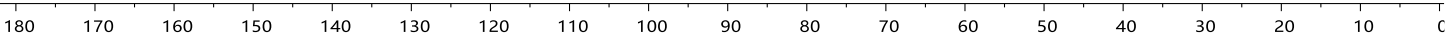




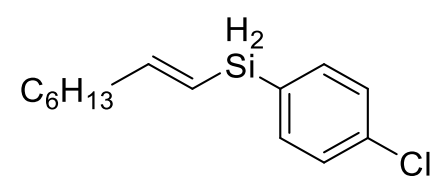
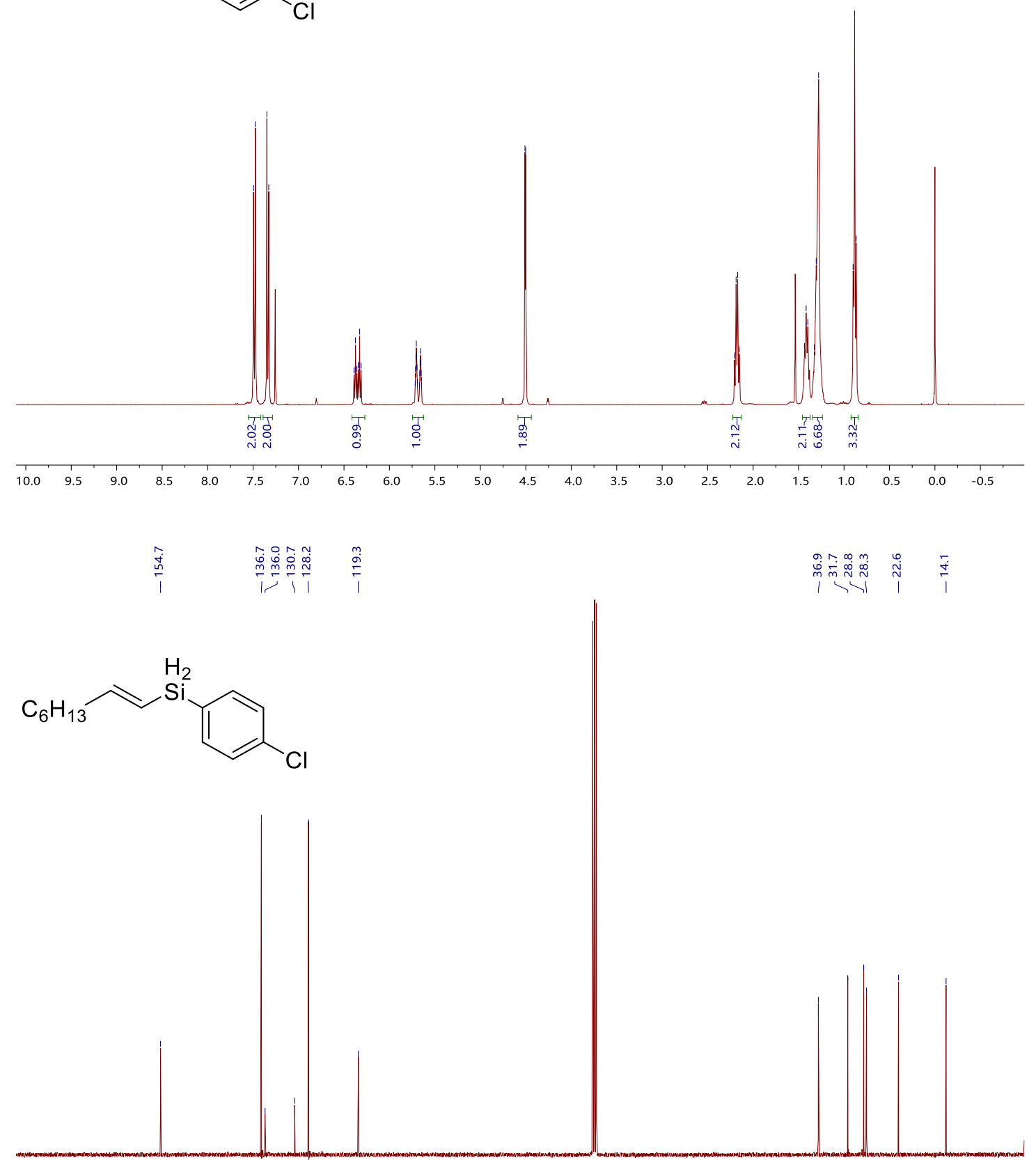

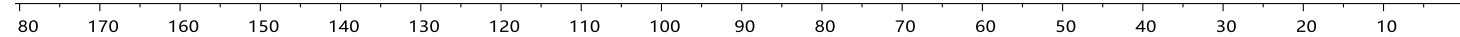


(4-chlorophenyl)(oct-1-en-2-yl)silane (4de)

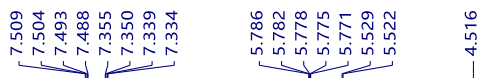

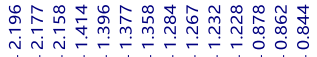

Cl

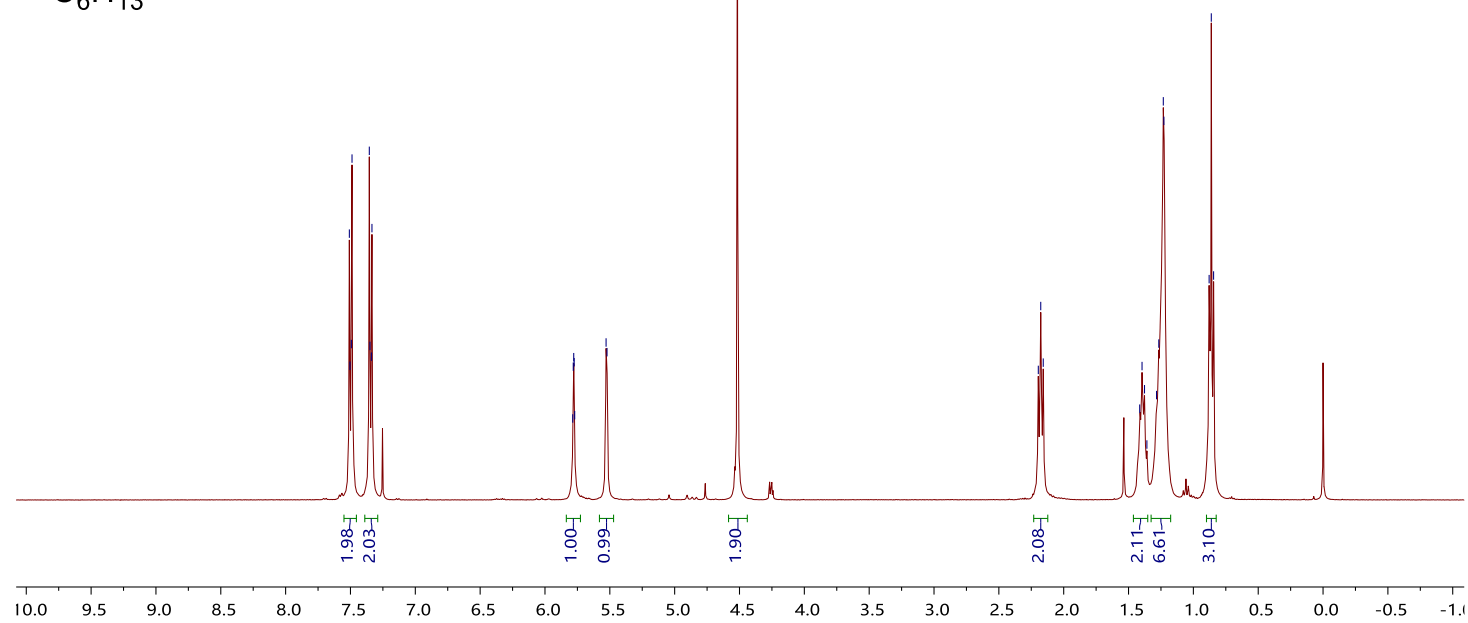

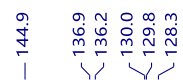

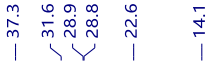

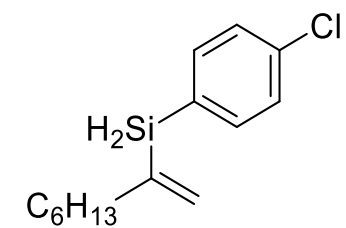

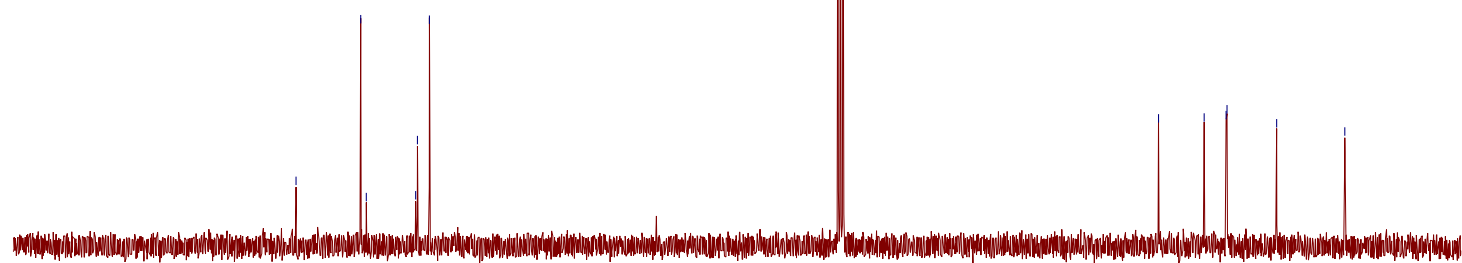

$\begin{array}{llllllllllllllllll}80 & 170 & 160 & 150 & 140 & 130 & 120 & 110 & 100 & 90 & 80 & 70 & 60 & 50 & 40 & 30 & 20 & 10\end{array}$ 


\section{(E)-dodecyl(oct-1-en-1-yl)silane (3df)}

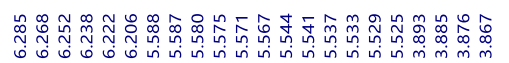

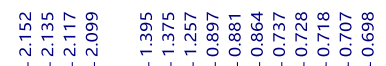

$\mathrm{C}_{6} \mathrm{H}_{13} \curvearrowright \mathrm{SiH}_{2} \mathrm{C}_{12} \mathrm{H}_{25}$
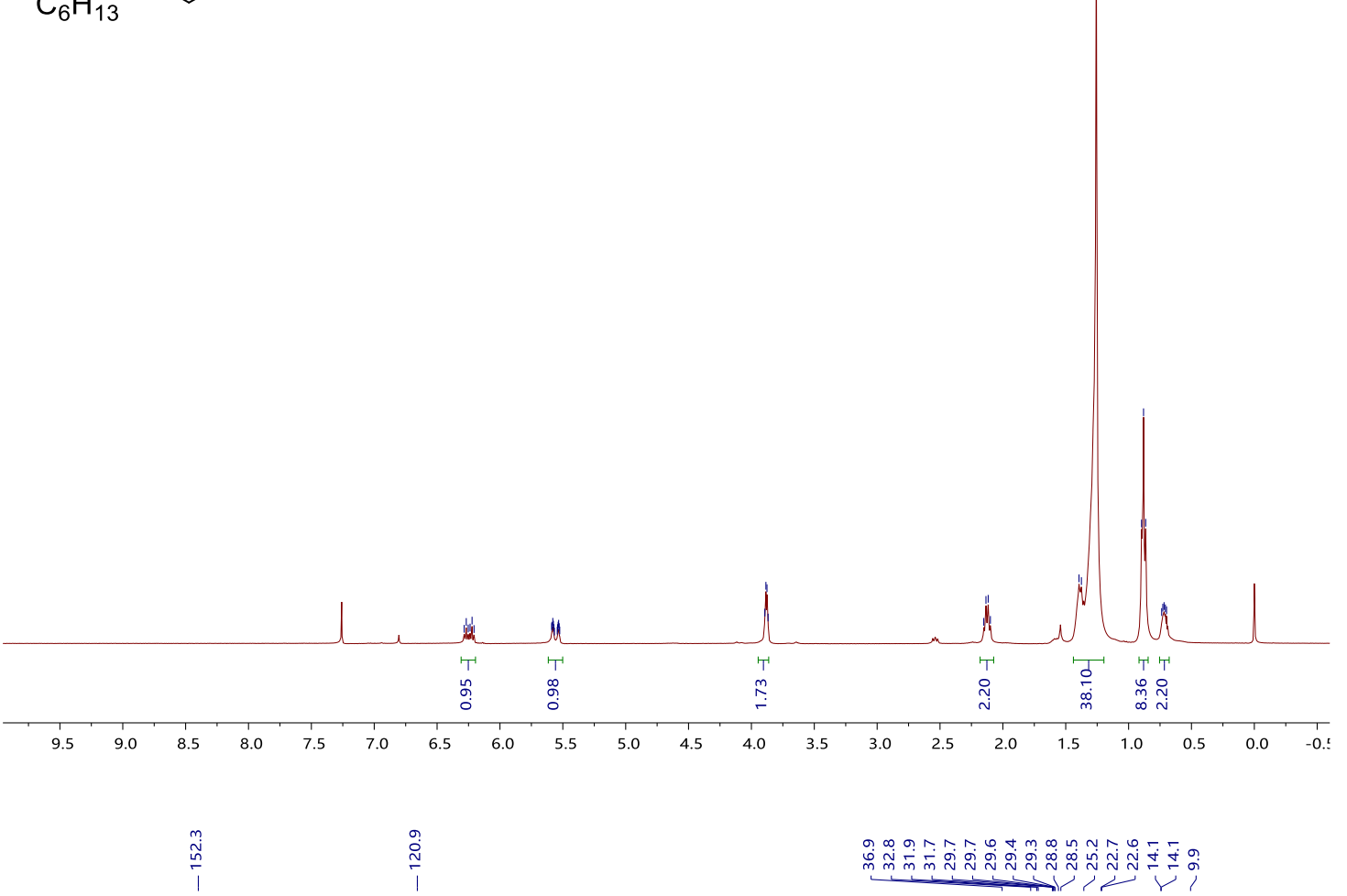

$\underbrace{0}$

$\mathrm{C}_{6} \mathrm{H}_{13} \leadsto \mathrm{SiH}_{2} \mathrm{C}_{12} \mathrm{H}_{25}$

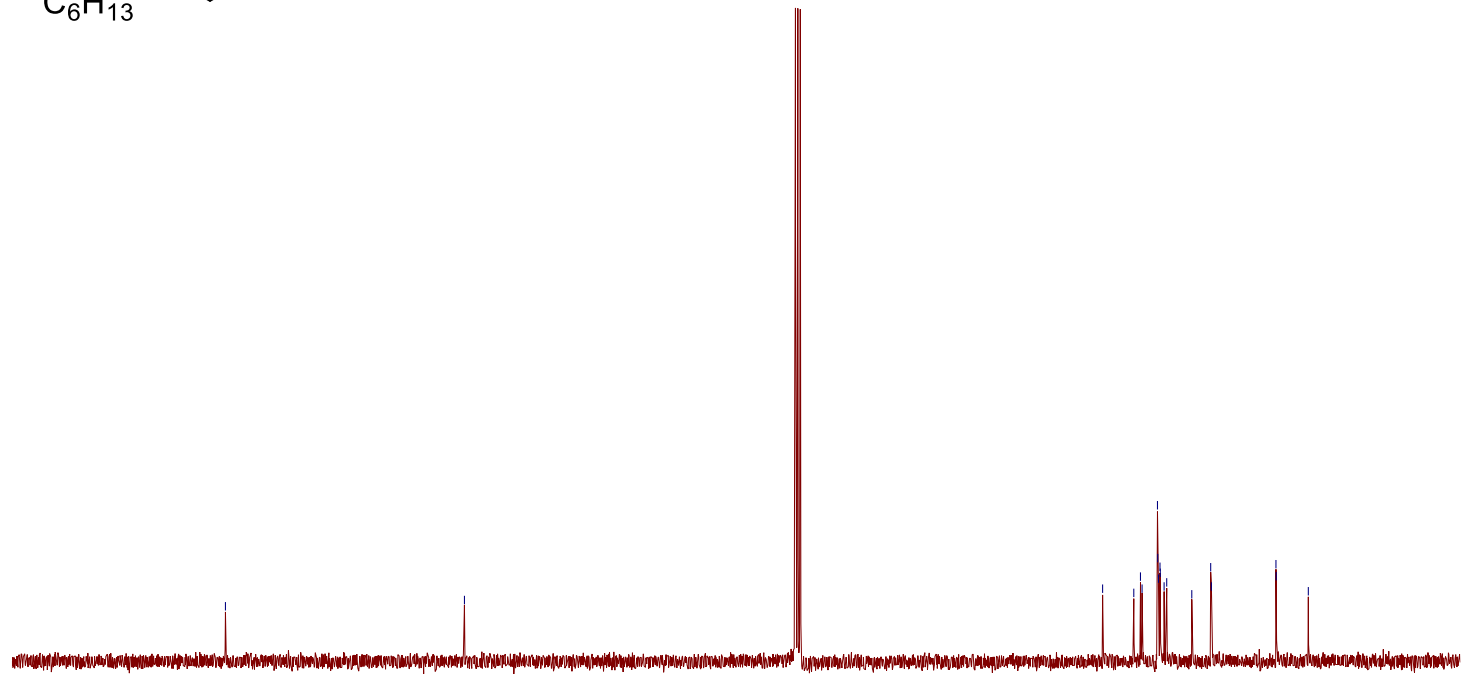

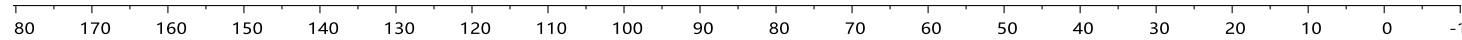


dodecyl(oct-1-en-2-yl)silane (4df)

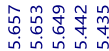

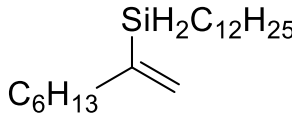
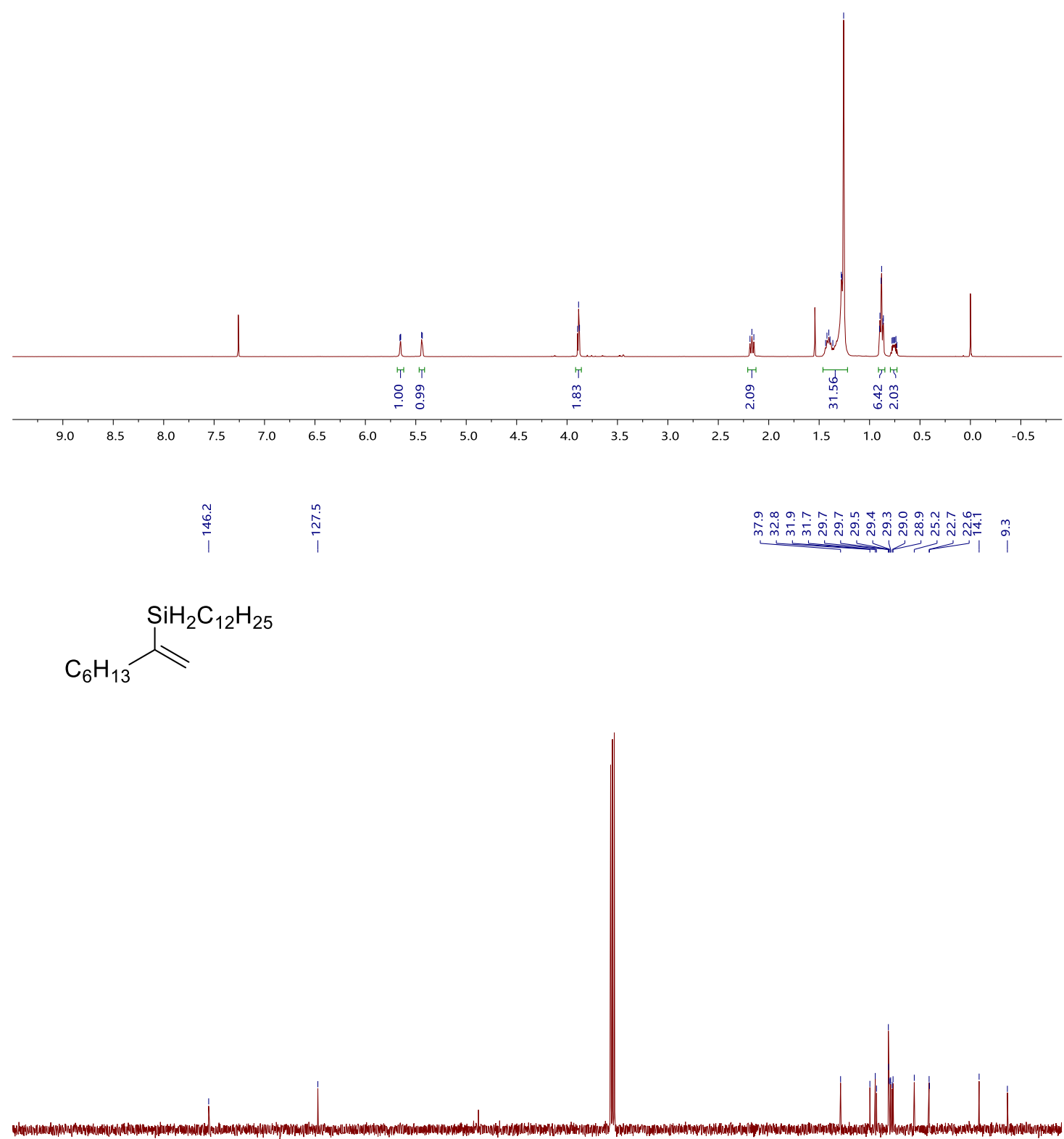
(E)-phenyl(1-phenylprop-1-en-2-yl)silane (3ea)

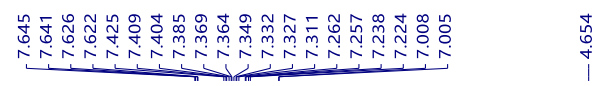

$\mathrm{CiH}_{\mathrm{Me}}^{\mathrm{SiH}_{2} \mathrm{Ph}}$

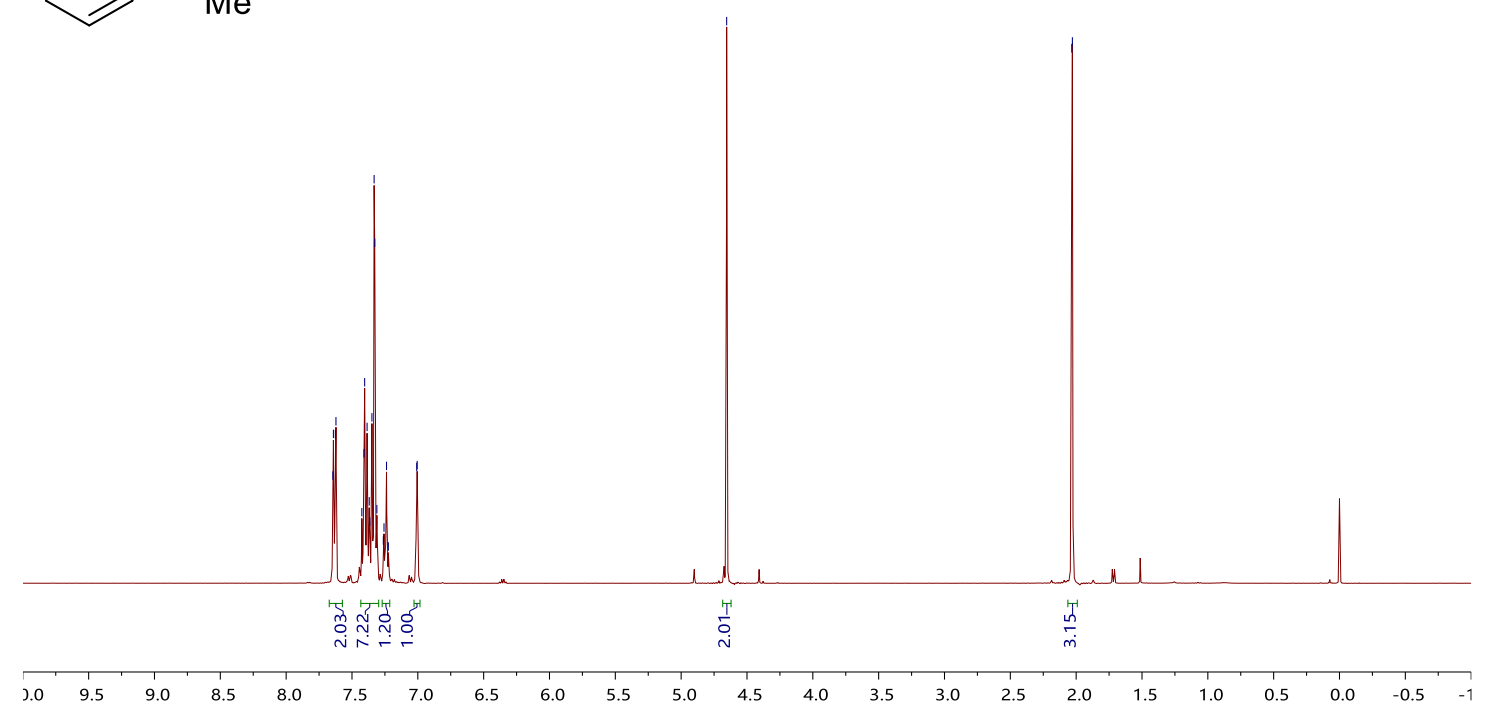

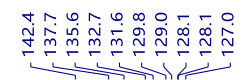

$\overbrace{M e}^{\mathrm{SiH}_{2} \mathrm{Ph}}$

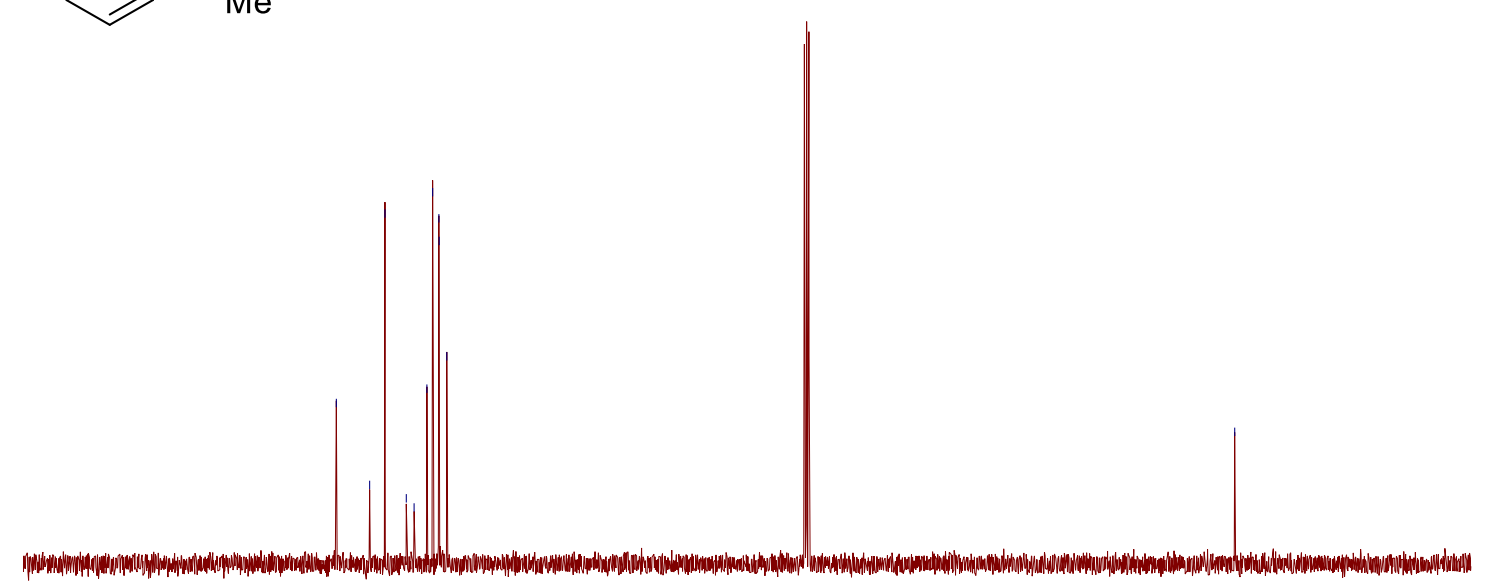

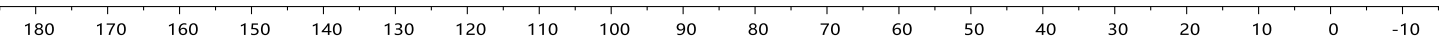


(E)-phenyl(1-phenylprop-1-en-1-yl)silane (4ea)

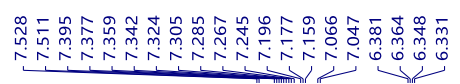<smiles>C/C=C(/[SH2+]c1ccccc1)c1ccccc1</smiles>

Me

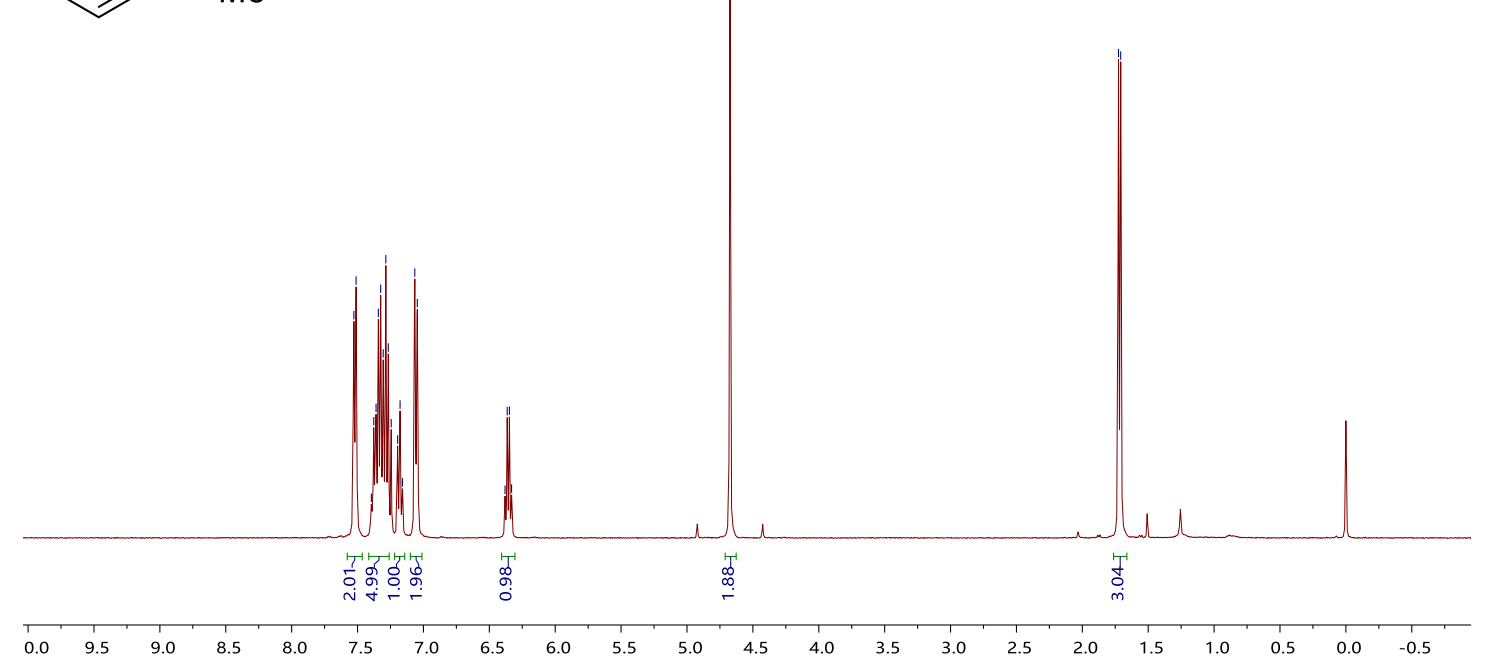

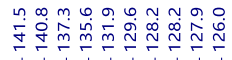

$\underbrace{-1}$

$\mathrm{Me}_{\mathrm{Me}}^{\mathrm{SiH}_{2} \mathrm{Ph}}$

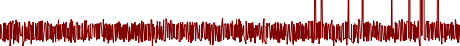

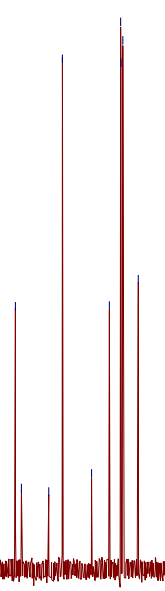

180

$\begin{array}{llllllll}170 & 160 & 150 & 140 & 130 & 120 & 110 & 100\end{array}$

$90 \quad 80$

$70 \quad 60$

$\begin{array}{lllll}40 & 30 & 20 & 10 & 0\end{array}$ 
$\mathrm{SiH}_{\mathrm{Me}}^{\mathrm{SiH}_{2} \mathrm{Ph}}$
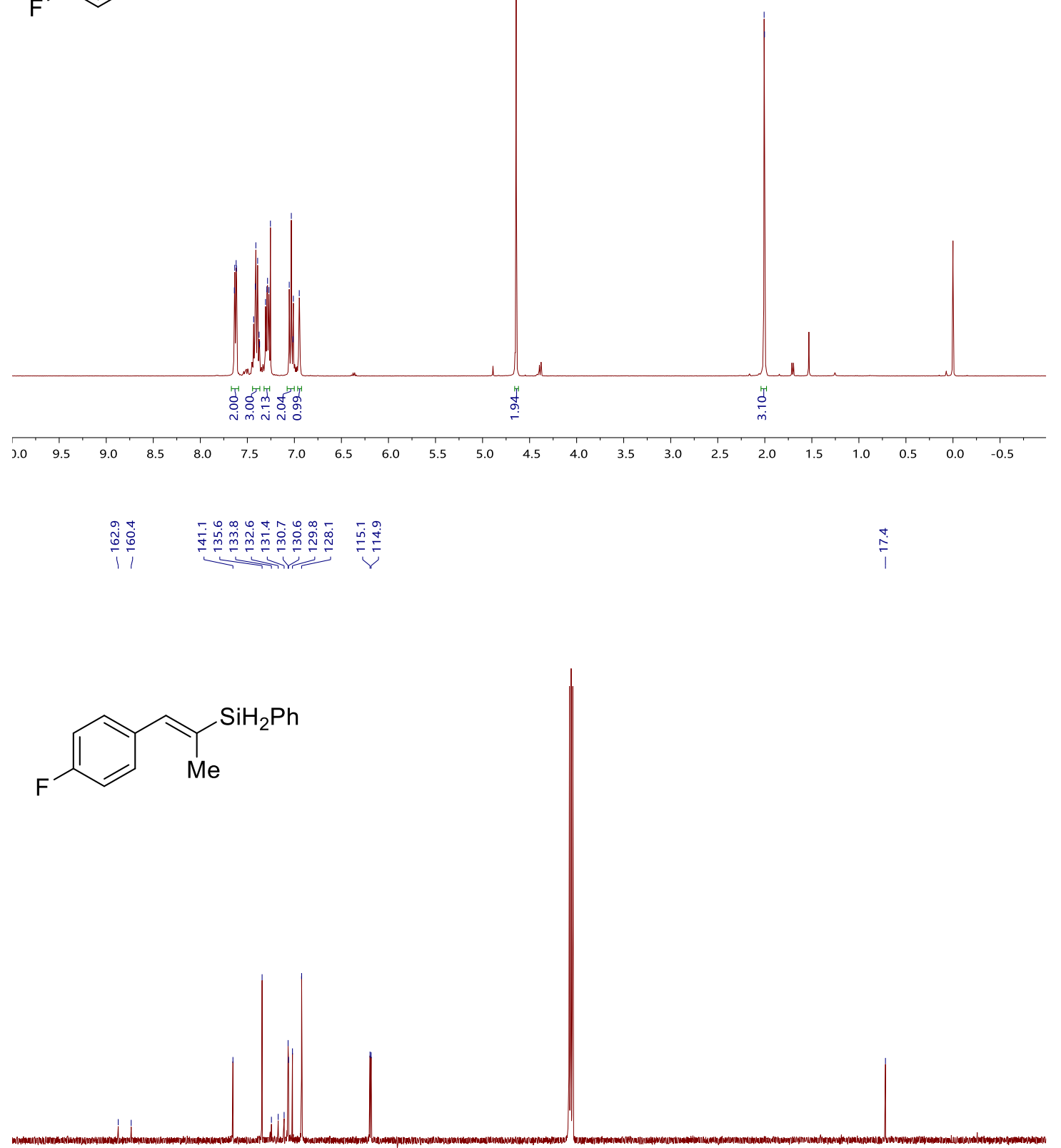

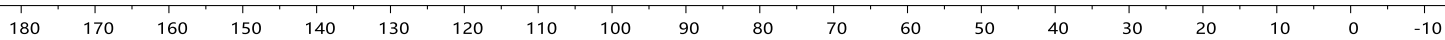




\section{(E)-(1-(4-fluorophenyl)prop-1-en-1-yl)(phenyl)silane (4eb)}<smiles>C/C=C(/c1ccccc1)c1ccc(F)cc1</smiles>

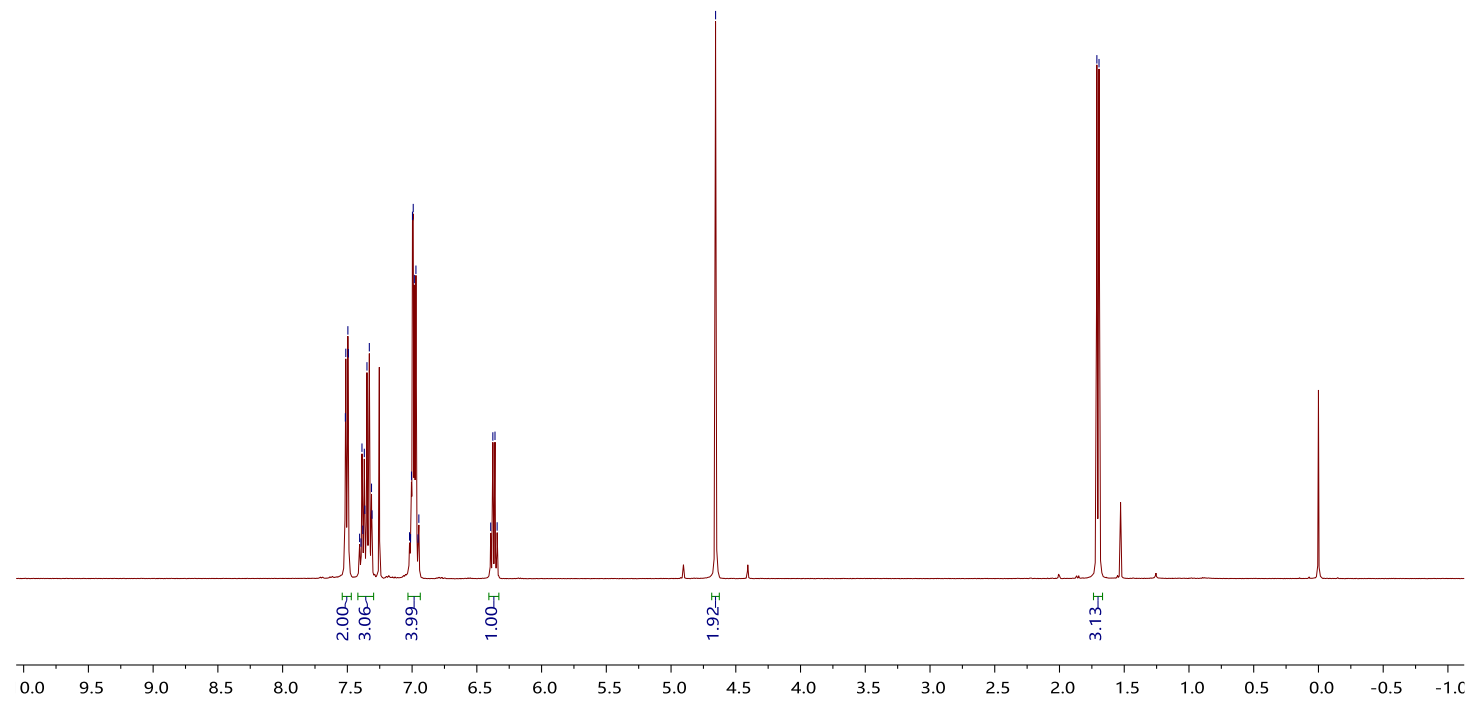

管家<smiles>C/C=C(/Sc1ccccc1)c1ccc(F)cc1</smiles>

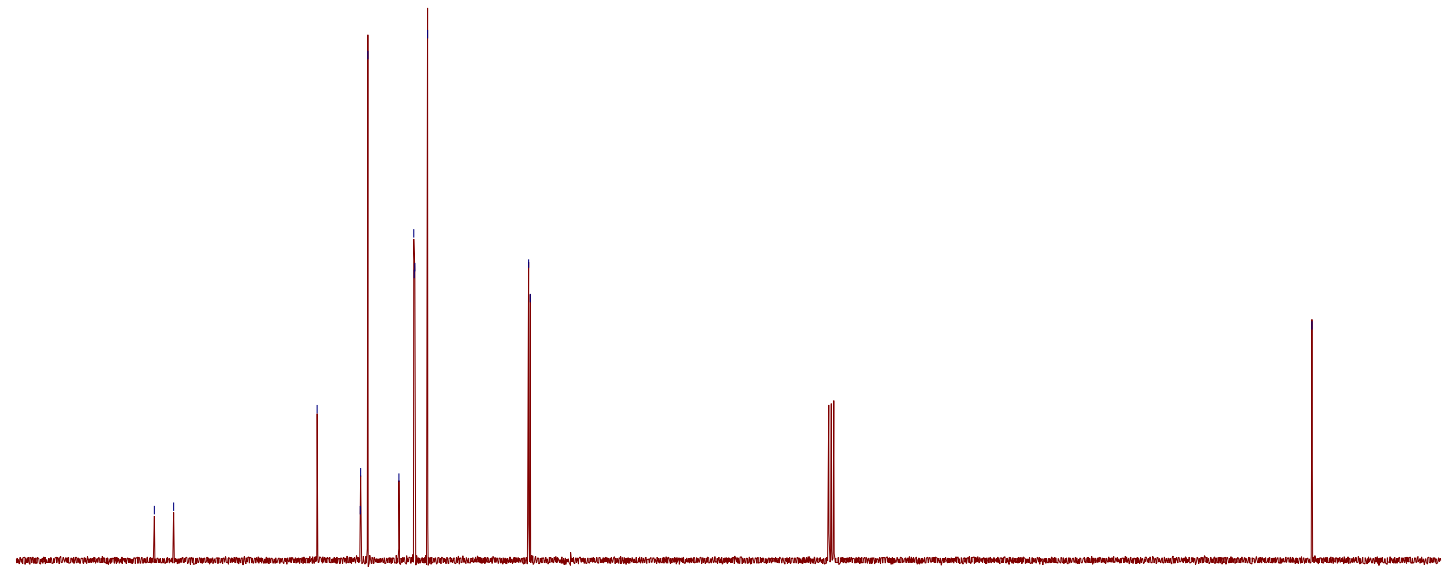

80

$170 \quad 160 \quad 150 \quad 140$

曽

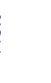

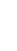


(E)-(1-(4-methoxyphenyl)prop-1-en-2-yl)(phenyl)silane (3ec)

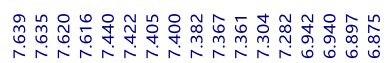<smiles>COc1ccc(/C=C(\C)[SH2+]c2ccccc2)cc1</smiles>

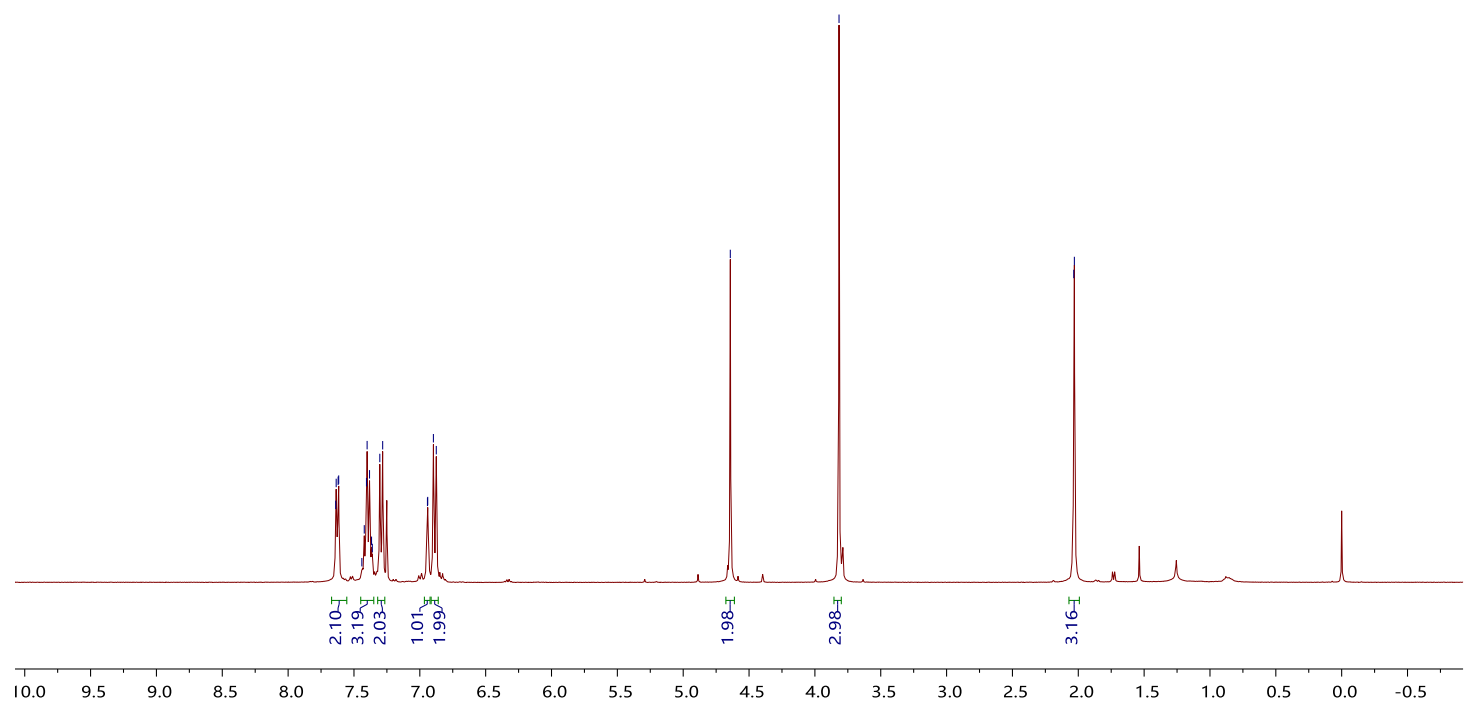

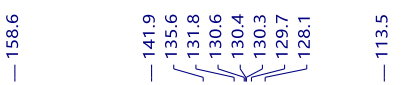<smiles>COc1ccc(/C=C(\C)[SH+]c2ccccc2)cc1</smiles>

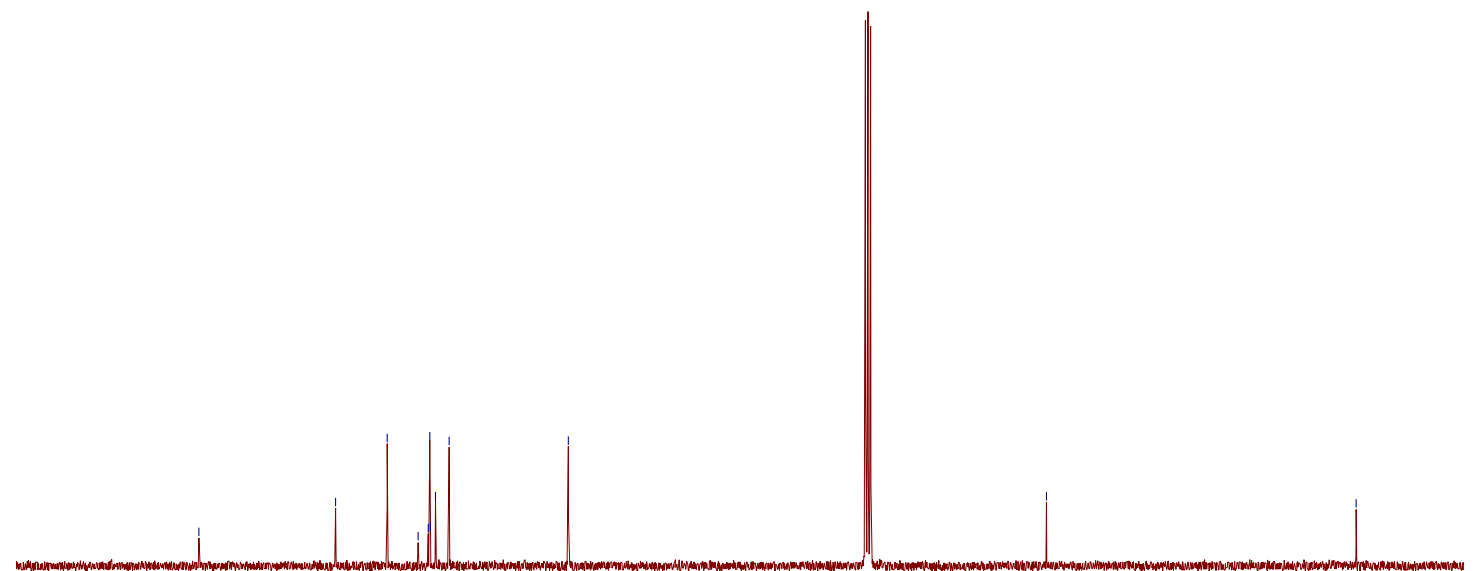

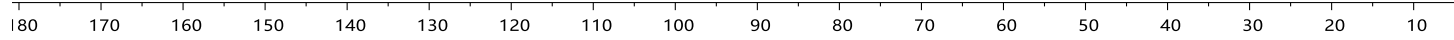


(E)-(1-(4-methoxyphenyl)prop-1-en-1-yl)(phenyl)silane (4ec)

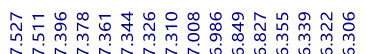

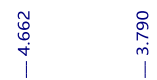

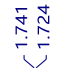

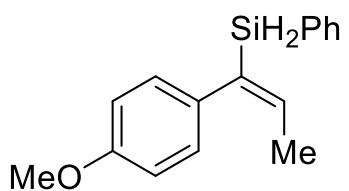

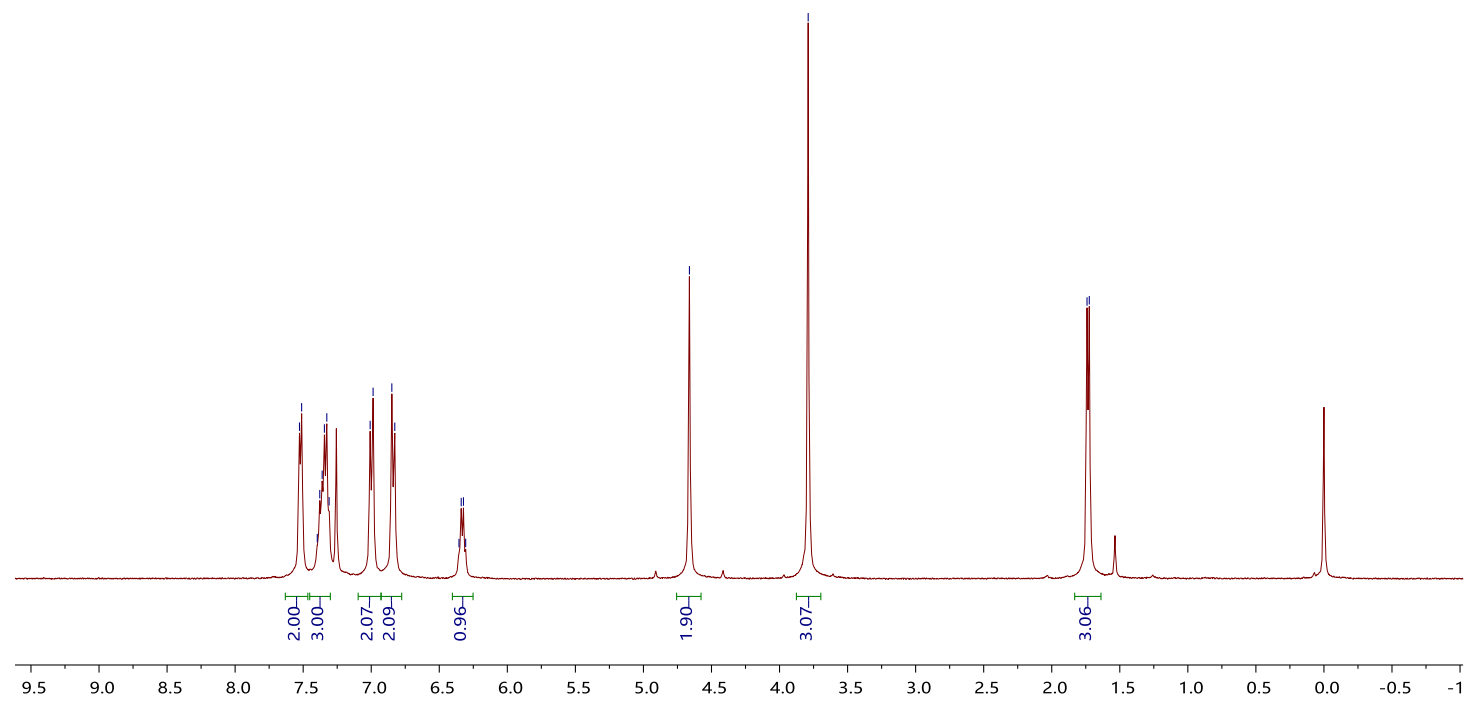

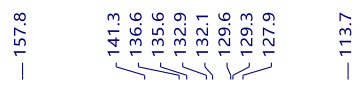

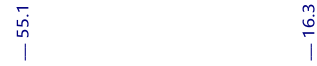

(l)

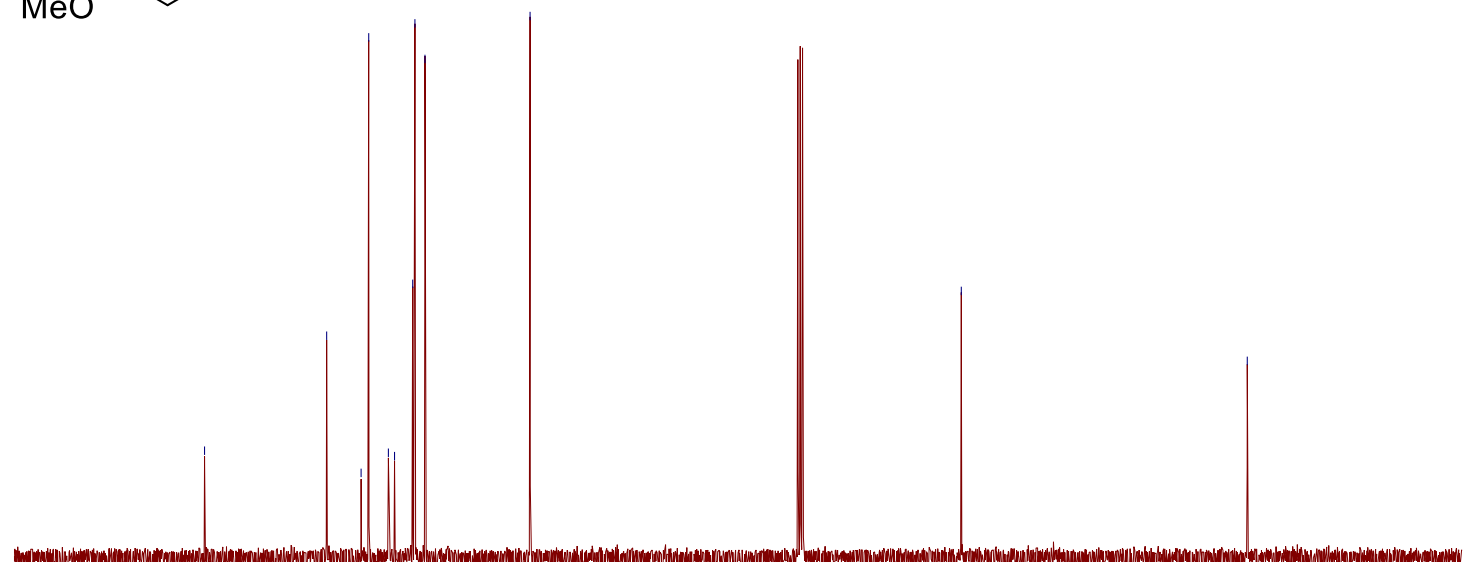

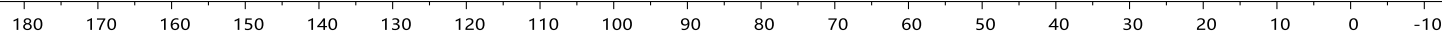


(E)-(1-([1,1'-biphenyl]-4-yl)prop-1-en-2-yl)(phenyl)silane (3ed)

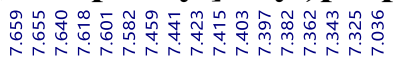<smiles>CC(=Cc1ccc(-c2ccccc2)cc1)Sc1ccccc1</smiles>

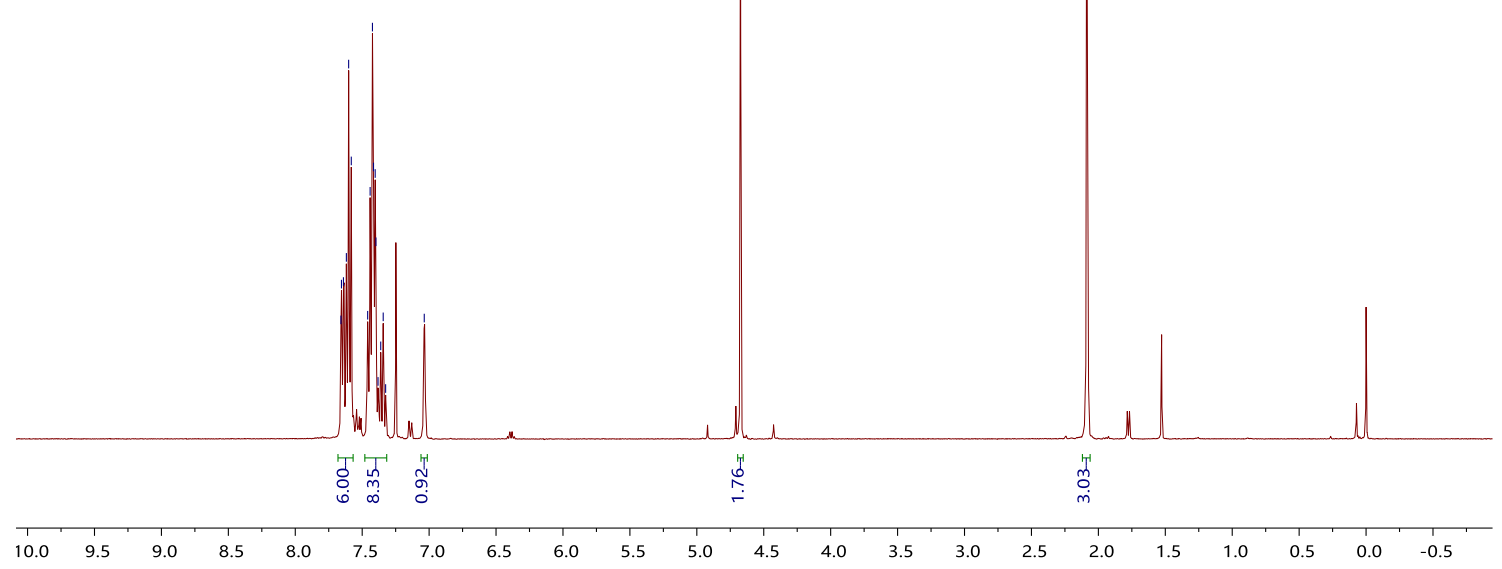

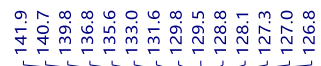<smiles>C/C(=C\c1ccc(-c2ccccc2)cc1)[SH2+]c1ccccc1</smiles>

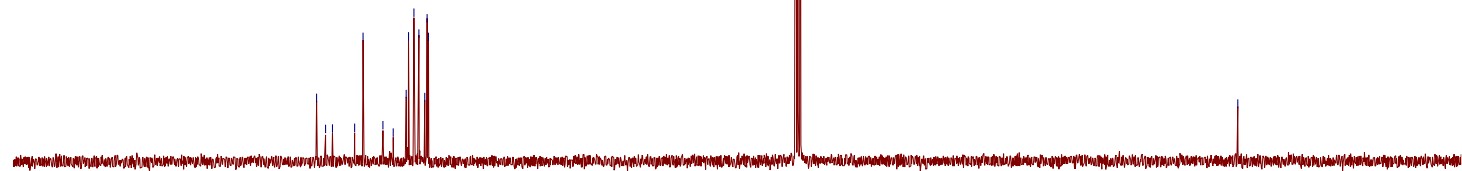

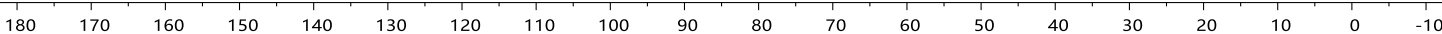


(E)-(1-([1,1'-biphenyl]-4-yl)prop-1-en-1-yl)(phenyl)silane (4ed)

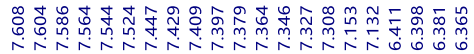<smiles>C/C=C(/Sc1ccccc1)c1ccc(-c2ccccc2)cc1</smiles>

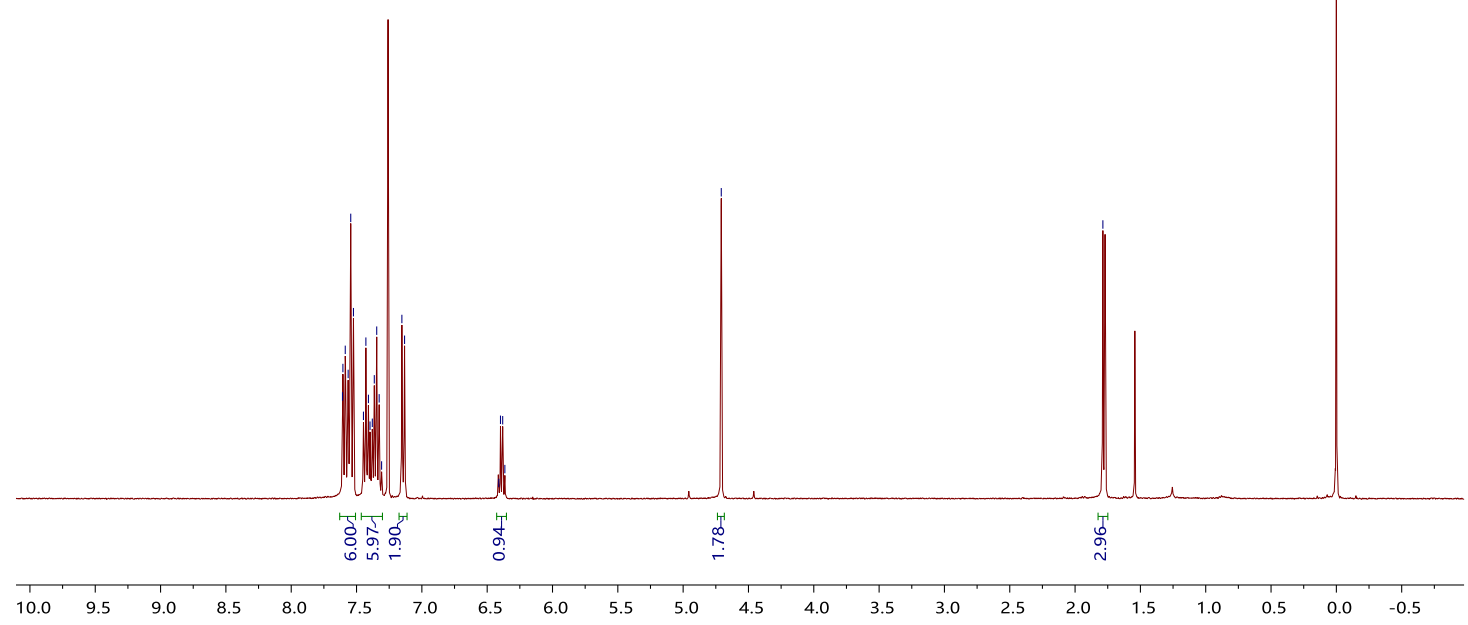

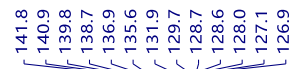<smiles>C/C=C(/[SH2+]c1ccccc1)c1ccc(-c2ccccc2)cc1</smiles>

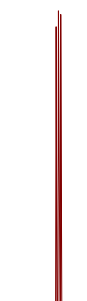


(E)-(1-(2-methoxyphenyl)prop-1-en-2-yl)(phenyl)silane (3ee)

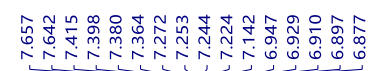<smiles>COc1ccccc1/C=C(\C)[SH2+]c1ccccc1</smiles>

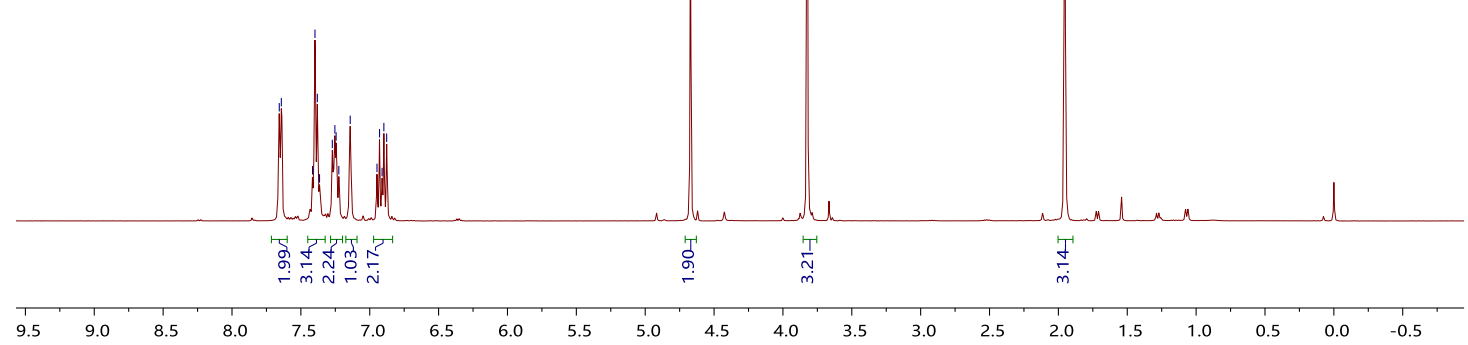

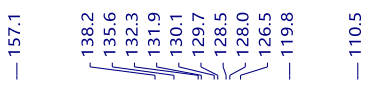

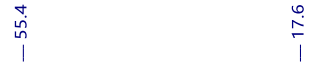<smiles>COc1ccccc1/C=C(\[Si])CSc1ccccc1</smiles>

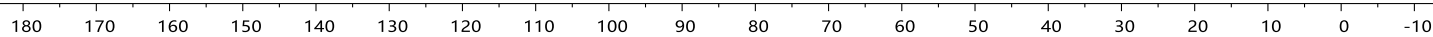


(E)-(1-(2-methoxyphenyl)prop-1-en-1-yl)(phenyl)silane (4ee)

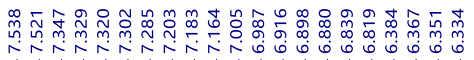

$\underset{\substack{i \\ \dot{0}}}{\substack{0 \\ \dot{m} \\ i}}$

立<smiles>C/C=C(\[SH+]c1ccccc1)c1ccccc1OC</smiles>

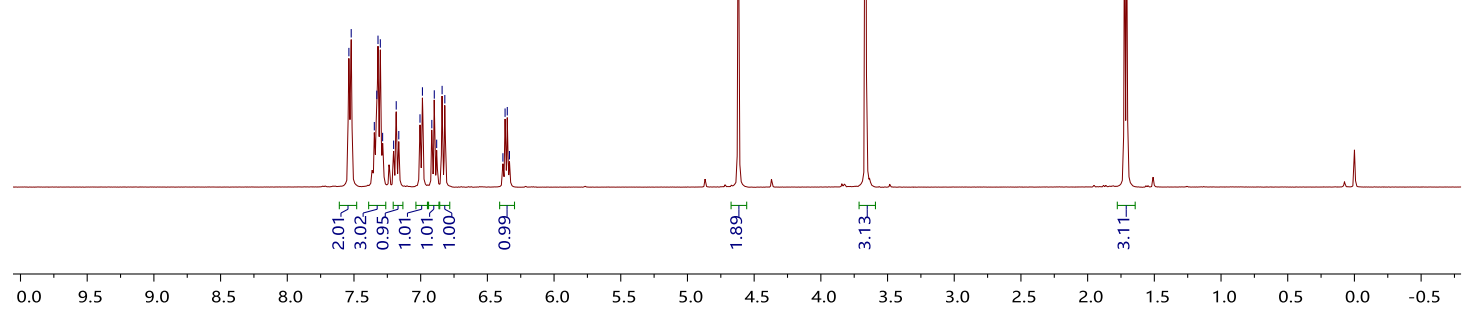

I

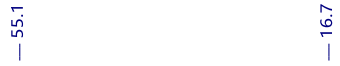<smiles>C/C=C(/[SH+]c1ccccc1)c1ccccc1OC</smiles> 
<smiles>CC/C(=C\c1ccccc1)[SiH2]c1ccccc1</smiles>

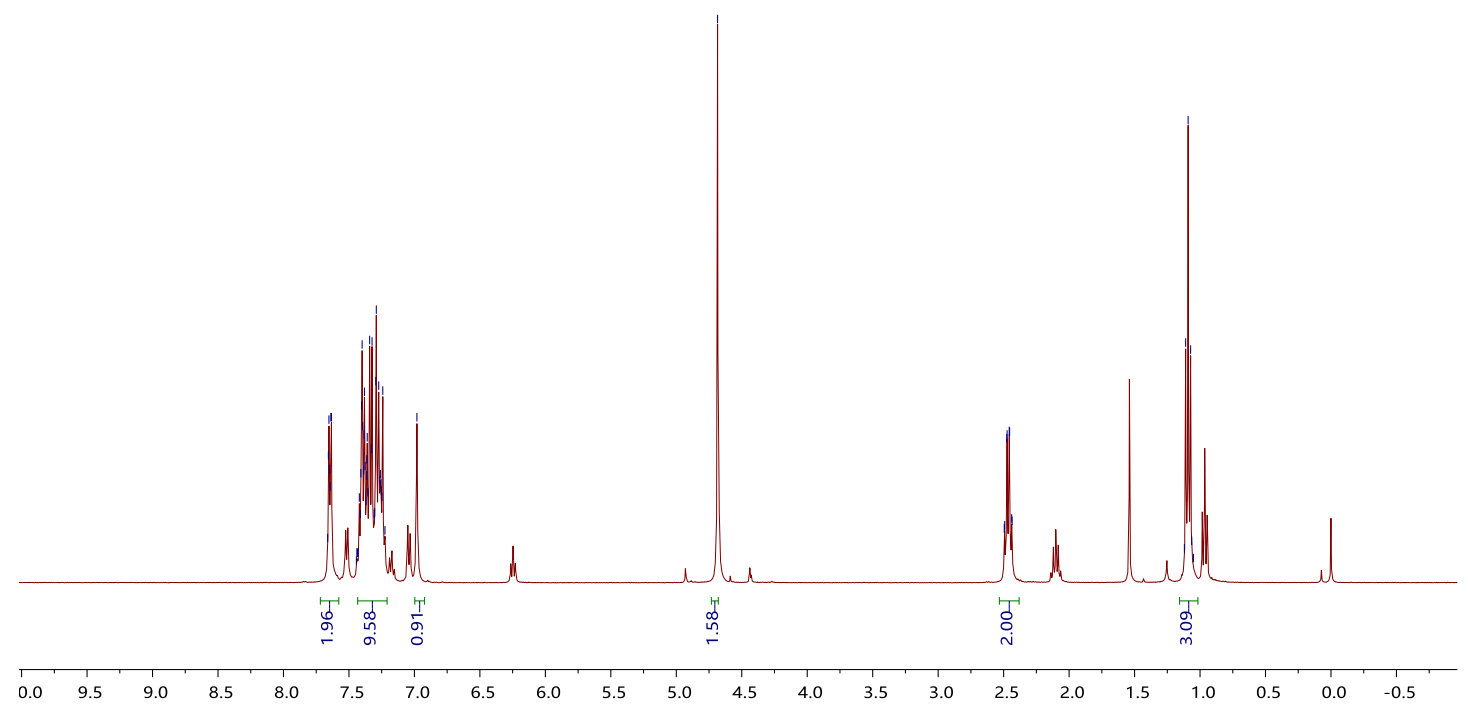

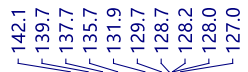<smiles>CC/C(=C\c1ccccc1)[SiH2]c1ccccc1</smiles>

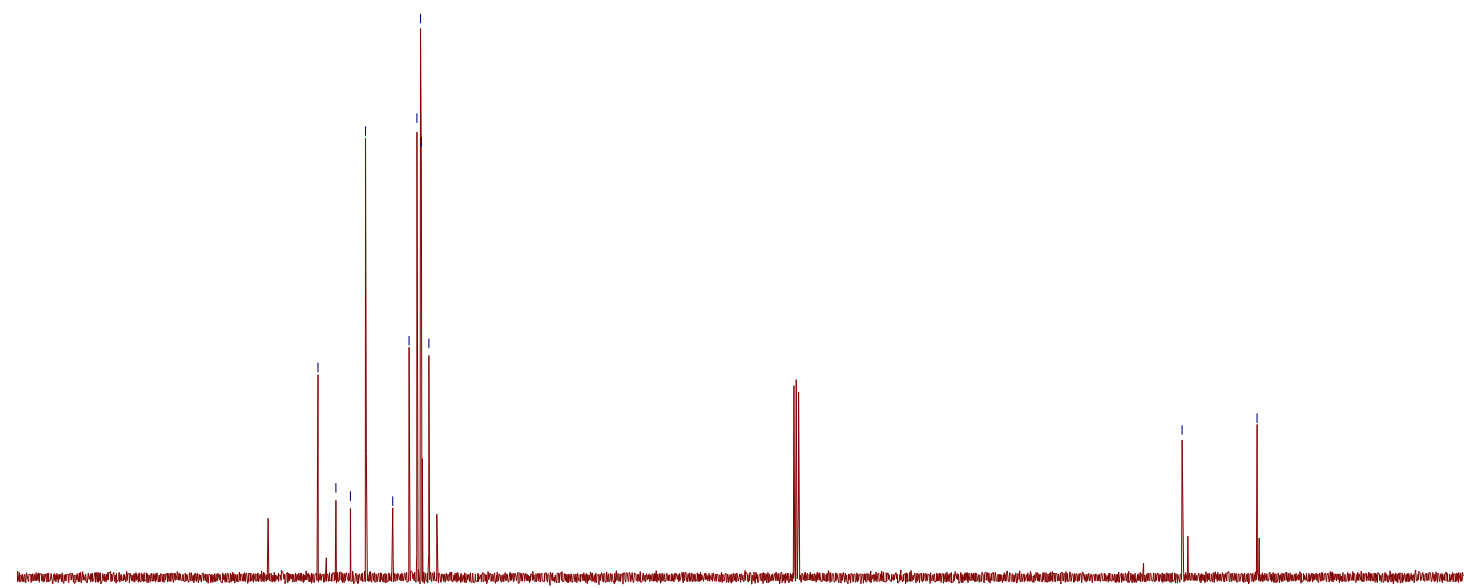

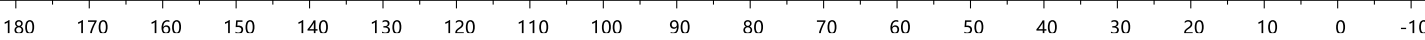


(E)-phenyl(1-phenylbut-1-en-1-yl)silane (4ef)

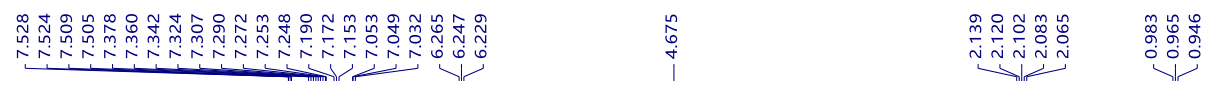<smiles>CC/C=C(\[SnH+])c1ccccc1</smiles>

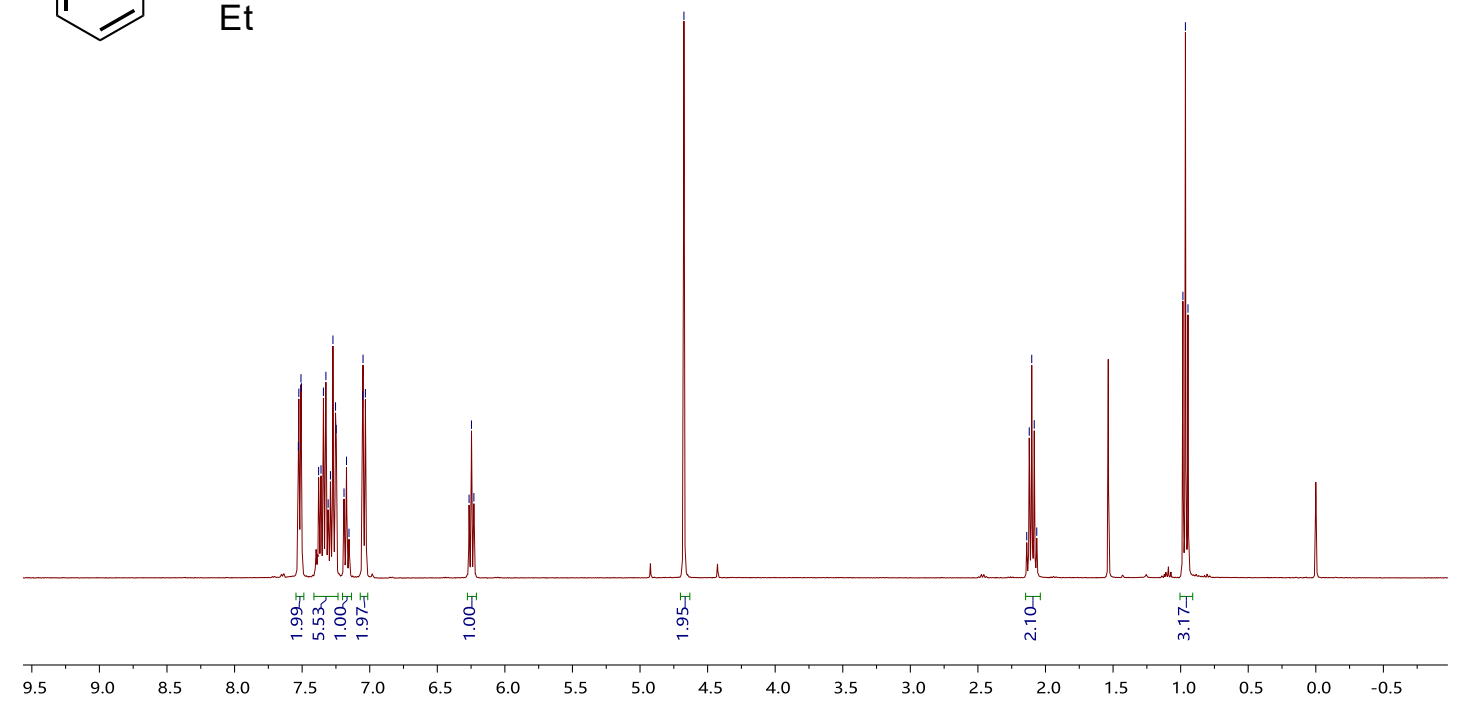

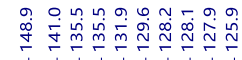

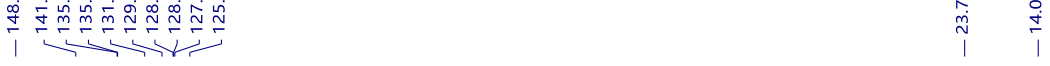

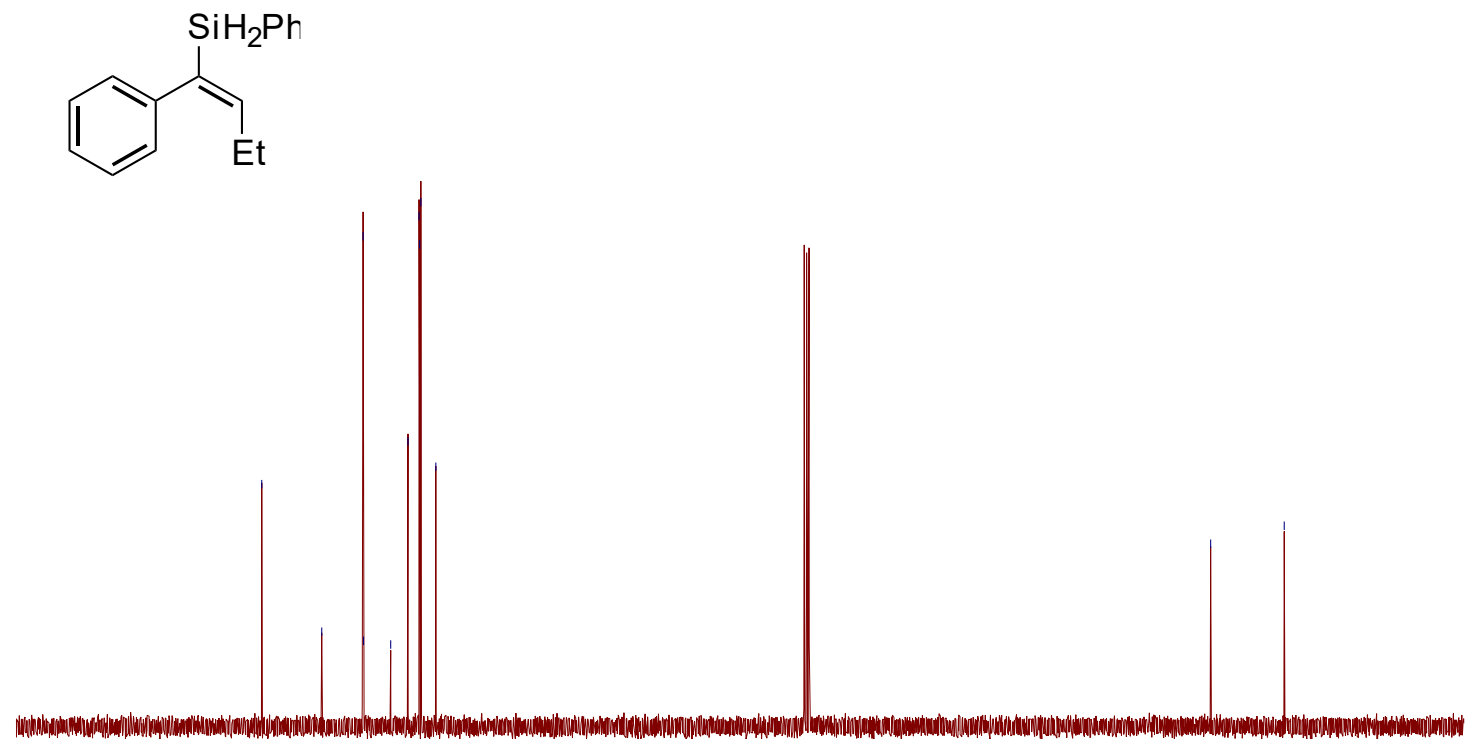

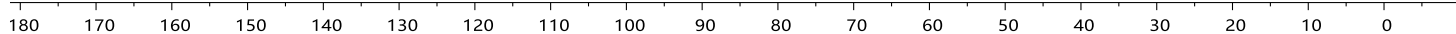


phenyl((2E,4E)-5-phenylpenta-2,4-dien-2-yl)silane (3eg)

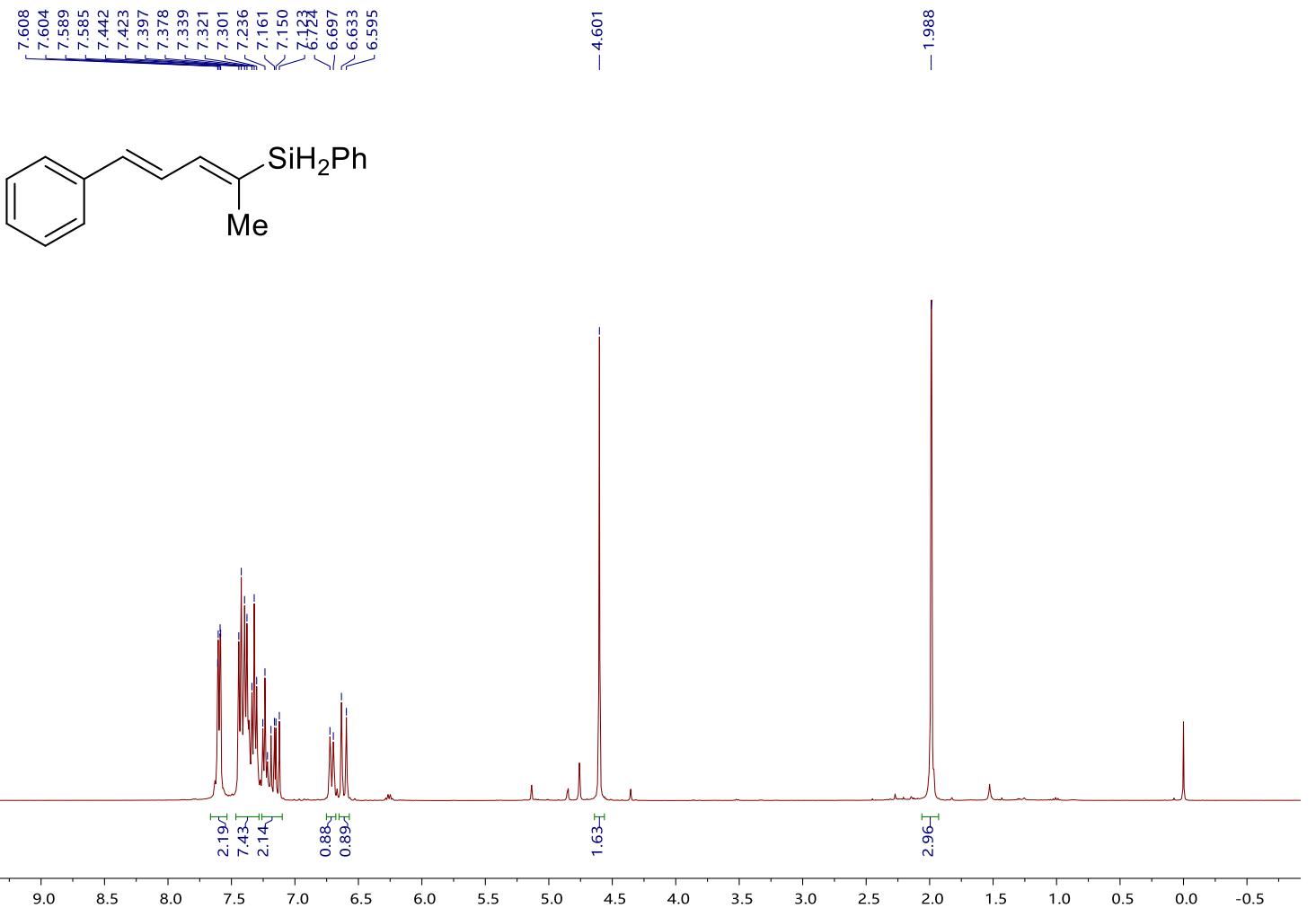

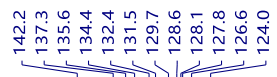<smiles>CC(=CC=Cc1ccccc1)Sc1ccccc1</smiles>

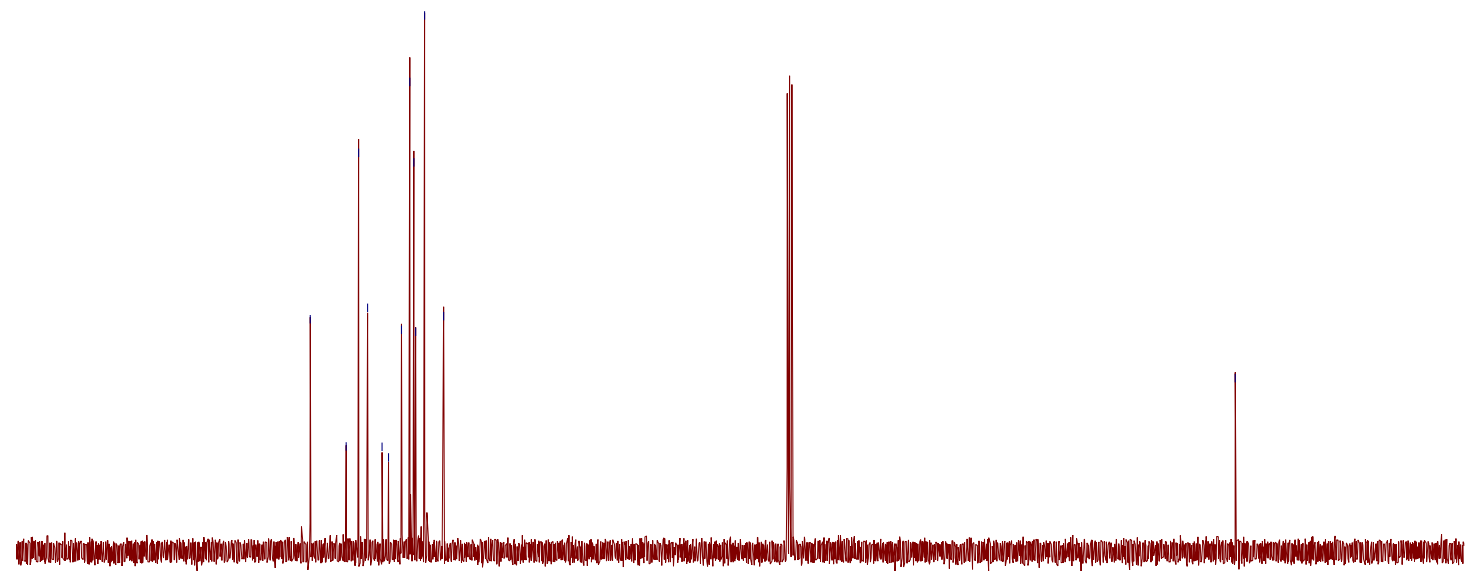

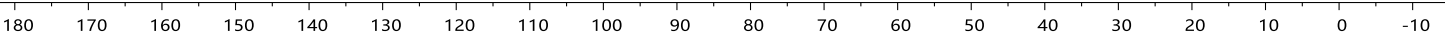


phenyl((1E,3E)-1-phenylpenta-1,3-dien-3-yl)silane (4eg)

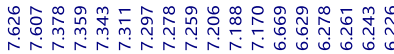<smiles>C/C=C(\C=C\c1ccccc1)c1ccccc1</smiles>

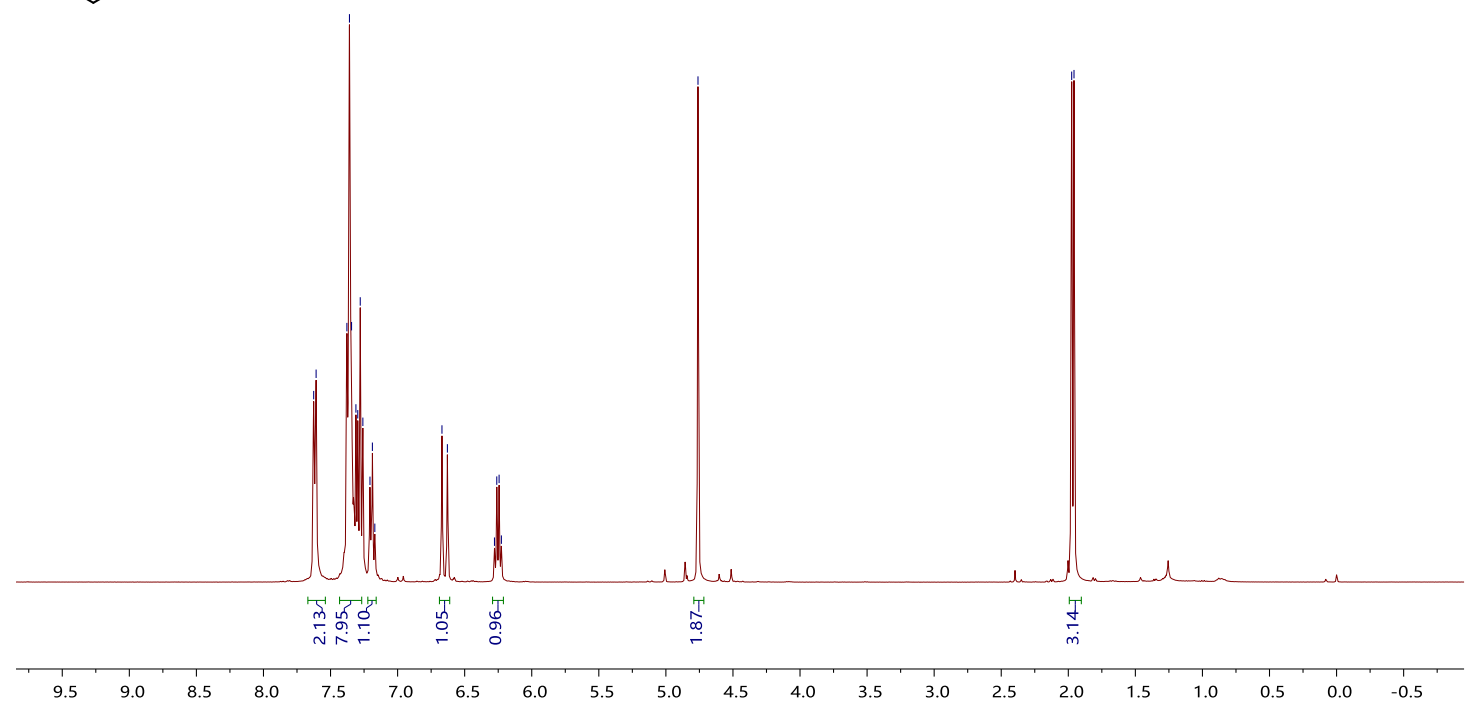

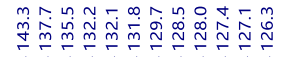<smiles>C/C=C(/C=C/c1ccccc1)c1ccccc1</smiles>

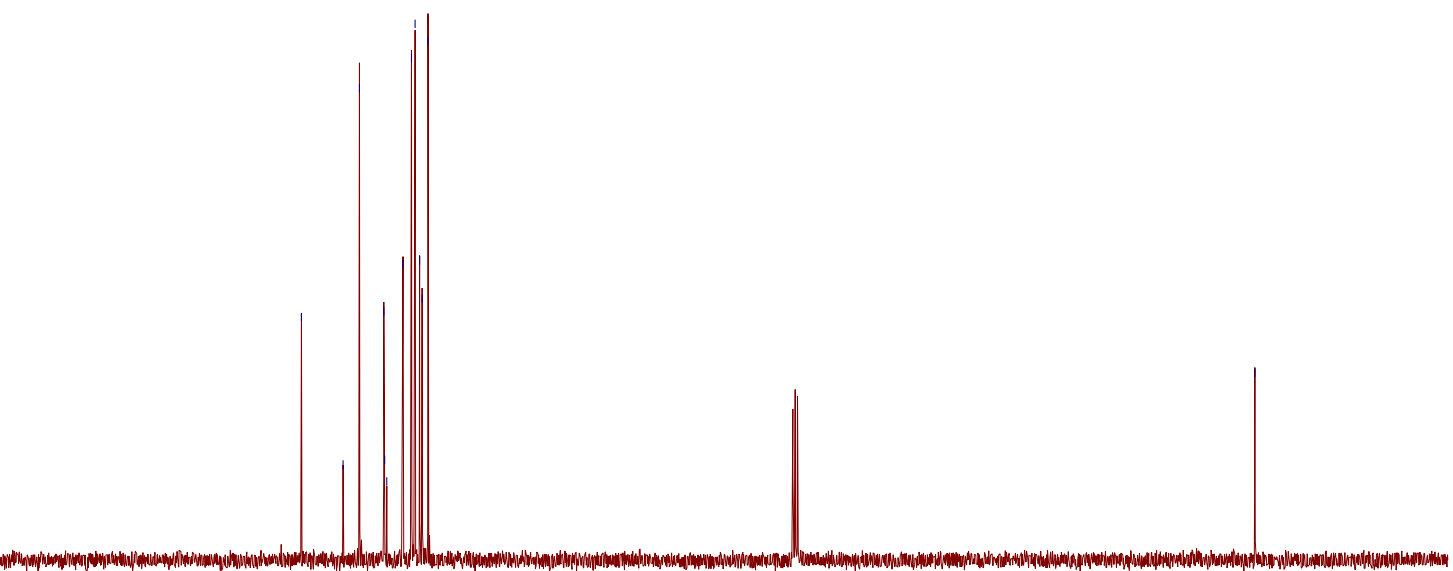

$\begin{array}{lllllllllllllllllll}180 & 170 & 160 & 150 & 140 & 130 & 120 & 110 & 100 & 90 & 80 & 70 & 60 & 50 & 40 & 30 & 20 & 10 & 1\end{array}$ 
dodecyl((2E,4E)-4-methyl-5-phenylpenta-2,4-dien-2-yl)silane (3eh)

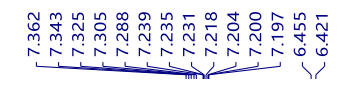

苋

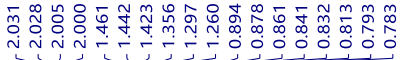

$\mathrm{M}_{\mathrm{Me}}^{\mathrm{SiH}_{2} \mathrm{C}_{12} \mathrm{H}_{25}}$
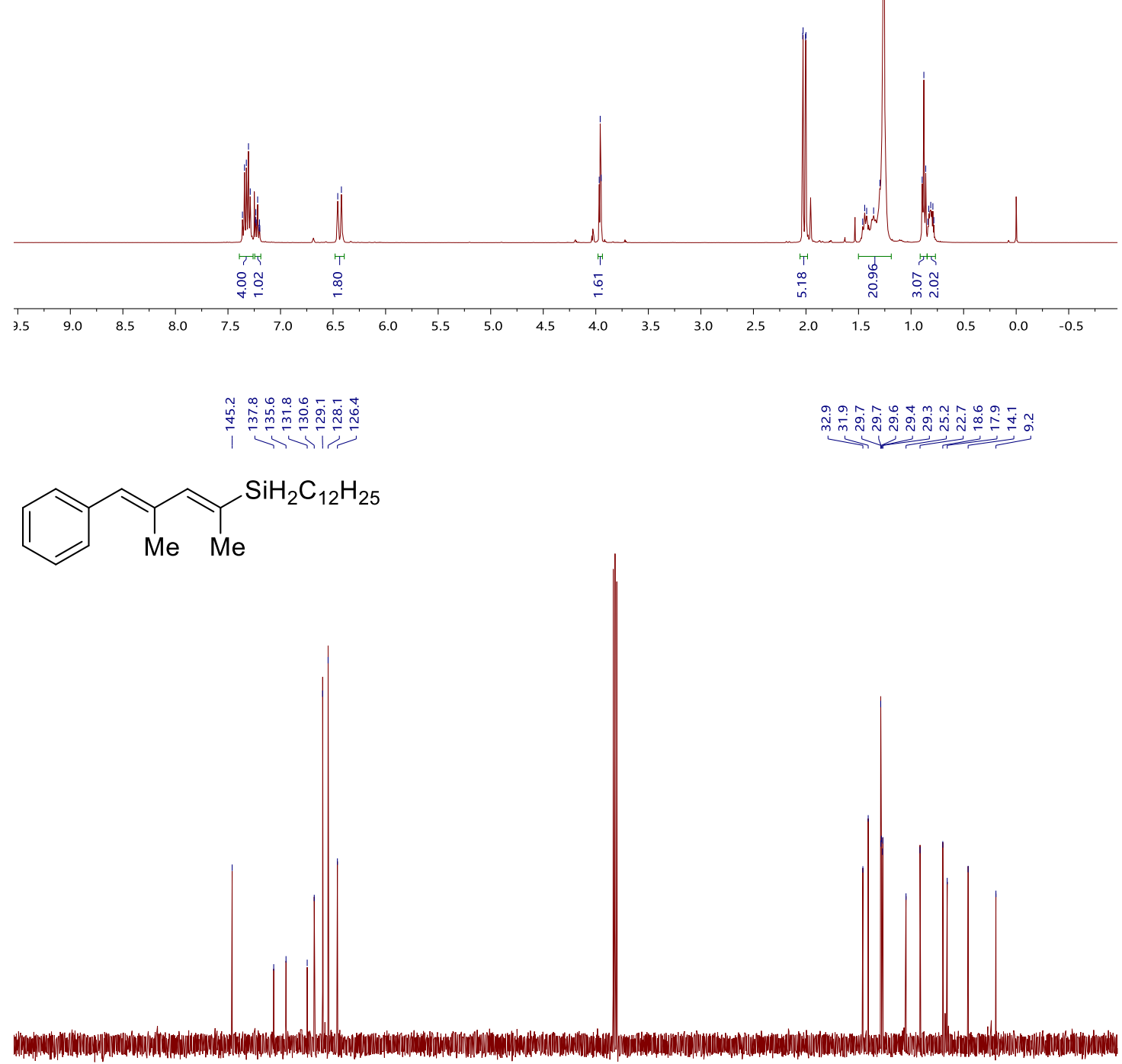

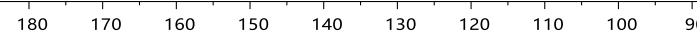

80

$60 \quad 50$

$40 \quad 30$

20 , 
((1E,3E)-2-methyl-1-phenylpenta-1,3-dien-3-yl)(phenyl)silane (4eh)

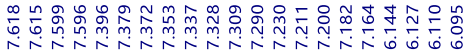

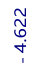

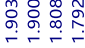<smiles>C/C=C(/[SH+]c1ccccc1)C(C)=Cc1ccccc1</smiles>

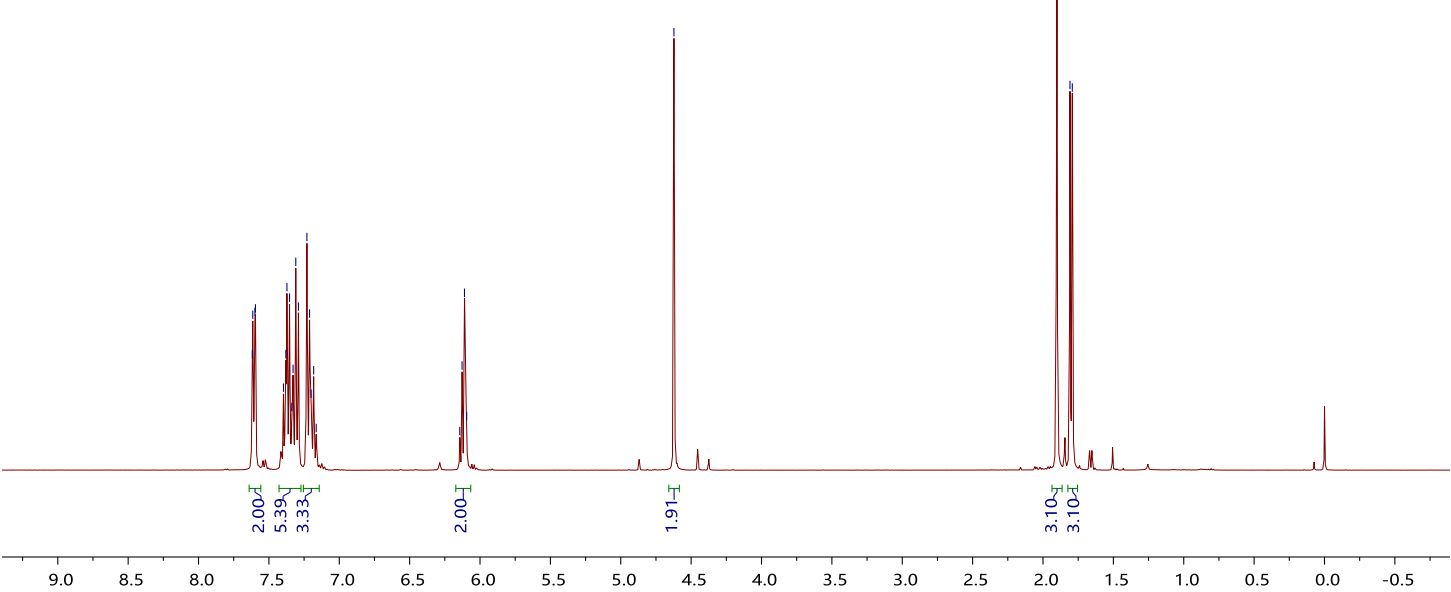

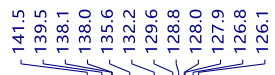<smiles>C/C=C(/[SH+]c1ccccc1)C(C)=Cc1ccccc1</smiles>

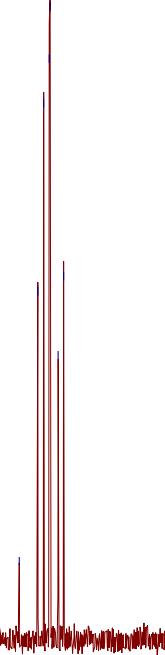

$\begin{array}{llllllllll}180 & 170 & 160 & 150 & 140 & 130 & 120 & 110 & 100 & 90\end{array}$

80

60

40

20

$0,-10$ 
(E)-(1-(cyclohex-1-en-1-yl)prop-1-en-2-yl)(phenyl)silane (3ei)

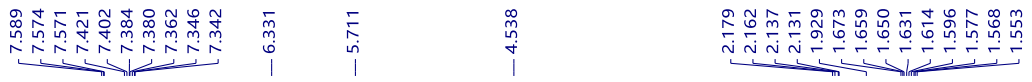

$\overbrace{M e}^{\mathrm{SiH}_{2} \mathrm{Ph}}$

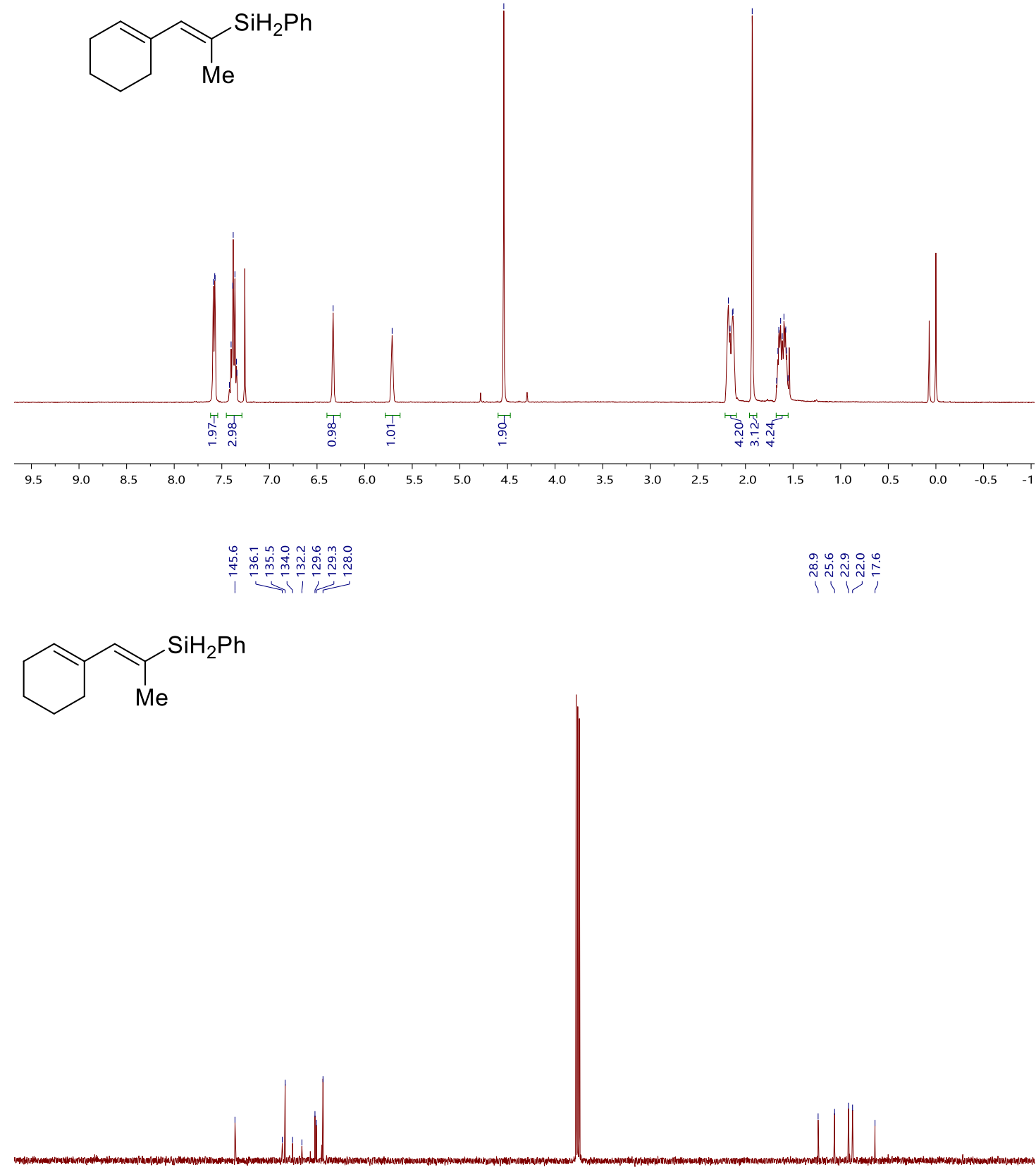

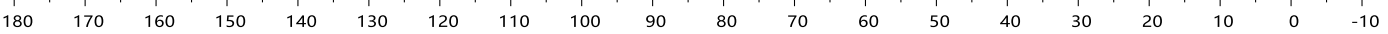




\section{(E)-(1-(cyclohex-1-en-1-yl)prop-1-en-1-yl)(phenyl)silane (4ei)}

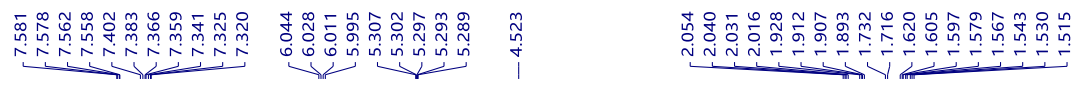<smiles>C/C=C(/Sc1ccccc1)C1=CCCCC1</smiles>
$\mathrm{Me}$

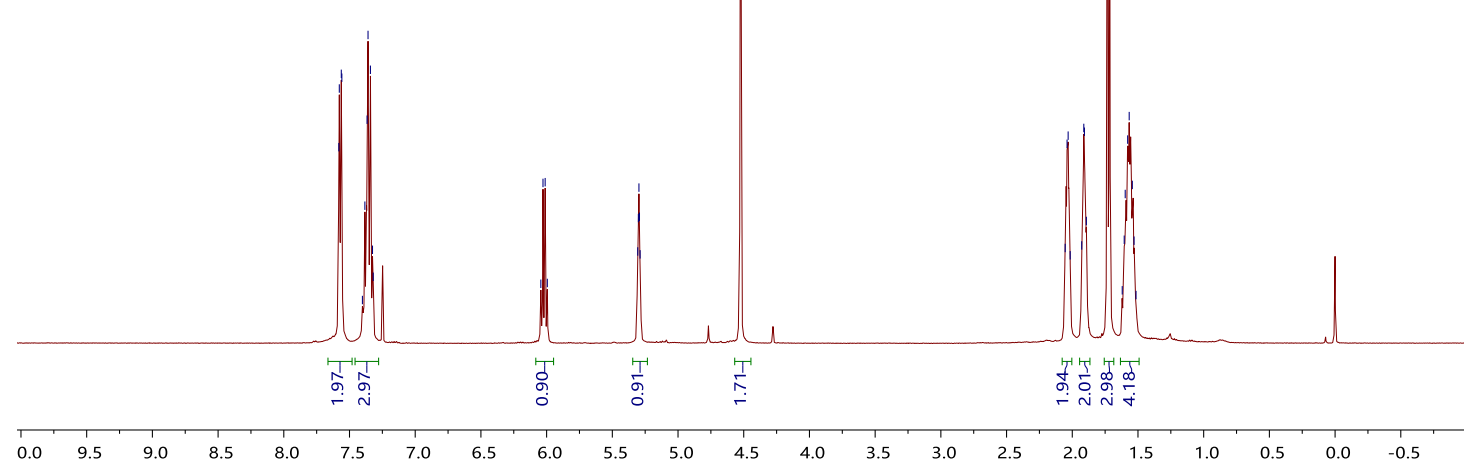

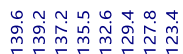

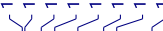

ปูำ<smiles>C/C=C(/[SH2+]c1ccccc1)C1=CCCCC1</smiles>

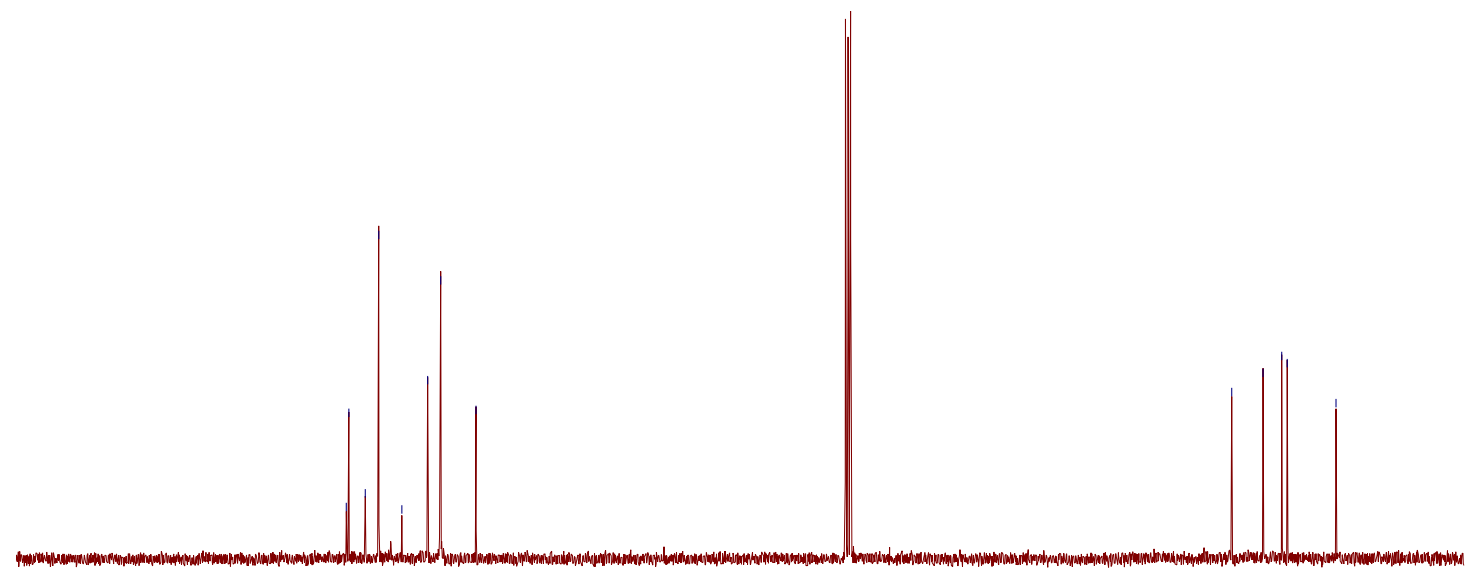


(E)-(4-methylbenzyl)(oct-2-en-2-yl)silane (3ej)

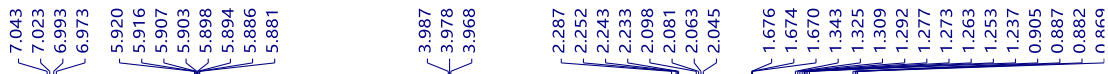

$\mathrm{C}_{5} \mathrm{H}_{11} \overbrace{\mathrm{Me}}^{\mathrm{Si}_{2}^{\mathrm{Me}}}$

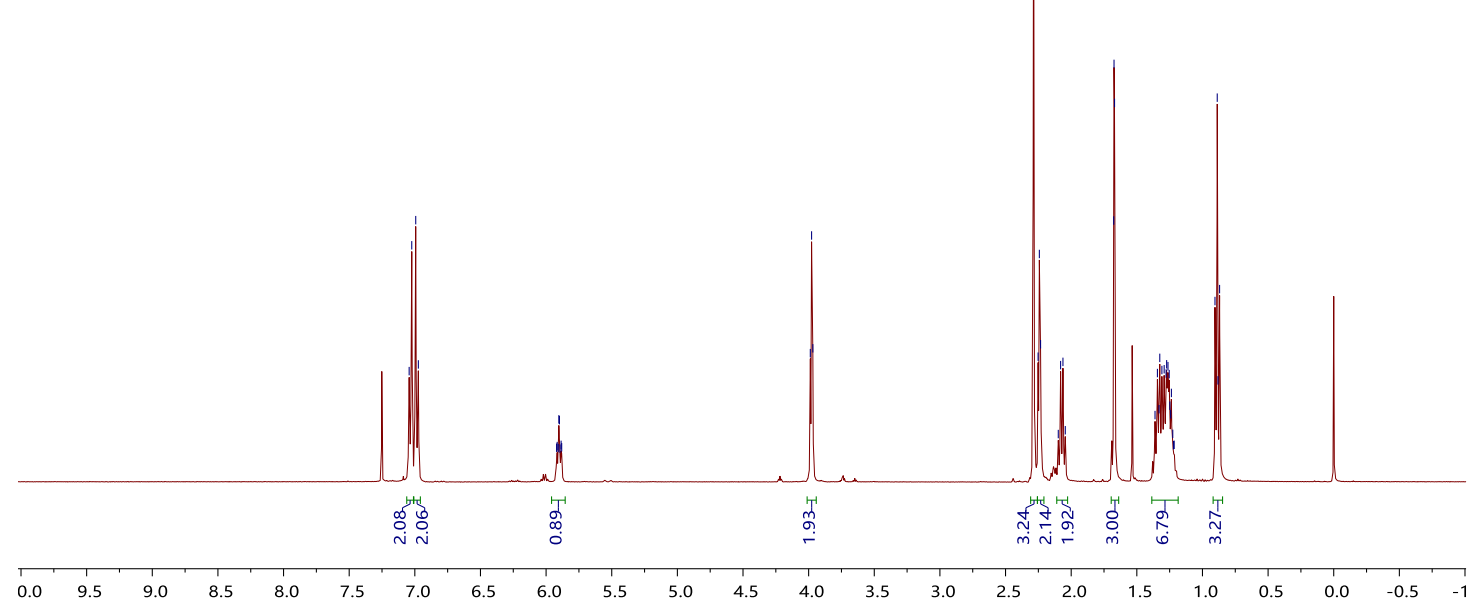

年

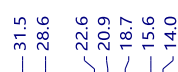

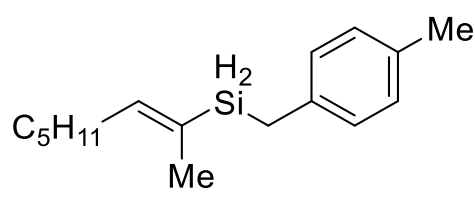

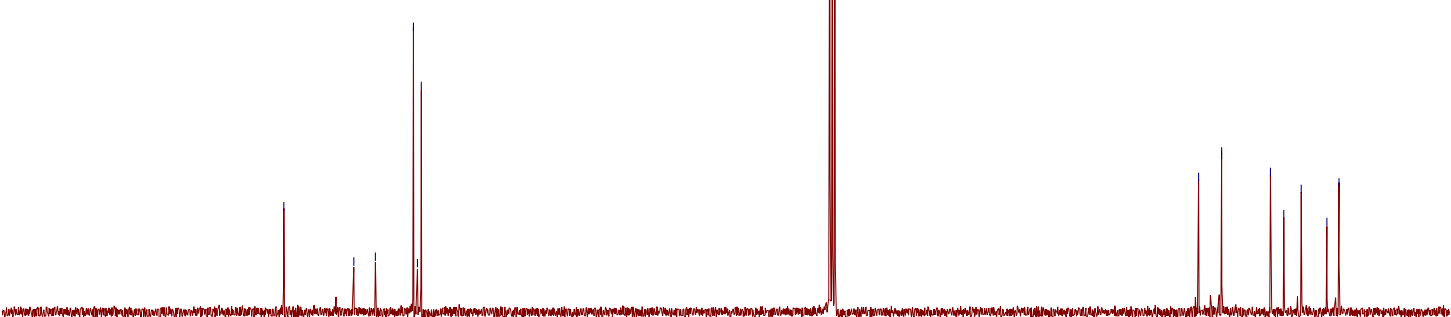

80

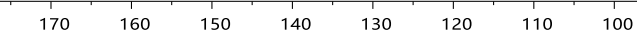

90

$\quad 70 \quad 60$

$50 \quad 40$

$30 \quad 20 \quad 10$ 
(E)-oct-2-en-3-yl(phenyl)silane (4ej)

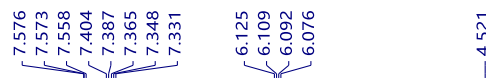

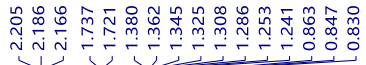<smiles>C/C=C(/[SnH+]c1ccccc1)[Hg]c1ccccc1</smiles>

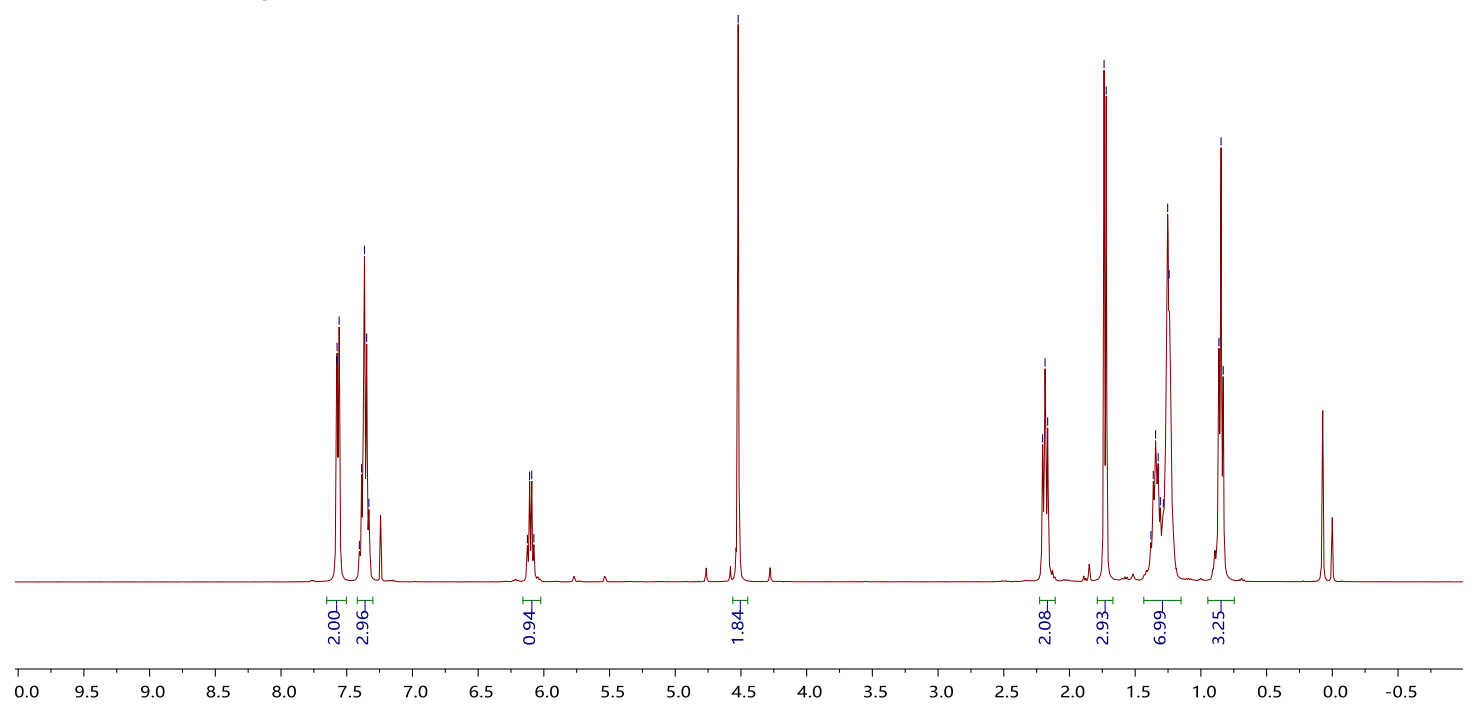

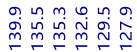

ऽ广।

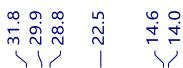<smiles>CC=C([Hg]c1ccccc1)[Hg]c1ccccc1</smiles>
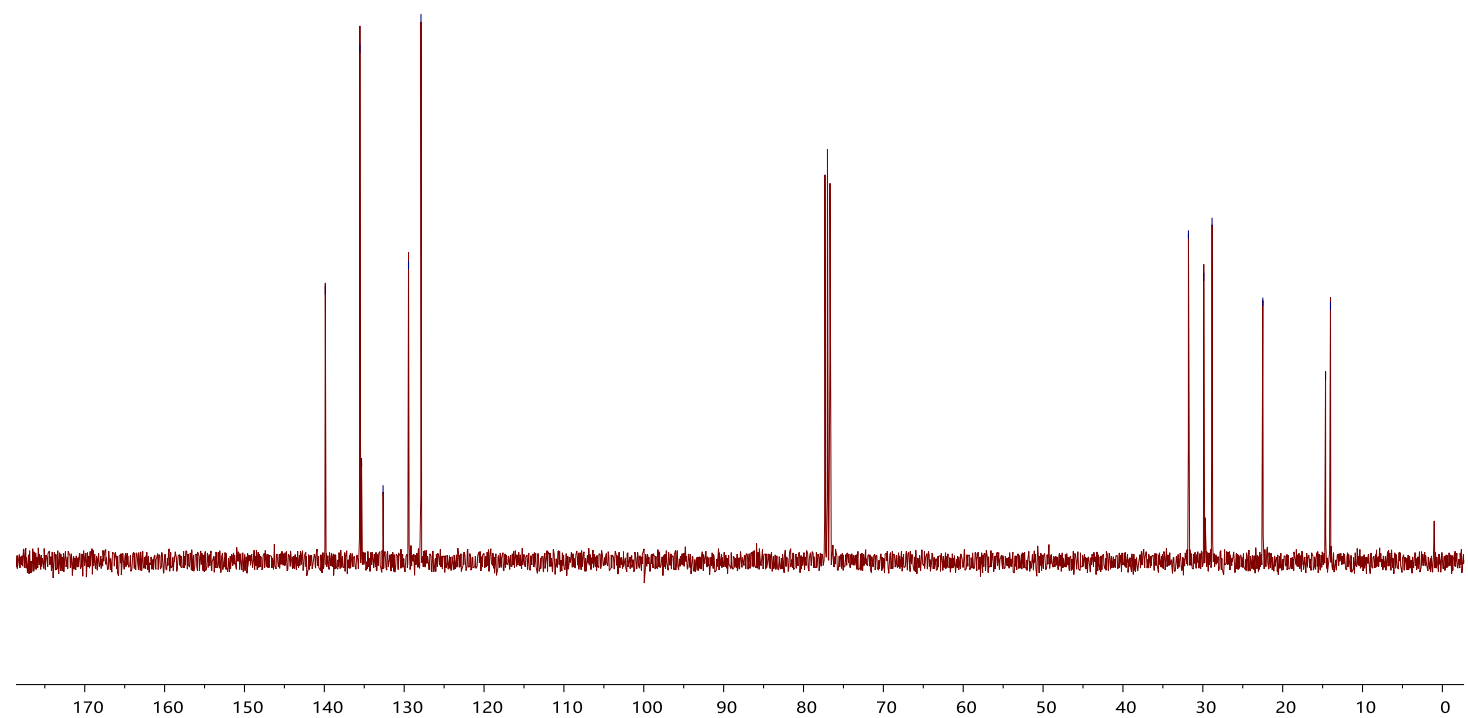
(E)-(4-methylbenzyl)(6-phenylhex-2-en-2-yl)silane (3ek)

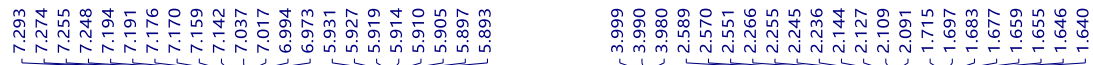

$\overbrace{M e}^{M e}$

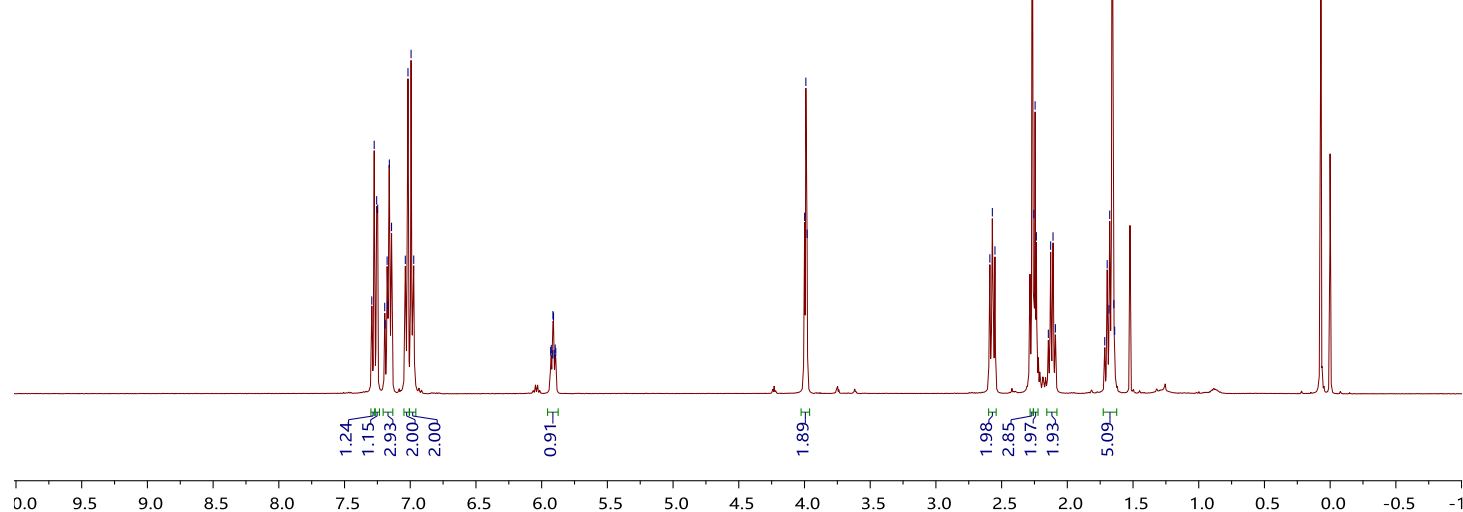

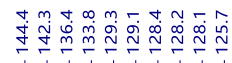

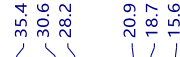<smiles>CC(=CCCCc1ccccc1)[SiH2]Cc1ccc(C)cc1</smiles>

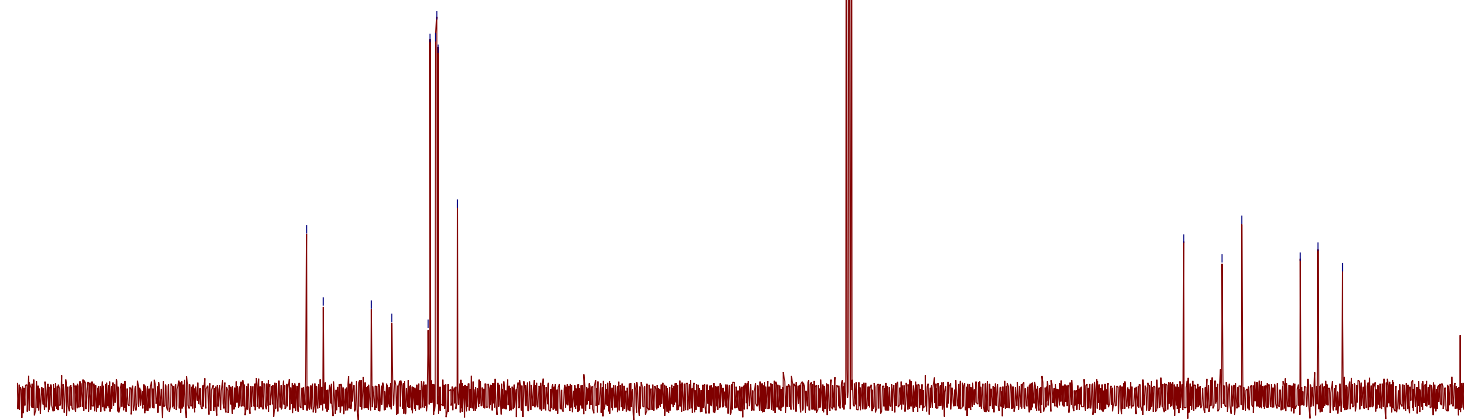

80

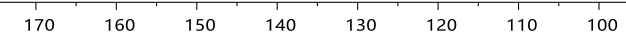

$90 \quad 80$

$70 \quad 60$

$50 \quad 40$

$30 \quad 20 \quad 10$ 
(E)-phenyl(6-phenylhex-2-en-3-yl)silane (4ek)

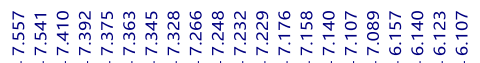<smiles>CC=C(CCCc1ccccc1)C[SnH+]c1ccccc1</smiles>

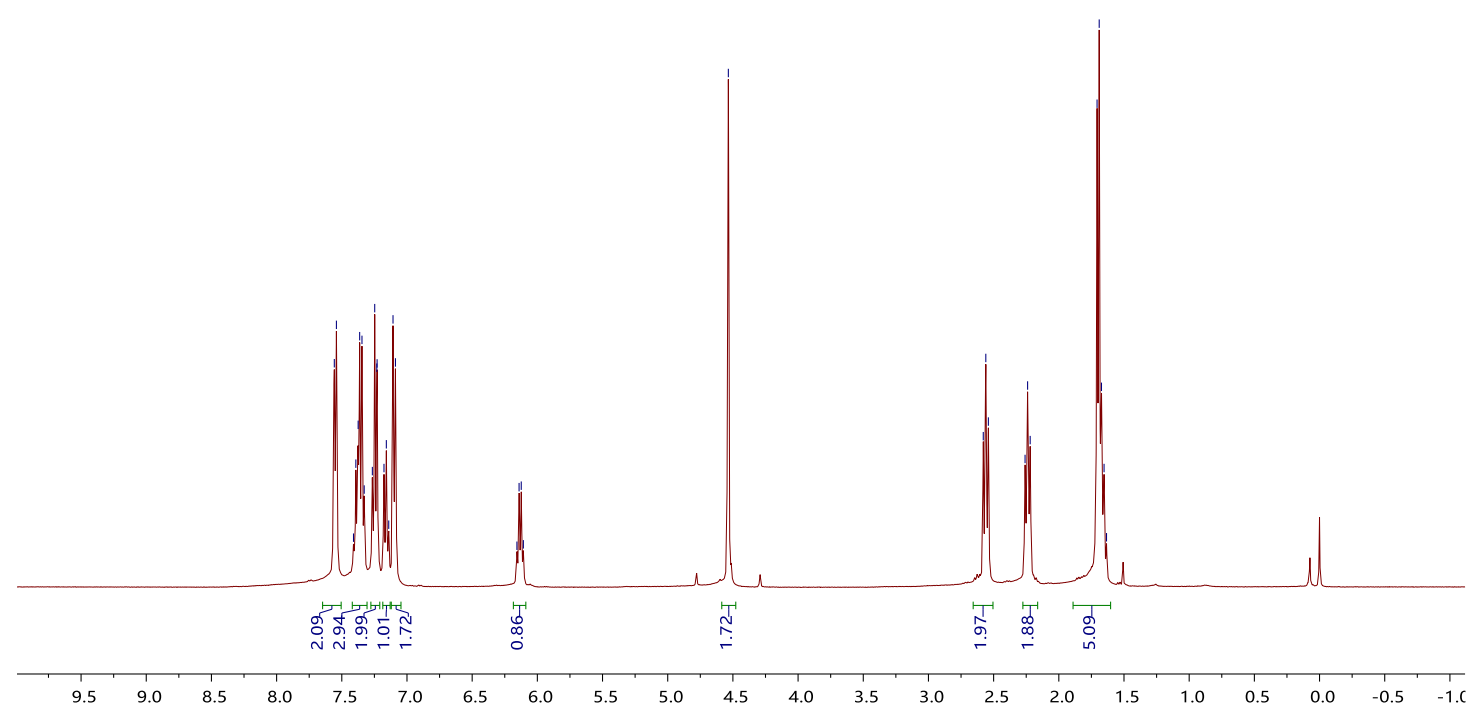

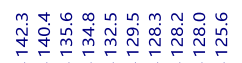

$\underbrace{1}$

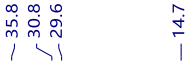<smiles>CC=C(CCCc1ccccc1)CSc1ccccc1</smiles>

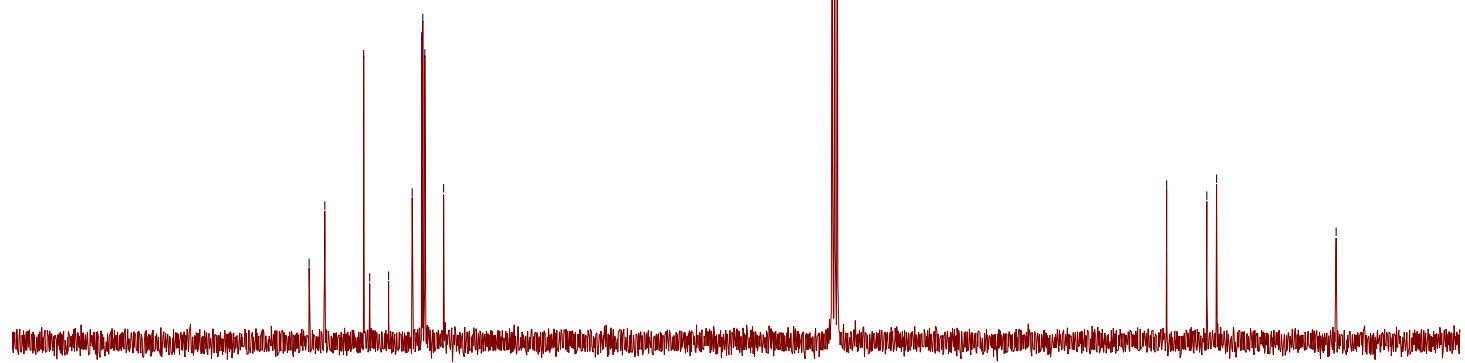

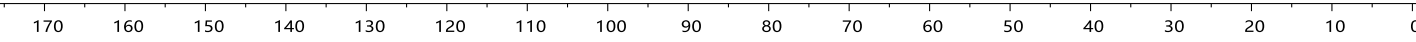


(E)-(1-cyclohexylprop-1-en-2-yl)(phenyl)silane (3el)

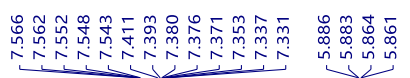

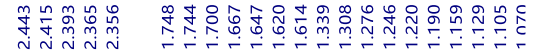

$\overbrace{\mathrm{Me}}^{\mathrm{SiH}_{2} \mathrm{Ph}}$

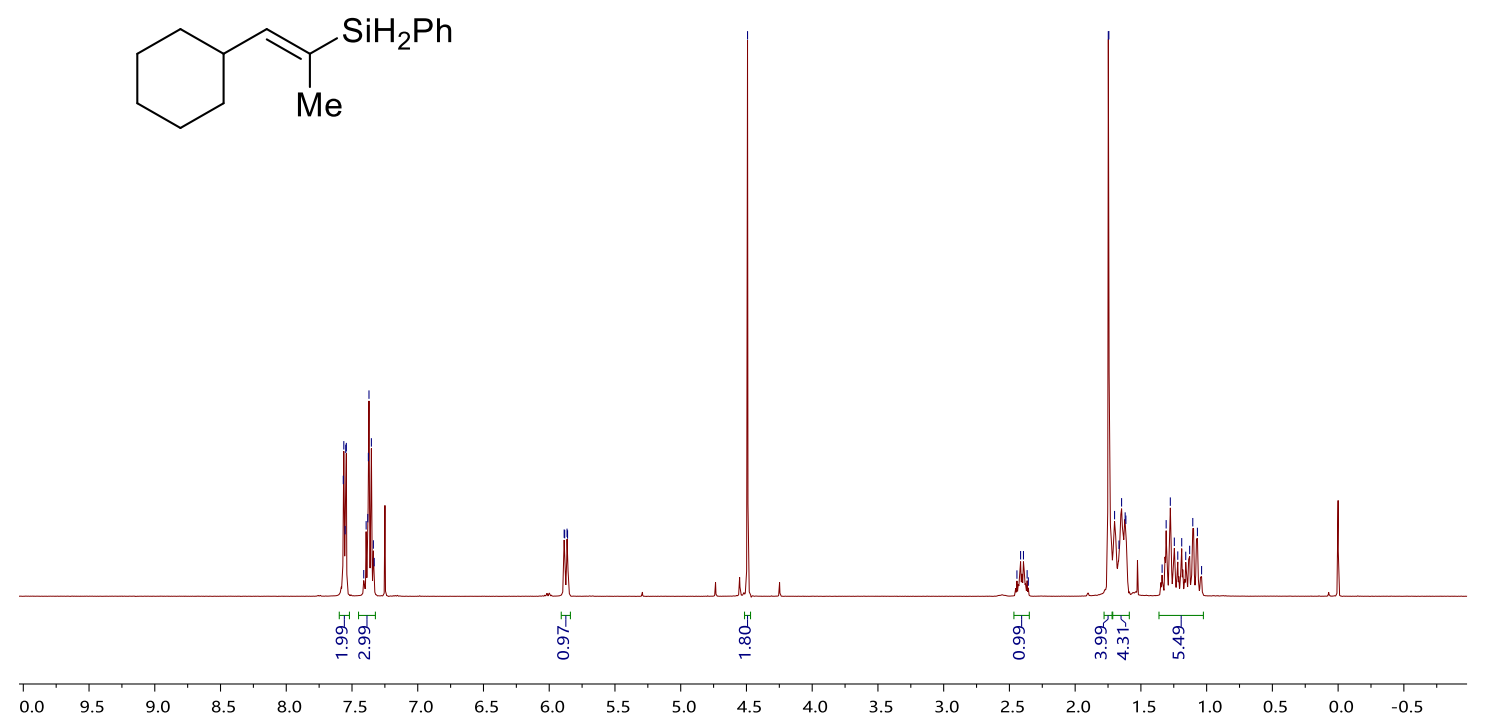

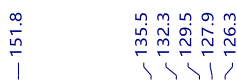

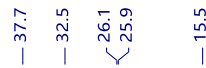

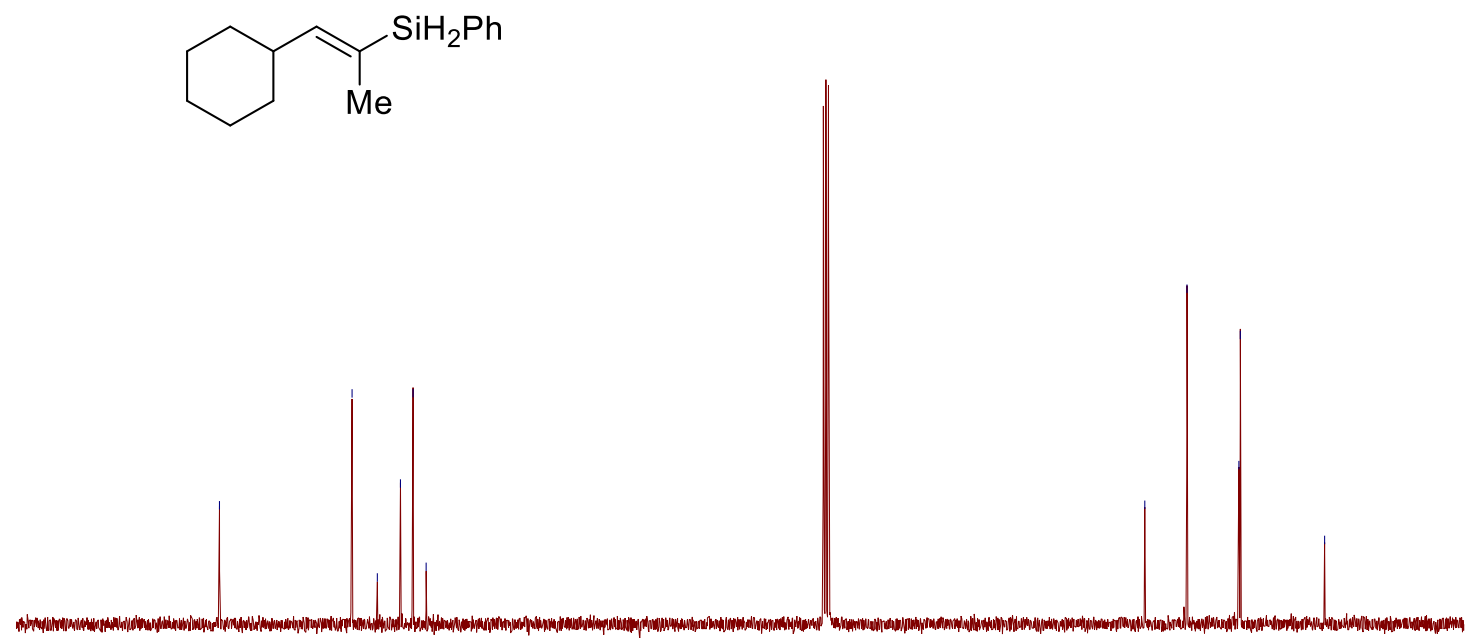

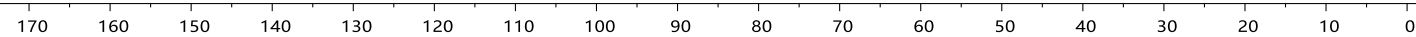


(E)-(1-cyclohexylprop-1-en-1-yl)(phenyl)silane (4el)

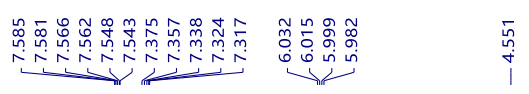

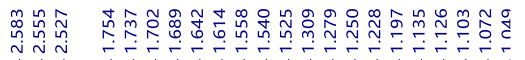<smiles>C/C=C(/[SH2+]c1ccccc1)C1CCCCC1</smiles>

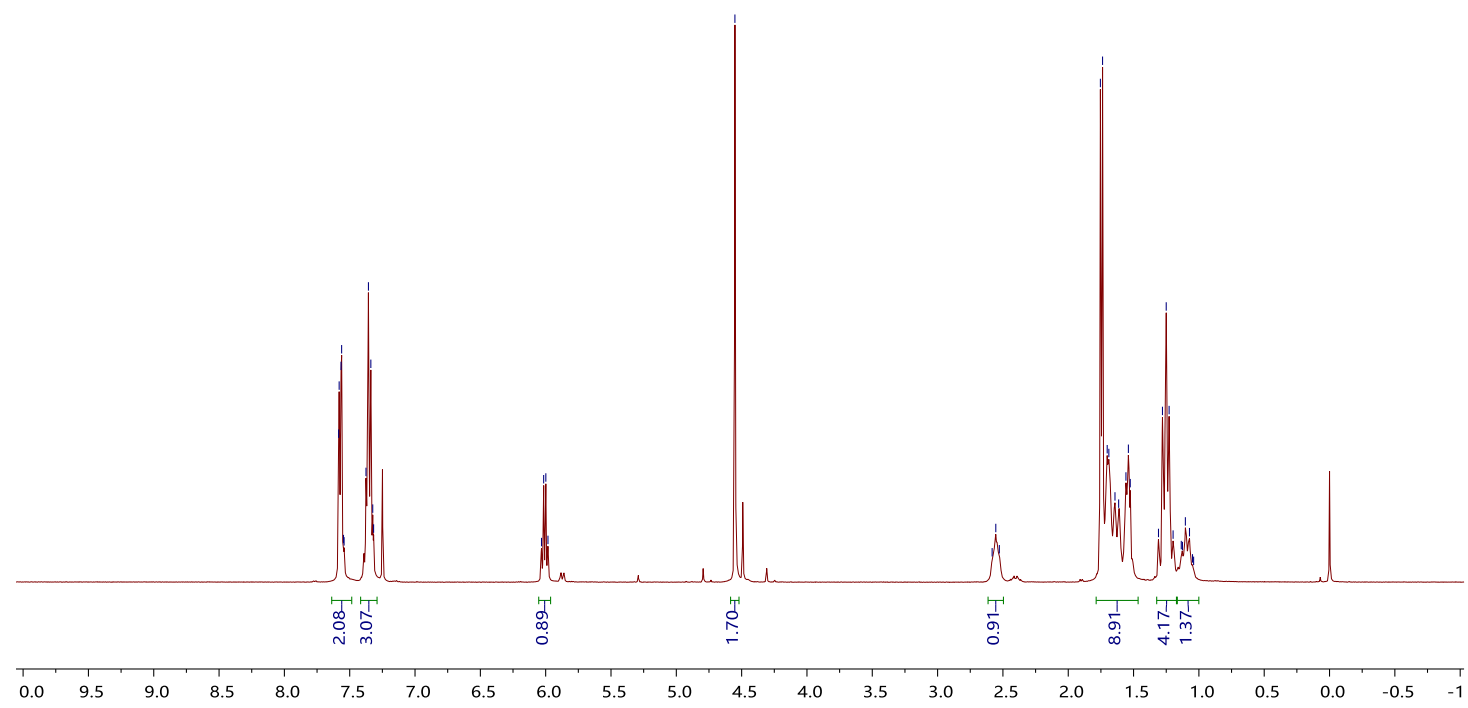

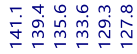

广Т

产<smiles>C/C=C(/[SH2+]c1ccccc1)C1CCCCC1</smiles>

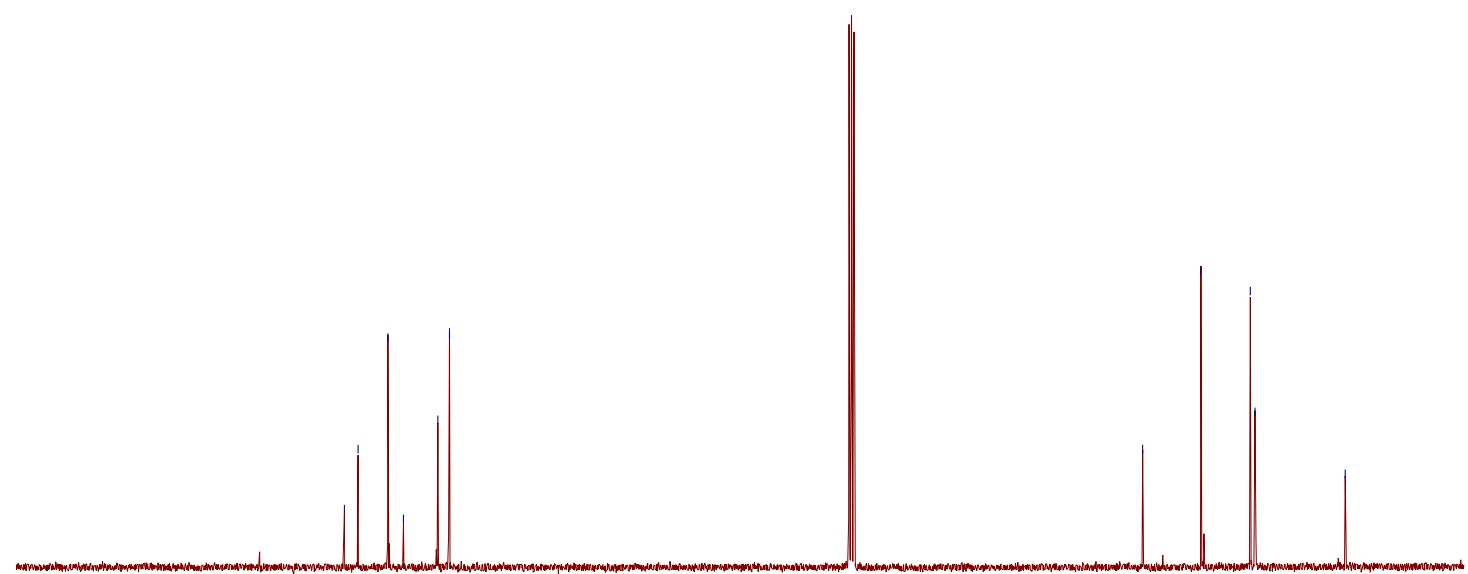

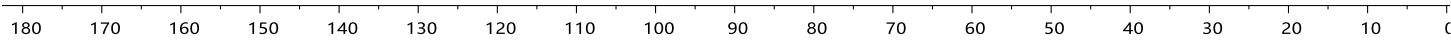


(E)-(4,4-dimethylpent-2-en-2-yl)(dodecyl)silane (3em)
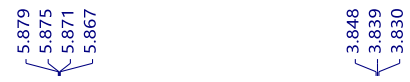

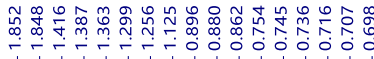

${ }^{t} \mathrm{Bu} \overbrace{\mathrm{Me}}^{\mathrm{SiH}_{2} \mathrm{C}_{12} \mathrm{H}_{25}}$
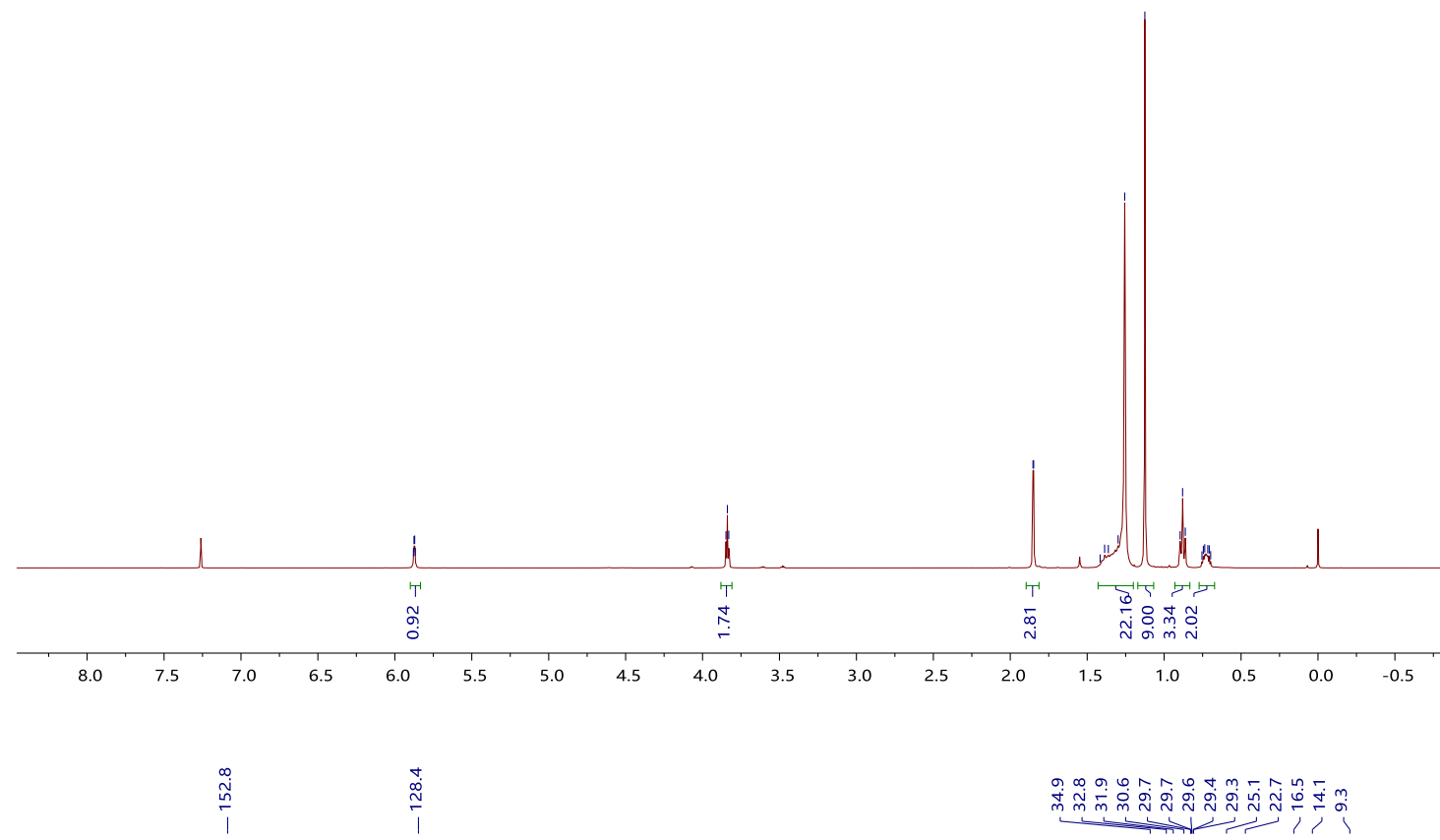

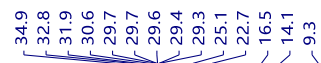
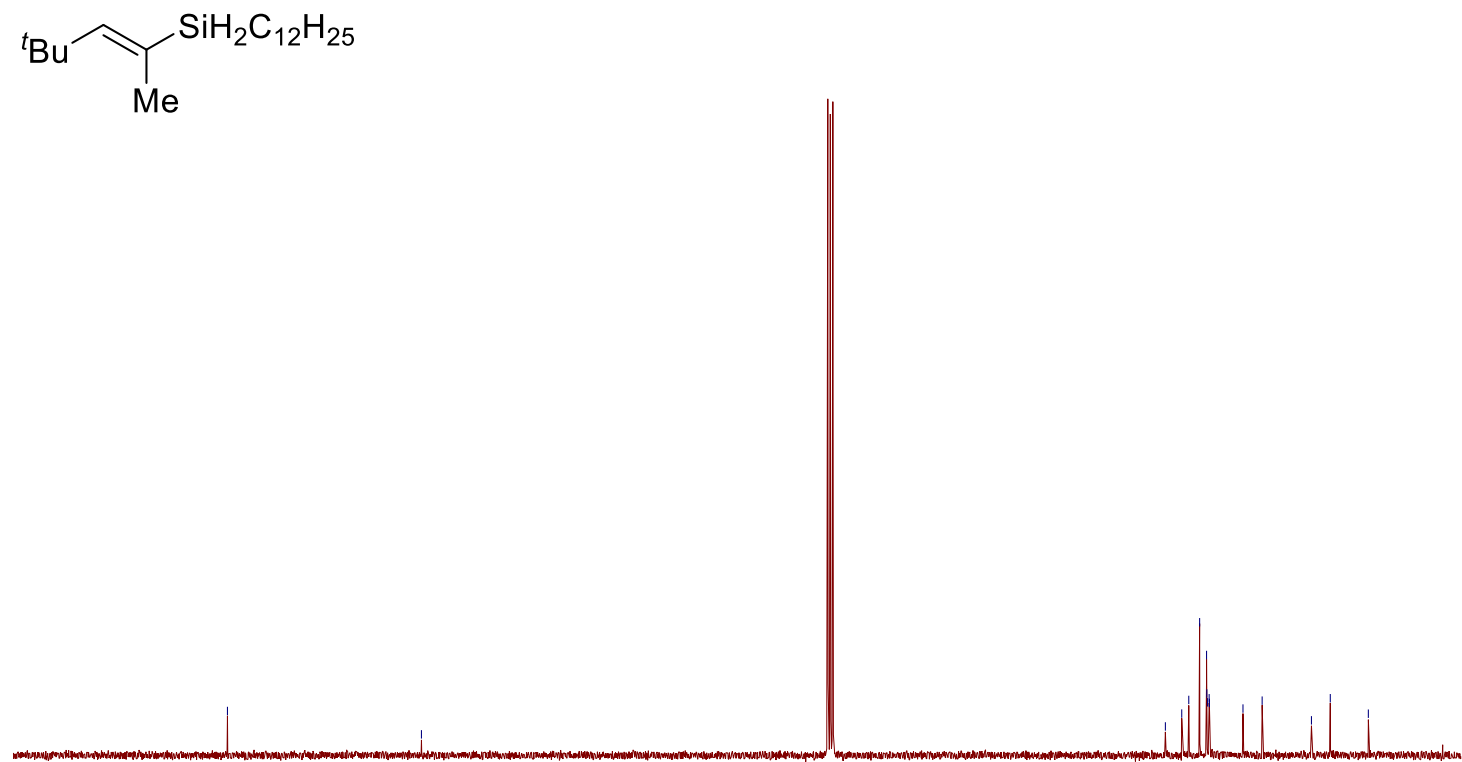

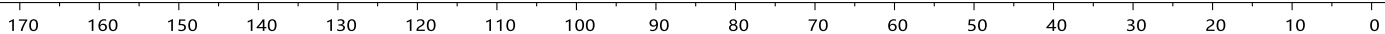


(E)-(4,4-dimethylpent-2-en-3-yl)(phenyl)silane (4em)

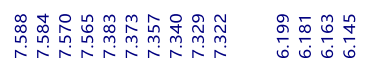

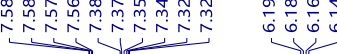

㞭<smiles>C/C=C(\[SH2+]c1ccccc1)C(C)(C)C</smiles>

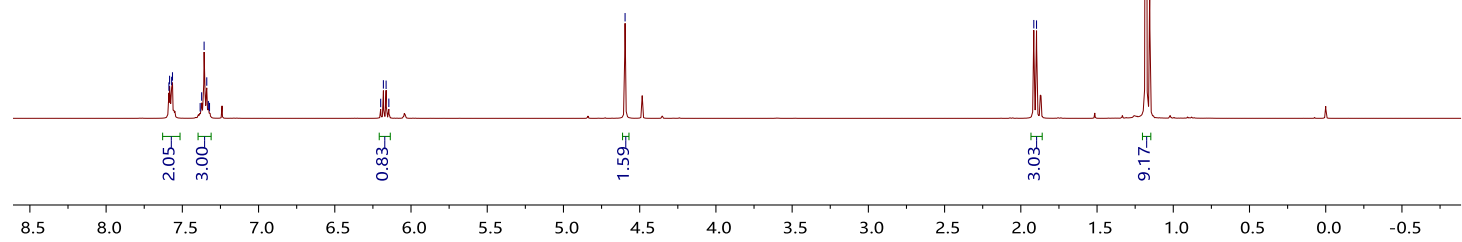

竎等

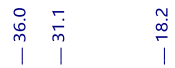<smiles>C/C=C(/[SH2+]c1ccccc1)C(C)(C)C</smiles>

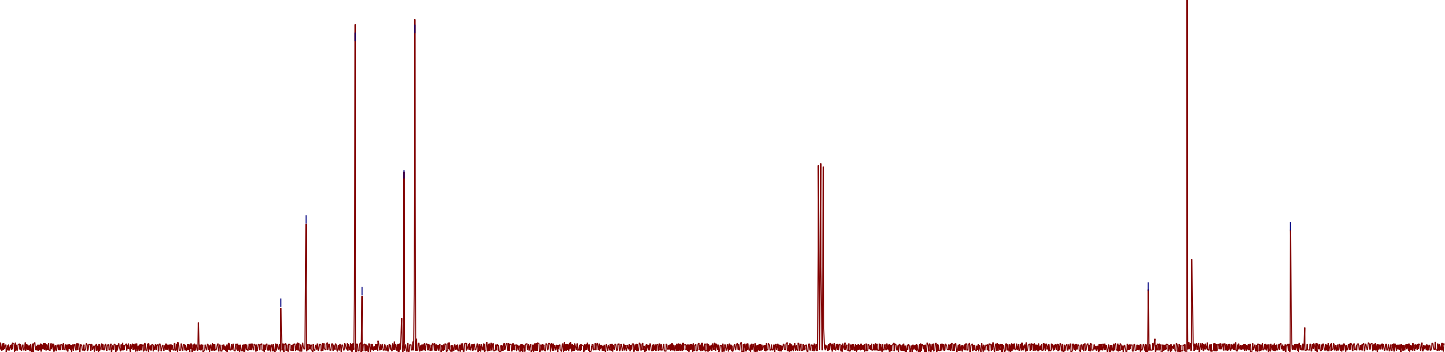

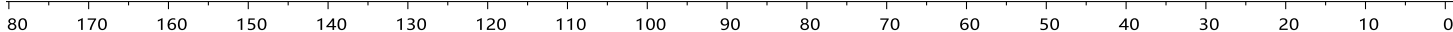


(E)-(1-cyclohexylbut-1-en-2-yl)(dodecyl)silane (3en)

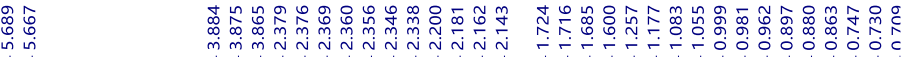

$ح_{\mathrm{Et}}^{\mathrm{SiH}_{2} \mathrm{C}_{12} \mathrm{H}_{25}}$

$\underset{\longrightarrow}{ }$

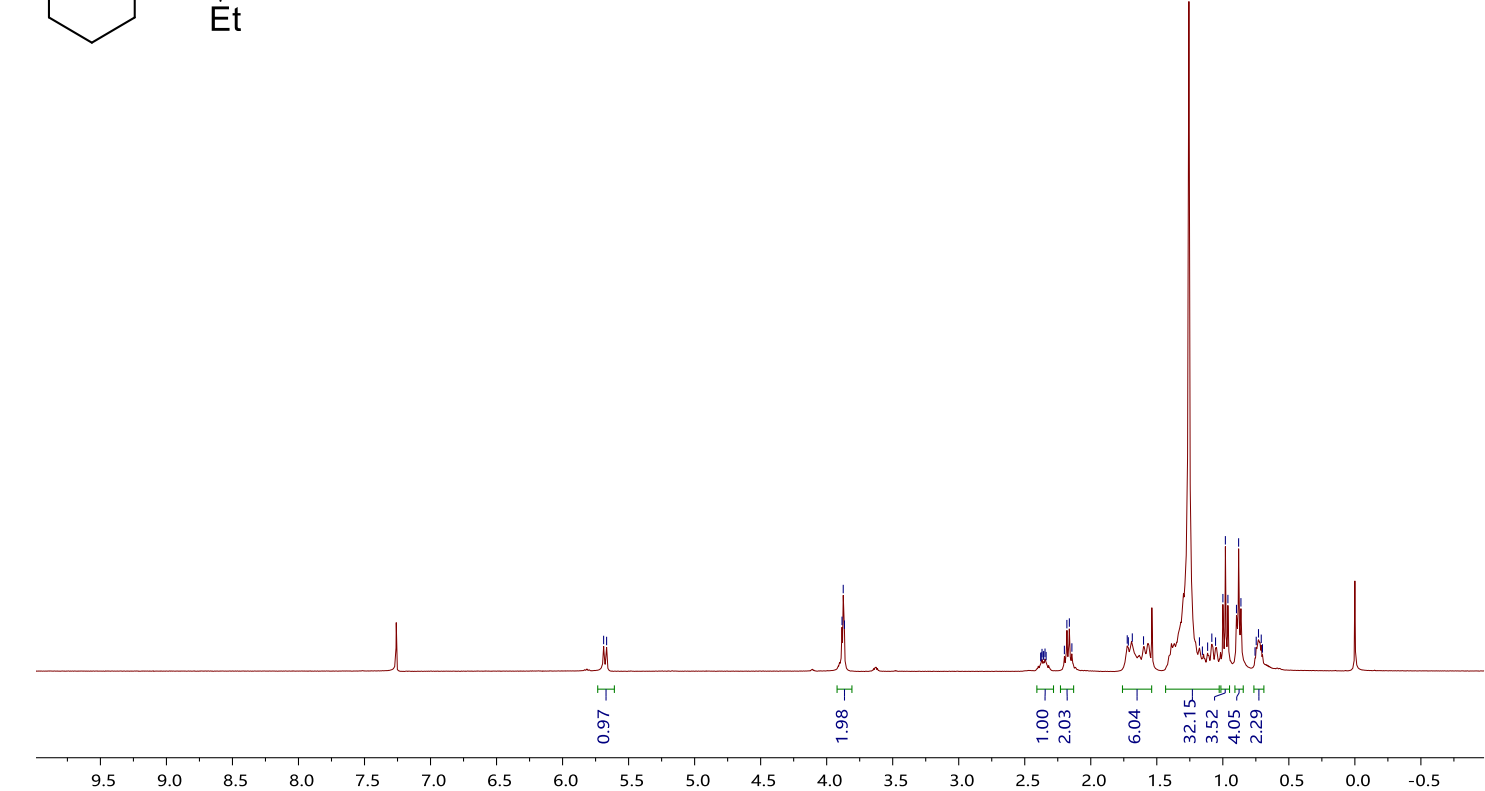

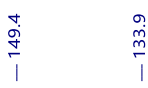

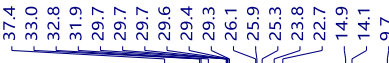

$\overbrace{\mathrm{Et}}^{\mathrm{SiH}_{2} \mathrm{C}_{12} \mathrm{H}_{25}}$ 
(E)-(1-cyclohexylbut-1-en-1-yl)(phenyl)silane (4en)

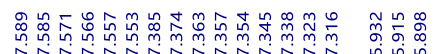

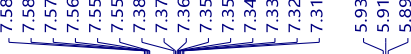

等

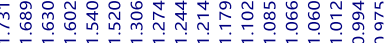<smiles>CC/C=C(/[SH+]c1ccccc1)C1CCCCC1</smiles>

Et

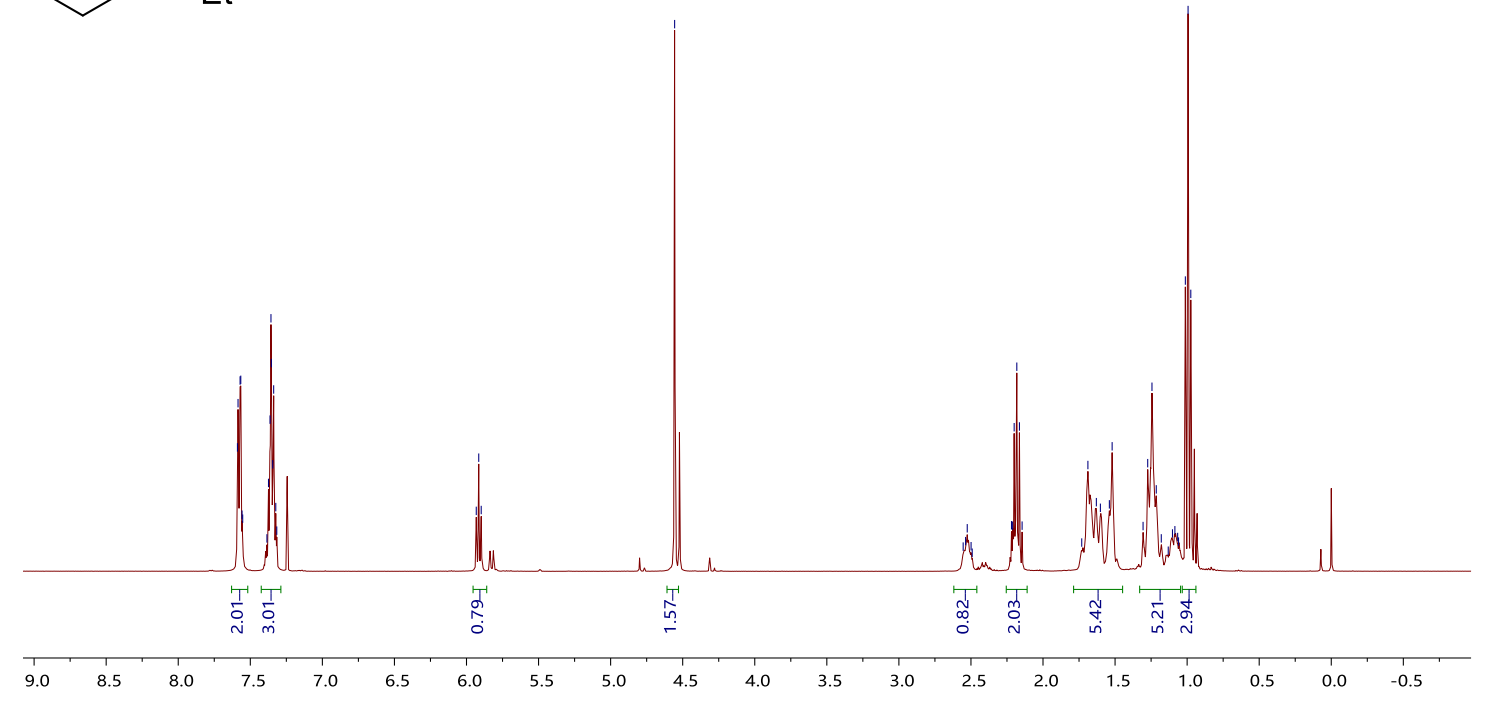

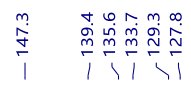

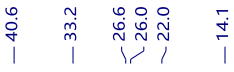<smiles>C/C=C(/[SH+]c1ccccc1)C1CCCCC1</smiles>

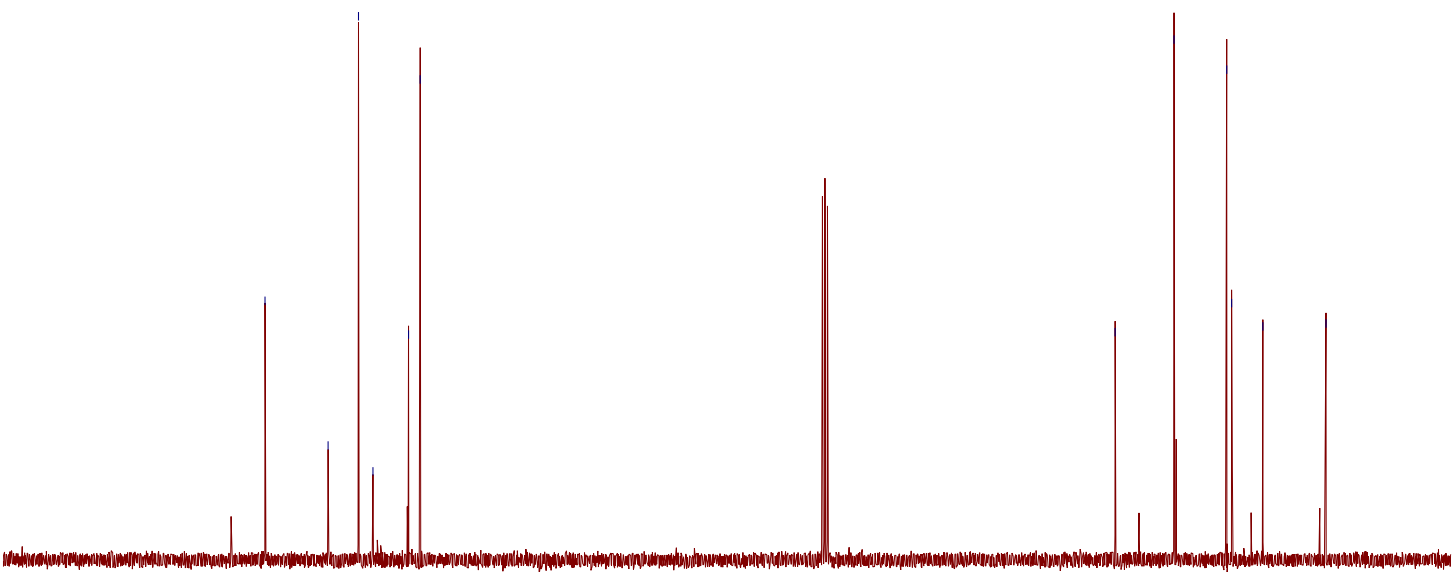

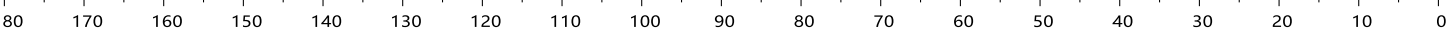


(E)-(2-methyloct-3-en-4-yl)(phenyl)silane (3eo)

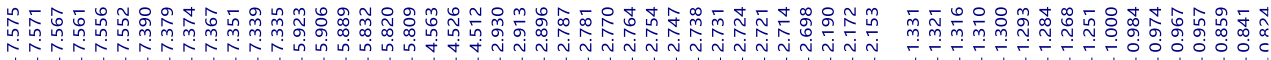<smiles>C=C(C)C=C(CCC)CSc1ccccc1</smiles>

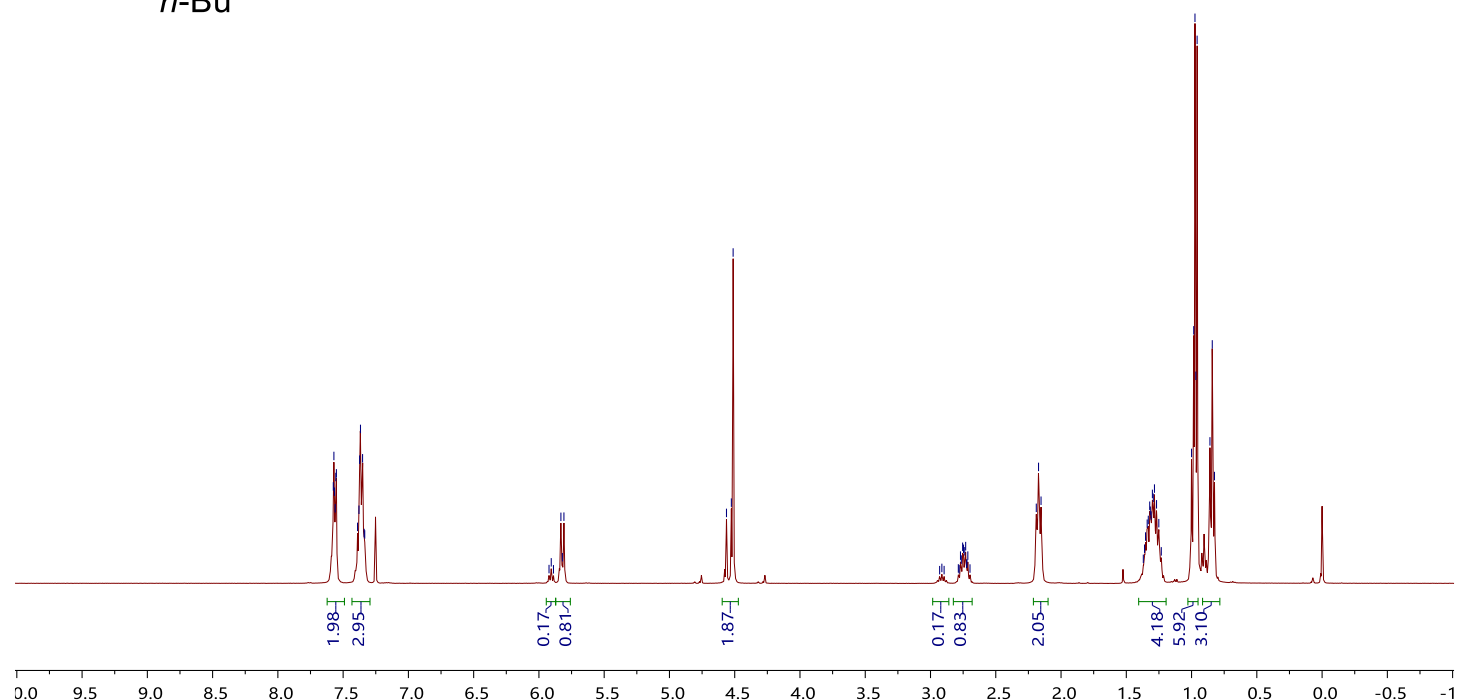

$\underbrace{m}_{\substack{m \\ m}}$

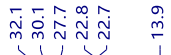<smiles>C=C(C)C=C(CCC)CSc1ccccc1</smiles>

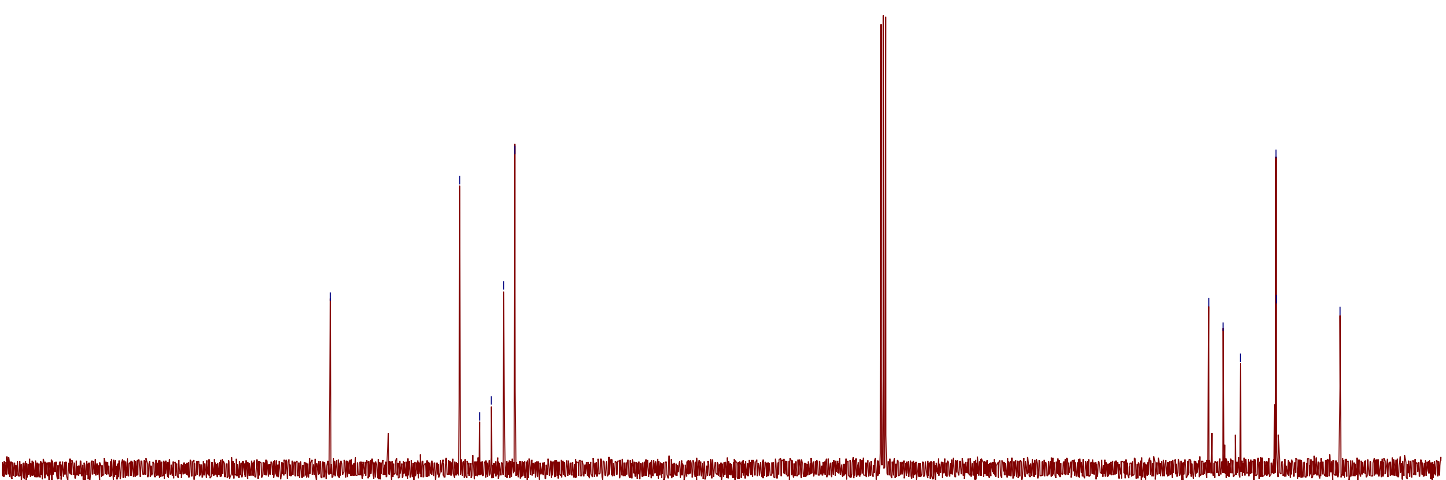




\section{(E)-(2-methyloct-3-en-3-yl)(phenyl)silane (4eo)}

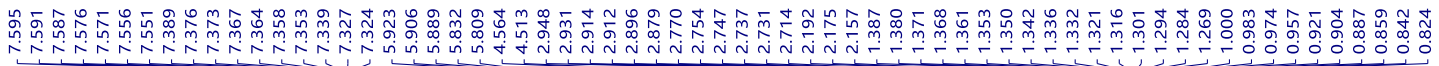<smiles>CCCCC(C)=Cc1ccccc1</smiles>

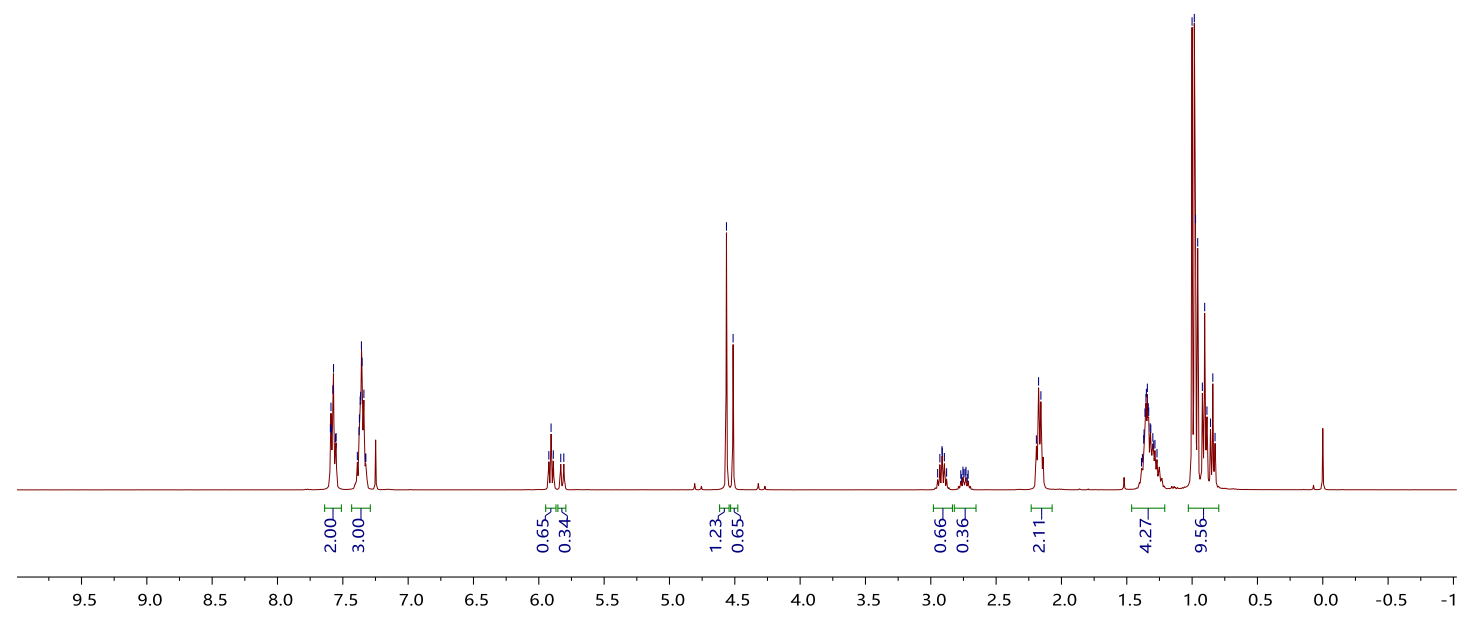

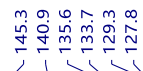

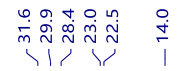<smiles>CCCCCCCCCC(C)CC</smiles>

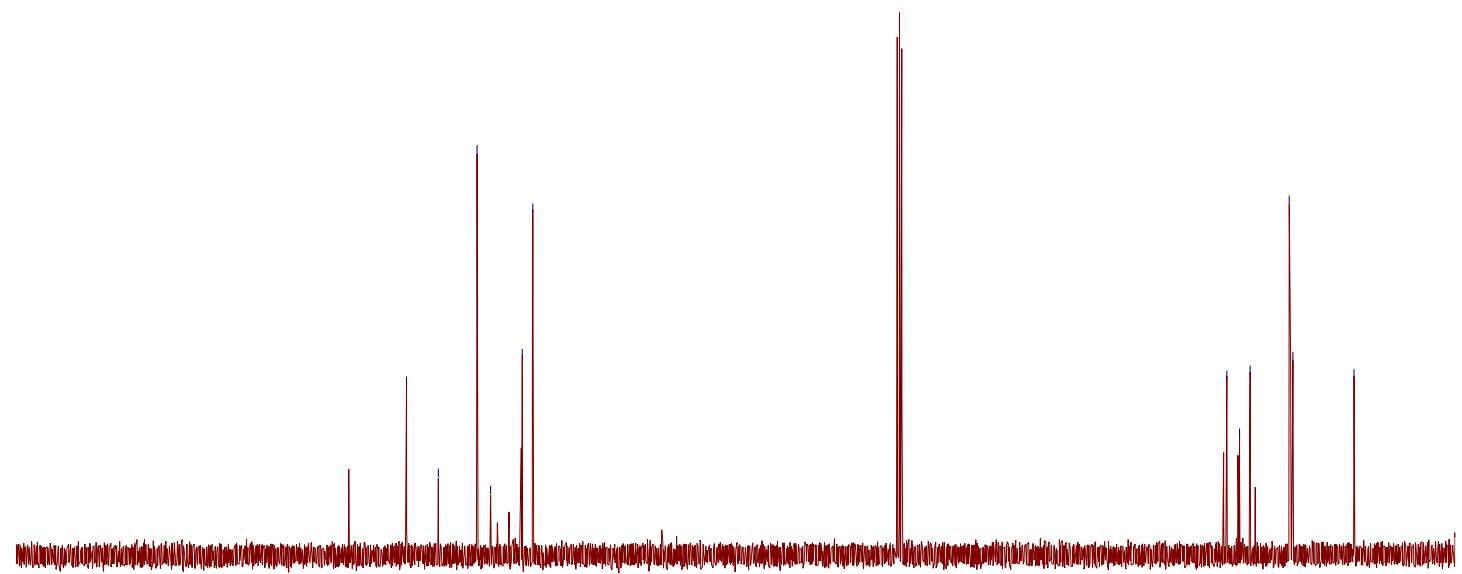

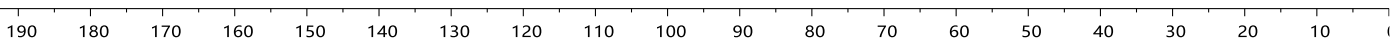




\section{(E)-(oct-1-en-1-yl-2- $d$ ) (phenyl)silane- $d_{2}$ (3ab- $d$ )}

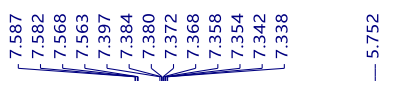

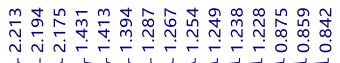

$\mathrm{C}_{6} \mathrm{H}_{13} \overbrace{\mathrm{H}}^{\mathrm{D}} \mathrm{SiD}_{2} \mathrm{Ph}$

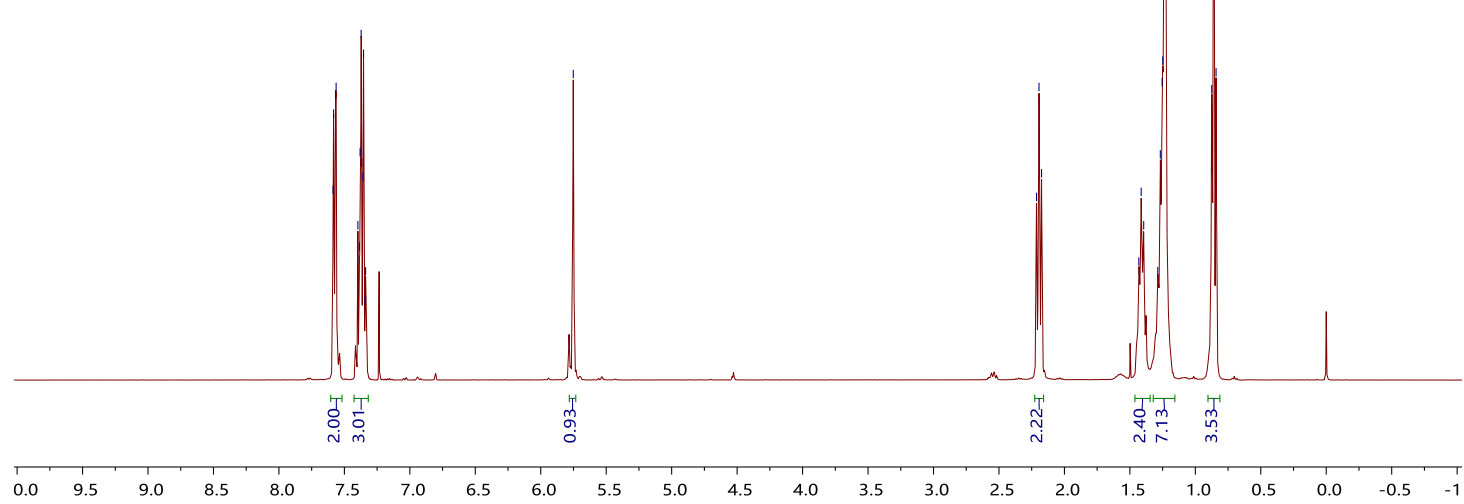

I

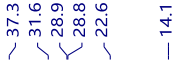<smiles>[2H]/C(=C\c1ccccc1)[SeH]c1ccccc1</smiles>

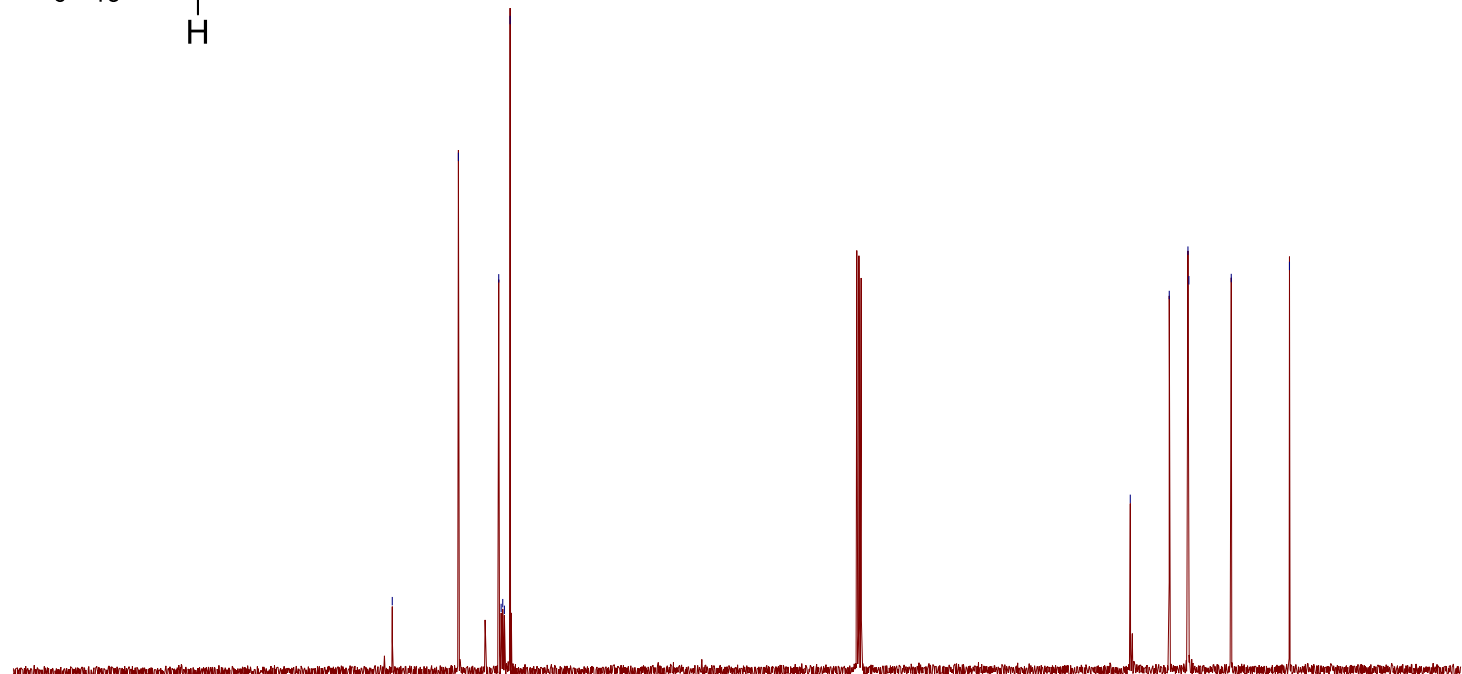

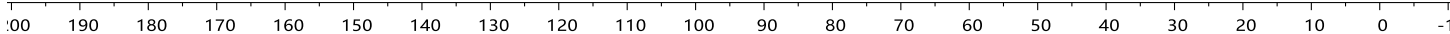


(Z)-(oct-1-en-2-yl-1- $d$ )(phenyl)silane- $d_{2}$ (4ab- $d$ )

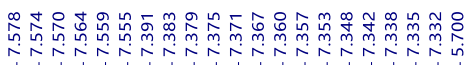

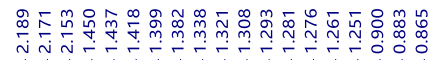

$\mathrm{C}_{6} \mathrm{H}_{13} \overbrace{\mathrm{H}}^{\mathrm{SiD} P h}$

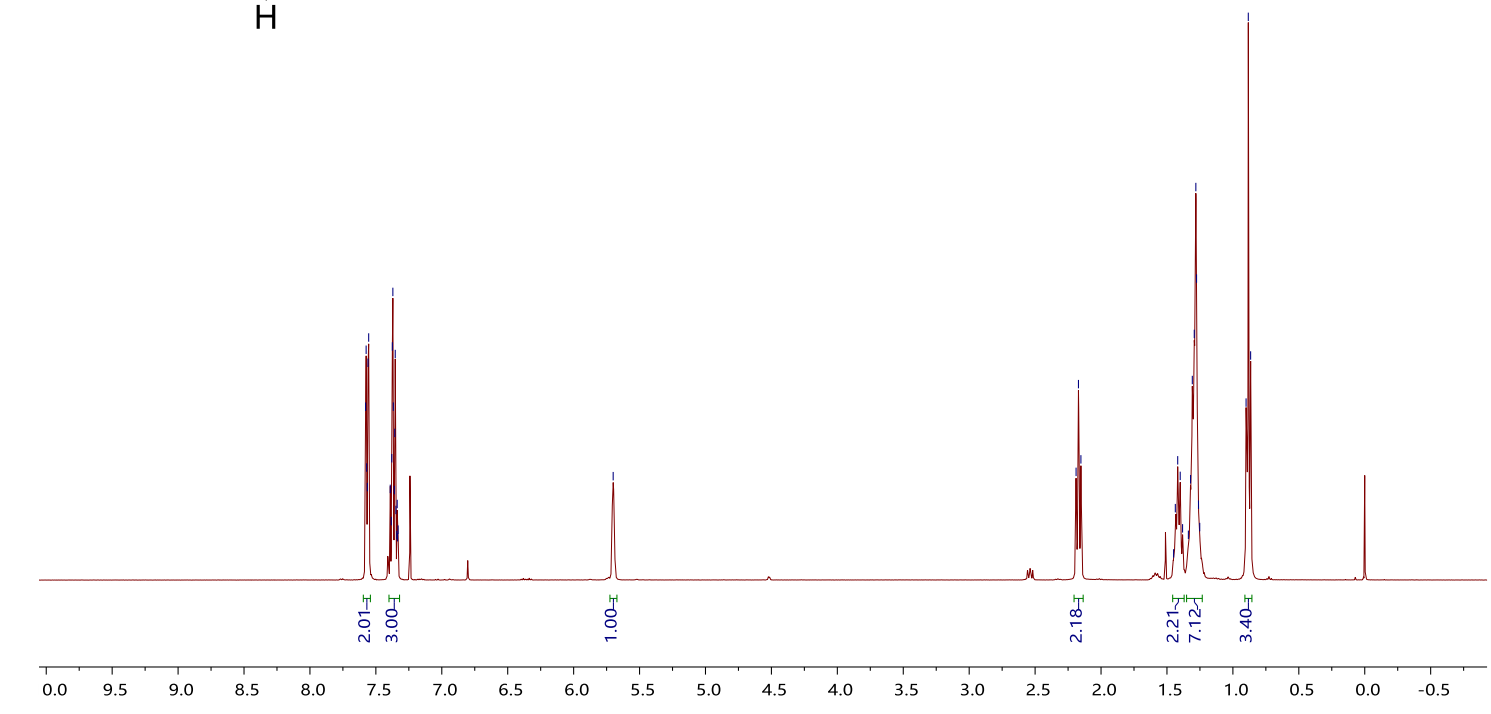

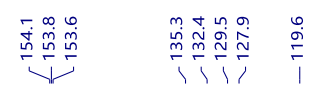

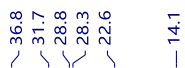

$\mathrm{C}_{6} \mathrm{H}_{13} \overbrace{\mathrm{H}}^{\mathrm{SiD} P h}$

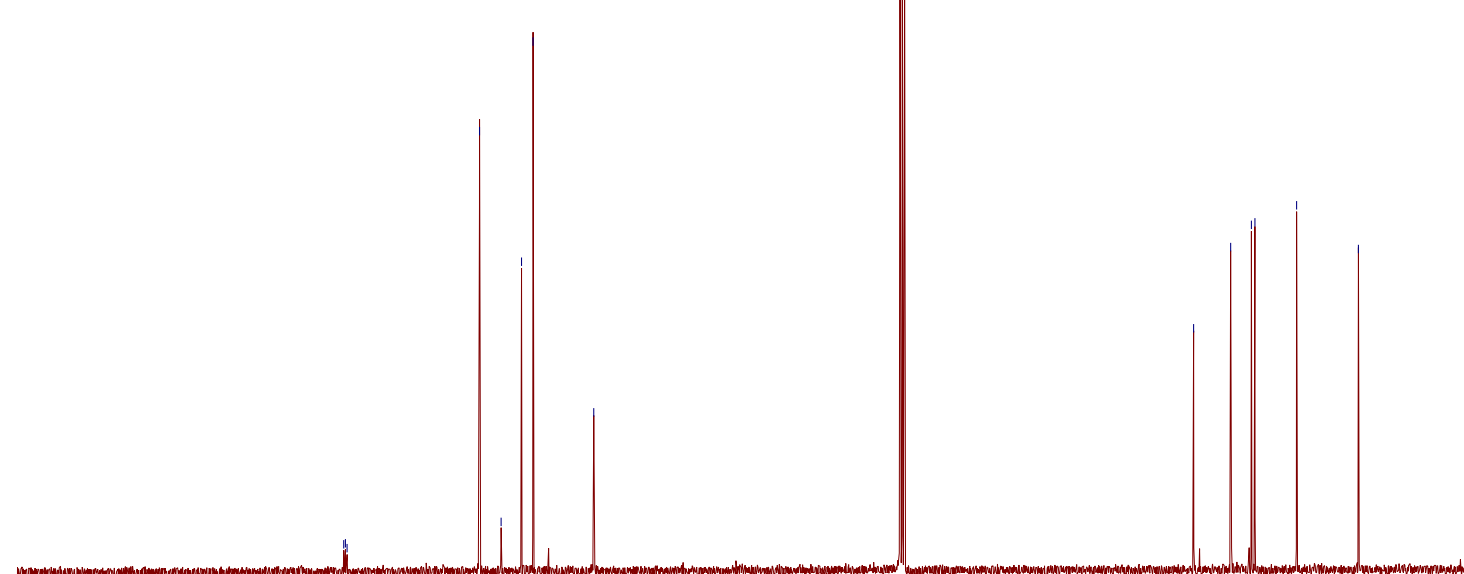

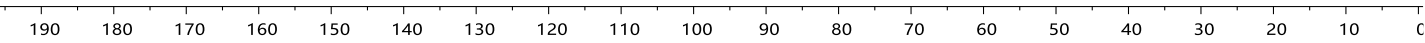




\section{(E)-phenyl(5-phenylpent-1-en-1-yl-1- $d$ ) silane (3aa- $d$-A)}

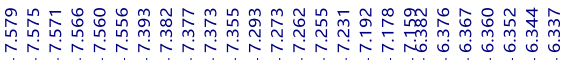

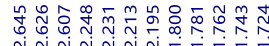

$\overbrace{\mathrm{Ph}\left(\mathrm{H}_{2} \mathrm{C}\right)_{3}}^{\mathrm{H}}=\mathrm{D}_{\mathrm{D}}^{\mathrm{SiH}_{2} \mathrm{Ph}}$
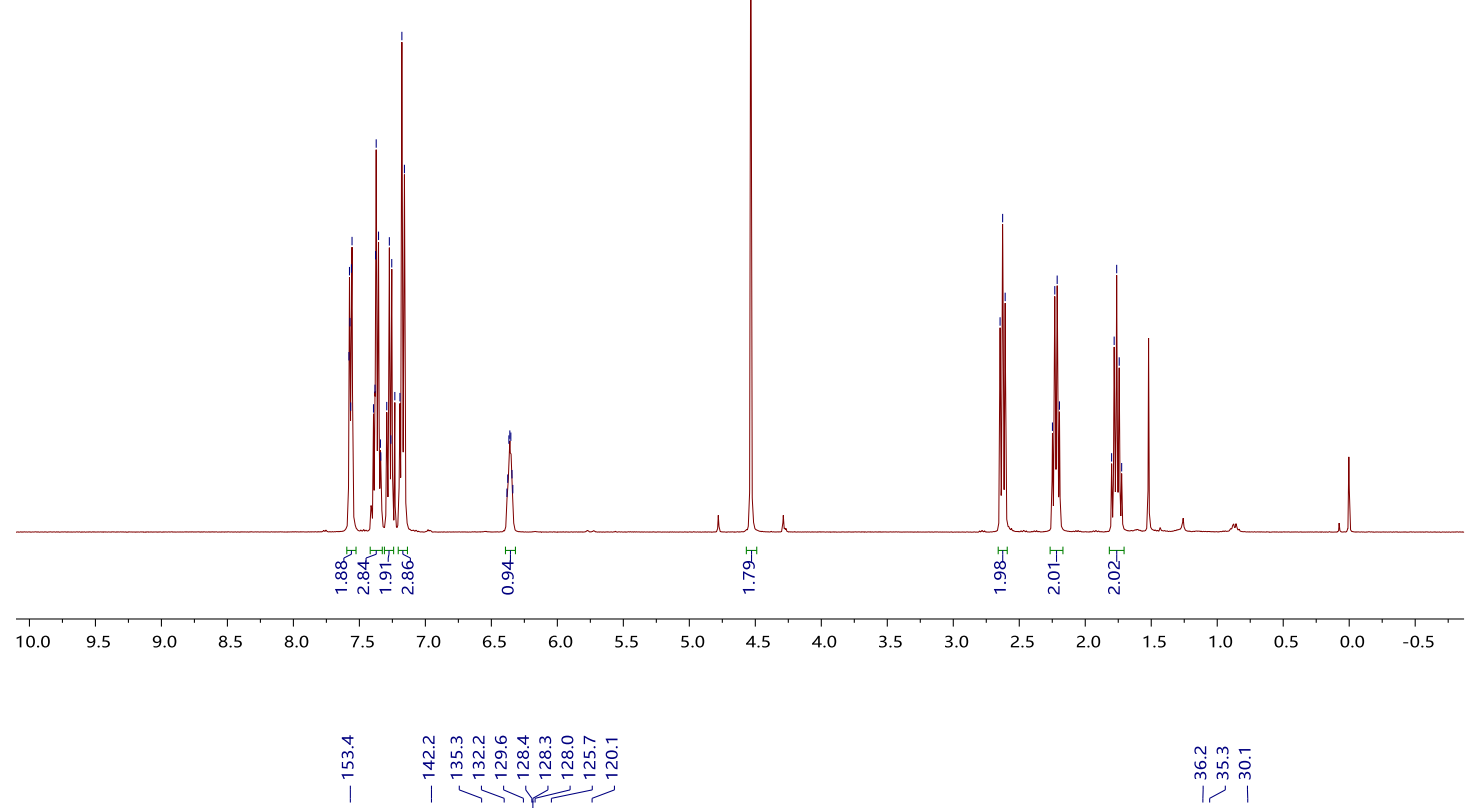<smiles>[2H]/C(=C\CCc1ccccc1)[SiH2]c1ccccc1</smiles>

$\mathrm{Ph}\left(\mathrm{H}_{2} \mathrm{C}\right)_{3}$

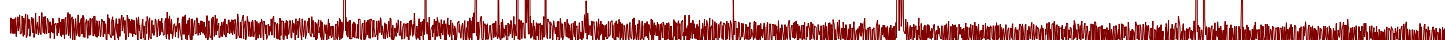

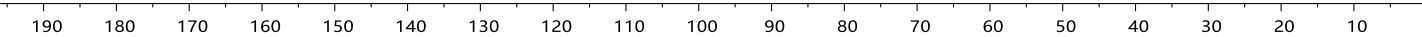




\section{(E)-phenyl(5-phenylpent-1-en-2-yl-1- $d$ ) silane (3aa- $d$-B)}

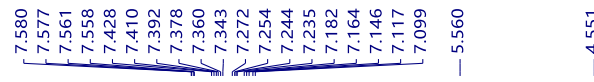

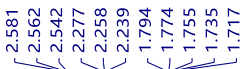

$\underset{\mathrm{Ph}\left(\mathrm{H}_{2} \mathrm{C}\right)_{3}}{\mathrm{PhH}_{2} \mathrm{Si}}=\mathrm{D}_{\mathrm{D}}^{\mathrm{H}}$

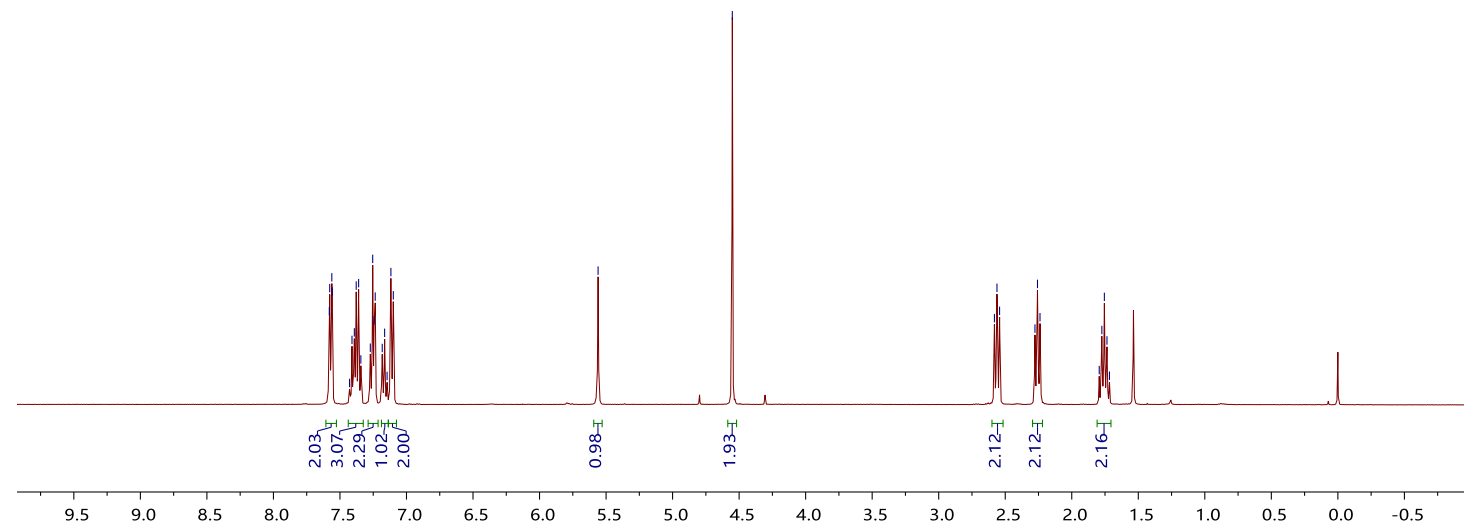

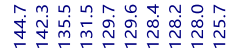

113

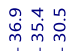

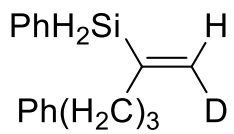

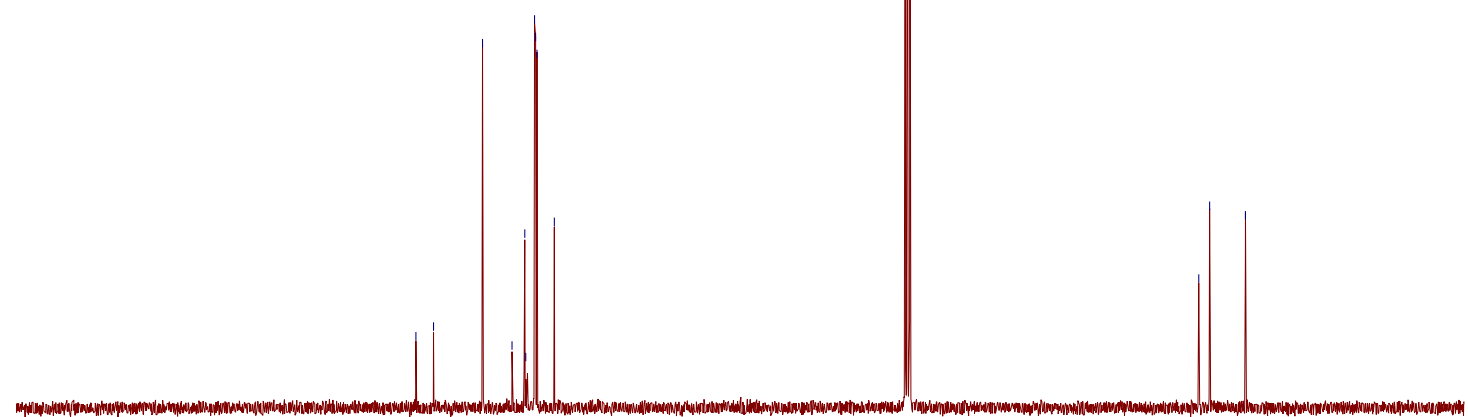

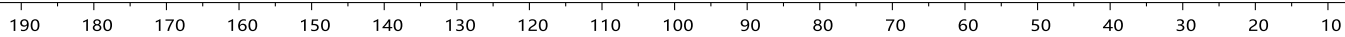




\section{(E)-phenyl(5-phenylpent-1-en-1-yl-2- $d$ ) silane- $d_{2}$ (3aa- $d$-C)}

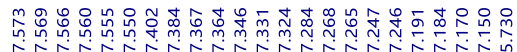

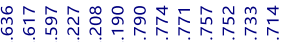

$\mathrm{Ph}\left(\mathrm{H}_{2} \mathrm{C}\right)_{3}{ }_{\mathrm{H}}^{\mathrm{SiD}}$
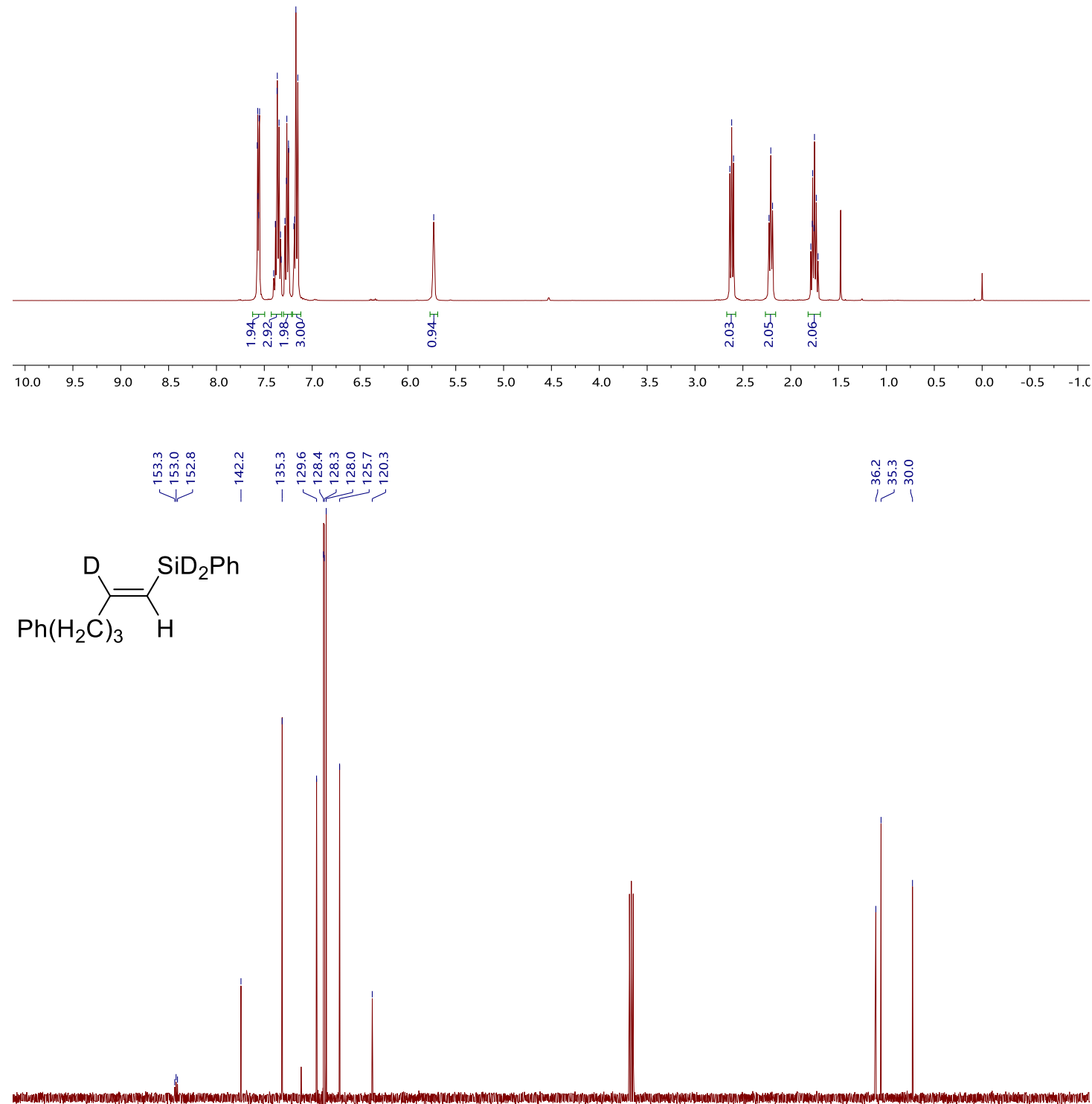

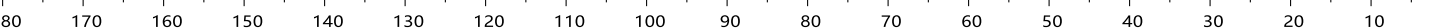




\section{(Z)-phenyl(5-phenylpent-1-en-2-yl-1- $d$ ) silane- $d_{2}$ (3aa- $d$-D)}

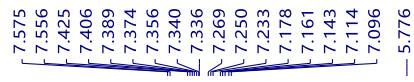

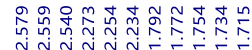

$\underset{P h\left(\mathrm{H}_{2} \mathrm{C}\right)_{3}}{\mathrm{PhD}_{2} \mathrm{Si}}=\stackrel{\mathrm{D}}{\mathrm{D}}$

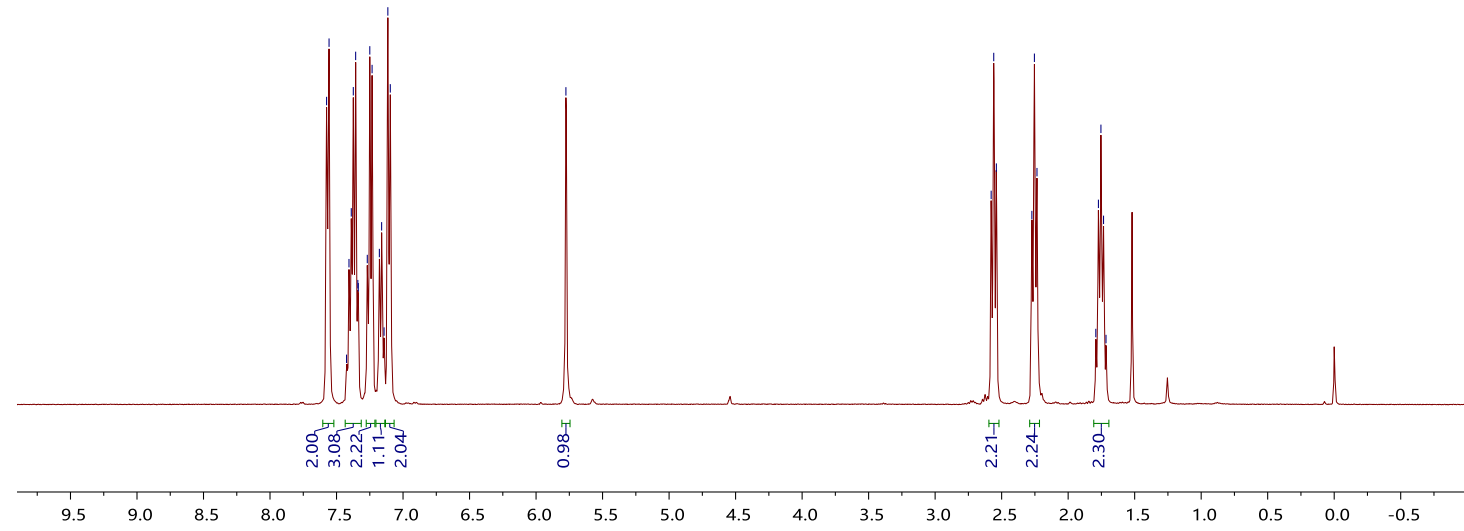

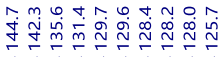

17

${ }_{\mathrm{Ph}\left(\mathrm{H}_{2} \mathrm{C}\right)_{3}}^{\mathrm{PhD}_{2} \mathrm{Si}}=\mathrm{H}_{\mathrm{H}}^{\mathrm{D}}$

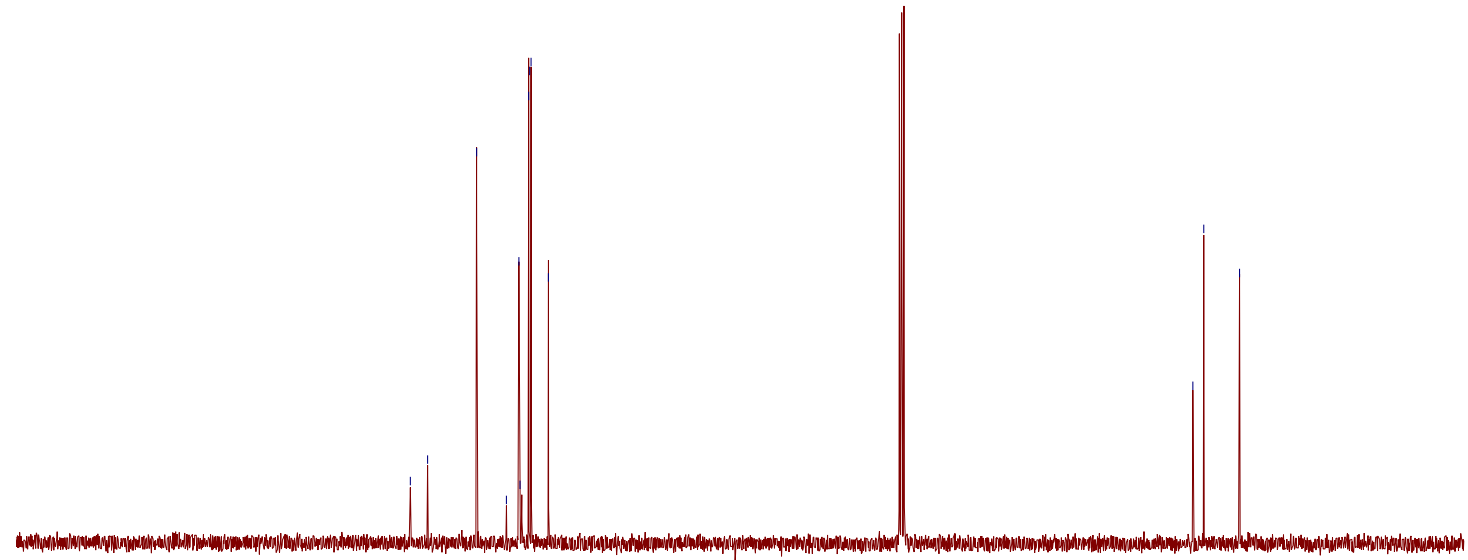

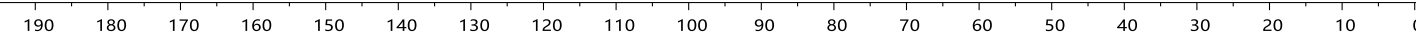




\section{References}

${ }^{1}$ Emerson, C. R.; Zakharov, L. N.; Blakemore, P. R. Investigation of functionalized $\alpha-$ chloroalkyllithiums for a stereospecific reagent-controlled homologation approach to the analgesic alkaloid (-)-epibatidine. Chem. Eur. J. 2013, 19, 16342-16356.

${ }^{2}$ Rivara, M.; Patel, M. K.; Amori, L.; Zuliani, V. Inhibition of $\mathrm{Na}_{\mathrm{v}} 1.6$ sodium channel currents by a novel series of 1,4-disubstituted-triazole derivatives obtained via copper-catalyzed click chemistry. Bioorg. Med. Chem. Lett. 2012, 22, 6401-6404.

3 Yamashige, R.; Kimoto, M.; Takezawa, Y.; Sato, A.; Mitsui, T.; Yokoyama, S.; Hirao, I. Highly specific unnatural base pair systems as a third base pair for PCR amplification. Nucleic Acids Res. 2012, 40, 2793-2806.

${ }^{4}$ Ghani, S. B. A.; Chapman, J. M.; Figadère, B.; Herniman, J. M.; Langley, G. J.; Niemann, S.; Brown, R. C. D. Total synthesis and stereochemical assignment of cisuvariamicin i and cis-reticulatacin. J. Org. Chem. 2009, 74, 6924-6928.

5 Weiss, H. M.; Touchette, K. M.; Angell, S.; Khan, J. The concerted addition of HBr to aryl alkynes; orthogonal pi bond selectivity. Org. Biomol. Chem. 2003, 1, 21522156.

6 Swenson, A. K.; Higgins, K. E.; Brewer, M. G.; Brennessel, W. W.; Coleman, M. G. Highly selective synthesis of tetra-substituted furans and cyclopropenes: copper(I)catalyzed formal cycloadditions of internal aryl alkynes and diazoacetates. Org. Biomol. Chem. 2012, 10, 7483-7486.

7 Presset, M.; Oehlrich, D.; Rombouts, F.; Molander, G. A. Copper-mediated radical trifluoromethylation of unsaturated potassium organotrifluoroborates. J. Org. Chem. 2013, 78, 12837-12843.

8 Park, S.; Malcolmson, S. J. Development and mechanistic investigations of enantioselective Pd-catalyzed intermolecular hydroaminations of internal dienes. ACS. Catal. 2018, 8, 8468-8476.

${ }^{9}$ Liu, Y.-Y.; Yang, X.-H.; Huang, X.-C.; Wei, W.-T.; Song, R.-J.; Li, J.-H. Palladiumcatalyzed methylation of alkynyl $\mathrm{C}(\mathrm{sp})-\mathrm{H}$ bonds with dimethyl sulfonium ylides. $J$. Org. Chem. 2013, 78, 10421-10426.

10 Kuritani, M.; Tashiro, S.; Shionoya, M. Heterodinuclear metal arrangement in a flat macrocycle with two chemically-equivalent metal chelating sites. Inorg. Chem. 2012, $51,1508-1515$.

${ }^{11}$ Hu, M.-Y.; He, Q.; Fan, S.-J.; Wang, Z.-C.; Liu, L.-Y.; Mu, Y.-J.; Peng, Q.; Zhu, S.F. Ligands with 1,10-phenanthroline scaffold for highly regioselective iron-catalyzed alkene hydrosilylation. Nat. Commun. 2018, 9, 221-231.

${ }^{12}$ Hu, M.-Y.; Lian, J.; Sun, W.; Qiao, T.-Z.; Zhu, S.-F. Iron-catalyzed dihydrosilylation of alkynes: efficient access to geminal bis(silanes). J. Am. Chem. Soc. 2019, 141, 4579-4583.

${ }^{13}$ Ding, S.; Song, L.-J.; Chung, L.W.; Zhang, X.-H.; Sun, J.-W.; Wu, Y.-D. Ligand- 
controlled remarkable regio- and stereodivergence in intermolecular hydrosilylation of internal alkynes: experimental and theoretical studies. J. Am. Chem. Soc. 2013, $135,13835-13842$.

${ }^{14}$ Li, R.-H.; An, X.-M.; Yang, Y.; Li, D.-C.; Hu, Z.-L.; Zhan, Z.-P. Highly regio- and stereoselective heterogeneous hydrosilylation of terminal alkynes over cobaltmetalated porous organic polymer. Org. Lett. 2018, 20, 5023-5026.

15 Zong, Z.-J.; Yu, Q.-W.; Sun, N.; Hu, B.-X.; Shen, Z.-L.; Hu, X.-Q.; Jin, L.-Q. Bidentate geometry-constrained iminopyridyl ligands in cobalt catalysis: highly Markovnikov-selective hydrosilylation of alkynes. Org. Lett. 2019, 21, 5767-5772.

$16 \mathrm{Wu}, \mathrm{C}$; Teo, W. J.; Ge, S. Cobalt-catalyzed (E)-selective anti-Markovnikov hydrosilylation of terminal alkynes. ACS Catal. 2018, 8, 5896-5900.

17 Zhang, S.; Ibrahim, J. J.; Yang, Y. An NNN-pincer-cobalt complex catalyzed highly Markovnikov selective alkyne hydrosilylation. Org. Lett. 2018, 20, 6265-6269.

${ }^{18} \mathrm{Wu}$, G.; Chakraborty, U.; von Wangelin, A. J. Regiocontrol in the cobalt-catalyzed hydrosilylation of alkynes. Chem. Commun. 2018, 54, 12322-12325.

19 Dash, A. K.; Gourevich, I.; Wang, J.-Q.; Wang, J.-X.; Kapon, M.; Eisen, M. S. The catalytic effect in opening an organoactinide metal coordination sphere: regioselective dimerization of terminal alkynes and hydrosilylation of alkynes and alkenes with $\mathrm{PhSiH} 3$ promoted by $\mathrm{Me}_{2} \mathrm{SiCp}^{\prime \prime}{ }_{2} \mathrm{Th}^{\mathrm{n}} \mathrm{Bu}_{2}$. Organometallics 2001, 20, 5084-5104.

${ }^{20}$ Lim, D. S. W.; Anderson, E. A. Synthesis of vinylsilanes. Synthesis 2012, 44, 9831010.

${ }^{21}$ Levine, D. S.; Tilley, T. D.; Andersen, R. A. Efficient and selective catalysis for hydrogenation and hydrosilation of alkenes and alkynes with PNP complexes of scandium and yttrium. Chem. Commun. 2017, 53, 11881-11884.

${ }^{22}$ Molander, G. A.; Retsch, W. H. Selective hydrosilylation of alkynes catalyzed by an organoyttrium complex. Organometallics 1995, 14, 4570-4575.

${ }^{23}$ Kapat, A.; Sperger, T.; Guven, S.; Schoenebeck, F. E-olefins through intramolecular radical relocation. Science 2019, 363, 391-396.

${ }^{24}$ See, J. Y.; Yang, H.; Zhao, Y.; Wong, M. W.; Ke, Z.; Yeung, Y.-Y. Desymmetrizing enantio- and diastereoselective selenoetherification through supramolecular catalysis. ACS Catal. 2018, 8, 850-858.

${ }^{25}$ Okada, H.; Mori, T.; Saikawa, Y.; Nakata, M. Formation of $\alpha$-hydroxyketones via irregular Wittig reaction. Tetrahedron Lett. 2009, 50, 1276-1278.

${ }^{26}$ Kang, S.; Han, J.; Lee, E. S.; Choi, E. B.; Lee, H.-K. Enantioselective synthesis of cyclic sulfamidates by using chiral rhodium-catalyzed asymmetric transfer hydrogenation. Org. Lett. 2010, 12, 4184-4187.

${ }^{27}$ Kinoshita, H.; Takahashi, H.; Miura, K. Regioselective synthesis of multisubstituted benzenes by palladium-catalyzed intermolecular reaction of $\beta$-iodo- $\beta$-silylstyrenes with alkynes. Org. Lett. 2013, 15, 2962-2965.

${ }^{28}$ Fang, Z. X.; Liao, P.-C.; Yang, Y.-L.; Yang, F.-L.; Chen, Y.-L.; Lam, Y.; Hua, K.-F.; $\mathrm{Wu}, \mathrm{S}$. Synthesis and biological ev\{alation of polyenylpyrrole derivatives as 
anticancer agents acting through caspases-dependent apoptosis. J. Med. Chem. 2010, $53,7967-7978$.

${ }^{29}$ Liu, D.; Li, X.-M.; Meng, Li.; Li, C.-S.; Gao, S.-S.; Shang, Z.; Proksch, P.; Huang, C.-G.; Wang, B.-G. Nigerapyrones A-H, $\alpha$-pyrone derivatives from the marine mangrove-derived endophytic fungus aspergillus niger MA-132. J. Nat. Prod. 2011, 74, 1787-1791.

30 Frisch, M. J.; Trucks, G. W.; Schlegel, H. B.; Scuseria, G. E.; Robb, M. A.; Cheeseman, J. R.; Scalmani, G.; Barone, V.; Mennucci, B.; Petersson, G. A.; Nakatsuji, H.; Caricato, M.; Li, X.; Hratchian, H. P.; Izmaylov, A. F.; Bloino, J.; Zheng, G.; Sonnenberg, J. L.; Hada, M.; Ehara, M.; Toyota, K.; Fukuda, R.; Hasegawa, J.; Ishida, M.; Nakajima, T.; Honda, Y.; Kitao, O.; Nakai, H.; Vreven, T.; Montgomery J. A., Jr.; Peralta, J. E.; Ogliaro, F.; Bearpark, M.; Heyd, J. J.; Brothers, E.; Kudin, K. N.; Staroverov, V. N.; Kobayashi, R.; Normand, J.; Raghavachari, K.; Rendell, A.; Burant, J. C.; Iyengar, S. S.; Tomasi, J.; Cossi, M.; Rega, N.; Millam, J. M.; Klene, M.; Knox, J. E.; Cross, J. B.; Bakken, V.; Adamo, C.; Jaramillo, J.; Gomperts, R.; Stratmann, R. E.; Yazyev, O.; Austin, A. J.; Cammi, R.; Pomelli, C.; Ochterski, J. W.; Martin, R. L.; Morokuma, K.; Zakrzewski, V. G.; Voth, G. A.; Salvador, P.; Dannenberg, J. J.; Dapprich, S.; Daniels, A. D.; Farkas, Ö.; Foresman, J. B.; Ortiz, J. V.; Cioslowski, J; Fox, D. J. Gaussian 09, Revision D.01, Gaussian, Inc., Wallingford CT, 2009.

${ }^{31}$ Chai, J.-D.; Head-Gordon, M. Long-range corrected hybrid density functionals with damped atom-atom dispersion corrections. Phys. Chem. Chem. Phys. 2008, 10, 6615-6620.

32 Schäfer, A.; Horn, H.; Ahlrichs, R. Fully optimized contracted gaussian basis sets for atoms Li to Kr. J. Chem. Phys. 1992, 97, 2571-2577.

33 Schäfer, A.; Huber, C.; Ahlrichs, R. Fully optimized contracted gaussian basis sets of triple zeta valence quality for atoms Li to Kr. J. Chem. Phys. 1994, 100, 58295835.

34 Weigend, F.; Ahlrichs, R. Balanced basis sets of split valence, triple zeta valence and quadruple zeta valence quality for $\mathrm{H}$ to $\mathrm{Rn}$ : design and assessment of accuracy. Phys. Chem. Chem. Phys. 2005, 7, 3297-3305.

35 Weigend, F. Accurate coulomb-fitting basis sets for H to Rn. Phys. Chem. Chem. Phys. 2006, 8, 1057-1065.

${ }^{36}$ Cossi, M.; Rega, N.; Scalmani, G.; Barone, V. Energies, structures, and electronic properties of molecules in solution with the C-PCM solvation model. J. Comput. Chem. 2003, 24, 669-681.

${ }^{37}$ Barone, V.; Cossi, M. Quantum calculation of molecular energies and energy gradients in solution by a conductor solvent model. J. Phys. Chem. A 1998, 102, 1995-2001.

38 Zhao, Y.; Truhlar, D. G. The M06 suite of density functionals for main group thermochemistry, thermochemical kinetics, noncovalent interactions, excited states, and transition elements: two new functionals and systematic testing of four M06class functionals and 12 other functiona. Theor. Chem. Acc. 2008, 120, 215-241. 
39 Marenich, A. V.; Cramer, C. J.; Truhlar, D. G. Universal solvation model based on solute electron density and on a continuum model of the solvent defined by the bulk dielectric constant and atomic surface tensions. J. Phys. Chem. B 2009, 113, 63786396.

40 Hratchian, H. P.; Schlegel, H. B. Accurate reaction paths using a hessian based predictor-corrector integrator. J. Chem. Phys. 2004, 120, 9918-9924.

${ }^{41}$ Hratchian, H. P.; Schlegel, H. B. Using Hessian updating to increase the efficiency of a Hessian based predictorcorrector reaction path following method. J. Chem. Theory Comput. 2005, 1, 61-69.

42 Legault, C. Y. CYLview, 1.0b (Université de Sherbrooke, 2009); http://www.cylview.org. 